\title{
民USGS
}

science for a changing world

\section{PRELIMINARY COMPILATION OF DATA FOR SELECTED OIL TEST WELLS IN NORTHERN CALIFORNIA}

By

EARL E. BRABB, CHARLES POWELL, II, and THOMAS M. BROCHER U.S. Geological Survey, 345 Middlefield Rd., Menlo Park, CA 94025

Open-file Report 01-152

This report is preliminary and has not been reviewed for conformity with U.S. Geological Survey editorial standards or with the North American Stratigraphic Code. Any use of trade, product, or firm names is for descriptive purpose only and does not imply endorsement by the U.S. Government

U.S. DEPARTMENT OF THE INTERIOR

U.S. GEOLOGICAL SURVEY 


\section{INTRODUCTION}

Oil test wells can provide information on the depth, age, inclination, porosity, permeability, density, faulting, folding, and organic content of geologic formations mapped at the surface, or on units not recognized in surface outcrops. Formation density, as expressed in sonic and density logs commonly obtained when wells are drilled, has become increasingly important in making a crustal-scale 3-D seismic velocity model for the San Francisco Bay region. This model will be used for the calculation of realistic strong-ground motion synthetic seismograms (Brocher and others, 1997), and to determine the geometry of the basement surface beneath Tertiary basins (Jachens and others, 1997). The availability of this density and other information for oil test wells has, until recently, been restricted for competitive reasons, but several petroleum companies have recently made these data available. Accordingly, we began in 1992 to obtain these data to help prepare new geologic maps and geophysical models for the San Francisco Bay region, and to share the information with the public.

\section{AREA COVERED}

This report contains brief descriptions of information and materials available for 1,550 oil exploration and production wells in the following counties: Alameda (42), Butte (31), Colusa (103), Contra Costa (102), Glenn (103), Humboldt (33), Marin (6), Mendocino (2), Merced (33), Monterey (172), Napa (5), Placer (2), Sacramento (72), San Benito (51), San Joaquin (164), San Mateo (73), Santa Clara (8), Santa Cruz (23), Shasta (3), Siskiyou (1), Solano (251), Sonoma (10), Stanislaus (29), Sutter (59), Tehama (59), and Yolo (113).

\section{TYPES OF DATA}

We have been most interested in wells that have sonic and density logs, depths to the various geologic formations, information on lithology, the names, character, and diversity of microfossils, the original slides or vials with foraminifers or nannoplankton, and paleo logs that indicate the character of the microfossils at various depths. Where available, we have also collected electrical logs (E logs) and mud logs, which show the drilling rate, visual porosity, lithology, oil and gas content, physical properties and chemistry of the drilling mud, and a description of the cuttings. In the database, density logs are included in the space allocated for sonic logs.

The most useful paleo logs (shortened from paleontology logs) have an electrical $\log$ on the left side, formation names and biostratigraphic stages, zones, and ages in the middle, and a description of fossils and unusual minerals in a column on the right. Many paleo logs also have information that was not transferred to this summary, such as strike and dips of bedding and the character of other fossils from the well.

Many slides with foraminifers (shortened to forams for brevity throughout this report) from cores and cuttings were received with no accompanying data. Presumably data for these wells are available from Jennings and Hart (1956), the 
California Division of Oil and Gas, published reports in libraries, or from commercial oil-well data companies.

Nearly every slide has 3 circular compartments for forams. Most of these compartments have only loose forams, but a few picked and mounted material. Vials contain mainly loose forams, silt, and sand grains.

Many more wells are available than shown in this list. Wells drilled prior to 1950 were generally omitted if the data are sparse. Conversely, some well files are enormous, occupying several feet of shelf space in an oil company library, but we copied only the data of most interest to our projects.

\section{ARRANGEMENT OF THE DATA IN THIS REPORT}

Within each county, the data are arranged alphabetically by lease name followed by the name of the company responsible for the well. Because both names may have changed over time, we have provided former names when known. The exceptions are Chevron, formerly Standard; Arco, formerly Richfield ; Exxon, formerly Humble; Texaco, formerly The Texas Co.; and Unocal, formerly Union.

The section, township and range are from the data provided, and were checked only for wells of interest to our projects. The number provided at the beginning of the geology notes section is a company reference number for the well. The rest of the information in the geology notes was taken mainly from data obtained from the various oil companies.

\section{PROBLEMS WITH THE DATA}

Almost every entry in our report may have errors, even such things as section, township, range, county, quadrangle name, and drilling depth. The depths and ages given for formations are particularly subjective. Few of these formations are defined in accordance with the North American Stratigraphic code, and their position in a well may be defined by correlation with nearby wells, by paleontologic or mineral content, by geochemical or geophysical character, or by thermal maturation. Zone and stage assignments generally follow those of Laiming (1943), Goudkoff (1945), Kleinpell (1938) and Mallory (1959), but Poore (1980), Almgren (1986), Almgren and others (1988), and several others have pointed out major difficulties in using these stratigraphic standards. Moreover, some oil company micropaleontologists have not made rigorous distinctions between paleontologic and lithologic terms. The use of the term "Capay", for example, could mean a rock correlative with coarse clastic rocks in the Capay's type area but it is almost certainly a faunal term for a shale with Eocene faunas correlative with the $\mathrm{C}$ zone of Laiming. If an electrical log is provided, the lithology can be checked, but many paleo logs do not have electrical logs.

Many of the ages on the paleo logs are obsolete. The Paleocene was not recognized in California until the late 1930's, and the zone assignments have also changed. We have attempted to update this information, so that the data in our report do not correspond exactly with the original data. Similarly, we have inferred lithologic information for some names when the lithology is not provided in the data, such as shale for the Capay and sandstone for the Markley. 
The Domengine, however, may mean a glauconitic sandstone, a white sandstone, or a sandstone and shale sequence. An electrical log is very helpful in checking names used by paleontologists.

The quality of the foram faunas (sparse, fairly good, and good) reflects a casual look at the list of forams on the well log. The quality is affected by the spacing of the washed samples, commonly 20 feet but as large as thousands of feet, or the samples may never have been washed. Quality will also be affected by washing techniques, character of preservation, and how the samples were obtained. Some companies for example, will only loan or give incomplete or poorer parts of their collection to another company. Core samples generally yield better preserved specimens than ditch samples.

Reworking of forams into younger formations and caving of forams into older formations is apparently common in many wells. Lab contamination is also common. Hopefully, the paleontologist will make comments on the paleo log about these occurrences.

The slides may be numbered consecutively, but the shallowest and deepest samples may not be in order. Moreover, the numbers on the slide may be interspersed with letters, so that the highest number may not be the total number of slides. Some slides are marked with depths greater than the total depth given for the well.

The problem of correlating between written data and foram slides is especially difficult because the foram slides provide the original names and the written data the newer names, and neither may have any of the same names. Several wells have the same lease name but different company names, and the data may be insufficient to determine if the wells are the same or different.

For all of these reasons, our report should not be used as an authoritative source, but only as a general guide to the kind of information available in selected company files.

\section{ACKNOWLEDGEMENTS}

We are most grateful to the paleontologists, geologists, and managers with Chevron, Unocal, and Exxon, who provided most of the materials for this report, or allowed us access to their files. We are also grateful to personnel in Texaco, Arco and Shell for their contributions. Richard Stanley, U. S. Geological Survey, Richard Thorup and Alvin Almgren, consultants, and Joseph Clark, Indiana University of Pennsylvania, kindly donated well logs and other materials for our project. Bette Hamachi organized the files several years ago, and Louise Dickerson made an early draft of information available from a published report by Jennings and Hart (1956). U. S. Geological Survey volunteers John Parker, Larry Dickerson (deceased), William West, Karen Wild, Purty Dorn, Sonoko Migitaka, Domenique Granier, and others helped in many ways.

\section{REFERENCES}

Almgren, A. A., 1986, Benthic foraminiferal zonation and correlations of Upper Cretaceous strata of the Great Valley of California, in Abbot, P. L.. ed., Cretaceous stratigraphy, western North America: Society of Economic Paleontologists and Mineralogists, Pacific Section, p. 137-152. 
Almgren, A. A., Filewicz, M. V., and Heitman, H. L., 1988, Lower Tertiary foraminiferal and calcareous nannoplankton zonation of California, and overview and rekcommendation, in Fileewicz, M. V., and Squires, R. L., eds., Paleogene stratigraphy, West Coast of North America: Society of Economic Paleontologists and Mineralogists, Pacific Section, p. 83-105.

Brocher, T. M., and others, 1997, A crustal-scale 3-D seismic velocity model for the San Francisco Bay area, California: American Geophysical Union Fall Meeting 1997, abstract S12B-3.

Goudkoff, P. P., 1945, Stratigraphic relations of Upper cretaceous in Great Valley, California: American Association of Petroleum Geologists Bulletin, v. 29 , p. $956-1007$.

Jachens, R. C., and others, 1997, the basement interface: San Francisco Bay area, 3-D seismic velocity model: American Geophysical Union Fall Meeting 1997, abstract S12B-4.

Jennings, C. W., and Hart, E. W., 1956, Exploratory wells drilled outside of oil and gas fields in California to December 1953: California Division of Mines and Geology Special Report 45, 104p.

Kleinpell, R. M., 1938, Miocene stratigraphy of California: American Association of Petroleum Geologists, Tulsa. Oklahoma, $450 \mathrm{p}$.

Laiming, Boris, 1940, Foraminiferal correlations in Eocene of San Joaquin Valley, California: American Association of Petroleum Geologists Bulletin, v. 24, p. 1923-1939.

Mallory, V. S., 1959, Lower Tertiary stratigraphy of the California Coast Ranges: American Association of Petroleum Geologists, Tulsa, Oklahoma, 416p.

Poore, R. Z., 1980, Age and correlation of California Paleogene benthic foraminiferal stages: U. S. Geological Survey Professional Paper 1162-C, 8p. 
SELECTED OIL TEST WELLS IN CENTRAL CALIFORNIA DATABASE County

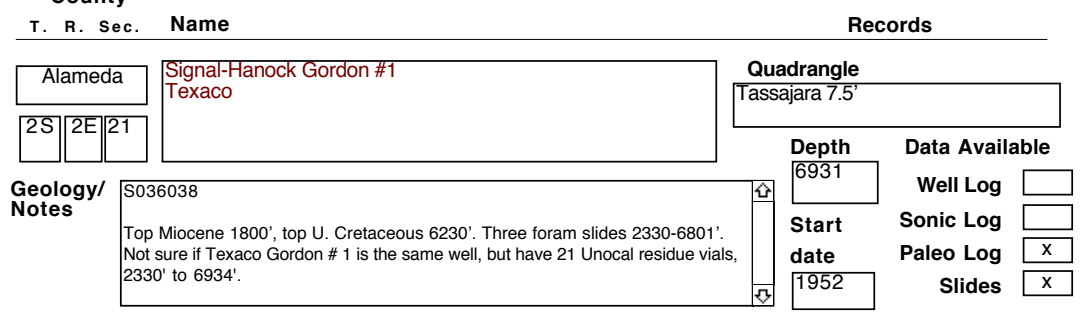

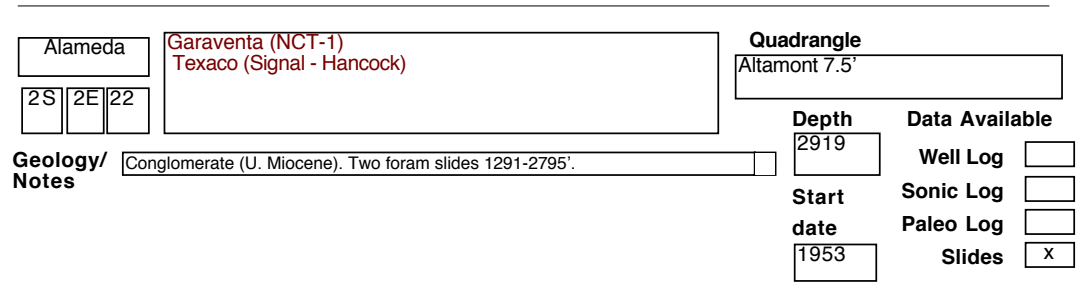

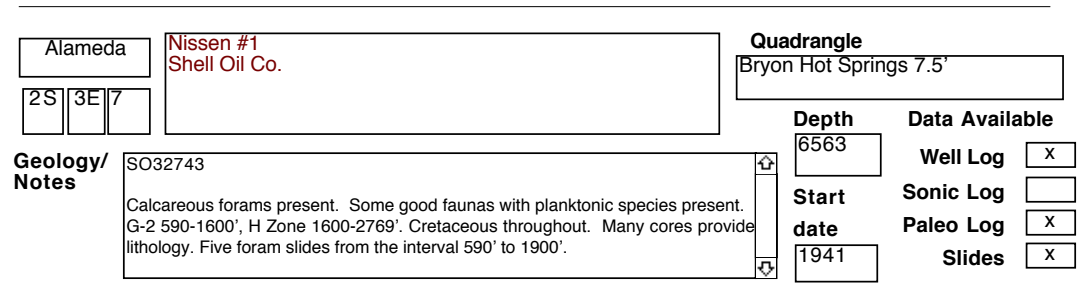

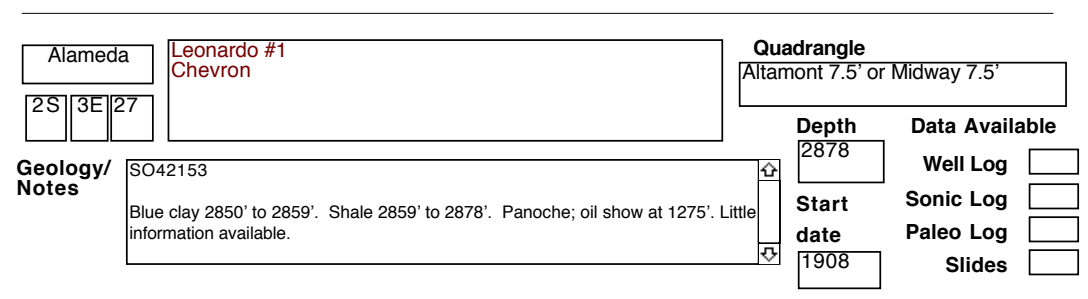

\begin{tabular}{l|l|l|l|l|l|}
\hline Alameda & $\begin{array}{l}\text { Wagoner \#1 } \\
\text { Texaco }\end{array}$ & \multicolumn{2}{|c|}{$\begin{array}{l}\text { Quadrangle } \\
\text { Livermore 7.5' }\end{array}$} \\
\hline
\end{tabular}


SELECTED OIL TEST WELLS IN CENTRAL CALIFORNIA DATABASE County

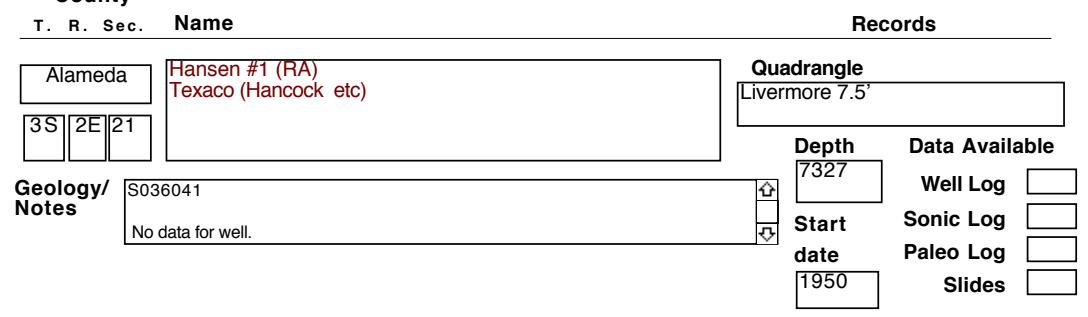

\begin{tabular}{|c|c|c|c|c|c|}
\hline Alameda & \multirow[t]{2}{*}{$\begin{array}{l}\text { Wente (NCT-1) \#1 } \\
\text { Texaco (Hancock etc) }\end{array}$} & \multicolumn{4}{|c|}{$\begin{array}{r}\text { Quadrangle } \\
\text { Altamont 7.5' }\end{array}$} \\
\hline \begin{tabular}{|l|l|l|}
$3 \mathrm{~S}$ & $2 \mathrm{E}$ & 27 \\
\end{tabular} & & & \multirow{2}{*}{$\begin{array}{l}\text { Depth } \\
7062\end{array}$} & \multicolumn{2}{|c|}{ Data Available } \\
\hline \multirow{2}{*}{$\begin{array}{l}\text { Geology/ } \\
\text { Notes }\end{array}$} & & ڤ & & Well Log & $\mathrm{X}$ \\
\hline & $\begin{array}{l}\text { covery well of Hospital Nose Field; } 350 \mathrm{mcf} \text { gas/day from } 5057 \text { '-5180 'and } \\
\text { 0'-5280' within Wente Sd. Cores and sidewall samples taken. Paleo mostly } \\
\text { ren except for Upper Cret. Bolivina incrassasa Zone fauna at } 5010 \text { '. Three } \\
\text { m slides 3364-5680'. }\end{array}$ & & $\begin{array}{l}\begin{array}{l}\text { Start } \\
\text { date }\end{array} \\
1952\end{array}$ & $\begin{array}{r}\text { Sonic Log } \\
\text { Paleo Log } \\
\text { Slides }\end{array}$ & $\mathrm{x}$ \\
\hline
\end{tabular}

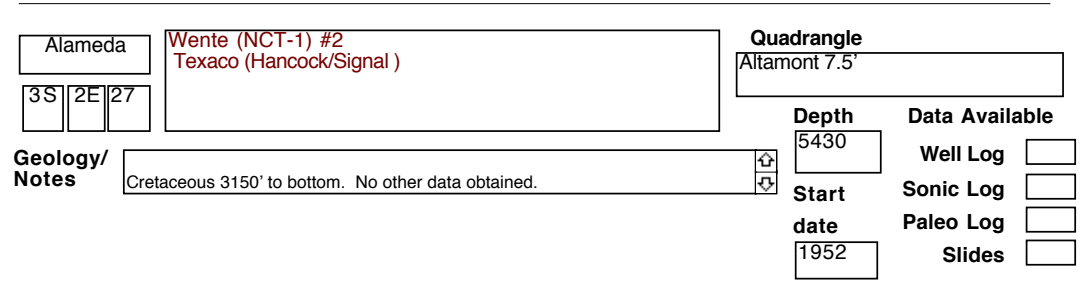

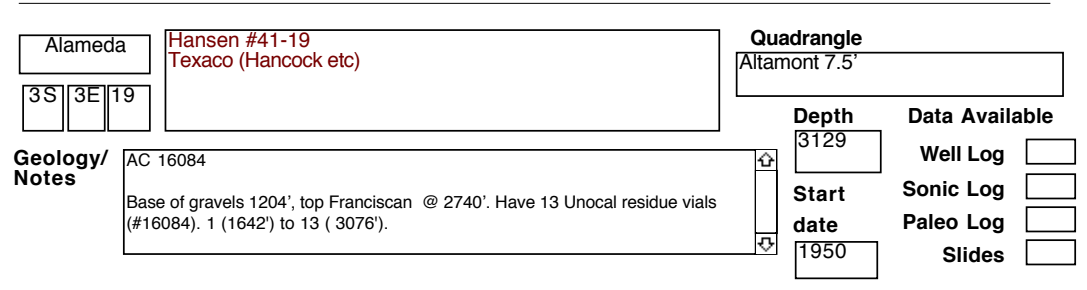

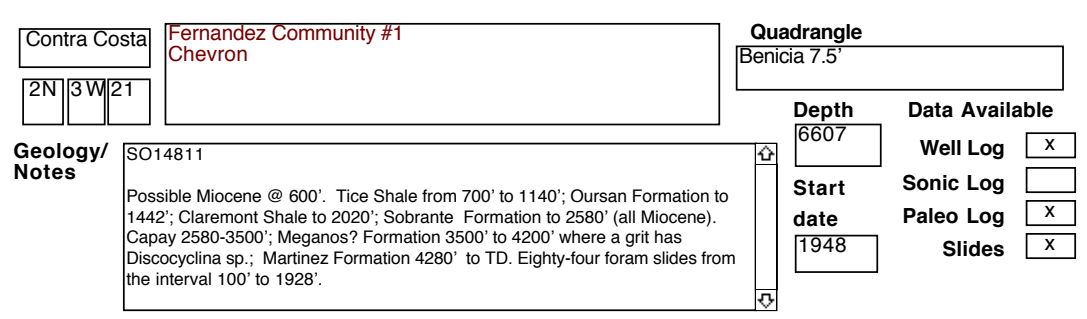




\section{SELECTED OIL TEST WELLS IN CENTRAL CALIFORNIA DATABASE}

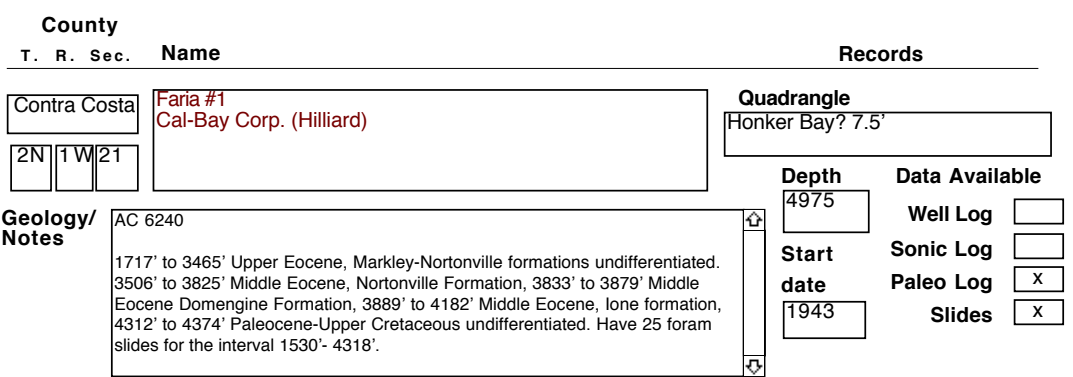

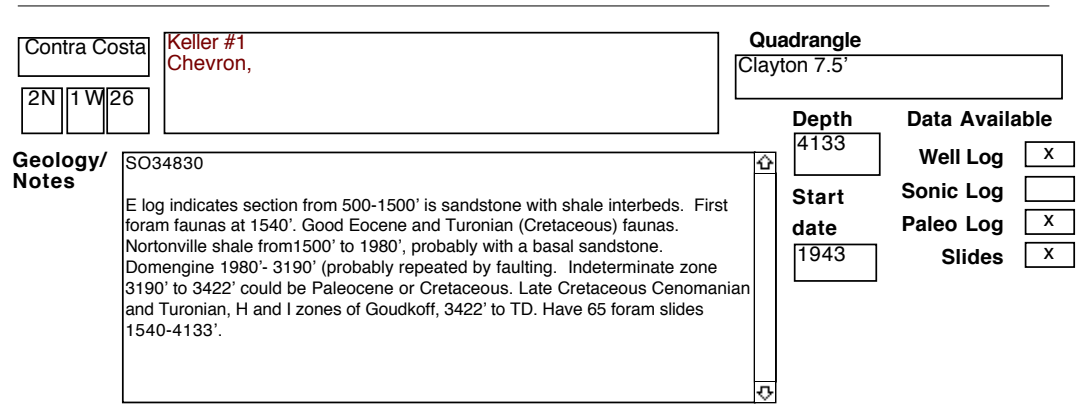

\begin{tabular}{|c|c|c|c|c|}
\hline Contra Costa & \multirow[t]{2}{*}{$\begin{array}{l}\text { Porter Sesnon \#1 } \\
\text { Chevron (Gulf) }\end{array}$} & \multicolumn{3}{|c|}{$\begin{array}{c}\text { Quadrangle } \\
\text { Brentwood 7.5' }\end{array}$} \\
\hline \begin{tabular}{|l|l|l|}
$2 \mathrm{~N}$ & $2 \mathrm{E}$ & 25 \\
\end{tabular} & & Depth & Data Availab & \\
\hline $\begin{array}{l}\text { Geology/ } \\
\text { Notes }\end{array}$ & $\begin{array}{l}\text { S034067 } \\
\text { Base of late Eocene A-1 zone Markley sandstone 4510'. Nortonville shale } \\
\text { 4510-4980'. Domengine green (probably glauconitic) sandstone 4980-5230'. } \\
\text { Domengine white sandstone } 5230-5670 \text { '. Capay shale of early Eocene age } \\
5670-6360 \text { ' has common to abundant planktic forams. Top of Paleocene ' } 2 \text { zone } \\
\text { Maganos } 6360 \text { ' extends indefinite distance downward to } 8730 \text { ' where first } \\
\text { definite Cretaceous faunas were found. Part of this interval may be Martinez } \\
\text { formation, Paleocene. Cretaceous shale 8730'-TD. Have mud log. }\end{array}$ & $\begin{array}{l}\text { Start } \\
\text { date }\end{array}$ & $\begin{array}{r}\text { Well Log } \\
\text { Sonic Log } \\
\text { Paleo Log } \\
\text { Slides }\end{array}$ & $\begin{array}{l}x \\
x \\
\end{array}$ \\
\hline
\end{tabular}

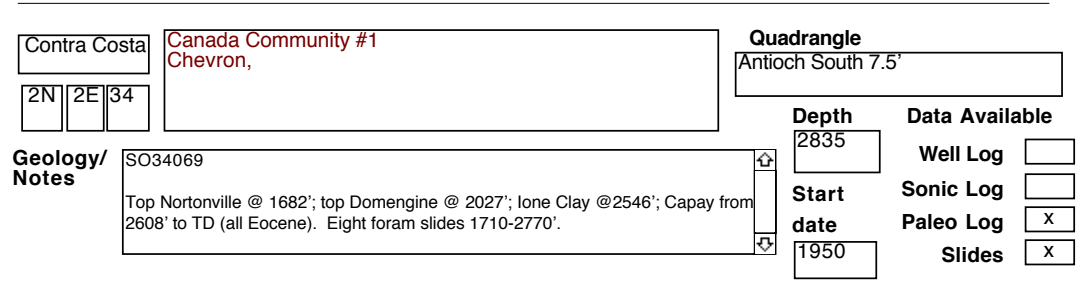

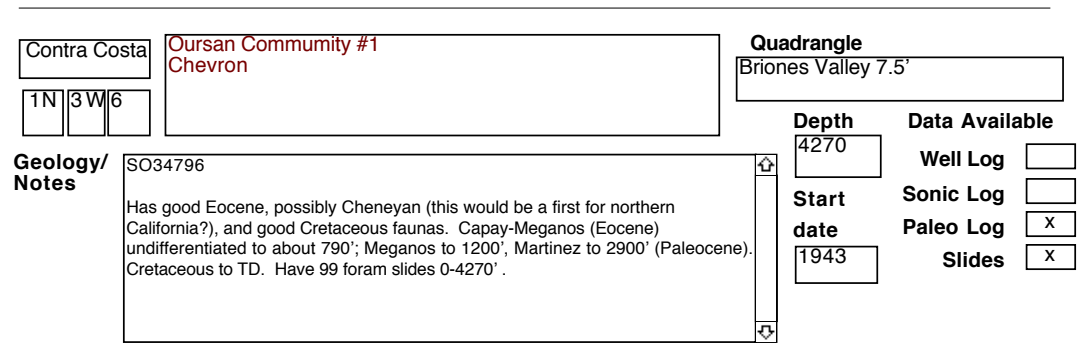


SELECTED OIL TEST WELLS IN CENTRAL CALIFORNIA DATABASE

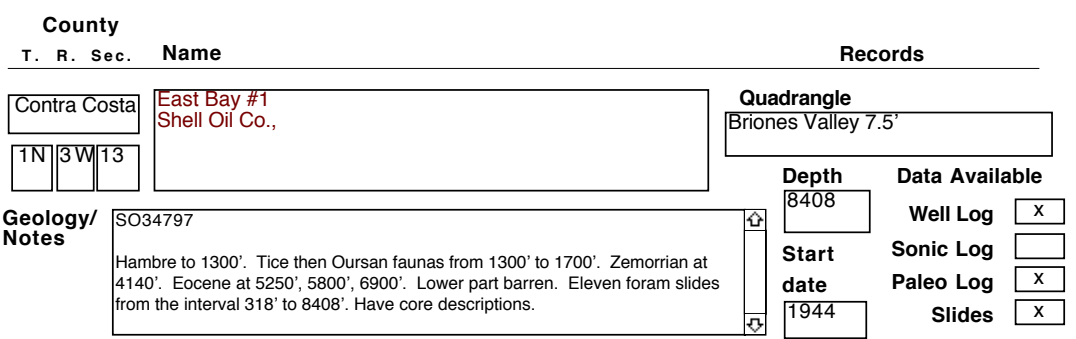

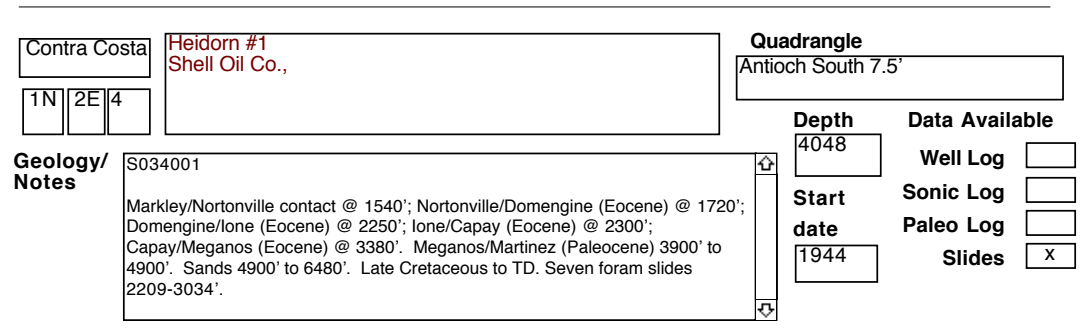

\begin{tabular}{|c|c|c|c|c|}
\hline \multirow{2}{*}{ 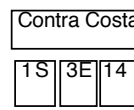 } & \multirow{2}{*}{$\begin{array}{l}\text { Sproule \#1 } \\
\text { Chevron }\end{array}$} & \multicolumn{3}{|c|}{$\begin{array}{l}\text { Quadrangle } \\
\text { Clifton Court Forebay 7.5' }\end{array}$} \\
\hline & & \multirow{2}{*}{$\begin{array}{l}\text { Depth } \\
6070\end{array}$} & \multicolumn{2}{|c|}{ Data Available } \\
\hline \multirow{3}{*}{$\begin{array}{l}\text { Geology/ } \\
\text { Notes }\end{array}$} & $\longdiv { 3 2 6 9 7 }$ & & Well Log & $\mathrm{x}$ \\
\hline & od Eocene faunas. Basalt and basaltic conglomerate reported from upper part & Start & Sonic Log & $x$ \\
\hline & $\begin{array}{l}\text { pay to } 5480^{\prime} \text { (all Eocene). Cretaceous to TD. Thirty-two slides from the interval } \\
60^{\prime} \text { to } 6045^{\prime} \text {. Some lithology information from sidewall cores. }\end{array}$ & 1949 & Slides & $\bar{x}$ \\
\hline
\end{tabular}

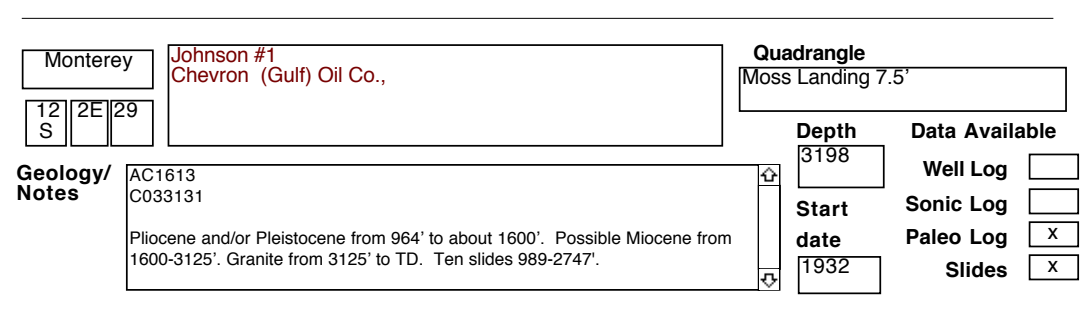

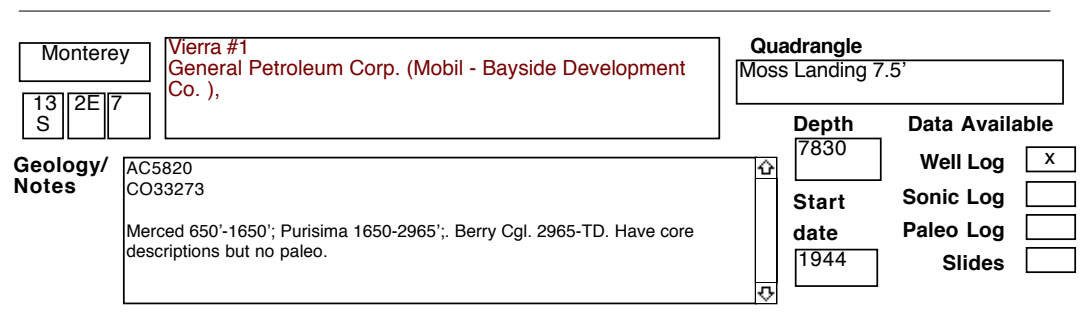


SELECTED OIL TEST WELLS IN CENTRAL CALIFORNIA DATABASE County

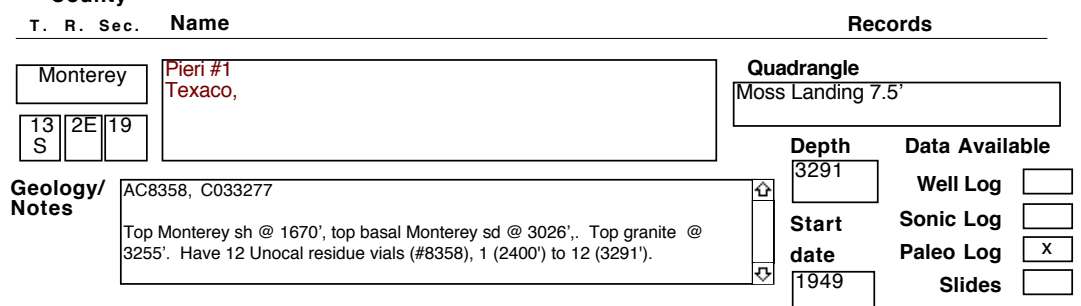

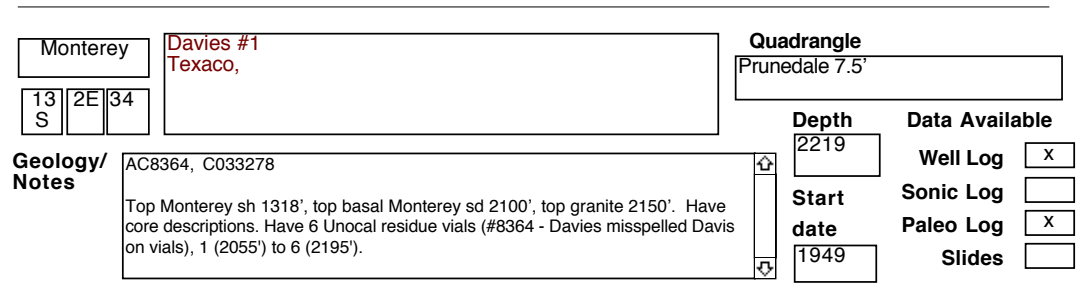

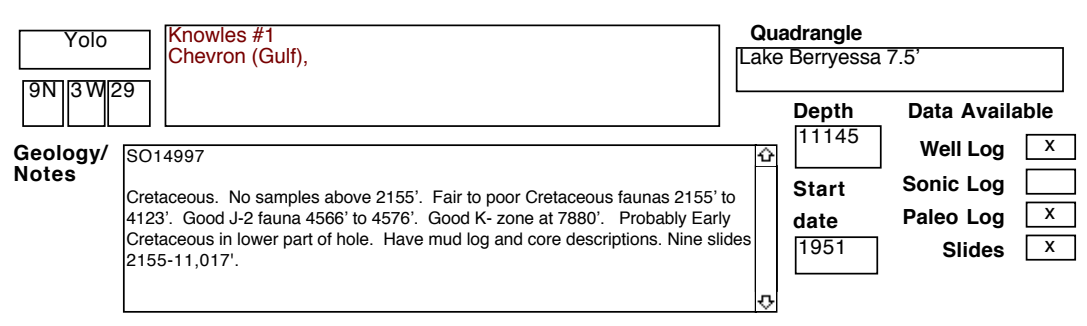

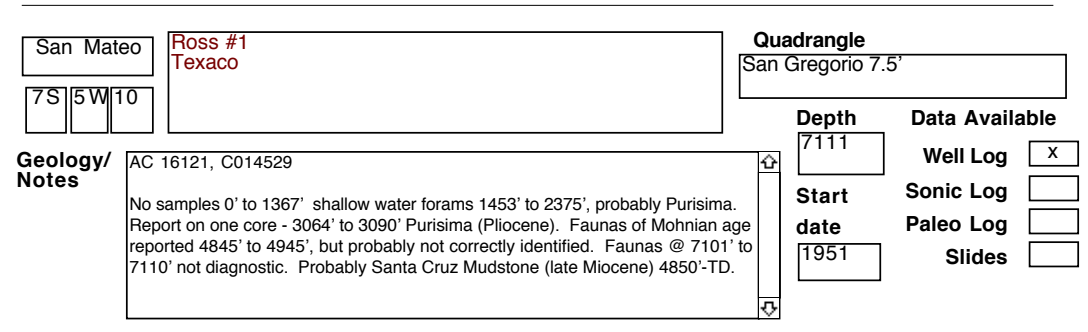

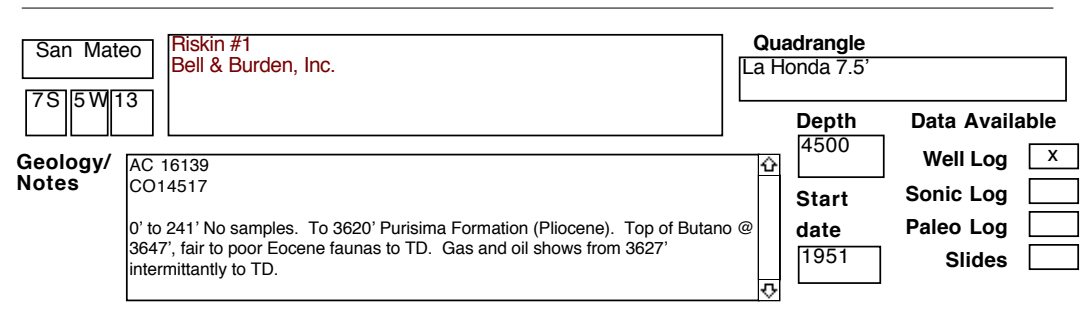


SELECTED OIL TEST WELLS IN CENTRAL CALIFORNIA DATABASE County

\begin{tabular}{|c|c|c|c|}
\hline T. R. Sec. & Name & \multicolumn{2}{|c|}{ Records } \\
\hline \begin{tabular}{|l|} 
Santa Clara \\
\end{tabular} & \multirow[t]{2}{*}{$\begin{array}{l}\text { Gilroy City \#1 } \\
\text { Leroy C. Gould, }\end{array}$} & \multicolumn{2}{|c|}{$\begin{array}{c}\text { Quadrangle } \\
\text { Chittenden } 7.5^{\prime}\end{array}$} \\
\hline \begin{tabular}{|l|l||l|}
11 & $4 \mathrm{E}$ & 9 \\
$\mathrm{~S}$ & & \\
\end{tabular} & & \multirow{2}{*}{$\begin{array}{l}\text { Depth } \\
3520\end{array}$} & Data Available \\
\hline \multirow{3}{*}{\multicolumn{2}{|c|}{$\begin{array}{l}\text { Geology/ Top of Franciscan basement rocks at } 1875^{\prime} \text {. } \\
\text { Notes }\end{array}$}} & & Well Log \\
\hline & & Start & Sonic Log \\
\hline & & $\begin{array}{l}\text { date } \\
1953\end{array}$ & $\begin{array}{r}\text { Paleo Log } \\
\text { Slides }\end{array}$ \\
\hline
\end{tabular}

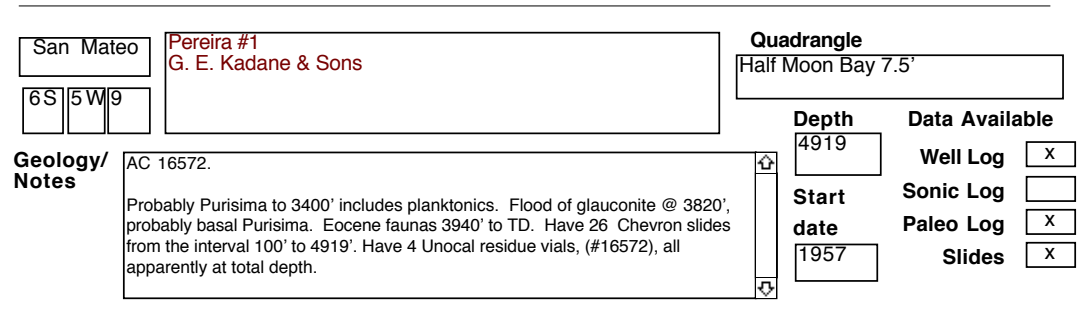

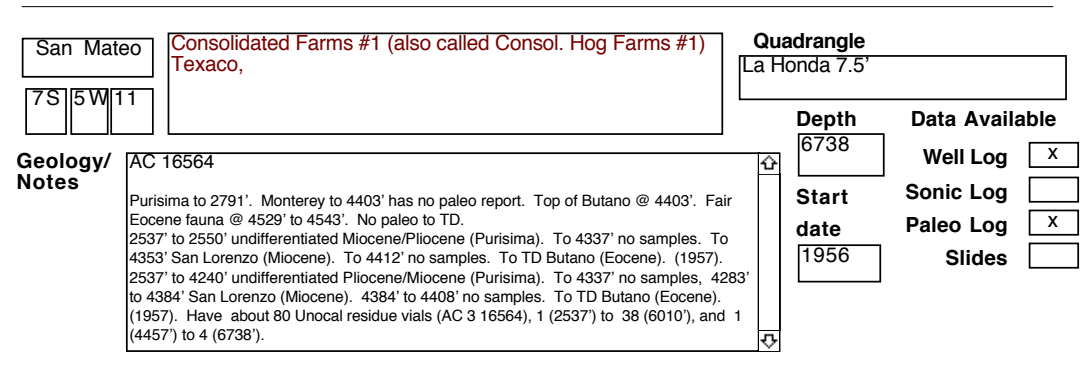

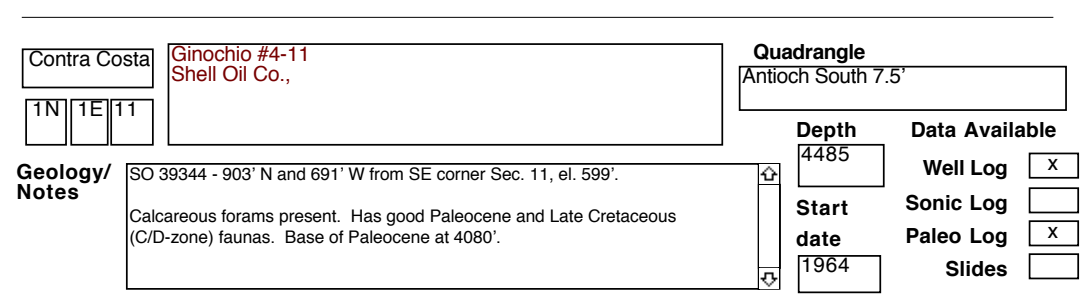

\begin{tabular}{l|l|l|l|l|l|}
\hline Elworthy \#1 \\
Texaco,
\end{tabular}


SELECTED OIL TEST WELLS IN CENTRAL CALIFORNIA DATABASE

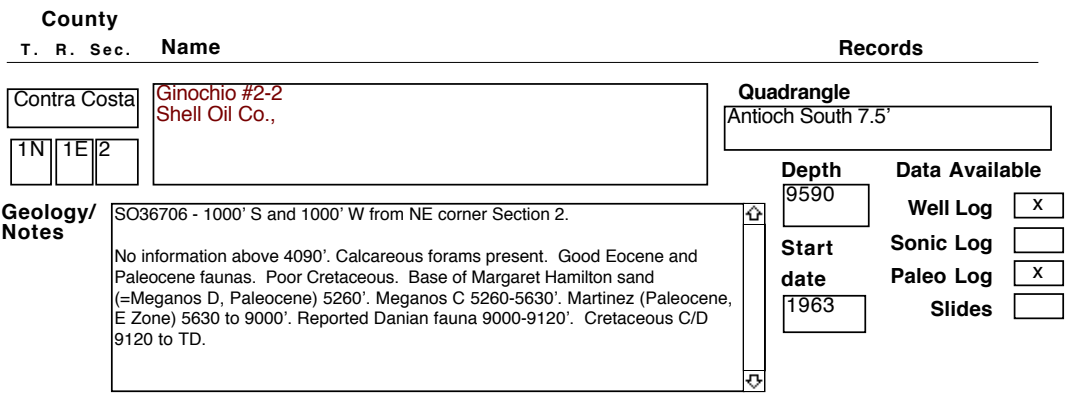

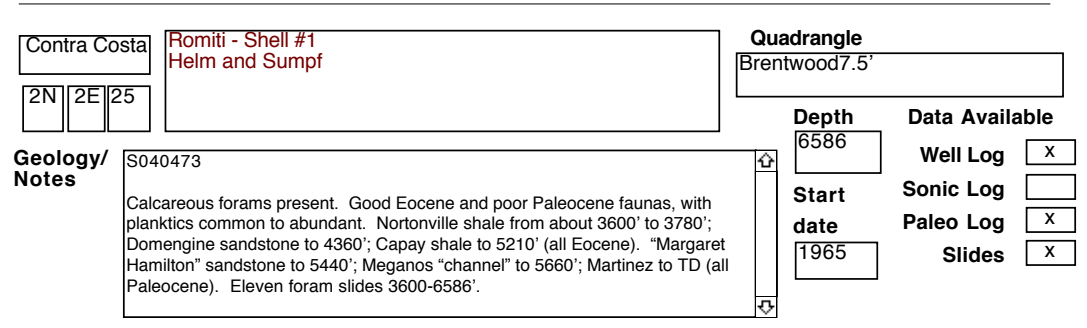

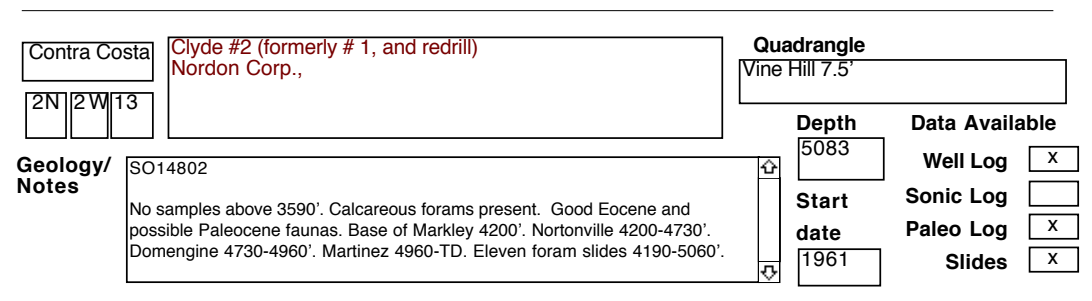

\begin{tabular}{l|l|l|l|l|l|l|}
\hline McCulloch-Soite \#1 \\
McCulloch Oil Corp.,
\end{tabular}

\begin{tabular}{|c|c|c|c|c|c|}
\hline \multirow{2}{*}{\begin{tabular}{|l|} 
Contra Costa \\
\begin{tabular}{|l|l|l|}
$1 S$ & $3 E$ & 19 \\
\end{tabular}
\end{tabular}} & \multirow{2}{*}{$\begin{array}{l}\text { Souza \#1 (Louis) } \\
\text { Davidor \& Davidor }\end{array}$} & \multicolumn{4}{|c|}{$\begin{array}{l}\text { Quadrangle } \\
\text { Bryon Hot Springs 7.5' }\end{array}$} \\
\hline & & & \multirow{2}{*}{$\begin{array}{l}\text { Depth } \\
3911\end{array}$} & \multicolumn{2}{|c|}{ Data Available } \\
\hline \multirow{2}{*}{$\begin{array}{l}\text { Geology/ } \\
\text { Notes }\end{array}$} & 32698 & 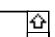 & & Well Log & \\
\hline & $\begin{array}{l}\text { careous forams present. Fairly good Cretaceous faunas. Cretaceous from } \\
0^{\prime} \text { to TD. Have } 29 \text { foram slides } 670-3911 \text {. }\end{array}$ & $\theta$ & $\begin{array}{l}\text { Start } \\
\text { date } \\
1957\end{array}$ & $\begin{array}{r}\text { Sonic Log } \\
\text { Paleo Log } \\
\text { Slides }\end{array}$ & $\frac{x}{x}$ \\
\hline
\end{tabular}


SELECTED OIL TEST WELLS IN CENTRAL CALIFORNIA DATABASE

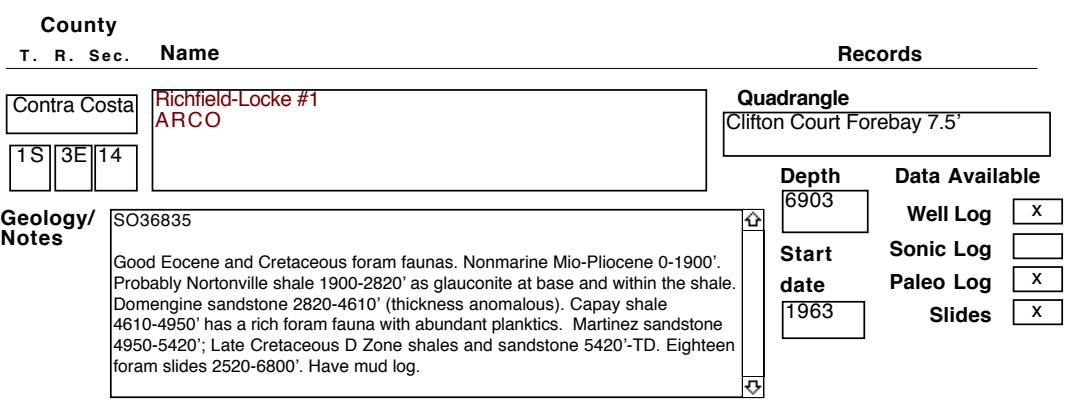

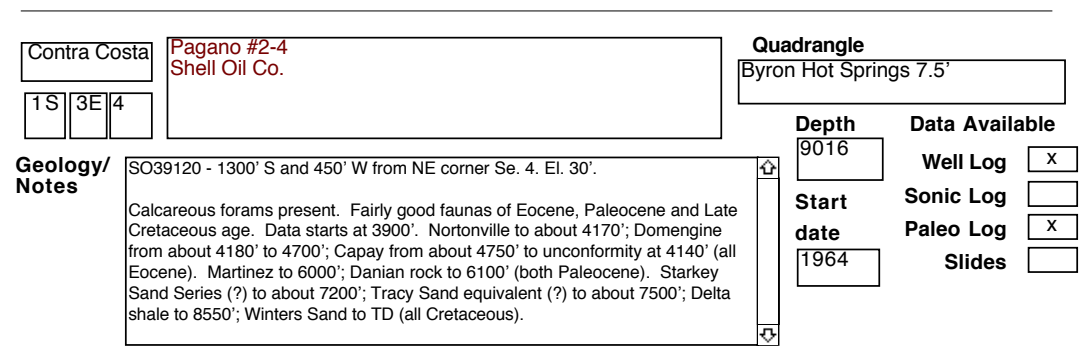

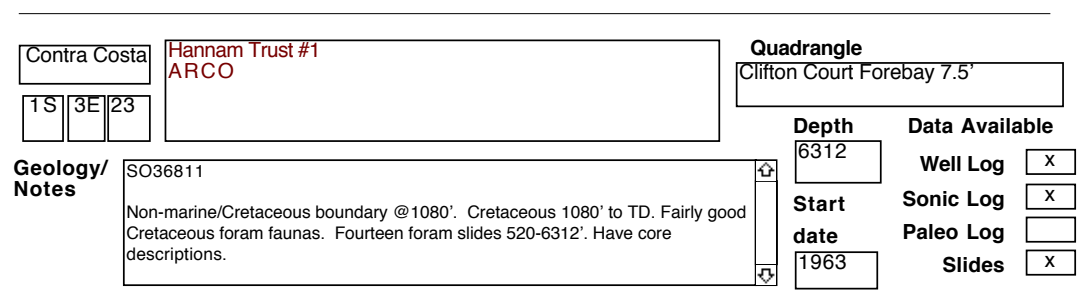

Geology/
$\begin{aligned} & \text { Notes } \\ & \text { SO36811- 1000' N and 1650' W from SE corner Sec. 23, el 44'. }\end{aligned}$
$\begin{aligned} & \text { Base of Miocene nonmarine rocks at 1070'. Late Cretaceous C and/or D zone } \\ & \text { forams plus Baculites and Inoceramus prisms in shale 1070-2400'. Sandstone } \\ & \text { Sand shale with D-1, D-2 and E zone faunas 2400'-TD. Probable fault at 3100' } \\ & \text { Sepeats part of section. }\end{aligned}$

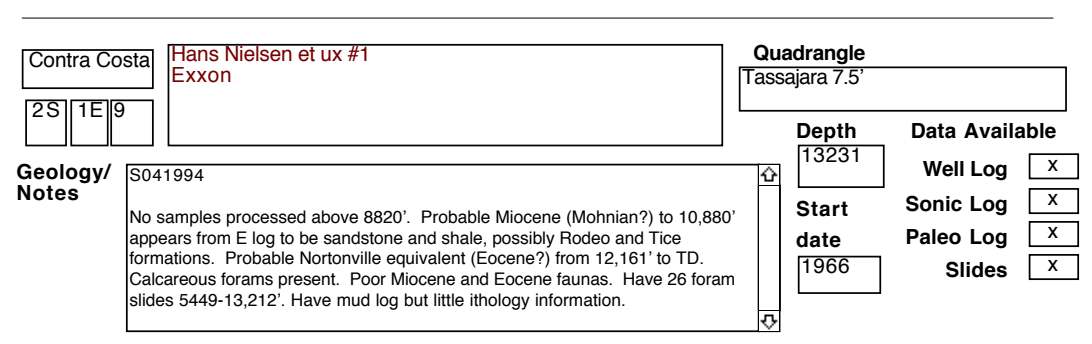


SELECTED OIL TEST WELLS IN CENTRAL CALIFORNIA DATABASE

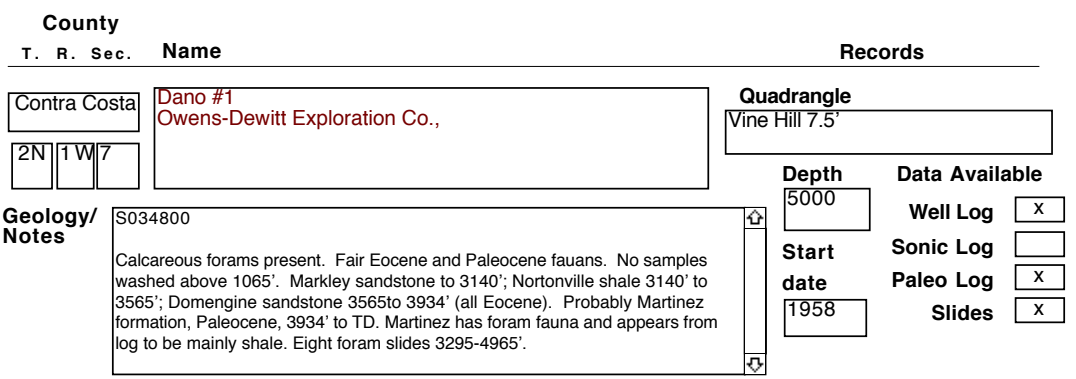

\begin{tabular}{l|l|l|l|l|}
\hline Vlach Unit \#1 \\
Trico Oil \& Gas Co.
\end{tabular}

\begin{tabular}{|c|c|c|c|c|c|}
\hline \multirow{2}{*}{$\begin{array}{l}\text { Contra Costa } \\
2 \mathrm{~N} / \mathrm{W} 15 \\
\end{array}$} & \multirow{2}{*}{$\begin{array}{l}\text { Alves \# } 1 \\
\text { Trico Oil \& Gas Co., }\end{array}$} & \multicolumn{4}{|c|}{$\begin{array}{c}\text { Quadrangle } \\
\text { |Honker Bay 7.5' }\end{array}$} \\
\hline & & \multirow{2}{*}{\multicolumn{2}{|c|}{$\begin{array}{l}\text { Depth } \\
7076\end{array}$}} & \multicolumn{2}{|c|}{ Data Available } \\
\hline \multirow{3}{*}{$\begin{array}{l}\text { Geology/ } \\
\text { Notes }\end{array}$} & 036873 & & & Well Log & $x$ \\
\hline & & & Start & Sonic Log & \\
\hline & $\begin{array}{l}\text { tood Eocene and fair Paleocene faunas. Markley sands from } 3700^{\prime} \text { to } 4670 \text { '; } \\
\text { lortonville to 5040', Domengine to about 5470' (all Eocene). Martinez } \\
\text { 'aleocene) to about } 6750^{\prime} \text {, uncertain to TD. }\end{array}$ & & $\begin{array}{l}\text { date } \\
1964\end{array}$ & $\begin{array}{l}\text { Paleo Log } \\
\text { Slides }\end{array}$ & $x$ \\
\hline
\end{tabular}

\begin{tabular}{l|l|l|l|l|l|}
\hline Keller Estate \#1 \\
Cameron, (George E., Jr.)
\end{tabular}

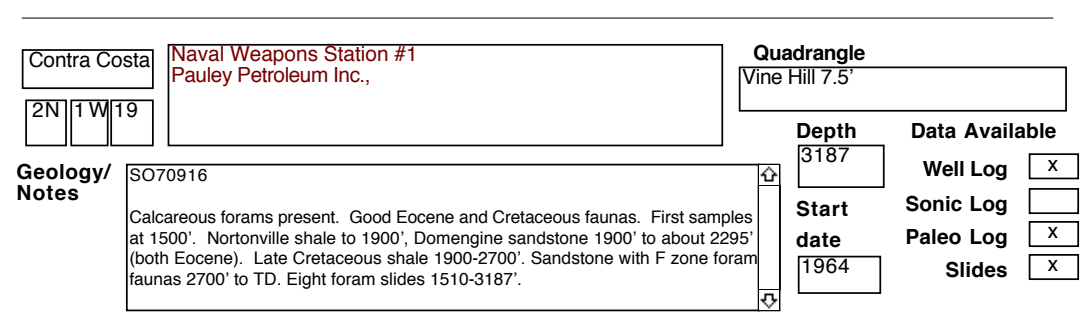


SELECTED OIL TEST WELLS IN CENTRAL CALIFORNIA DATABASE

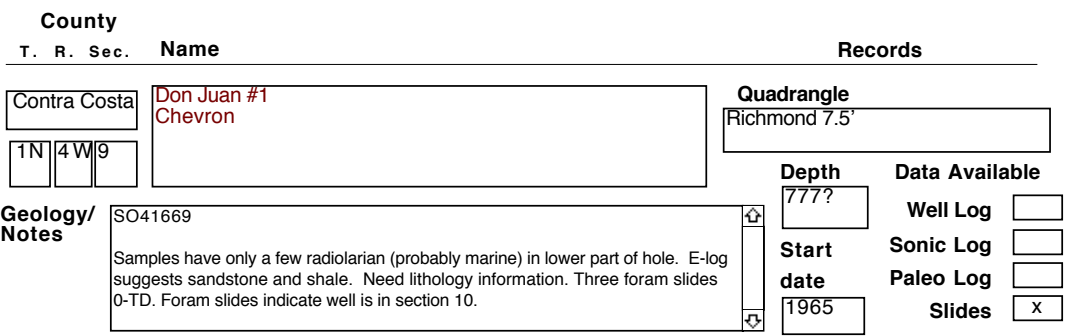

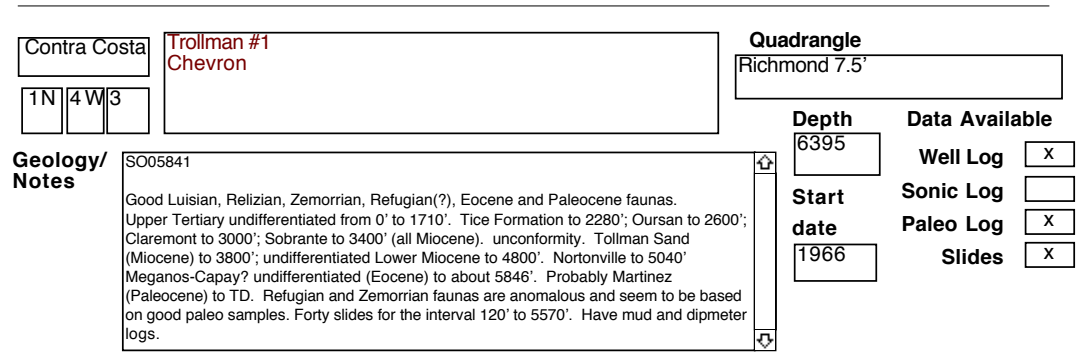

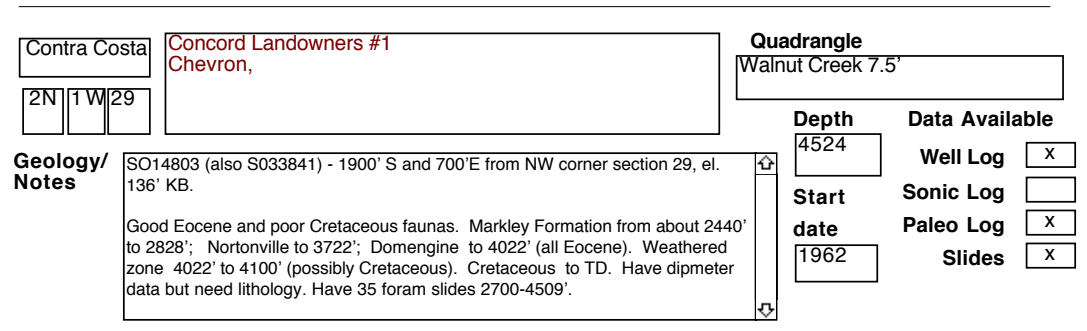

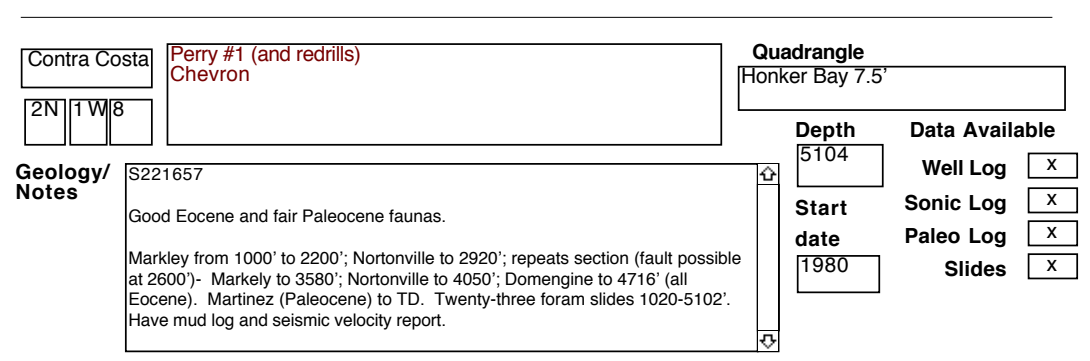

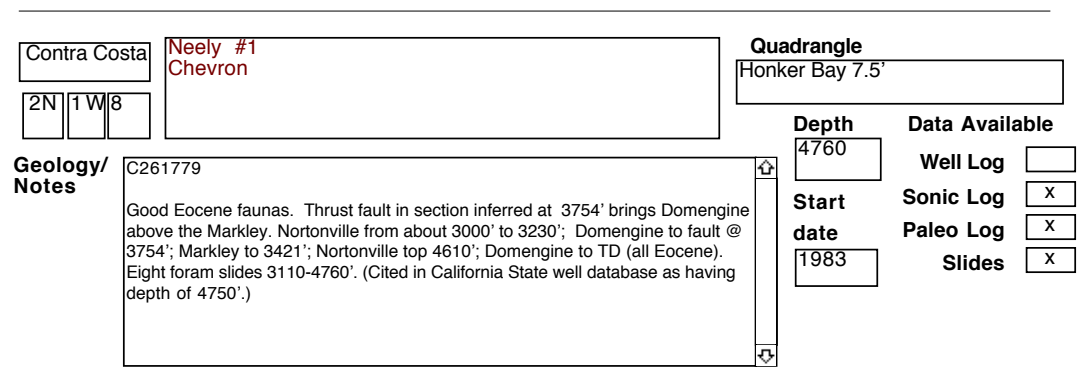


SELECTED OIL TEST WELLS IN CENTRAL CALIFORNIA DATABASE

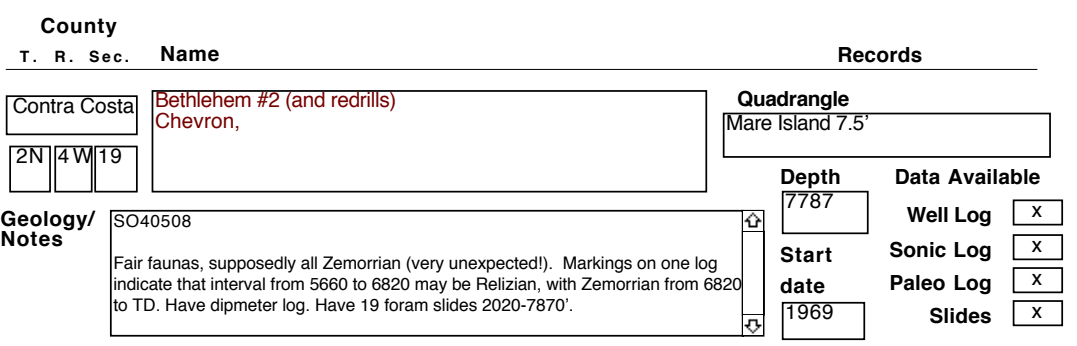

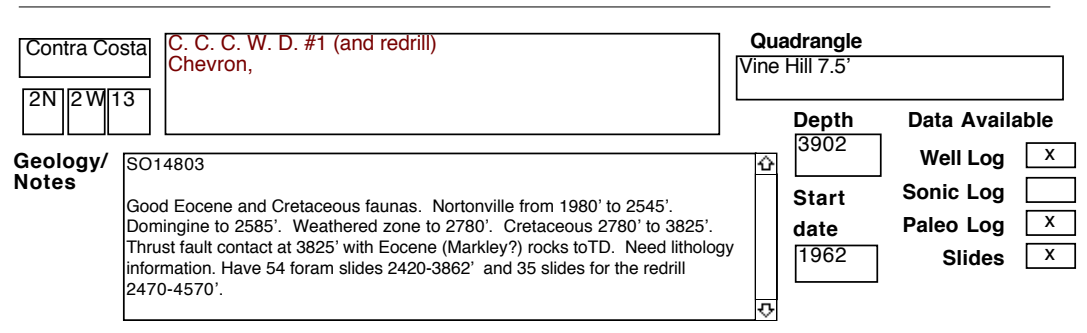

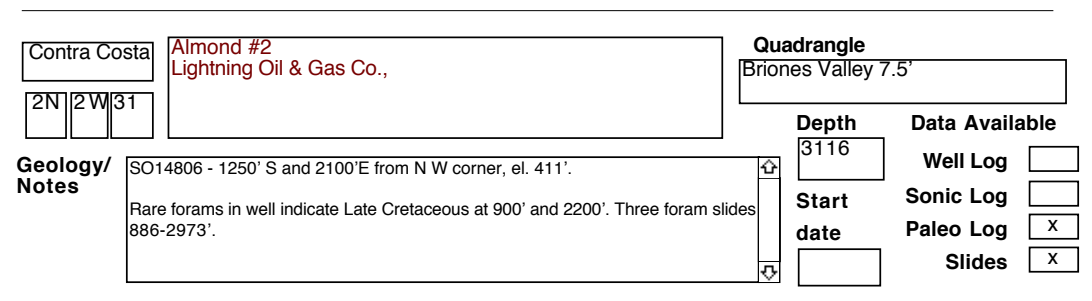

\begin{tabular}{l|l|l|l|l|l|l|}
\hline Cantra Costa & $\begin{array}{l}\text { Marsten et al \#1 } \\
\text { Chevron, }\end{array}$ \\
\hline
\end{tabular}

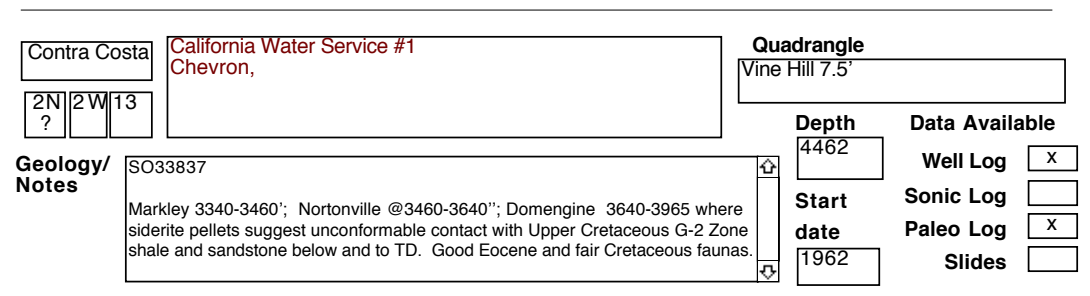


SELECTED OIL TEST WELLS IN CENTRAL CALIFORNIA DATABASE County

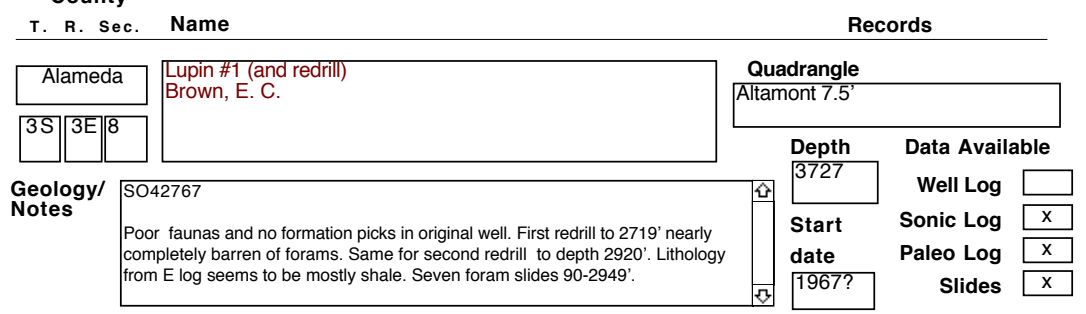

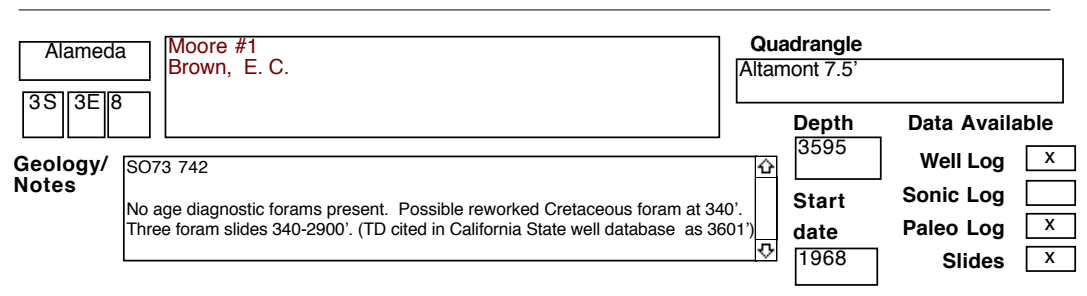

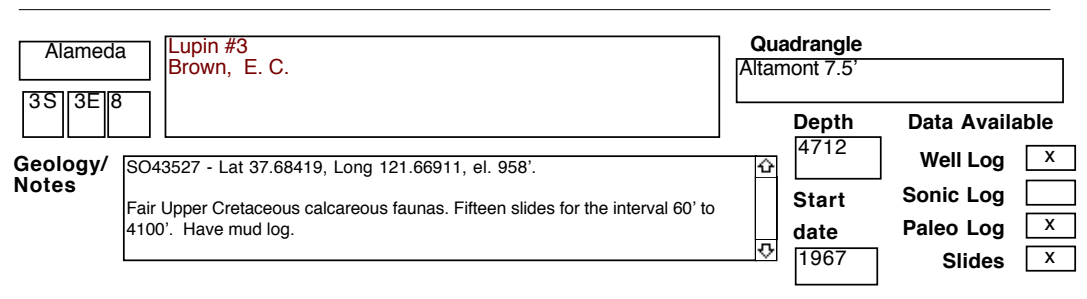

\begin{tabular}{l|l|l|l|l|l|}
\hline Alameda & $\begin{array}{l}\text { Christensen \#1 } \\
\text { Texaco (Hancock /Signal) }\end{array}$ \\
\hline
\end{tabular}

\begin{tabular}{|c|c|c|c|c|}
\hline Alameda & \multirow[t]{2}{*}{$\begin{array}{l}\text { Mulqueeny \#1 } \\
\text { Trico Oil \& Gas Co. }\end{array}$} & \multicolumn{3}{|c|}{$\begin{array}{r}\text { Quadrangle } \\
\text { Midway 7.5' }\end{array}$} \\
\hline \begin{tabular}{|l|l|l|}
$3 S$ & $3 E$ \\
\end{tabular} & & \multirow{2}{*}{$\begin{array}{l}\text { Depth } \\
5020\end{array}$} & \multicolumn{2}{|c|}{ Data Available } \\
\hline \multirow{4}{*}{$\begin{array}{l}\text { Geology/ } \\
\text { Notes }\end{array}$} & 10270 & & Well Log & $\mathrm{x}$ \\
\hline & careous forams present. Fair faunas, mostly sparse. Cretaceous from 1470 ' to & Start & Sonic Log & $\mathrm{x}$ \\
\hline & Fourteen foram slides 1470-5020'. & date & Paleo Log & $\mathrm{x}$ \\
\hline & & 1967 & Slides & $\mathrm{x}$ \\
\hline
\end{tabular}


SELECTED OIL TEST WELLS IN CENTRAL CALIFORNIA DATABASE County

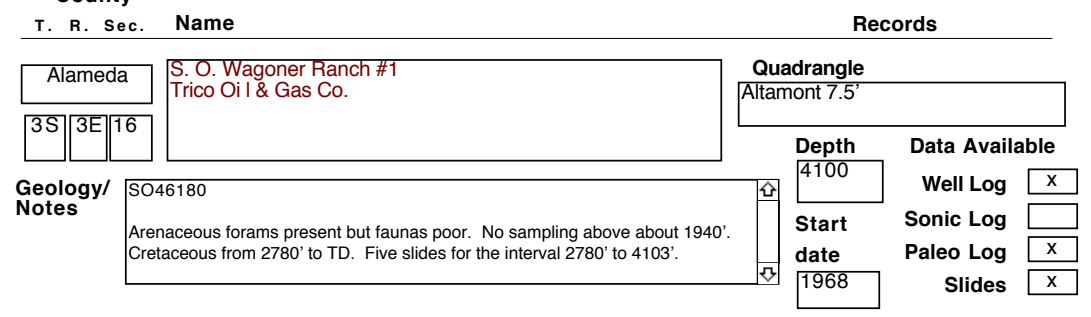

\begin{tabular}{l|l|l|l|l|l|}
\hline Alameda & $\begin{array}{l}\text { Tesla \#1 } \\
\text { R. W. McBurney (Crestmont Oil Co.) }\end{array}$ \\
\hline
\end{tabular}

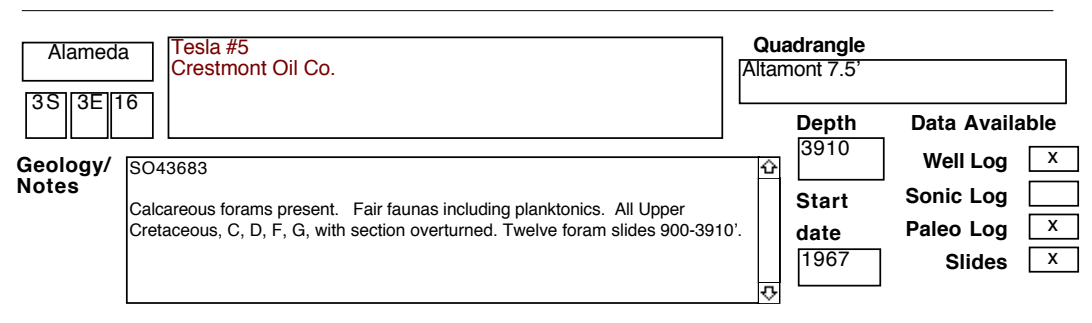

\begin{tabular}{|l|l|l|l|l|l|l|}
\hline Alameda & Wagoner \#1 \\
\hline
\end{tabular}

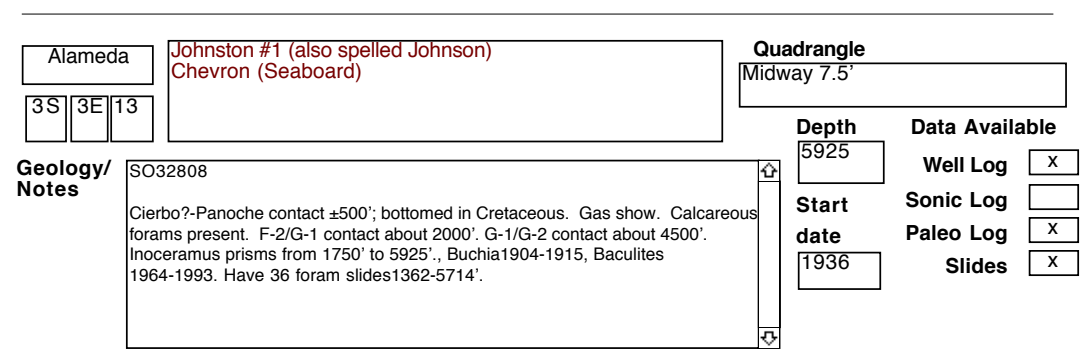


SELECTED OIL TEST WELLS IN CENTRAL CALIFORNIA DATABASE County

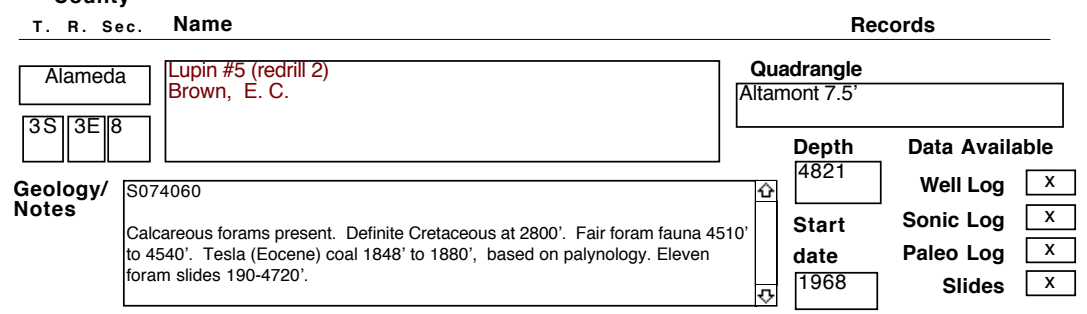

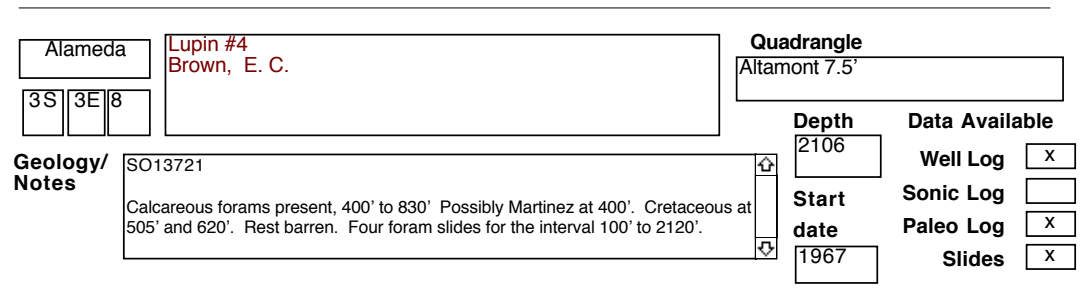

\begin{tabular}{|c|c|c|c|c|}
\hline Alamed & \multirow[t]{2}{*}{$\begin{array}{l}\text { Nissen \#4 } \\
\text { McCulloch Oil Co. }\end{array}$} & \multicolumn{3}{|l|}{$\begin{array}{r}\text { Quadrangle } \\
\text { Altamont 7.5' }\end{array}$} \\
\hline${ }^{3 S}\left\|^{3 \mathrm{E}}\right\|^{7}$ & & \multirow{2}{*}{$\begin{array}{l}\text { Depth } \\
3093\end{array}$} & \multicolumn{2}{|c|}{ Data Available } \\
\hline \multirow{3}{*}{$\begin{array}{l}\text { Geology/ } \\
\text { Notes }\end{array}$} & $\mathbf{S 0 7 3 4 0 5}$ & & Well Log & $\bar{x}$ \\
\hline & Calcareous forams present. Barren above 1040'. First Cretaceous, Inoceramus? & Start & Sonic Log & \\
\hline & $\begin{array}{l}\text { at } 1310^{\prime} \text {. Rare Cretaceous forams at } 1400^{\prime} \text { and } 1490^{\prime} \text {. Anomalous Tesla "coal" } \\
\text { (Eocene) at } 2400^{\prime} \text { ', possible Cretaceous D zones at 3083', near TD. }\end{array}$ & $\begin{array}{l}\text { date } \\
1943 ?\end{array}$ & $\begin{array}{r}\text { Paleo Log } \\
\text { Slides }\end{array}$ & $\mathrm{x}$ \\
\hline
\end{tabular}

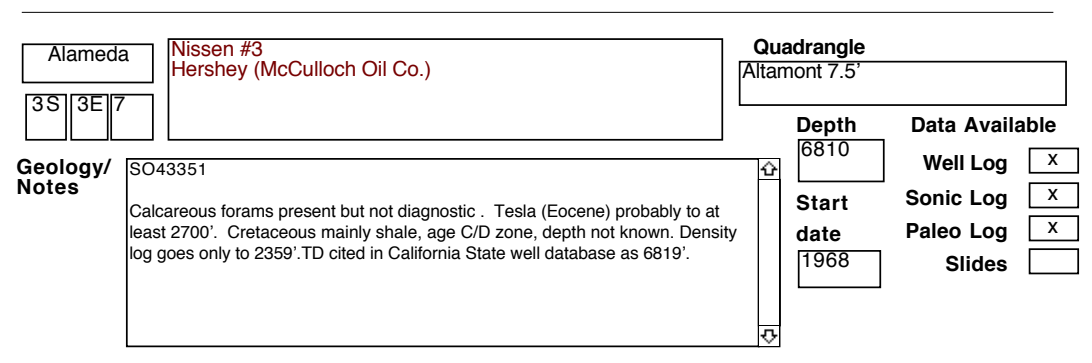

\begin{tabular}{|l|l|l|l|l|l|l|l|}
\hline Alameda & $\begin{array}{l}\text { Nissen \#1 } \\
\text { McCulloch Oil Co. }\end{array}$ \\
\hline
\end{tabular}


SELECTED OIL TEST WELLS IN CENTRAL CALIFORNIA DATABASE County

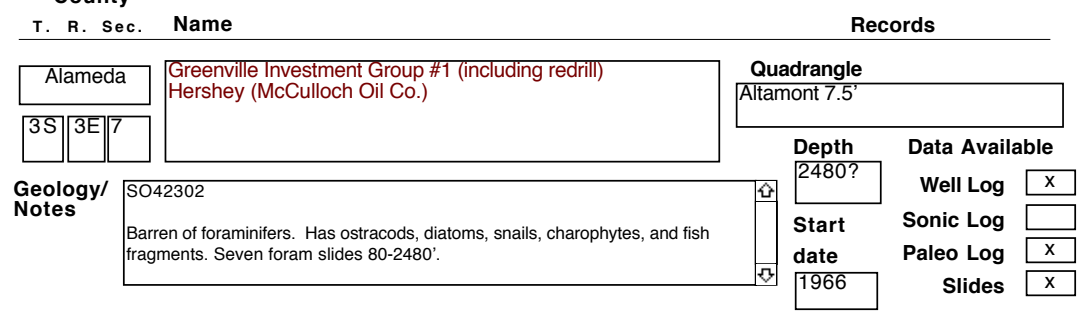

\begin{tabular}{|c|c|c|c|c|c|}
\hline Alameda & \multirow[t]{2}{*}{$\begin{array}{l}\text { Smith et ux \#2 } \\
\text { McCulloch Oil Co. }\end{array}$} & \multicolumn{4}{|c|}{$\begin{array}{l}\text { Quadrangle } \\
\text { Altamont } 7.5^{\prime}\end{array}$} \\
\hline \begin{tabular}{|l|l|l|l|l|l|}
$3 S$ & $3 E$ & 6 \\
\end{tabular} & & \multirow{2}{*}{\multicolumn{2}{|c|}{$\begin{array}{l}\text { Depth } \\
5000\end{array}$}} & \multicolumn{2}{|c|}{ Data Available } \\
\hline \multirow{3}{*}{$\begin{array}{l}\text { Geology/ } \\
\text { Notes }\end{array}$} & 73764 & & & Well Log & $\mathrm{x}$ \\
\hline & amples barren of forams. & \multirow[t]{2}{*}{0} & \multirow{2}{*}{$\begin{array}{l}\text { Start } \\
\text { date } \\
1968\end{array}$} & Sonic Log & \\
\hline & & & & $\begin{array}{r}\text { Paleo Log } \\
\text { Slides }\end{array}$ & $\mathrm{X}$ \\
\hline
\end{tabular}

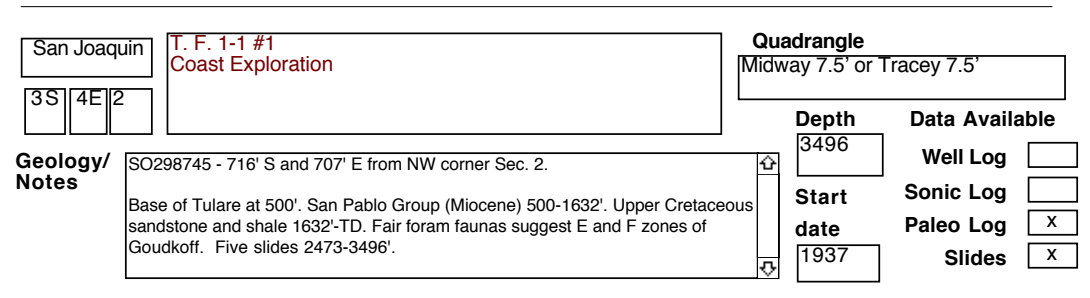

\begin{tabular}{l|l|l|l|l|l|}
\hline Alameda & $\begin{array}{l}\text { Maud Stanley \#1 } \\
\text { Texaco }\end{array}$ \\
\hline
\end{tabular}

\begin{tabular}{l|l|l|l|l|l|}
\hline Gethlehem \#1 \\
Chevron,
\end{tabular}




\section{SELECTED OIL TEST WELLS IN CENTRAL CALIFORNIA DATABASE}

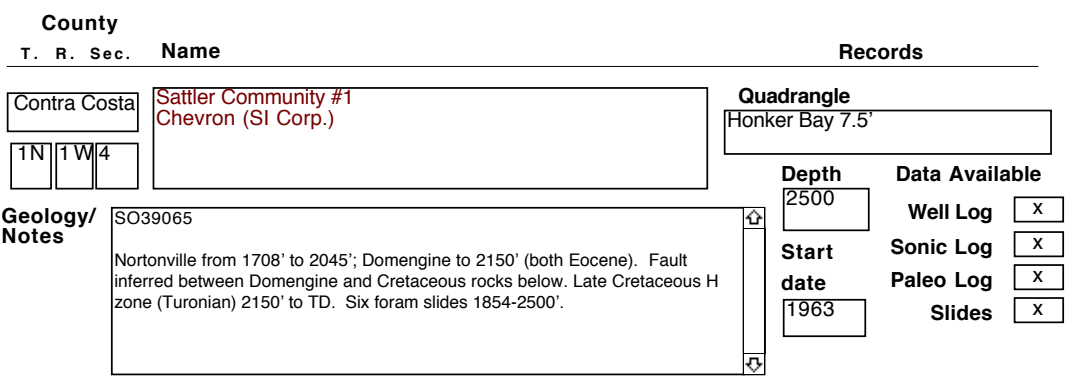

\begin{tabular}{l|l|l|l|l|l|}
\hline Contra Costa & $\begin{array}{l}\text { Seeno Scott \#2 } \\
\text { Chevron }\end{array}$ & \multicolumn{2}{|c|}{ Quadrangle } \\
\hline
\end{tabular}

\begin{tabular}{|c|c|c|c|c|c|}
\hline Contra Costa & \multirow[t]{2}{*}{$\begin{array}{l}\text { Gumpert \#1 } \\
\text { Chevron }\end{array}$} & \multicolumn{4}{|c|}{$\begin{array}{l}\text { Quadrangle } \\
\text { Diablo } 7.5\end{array}$} \\
\hline \begin{tabular}{|l|l|l|l|l|}
$2 S$ & $1 E$ & 17
\end{tabular} & & & \multirow{2}{*}{$\begin{array}{l}\text { Depth } \\
8081\end{array}$} & \multicolumn{2}{|c|}{ Data Available } \\
\hline \multirow{4}{*}{$\begin{array}{l}\text { Geology/ } \\
\text { Notes }\end{array}$} & 03798 & 它 & & Well Log & $\mathrm{x}$ \\
\hline & inly fish fragments ostracods and charanhytes throughout well with several & & Start & Sonic Log & $\mathrm{x}$ \\
\hline & ams that are considered to be reworked. A few Amnicola-type snails are & & date & Paleo Log & $\mathrm{x}$ \\
\hline & $\begin{array}{l}\text { sent and are considered to be similar to those in the Tulare formation of } \\
\text { istocene age. E log suggests lithology almost all shale. Have } 21 \text { slides }\end{array}$ & & 1964 & Slides & $\mathrm{x}$ \\
\hline
\end{tabular}

\begin{tabular}{l|l|l|l|l|l|}
\hline Contra Costa & $\begin{array}{l}\text { Collier \#1 } \\
\text { Cullinan, }\end{array}$ & \multicolumn{2}{c|}{ Quadrangle } \\
\hline
\end{tabular}

\begin{tabular}{l|l|l|l|l|l|}
\hline Contra Costa & $\begin{array}{l}\text { Las Trampas Unit \#A1 } \\
\text { McCulloch Oil Corp. (ARCO) }\end{array}$ \\
\hline
\end{tabular}


SELECTED OIL TEST WELLS IN CENTRAL CALIFORNIA DATABASE

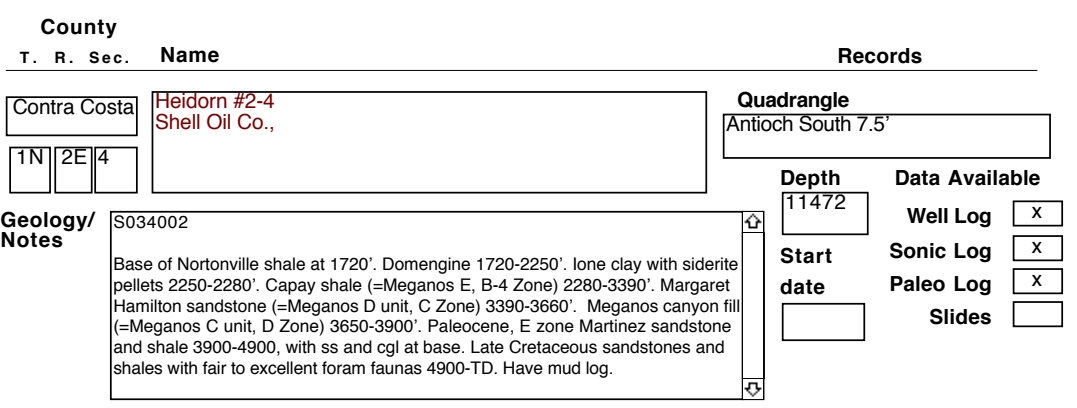

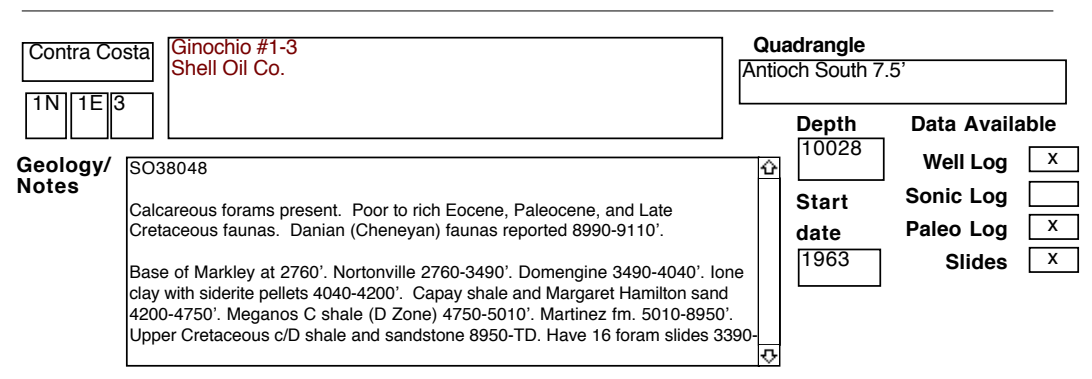

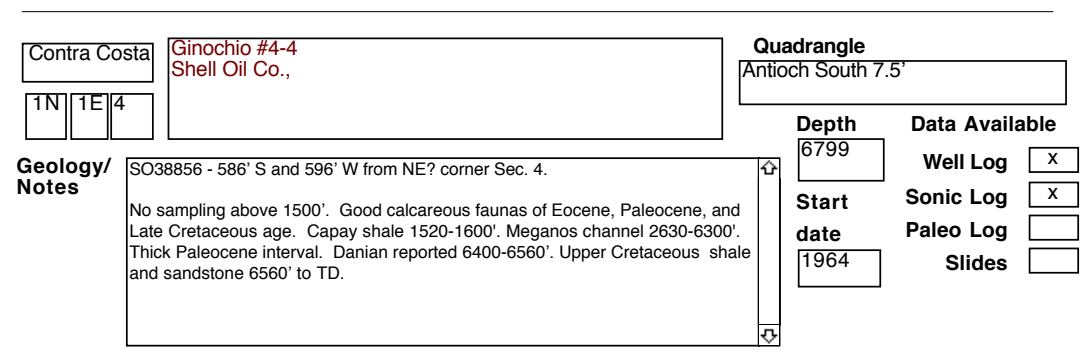

\begin{tabular}{l|l|l|l|l|l|}
\hline Giggins \#1-12 \\
Shell Oil Co.,
\end{tabular}

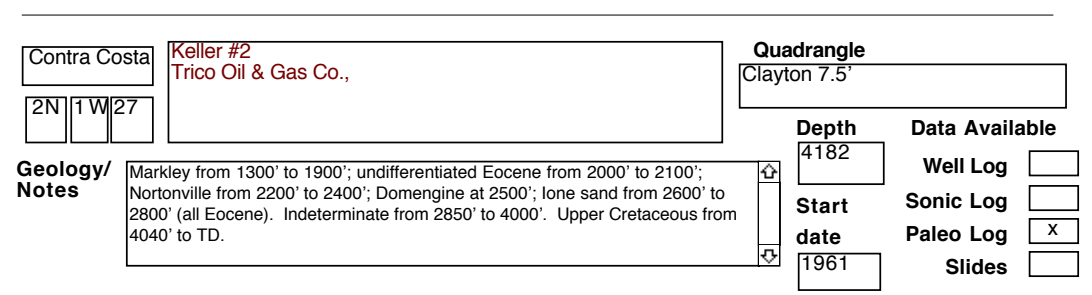


SELECTED OIL TEST WELLS IN CENTRAL CALIFORNIA DATABASE County

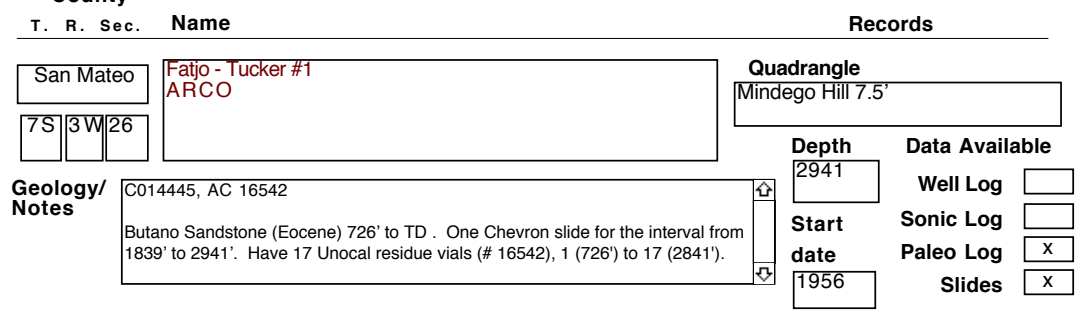

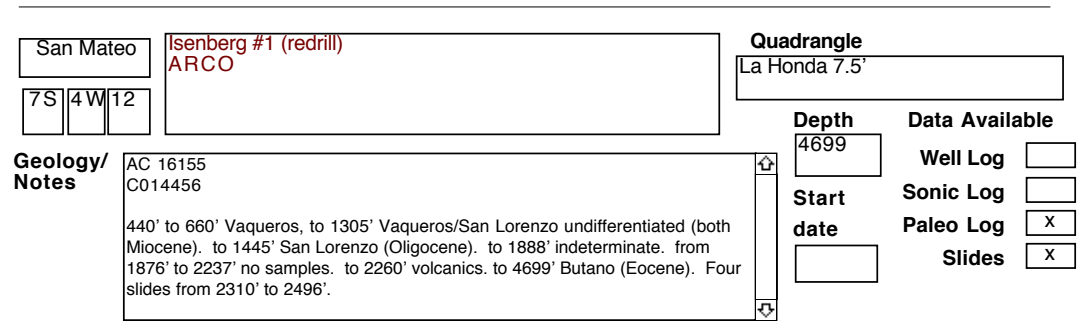

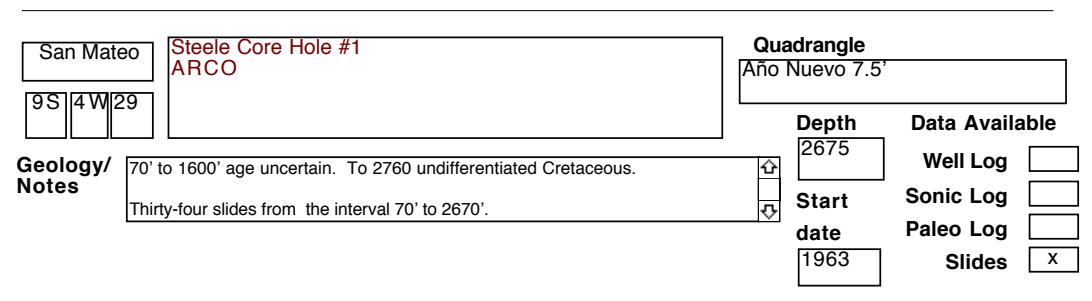

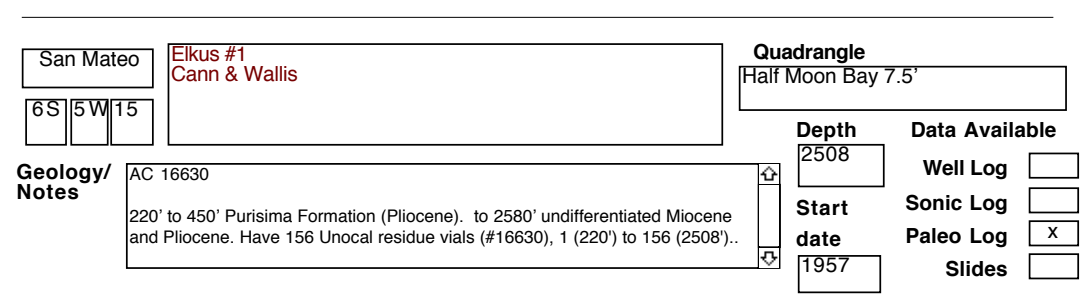

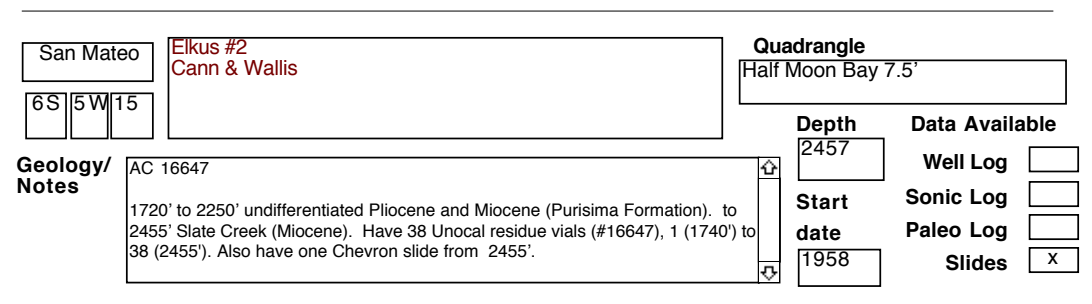


SELECTED OIL TEST WELLS IN CENTRAL CALIFORNIA DATABASE County

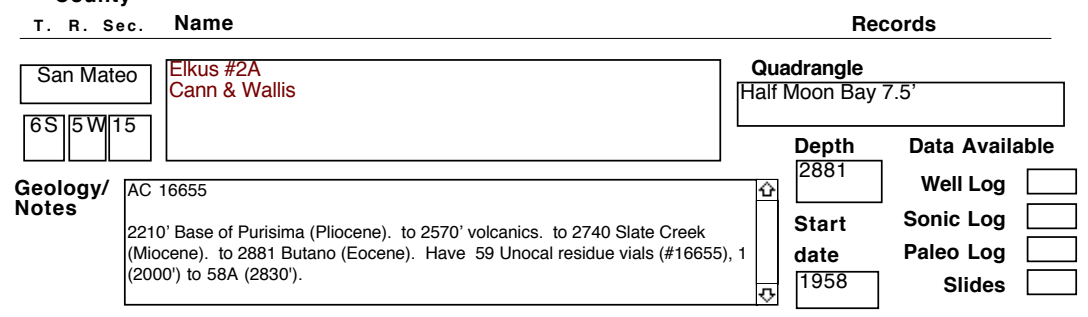

\begin{tabular}{l|l|l|l|l|l|}
\hline San Mateo & $\begin{array}{l}\text { ElkuS \#3 } \\
\text { Cann \& Wallis }\end{array}$ \\
\hline
\end{tabular}

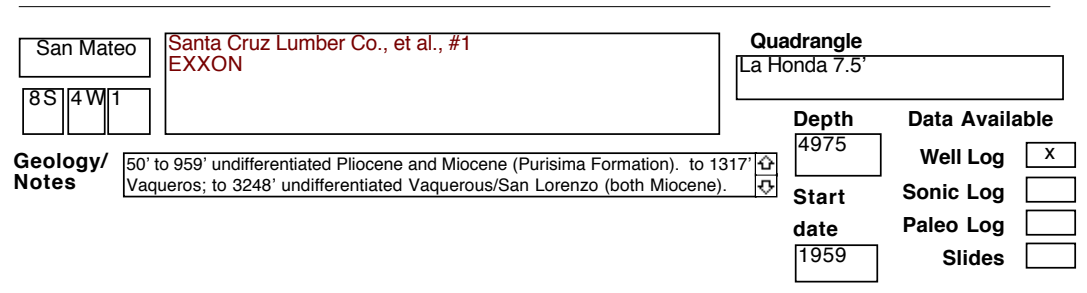

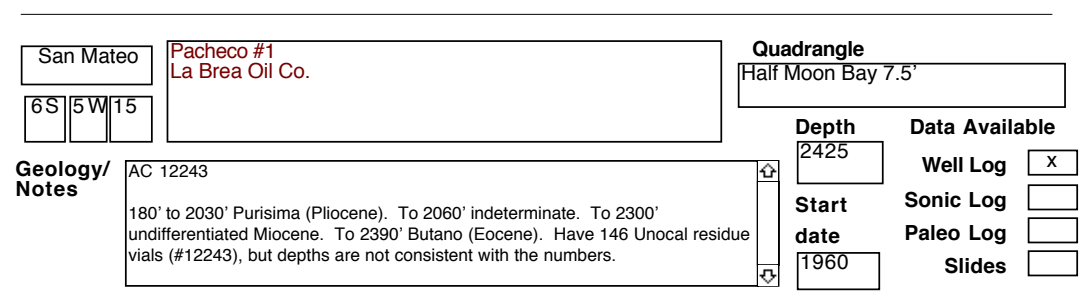

\begin{tabular}{|c|c|c|c|c|c|}
\hline San Mateo & \multirow[t]{2}{*}{$\begin{array}{l}\text { Tener \#1 } \\
\text { M. J. \& O., Inc. }\end{array}$} & \multicolumn{4}{|c|}{$\begin{array}{l}\text { Quadrangle } \\
\text { Half Moon Bay 7.5' }\end{array}$} \\
\hline \begin{tabular}{|l|l|l|l|l|}
$6 S$ & $5 W$
\end{tabular} & & \multirow{2}{*}{\multicolumn{2}{|c|}{$\begin{array}{l}\text { Depth } \\
2460\end{array}$}} & \multicolumn{2}{|l|}{ Data Available } \\
\hline \multirow{3}{*}{$\begin{array}{l}\text { Geology/ } \\
\text { Notes }\end{array}$} & ' to 2120' undifferentiated Miocene and Pliocene (Purisima Formation). to & & & Well Log & \\
\hline & & & Start & Sonic Log & \\
\hline & & & date & $\begin{array}{r}\text { Paleo Log } \\
\text { Slides }\end{array}$ & $x$ \\
\hline
\end{tabular}


SELECTED OIL TEST WELLS IN CENTRAL CALIFORNIA DATABASE County

\begin{tabular}{|c|c|c|c|c|}
\hline T. R. Sec. & Name & \multicolumn{3}{|c|}{ Records } \\
\hline San Mateo & \multirow{3}{*}{$\begin{array}{l}\text { Neaves de Hoff \#1 } \\
\text { Neaves Petreoleum Development Co., }\end{array}$} & \multirow{2}{*}{\multicolumn{3}{|c|}{$\begin{array}{l}\text { Quadrangle } \\
\text { Half Moon Bay 7.5 }\end{array}$}} \\
\hline \multirow[t]{2}{*}{$6 \mathrm{~S} 5 \mathrm{~W} 10$} & & \multirow{3}{*}{\multicolumn{2}{|c|}{$\begin{array}{r}\text { Depth } \\
\text { (仓) } 3757 \\
\end{array}$}} & \multirow{2}{*}{ Data Available } \\
\hline & & & & \\
\hline \multirow{3}{*}{$\begin{array}{l}\text { Geology/ } \\
\text { Notes }\end{array}$} & to 3560 ' Purisima (Pliocene). To 3730 ' undifferentiated Pliocene/Miocene & & & Well Log \\
\hline & risima Formation). & \multirow{2}{*}{ 更 } & Start & Sonic Log \\
\hline & & & $\begin{array}{l}\text { date } \\
1958\end{array}$ & $\begin{array}{r}\text { Paleo Log } \\
\text { Slides }\end{array}$ \\
\hline
\end{tabular}

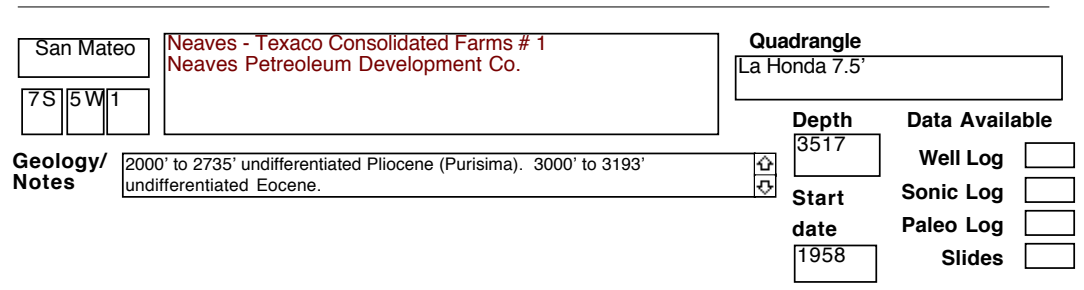

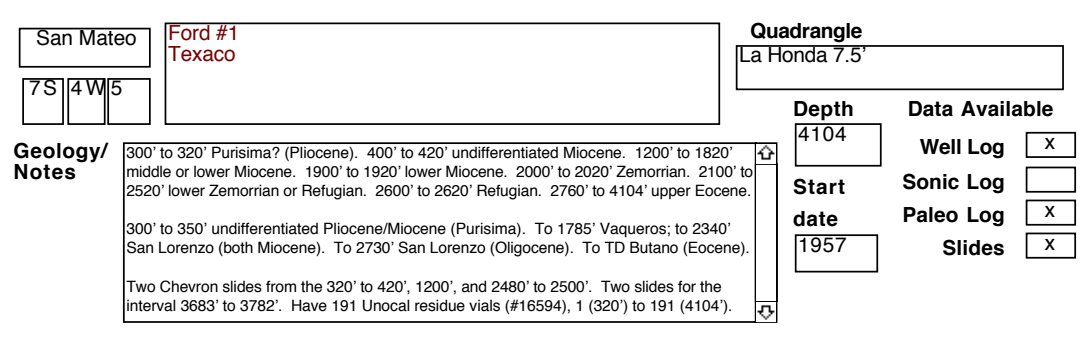

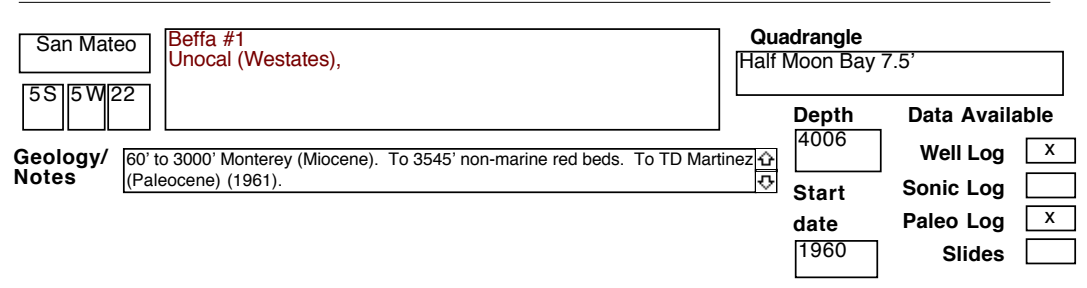

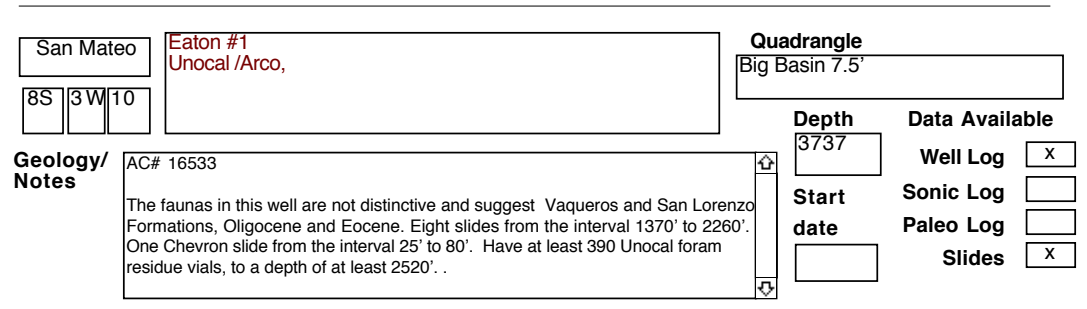




\section{SELECTED OIL TEST WELLS IN CENTRAL CALIFORNIA DATABASE}

\begin{tabular}{|c|c|c|c|}
\hline $\begin{array}{l}\text { County } \\
\text { T. R. Sec. }\end{array}$ & Name & \multicolumn{2}{|c|}{ Records } \\
\hline San Mateo & \multirow[t]{2}{*}{$\begin{array}{l}\text { Elkus \#1-15 } \\
\text { Unocal }\end{array}$} & \multicolumn{2}{|c|}{$\begin{array}{l}\text { Quadrangle } \\
\text { Half Moon Bay 7.5' }\end{array}$} \\
\hline $6 \mathrm{~S} / 5 \mathrm{~W} / 15$ & & \multirow{2}{*}{$\begin{array}{l}\text { Depth } \\
3279\end{array}$} & Data Available \\
\hline \multirow{2}{*}{\multicolumn{2}{|c|}{$\begin{array}{l}\text { Geology/ No data received. } \\
\text { Notes }\end{array}$}} & & Well Log \\
\hline & & $\begin{array}{l}\begin{array}{l}\text { Start } \\
\text { date }\end{array} \\
1968\end{array}$ & $\begin{array}{r}\text { Sonic Log } \\
\text { Paleo Log } \\
\text { Slides }\end{array}$ \\
\hline
\end{tabular}

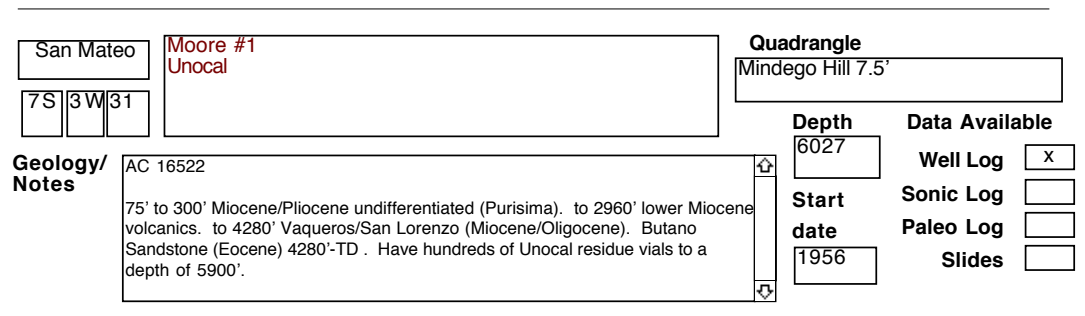

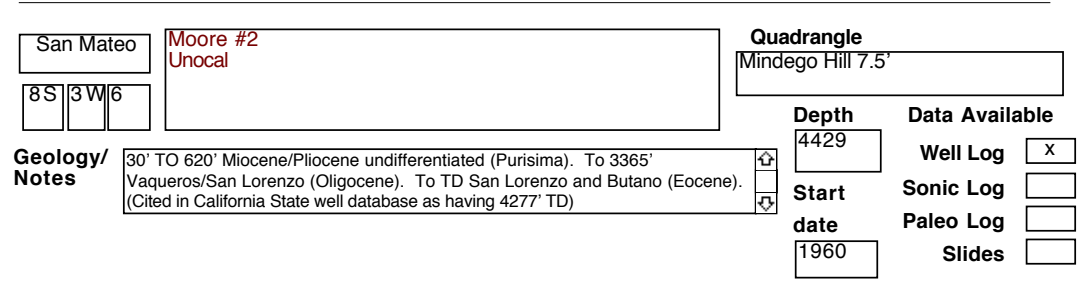

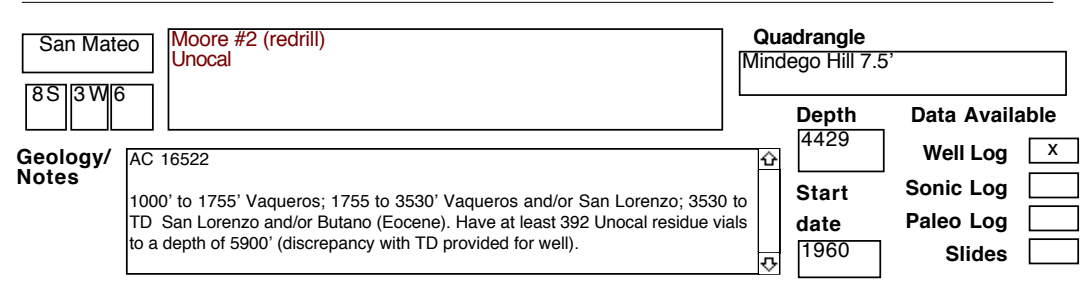

\begin{tabular}{|c|c|c|c|c|c|}
\hline San Mateo & \multirow[t]{2}{*}{$\begin{array}{l}\text { Union-Richfield-Costa \#2 (and redrill) } \\
\text { Costa Loma }\end{array}$} & \multicolumn{4}{|c|}{$\begin{array}{r}\text { Quadrangle } \\
\text { Big Basin 7.5 }\end{array}$} \\
\hline \multicolumn{3}{|c|}{\begin{tabular}{|l|l|l|}
$8 S$ & $3 \mathrm{~W}$ & 14 \\
\end{tabular}} & Depth & & \\
\hline \multirow{3}{*}{$\begin{array}{l}\text { Geology/ } \\
\text { Notes }\end{array}$} & 16481x. & 0 & & Well Log & $x$ \\
\hline & $\begin{array}{l}\text { Chevron slides from the interval } 35^{\prime} \text { to } 3490^{\prime} \text {. Have perhaps } 300 \text { Unocal } \\
\text { idue vials ( } \# 16481 \text { ), but many are not numbered consecutively. }\end{array}$ & & $\begin{array}{l}\text { Start } \\
\text { date }\end{array}$ & $\begin{array}{l}\text { Sonic Log } \\
\text { Paleo Log }\end{array}$ & \\
\hline & & & & Slides & $\bar{x}$ \\
\hline
\end{tabular}


SELECTED OIL TEST WELLS IN CENTRAL CALIFORNIA DATABASE County

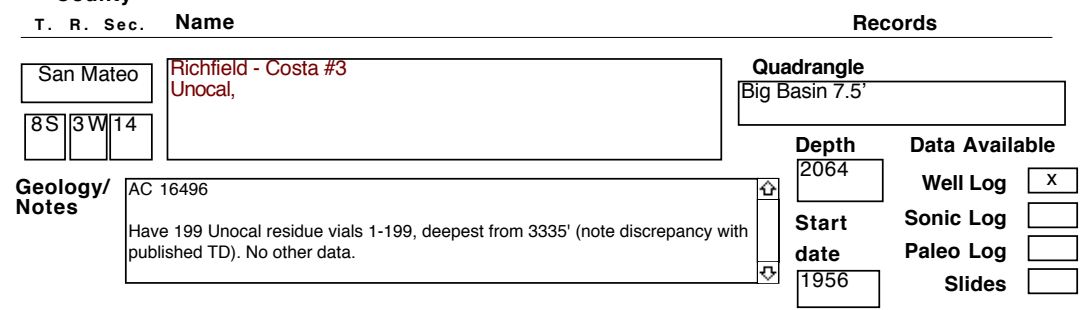

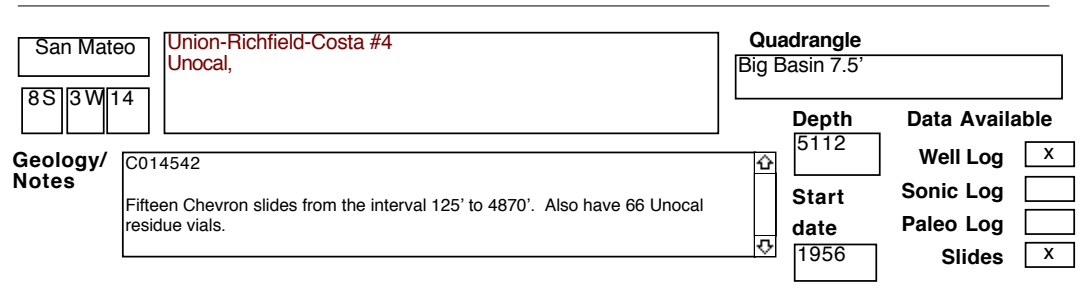

\begin{tabular}{|c|c|c|c|c|c|}
\hline San Mateo & \multirow[t]{2}{*}{ Chevron (Gulf) Oil Co. } & \multicolumn{4}{|c|}{$\begin{array}{l}\text { Quadrangle } \\
\text { Mindego Hill 7.5' }\end{array}$} \\
\hline${ }^{85} \|^{3 \mathrm{~V}}$ & & & \multirow{2}{*}{$\begin{array}{l}\text { Depth } \\
4430 ?\end{array}$} & \multicolumn{2}{|c|}{ Data Available } \\
\hline \multirow{4}{*}{$\begin{array}{l}\text { Geology/ } \\
\text { Notes }\end{array}$} & \multirow{4}{*}{\multicolumn{2}{|c|}{\begin{tabular}{|l|} 
AC \# 16526, AC 16539 \\
Purisima (Miocene/Pliocene undifferentiated) $80^{\prime}$ to $835^{\prime}$. Vaqueros Sandstone \\
(Oligocene) 835-950'. To $1390^{\prime}$ 'Miocene volcanics $950-1390^{\prime}$. To $1755^{\prime}$ \\
Vaqueros Sandstone 1390-1755'. To 3460' Vaqueros or San Lorenzo Formation \\
1755 to 3848'. 3848' top of Butano Sandstone (Eocene). Two slides from the \\
interval 340' to 3150'. Four slides from the interval 1639' to $4430^{\prime}$. One Chevron \\
slide from 118'. Approx 1,000 vials with residues and/or ditch samples.
\end{tabular}}} & & Well Log & $\mathrm{x}$ \\
\hline & & & Start & Sonic Log & \\
\hline & & & date & Paleo Log & $x$ \\
\hline & & & & & \\
\hline
\end{tabular}

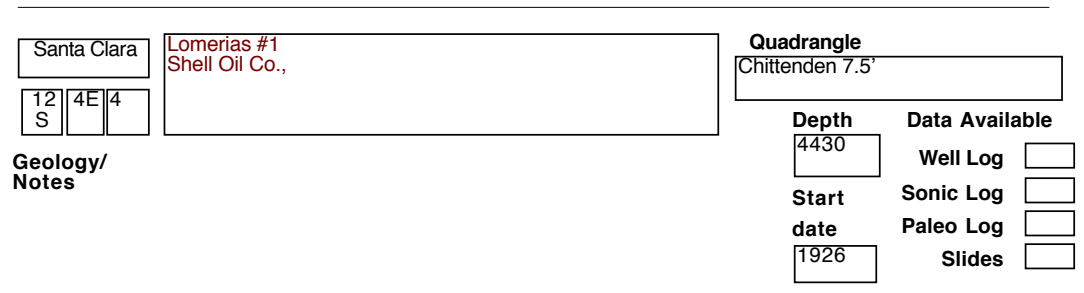

\begin{tabular}{|c|c|c|c|c|c|}
\hline Santa Clara & \multirow[t]{2}{*}{$\begin{array}{l}\text { Wauganen \#1 } \\
\text { Unocal, }\end{array}$} & \multicolumn{4}{|c|}{$\begin{array}{l}\text { Quadrangle } \\
\text { Watsonville 7.5' }\end{array}$} \\
\hline $\begin{array}{l}1 \\
S\end{array}\left\|^{3 E}\right\|^{2 t}$ & & \multirow{2}{*}{\multicolumn{2}{|c|}{$\begin{array}{l}\text { Depth } \\
5764\end{array}$}} & \multicolumn{2}{|c|}{ Data Available } \\
\hline \multirow{2}{*}{$\begin{array}{l}\text { Geology/ } \\
\text { Notes }\end{array}$} & \# 16311 & & & Well Log & $x$ \\
\hline & $\begin{array}{l}\text { to } 3880 \text { ' Slate Creek/Vaqueros (Miocene). to } 6426 \text { ' Vaqueros (Miocene). } \\
\text { ve Unocal residue vials } 3\left(30^{\prime}\right) \text { to } 378\left(3740^{\prime}\right) \text {. Have residues for } 3 \text { sidewall } \\
\text { mples, } 4249-6107 \text { '. }\end{array}$ & & $\begin{array}{l}\text { Start } \\
\text { date } \\
1953 ?\end{array}$ & $\begin{array}{r}\text { Sonic Log } \\
\text { Paleo Log } \\
\text { Slides }\end{array}$ & \\
\hline
\end{tabular}


SELECTED OIL TEST WELLS IN CENTRAL CALIFORNIA DATABASE County

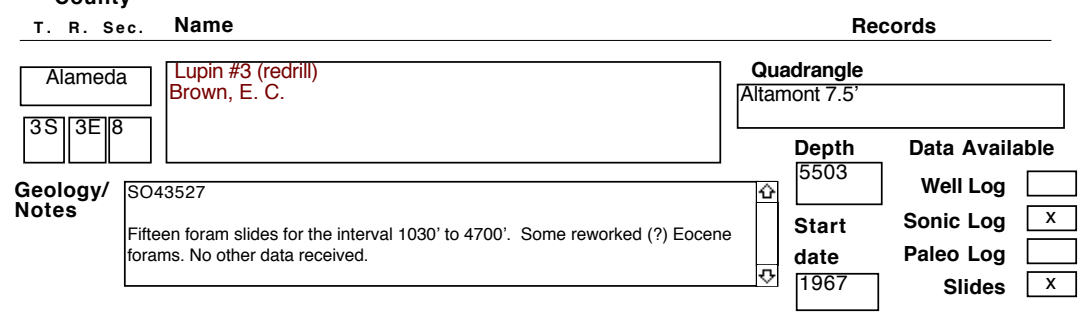

\begin{tabular}{|c|c|c|c|c|c|}
\hline \multirow{2}{*}{$\begin{array}{l}\text { Contra Costa } \\
2 \mathrm{~N} / 2 \mathrm{~W} / 24\end{array}$} & \multirow{2}{*}{$\begin{array}{l}\text { Boylan \#1 } \\
\text { Chevron, }\end{array}$} & \multicolumn{4}{|c|}{$\begin{array}{l}\text { Quadrangle } \\
\text { Vine Hill } 7.5^{\prime}\end{array}$} \\
\hline & & & \multirow{2}{*}{$\begin{array}{l}\text { Depth } \\
4442\end{array}$} & \multicolumn{2}{|c|}{ Data Available } \\
\hline \multirow{5}{*}{$\begin{array}{l}\text { Geology/ } \\
\text { Notes }\end{array}$} & 33853 & 今 & & Well Log & $\mathrm{x}$ \\
\hline & nema and Markley formations 0-1550. Nortonville shale $1550-1900^{\prime}$. & & Start & Sonic Log & $\mathrm{x}$ \\
\hline & mengine missing by faulting. Nortonville rests directly on Late Cretaceous $\mathrm{E}$ & & date & Paleo Log & $\mathrm{x}$ \\
\hline & $\begin{array}{l}10^{\prime}-3000^{\prime} \text {, where a fault brings these rocks in contact with Albian and } \\
\text { nomanian sandstones and shales. Have } 48 \text { foram slides } 1020-4442 \text { '. }\end{array}$ & & 1962 & Slides & $\mathrm{x}$ \\
\hline & & 可 & & & \\
\hline
\end{tabular}

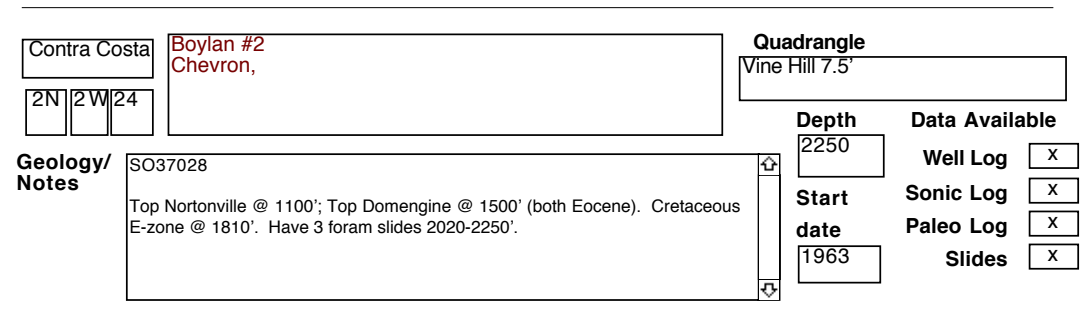

\begin{tabular}{|c|c|c|c|c|c|}
\hline Contra Costa & \multirow[t]{2}{*}{$\begin{array}{l}\text { Las Trampas Unit A \#2 } \\
\text { Phillips Petroleum Co., }\end{array}$} & \multicolumn{4}{|c|}{$\begin{array}{l}\text { Quadrangle } \\
\begin{array}{|l}\text { Las Trampas Ridge 7.5' }\end{array}\end{array}$} \\
\hline \begin{tabular}{|l|l|l|}
$1 S$ & 24 \\
\end{tabular} & & \multirow{2}{*}{\multicolumn{2}{|c|}{$\begin{array}{r}\text { Depth } \\
8535\end{array}$}} & \multicolumn{2}{|c|}{ Data Available } \\
\hline \multirow{3}{*}{$\begin{array}{l}\text { Geology/ } \\
\text { Notes }\end{array}$} & \multirow{3}{*}{$\begin{array}{l}\text { ave slides or the interval } 108^{\prime} \text { to } 8535^{\prime} \text {. Oursan (Miocene)660-960'. Base of } \\
\text { obrante inferred at } 4945 \text { '. Have mud log and } 46 \text { foram slides } 108-8535 \text { ' (slides } \\
\text { 5-37 missing). }\end{array}$} & & & Well Log & $\mathrm{x}$ \\
\hline & & & Start & Sonic Log & \\
\hline & & & 1964 & Slides & $\mathrm{x}$ \\
\hline
\end{tabular}

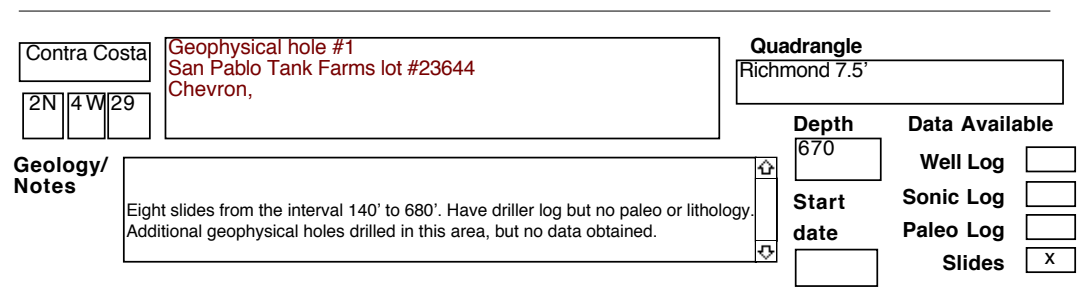


SELECTED OIL TEST WELLS IN CENTRAL CALIFORNIA DATABASE County

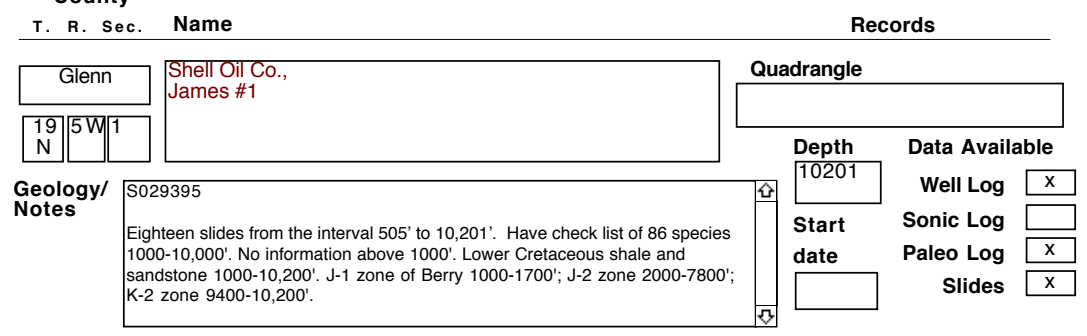

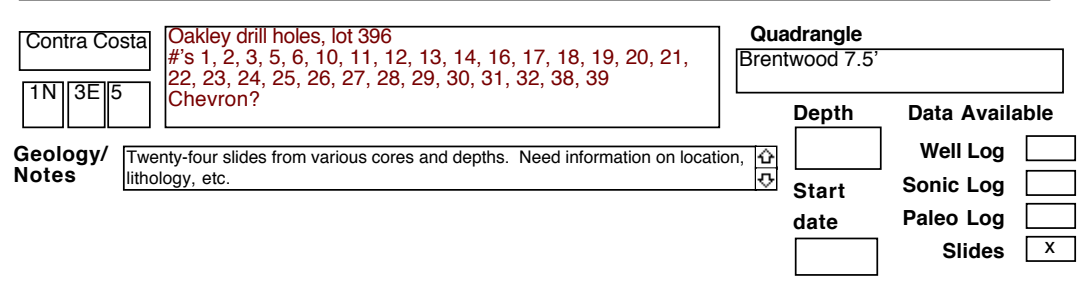

\begin{tabular}{l|l|l|l|l|l|l|}
\hline Contra Costa & $\begin{array}{l}\text { Hofman et al \#1 } \\
\text { Chevron, }\end{array}$ \\
\hline
\end{tabular}

\begin{tabular}{|c|c|c|c|c|c|}
\hline \multirow{2}{*}{\begin{tabular}{|l|} 
Contra Costa \\
$2 \mathrm{~N}$ \\
\end{tabular}} & \multirow{2}{*}{$\begin{array}{l}\text { Ginochio \#2 } \\
\text { Pacific Gas \&Electric (formerly McCulloch Oil ) }\end{array}$} & \multicolumn{4}{|c|}{$\begin{array}{c}\text { Quadrangle } \\
\text { Walnut Creek 7.5' }\end{array}$} \\
\hline & & \multirow{2}{*}{\multicolumn{2}{|c|}{$\begin{array}{l}\text { Depth } \\
4910 ? \\
\text { Start } \\
\text { date }\end{array}$}} & \multicolumn{2}{|c|}{ Data Available } \\
\hline $\begin{array}{l}\text { Geology/ } \\
\text { Notes }\end{array}$ & $\begin{array}{l}\text { per Eocene } 2150^{\prime} \text { to } 2870^{\prime} \text {. Nortonville @ } 2930^{\prime} \text { to } 3000^{\prime} \text {. Martinez } \\
00-3140^{\prime} \text {. Fault at } 3140 \text { brings Martinez over Domengine. Domengine } \\
40-4075 \text {. Fault at } 4075 \text { ' brings Domengine over Nortonville. Nortonville from } \\
75 \text { to } 4485 \text {. Domengine from } 4485 \text { 't } 4745^{\prime} \text { (both Eocene). Martinez } \\
45-T D \text {. Have mud log. Ten foram slides } 3020-4810^{\prime} \text {. }\end{array}$ & & & $\begin{array}{l}\text { Well Log } \\
\text { Sonic Log } \\
\text { Paleo Log }\end{array}$ & $\frac{x}{x}$ \\
\hline
\end{tabular}

\begin{tabular}{|c|c|c|c|c|c|}
\hline Contra Costa & \multirow[t]{2}{*}{\begin{tabular}{|l} 
Vierra \#1 \\
Phillips Petroleum Co.,
\end{tabular}} & \multicolumn{4}{|c|}{$\begin{array}{l}\text { Quadrangle } \\
\text { Byron Hot Springs 7.5' }\end{array}$} \\
\hline \begin{tabular}{|l|l|l|}
$1 S$ & $3 E$ & 9
\end{tabular} & & \multirow{2}{*}{\multicolumn{2}{|c|}{$\begin{array}{l}\text { Depth } \\
\text { is } 8733\end{array}$}} & \multicolumn{2}{|l|}{ Data Available } \\
\hline \multirow{3}{*}{$\begin{array}{l}\text { Geology/ } \\
\text { Notes }\end{array}$} & 3867 & & & Well Log & \\
\hline & d logs but no paleo or formation information available. Have mud log. & (a) & Start & Sonic Log & $\mathrm{x}$ \\
\hline & & & $\begin{array}{l}\text { date } \\
1981\end{array}$ & Slides & \\
\hline
\end{tabular}


SELECTED OIL TEST WELLS IN CENTRAL CALIFORNIA DATABASE County

\begin{tabular}{|c|c|c|c|c|}
\hline T. R. Sec. & Name & \multicolumn{3}{|c|}{ Records } \\
\hline Alameda & \multirow[t]{2}{*}{\begin{tabular}{|l} 
Livermore \#1 \\
Buttes
\end{tabular}} & \multicolumn{3}{|l|}{$\begin{array}{r}\text { Quadrangle } \\
\text { Altamont 7.5' }\end{array}$} \\
\hline $2 \mathrm{~S}\left\|^{2 \mathrm{E}}\right\|^{27}$ & & \multirow{2}{*}{$\begin{array}{l}\text { Depth } \\
3837\end{array}$} & \multicolumn{2}{|c|}{ Data Available } \\
\hline \multirow{3}{*}{\multicolumn{2}{|c|}{$\begin{array}{l}\text { Geology/ Need information } \\
\text { Notes }\end{array}$}} & & Well Log & \\
\hline & & Start & Sonic Log & $\mathrm{x}$ \\
\hline & & $\begin{array}{l}\text { date } \\
1968 ?\end{array}$ & $\begin{array}{r}\text { Paleo Log } \\
\text { Slides }\end{array}$ & \\
\hline
\end{tabular}

\begin{tabular}{|c|c|c|c|c|c|}
\hline Alameda & \multirow[t]{2}{*}{$\begin{array}{l}\text { Wisner Unit 1 \#2 and } 3 \\
\text { Castle }\end{array}$} & \multicolumn{4}{|c|}{$\begin{array}{r}\text { Quadrangle } \\
\text { Altamont 7.5' }\end{array}$} \\
\hline \begin{tabular}{|l|l|l|l|}
$3 S$ & $3 \mathrm{E}$ \\
\end{tabular} & & \multirow{2}{*}{\multicolumn{2}{|c|}{$\begin{array}{l}\text { Depth } \\
3091\end{array}$}} & \multicolumn{2}{|c|}{ Data Available } \\
\hline \multirow{3}{*}{$\begin{array}{l}\text { Geology/ } \\
\text { Notes }\end{array}$} & \multirow{3}{*}{ Need more information } & & & Well Log & \\
\hline & & & Start & Sonic Log & $\mathrm{x}$ \\
\hline & & & $\begin{array}{l}\text { date } \\
1983\end{array}$ & $\begin{array}{r}\text { Paleo Log } \\
\text { Slides }\end{array}$ & \\
\hline
\end{tabular}

\begin{tabular}{|c|c|c|c|c|c|}
\hline Alameda & \multirow[t]{2}{*}{$\begin{array}{l}\text { SPRR \#1 } \\
\text { Capitol Oil }\end{array}$} & \multicolumn{4}{|c|}{$\begin{array}{r}\text { Quadrangle } \\
\text { Altamont 7.5' }\end{array}$} \\
\hline \begin{tabular}{|l|l|l|}
$3 S$ & $2 E$ & 1
\end{tabular} & & \multirow{2}{*}{\multicolumn{2}{|c|}{$\begin{array}{l}\text { Depth } \\
4502\end{array}$}} & \multicolumn{2}{|c|}{ Data Available } \\
\hline \multirow{3}{*}{$\begin{array}{l}\text { Geology/ } \\
\text { Notes }\end{array}$} & \multirow{3}{*}{ eed more information. } & & & Well Log & \\
\hline & & . & Start & Sonic Log & $x$ \\
\hline & & & date & Paleo Log & \\
\hline
\end{tabular}

\begin{tabular}{|c|c|c|c|c|c|}
\hline Alameda & \multirow[t]{2}{*}{$\begin{array}{l}\text { Garvanta } 68-22 \\
\text { Chowchilla Gas Co. }\end{array}$} & \multicolumn{4}{|c|}{$\begin{array}{r}\text { Quadrangle } \\
\text { Altamont } 7.5^{\prime}\end{array}$} \\
\hline \begin{tabular}{|l|l|l}
$2 S$ & $2 E$ \\
\end{tabular} & & \multirow{2}{*}{\multicolumn{2}{|c|}{$\begin{array}{l}\text { Depth } \\
2575\end{array}$}} & \multicolumn{2}{|c|}{ Data Available } \\
\hline \multirow{3}{*}{$\begin{array}{l}\text { Geology/ } \\
\text { Notes }\end{array}$} & \multirow{3}{*}{ Need more information } & & & Well Log & \\
\hline & & 急 & Start & Sonic Log & $x$ \\
\hline & & & $\begin{array}{l}\text { date } \\
1968\end{array}$ & $\begin{array}{r}\text { Paleo Log } \\
\text { Slides }\end{array}$ & \\
\hline
\end{tabular}

\begin{tabular}{|c|c|c|c|c|c|}
\hline Alameds & \multirow[t]{2}{*}{$\begin{array}{l}\text { Miller-Richards \#1-1 } \\
\text { Hershey Oil Co. }\end{array}$} & \multicolumn{4}{|c|}{$\begin{array}{r}\text { Quadrangle } \\
\text { Altamont 7.5' }\end{array}$} \\
\hline \begin{tabular}{|l|l|l|l|}
$3 S$ & $2 E$
\end{tabular} & & \multirow{2}{*}{\multicolumn{2}{|c|}{$\begin{array}{l}\text { Depth } \\
6313\end{array}$}} & \multicolumn{2}{|c|}{ Data Available } \\
\hline \multirow{3}{*}{$\begin{array}{l}\text { Geology/ } \\
\text { Notes }\end{array}$} & \multirow{3}{*}{ Need more information } & & & Well Log & \\
\hline & & (3) & Start & Sonic Log & $x$ \\
\hline & & & $\begin{array}{l}\text { date } \\
1981\end{array}$ & $\begin{array}{r}\text { Paleo Log } \\
\text { Slides }\end{array}$ & \\
\hline
\end{tabular}


SELECTED OIL TEST WELLS IN CENTRAL CALIFORNIA DATABASE County

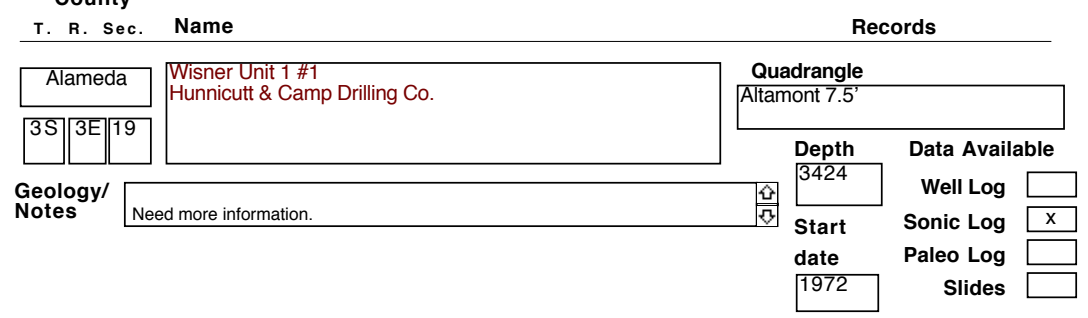

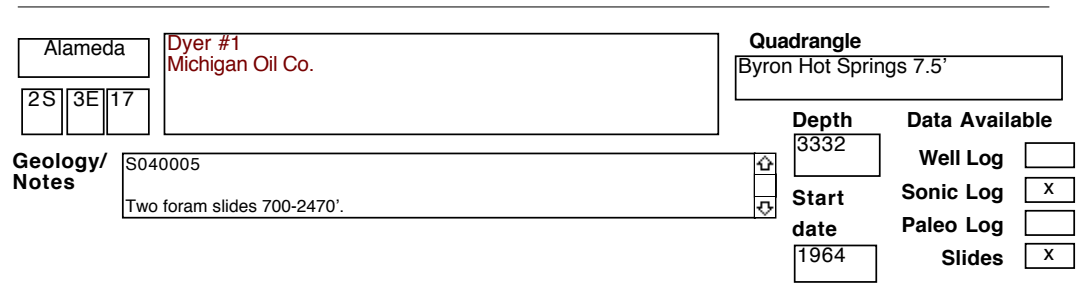

\begin{tabular}{|c|c|c|c|c|c|}
\hline Alameda & \multirow[t]{2}{*}{$\begin{array}{l}\text { Guidotti \#1 } \\
\text { McCulloch Oil Corp. }\end{array}$} & \multicolumn{4}{|c|}{$\begin{array}{r}\text { Quadrangle } \\
\text { Altamont } 7.5^{\prime}\end{array}$} \\
\hline $3 S|3 E| 6$ & & & Depth & Data Availa & \\
\hline \multirow{3}{*}{$\begin{array}{l}\text { Geology/ } \\
\text { Notes }\end{array}$} & \multirow{3}{*}{ eed additional information } & 今 & & Well Log & \\
\hline & & & Start & Sonic Log & $\mathrm{x}$ \\
\hline & & & date & Paleo Log & \\
\hline
\end{tabular}

\begin{tabular}{|c|c|c|c|c|}
\hline Alameda & \multirow[t]{2}{*}{$\begin{array}{l}\text { Castello \#1-13 } \\
\text { Superior Oil Co. }\end{array}$} & \multicolumn{3}{|c|}{$\begin{array}{l}\text { Quadrangle } \\
\text { Clifton Court Forebay 7.5' }\end{array}$} \\
\hline \begin{tabular}{|l|l|l|}
$2 S$ & $3 E$ & 13
\end{tabular} & & \multirow{2}{*}{$\begin{array}{l}\text { Depth } \\
6004\end{array}$} & \multicolumn{2}{|c|}{ Data Available } \\
\hline \multirow{3}{*}{\multicolumn{2}{|c|}{$\begin{array}{l}\text { Geology/ Probably all Cretaceous, F-2, G-2. } \\
\text { Notes }\end{array}$}} & & Well Log & $\mathrm{x}$ \\
\hline & & Start & Sonic Log & $\mathrm{x}$ \\
\hline & & $\begin{array}{l}\text { date } \\
1964\end{array}$ & $\begin{array}{r}\text { Paleo Log } \\
\text { Slides }\end{array}$ & $\mathrm{x}$ \\
\hline
\end{tabular}

\begin{tabular}{|c|c|c|c|c|c|}
\hline Sonoma & \multirow[t]{2}{*}{$\begin{array}{l}\text { Wesley Haire \#2 } \\
\text { General Crude }\end{array}$} & \multicolumn{4}{|c|}{$\begin{array}{l}\text { Quadrangle } \\
\text { Cuttings Wharf (?) 7.5' }\end{array}$} \\
\hline $5 \mathrm{~N} 5 \mathrm{~W} 35$ & & \multirow{2}{*}{\multicolumn{2}{|c|}{$\begin{array}{l}\text { Depth } \\
5409\end{array}$}} & \multicolumn{2}{|c|}{ Data Available } \\
\hline \multirow{3}{*}{$\begin{array}{l}\text { Geology/ } \\
\text { Notes }\end{array}$} & \multirow{3}{*}{$\begin{array}{l}\text { Paleo and e log available for } 3700^{\prime} \text { to TD. Zemorrian shale } 4300 \text { to } 4865 \text { '. } \\
\text { Zemorrian sand } 4865 \text { to } 5110^{\prime} \text { '. Upper Cretaceous with Inoceramus prisms and } \\
\text { arenaceous foram fauna } 5110 \text { to TD. }\end{array}$} & & & Well Log & \\
\hline & & & Start & Sonic Log & \\
\hline & & 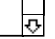 & $\begin{array}{l}\text { date } \\
1967\end{array}$ & Paleo Log & $x$ \\
\hline
\end{tabular}


SELECTED OIL TEST WELLS IN CENTRAL CALIFORNIA DATABASE County

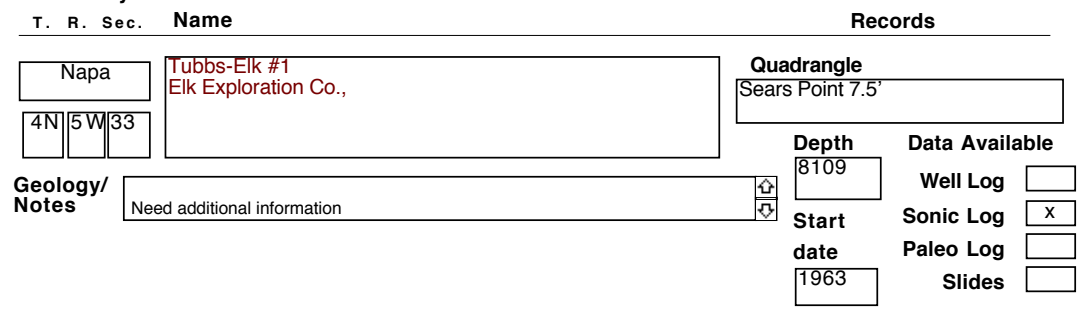

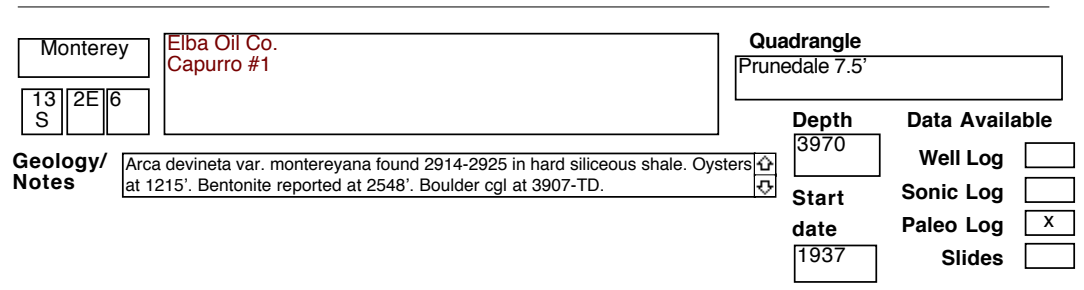

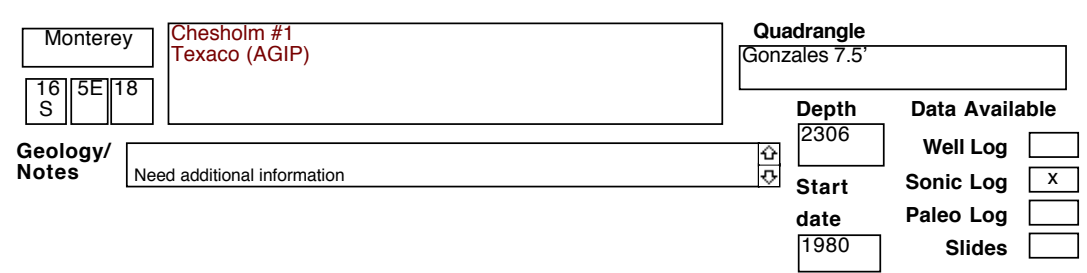

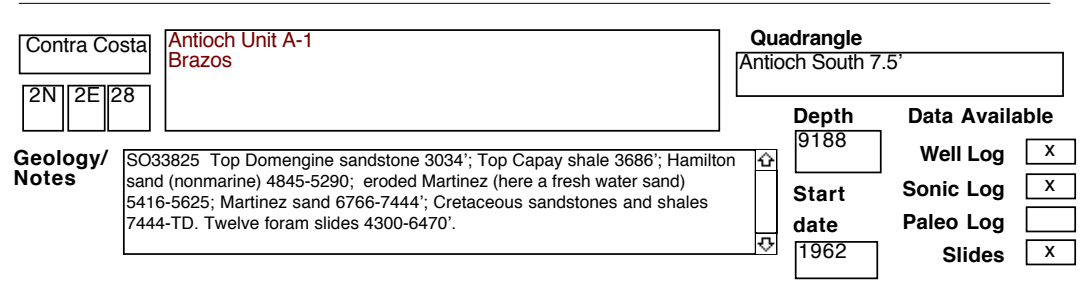

\begin{tabular}{|c|c|c|c|c|c|}
\hline Alameda & \multirow[t]{2}{*}{$\begin{array}{l}\text { Gomes \#1 } \\
\text { Brazos Oil Corp. }\end{array}$} & \multicolumn{4}{|c|}{$\begin{array}{l}\text { Quadrangle } \\
\text { Clifton Court Forebay 7.5' }\end{array}$} \\
\hline \begin{tabular}{|l|l|l|}
$2 S$ & 35 \\
\end{tabular} & & \multirow{2}{*}{\multicolumn{2}{|c|}{$\begin{array}{l}\text { Depth } \\
4013\end{array}$}} & \multicolumn{2}{|c|}{ Data Available } \\
\hline \multirow{3}{*}{$\begin{array}{l}\text { Geology/ } \\
\text { Notes }\end{array}$} & & & & Well Log & \\
\hline & ed additional information & 胢 & Start & Sonic Log & $\mathrm{x}$ \\
\hline & & & \begin{tabular}{|l} 
date \\
1962
\end{tabular} & $\begin{array}{r}\text { Paleo Log } \\
\text { Slides }\end{array}$ & \\
\hline
\end{tabular}


SELECTED OIL TEST WELLS IN CENTRAL CALIFORNIA DATABASE

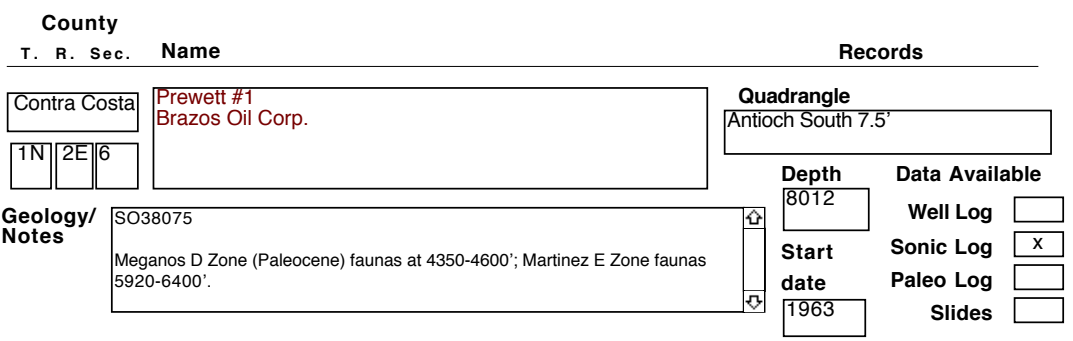

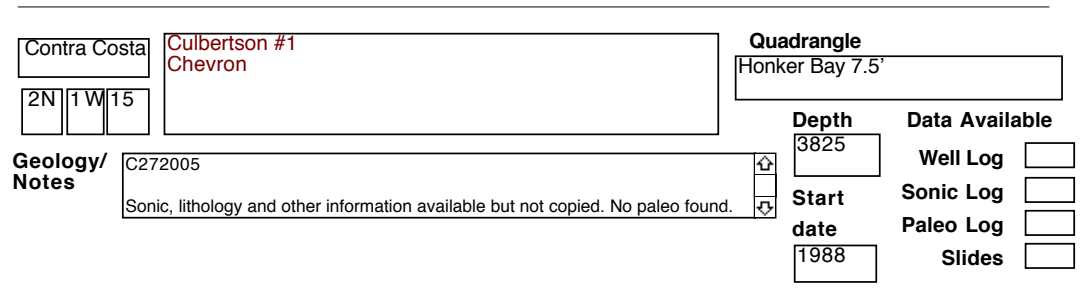

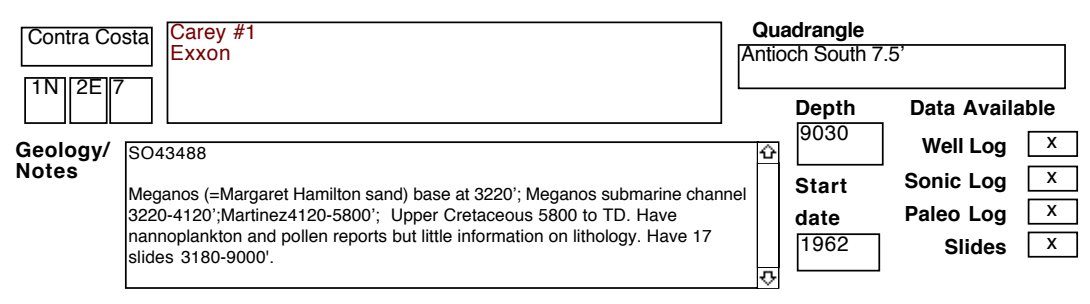

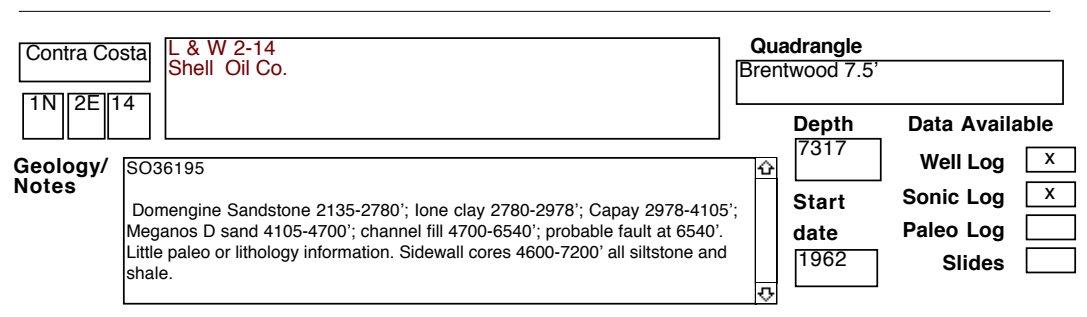

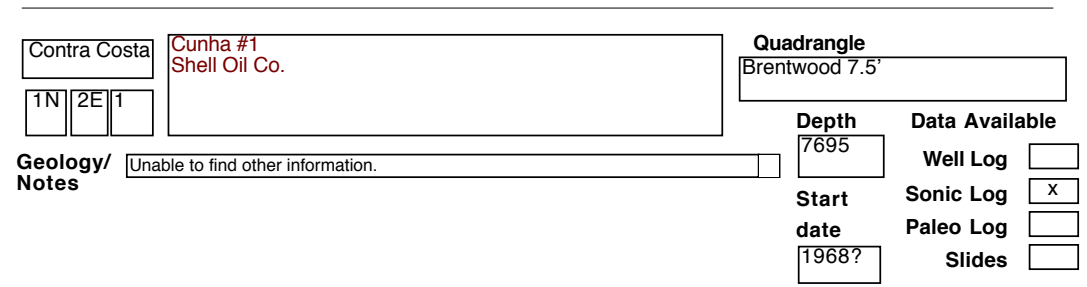




\section{SELECTED OIL TEST WELLS IN CENTRAL CALIFORNIA DATABASE}

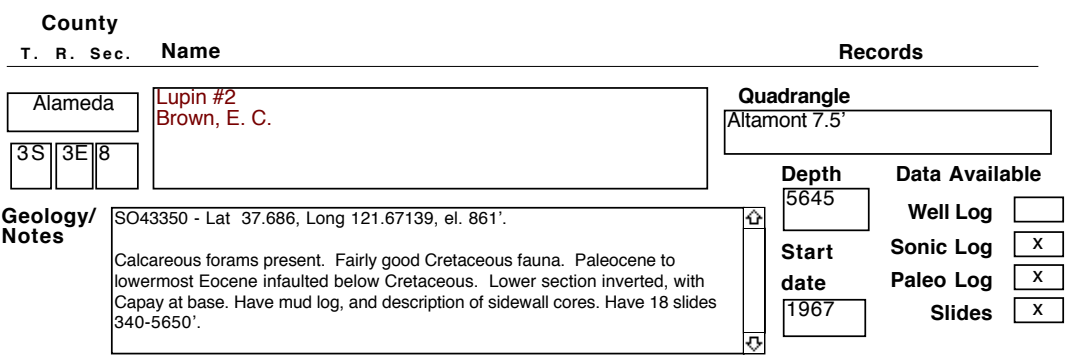

\begin{tabular}{|c|c|c|c|c|c|}
\hline Alameda & \multirow{2}{*}{$\begin{array}{l}\text { Signal-Hancock-Christensen \# } 1 \\
\text { Texaco }\end{array}$} & \multicolumn{4}{|c|}{ Quadrangle } \\
\hline \begin{tabular}{|l|l|l}
$2 S$ & 26 \\
\end{tabular} & & \multirow{2}{*}{\multicolumn{2}{|c|}{$\begin{array}{l}\text { Depth } \\
3511\end{array}$}} & \multicolumn{2}{|c|}{ Data Available } \\
\hline \multirow{2}{*}{$\begin{array}{l}\text { Geology/ } \\
\text { Notes }\end{array}$} & 36044 & & & Well Log & $\mathrm{x}$ \\
\hline & $\begin{array}{l}\text { dded in Orinda. Contact with Cretaceous at } 466 \text { '. Has good faunas of Late } \\
\text { aceous (Campanian, F-2 of Goudkoff) age. E log suggests sandstone and } \\
\text { e. }\end{array}$ & 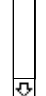 & $\begin{array}{l}\text { Start } \\
\text { date } \\
1953\end{array}$ & $\begin{array}{r}\text { Sonic Log } \\
\text { Paleo Log } \\
\text { Slides }\end{array}$ & $\mathrm{x}$ \\
\hline
\end{tabular}

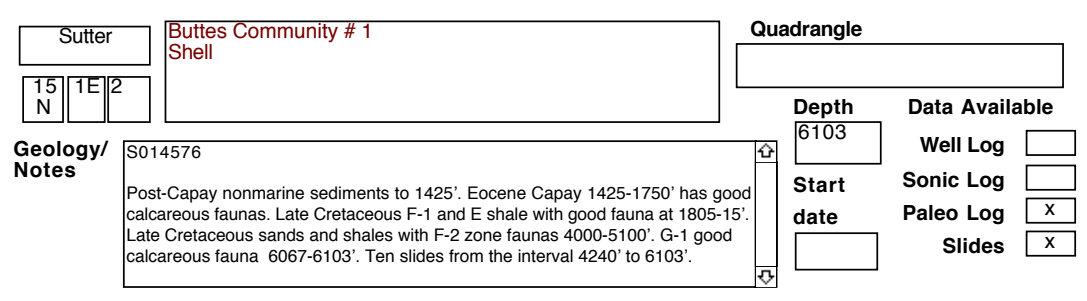

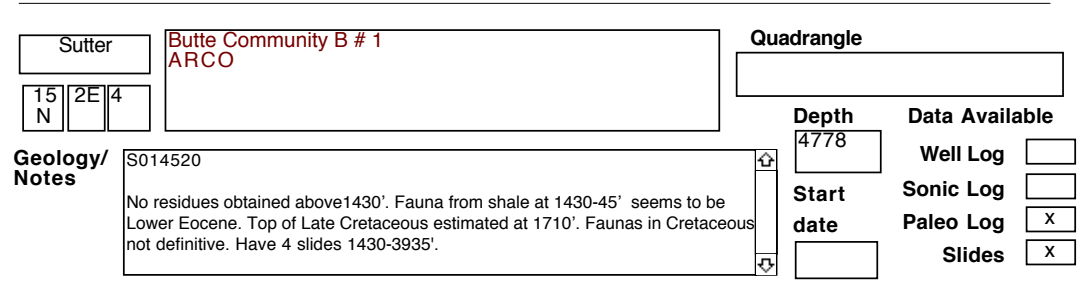

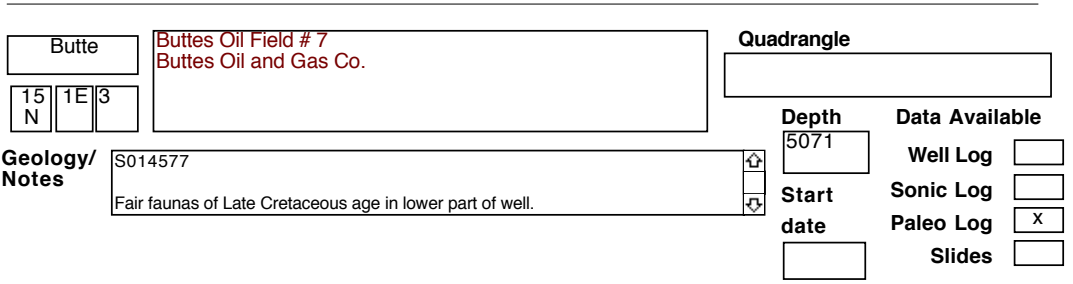




\section{SELECTED OIL TEST WELLS IN CENTRAL CALIFORNIA DATABASE}

County

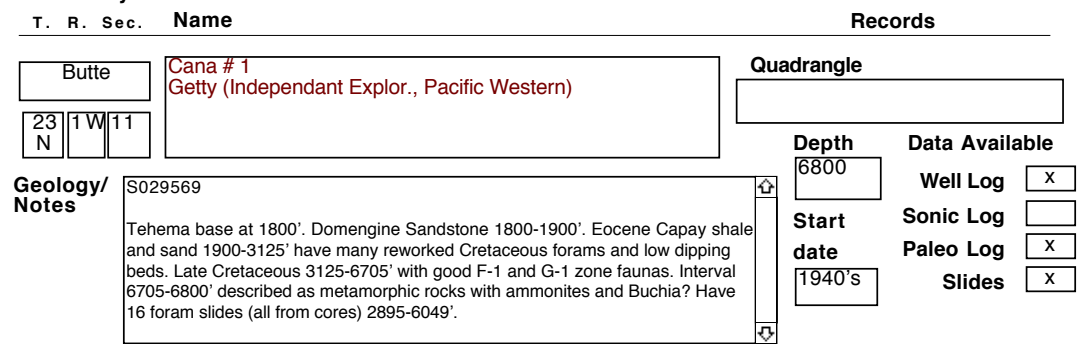

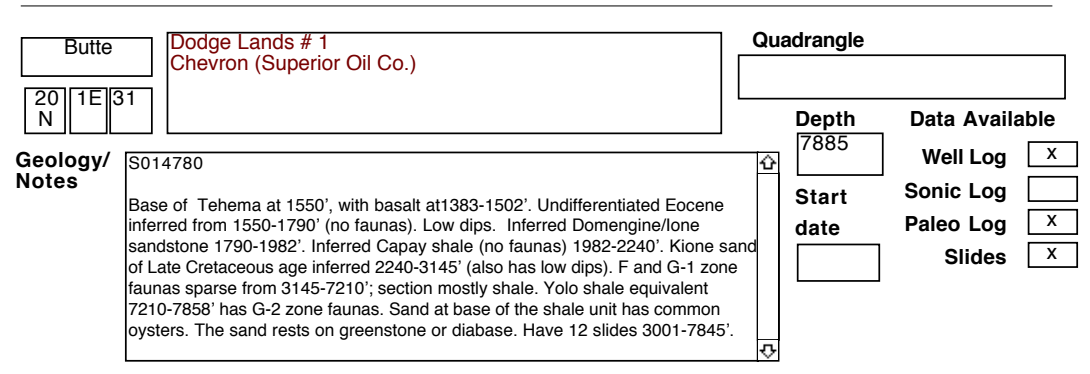

\begin{tabular}{l|l|l|l|l|l|l|l|}
\hline \multicolumn{1}{|c|}{ Butte } & $\begin{array}{l}\text { Donohoe Fee \# } 1 \\
\text { Chevron }\end{array}$ \\
\hline
\end{tabular}

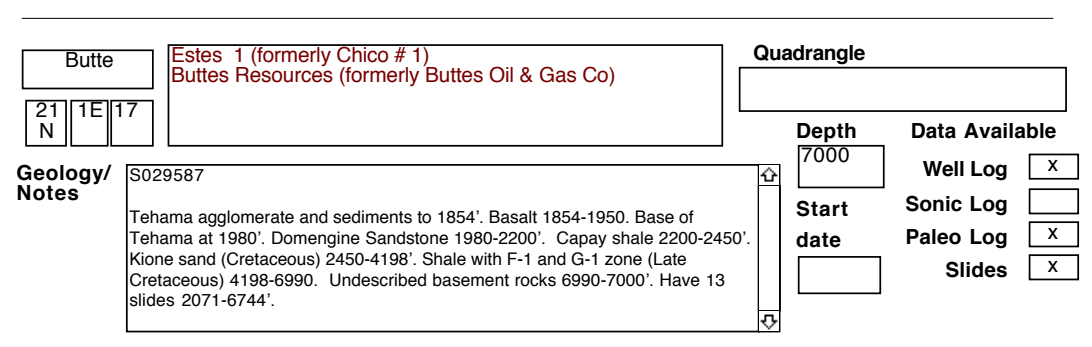

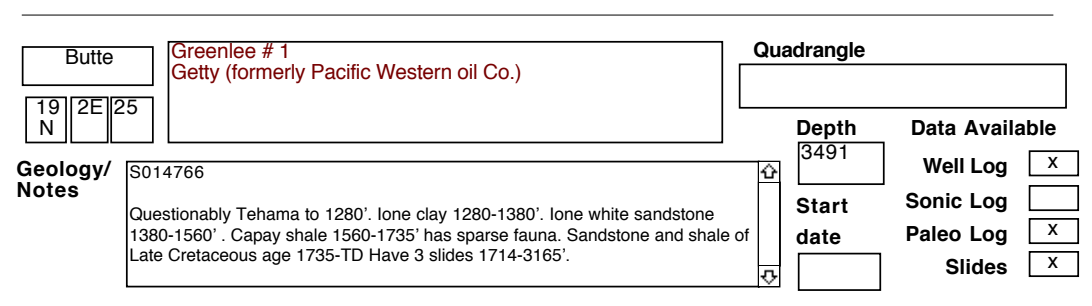




\section{SELECTED OIL TEST WELLS IN CENTRAL CALIFORNIA DATABASE}

County

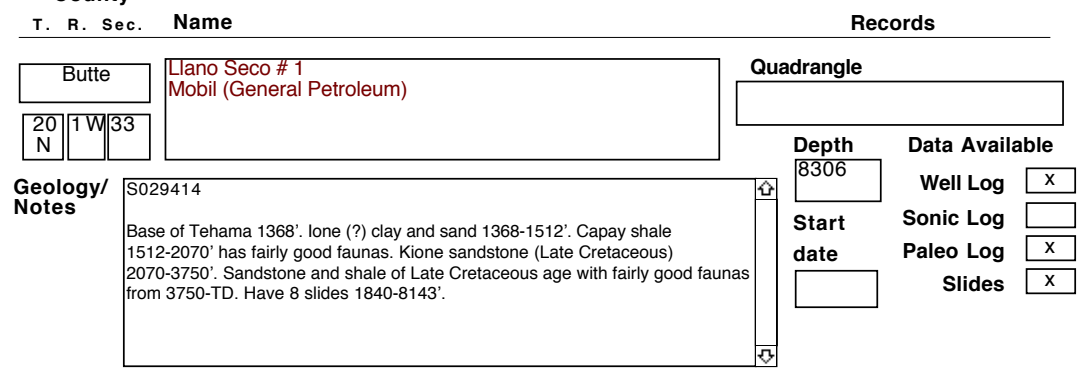

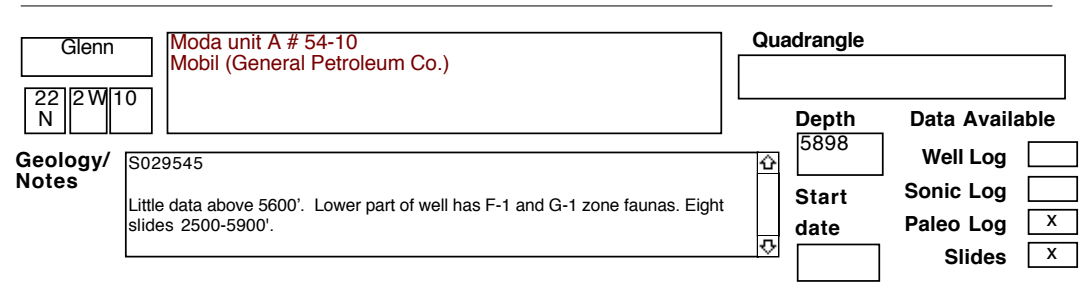

\begin{tabular}{l|l|l|l|l|l|l|}
\hline \multicolumn{1}{|c|}{ Butte } & $\begin{array}{l}\text { Odell \# } 1 \\
\text { Chevron }\end{array}$ & \multicolumn{2}{l|}{ Quadrangle } \\
\hline
\end{tabular}

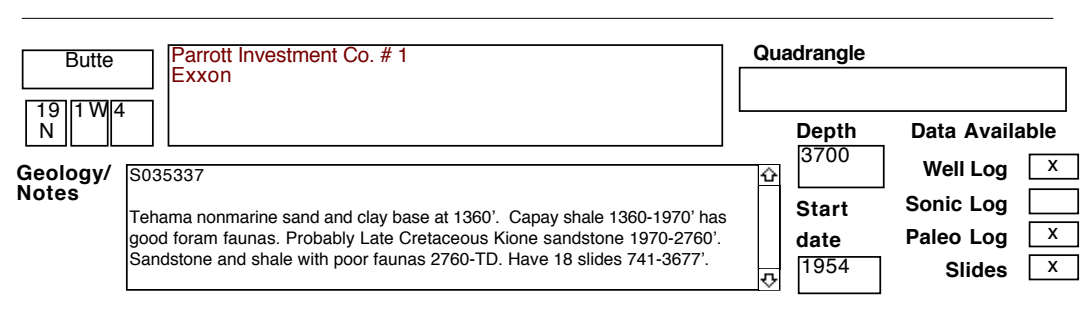

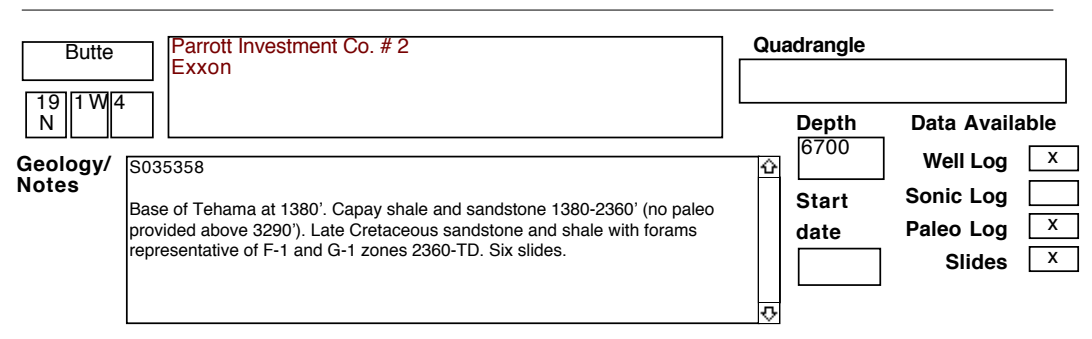


SELECTED OIL TEST WELLS IN CENTRAL CALIFORNIA DATABASE County

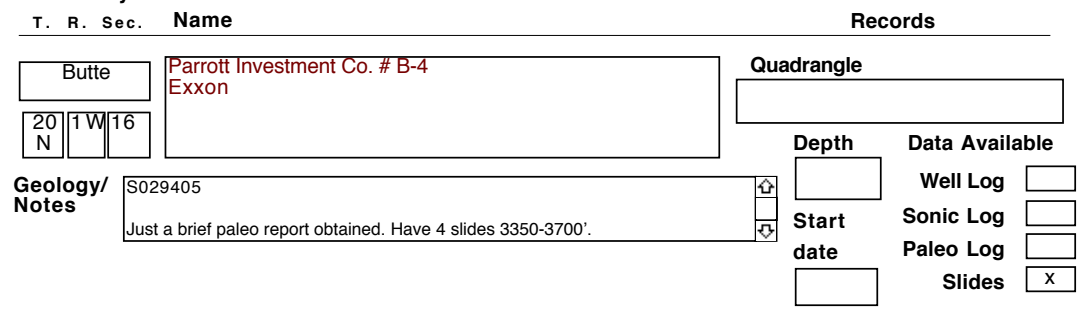

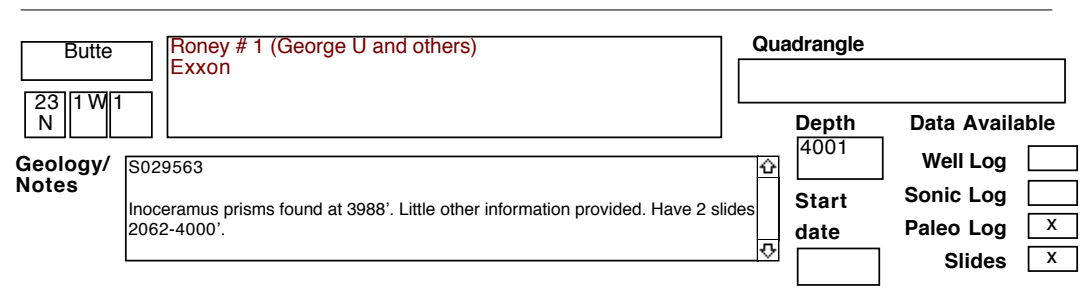

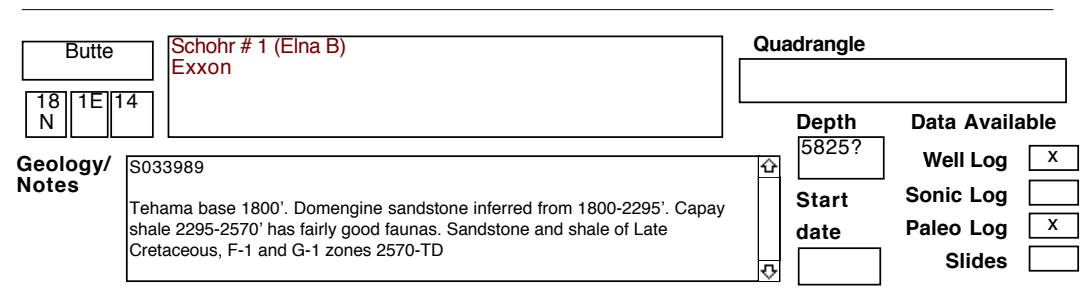

\begin{tabular}{|l|l|l|l|l|l|l|}
\hline \multicolumn{1}{|c|}{$\begin{array}{l}\text { Scammon \# 14-18 } \\
\text { Unocal }\end{array}$} \\
\hline
\end{tabular}

\begin{tabular}{l|l|l|l|l|l|l|}
\hline \multicolumn{1}{|c|}{$\begin{array}{l}\text { Wahi \# 1 } \\
\text { Chevron }\end{array}$} \\
\hline
\end{tabular}


SELECTED OIL TEST WELLS IN CENTRAL CALIFORNIA DATABASE County

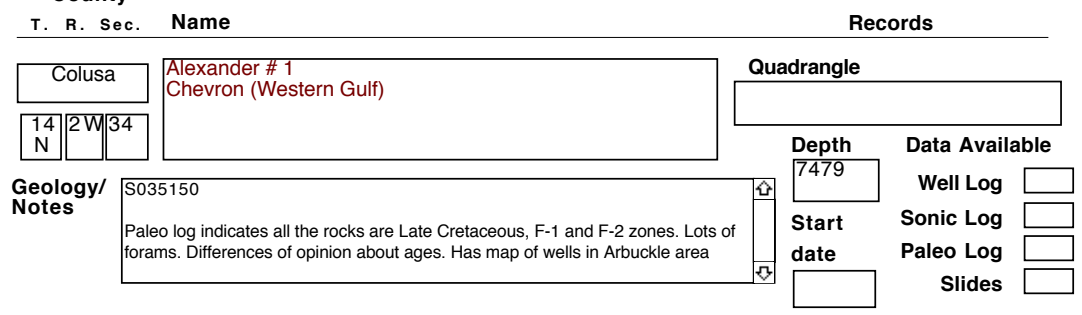

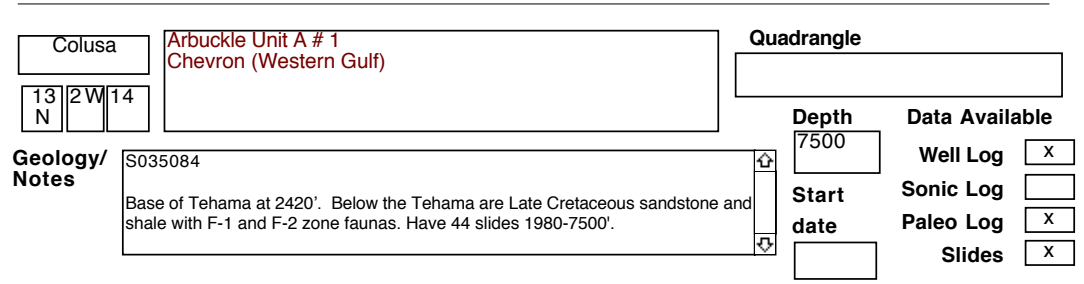

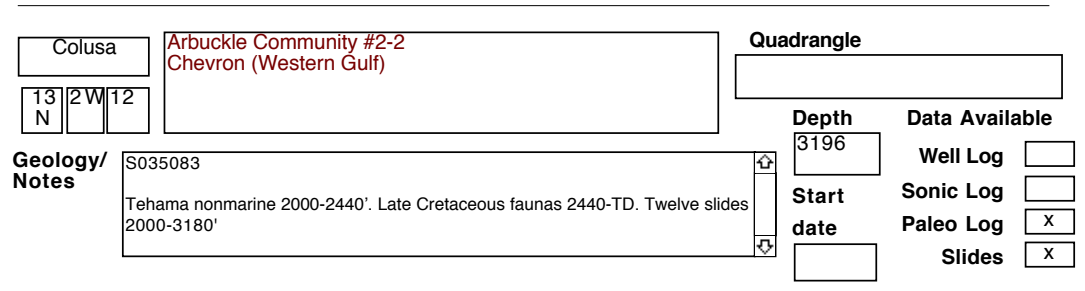

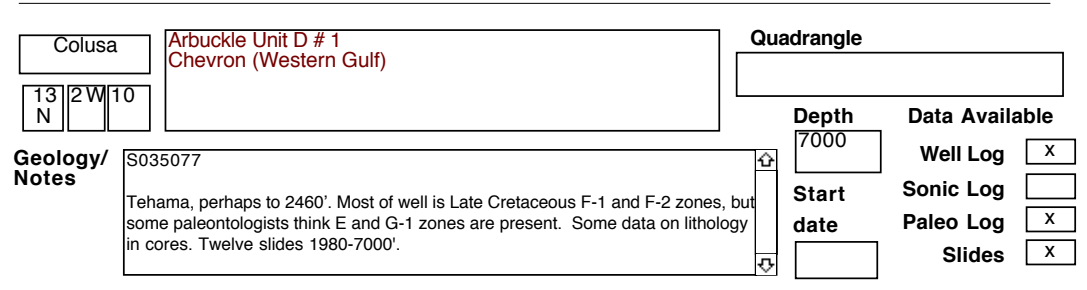

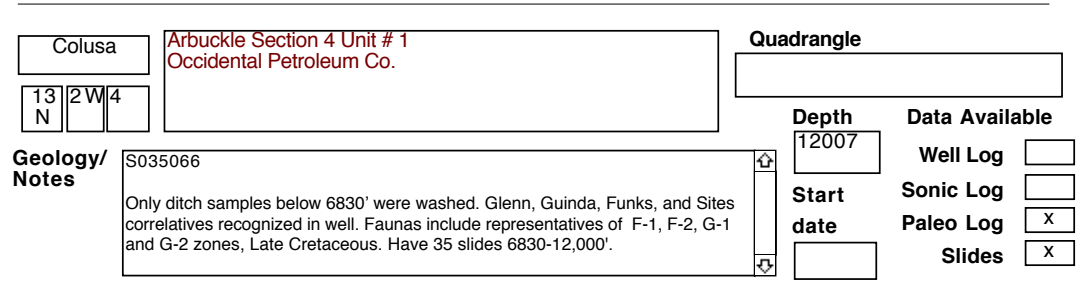


SELECTED OIL TEST WELLS IN CENTRAL CALIFORNIA DATABASE County

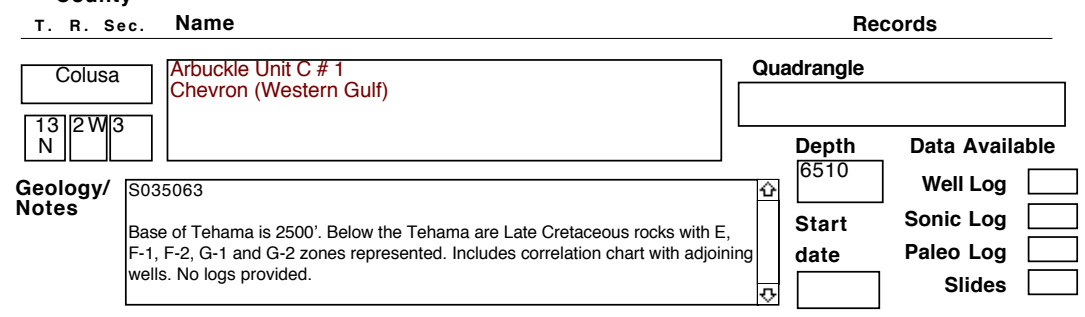

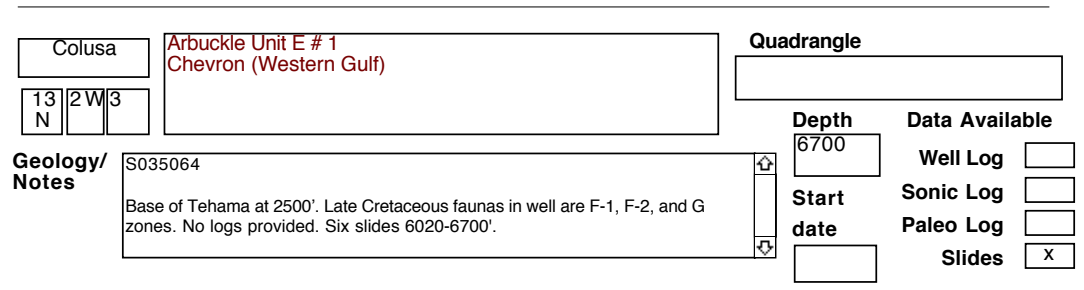

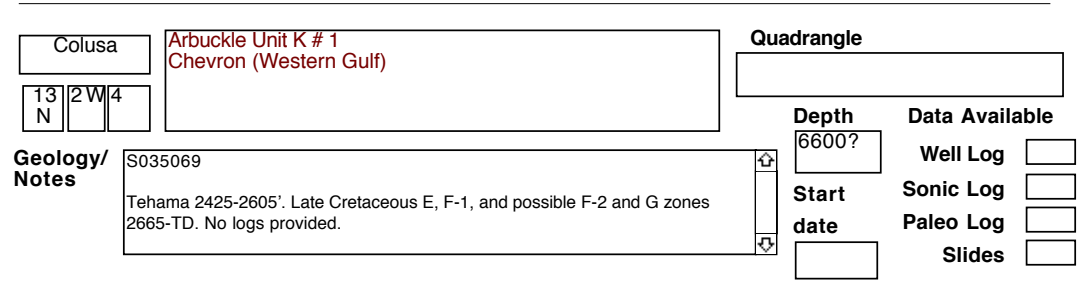

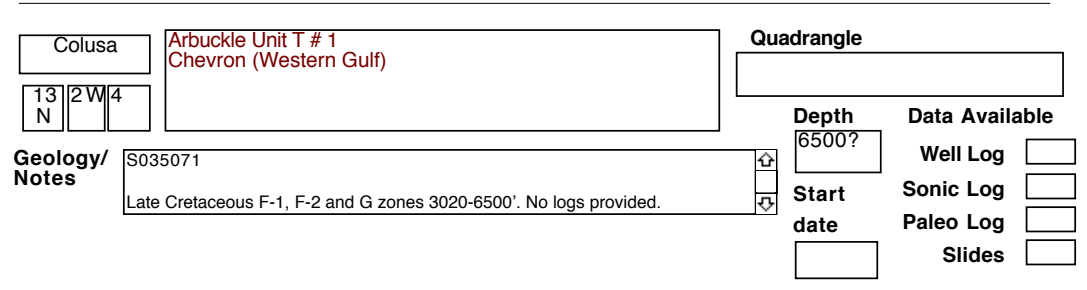

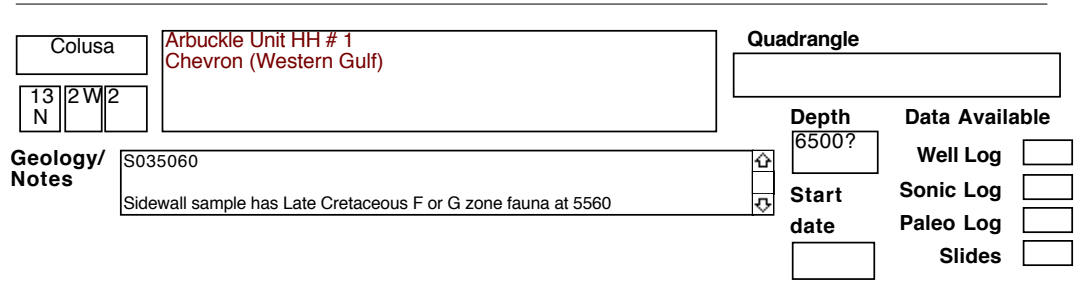


SELECTED OIL TEST WELLS IN CENTRAL CALIFORNIA DATABASE County

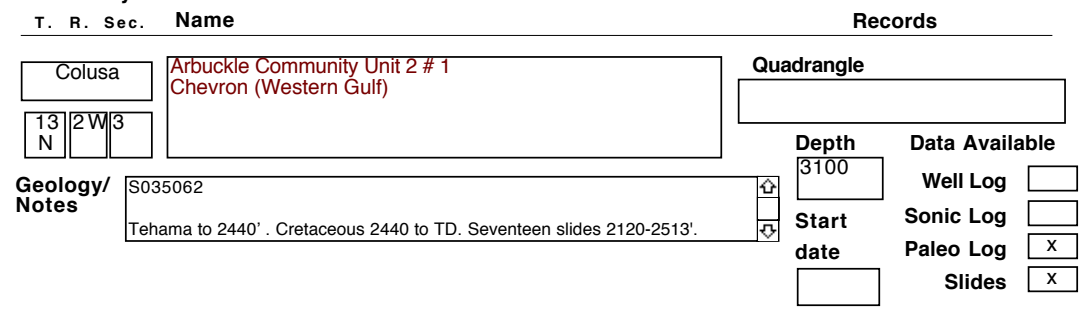

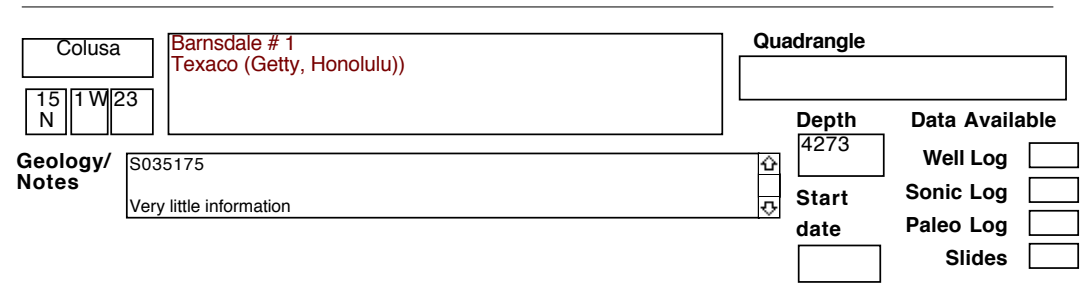

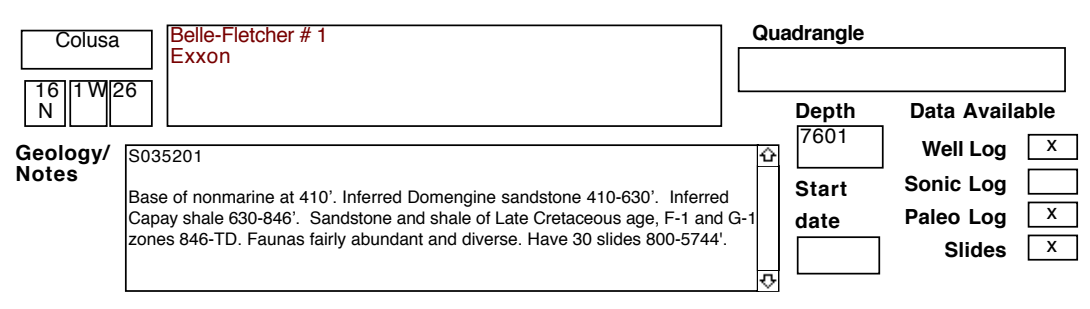

\begin{tabular}{l|l|l|l|l|l|l|}
\hline \multicolumn{1}{|c|}{$\begin{array}{l}\text { Colusa } \\
\text { Exxon }\end{array}$} \\
\hline
\end{tabular}

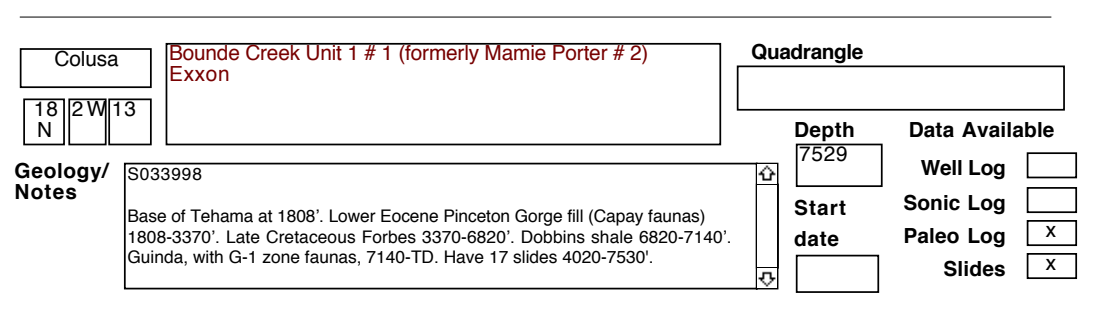


SELECTED OIL TEST WELLS IN CENTRAL CALIFORNIA DATABASE County

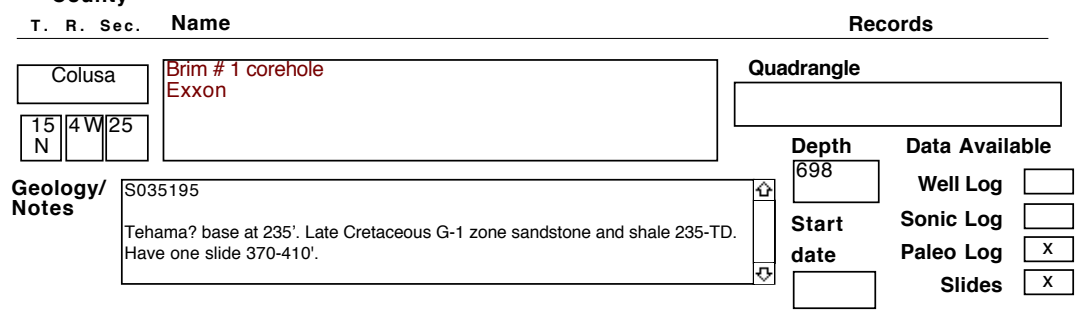

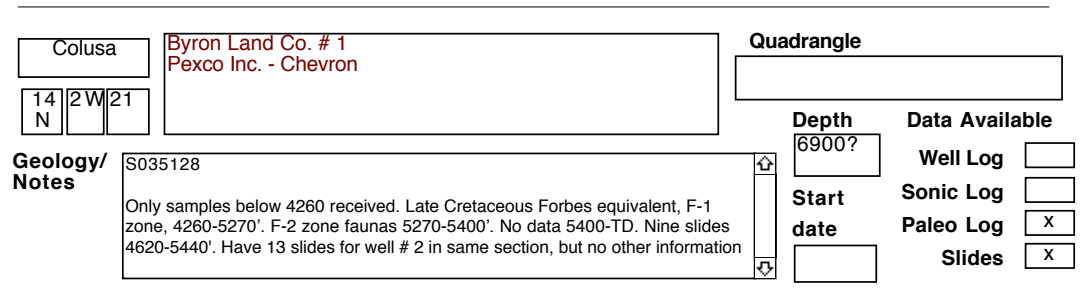

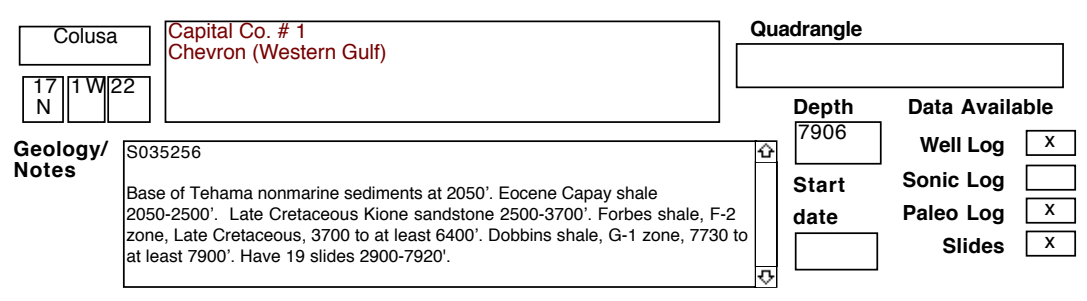

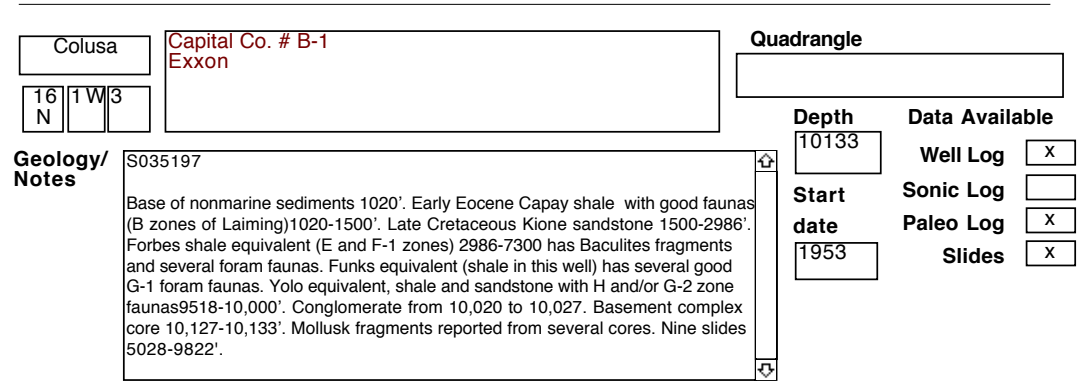

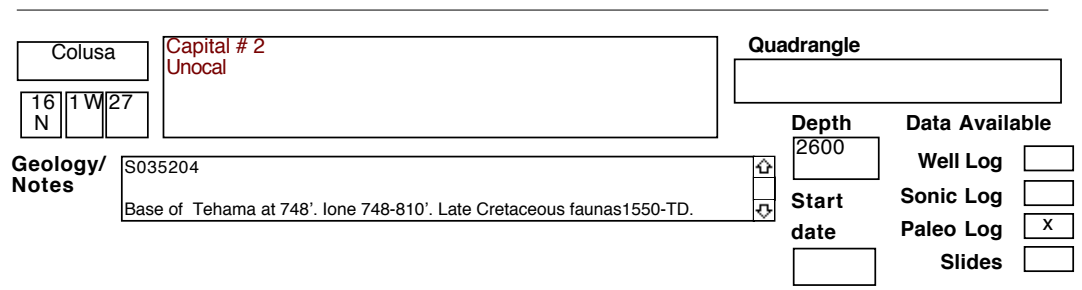




\section{SELECTED OIL TEST WELLS IN CENTRAL CALIFORNIA DATABASE}

County

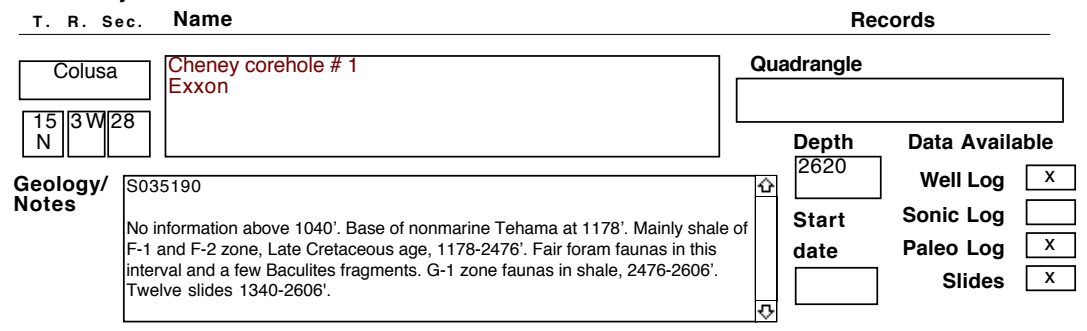

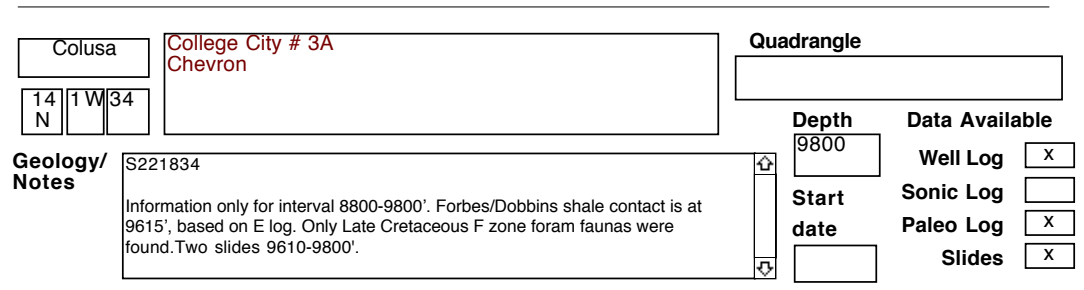

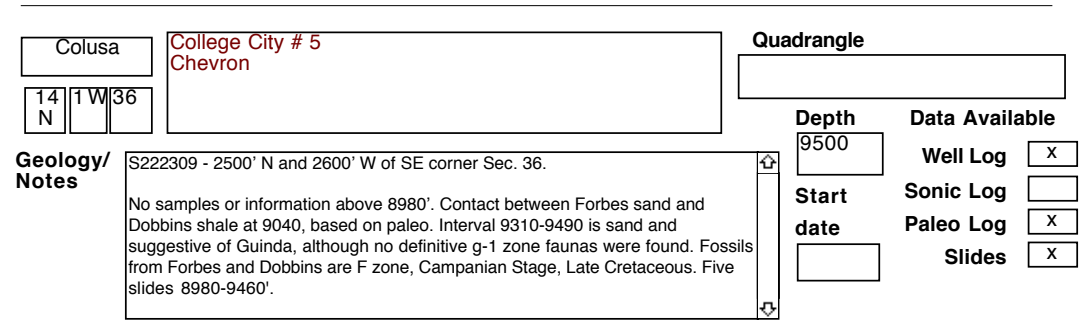

\begin{tabular}{|l|l|l|l|l|l|}
\hline Colusa & $\begin{array}{l}\text { Colusa \# 1 } \\
\text { Amerada Hess Corp }\end{array}$ \\
\hline
\end{tabular}




\section{SELECTED OIL TEST WELLS IN CENTRAL CALIFORNIA DATABASE}

County

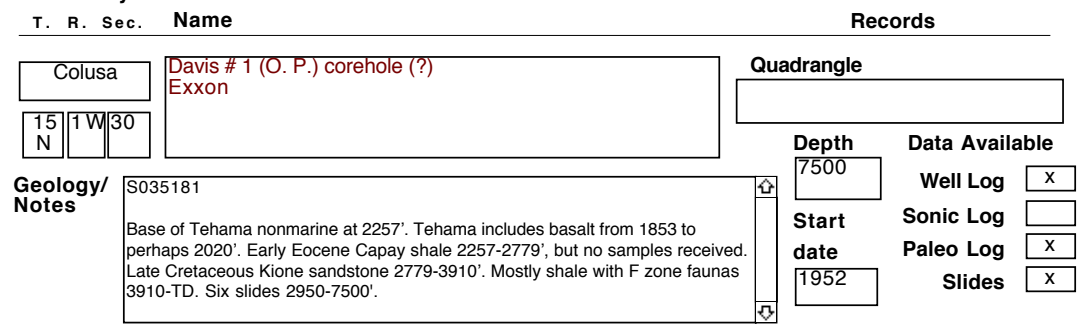

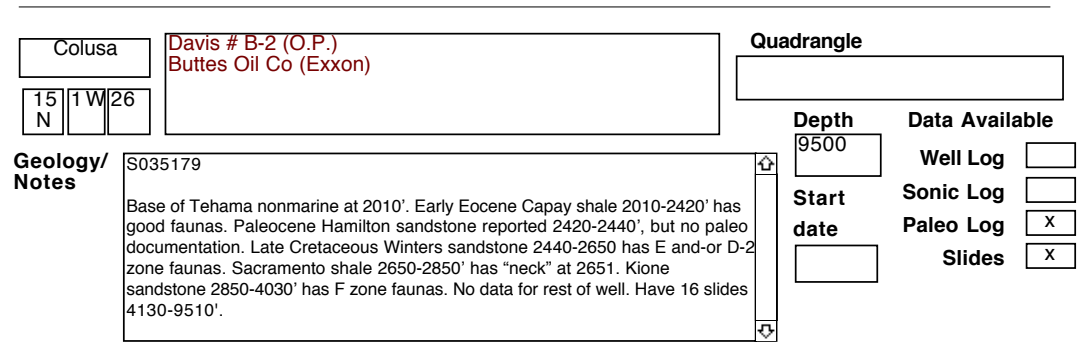

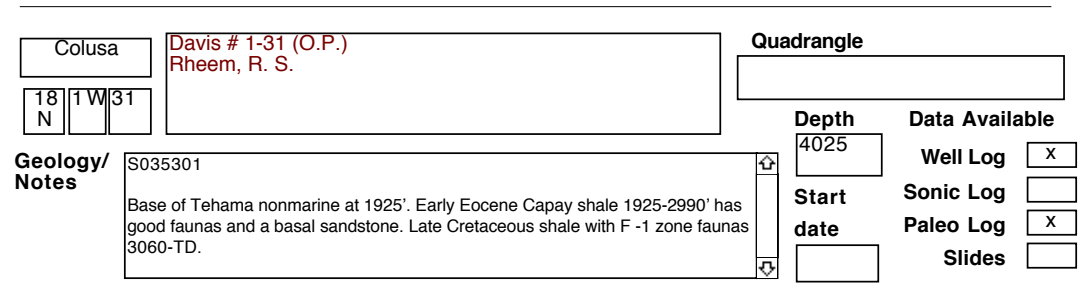

\begin{tabular}{l|l|l|l|l|l|l|}
\hline Colusa & $\begin{array}{l}\text { Dobbins Unit \# 1 } \\
\text { Sunray Mid-Continent Oil Co. }\end{array}$ \\
\hline
\end{tabular}

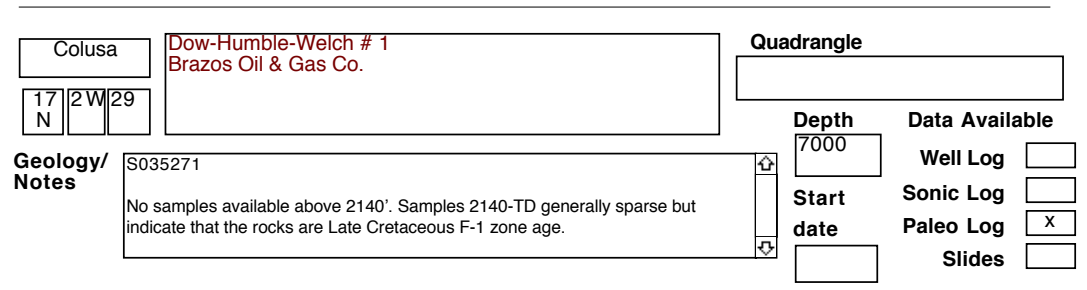


SELECTED OIL TEST WELLS IN CENTRAL CALIFORNIA DATABASE County

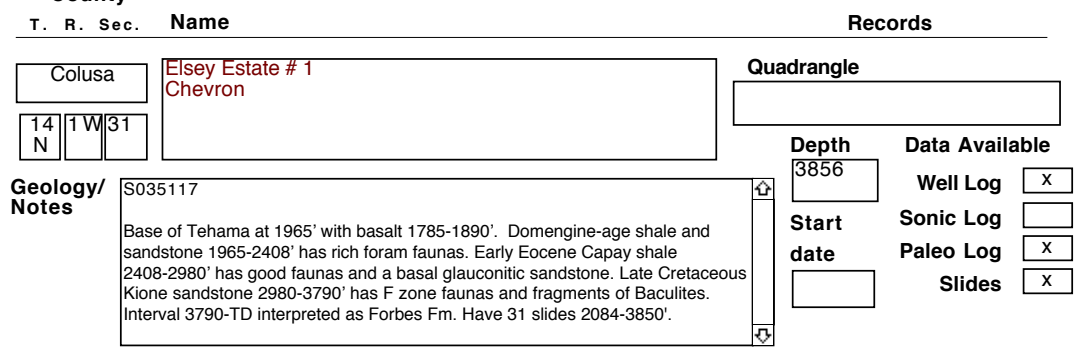

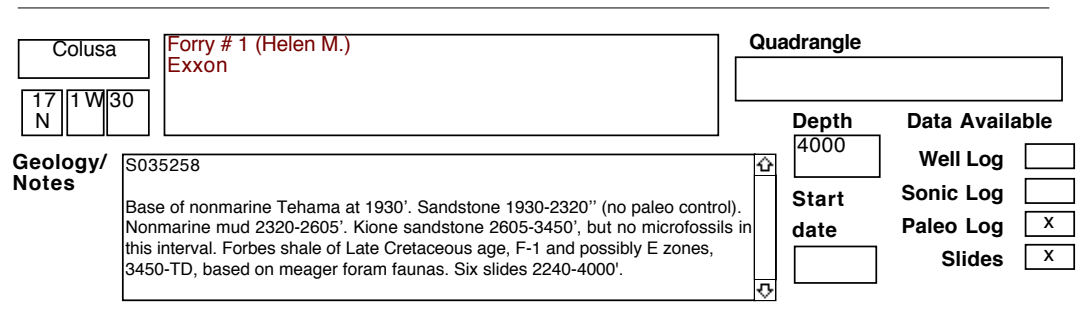

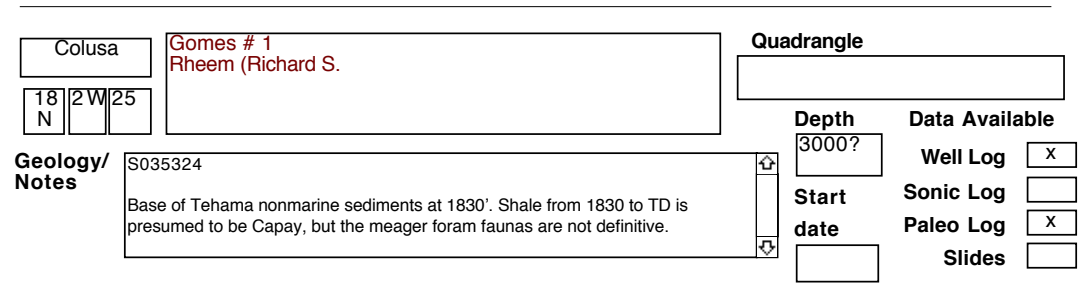

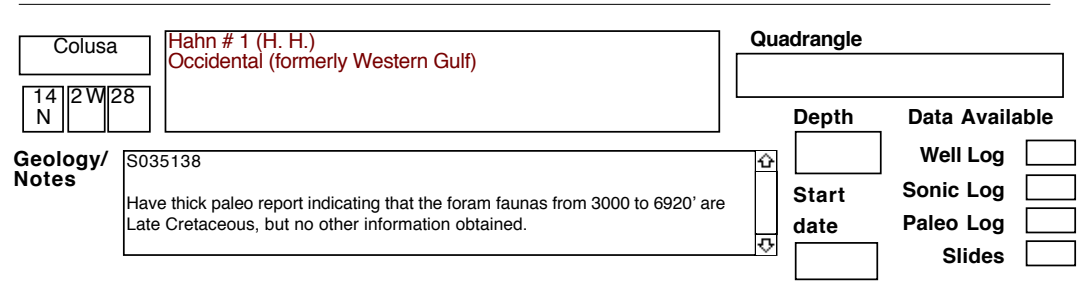

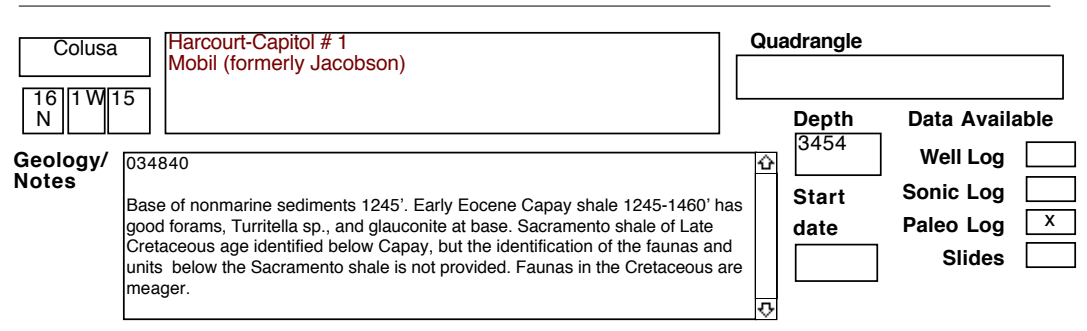


SELECTED OIL TEST WELLS IN CENTRAL CALIFORNIA DATABASE County

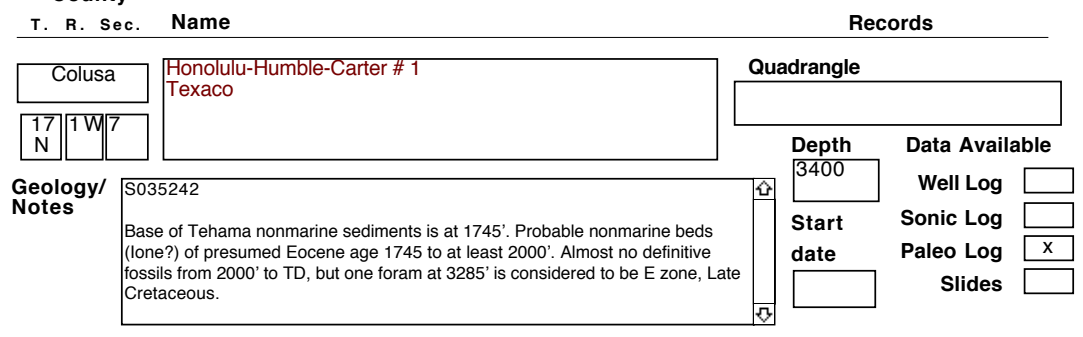

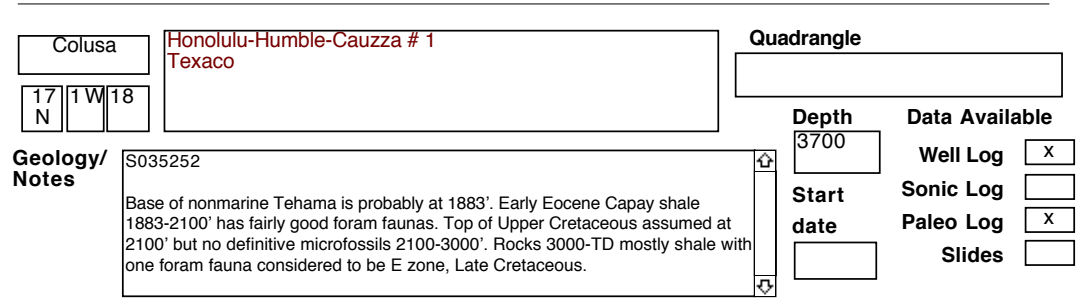

\begin{tabular}{l|l|l|l|l|l|l|}
\hline Colusa & $\begin{array}{l}\text { Honolulu-Humble-Cox Aneita S unit \# 1 } \\
\text { Texaco }\end{array}$ \\
Geology/ & $\begin{array}{l}\text { S035253 } \\
\text { Base of Tehama nonmarine sediments considererd to be at 1850', but the rocks } \\
\text { from 1850 to 3427' have no foram faunas and appear to be nonmarine. They are } \\
\text { considered in the log to be Late Cretaceous. At 3427' to TD, the rocks contain } \\
\text { forams considered correlative with the E zone, Late Cretaceous. }\end{array}$ \\
\hline
\end{tabular}

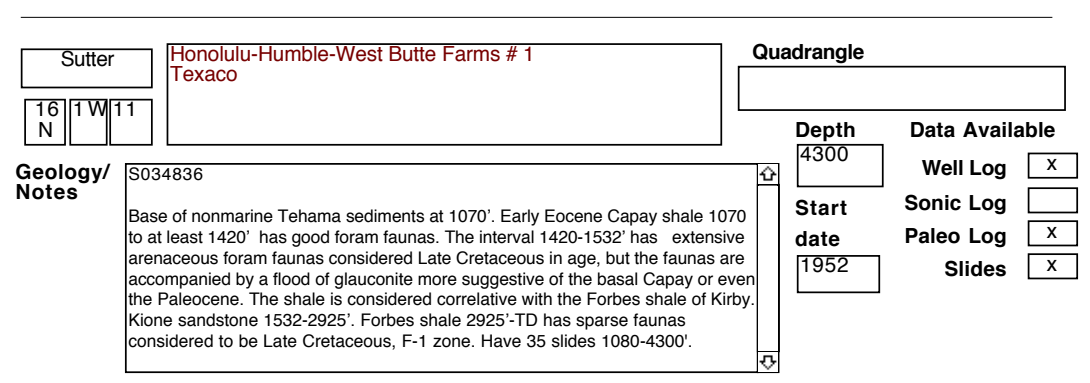

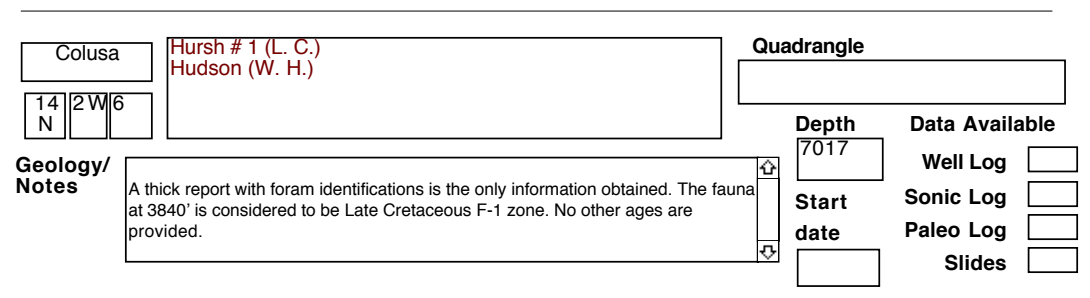




\section{SELECTED OIL TEST WELLS IN CENTRAL CALIFORNIA DATABASE}

County

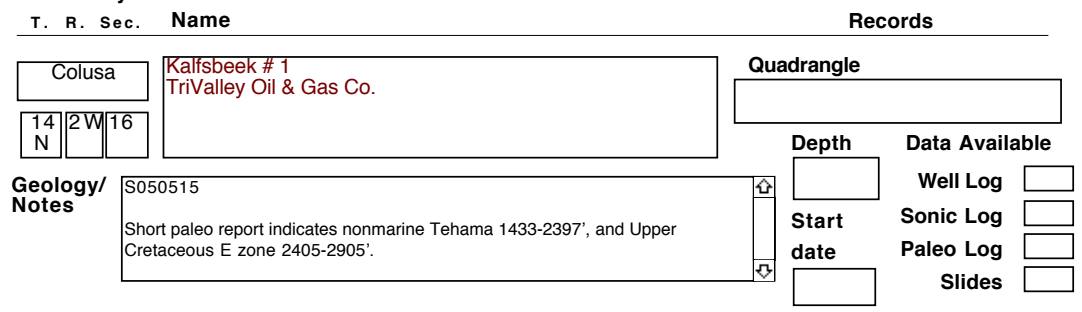

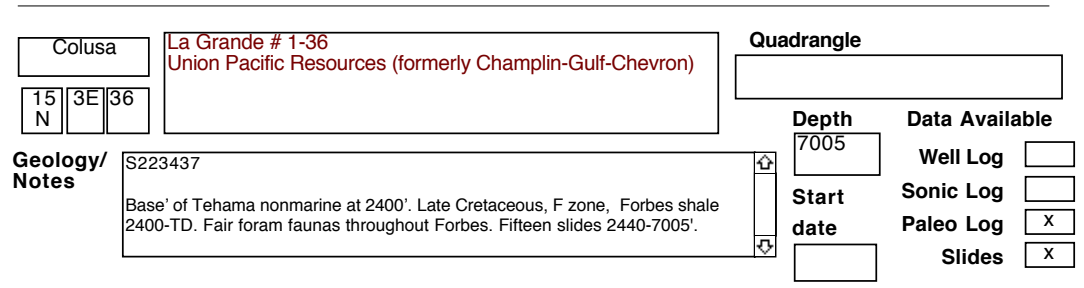

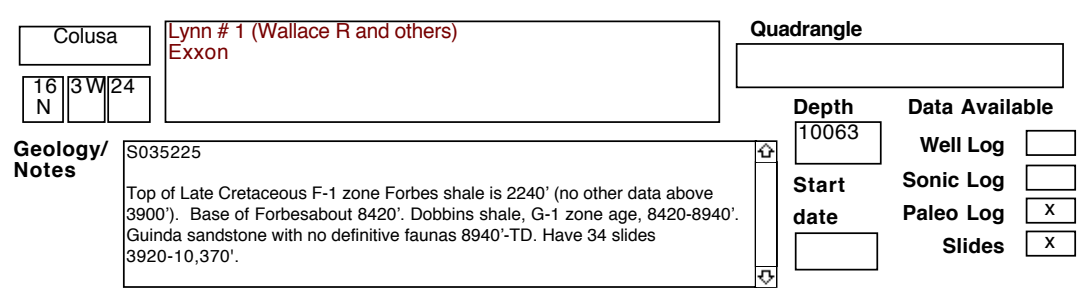

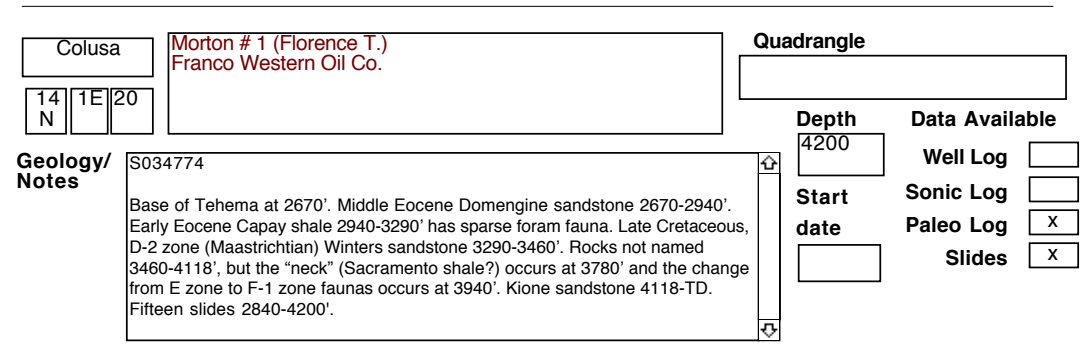

\begin{tabular}{l|l|l|l|l|l|}
\hline \multicolumn{1}{|c|}{ Colusa } & $\begin{array}{l}\text { Pecht \# 1 } \\
\text { Chevron }\end{array}$ \\
\hline
\end{tabular}


SELECTED OIL TEST WELLS IN CENTRAL CALIFORNIA DATABASE County

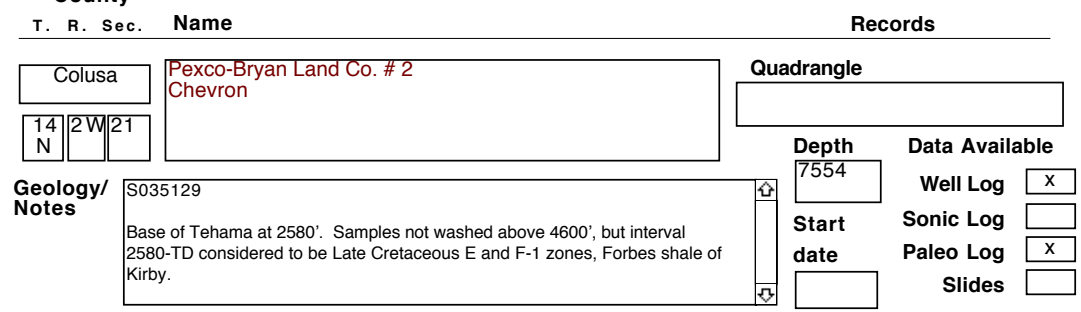

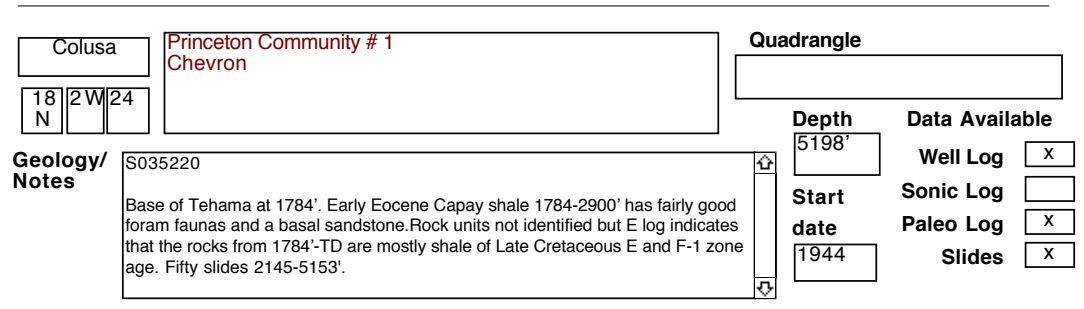

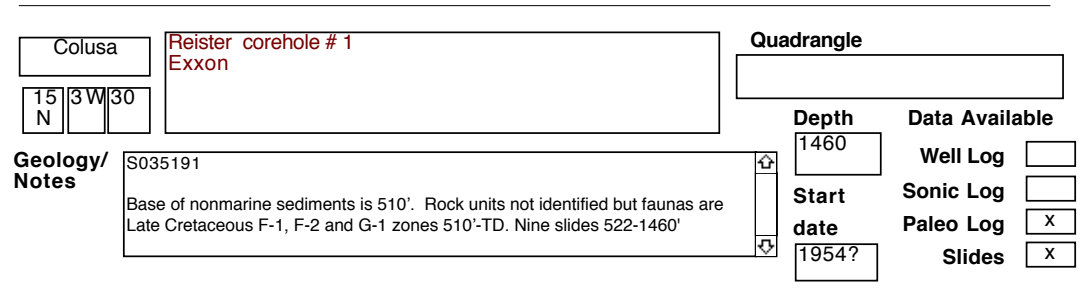

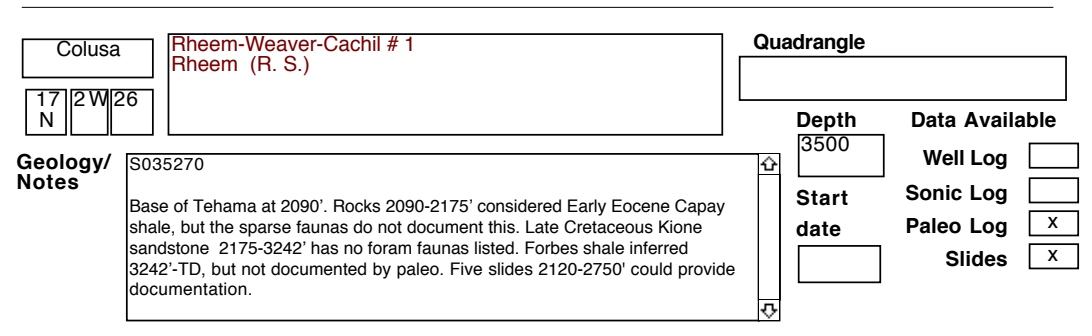

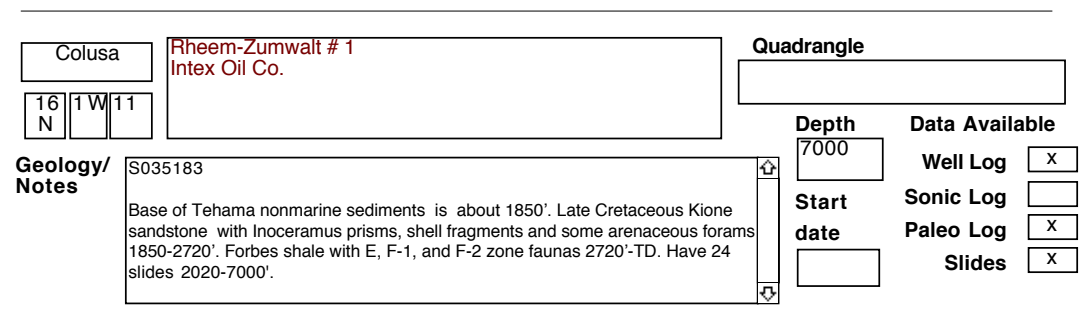


SELECTED OIL TEST WELLS IN CENTRAL CALIFORNIA DATABASE County

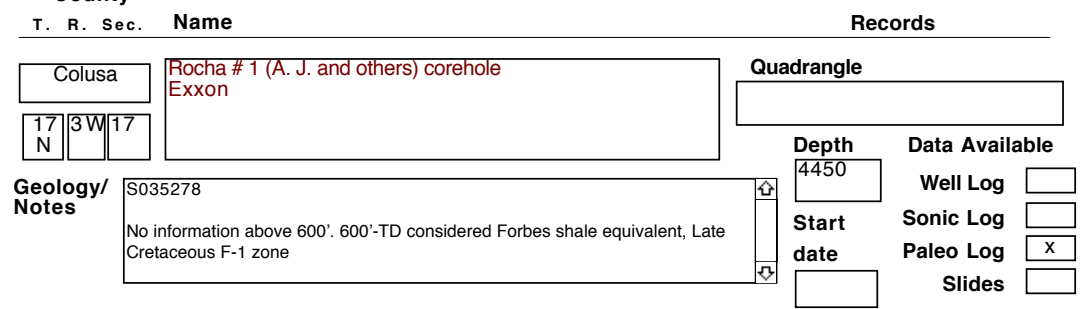

\begin{tabular}{l|l|l|l|l|l|}
\hline Colusa & $\begin{array}{l}\text { Sacramento Outing Club Unit B\# } \\
\text { Occidental Petroleum }\end{array}$ \\
\hline
\end{tabular}

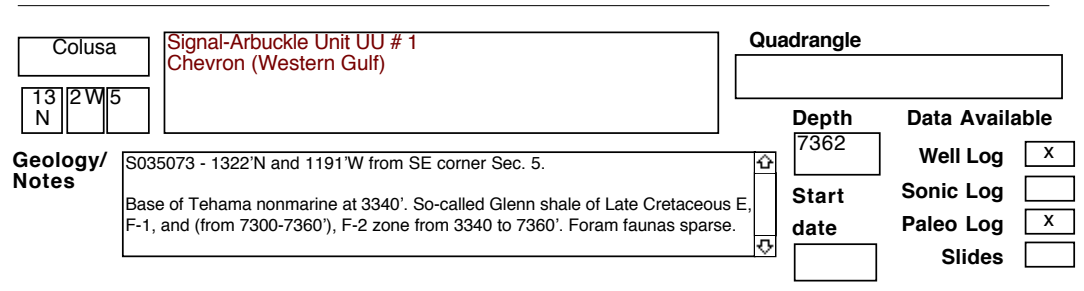

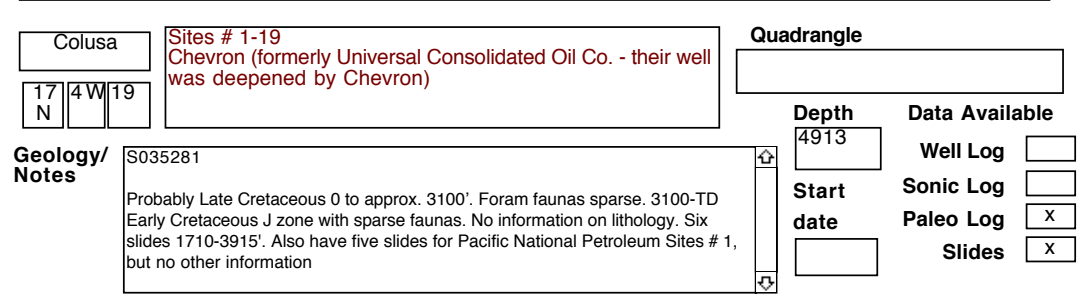

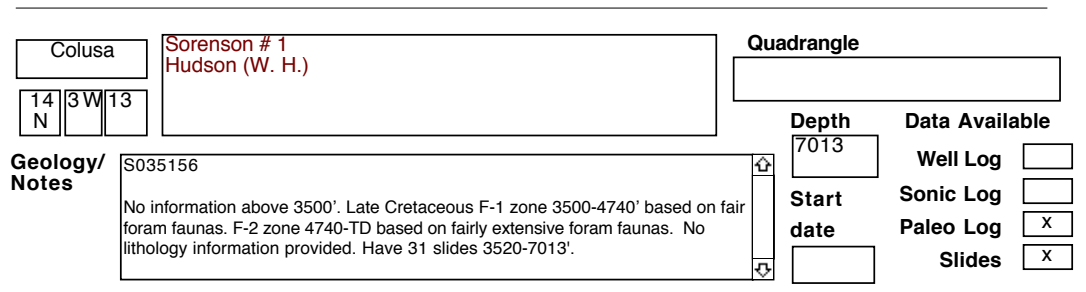


SELECTED OIL TEST WELLS IN CENTRAL CALIFORNIA DATABASE County

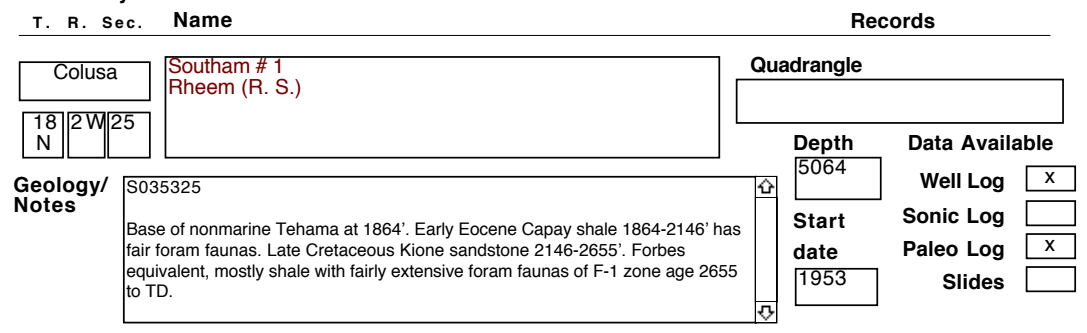

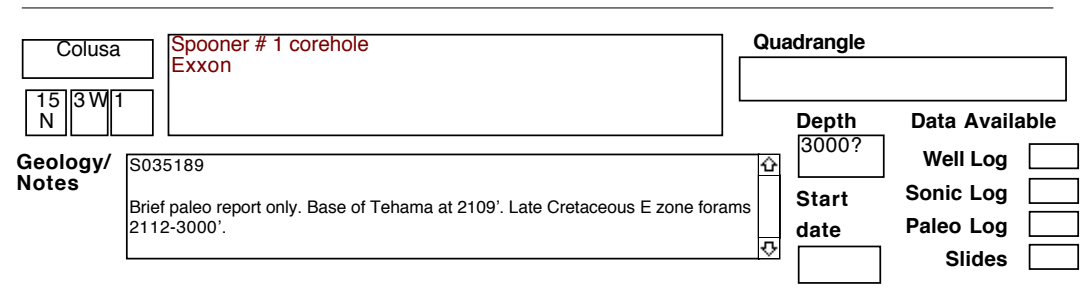

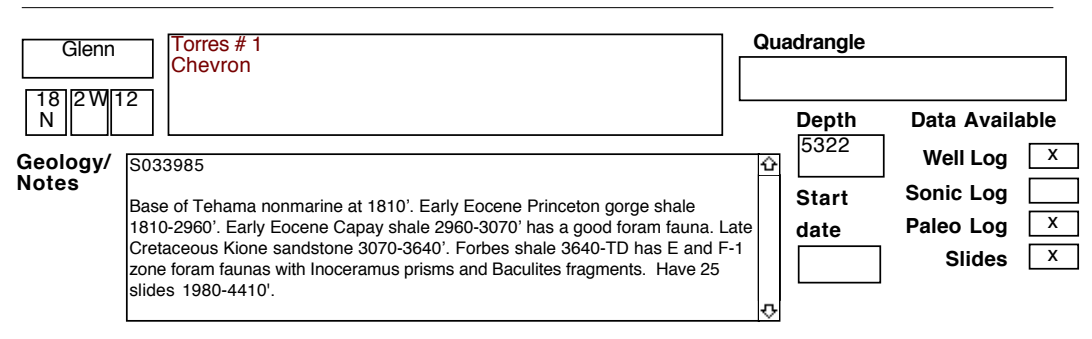

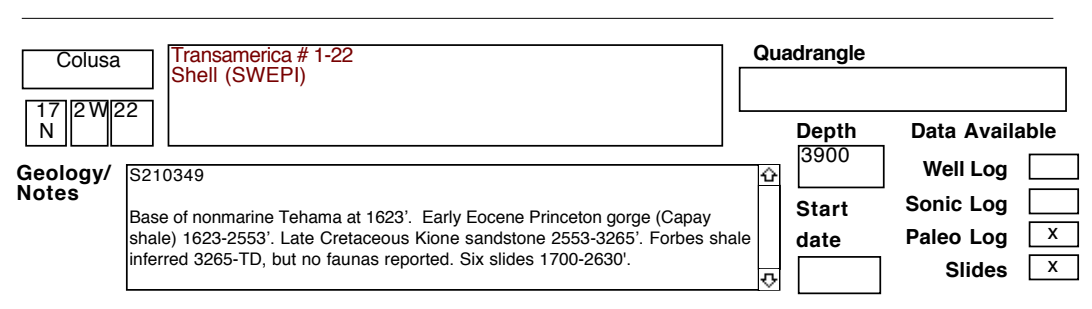

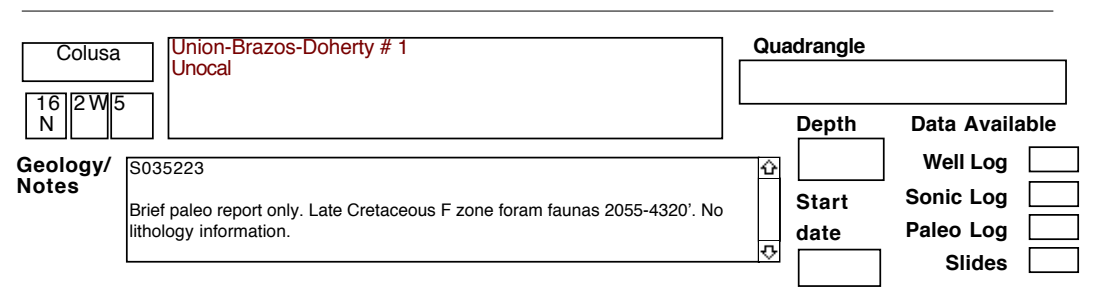


SELECTED OIL TEST WELLS IN CENTRAL CALIFORNIA DATABASE County

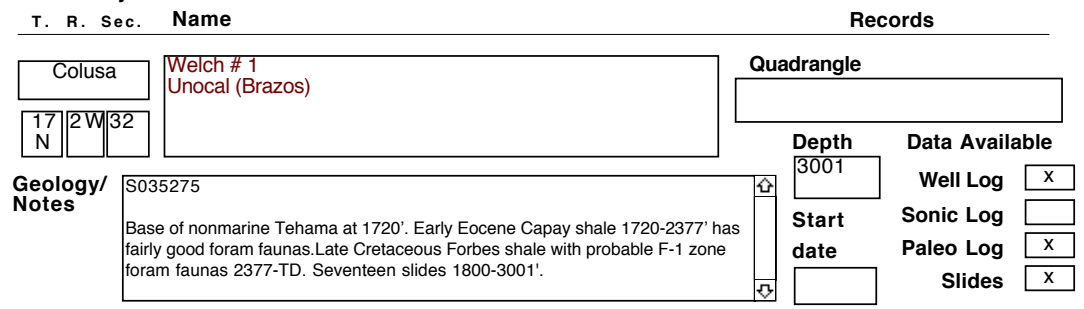

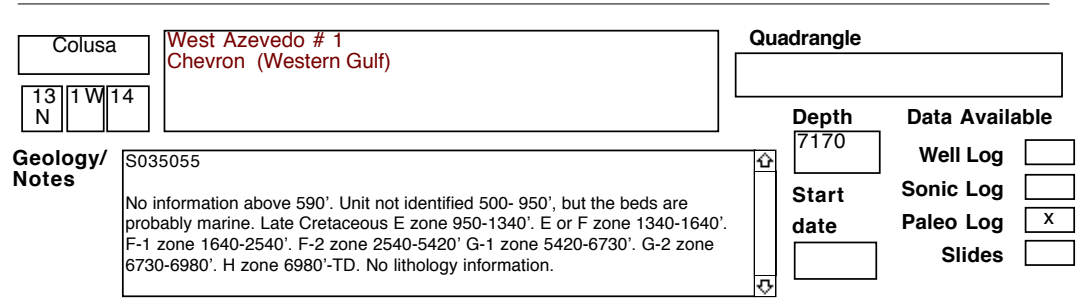

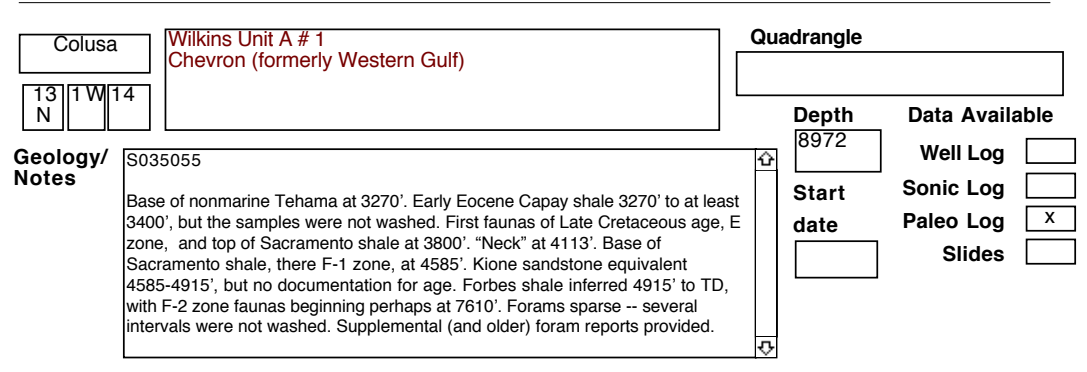

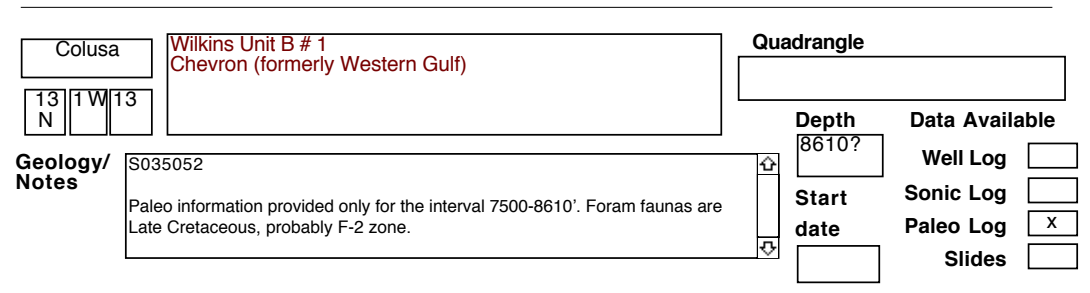

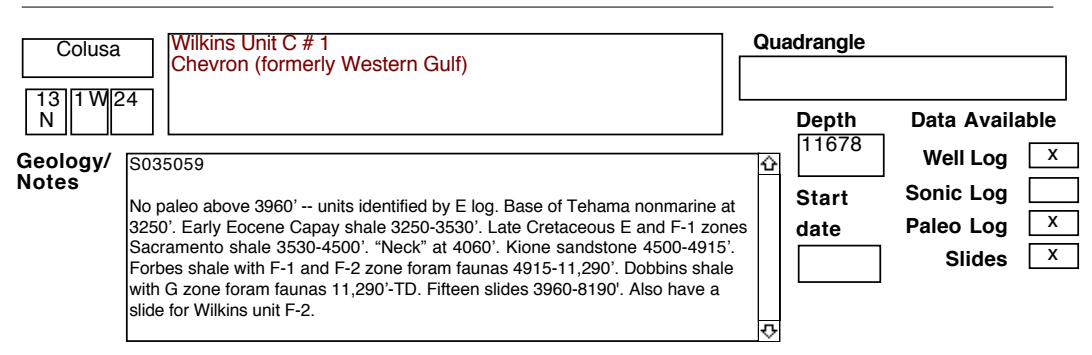


SELECTED OIL TEST WELLS IN CENTRAL CALIFORNIA DATABASE County

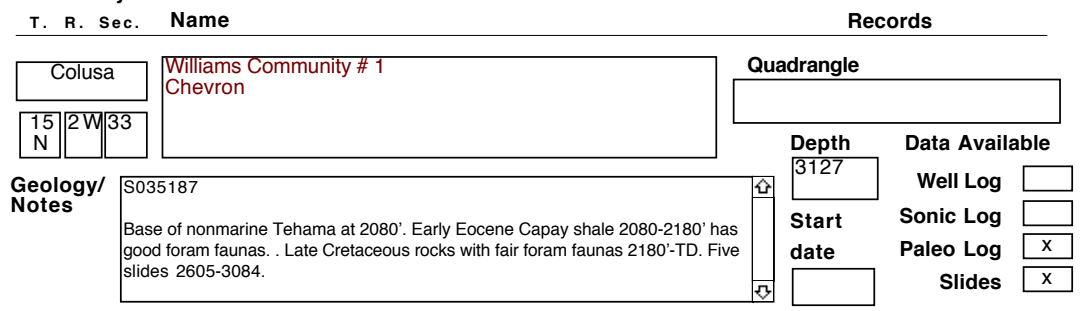

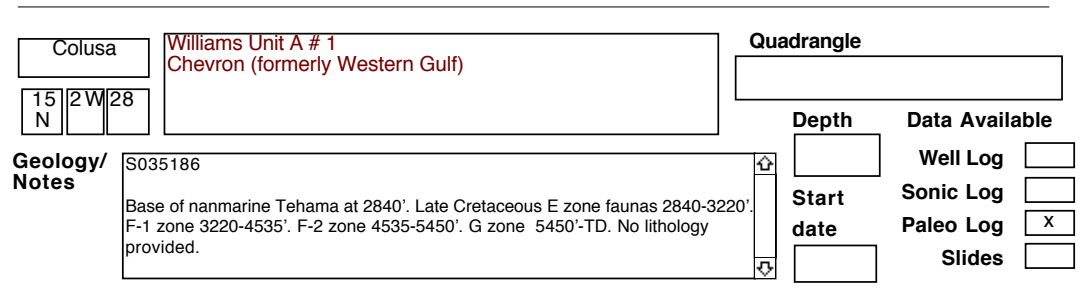

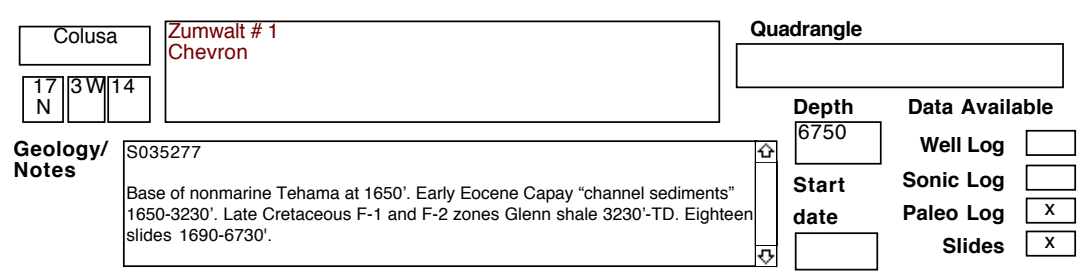

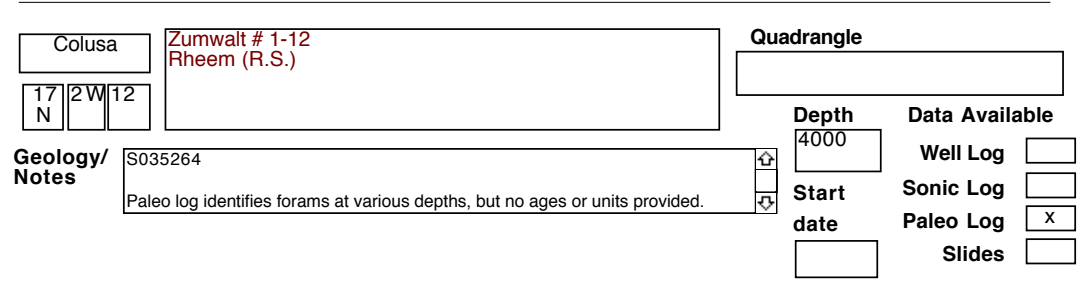

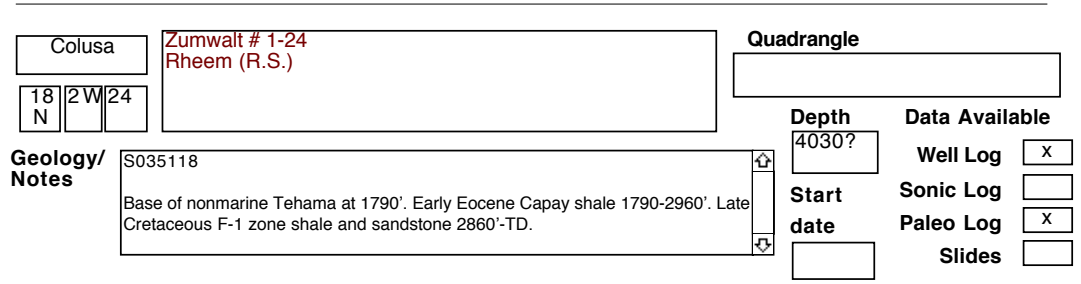


SELECTED OIL TEST WELLS IN CENTRAL CALIFORNIA DATABASE County

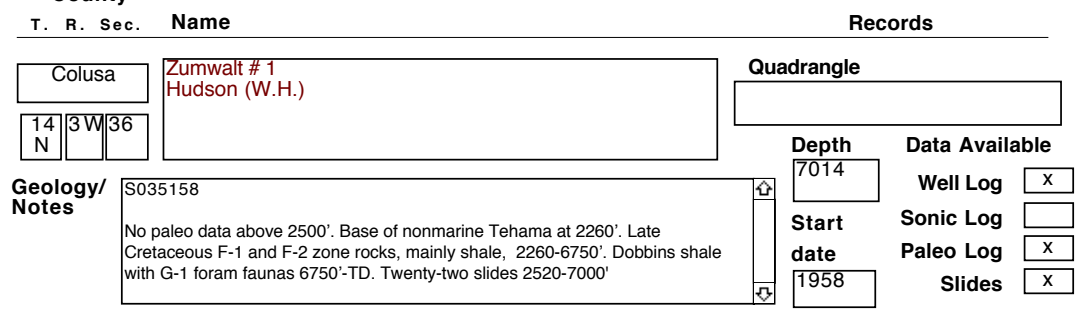

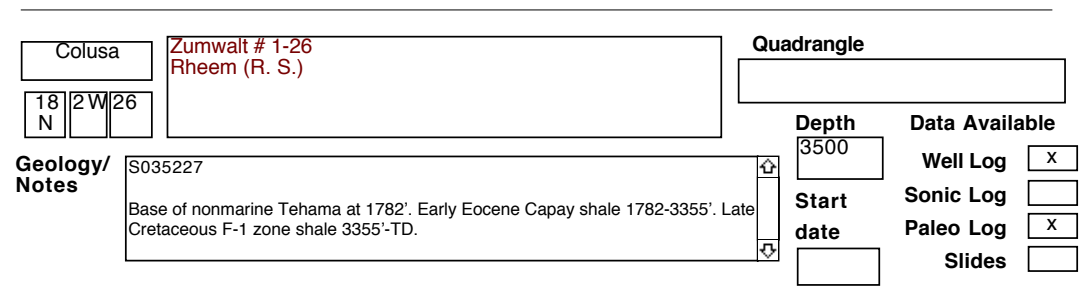

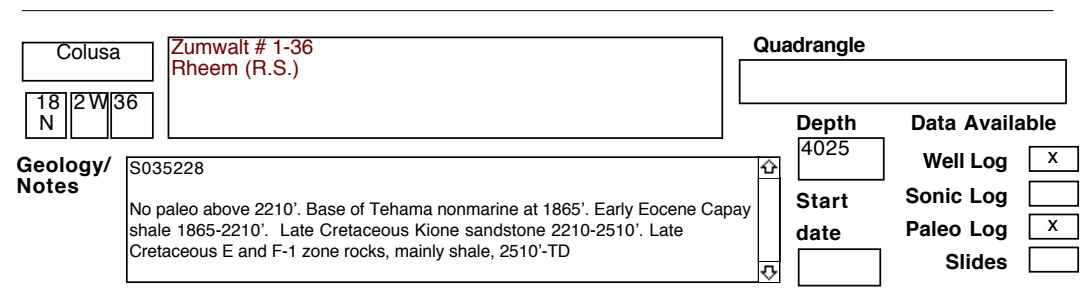

\begin{tabular}{|l|l|l|l|l|l|l|}
\hline Colusa & $\begin{array}{l}\text { Zumwalt \# 2 } \\
\text { Hudson (W.H.) }\end{array}$ \\
\hline
\end{tabular}

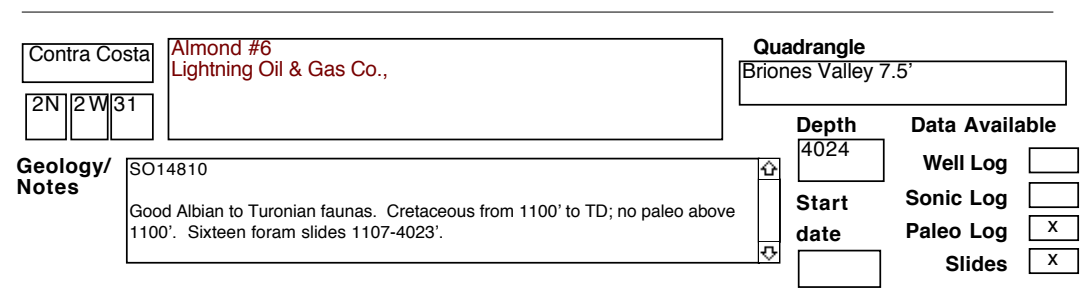




\section{SELECTED OIL TEST WELLS IN CENTRAL CALIFORNIA DATABASE}

\begin{tabular}{|c|c|c|}
\hline $\begin{array}{l}\text { County } \\
\text { T. R. Sec. }\end{array}$ & Name & \\
\hline Contra Costa & \multirow[t]{2}{*}{$\begin{array}{l}\text { Amerada-Baldocchi et al \# } 1 \\
\text { Occidental Petroleum Co. }\end{array}$} & $\begin{array}{r}\text { Qua } \\
\text { Wooc }\end{array}$ \\
\hline \begin{tabular}{|l|l||l|}
$2 \mathrm{~N}$ & $3 \mathrm{E}$ & 36 \\
\end{tabular} & & \\
\hline $\begin{array}{l}\text { Geology/ So } \\
\text { Notes }\end{array}$ & $\begin{array}{l}40467 \\
\text { data above 6100'. Inferred Meganos (Eocene) channel sands 5730-5830'. } \\
\text { it definite Late Cretaceous (C or D zone) foram fauna at 6950'. Beds } \\
\text { 30-6900' have planktic forams resembling those in the Danian stage. Mostly } \\
\text { idstone 6230-7710'. Mostly shale of Late Cretaceous age 7710-9390'. Mostly } \\
\text { idstone 9390-TD. }\end{array}$ & tly \\
\hline
\end{tabular}

Records

Quadrangle

Woodward Island

\begin{tabular}{|c|c|c|}
\hline Depth & \multicolumn{2}{|c|}{ Data Available } \\
\hline 9550 & Well Log & $\mathrm{x}$ \\
\hline Start & Sonic Log & \\
\hline date & Paleo Log & $x$ \\
\hline
\end{tabular}

\begin{tabular}{|l|l|l|}
\hline Contra Costa & $\begin{array}{l}\text { Amerada-Honegger \# 1-34 } \\
\text { Buttes Resources Co. }\end{array}$ \\
\hline $2 \mathrm{~N}$ & $3 \mathrm{E}$ & 34 \\
\hline
\end{tabular}

Geology/ $\$ 042299$

Notes

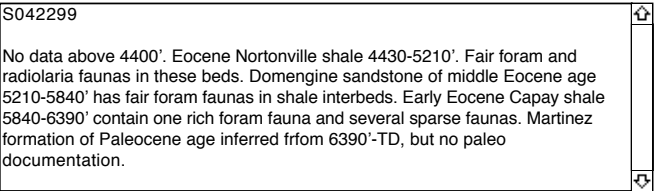

\begin{tabular}{|c|c|c|c|}
\hline \multicolumn{4}{|c|}{ Quadrangle } \\
\hline & \multirow{2}{*}{$\begin{array}{l}\text { Depth } \\
8000\end{array}$} & \multicolumn{2}{|c|}{ Data Available } \\
\hline \multirow{3}{*}{ 象 } & & Well Log & $\mathrm{x}$ \\
\hline & Start & Sonic Log & \\
\hline & date & Paleo Log & $\mathrm{x}$ \\
\hline & & Slides & \\
\hline
\end{tabular}

(

$\sqrt{3}$

Slides

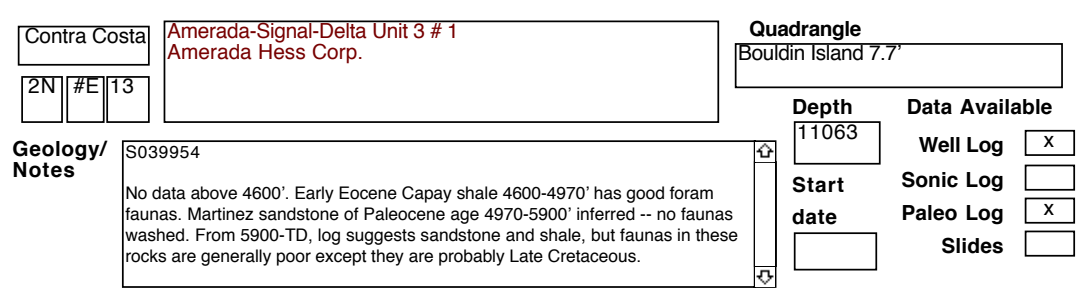

\begin{tabular}{l|l|l|l|l|l|}
\hline Avon Unit \# 1 \\
McCulloch (formerly Getty Oil Co. and Tidewater Assoc)
\end{tabular}

\begin{tabular}{|c|c|}
\hline Contra Costa & $\begin{array}{l}\text { Baker et al \#1 } \\
\text { Texaco (formerly Getty and Tidewater) }\end{array}$ \\
\hline $2 \mathrm{~N} / 2 \mathrm{~V} / 11$ & \\
\hline
\end{tabular}

Geology/ S014799

Notes

No information above $3100^{\prime}$ and no foram faunas above $5400^{\prime}$. Markley sandstone

inferred 4470-5420'. Nortonville shale of late Eocene age 5280-5820' inferred

from log and from fair foram faunas. Domengine sandstgone 5820-6178'

Martinez formation (log suggests shale) 6178'-TD has arenaceous forams only.

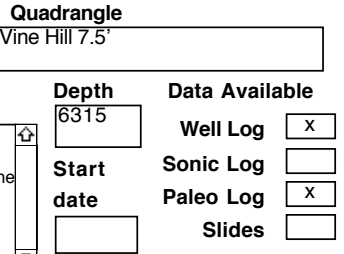


SELECTED OIL TEST WELLS IN CENTRAL CALIFORNIA DATABASE County

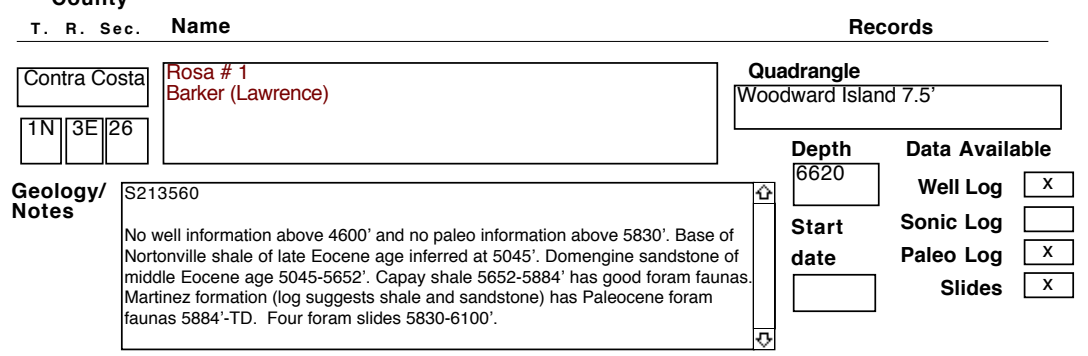

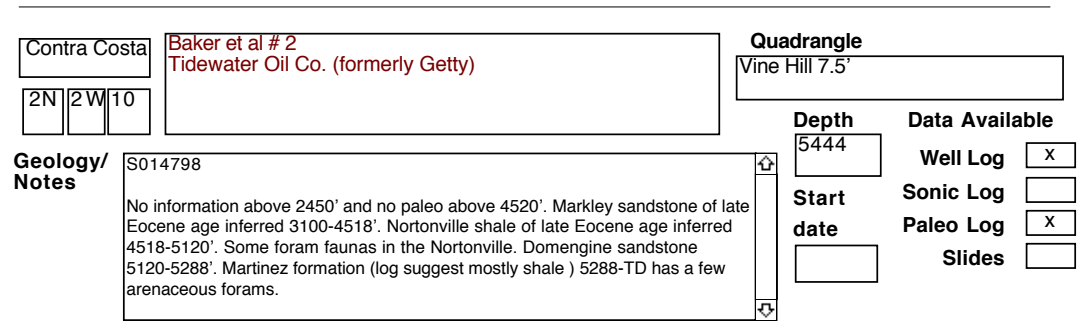

\begin{tabular}{l|l|l|l|l|l|}
\hline Contra Costa & $\begin{array}{l}\text { Baldwin-Soite \# 1 } \\
\text { Baldwin (John) }\end{array}$ \\
\hline
\end{tabular}

\begin{tabular}{l|l|l|l|l|}
\hline Bethel Island Community \# 1 \\
Texaco (formerly Tidewater, Trico, and Getty)
\end{tabular}

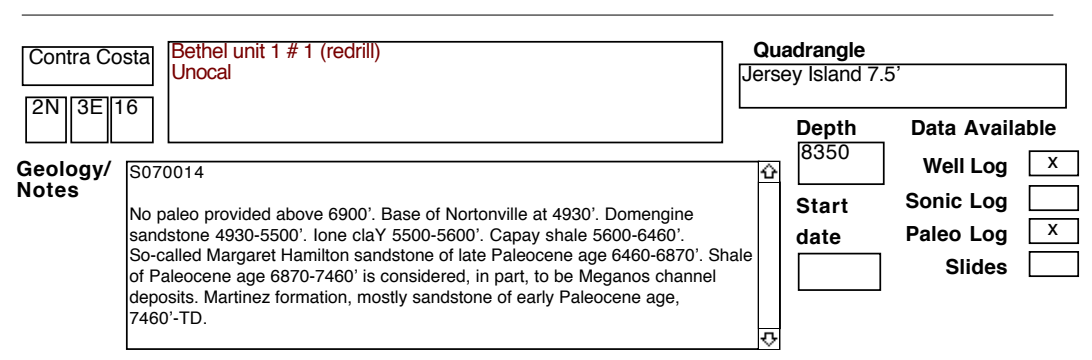




\section{SELECTED OIL TEST WELLS IN CENTRAL CALIFORNIA DATABASE}

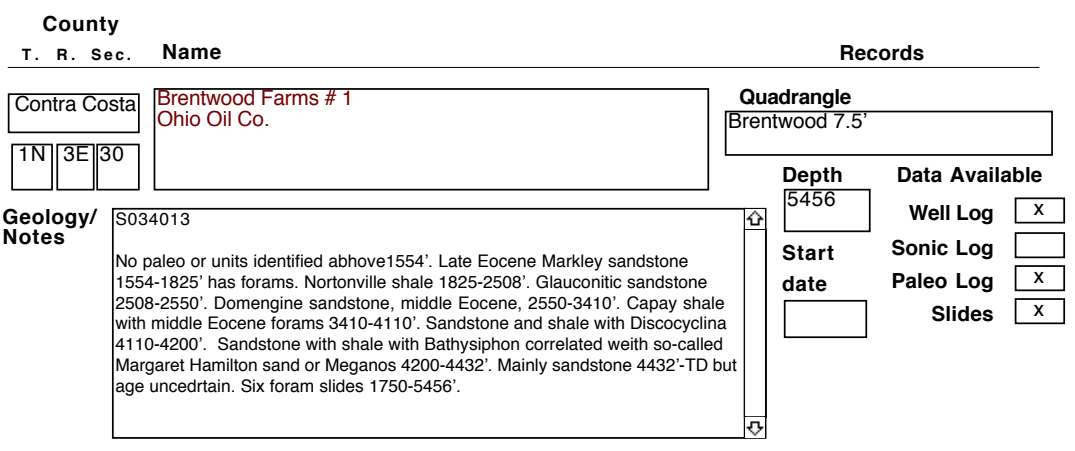

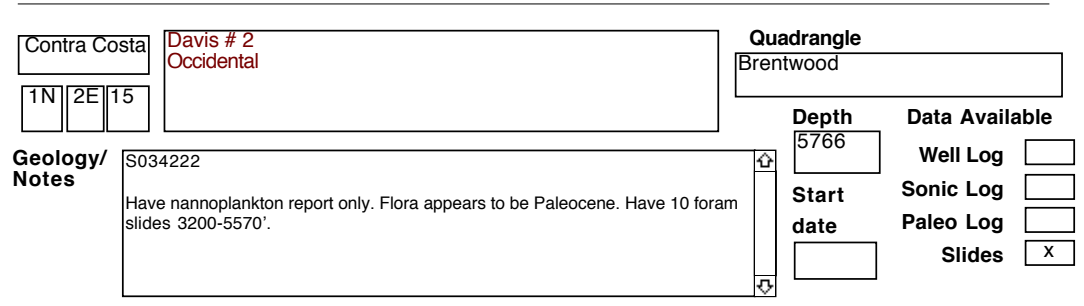

\begin{tabular}{|c|c|c|c|c|c|}
\hline \multicolumn{2}{|c|}{ Contra Costa } & \multirow[t]{2}{*}{$\begin{array}{l}\text { Delta Properties \# 3-1 } \\
\text { UNOCAL? (Amerada) }\end{array}$} & \multicolumn{3}{|c|}{$\begin{array}{l}\text { Quadrangle } \\
\text { Jersey Island }\end{array}$} \\
\hline \begin{tabular}{|l|l|}
$2 \mathrm{~N}$ & $3 \mathrm{E}$ \\
\end{tabular} & & & $\begin{array}{l}\text { Depth } \\
11060 ?\end{array}$ & \multicolumn{2}{|l|}{ Data Available } \\
\hline $\begin{array}{l}\text { Geology/ } \\
\text { Notes }\end{array}$ & $\begin{array}{l}\text { S039 } \\
\text { No in } \\
4960 \\
5610 \\
\text { shale } \\
7570 \\
4780 \\
\text { slide }\end{array}$ & 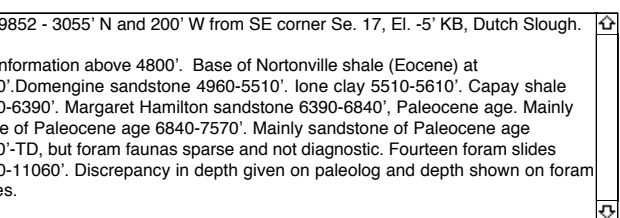 & $\begin{array}{l}11060 ? \\
\text { Start } \\
\text { date } \\
1964\end{array}$ & $\begin{array}{r}\text { Well Log } \\
\text { Sonic Log } \\
\text { Paleo Log } \\
\text { Slides }\end{array}$ & $\begin{array}{l}x \\
x\end{array}$ \\
\hline
\end{tabular}

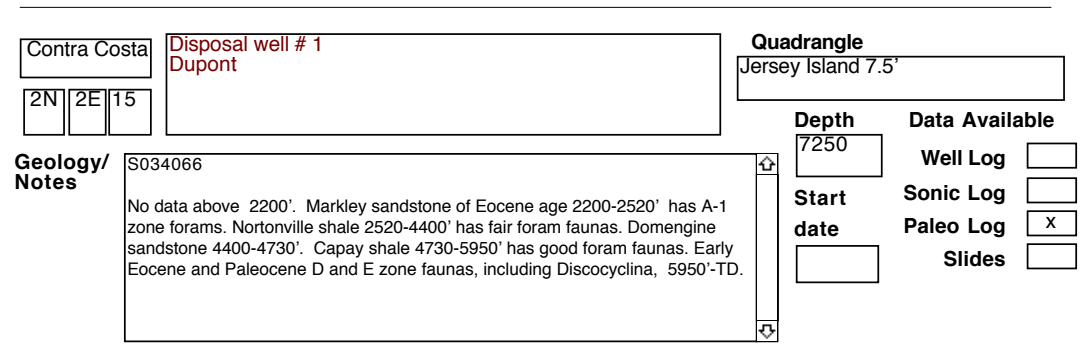

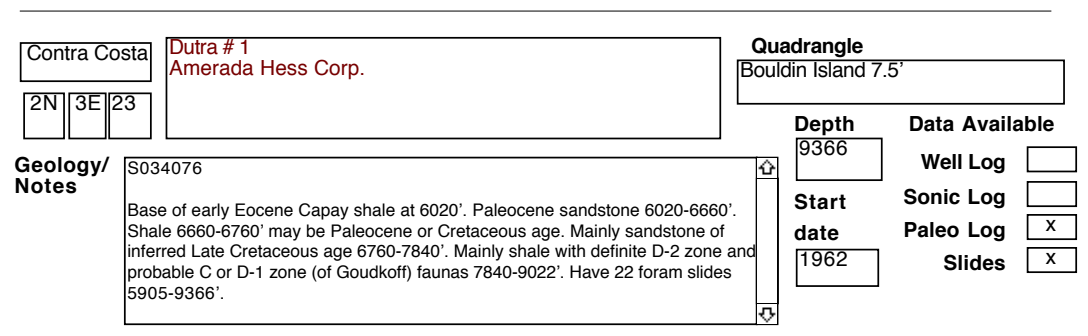




\section{SELECTED OIL TEST WELLS IN CENTRAL CALIFORNIA DATABASE}

\begin{tabular}{|c|c|c|c|}
\hline $\begin{array}{l}\text { County } \\
\text { T. R. Sec. }\end{array}$ & Name & \multicolumn{2}{|c|}{ Records } \\
\hline Contra Costa & \multirow[t]{2}{*}{$\begin{array}{l}\text { Delta Properties \#2 } \\
\text { Unocal (formerly Phillips and Aminoil) }\end{array}$} & \multicolumn{2}{|c|}{$\begin{array}{l}\text { Quadrangle } \\
\text { Jersey Island 7.5' }\end{array}$} \\
\hline \begin{tabular}{|l|l||l|l}
$2 \mathrm{~N}$ & $3 \mathrm{E}$ & 17 \\
\end{tabular} & & Depth & Data Available \\
\hline $\begin{array}{l}\text { Geology/ } \\
\text { Notes }\end{array}$ & $\begin{array}{l}9854 \\
\text { y formation names and ages provided. Base of Nortonville shale at } 4940 \text { '. } \\
\text { nengine sandstone } 4940-5450^{\prime} \text {. Ione clay } 5450-5590^{\prime} \text {. Capay shale } \\
0-6250^{\prime} \text {. Eocene D zone units (Margaret Hamilton sand and Meganos) } \\
0-6950^{\prime} \text {. E zone Paleocene units } 6950 \text { '-TD. }\end{array}$ & $\begin{array}{l}\text { Start } \\
\text { date } \\
1970 ?\end{array}$ & $\begin{array}{r}\text { Well Log } \\
\text { Sonic Log } \\
\text { Paleo Log } \\
\text { Slides }\end{array}$ \\
\hline
\end{tabular}

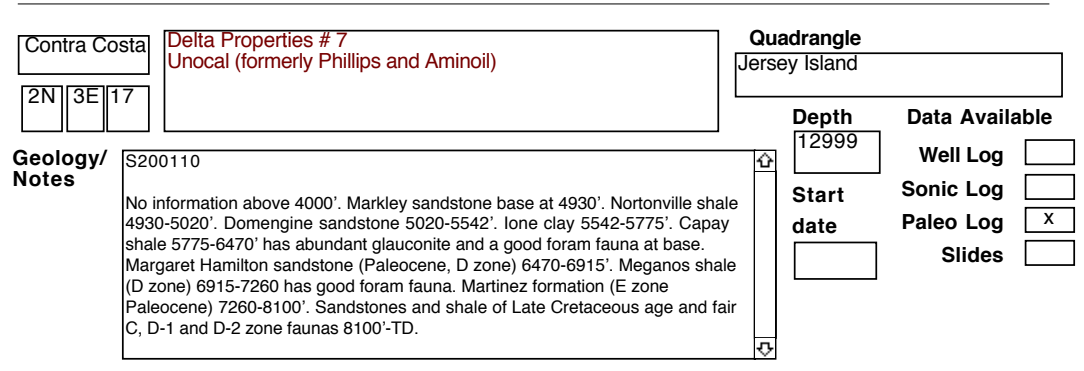

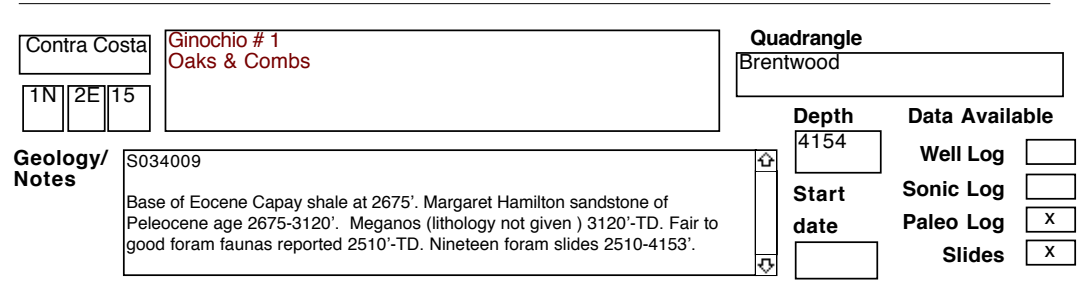

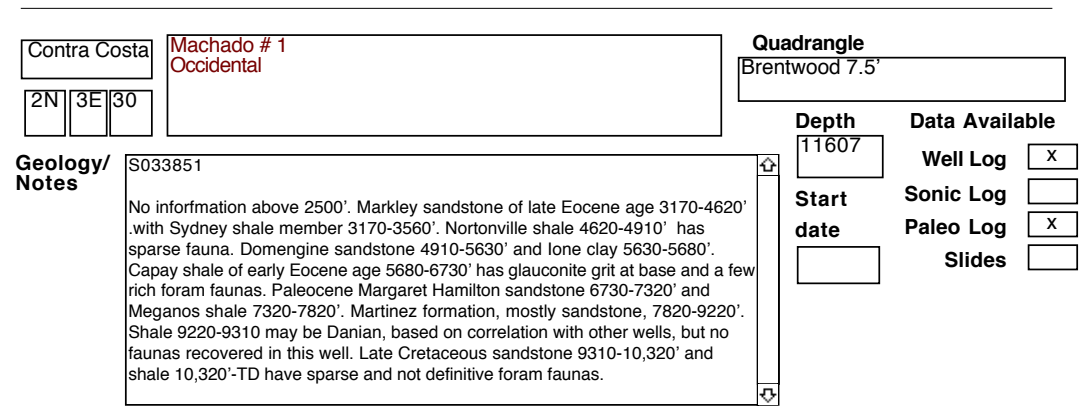

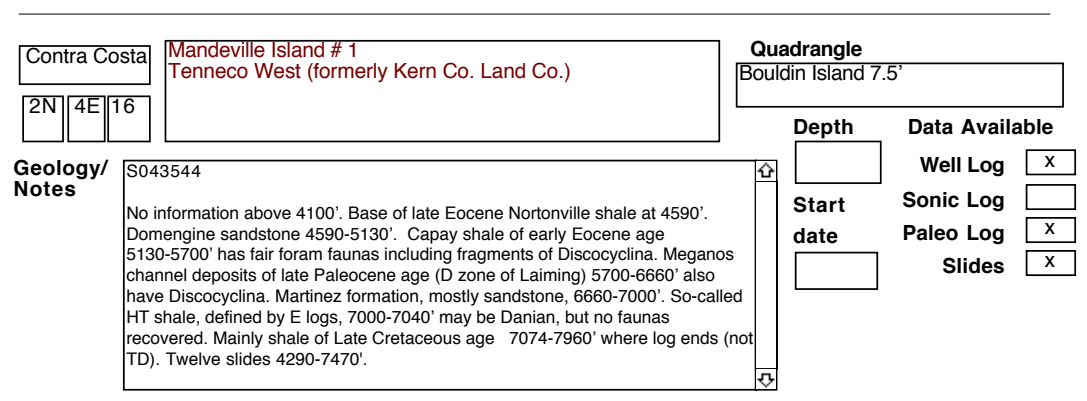


SELECTED OIL TEST WELLS IN CENTRAL CALIFORNIA DATABASE

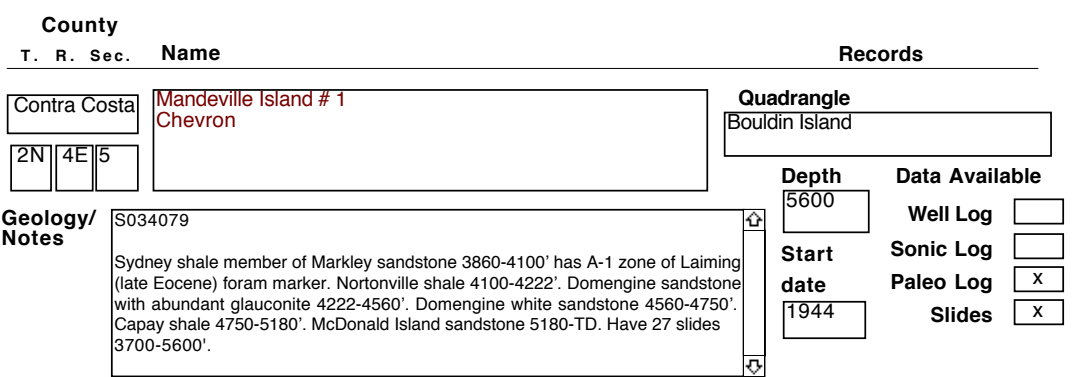

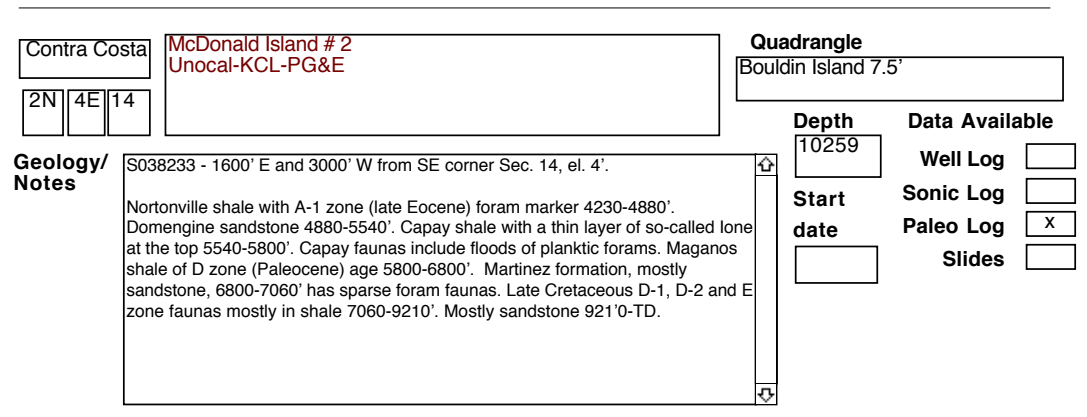

\begin{tabular}{|c|c|c|c|c|c|}
\hline Contra Costa & \multirow[t]{2}{*}{$\begin{array}{l}\text { Richfield-Hannum Trust \#2 } \\
\text { Santa Fe Oil Co. \& ARCO, }\end{array}$} & \multicolumn{4}{|c|}{$\begin{array}{l}\text { Quadrangle } \\
\text { Clifton Court Forebay 7.5' }\end{array}$} \\
\hline $15\left\|^{3 E}\right\|^{26}$ & & \multirow{2}{*}{\multicolumn{2}{|c|}{$\begin{array}{l}\text { Depth } \\
6968\end{array}$}} & \multicolumn{2}{|c|}{ Data Available } \\
\hline \multirow{3}{*}{$\begin{array}{l}\text { Geology/ } \\
\text { Notes }\end{array}$} & 37466 & & & Well Log & $x$ \\
\hline & Icareous forams present. Fairly good late Cretaceous faunas with $\mathrm{D}, \mathrm{E}$ and $\mathrm{F}$ & & Start & Sonic Log & \\
\hline & nes of Goudkoff represented. First sample at 560'. Cretaceous to TD. & & date & $\begin{array}{r}\text { Paleo Log } \\
\text { Slides }\end{array}$ & $x$ \\
\hline
\end{tabular}

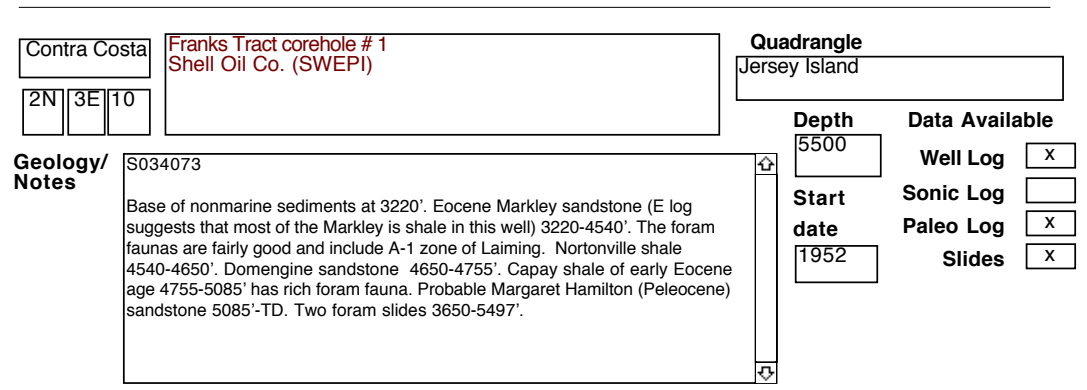

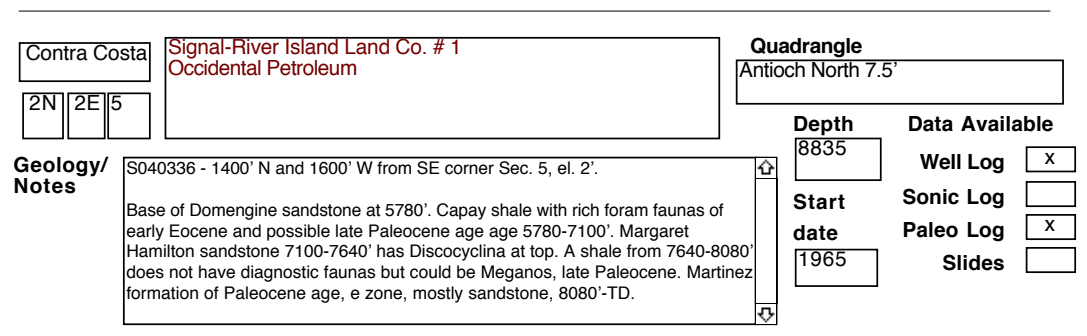


SELECTED OIL TEST WELLS IN CENTRAL CALIFORNIA DATABASE County

\begin{tabular}{|c|c|c|c|c|c|}
\hline T. R. Sec & Name & \multicolumn{4}{|c|}{ Records } \\
\hline Glenn & Altofer corehole \# 1 & Qua & drangle & & \\
\hline \begin{tabular}{|l|l|l|}
22 & $4 \mathrm{~W}$ & 14 \\
$\mathrm{~N}$ & & \\
\end{tabular} & & & Depth & Data Availab & \\
\hline $\begin{array}{l}\text { Geology/ } \\
\text { Notes }\end{array}$ & $\begin{array}{l}29557 \\
\text { e of nonmarine Tehama at } 1250 \text { '. Late Cretaceous E? and F-1 zone of } \\
\text { udkoff } 1250^{\prime}-T D \text {. Fair foram faunas, Baculites fragments, and Inoceramus } \\
\text { ms. }\end{array}$ & 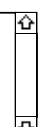 & $\begin{array}{l}3488 \\
\text { Start } \\
\text { date }\end{array}$ & $\begin{array}{r}\text { Well Log } \\
\text { Sonic Log } \\
\text { Paleo Log } \\
\text { Slides }\end{array}$ & $\mathrm{x}$ \\
\hline
\end{tabular}

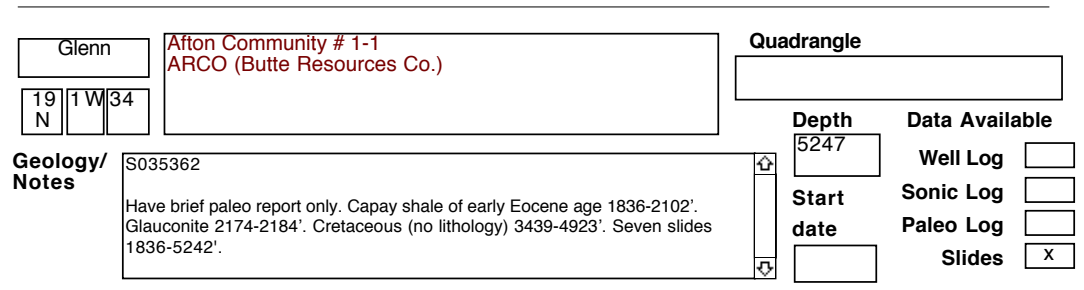

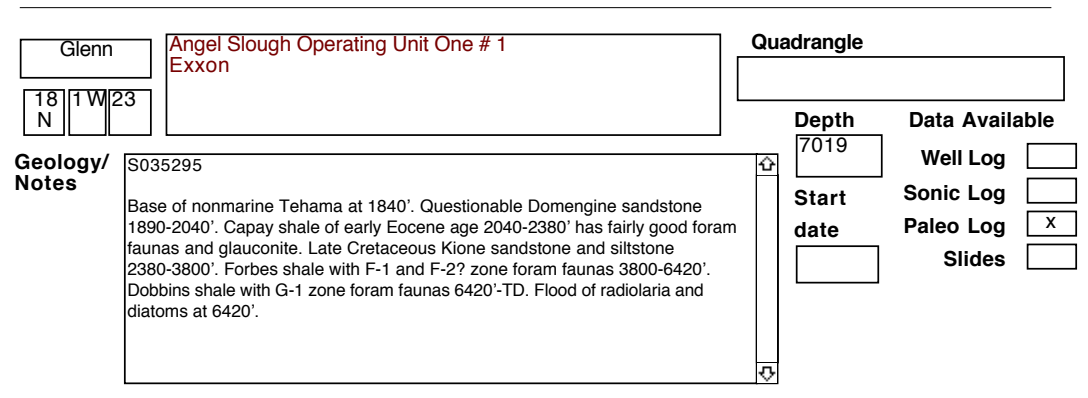

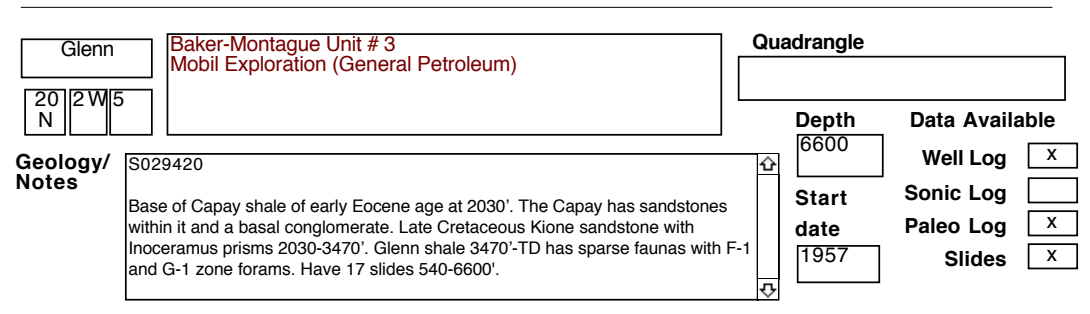

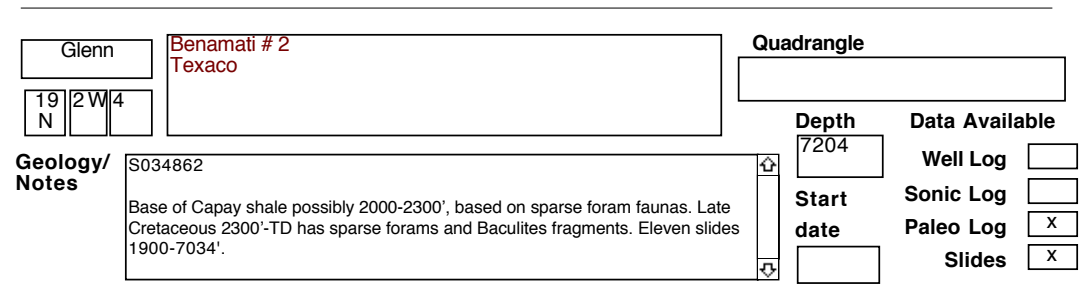


SELECTED OIL TEST WELLS IN CENTRAL CALIFORNIA DATABASE County

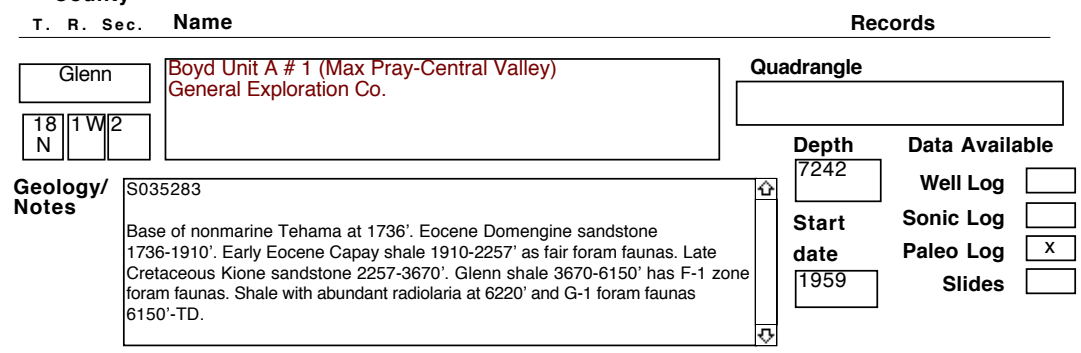

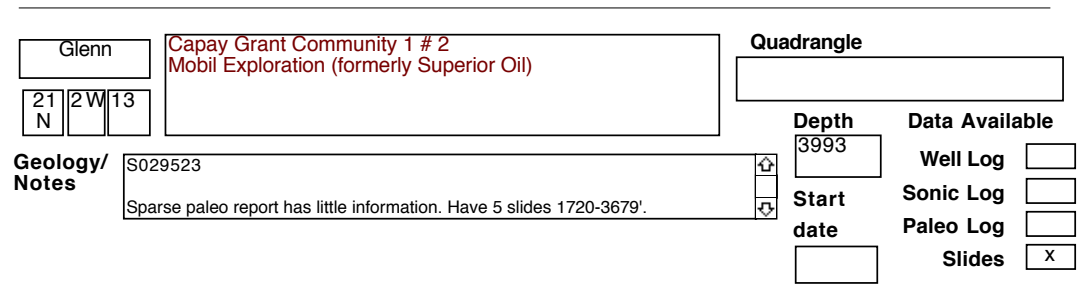

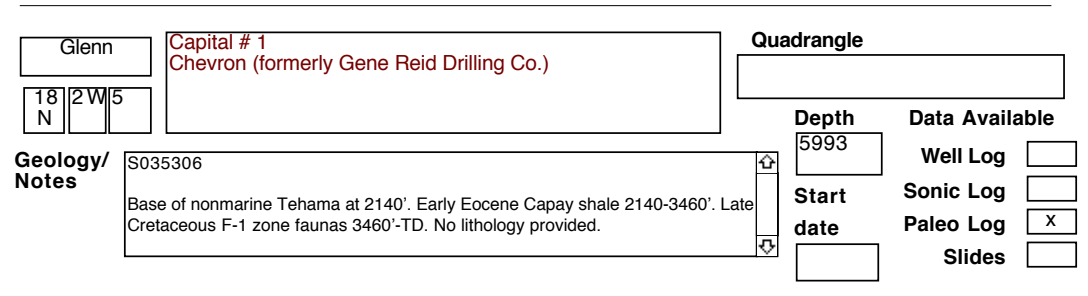

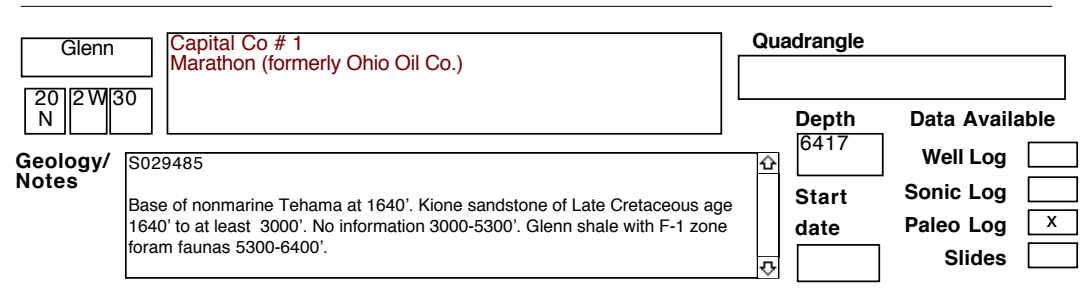

\begin{tabular}{l|l|l|l|l|}
\hline \multicolumn{1}{|c|}{$\begin{array}{l}\text { Capital \#2 } \\
\text { Chevron (formerly Western Gulf) }\end{array}$} \\
\hline
\end{tabular}


SELECTED OIL TEST WELLS IN CENTRAL CALIFORNIA DATABASE County

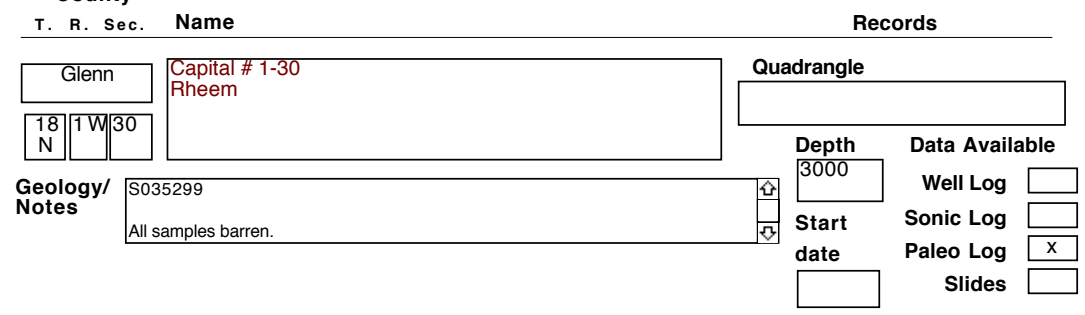

\begin{tabular}{l|l|l|l|l|l|}
\hline \multicolumn{1}{|c|}{$\begin{array}{l}\text { Capital \# 1-31 } \\
\text { Rheem }\end{array}$} & \multicolumn{2}{l|}{ Quadrangle } \\
\hline
\end{tabular}

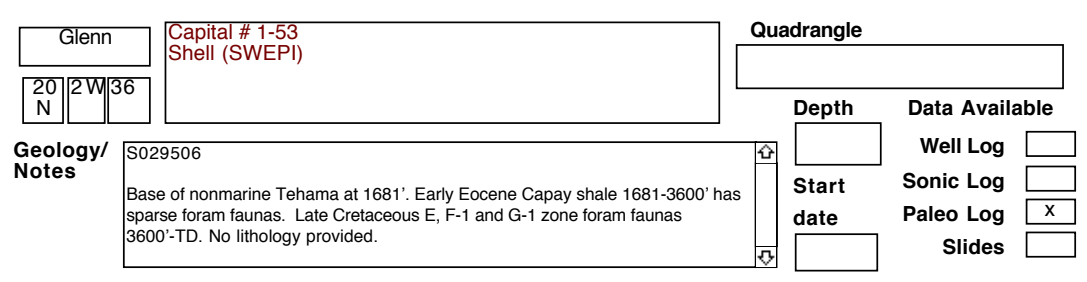

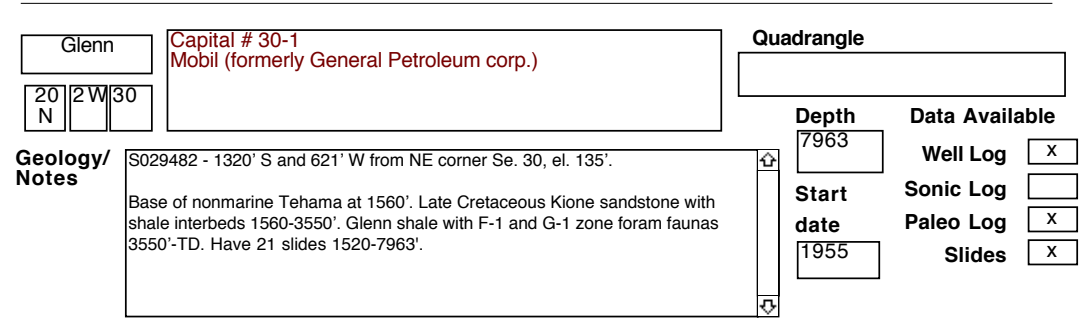

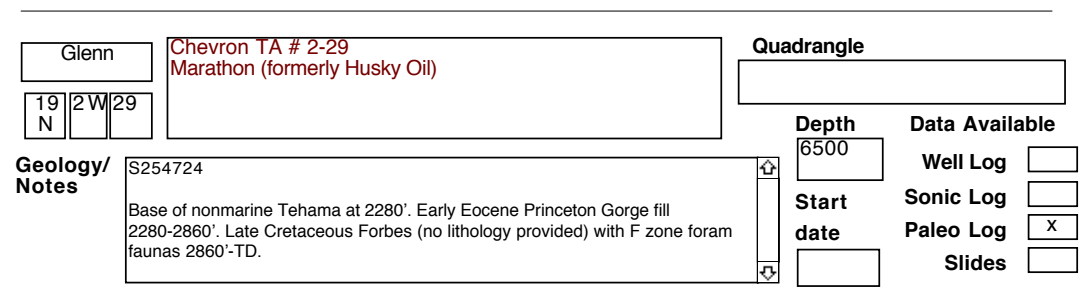




\section{SELECTED OIL TEST WELLS IN CENTRAL CALIFORNIA DATABASE}

County

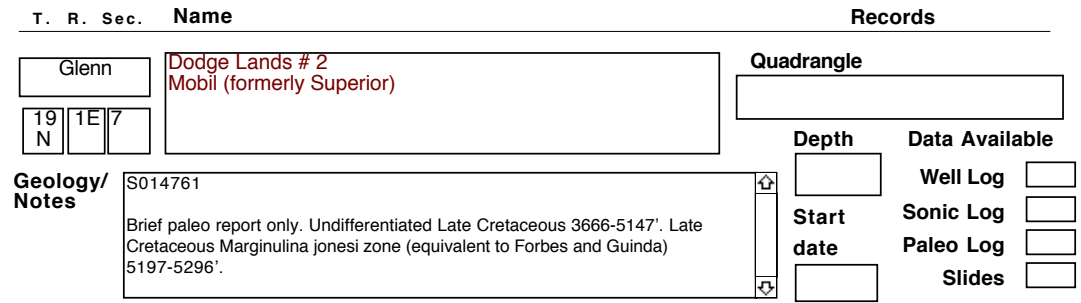

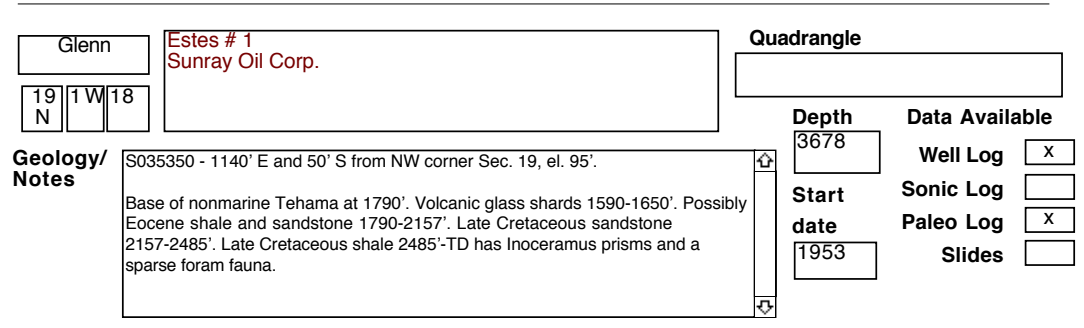

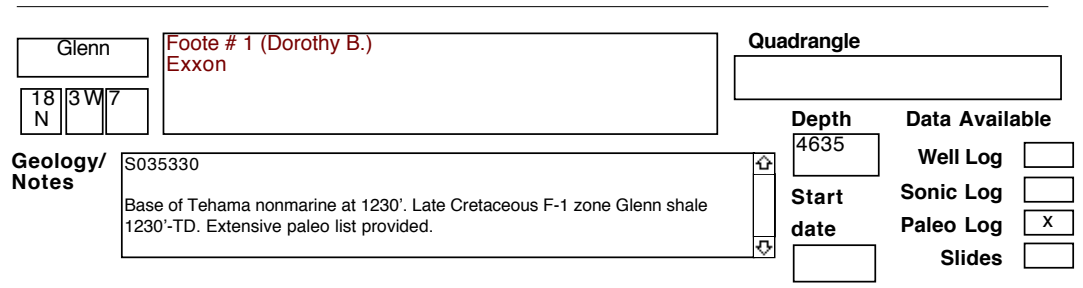

\begin{tabular}{|l|l|l|l|l|l|}
\hline \multicolumn{1}{|c|}{ Glenn } & $\begin{array}{l}\text { Fredericksen \# 1 } \\
\text { Exxon }\end{array}$ \\
\hline
\end{tabular}

\begin{tabular}{|c|c|c|c|c|}
\hline Glenn & Friesen \#1 & Qua & drangle & \\
\hline \begin{tabular}{|l|l|l|}
19 & $1 \mathrm{~W}$ & 19 \\
$\mathrm{~N}$ & & \\
\end{tabular} & & & Depth & Data Availabl \\
\hline $\begin{array}{l}\text { Geology/ } \\
\text { Notes }\end{array}$ & $\begin{array}{l}35351 \\
\text { ef paleo report only. Late Cretaceous Marginulina jonesi zone 3585-3778'. } \\
\text { culites fragments frequent in cores } 3750-3778 \text { '. Four slides } 3565-3778 \text { '. }\end{array}$ & 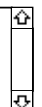 & $\begin{array}{l}\text { Start } \\
\text { date }\end{array}$ & $\begin{array}{l}\text { Well Log } \\
\text { Sonic Log } \\
\text { Paleo Log }\end{array}$ \\
\hline
\end{tabular}




\section{SELECTED OIL TEST WELLS IN CENTRAL CALIFORNIA DATABASE}

County

\begin{tabular}{|c|c|}
\hline Glenn & $\begin{array}{l}\text { Garnett-Knight \# } 1 \\
\text { Mobil (formerly General Petroleum) }\end{array}$ \\
\hline 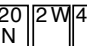 & \\
\hline
\end{tabular}

\section{Geology/ S029419}

Notes

Information from 1500-6500'. Base of Tehama nonmarine at 1690'. Capay or Tehama, mainly sandstone, 1690-2180'. Early Eocene Capay sandstone and shale and a basal conglomerate (2410-2695' ) 2180-2695'. Late Cretaceous Kione sandstone and shale 2695-4290'. Glenn shale with E, F, and G-1 zone foram faunas $4290-6500^{\prime}$. Fourteen slides $1760-6500^{\prime}$

\begin{tabular}{|c|c|c|c|}
\hline \multicolumn{4}{|c|}{ Records } \\
\hline \multicolumn{4}{|c|}{ Quadrangle } \\
\hline \multirow{4}{*}{$\varphi$} & Depth & Data Avail & \\
\hline & & Well Log & $\mathrm{x}$ \\
\hline & Start & Sonic Log & \\
\hline & date & Paleo Log & $\mathrm{x}$ \\
\hline & & Slides & $\mathrm{x}$ \\
\hline
\end{tabular}

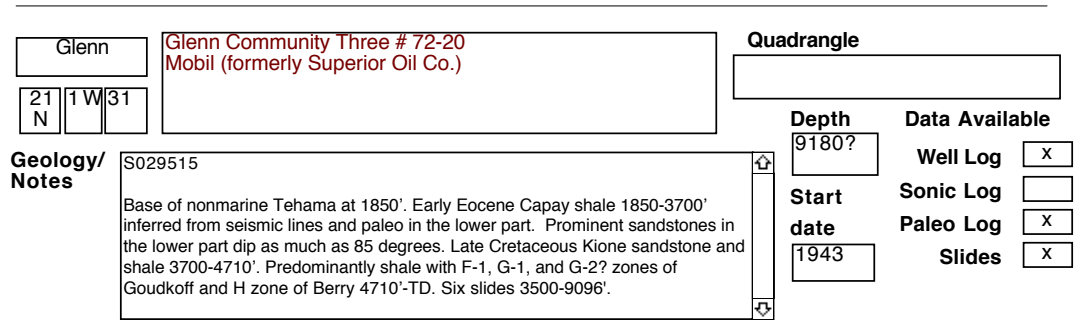

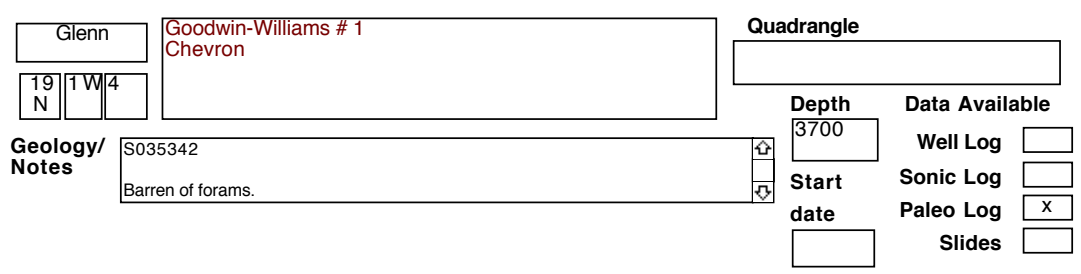

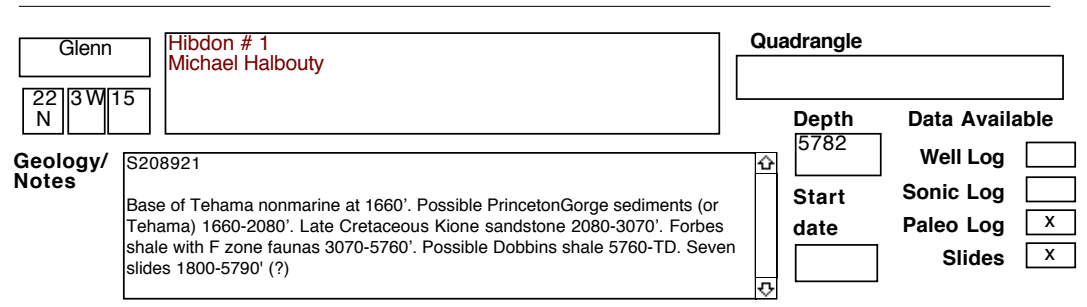

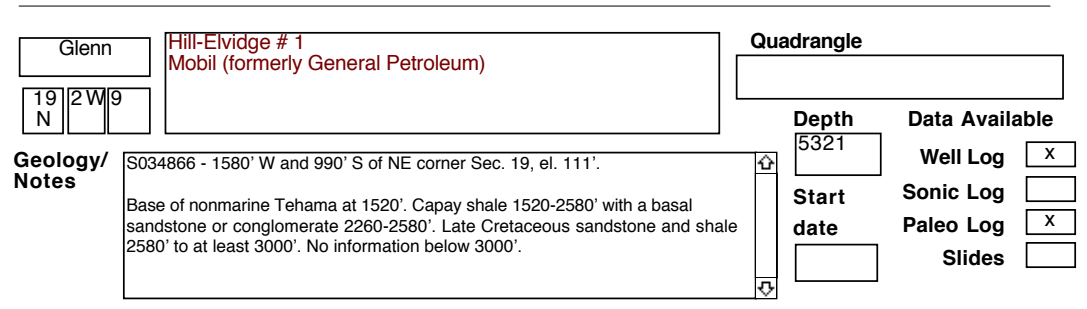


SELECTED OIL TEST WELLS IN CENTRAL CALIFORNIA DATABASE County

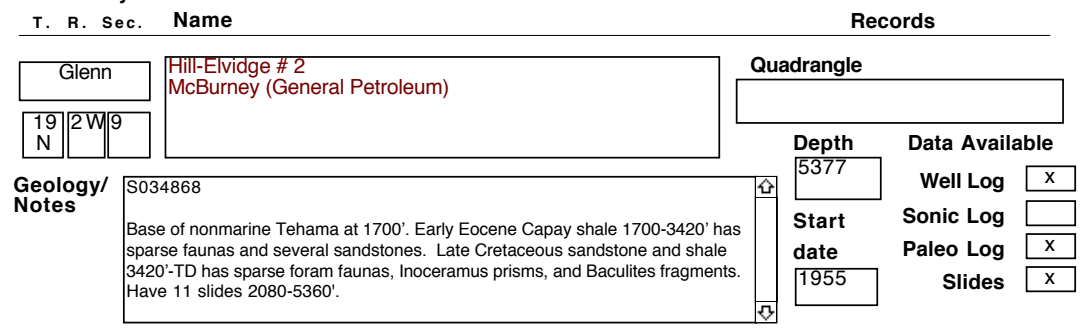

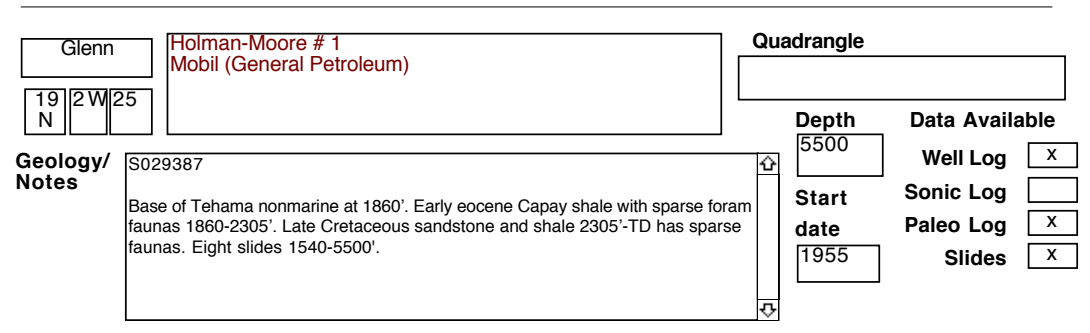

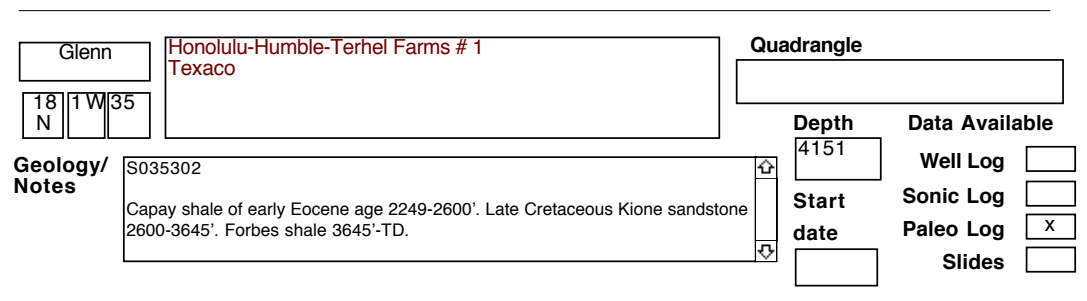

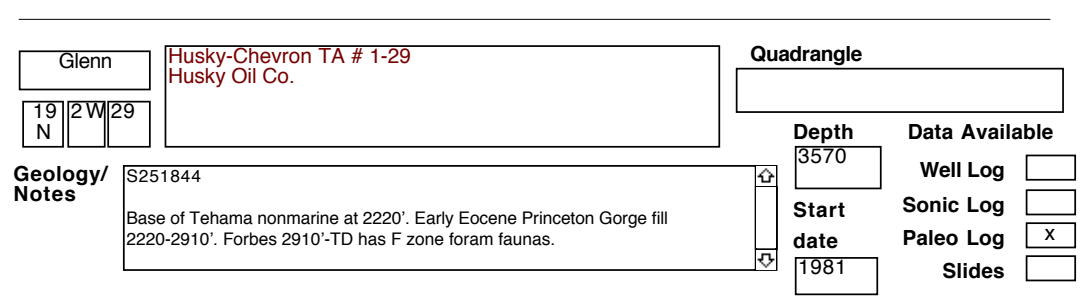

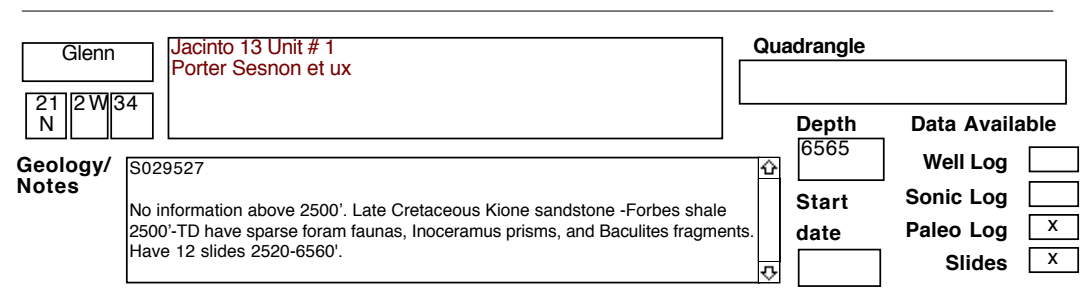


SELECTED OIL TEST WELLS IN CENTRAL CALIFORNIA DATABASE County

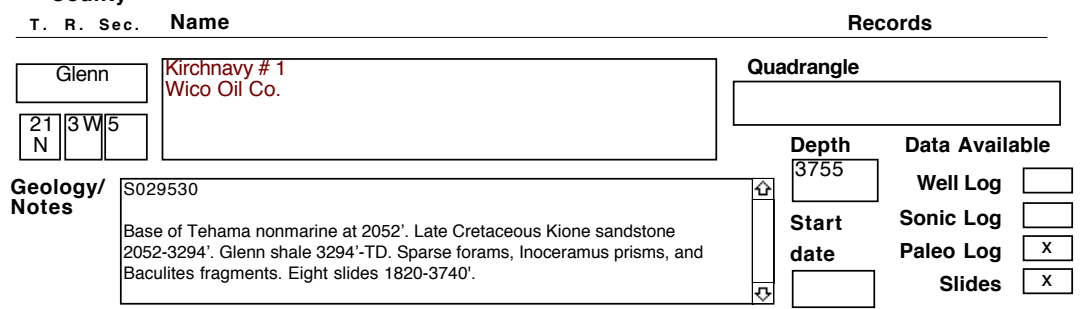

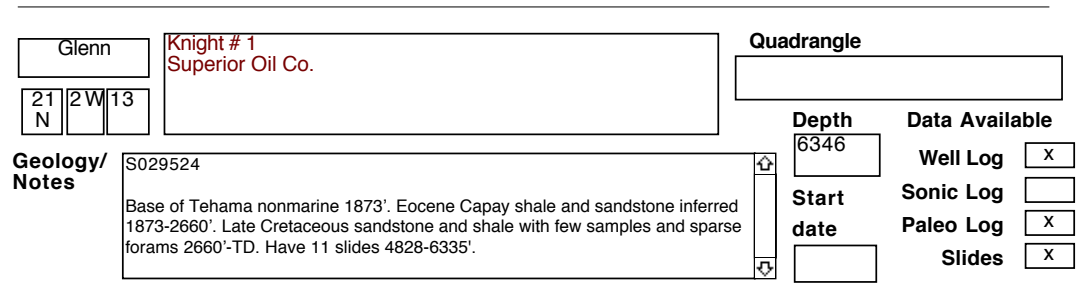

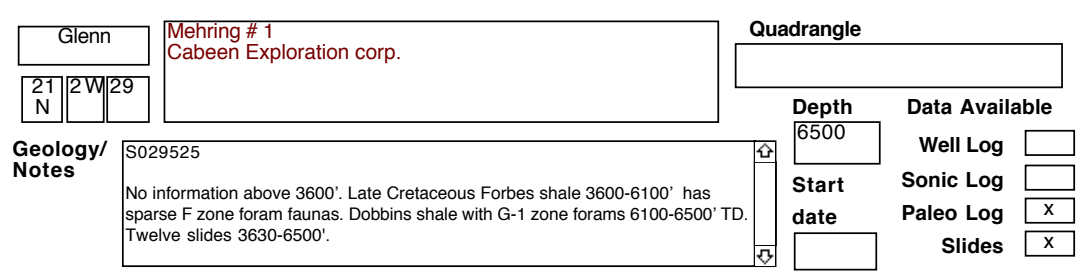

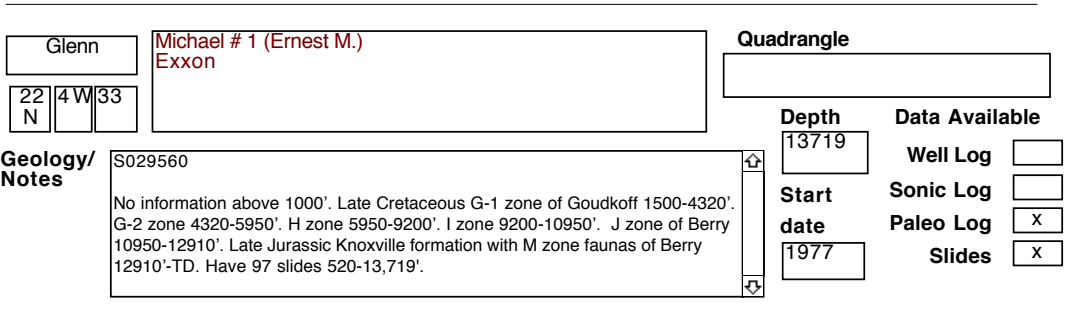

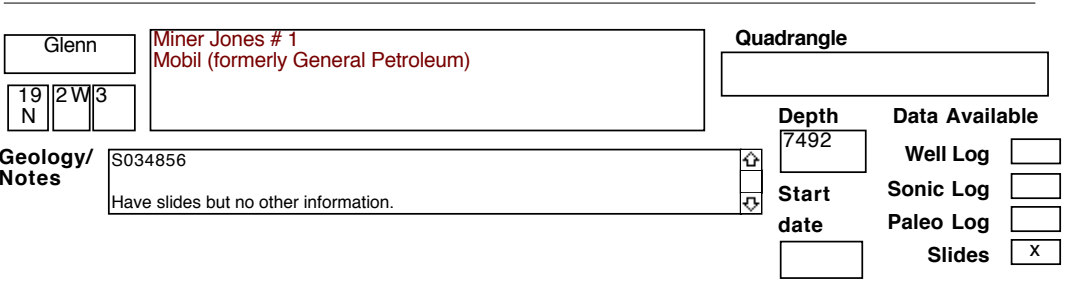


SELECTED OIL TEST WELLS IN CENTRAL CALIFORNIA DATABASE County

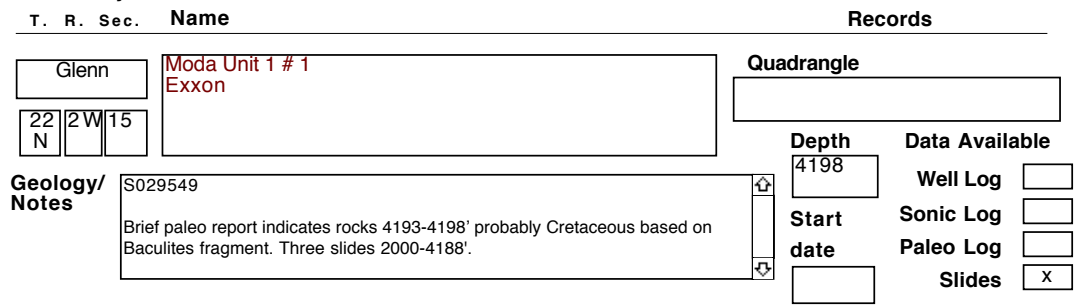

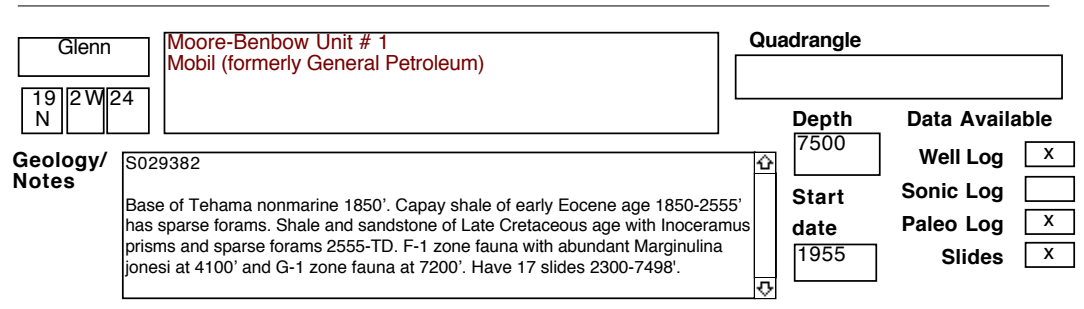

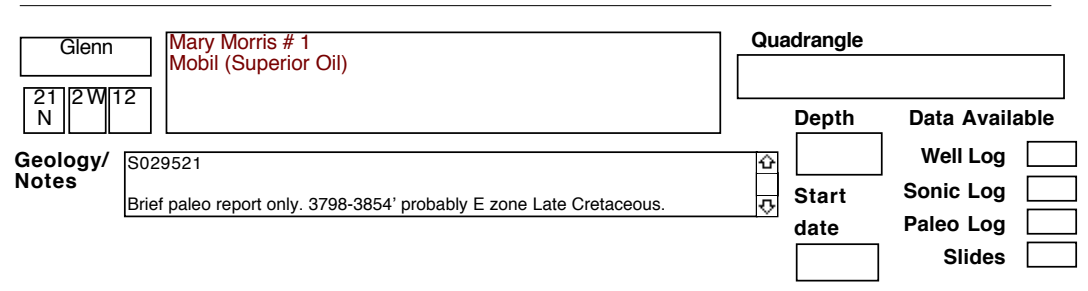

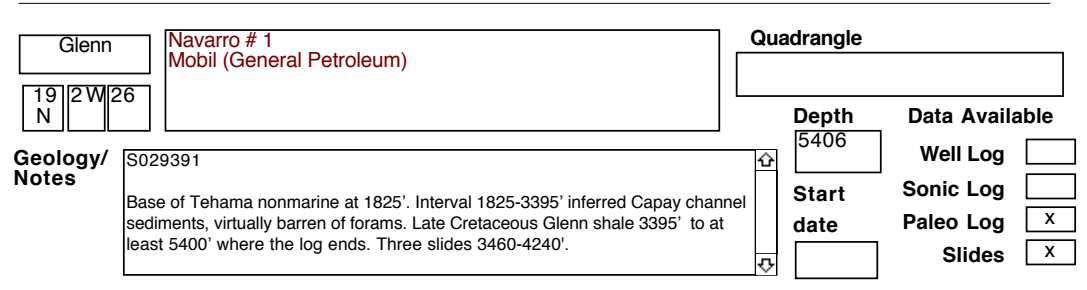

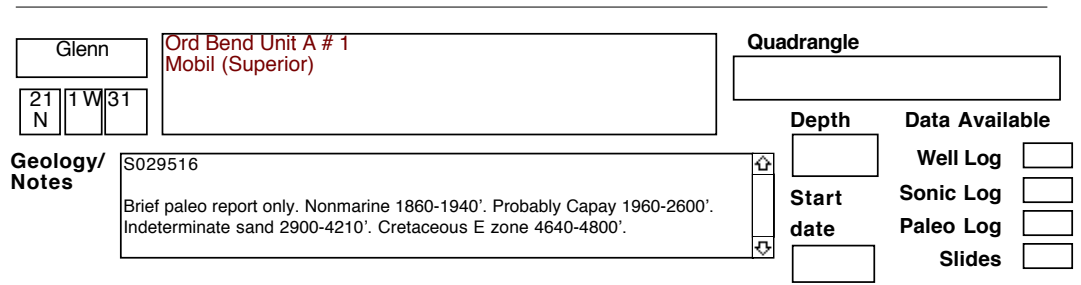


SELECTED OIL TEST WELLS IN CENTRAL CALIFORNIA DATABASE County

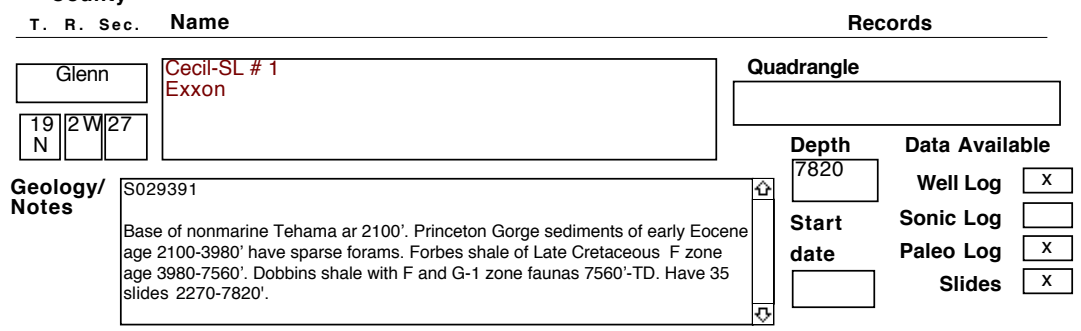

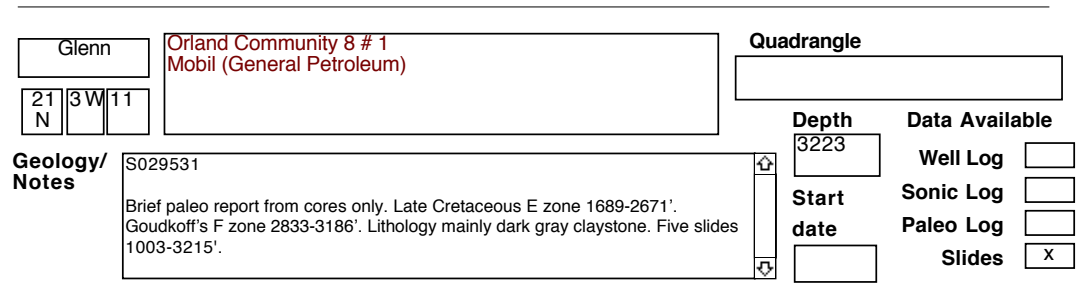

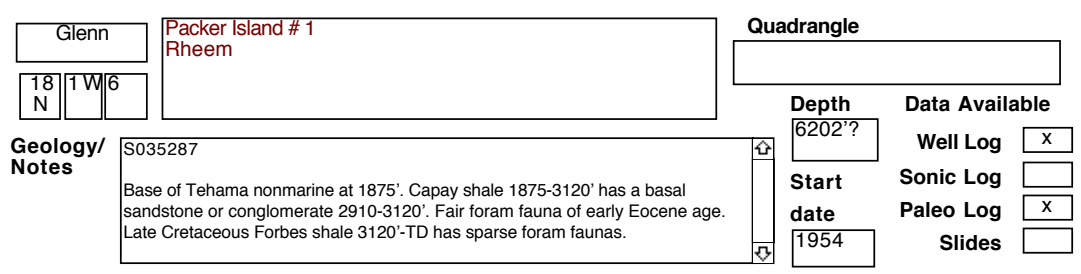

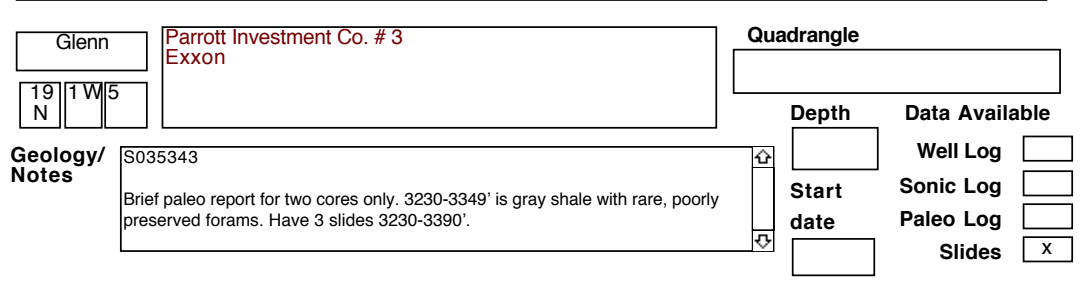

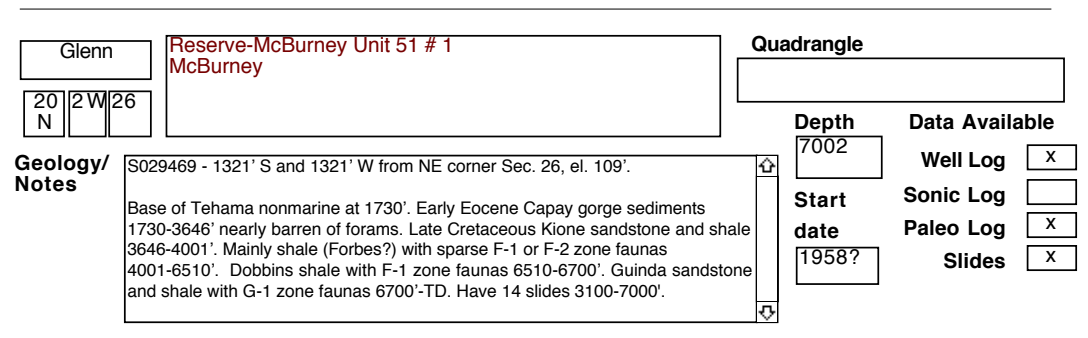


SELECTED OIL TEST WELLS IN CENTRAL CALIFORNIA DATABASE County

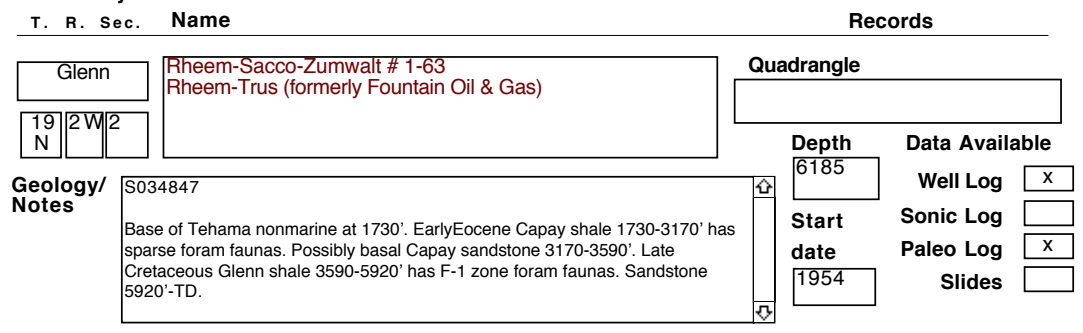

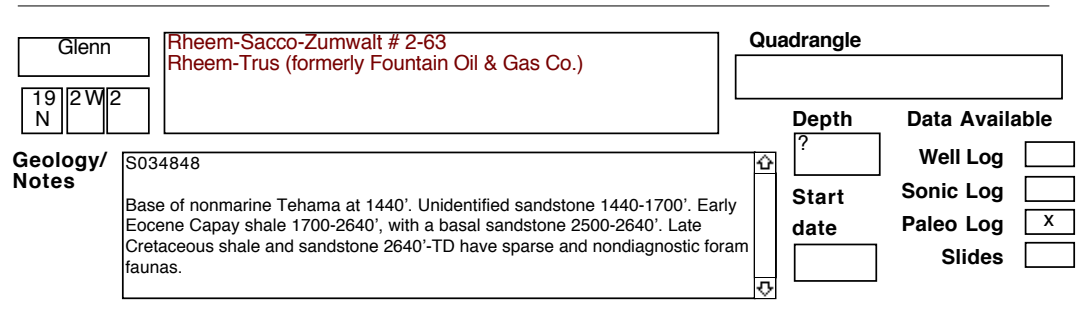

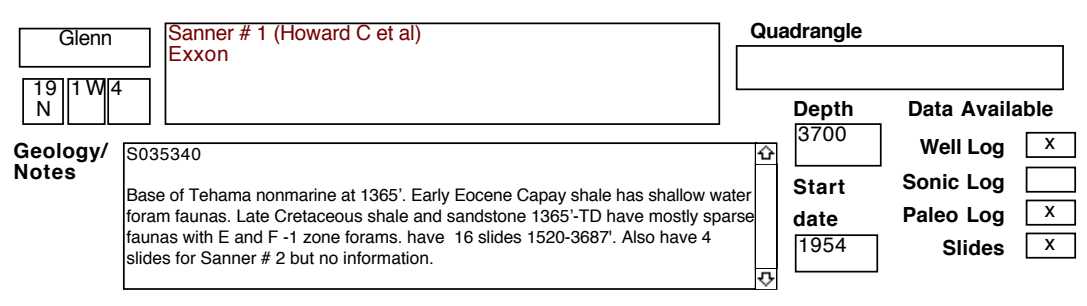

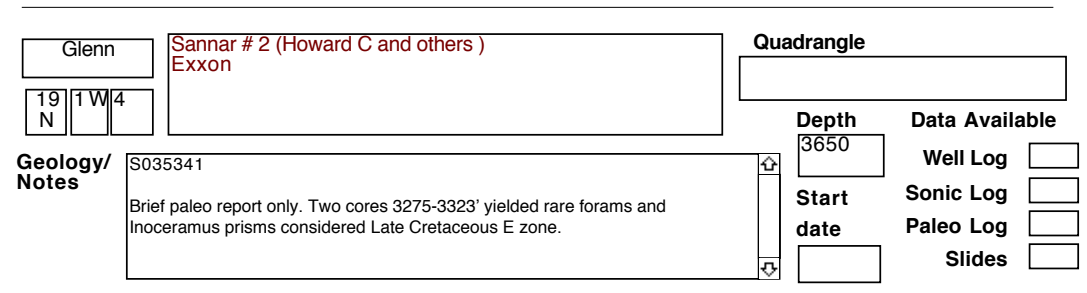

\begin{tabular}{|c|c|c|c|c|c|}
\hline Glenn & \multirow{2}{*}{$\begin{array}{l}\text { Section } 20 \text { Unit \# } 1 \\
\text { Mobil (General Petroleum) }\end{array}$} & \multicolumn{4}{|c|}{ Quadrangle } \\
\hline$\left.$\begin{tabular}{|l||l|l|}
$20 \mathrm{~V}$ & 2 \\
$\mathrm{~N}$
\end{tabular}\right|$^{2}$ & & \multirow{2}{*}{\multicolumn{2}{|c|}{$\begin{array}{l}\text { Depth } \\
5850\end{array}$}} & \multicolumn{2}{|c|}{ Data Available } \\
\hline \multirow{3}{*}{$\begin{array}{l}\text { Geology/ } \\
\text { Notes }\end{array}$} & 29449 & & & Well Log & $\mathrm{x}$ \\
\hline & se of Tehama nonmarine at $1375^{\prime}$. Late Cretaceous Kione sandstone and & & Start & Sonic Log & $\mathrm{x}$ \\
\hline & nas. Have 30 slides 1460-5850'. & & 1955 & Slides & $\mathrm{x}$ \\
\hline
\end{tabular}




\section{SELECTED OIL TEST WELLS IN CENTRAL CALIFORNIA DATABASE}

County

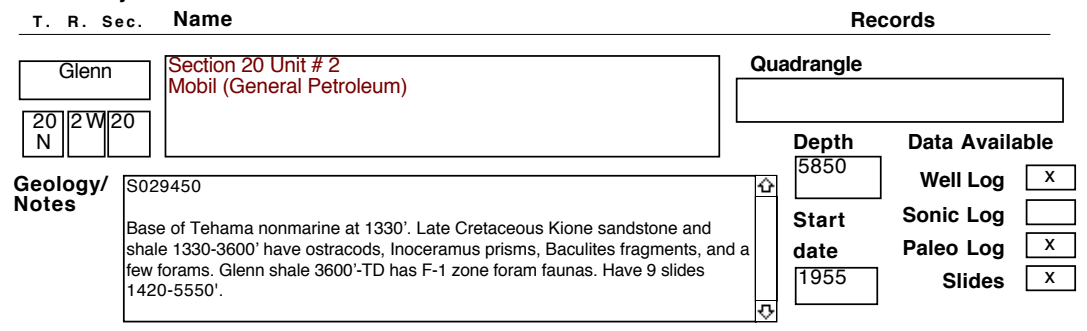

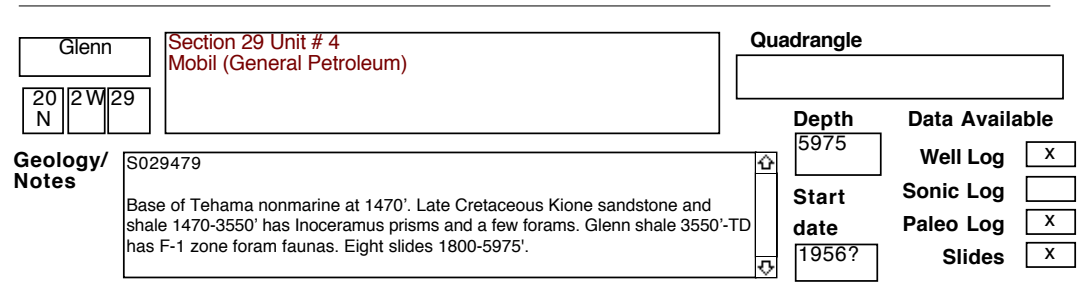

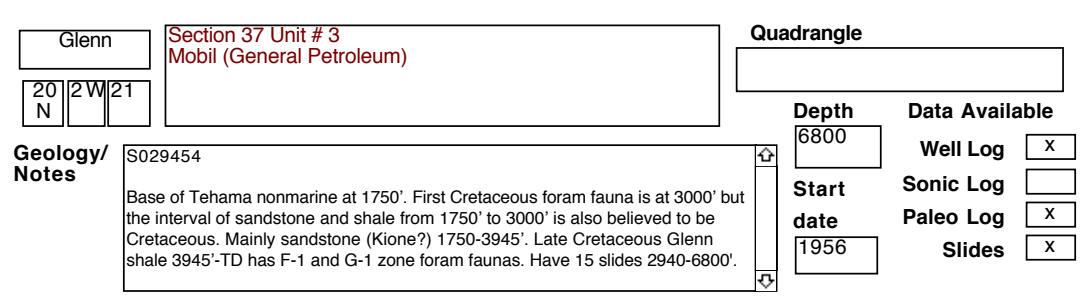

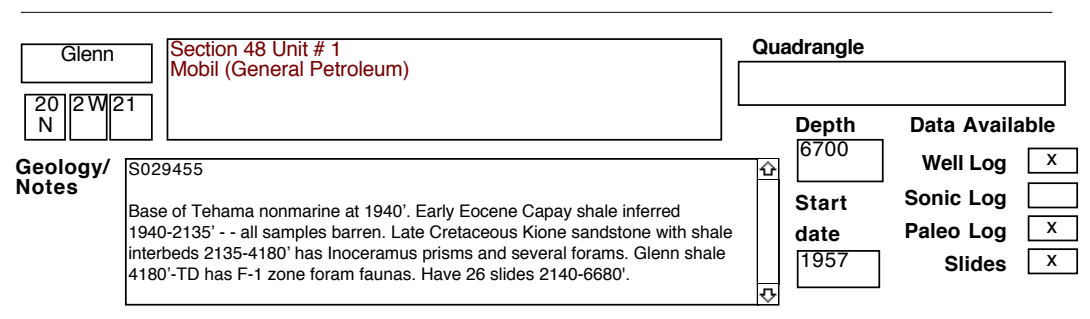

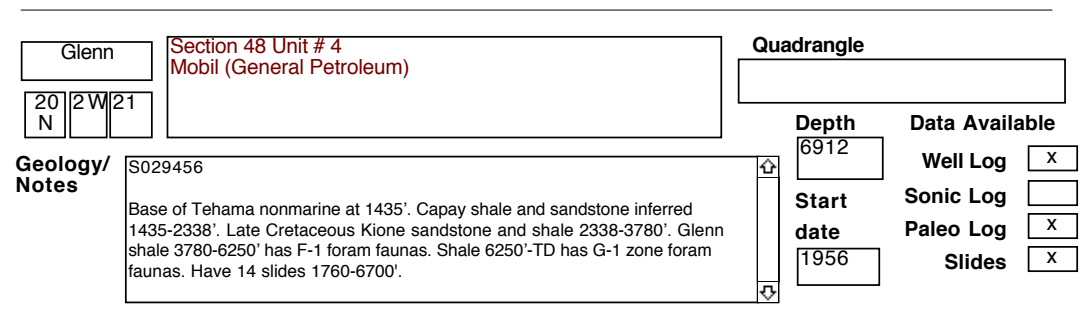




\section{SELECTED OIL TEST WELLS IN CENTRAL CALIFORNIA DATABASE}

County

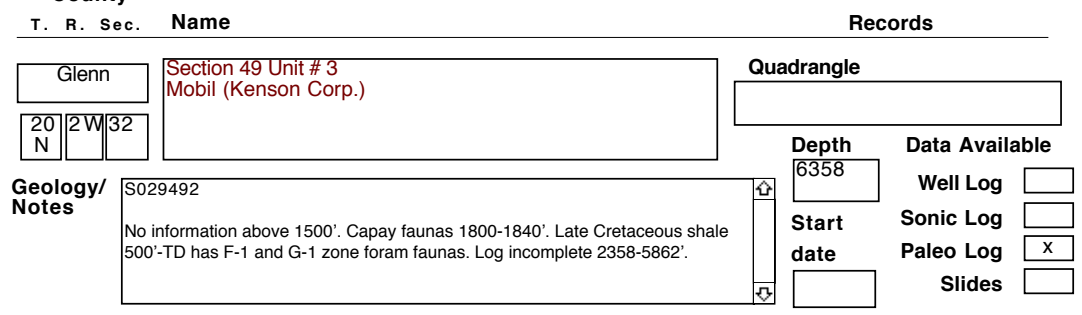

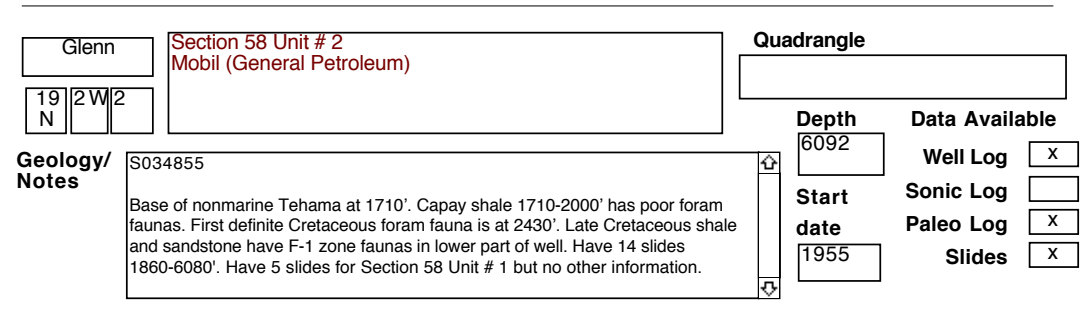

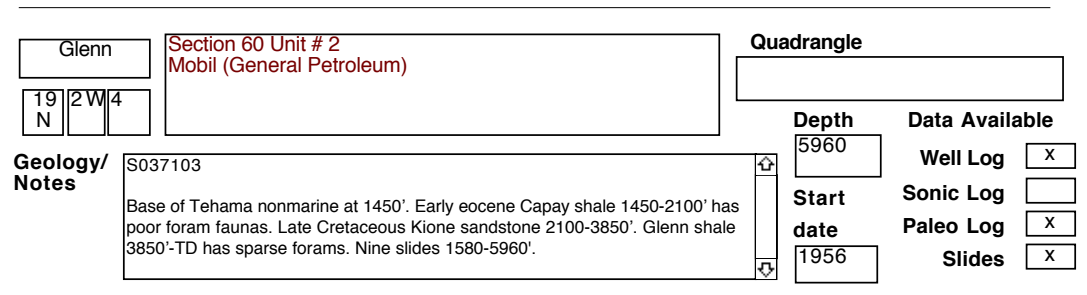

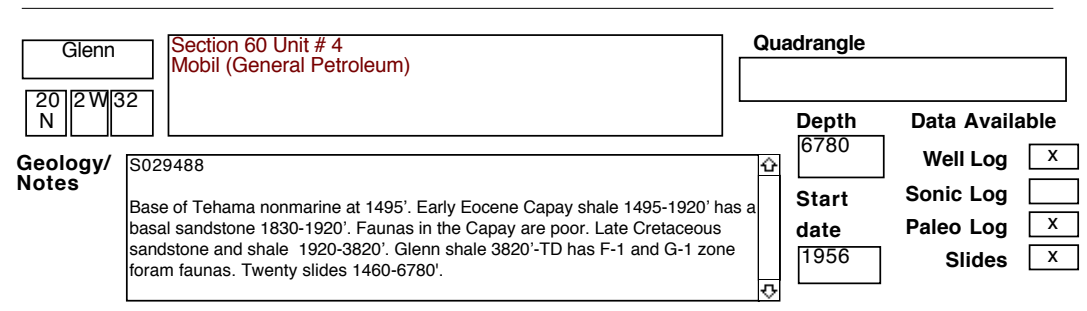

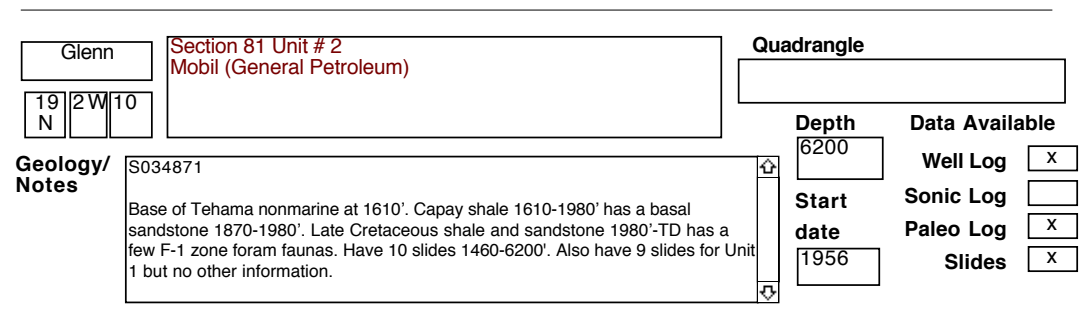


SELECTED OIL TEST WELLS IN CENTRAL CALIFORNIA DATABASE County

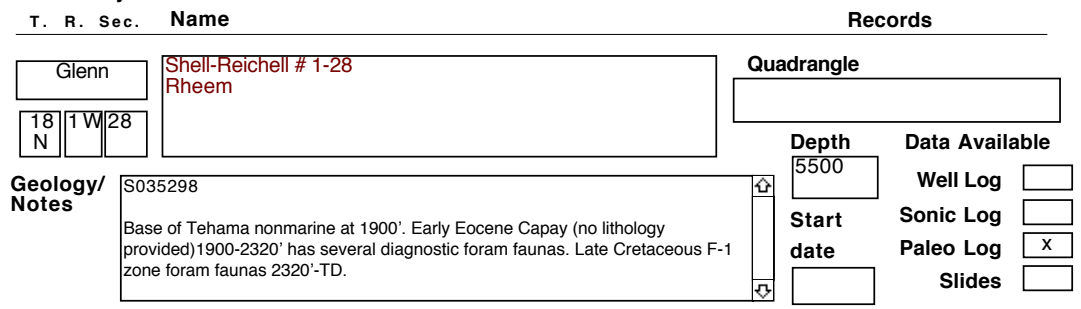

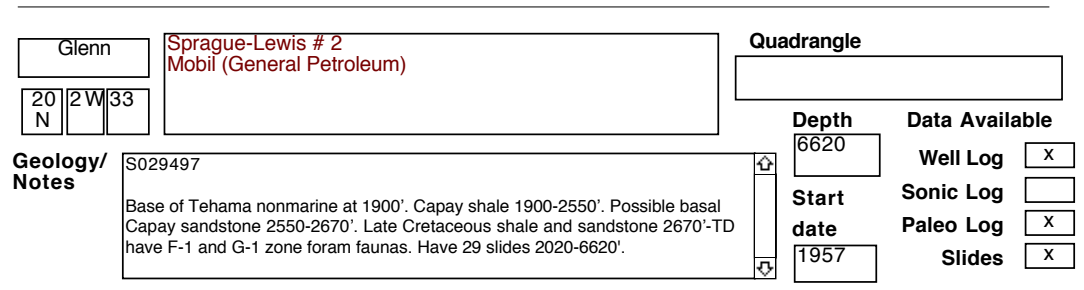

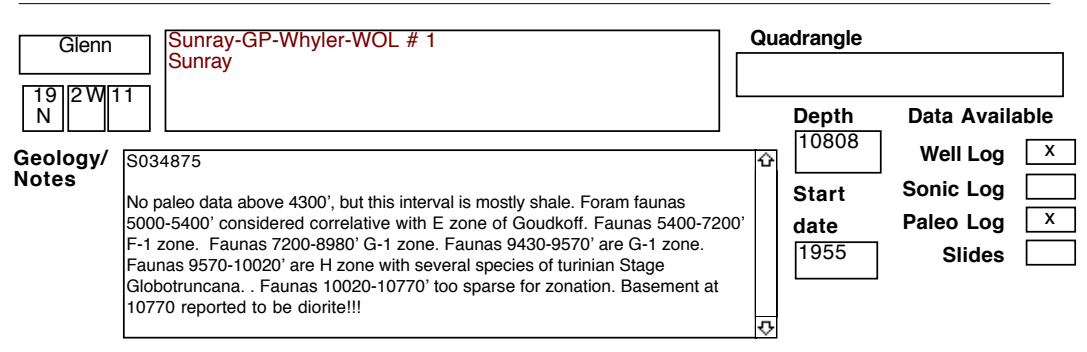

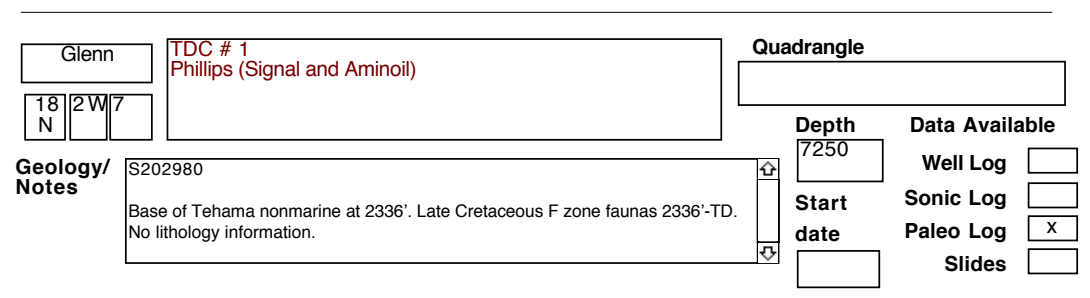

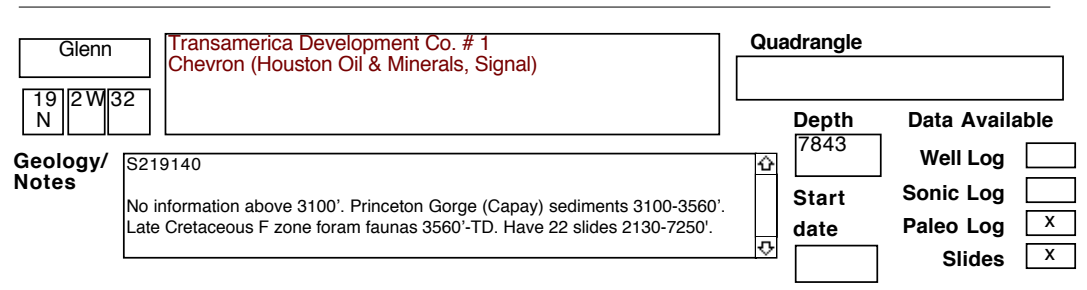


SELECTED OIL TEST WELLS IN CENTRAL CALIFORNIA DATABASE County

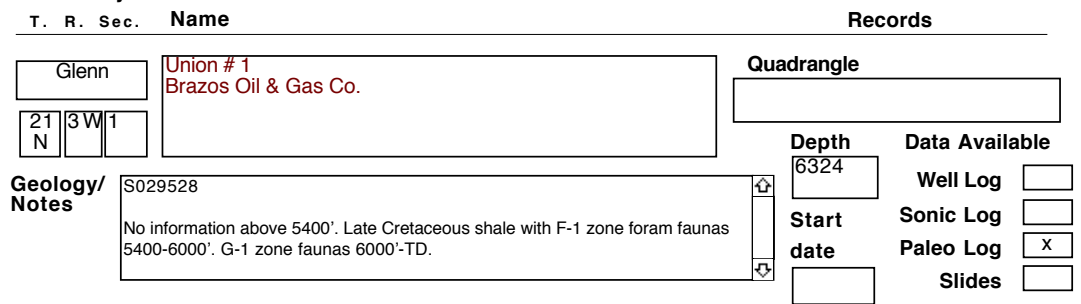

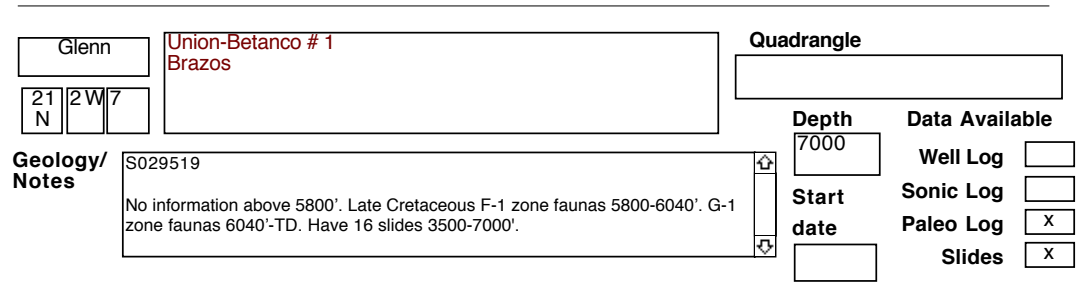

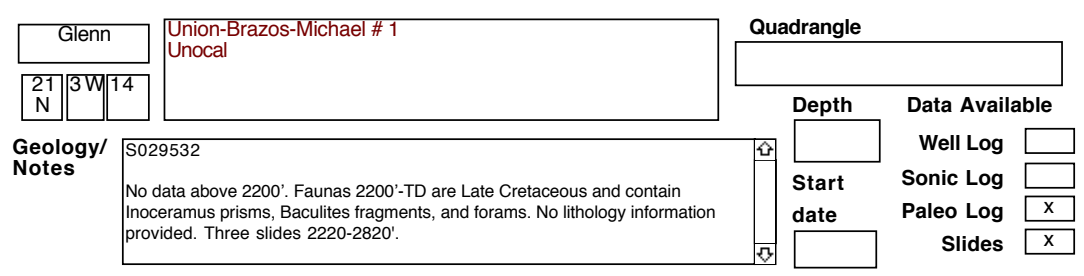

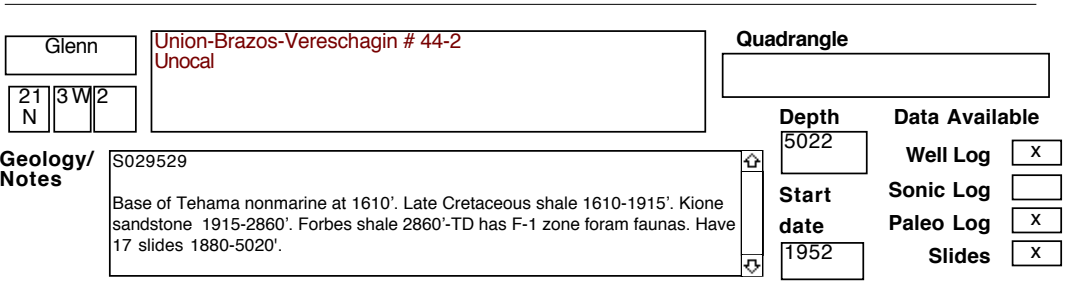

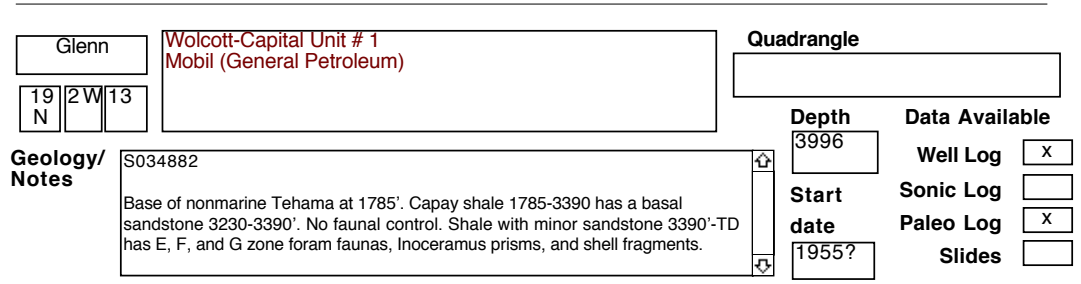


SELECTED OIL TEST WELLS IN CENTRAL CALIFORNIA DATABASE County

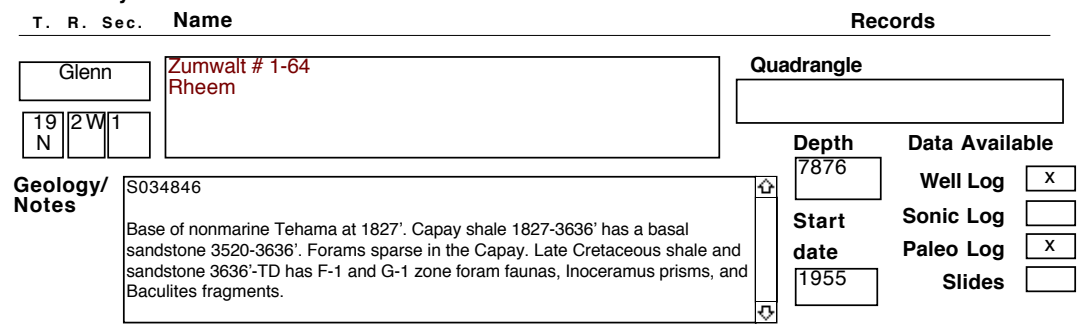

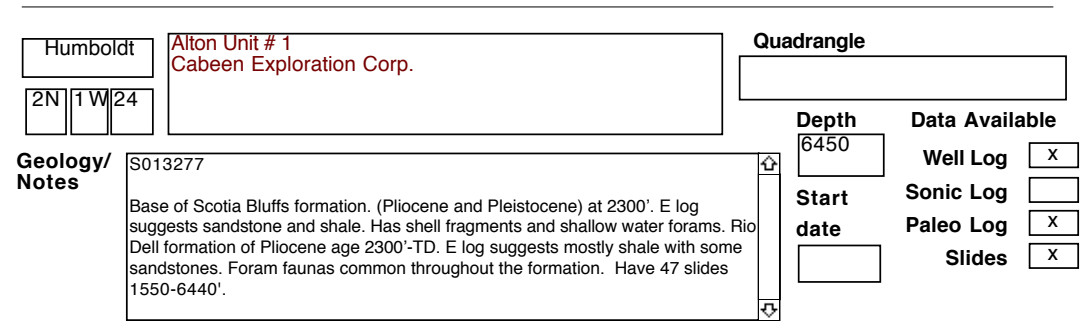

\begin{tabular}{l|l|l|l|}
\hline Humboldt & $\begin{array}{l}\text { Anderson \# } 1 \\
\text { Texaco }\end{array}$ & \multicolumn{2}{l|}{ Quadrangle } \\
\hline
\end{tabular}

\begin{tabular}{|c|c|c|c|c|c|}
\hline Humboldt & \multirow{2}{*}{$\begin{array}{l}\text { Bo-Peep \#1 } \\
\text { Bo-Peep Eureka Oil Co. }\end{array}$} & \multicolumn{4}{|c|}{ Quadrangle } \\
\hline $1 \mathrm{~S} \mid 2 \mathrm{~W} 21$ & & \multirow{2}{*}{\multicolumn{2}{|c|}{$\begin{array}{l}\text { Depth } \\
3281\end{array}$}} & \multicolumn{2}{|c|}{ Data Available } \\
\hline \multirow{4}{*}{$\begin{array}{l}\text { Geology/ } \\
\text { Notes }\end{array}$} & 39559 & & & Well Log & $\mathrm{x}$ \\
\hline & g suggests mainly sandstone with shale interbeds. Faunas mainly & & Start & Sonic Log & \\
\hline & $\begin{array}{l}\text { ths that are similar to those in rocks of late Miocene and early Pliocene age } \\
\text { ths }\end{array}$ & & date & Paleo Log & 垔 \\
\hline & arby. Have 26 slides 100-3280'. & & & Slides & $x$ \\
\hline
\end{tabular}

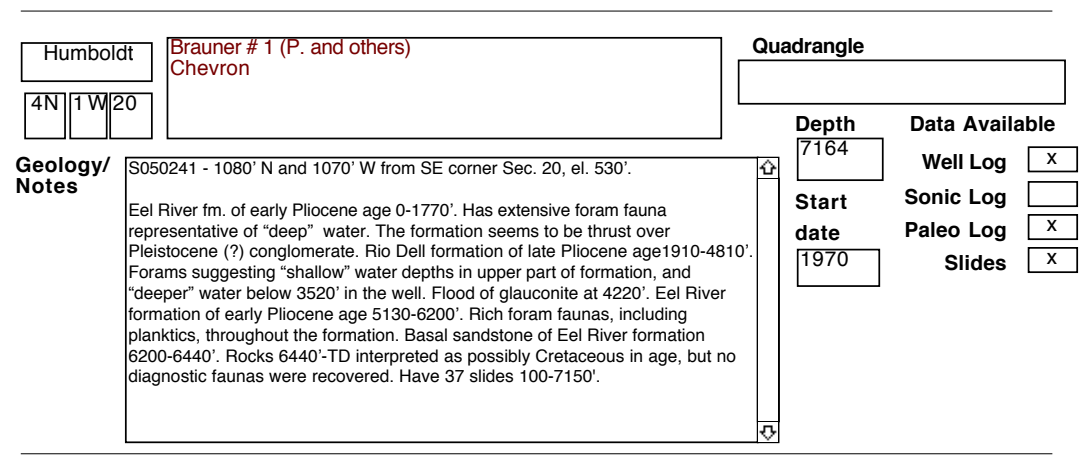




\section{SELECTED OIL TEST WELLS IN CENTRAL CALIFORNIA DATABASE}

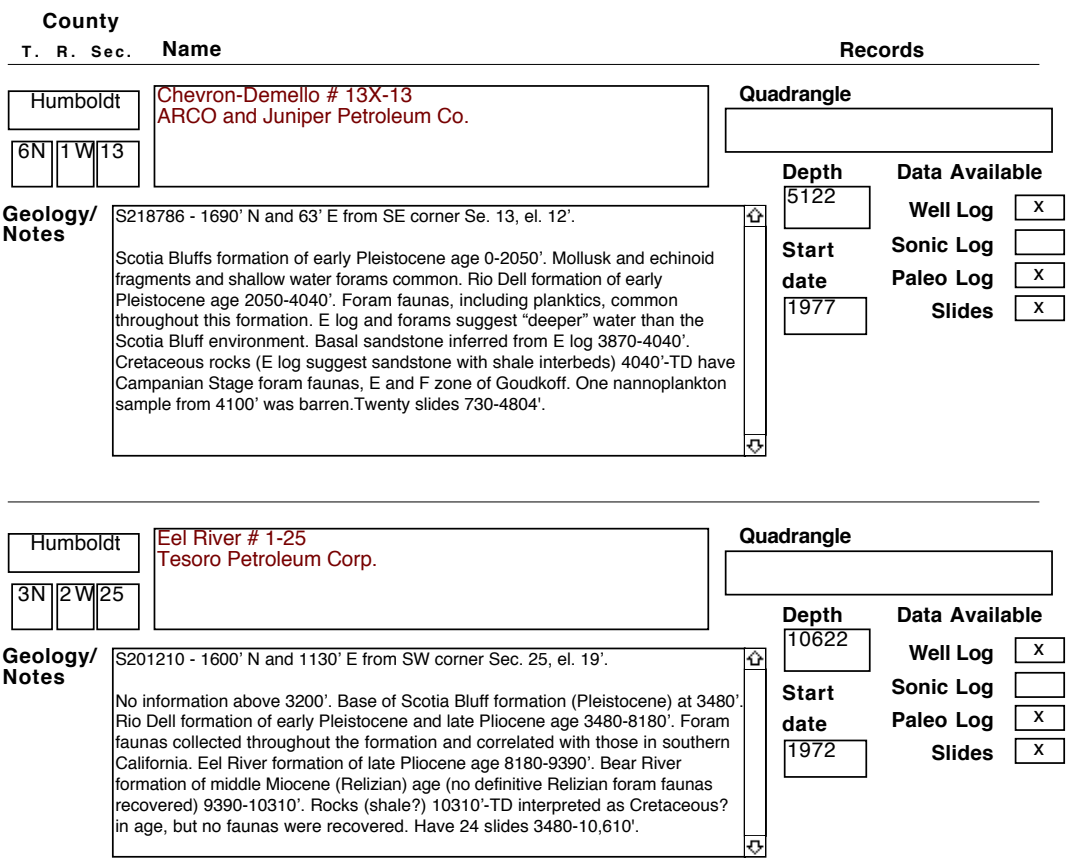

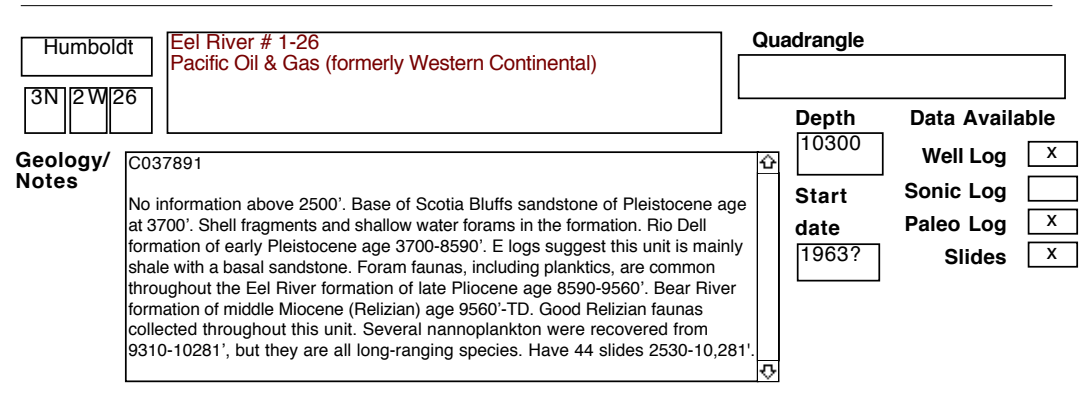

\begin{tabular}{l|l|l|l|l|l|}
\hline Humboldt & $\begin{array}{l}\text { Eureka \# 1 } \\
\text { Texaco }\end{array}$ \\
Geology/ \\
Notes
\end{tabular}

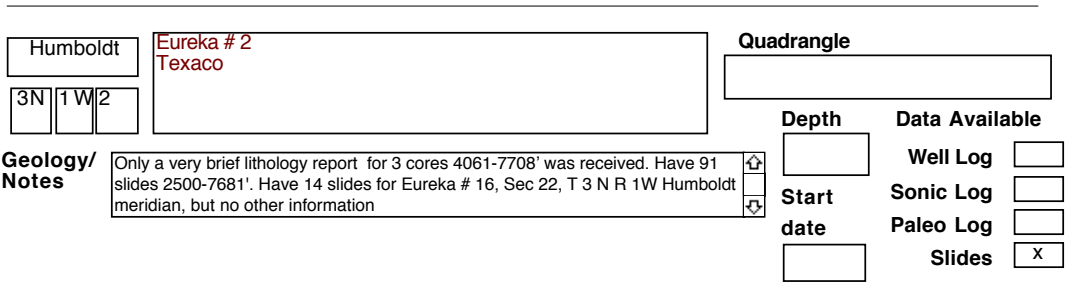


SELECTED OIL TEST WELLS IN CENTRAL CALIFORNIA DATABASE County

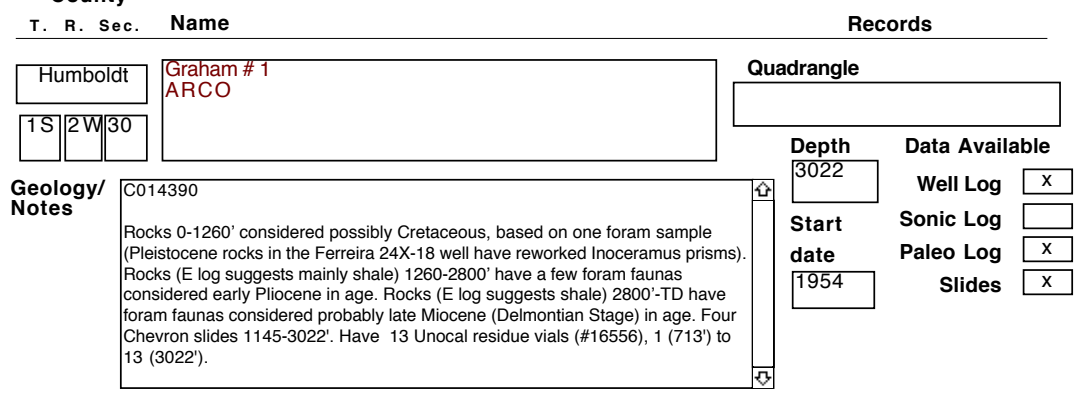

\begin{tabular}{l|l|l|l|l|l|}
\hline Humboldt & $\begin{array}{l}\text { Ferreira \# 24X-18 } \\
\text { Juniper Petroleum Co. }\end{array}$ \\
\hline
\end{tabular}

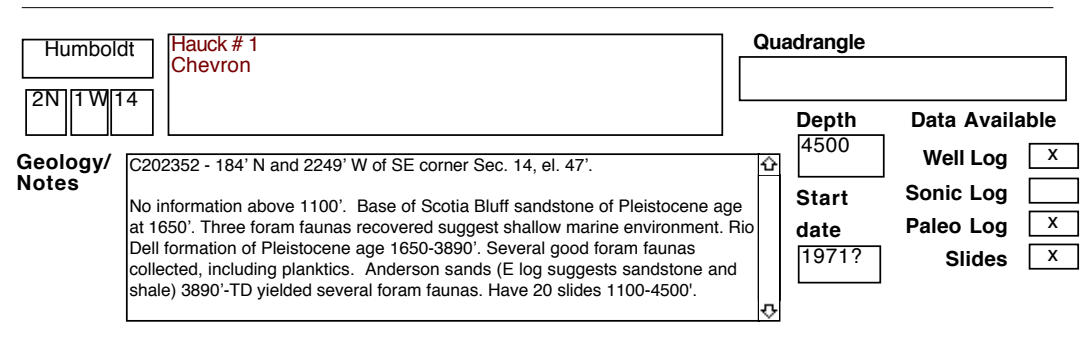

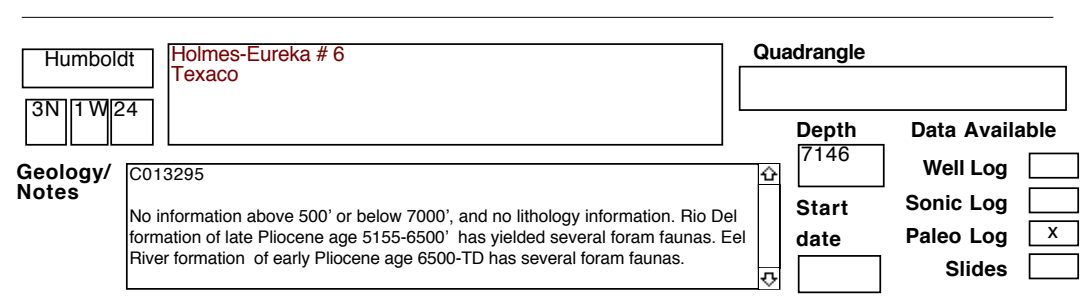

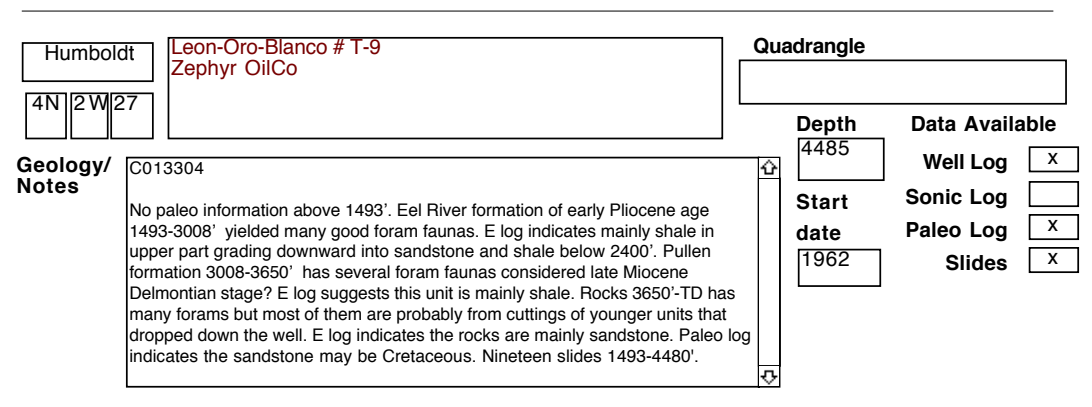




\section{SELECTED OIL TEST WELLS IN CENTRAL CALIFORNIA DATABASE}

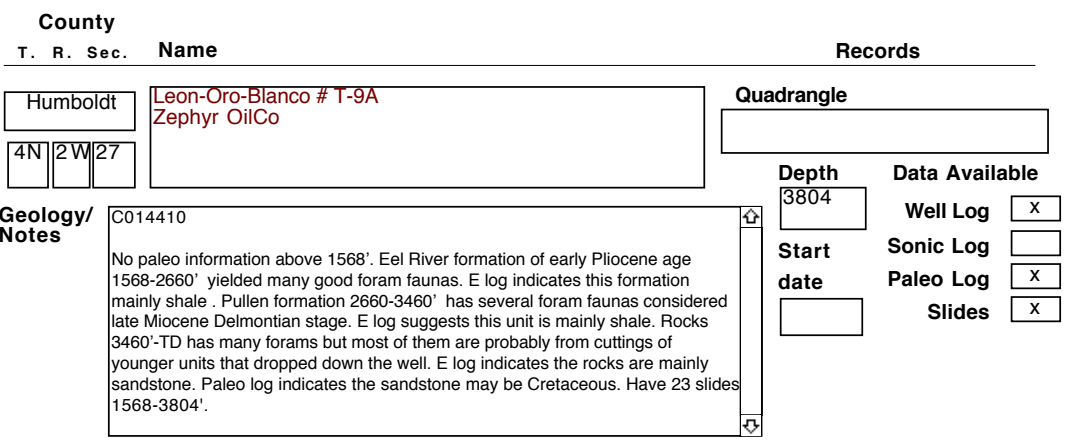

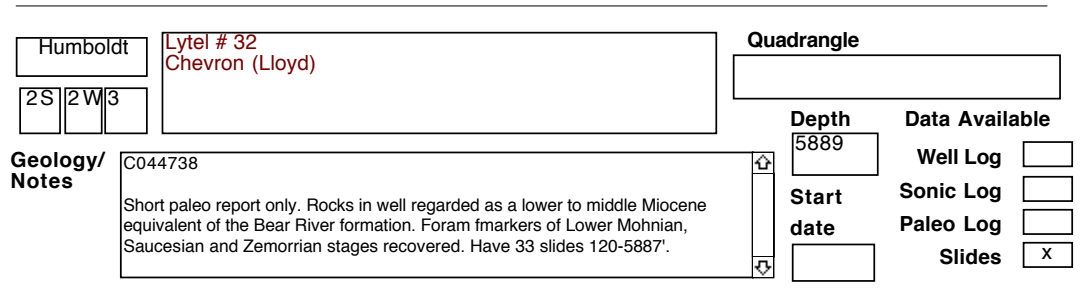

\begin{tabular}{|c|c|c|c|c|}
\hline Humbold & \multirow{2}{*}{$\begin{array}{l}\text { Quinn \# 1 } \\
\text { Texaco }\end{array}$} & \multicolumn{3}{|l|}{ Quadrangle } \\
\hline $4 \mathrm{~N} / 2 \mathrm{~W} / 36$ & & & \multicolumn{2}{|c|}{ Data Available } \\
\hline \multirow{4}{*}{$\begin{array}{l}\text { Geology/ } \\
\text { Notes }\end{array}$} & C013310 & 5590 & \multirow{3}{*}{$\begin{array}{r}\text { Well Log } \\
\text { Sonic Log } \\
\text { Paleo Log } \\
\text { Slides }\end{array}$} & $\mathrm{x}$ \\
\hline & Scotia Bluff sand 500-1000'. Rio Dell formation of early and late Pliocene age & Start & & \\
\hline & $1000-2610^{\prime}$. E log suggests sandstone and shale. Thrust fault inferred at 2610' & date & & $\mathrm{x}$ \\
\hline & $\begin{array}{l}\text { at } 4420^{\prime} \text { interpreted as a normal fault. Eel River formation } 4420-5310^{\prime} \text { has several } \\
\text { good foram faunas. E log suggests this unit is mainly shale becoming sandy } \\
\text { toward the base. Flood of glauconite } 5080-5086^{\prime} \text {. Late Miocene? shale } \\
5310-5420^{\prime} \text { has one foram fauna with several species. Sandstone } 5420^{\prime}-\text {-TD } \\
\text { interpreted as Cretaceous? based on sparse faunas that include species dropped } \\
\text { down from units higher in the well. Have } 26 \text { slides } 980-5580^{\prime} \text {. }\end{array}$ & 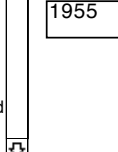 & & $\mathrm{x}$ \\
\hline
\end{tabular}

\begin{tabular}{l|l|l|l|l|}
\hline Humboldt & $\begin{array}{l}\text { Rocha \# 1 } \\
\text { B-N Development Co (formerly Blackwood-Nichols) }\end{array}$ \\
Geology/ \\
Notes
\end{tabular}

\begin{tabular}{l|l|l|l|l|l|l|}
\hline Humboldt & $\begin{array}{l}\text { Roscoe \# 1 } \\
\text { Independant Exploration Co. }\end{array}$ \\
\hline
\end{tabular}




\section{SELECTED OIL TEST WELLS IN CENTRAL CALIFORNIA DATABASE}

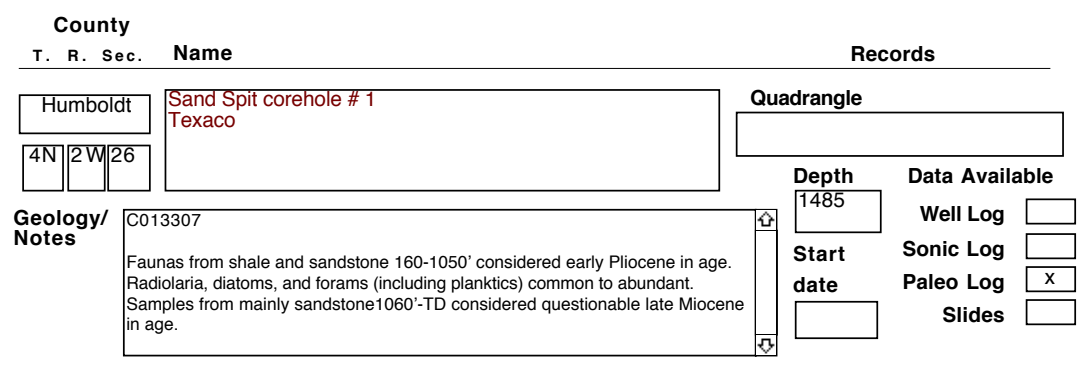

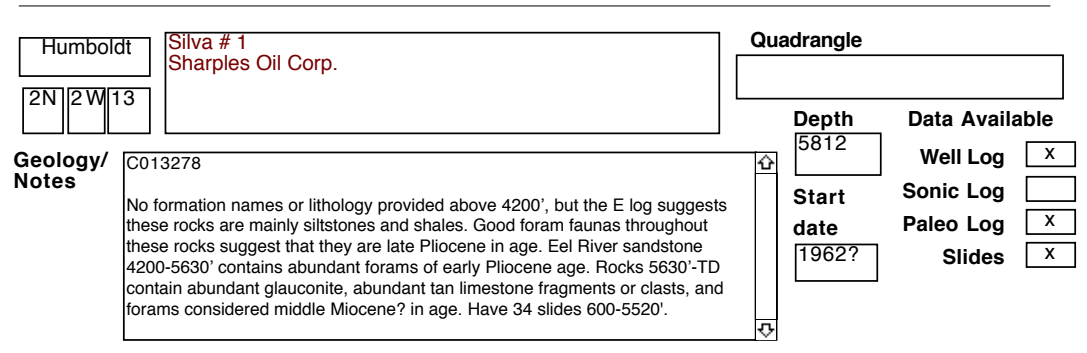

\begin{tabular}{l|l|l|l|l|l|l|}
\hline Humboldt & $\begin{array}{l}\text { Simpson Timber \# 1 } \\
\text { Chevron }\end{array}$ \\
\hline
\end{tabular}

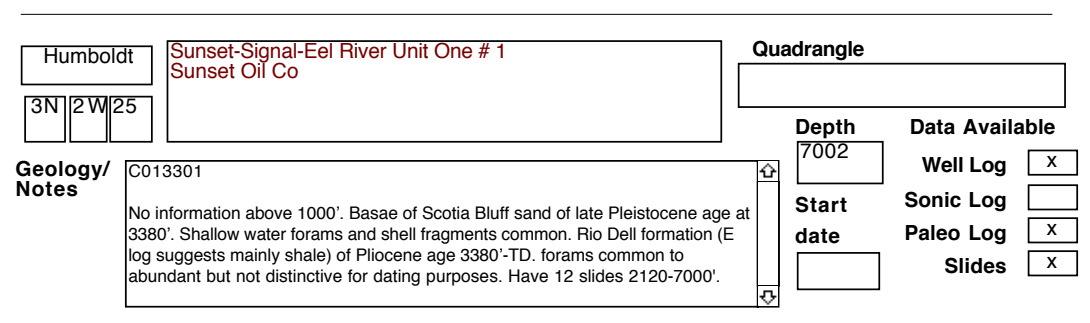

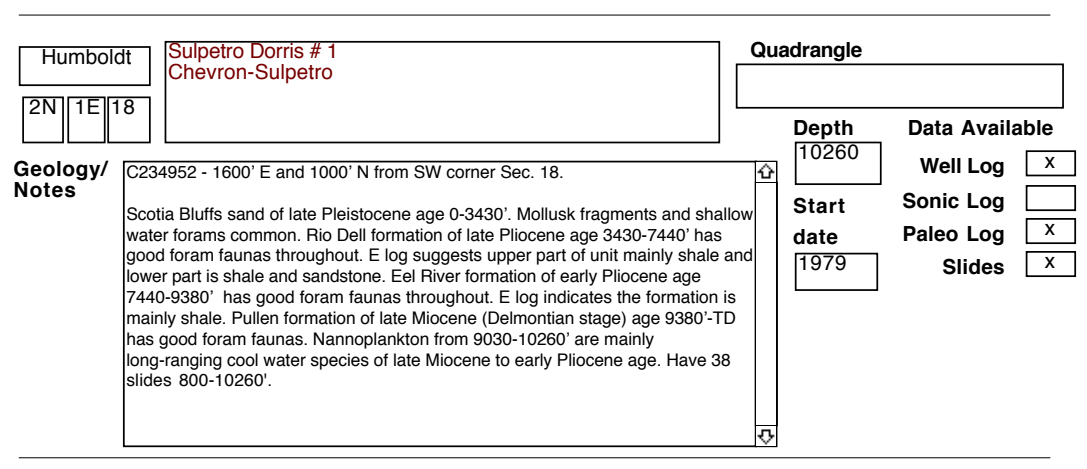


SELECTED OIL TEST WELLS IN CENTRAL CALIFORNIA DATABASE County

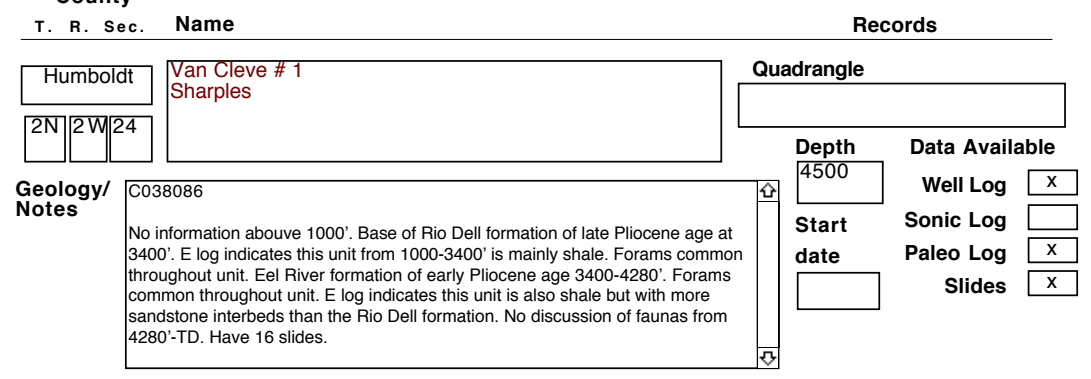

\begin{tabular}{l|l|l|l|l|l|l|l|}
\hline Humboldt & $\begin{array}{l}\text { Van Duzen \# 1 } \\
\text { Sharples Oil Corp. }\end{array}$ \\
Notes & $\begin{array}{l}\text { No paleo information above 2000'. Base of Rio Dell formation of late Pliocene age } \\
\text { at 5040'. E log suggests that this unit is mainly shale in the lower part grading } \\
\text { upward to mainly sandstone at 4200'. Shallow marine or brackish forams } \\
\text { common. Eel River formation of early Pliocene age 5040'-TD. E log suggests this } \\
\text { unit is mainly shale. Fair to good foram faunas throughout the unit. Have 21 slides } \\
\text { 2010-7820'. }\end{array}$
\end{tabular}

\begin{tabular}{l|l|l|l|l|l|l|}
\hline Humboldt & $\begin{array}{l}\text { Vicenus \# 1 (Bishop Estate) } \\
\text { Chevron }\end{array}$ \\
\hline
\end{tabular}

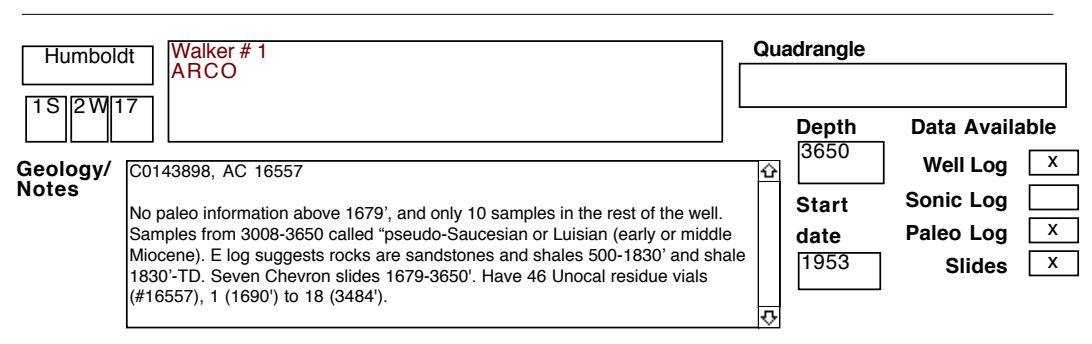

\begin{tabular}{|c|c|c|c|c|}
\hline Humboldt & \multirow{2}{*}{$\begin{array}{l}\text { Zanone \# } 1 \text { (slides labeled Zanoni) } \\
\text { ARCO }\end{array}$} & \multicolumn{3}{|c|}{ Quadrangle } \\
\hline $1 \mathrm{~S} / 3 \mathrm{~W} / 3$ & & \multirow{2}{*}{$\begin{array}{l}\text { Depth } \\
2560\end{array}$} & \multicolumn{2}{|c|}{ Data Available } \\
\hline \multirow{4}{*}{$\begin{array}{l}\text { Geology/ } \\
\text { Notes }\end{array}$} & 014391, AC 16558 & & Well Log & $\mathrm{x}$ \\
\hline & log suggests rocks in well are mainly shale with some sandstone interbeds and & Start & Sonic Log & \\
\hline & tervals. Foram samples considered late Pliocene in age. Four Chevron slides & date & Paleo Log & $\mathrm{x}$ \\
\hline & $\begin{array}{l}\text { us several misc. } \\
\text { und }\end{array}$ & 1953 & Slides & $\mathrm{x}$ \\
\hline
\end{tabular}


SELECTED OIL TEST WELLS IN CENTRAL CALIFORNIA DATABASE County

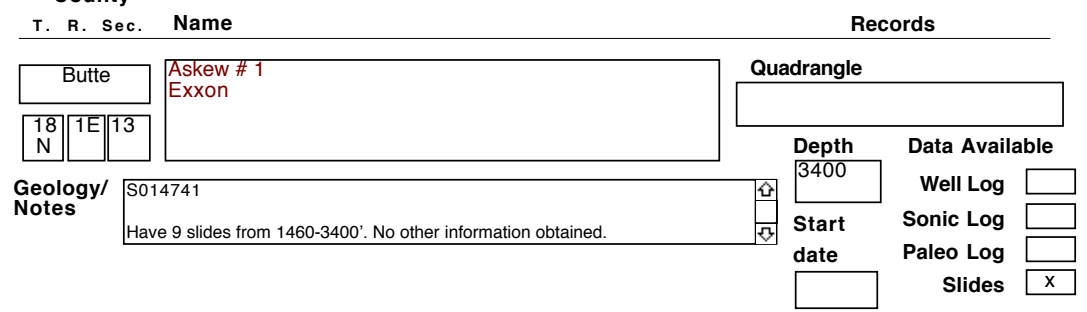

\begin{tabular}{l|l|l|l|l|l|}
\hline \multicolumn{1}{|c|}{$\begin{array}{l}\text { Beauchamp \# 1 } \\
\text { Exxon }\end{array}$} & \multicolumn{2}{l|}{ Quadrangle } \\
\hline
\end{tabular}

\begin{tabular}{|c|c|c|c|c|}
\hline Butte & Chico Community A-1 & Quadrangle & & \\
\hline \begin{tabular}{|c|c|c|}
21 & $1 \mathrm{E}$ & 8 \\
$\mathrm{~N}$ & & \\
\end{tabular} & (Chevron file indicates section is 23 for this well) & $\begin{array}{l}\text { Depth } \\
5300\end{array}$ & \multicolumn{2}{|c|}{ Data Available } \\
\hline $\begin{array}{l}\text { Geology/ } \\
\text { Notes }\end{array}$ & $\begin{array}{l}\text { S014788 } \\
\text { Have } 5 \text { slides } 4449-5297 \text { '. No other information available. Note that Chico \# } 1 \text { was } \\
\text { renamed Estes \# 1, but section is different and Chevron ID \# is different. }\end{array}$ & $\begin{array}{l}\text { Start } \\
\text { date }\end{array}$ & $\begin{array}{l}\text { Well Log } \\
\text { Sonic Log } \\
\text { Paleo Log }\end{array}$ & \\
\hline
\end{tabular}

\begin{tabular}{|l|l|l|l|l|}
\hline Butte? & $\begin{array}{l}\text { Donohoe Fee \# 2 } \\
\text { Chevron } \\
\text { (other wells with this name are in Kern Co.) }\end{array}$ \\
$\begin{array}{l}\text { Geology/ } \\
\text { Notes }\end{array}$ & Four slides 2100-2273'. No other information available. & Sell Log \\
\hline
\end{tabular}

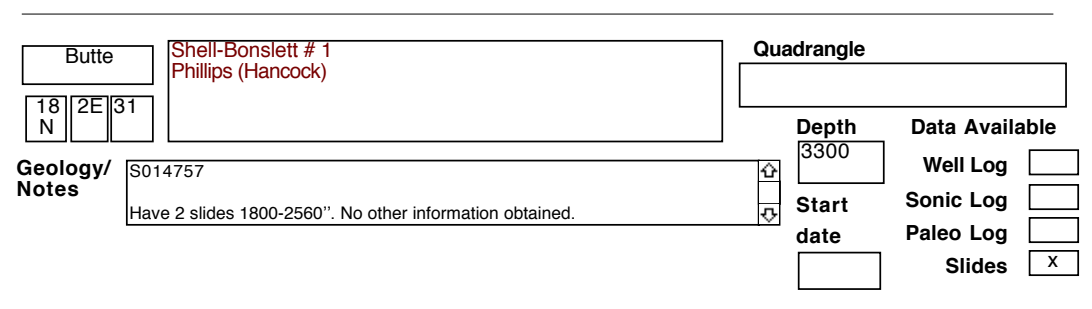


SELECTED OIL TEST WELLS IN CENTRAL CALIFORNIA DATABASE County

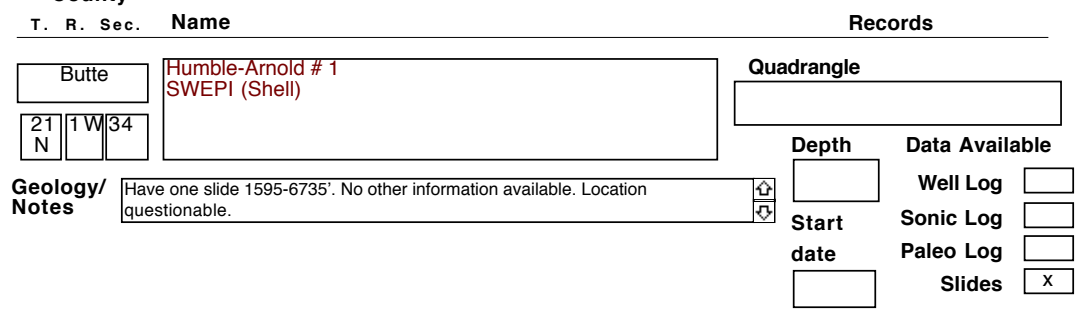

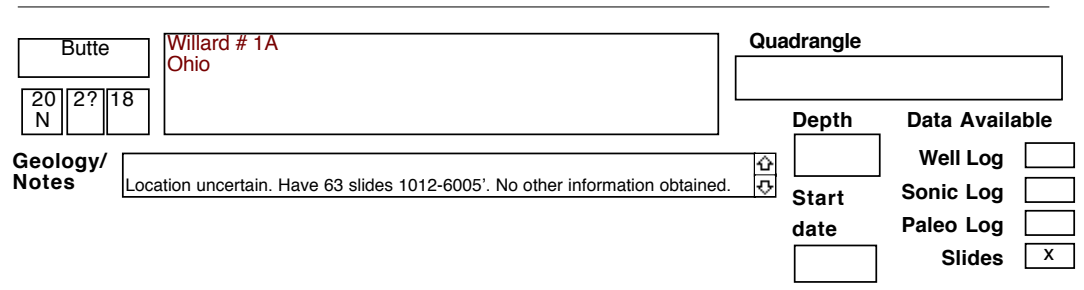

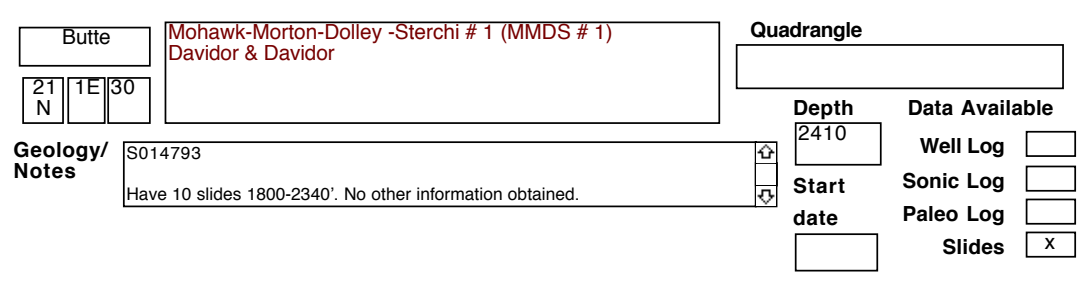

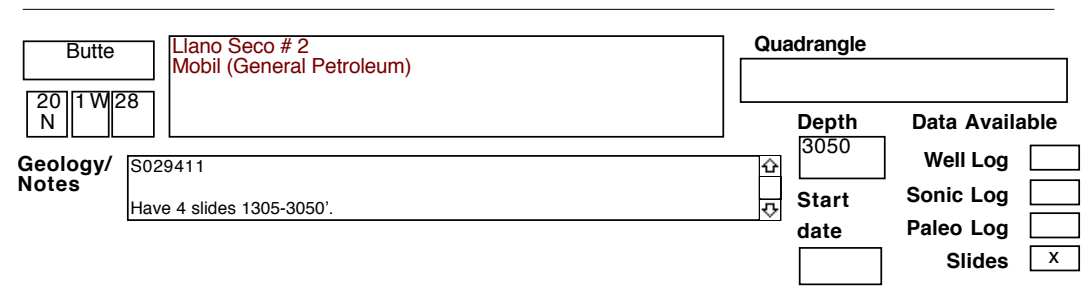

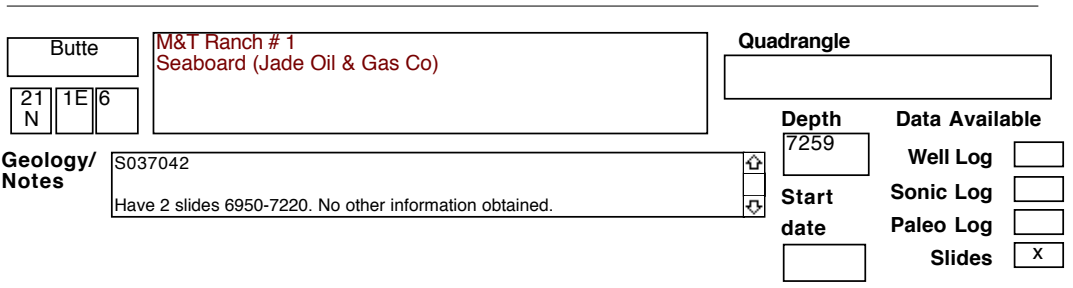


SELECTED OIL TEST WELLS IN CENTRAL CALIFORNIA DATABASE County

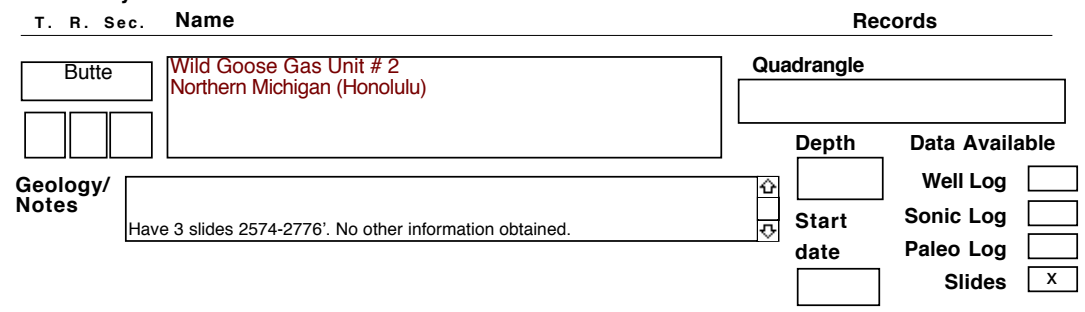

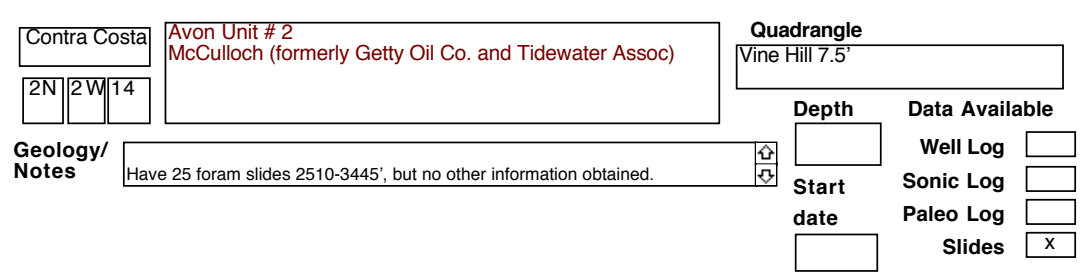

\begin{tabular}{l|l|l|l|l|}
\hline Contra Costa & $\begin{array}{l}\text { Delta Properties \# 6 } \\
\text { Unocal (Amerada) }\end{array}$ & \multicolumn{2}{l|}{ Quadrangle } \\
\hline
\end{tabular}

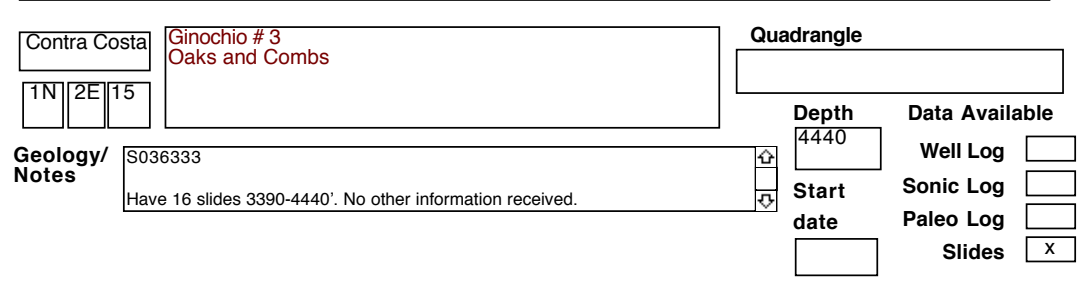

\begin{tabular}{|c|c|c|c|c|}
\hline Contra Costa & \multirow[t]{2}{*}{$\begin{array}{l}\text { Ronegger \# 1-34 } \\
\text { Buttes Oil Co. }\end{array}$} & \multicolumn{3}{|c|}{$\begin{array}{c}\text { Quadrangle } \\
\text { Brentwood 7.5' }\end{array}$} \\
\hline \multicolumn{4}{|l|}{\begin{tabular}{|l|l|l}
$2 \mathrm{~N}$ & $3 \mathrm{E}$ & 34 \\
\end{tabular}} & \\
\hline \multirow{4}{*}{\multicolumn{2}{|c|}{ Geology/ Ten foram slides $4520-8000^{\prime}$. No other information received. }} & & Well Log & \\
\hline & & Start & Sonic Log & \\
\hline & & date & Paleo Log & \\
\hline & & & Slides & $\mathrm{x}$ \\
\hline
\end{tabular}


SELECTED OIL TEST WELLS IN CENTRAL CALIFORNIA DATABASE

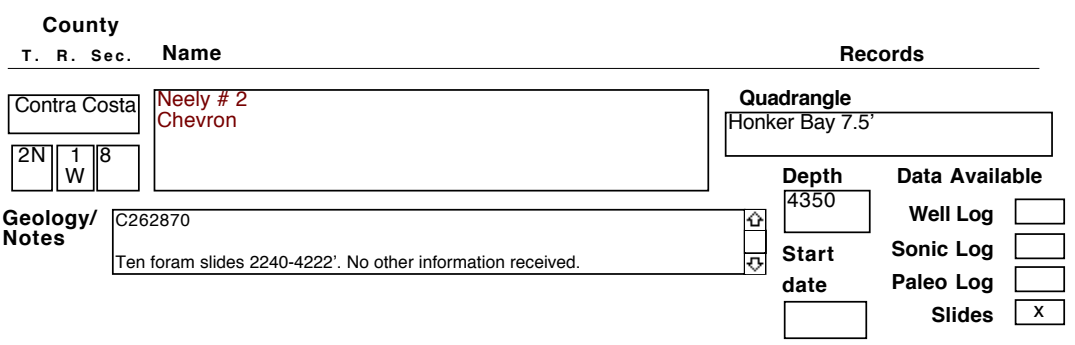

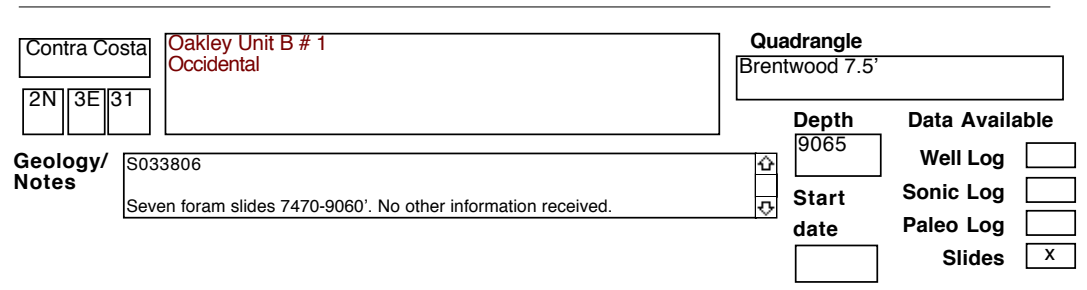

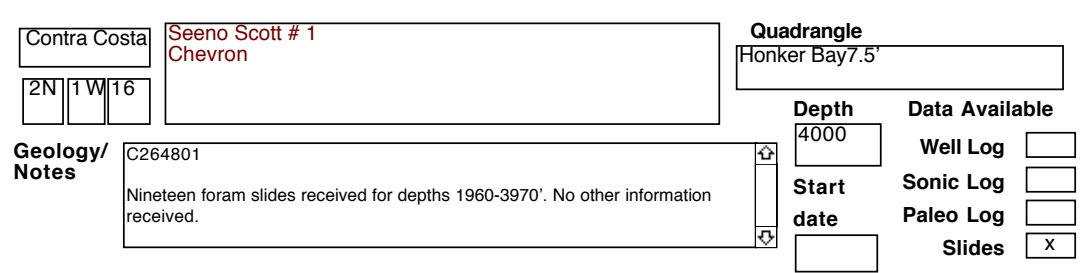

\begin{tabular}{|c|c|c|c|c|c|}
\hline \multirow{2}{*}{\begin{tabular}{|l} 
Contra Cost \\
$2 \mathrm{~N} / \mathrm{W} / 16$
\end{tabular}} & \multirow[t]{2}{*}{$\begin{array}{l}\text { Seeno Scott \# 3 } \\
\text { Chevron }\end{array}$} & \multicolumn{4}{|c|}{$\begin{array}{c}\text { Quadrangle } \\
\text { Honker Bay 7.5' }\end{array}$} \\
\hline & & & Depth & Data Availab & \\
\hline $\begin{array}{l}\text { Geology/ } \\
\text { Notes }\end{array}$ & $\begin{array}{l}269713 \\
\text { hirteen foram slides received for depths 2800--3970'. No other information } \\
\text { ceived. }\end{array}$ & 9 & $\begin{array}{l}\text { Start } \\
\text { date }\end{array}$ & $\begin{array}{l}\text { Well Log } \\
\text { Sonic Log } \\
\text { Paleo Log }\end{array}$ & \\
\hline
\end{tabular}

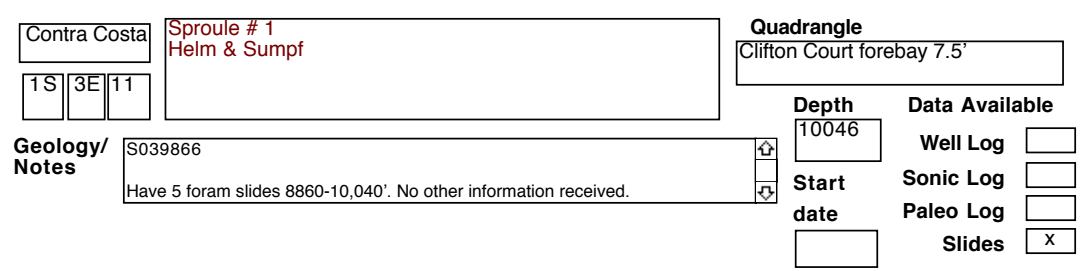


SELECTED OIL TEST WELLS IN CENTRAL CALIFORNIA DATABASE

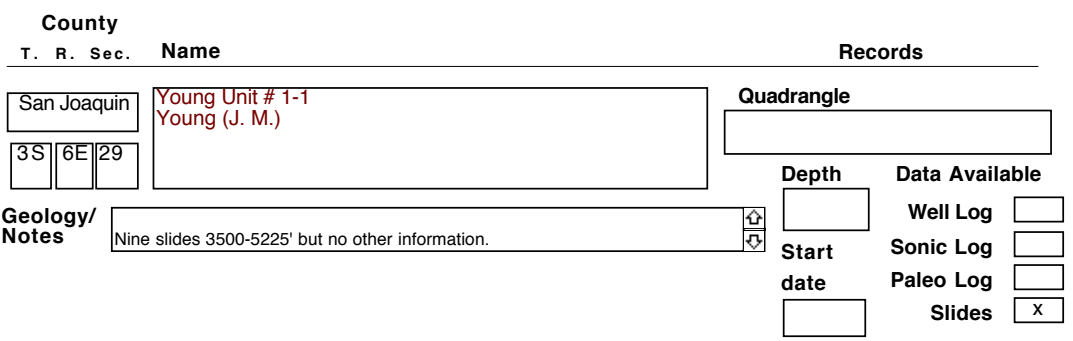

\begin{tabular}{l|l|l|l|l|}
\hline \multicolumn{1}{|c|}{$\begin{array}{l}\text { Conolusa } \\
\text { Texaco }\end{array}$} & \multicolumn{2}{|c|}{ Quadrangle } \\
\hline
\end{tabular}

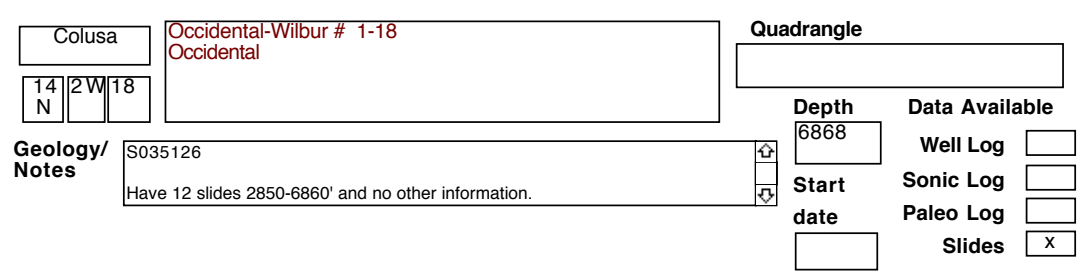

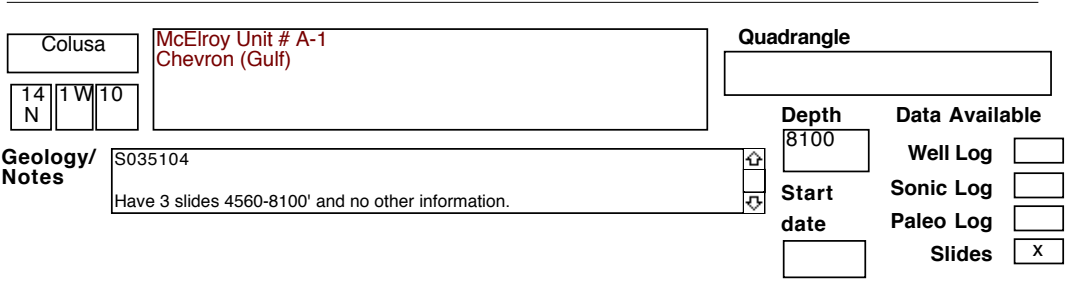

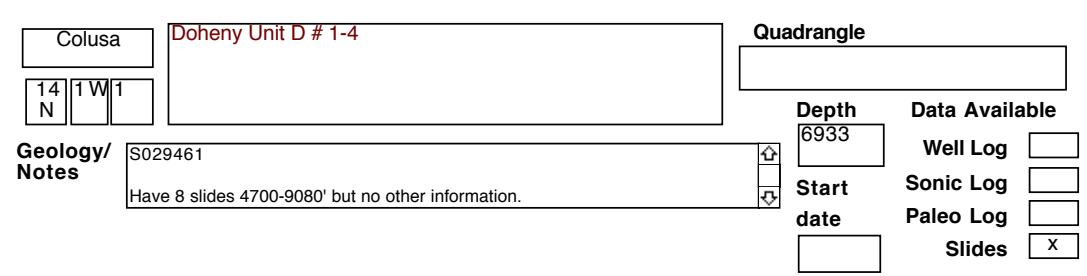


SELECTED OIL TEST WELLS IN CENTRAL CALIFORNIA DATABASE County

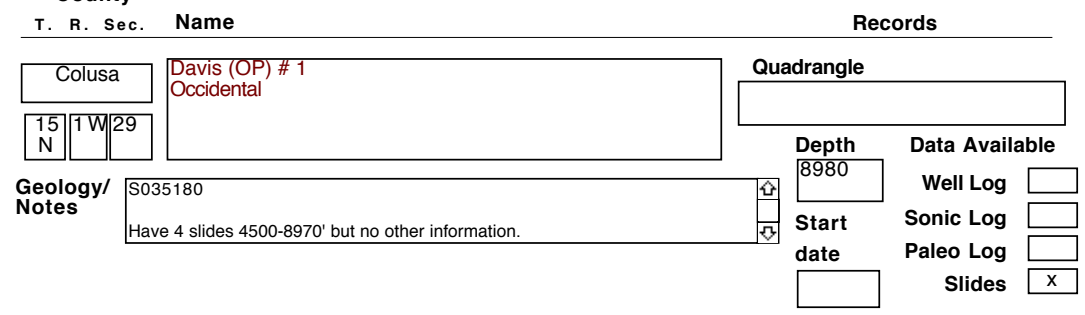

\begin{tabular}{|c|c|c|c|c|}
\hline Colusa & \multirow{2}{*}{$\begin{array}{l}\text { Canzza \#1 } \\
\text { Exxon }\end{array}$} & \multicolumn{3}{|l|}{ Quadrangle } \\
\hline \begin{tabular}{|l|l|l|l|}
17 & $1 \mathrm{~W}$ & 18 \\
$\mathrm{~N}$ & & \\
\end{tabular} & & & \multirow{2}{*}{\multicolumn{2}{|c|}{ Data Available }} \\
\hline \multirow{5}{*}{\multicolumn{2}{|c|}{$\begin{array}{l}\text { Geology/ Have } 3 \text { slides } 1844-3010^{\prime} \text { but no other information. } \\
\text { Notes }\end{array}$}} & & & \\
\hline & & & \multirow{4}{*}{$\begin{array}{r}\text { Well Log } \\
\text { Sonic Log } \\
\text { Paleo Log } \\
\text { Slides }\end{array}$} & \\
\hline & & Start & & \\
\hline & & date & & \\
\hline & & & & $\mathrm{x}$ \\
\hline
\end{tabular}

\begin{tabular}{|c|c|c|c|c|}
\hline Colusa & \multirow{2}{*}{\begin{tabular}{|l} 
CLC \# 1 \\
Amerada Hess
\end{tabular}} & \multicolumn{3}{|l|}{ Quadrangle } \\
\hline \begin{tabular}{|l|l|l|}
16 & $1 \mathrm{~W}$ & 34 \\
$\mathrm{~N}$
\end{tabular} & & \multirow[t]{2}{*}{ Depth } & \multicolumn{2}{|c|}{ Data Available } \\
\hline \multirow{3}{*}{$\begin{array}{l}\text { Geology/ } \\
\text { Notes }\end{array}$} & e two slides 2796-2976' but no other information. & & Well Log & \\
\hline & & Start & Sonic Log & \\
\hline & & date & Paleo Log & \\
\hline
\end{tabular}

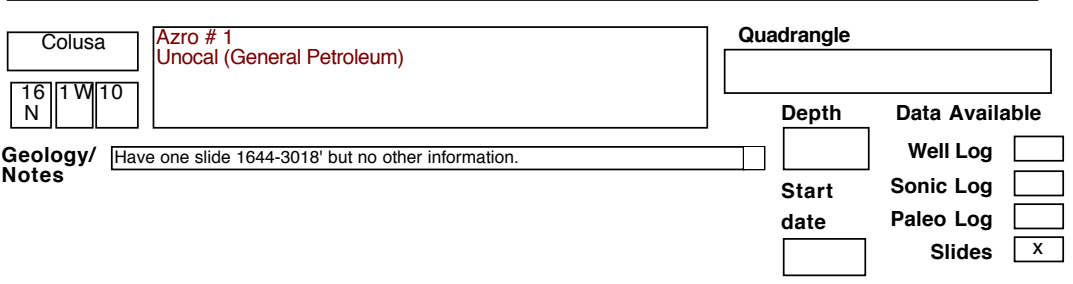

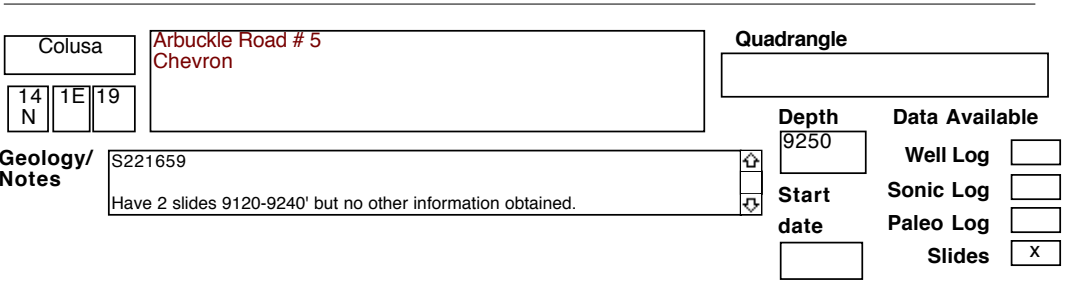


SELECTED OIL TEST WELLS IN CENTRAL CALIFORNIA DATABASE County

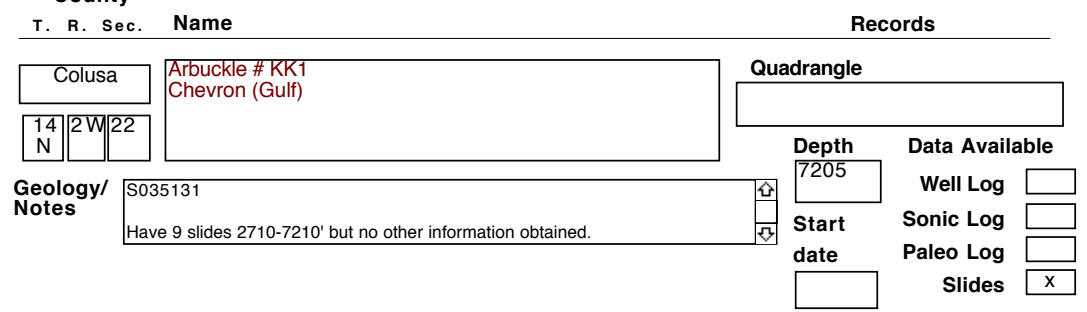

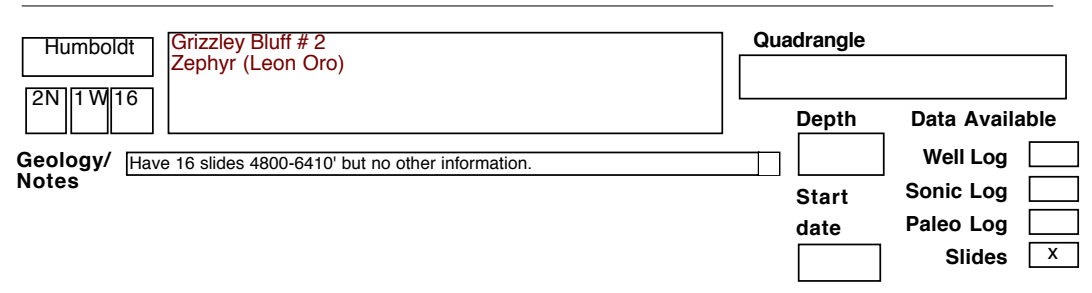

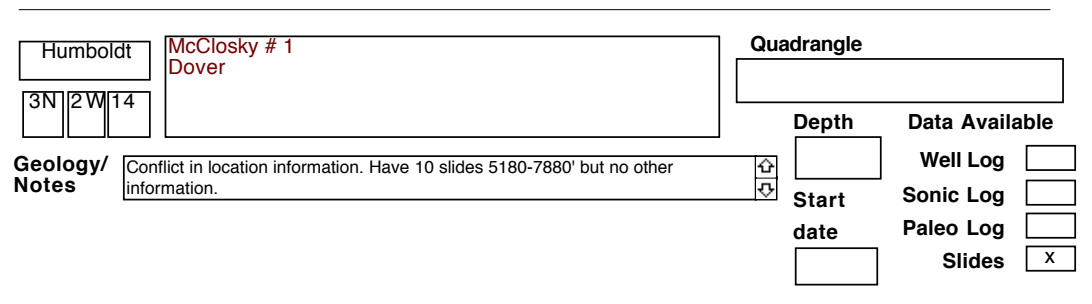

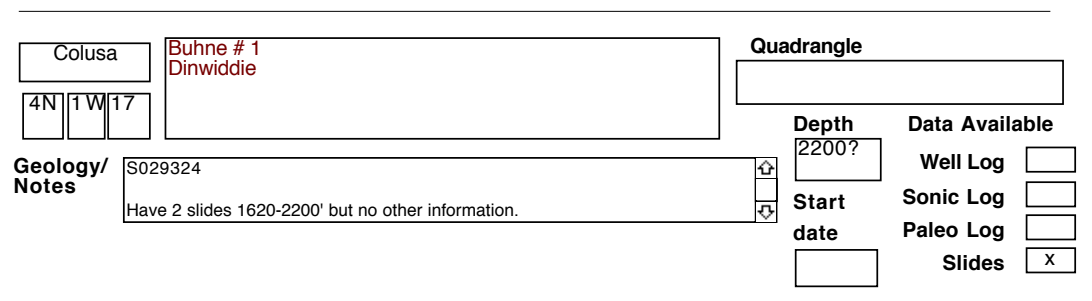

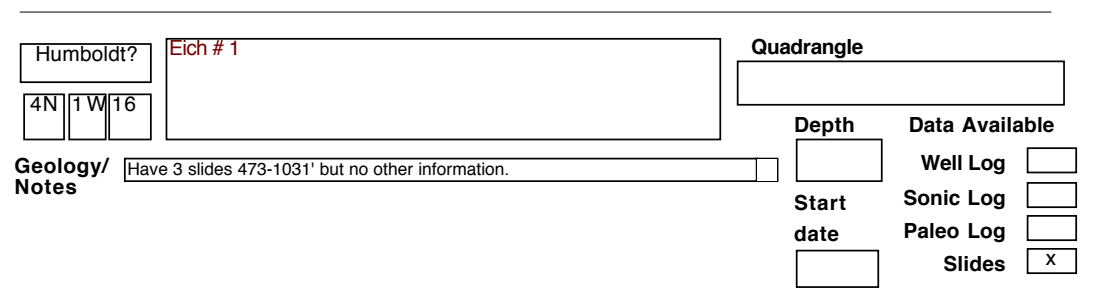


SELECTED OIL TEST WELLS IN CENTRAL CALIFORNIA DATABASE County

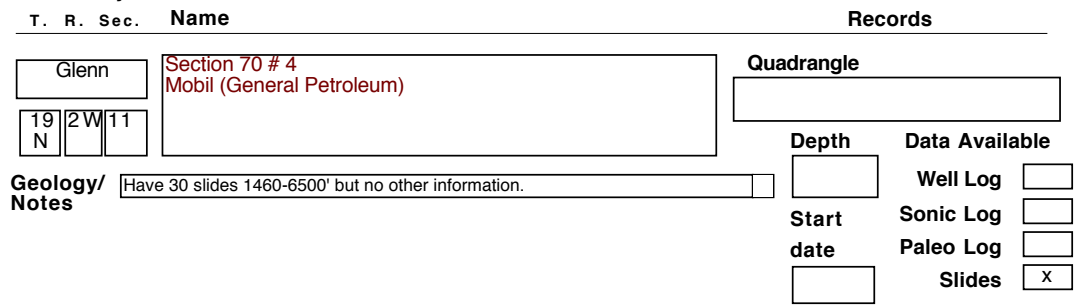

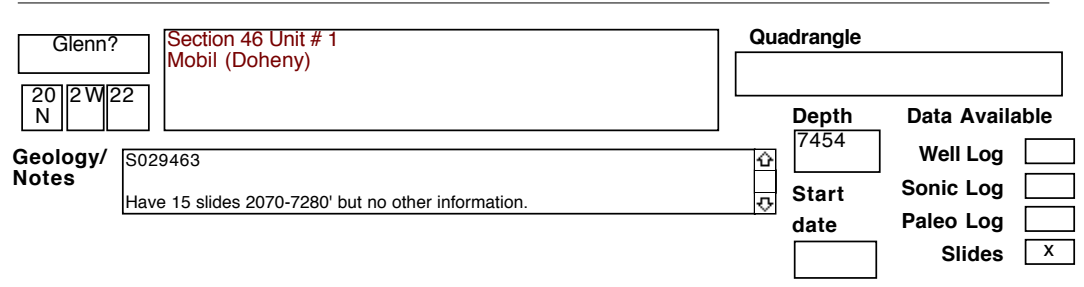

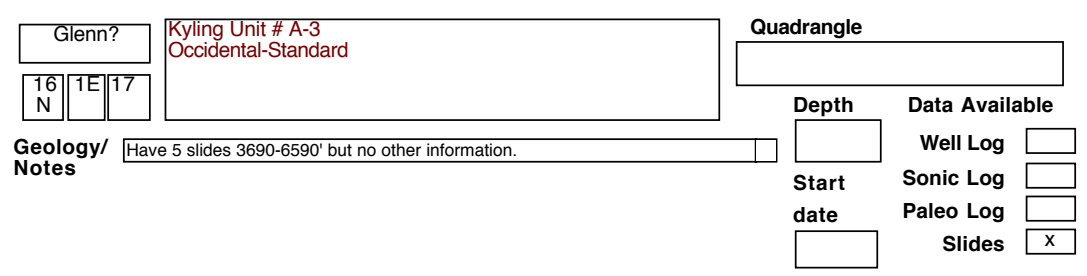

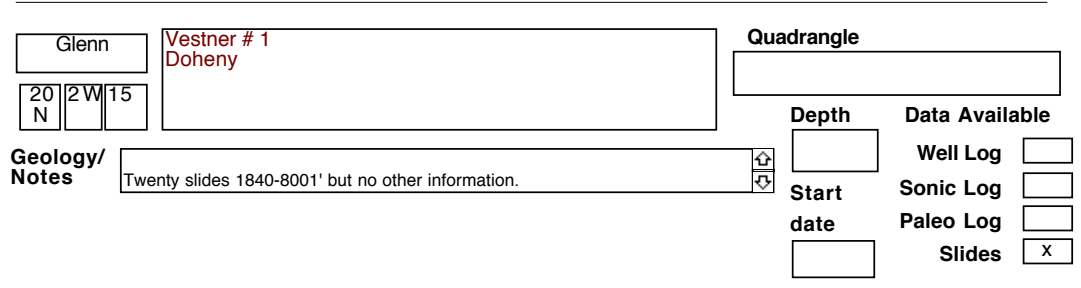

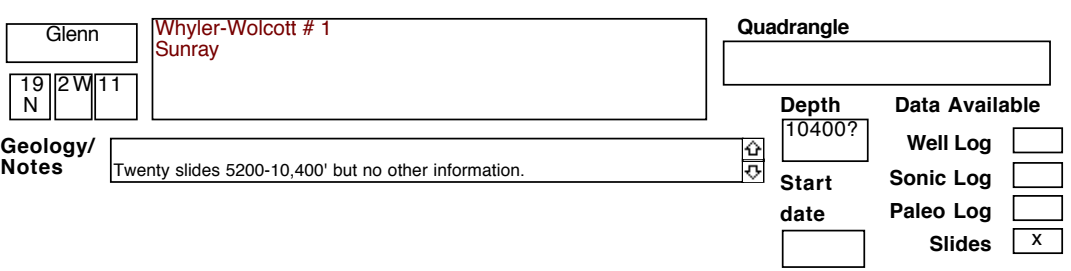


SELECTED OIL TEST WELLS IN CENTRAL CALIFORNIA DATABASE County

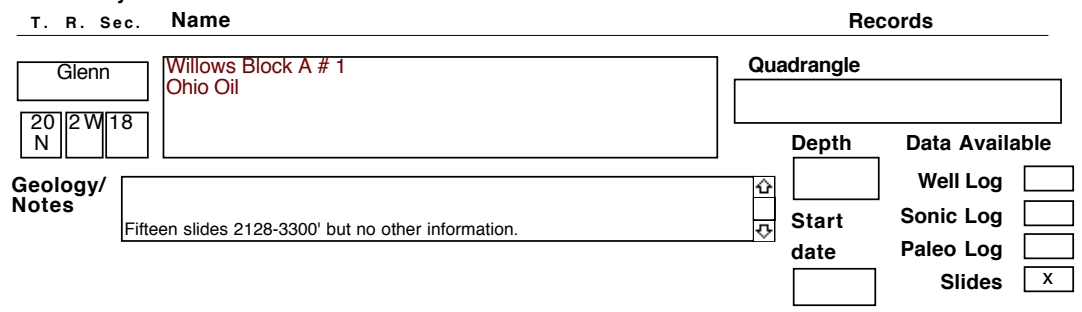

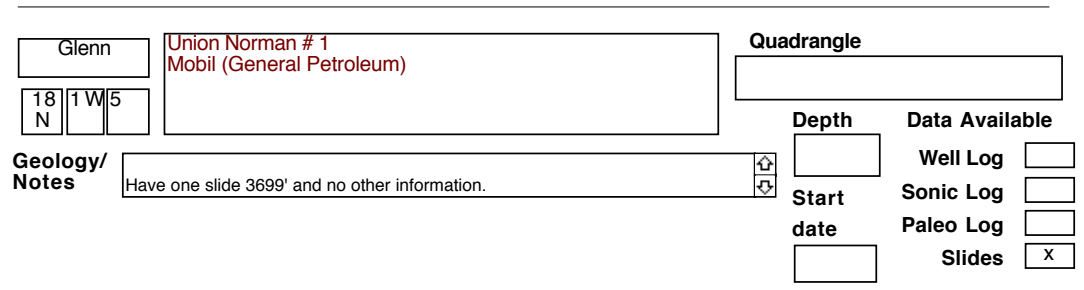

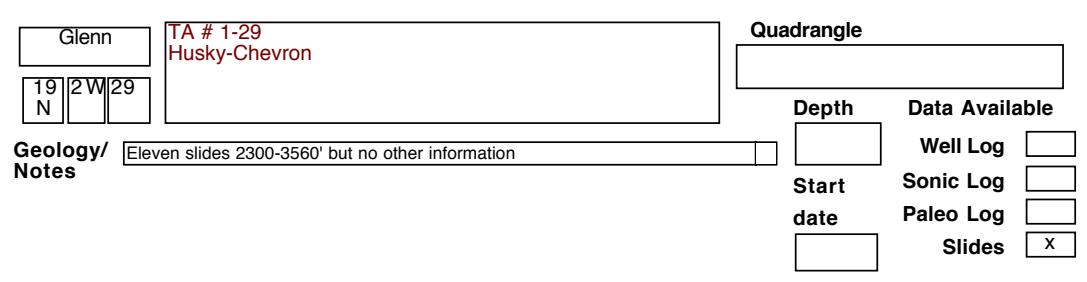

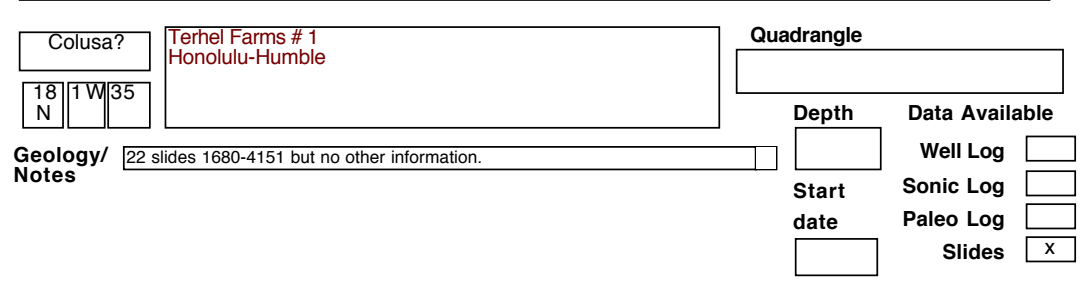

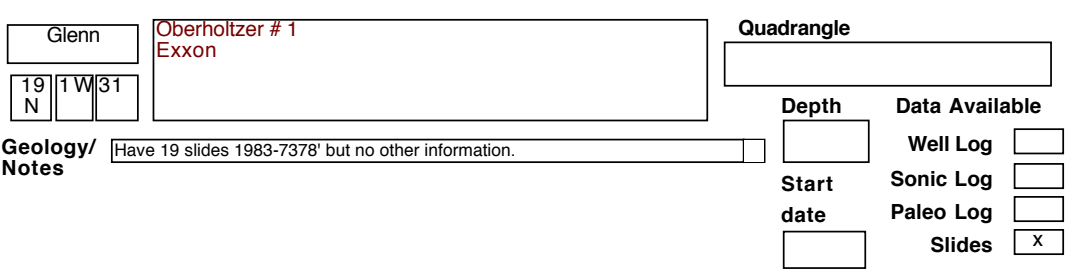


SELECTED OIL TEST WELLS IN CENTRAL CALIFORNIA DATABASE County

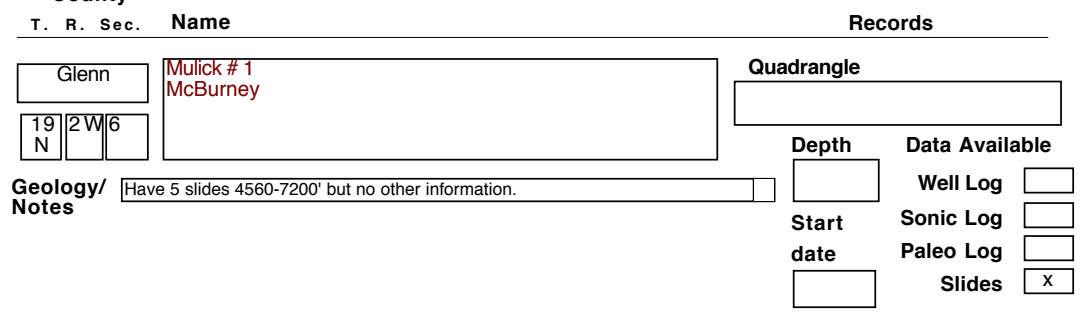

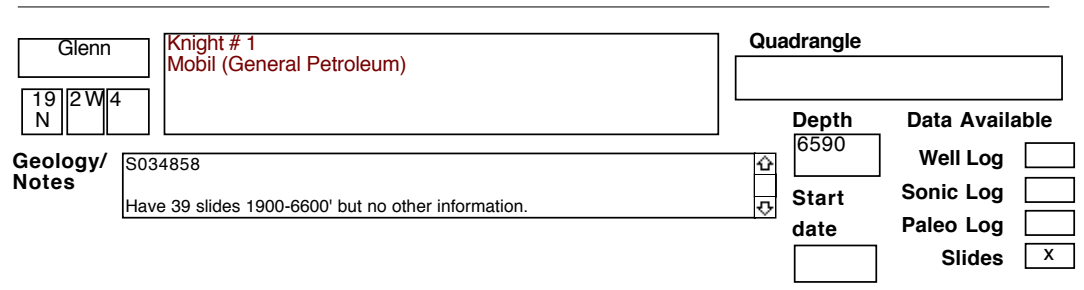

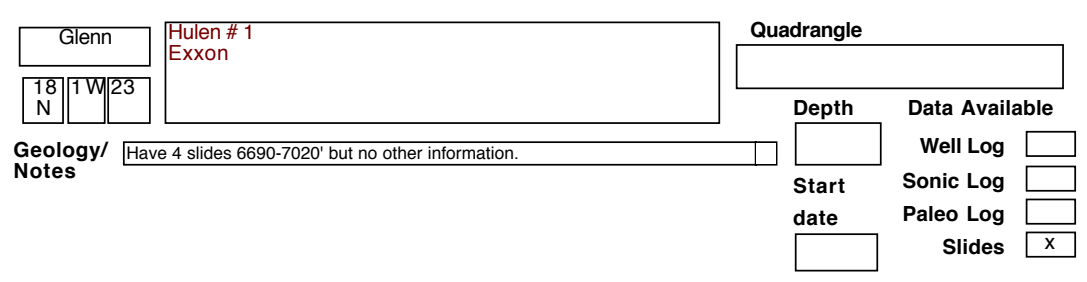

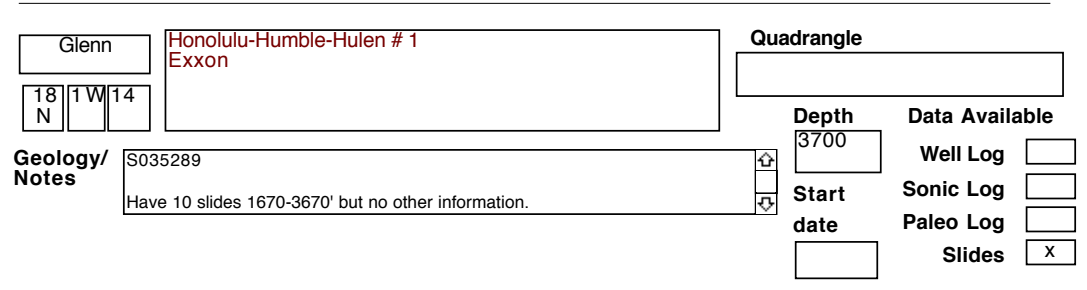

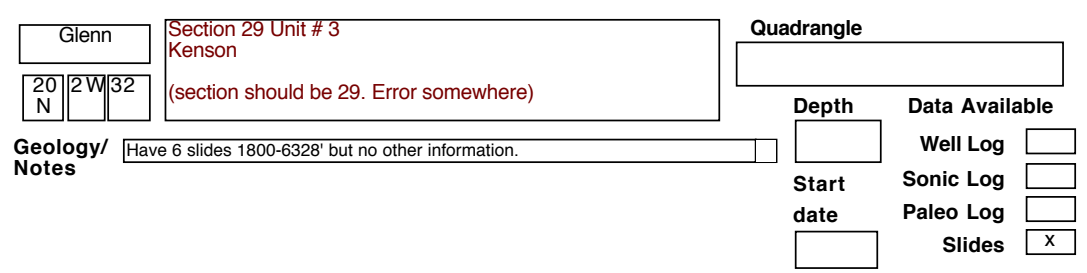


SELECTED OIL TEST WELLS IN CENTRAL CALIFORNIA DATABASE County

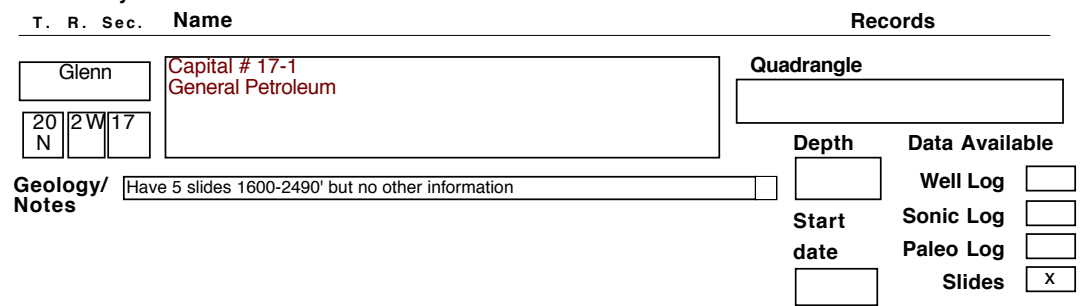

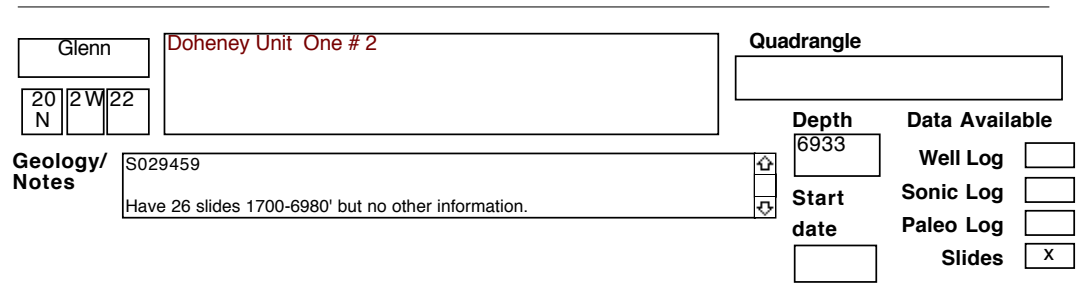

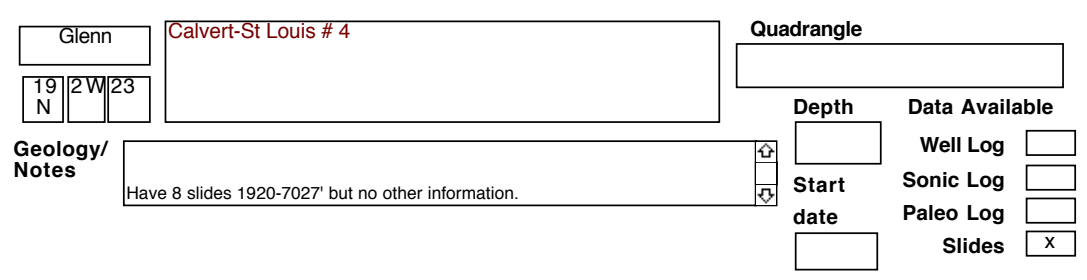

\begin{tabular}{l|l|l|l|l|l|}
\hline \multicolumn{1}{|c|}{ Glenn } & $\begin{array}{l}\text { Bertagna \# 31-32 } \\
\text { Franco Western }\end{array}$ & \multicolumn{2}{l|}{ Quadrangle } \\
\hline
\end{tabular}

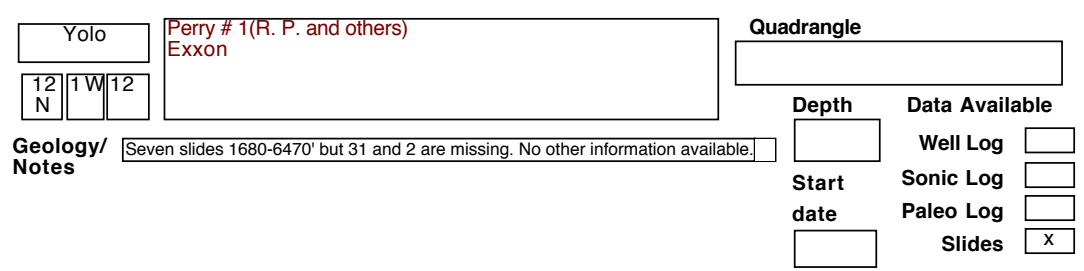


SELECTED OIL TEST WELLS IN CENTRAL CALIFORNIA DATABASE County

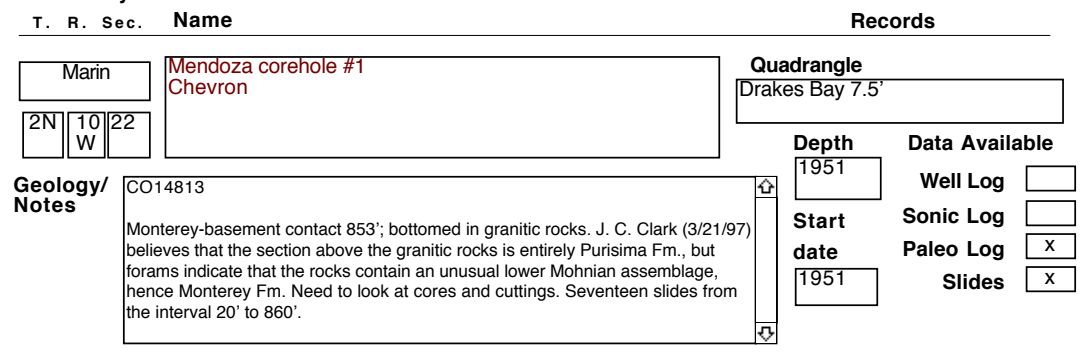

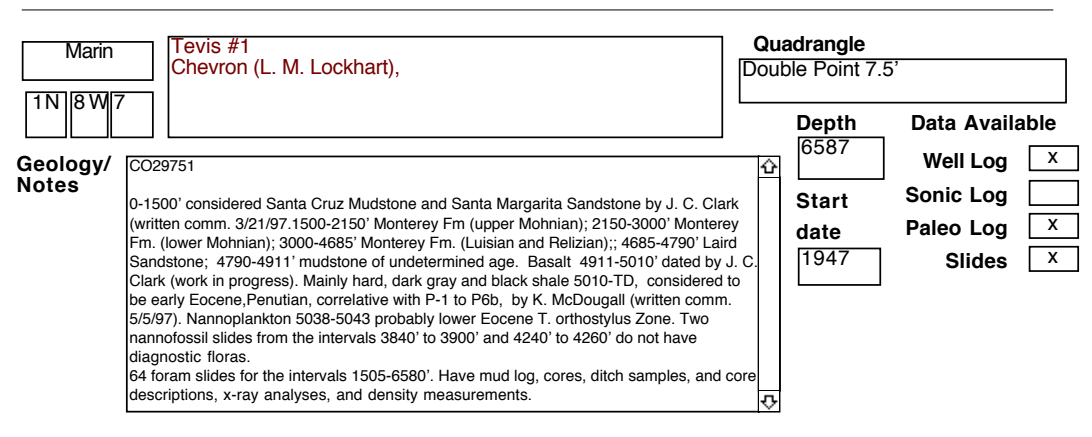

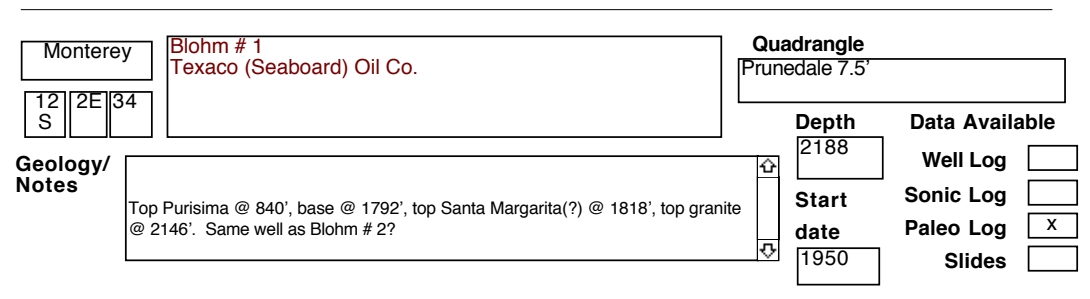

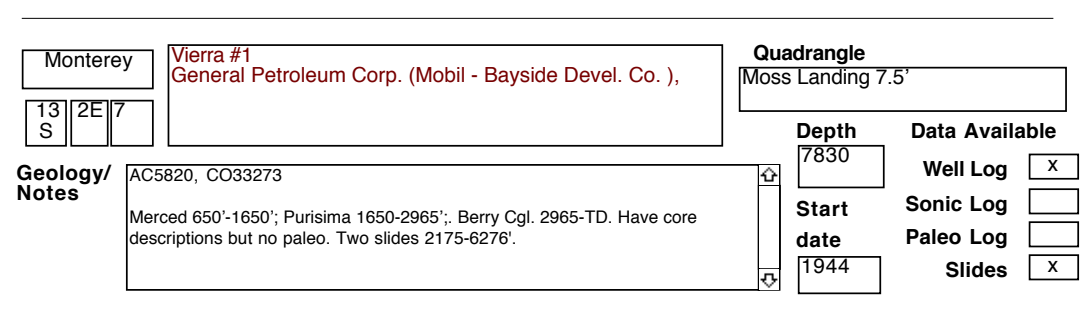

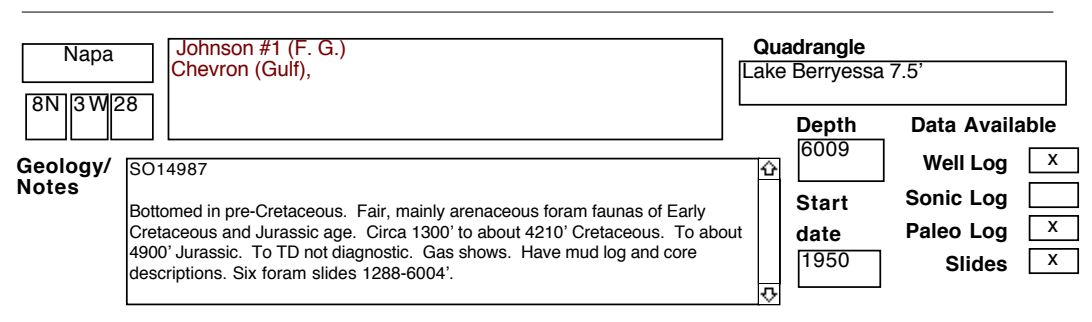


SELECTED OIL TEST WELLS IN CENTRAL CALIFORNIA DATABASE County

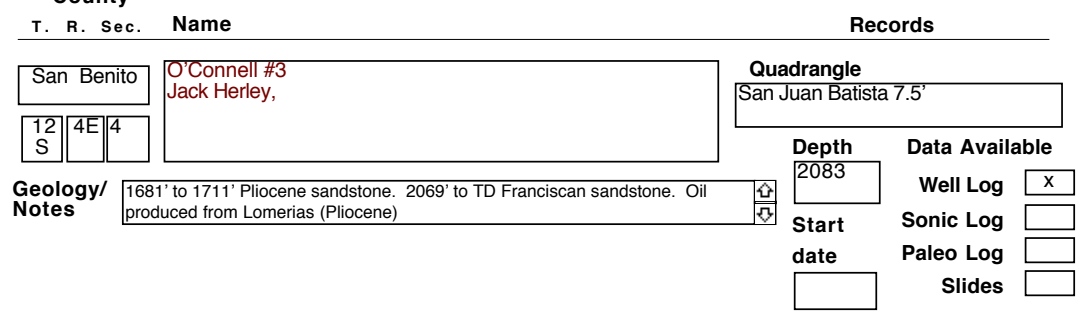

\begin{tabular}{|c|c|c|c|}
\hline \begin{tabular}{|l|} 
San Benito \\
\end{tabular} & $\begin{array}{l}\text { O'Connell \#2 } \\
\text { Jack Herley, }\end{array}$ & $\begin{array}{r}\text { Quadrangle } \\
\text { San Juan Bau }\end{array}$ & a 7.5 \\
\hline \begin{tabular}{|l|l|l|l|}
12 & $4 \mathrm{E}$ & \\
$\mathrm{S}$ & & \\
\end{tabular} & & \multirow{2}{*}{$\begin{array}{l}\text { Depth } \\
3578\end{array}$} & Data Available \\
\hline \multirow{3}{*}{\multicolumn{2}{|c|}{$\begin{array}{l}\text { Geology/ Pliocene/Franciscan contact at 2955'. } \\
\text { Notes }\end{array}$}} & & Well Log \\
\hline & & Start & Sonic Log \\
\hline & & $\begin{array}{l}\text { date } \\
1950\end{array}$ & $\begin{array}{r}\text { Paleo Log } \\
\text { Slides }\end{array}$ \\
\hline
\end{tabular}

\begin{tabular}{|c|c|c|c|c|c|}
\hline San Joaquin & \multirow[t]{2}{*}{$\begin{array}{l}\text { Mildred Island \#1 } \\
\text { Terry \& Jansen }\end{array}$} & \multicolumn{4}{|c|}{$\begin{array}{l}\text { Quadrangle } \\
\begin{array}{|l}\text { Woodward Island 7.5' }\end{array}\end{array}$} \\
\hline $2 \mathrm{~N}\left\|^{4 \mathrm{E}}\right\|^{34}$ & & \multirow{2}{*}{\multicolumn{2}{|c|}{ कepth }} & \multicolumn{2}{|c|}{ Data Available } \\
\hline \multirow{3}{*}{$\begin{array}{l}\text { Geology/ } \\
\text { Notes }\end{array}$} & \multirow{3}{*}{ op Capay sh 5600'. McDonald Island sd missing. } & & & Well Log & \\
\hline & & \multirow{2}{*}{ 勾 } & Start & Sonic Log & \\
\hline & & & $\begin{array}{l}\text { date } \\
1953\end{array}$ & $\begin{array}{r}\text { Paleo Log } \\
\text { Slides }\end{array}$ & $\mathrm{x}$ \\
\hline
\end{tabular}

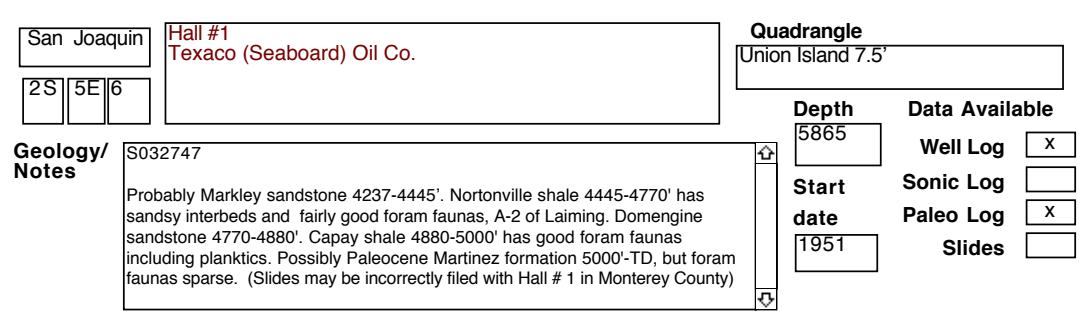

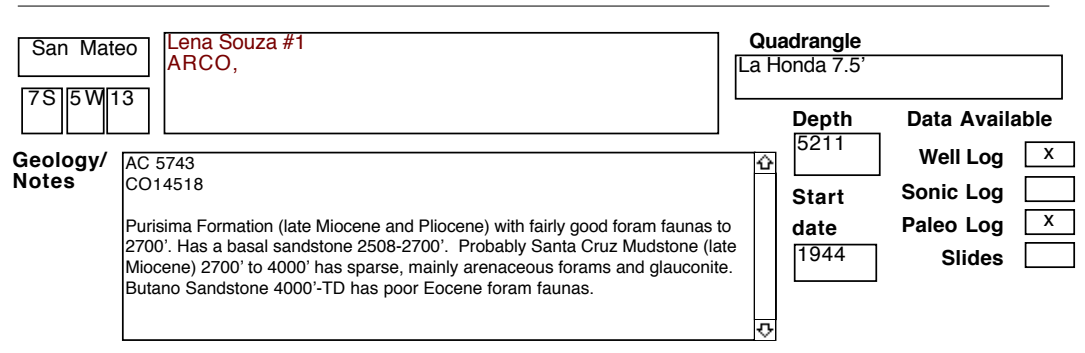


SELECTED OIL TEST WELLS IN CENTRAL CALIFORNIA DATABASE County

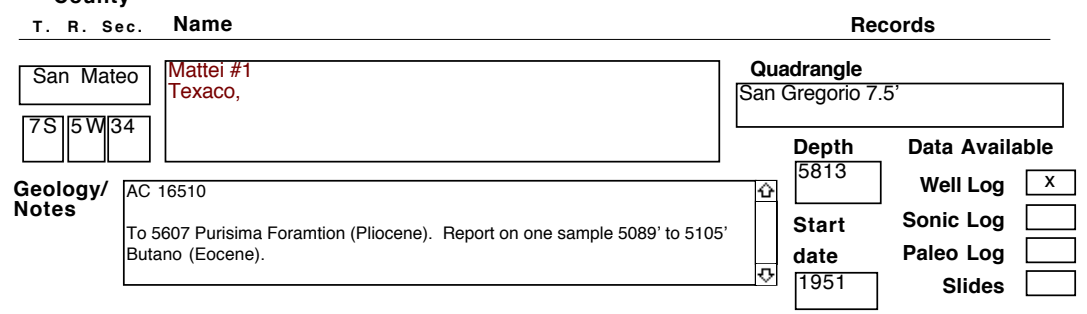

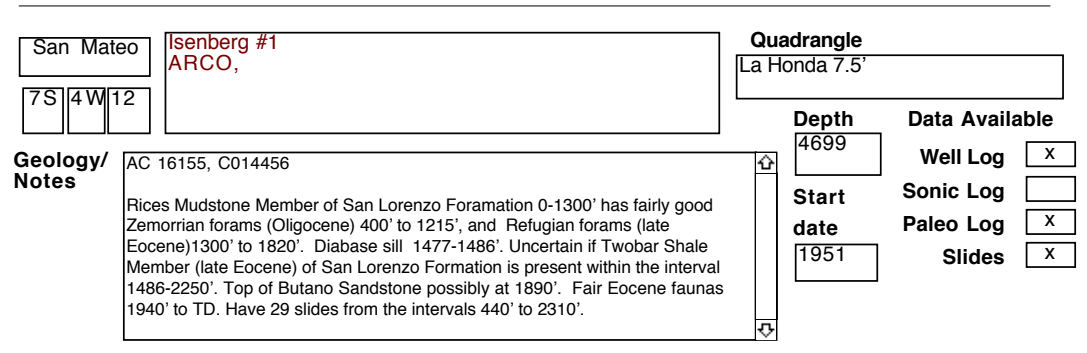

\begin{tabular}{|c|c|c|c|c|}
\hline San Mateo & \multirow[t]{2}{*}{$\begin{array}{l}\text { Y. M.. C. A. \#1 } \\
\text { Exxon (Jergins Oil Co.), }\end{array}$} & \multicolumn{3}{|l|}{$\begin{array}{l}\text { Quadrangle } \\
\begin{array}{|l}\text { La Honda 7.5' } \\
\end{array}\end{array}$} \\
\hline $15 \|\left.^{4} \mathrm{Vy}\right|^{26}$ & & \multirow{2}{*}{$\begin{array}{l}\text { Depth } \\
2510\end{array}$} & \multicolumn{2}{|c|}{ Data Available } \\
\hline \multirow{2}{*}{$\begin{array}{l}\text { Geology/ } \\
\text { Notes }\end{array}$} & $16015, \mathrm{C} 014506$ & & Well Log & $x$ \\
\hline & $\begin{array}{l}\text { isima Formation of late Miocene and Pliocene age } 0-767^{\prime} \text {. Lithology and } \\
\text { ams indicate that from } 767^{\prime} \text { to TD the well penetrated Lambert Shale, the } \\
\text { Idego volcanics, and possibly the San Lorenzo formation. Have } 26 \text { Unocal } \\
\text { idue vials (\#16015), } 2\left(915^{\prime}\right) \text { to } 27\left(2327^{\prime}\right) \text {. }\end{array}$ & $\begin{array}{l}\text { Start } \\
\text { date }\end{array}$ & $\begin{array}{r}\text { Sonic Log } \\
\text { Paleo Log } \\
\text { Slides }\end{array}$ & \\
\hline
\end{tabular}

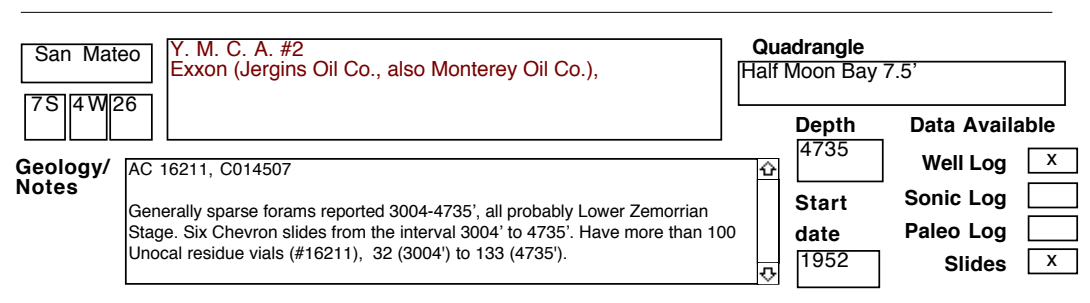

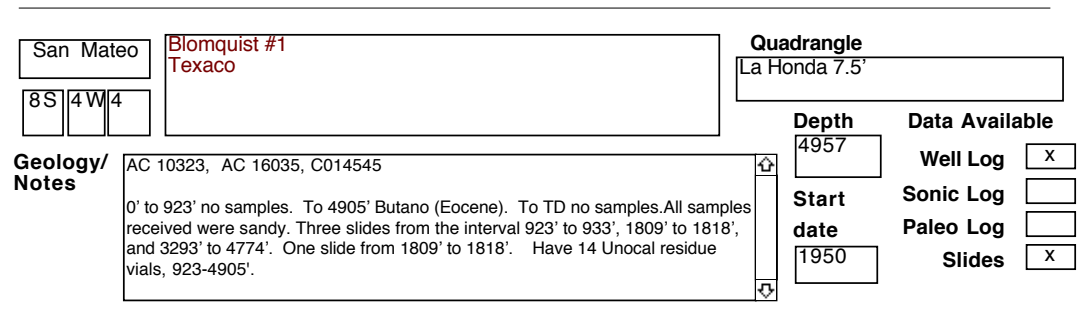


SELECTED OIL TEST WELLS IN CENTRAL CALIFORNIA DATABASE County

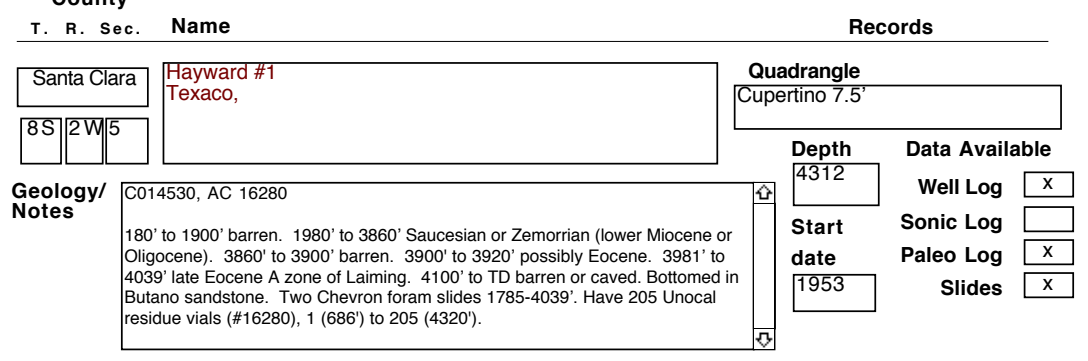

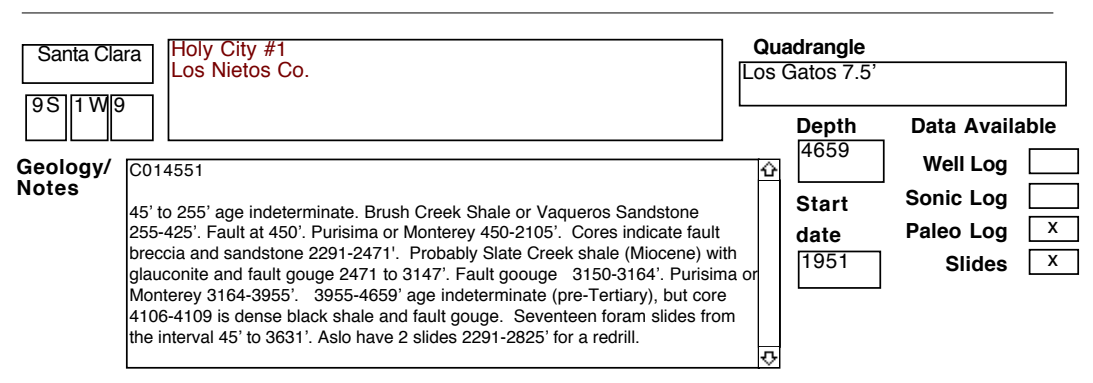

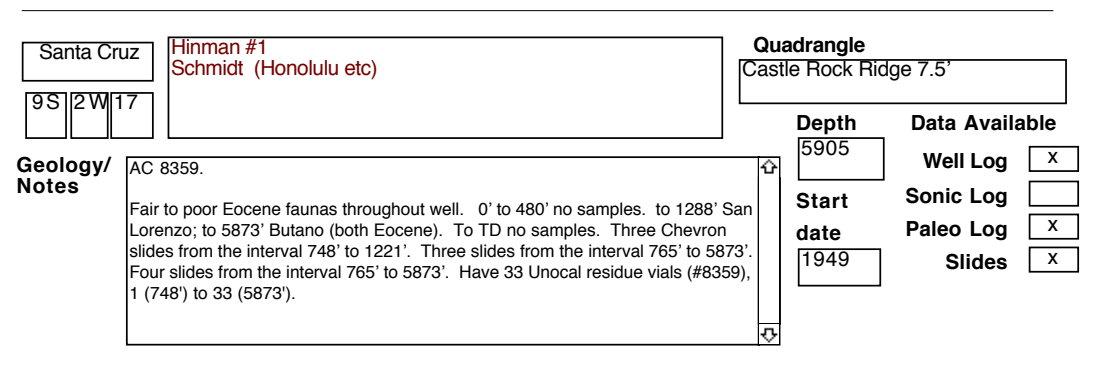

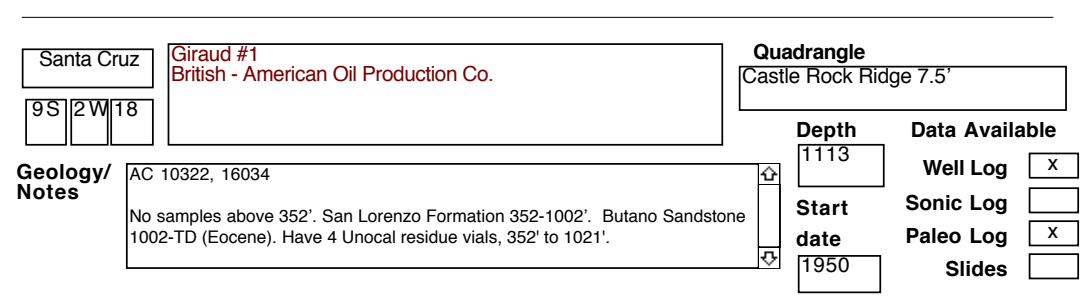

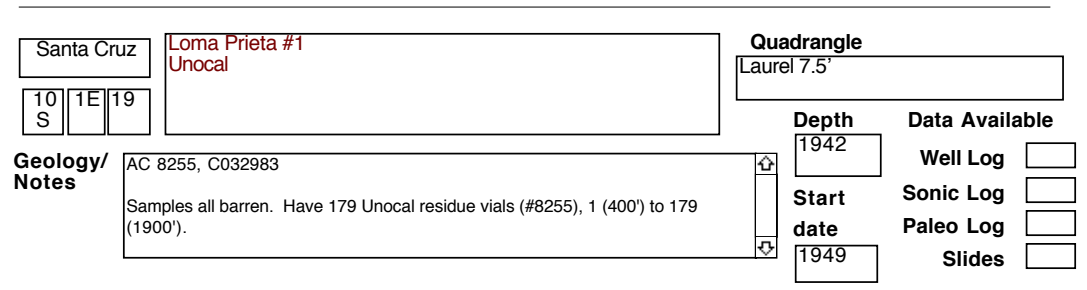


SELECTED OIL TEST WELLS IN CENTRAL CALIFORNIA DATABASE County

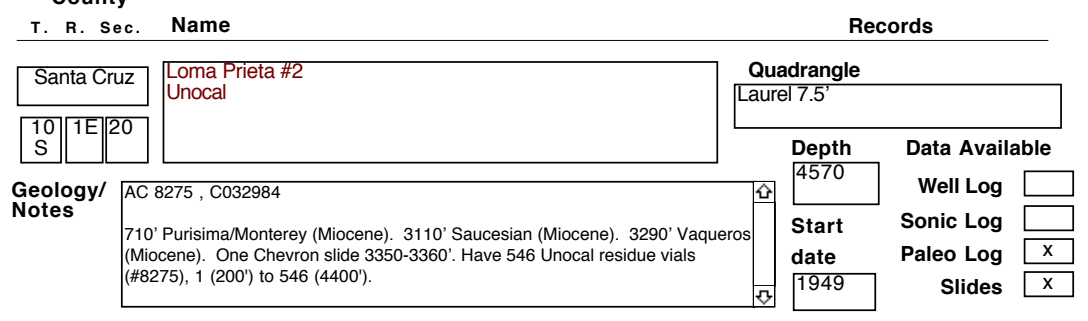

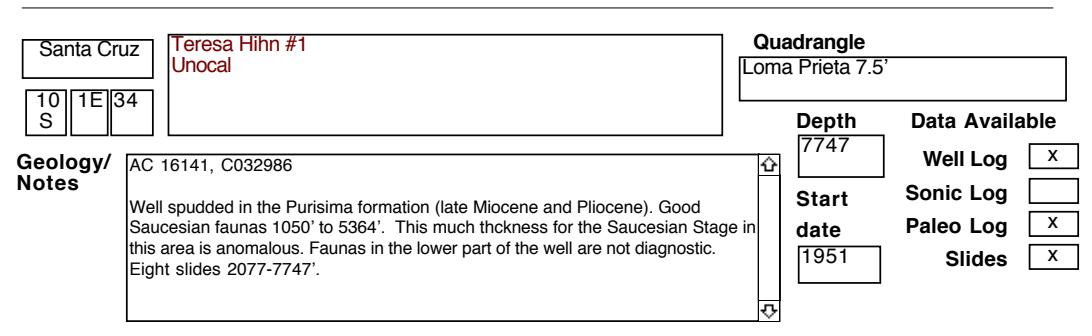

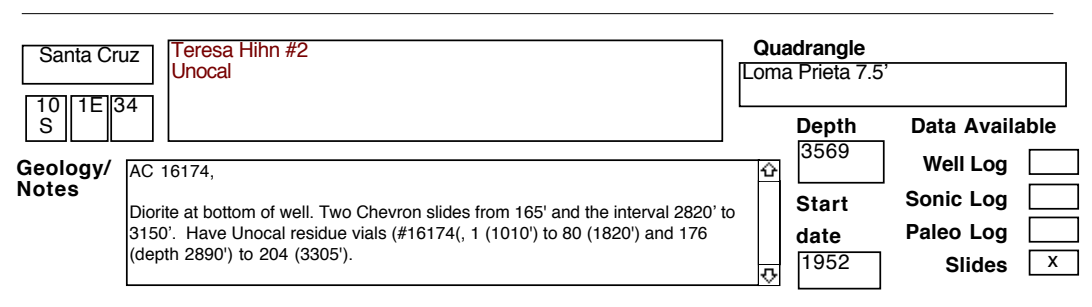

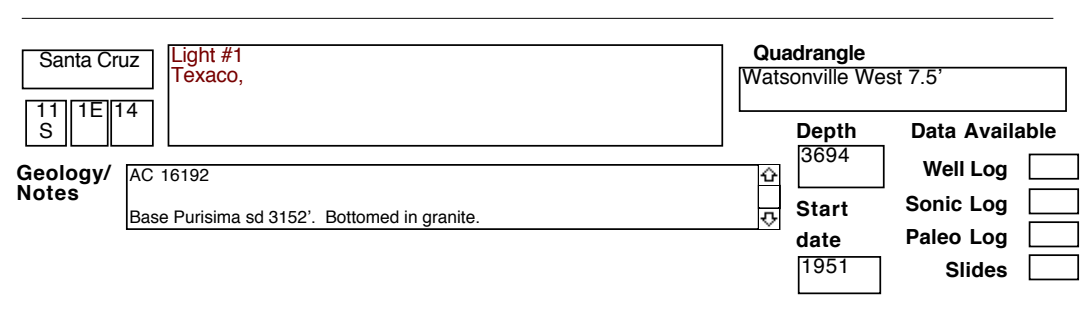

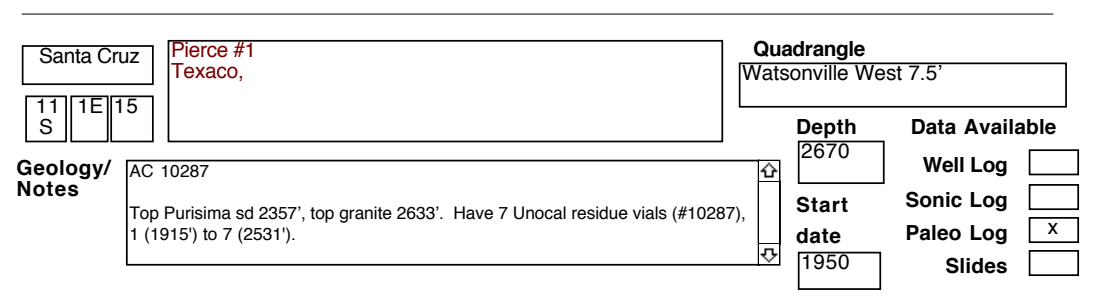


SELECTED OIL TEST WELLS IN CENTRAL CALIFORNIA DATABASE County

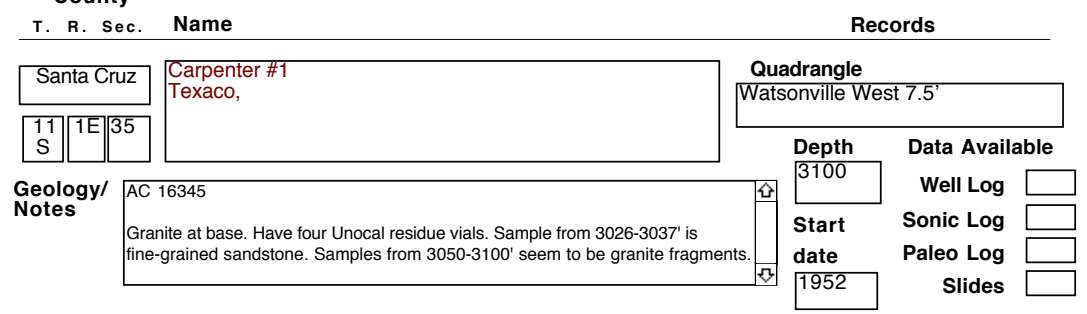

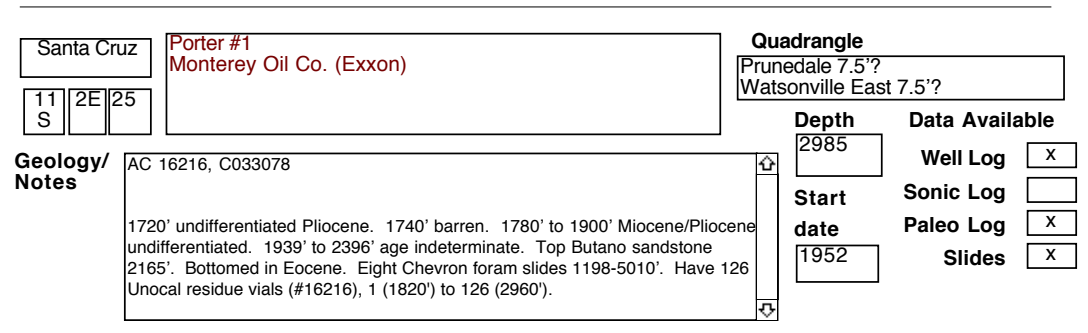

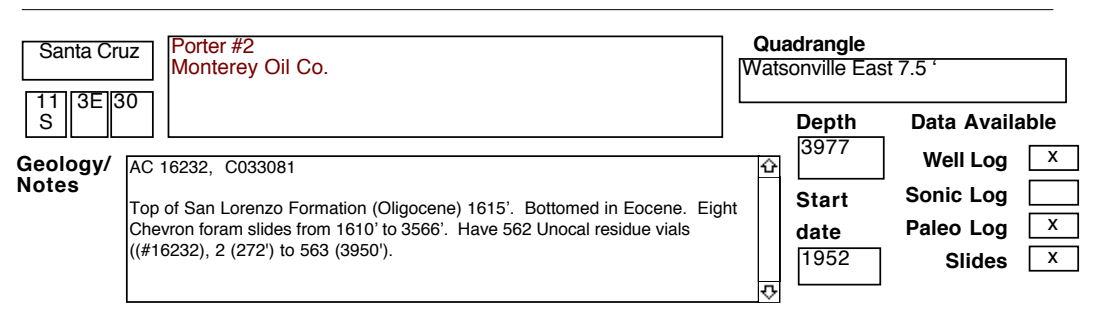

\begin{tabular}{l|l|l|l|l|l|l|}
\hline Santa Cruz & $\begin{array}{l}\text { Blake \#1 } \\
\text { Texaco }\end{array}$ & \multicolumn{2}{|c|}{ Quadrangle } \\
\hline
\end{tabular}

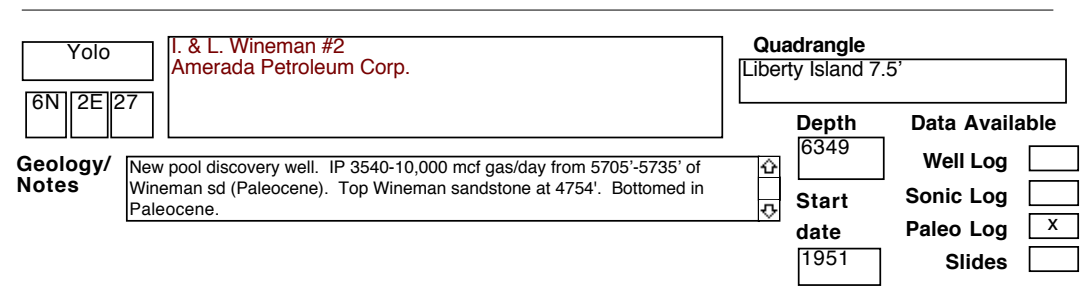


SELECTED OIL TEST WELLS IN CENTRAL CALIFORNIA DATABASE County

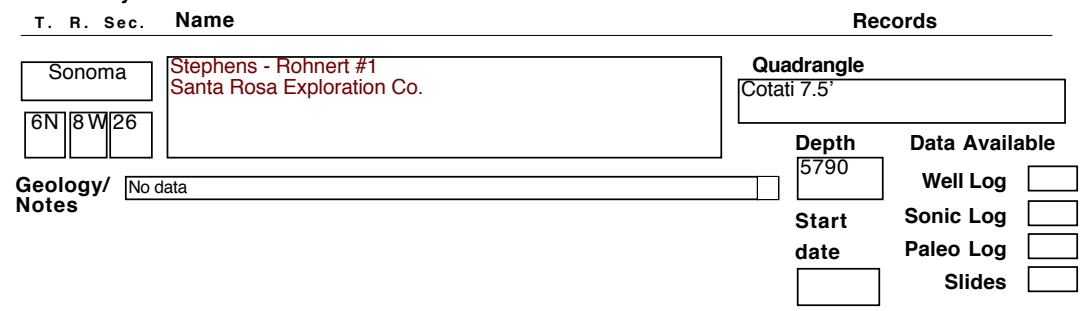

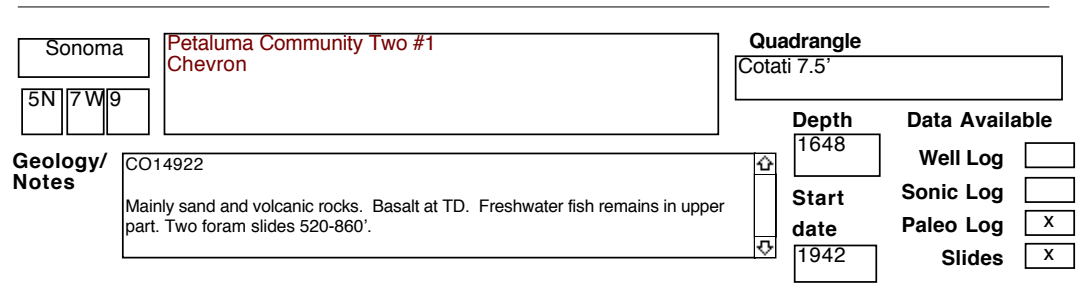

\begin{tabular}{l|l|l|l|l|l|}
\hline Sonoma & $\begin{array}{l}\text { Petaluma Community Three \#1 } \\
\text { Chevron }\end{array}$ & \multicolumn{2}{l|}{ Quadrangle } \\
\hline
\end{tabular}

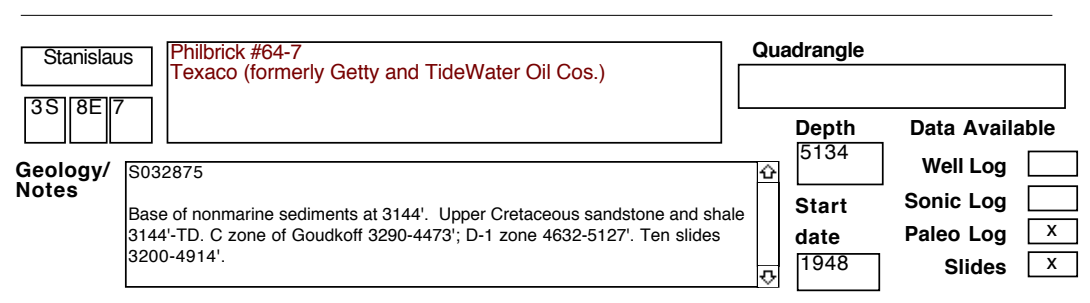

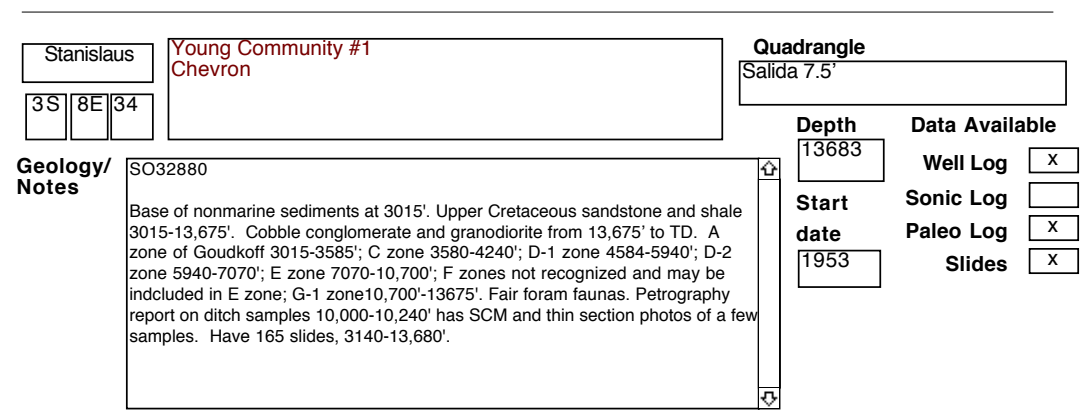


SELECTED OIL TEST WELLS IN CENTRAL CALIFORNIA DATABASE County

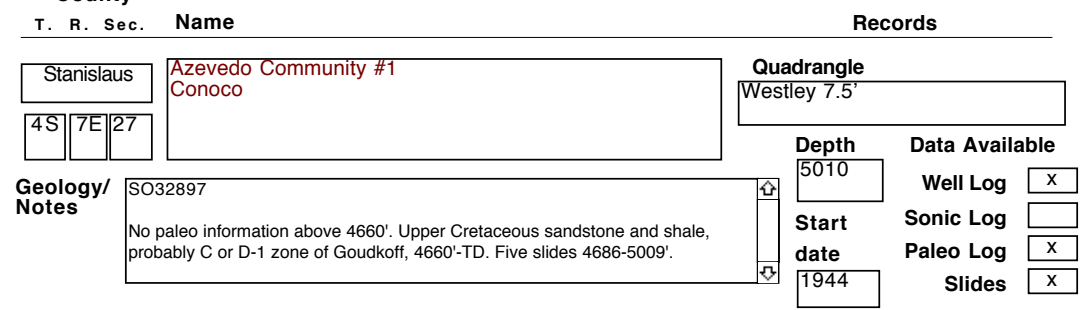

\begin{tabular}{|c|c|c|c|c|c|}
\hline Stanislaus & \multirow[t]{2}{*}{$\begin{array}{l}\text { K-2 \#1 } \\
\text { Coast Exploration Co. }\end{array}$} & \multicolumn{4}{|c|}{$\begin{array}{c}\text { Quadrangle } \\
\text { Brush Lake 7.5' }\end{array}$} \\
\hline $4 \mathrm{~S} / 9 \mathrm{E} 30$ & & & Depth & \multicolumn{2}{|c|}{ Data Available } \\
\hline \multirow{4}{*}{$\begin{array}{l}\text { Geology/ } \\
\text { Notes }\end{array}$} & 32902 & 它 & 631 & \multirow{4}{*}{$\begin{array}{r}\text { Well Log } \\
\text { Sonic Log } \\
\text { Paleo Log } \\
\text { Slides }\end{array}$} & $\mathrm{x}$ \\
\hline & se of nonmarine sediments at $3340^{\prime}$. Upper Cretaceous sandstone and shale & & Start & & \\
\hline & $\begin{array}{l}\text { 10'-TD have generally sparse forams. D-1 zone of Goudkoff } 6033^{\prime}-T D \text {. Ten } \\
\text { es from the interval } 3038^{\prime} \text { to } 6291^{\prime} \text {. }\end{array}$ & & date & & $\mathrm{x}$ \\
\hline & & (7) & & & $x$ \\
\hline
\end{tabular}

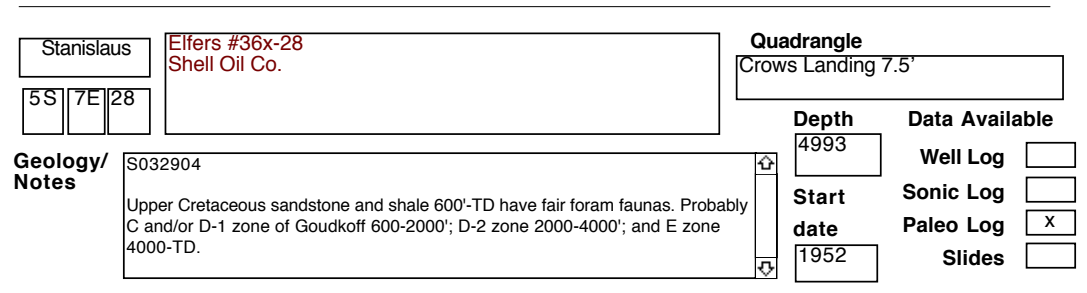

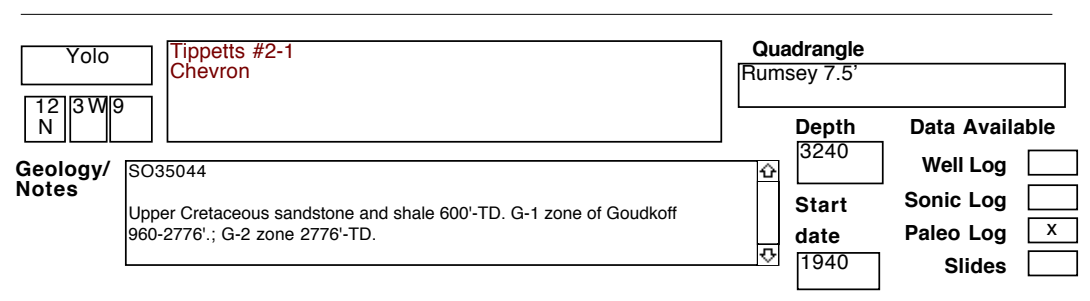

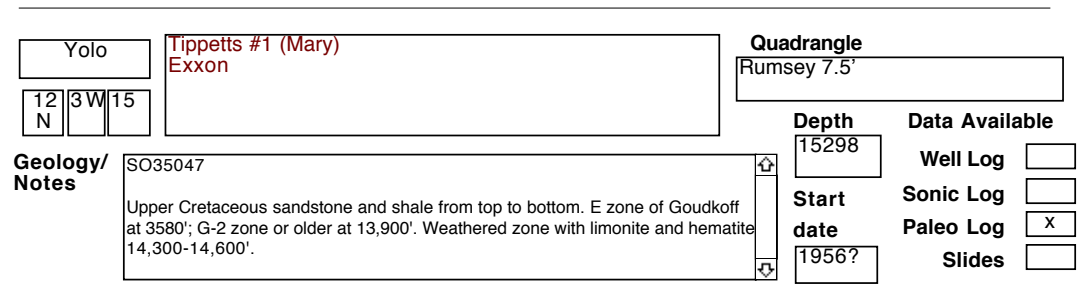


SELECTED OIL TEST WELLS IN CENTRAL CALIFORNIA DATABASE County

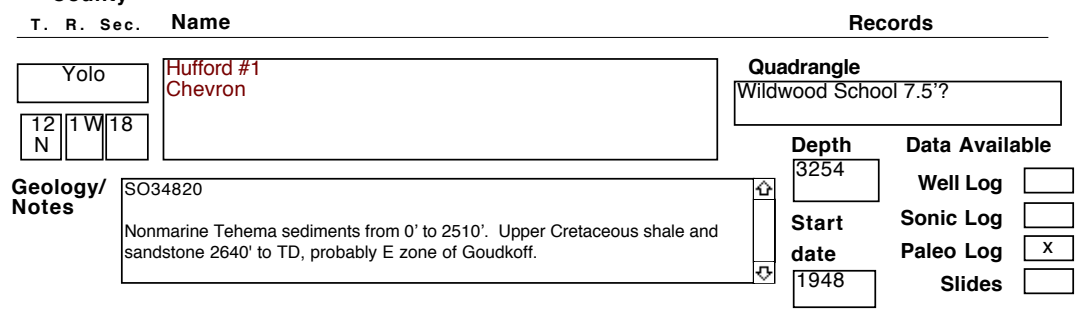

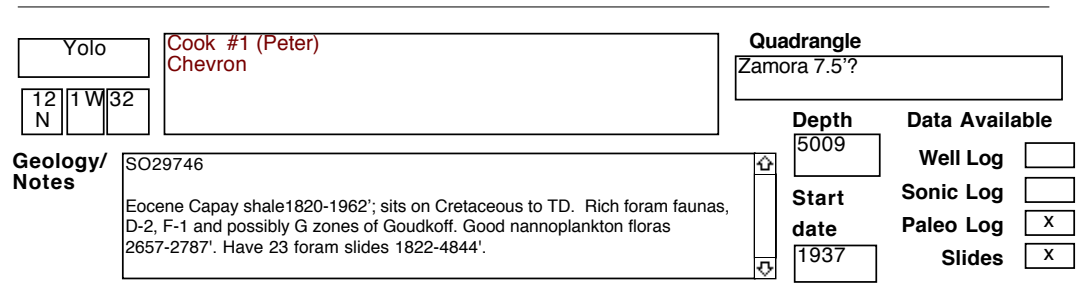

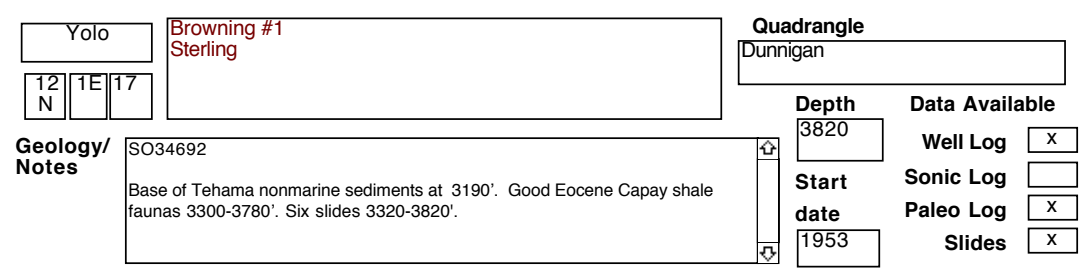

\begin{tabular}{|c|c|c|c|c|}
\hline Yolo & $\begin{array}{l}\text { Giguere \#1 } \\
\text { Chevron }\end{array}$ & \multicolumn{3}{|c|}{$\begin{array}{c}\text { Quadrangle } \\
\text { Bird Valley } 7.5\end{array}$} \\
\hline $\mathrm{N}$ & & \multirow{2}{*}{$\begin{array}{l}\text { Depth } \\
2750\end{array}$} & \multicolumn{2}{|c|}{ Data Available } \\
\hline \multirow{3}{*}{$\begin{array}{l}\text { Geology/ } \\
\text { Notes }\end{array}$} & $\mathbf{S O 3 5 0 0 9}$ & & Well Log & $\mathrm{x}$ \\
\hline & Base of nonmarine Tehama sediments at $1420^{\prime}$. Eocene sandstone and shale & Start & Sonic Log & \\
\hline & $\begin{array}{l}1420-1590^{\prime} \text {. Capay shale } 1590-2085^{\prime} \text {. Basal Capay grit } 2085-2123^{\prime} \text {. Upper } \\
\text { Cretaceous sandstone and shale } 2123^{\prime}-\text { TD have fair foram faunas correlative with } \\
\text { the D-2 zone of Goudkoff. Four slides } 1500-2610^{\prime} \text {. }\end{array}$ & $\begin{array}{l}\text { date } \\
1947\end{array}$ & $\begin{array}{r}\text { Paleo Log } \\
\text { Slides }\end{array}$ & $\mathrm{X}$ \\
\hline
\end{tabular}

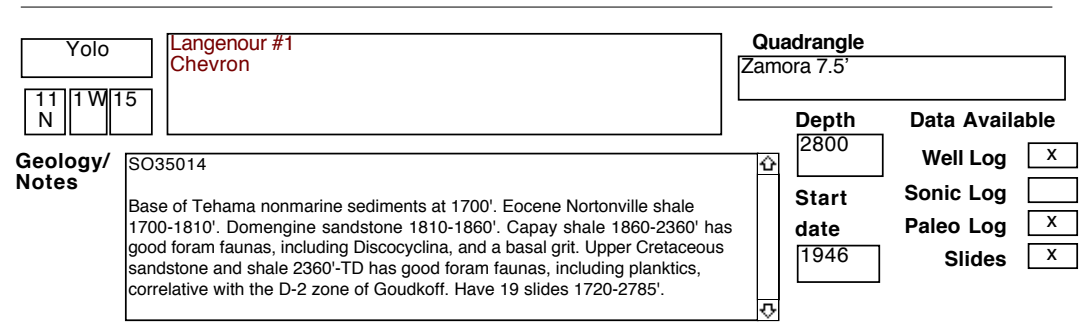


SELECTED OIL TEST WELLS IN CENTRAL CALIFORNIA DATABASE County

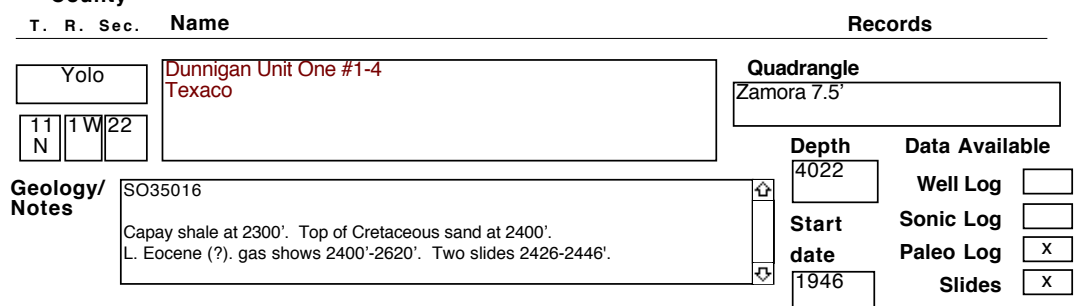

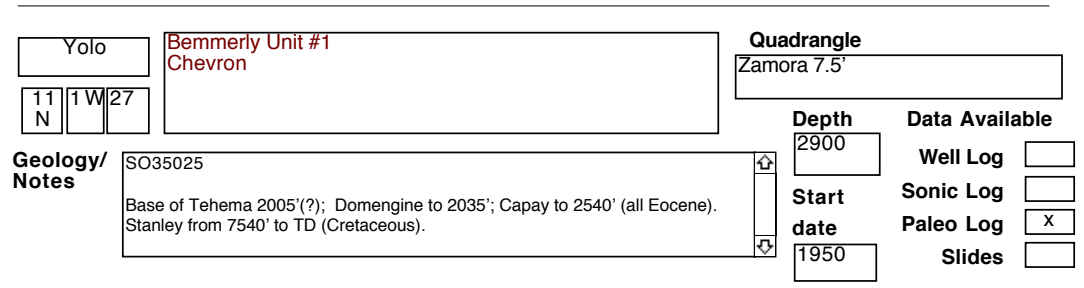

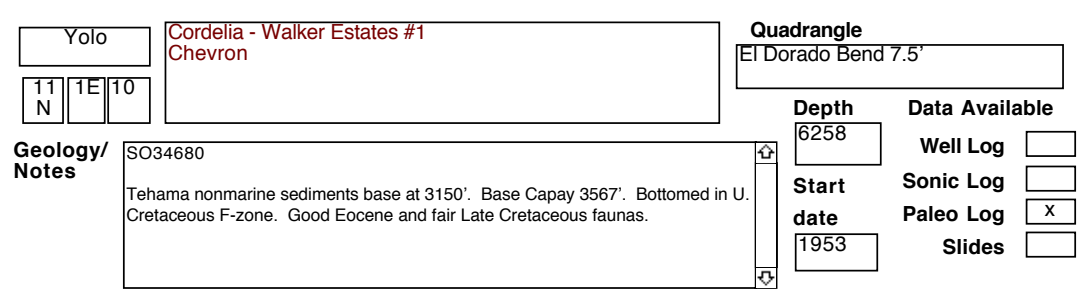

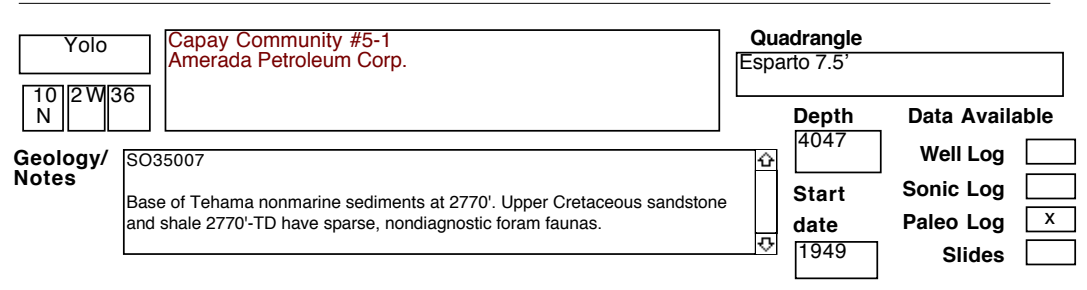

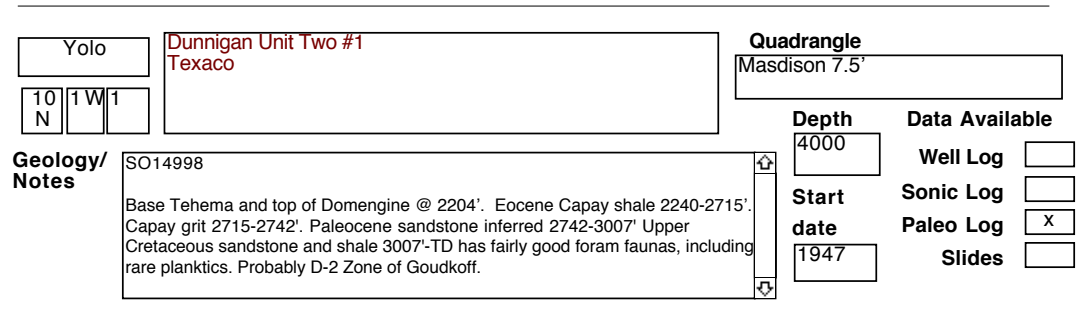


SELECTED OIL TEST WELLS IN CENTRAL CALIFORNIA DATABASE County

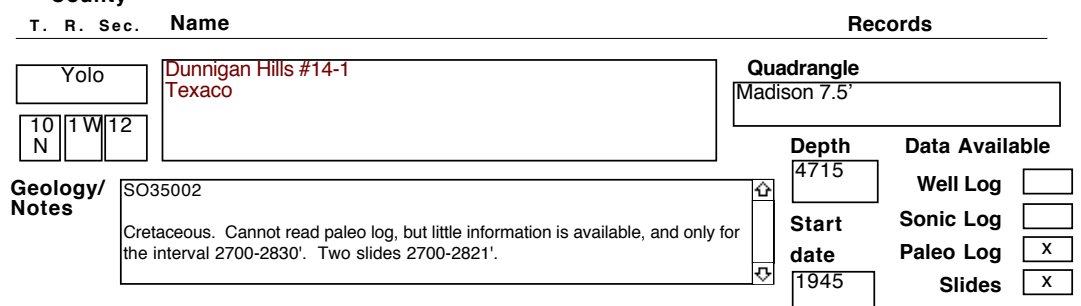

\begin{tabular}{l|l|l|l|l|}
\hline \multicolumn{1}{|c|}{ Yolo } & $\begin{array}{l}\text { Salisbury \#1 } \\
\text { Texaco }\end{array}$ & \multicolumn{2}{|c|}{ Quadrangle } \\
\hline
\end{tabular}

\begin{tabular}{|c|c|c|c|c|c|}
\hline Yolo & \multirow[t]{2}{*}{$\begin{array}{l}\text { Yolo Unit \#1 } \\
\text { Natural Gas Corp. of California }\end{array}$} & \multicolumn{4}{|c|}{$\begin{array}{l}\text { Quadrangle } \\
\text { Woodland } 7.5^{\prime} ?\end{array}$} \\
\hline 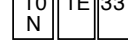 & & \multirow{2}{*}{\multicolumn{2}{|c|}{$\begin{array}{l}\text { Depth } \\
4100\end{array}$}} & \multicolumn{2}{|c|}{ Data Available } \\
\hline \multirow{3}{*}{$\begin{array}{l}\text { Geology/ } \\
\text { Notes }\end{array}$} & & & & Well Log & \\
\hline & $\begin{array}{l}\text { Nortonville sh } 2370 \text { ', top Domengine ss } 2520 \text { ', top Capay sh } 2732 \text { ', } \\
\text { tomed in Cretaceous ss and sh. }\end{array}$ & D & Start & Sonic Log & \\
\hline & & & $\begin{array}{l}\text { date } \\
1950\end{array}$ & $\begin{array}{r}\text { Paleo Log } \\
\text { Slides }\end{array}$ & $x$ \\
\hline
\end{tabular}

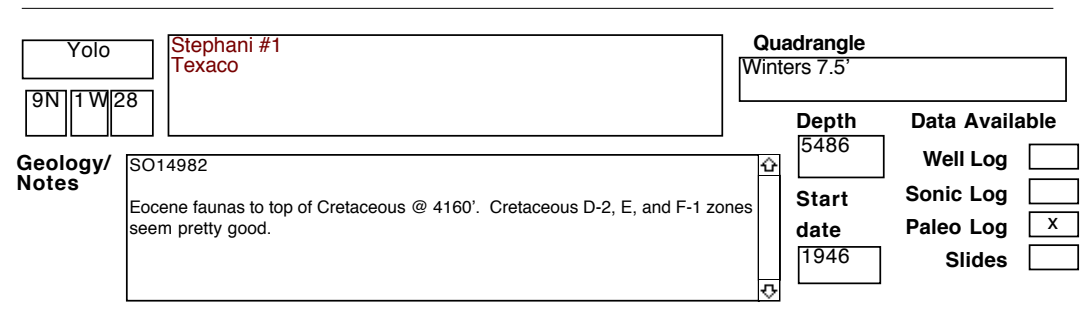

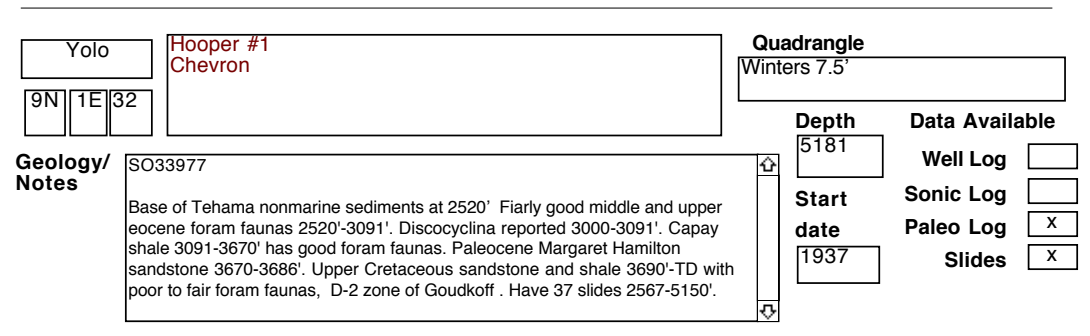


SELECTED OIL TEST WELLS IN CENTRAL CALIFORNIA DATABASE County

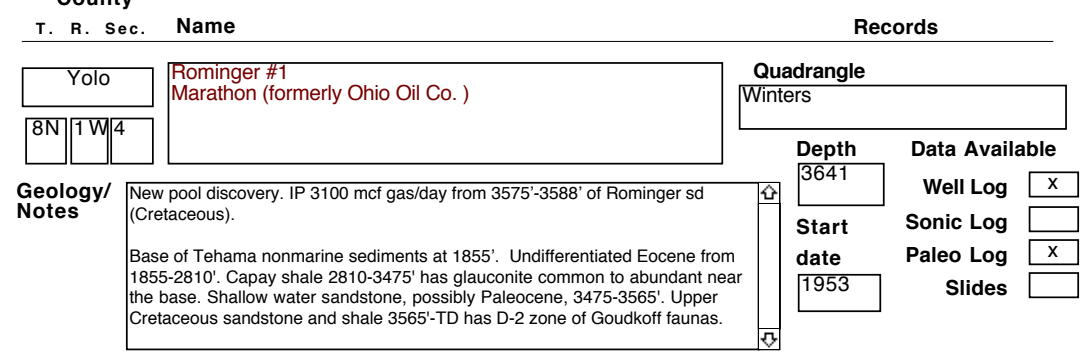

\begin{tabular}{|c|c|c|c|c|}
\hline Yolo & \multirow[t]{2}{*}{$\begin{array}{l}\text { Peters \#1 } \\
\text { Natural Gas Corp. of California }\end{array}$} & \multicolumn{3}{|l|}{$\begin{array}{l}\text { Quadrangle } \\
\text { Winters } 7.5^{\prime}\end{array}$} \\
\hline \multicolumn{4}{|l|}{$8 \mathrm{~N} / \mathrm{W} 9$} & \\
\hline \multirow{3}{*}{$\begin{array}{l}\text { Geology/ } \\
\text { Notes }\end{array}$} & e Capay 3426'. Bottomed in Cretaceou & & Well Log & \\
\hline & & Start & Sonic Log & \\
\hline & & $\begin{array}{l}\text { date } \\
1953\end{array}$ & $\begin{array}{r}\text { Paleo Log } \\
\text { Slides }\end{array}$ & $\mathrm{x}$ \\
\hline
\end{tabular}

\begin{tabular}{|c|c|c|c|c|c|}
\hline \multirow{2}{*}{\begin{tabular}{|c} 
Yolo \\
$8 \mathrm{~N} /\left.\mathrm{W}\right|^{12}$
\end{tabular}} & \multirow[t]{2}{*}{\begin{tabular}{|l} 
Wallace \#1 \\
Bender
\end{tabular}} & \multicolumn{4}{|c|}{$\begin{array}{l}\text { Quadrangle } \\
\text { Winters 7.5' }\end{array}$} \\
\hline & & \multirow{2}{*}{\multicolumn{2}{|c|}{$\begin{array}{l}\text { Depth } \\
4873\end{array}$}} & \multicolumn{2}{|c|}{ Data Available } \\
\hline \multirow{3}{*}{$\begin{array}{l}\text { Geology/ } \\
\text { Notes }\end{array}$} & & & & Well Log & \\
\hline & ie Capay-top Cretaceous 4375 ', McCune sd 4728 ', top unit sd 4826 '. & 0 & Start & Sonic Log & \\
\hline & & & $\begin{array}{l}\text { date } \\
1952\end{array}$ & $\begin{array}{r}\text { Paleo Log } \\
\text { Slides }\end{array}$ & $\mathrm{x}$ \\
\hline
\end{tabular}

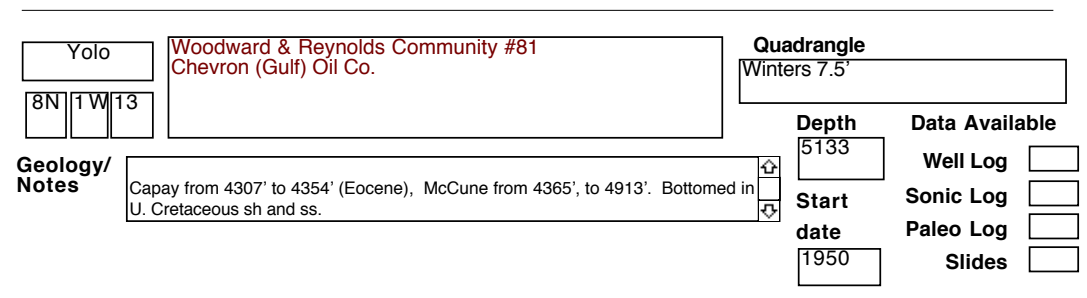

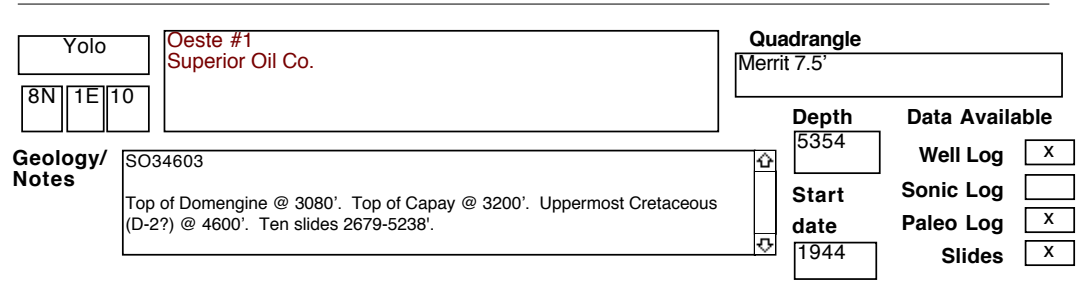




\section{SELECTED OIL TEST WELLS IN CENTRAL CALIFORNIA DATABASE}

County

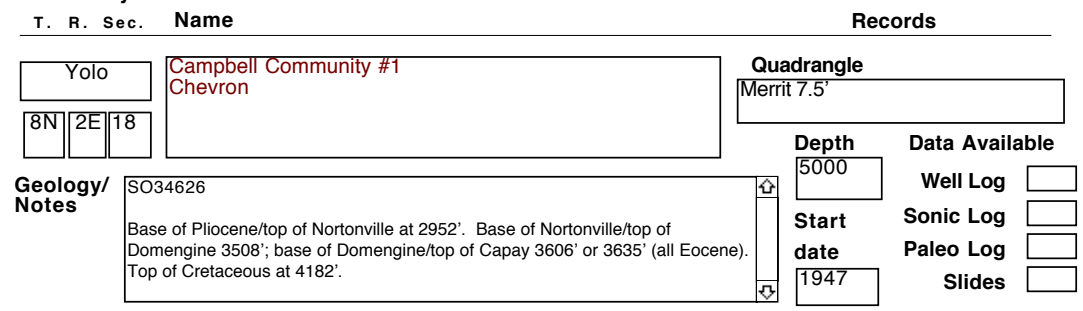

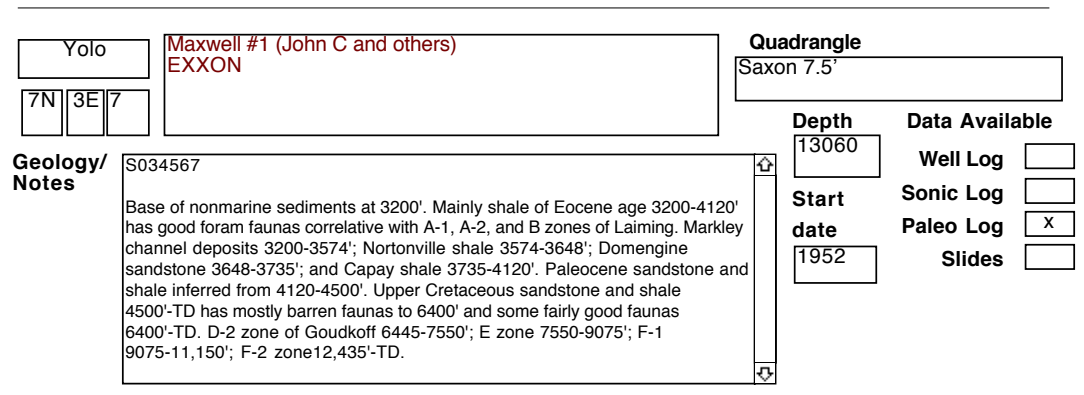

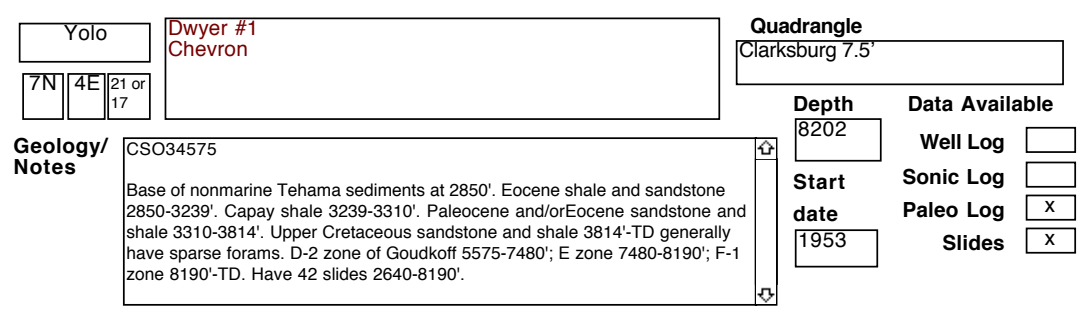

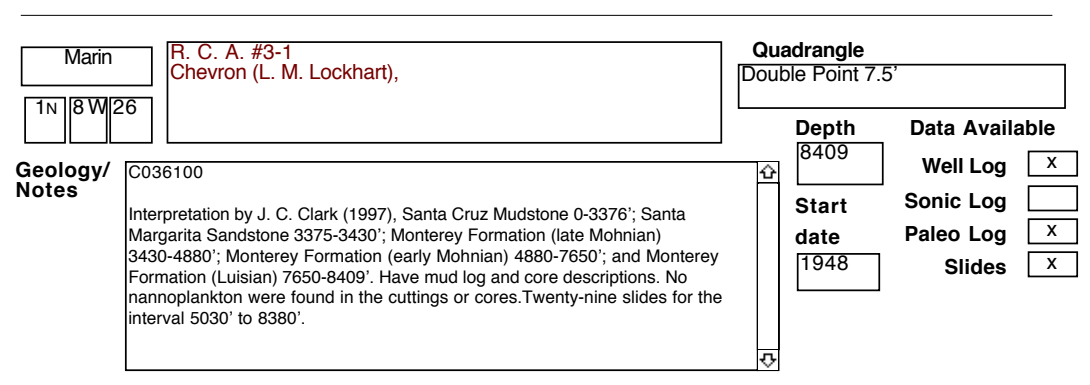

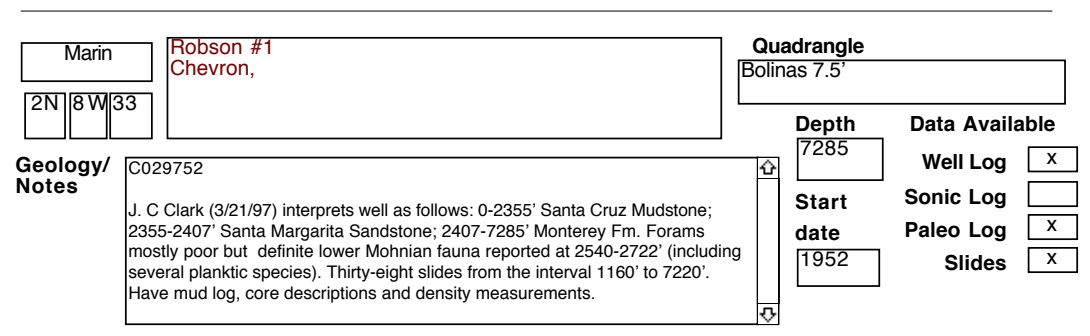


SELECTED OIL TEST WELLS IN CENTRAL CALIFORNIA DATABASE County

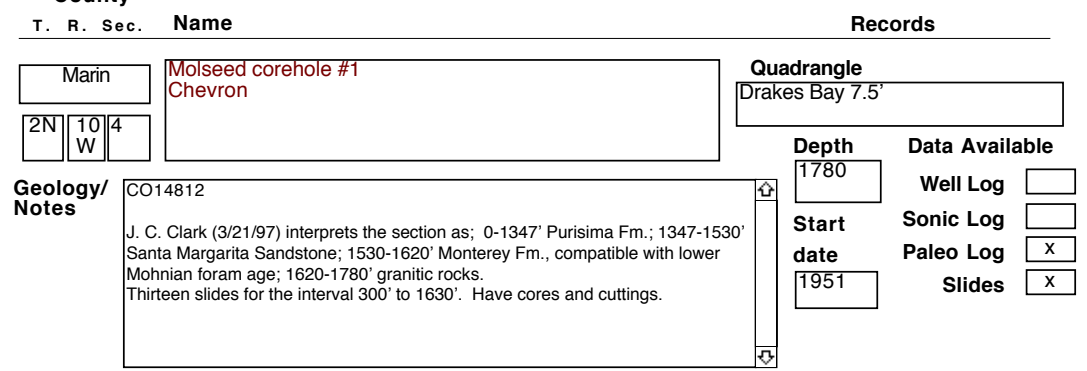

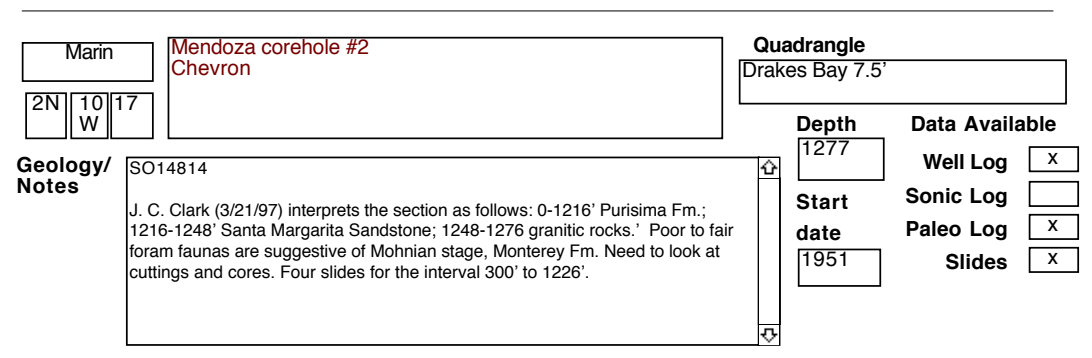

\begin{tabular}{l|l|l|l|l|l|}
\hline San Mateo & $\begin{array}{l}\text { Americo Oil Co. \#1 } \\
\text { Americo - Cabral }\end{array}$ & \multicolumn{2}{l|}{ Quadrangle } \\
\hline
\end{tabular}

\begin{tabular}{l|l|l|l|l|l|}
\hline Sonoma & $\begin{array}{l}\text { Wesey - Haire \#1 } \\
\text { General Crude Oil Co. }\end{array}$ & \multicolumn{2}{|c|}{ Quadrangle } \\
\hline
\end{tabular}

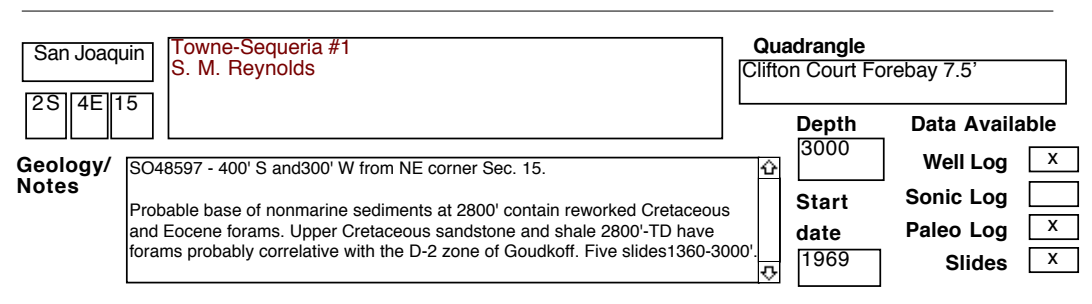




\section{SELECTED OIL TEST WELLS IN CENTRAL CALIFORNIA DATABASE}

\begin{tabular}{|c|c|c|c|c|}
\hline $\begin{array}{l}\text { County } \\
\text { T. R. Sec. }\end{array}$ & Name & \multicolumn{3}{|c|}{ Records } \\
\hline San Mateo & \multirow[t]{2}{*}{$\begin{array}{l}\text { Guiberson-Pereira \#1 } \\
\text { G. E. Kadane \& Sons }\end{array}$} & \multicolumn{3}{|c|}{$\begin{array}{l}\text { Quadrangle } \\
\begin{array}{|l}\text { Half Moon Bay 7.5' }\end{array}\end{array}$} \\
\hline $6 \mathrm{~S} 5 \mathrm{~W} 9$ & & \multirow{2}{*}{$\begin{aligned} & \text { Depth } \\
& 4919\end{aligned}$} & \multicolumn{2}{|c|}{ Data Available } \\
\hline \multirow{2}{*}{$\begin{array}{l}\text { Geology/ } \\
\text { Notes }\end{array}$} & $16572, \mathrm{C} 014415$ & & Well Log & $x$ \\
\hline & $\begin{array}{l}\text { bably Purisima faunas to } 3400^{\prime} \text { including planktonics. Flood of glauconite at } \\
0^{\prime} \text { probably basal Purisima. Volcanic rocks } 3820-3870 \text { '. Eocene faunas } 3940 \\
\text { 'D. Twenty-three slides from the interval } 100^{\prime} \text { to } 4704^{\prime} \text {, plus one from the } \\
\text { rval } 3860^{\prime} \text { to } 3920^{\prime} \text {. }\end{array}$ & $\begin{array}{l}\text { Start } \\
\text { date } \\
1957\end{array}$ & $\begin{array}{l}\text { Sonic Log } \\
\text { Paleo Log } \\
\text { Slides }\end{array}$ & $\begin{array}{l}x \\
x \\
\end{array}$ \\
\hline
\end{tabular}

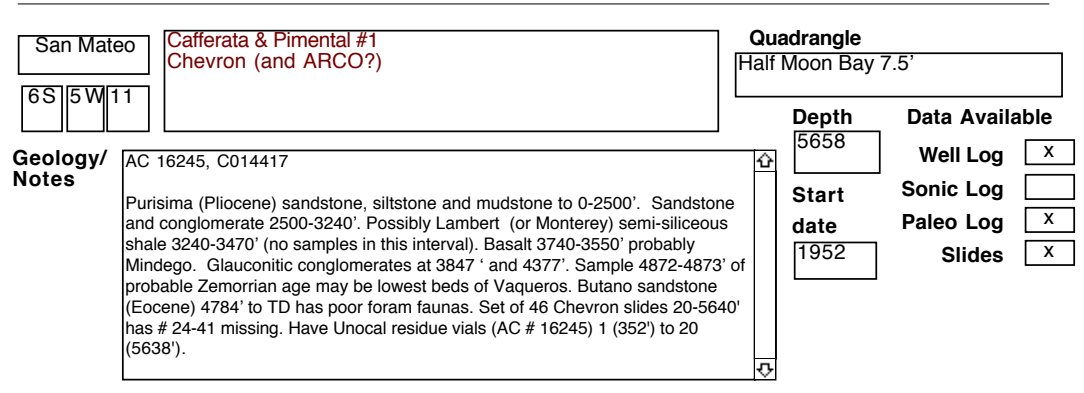

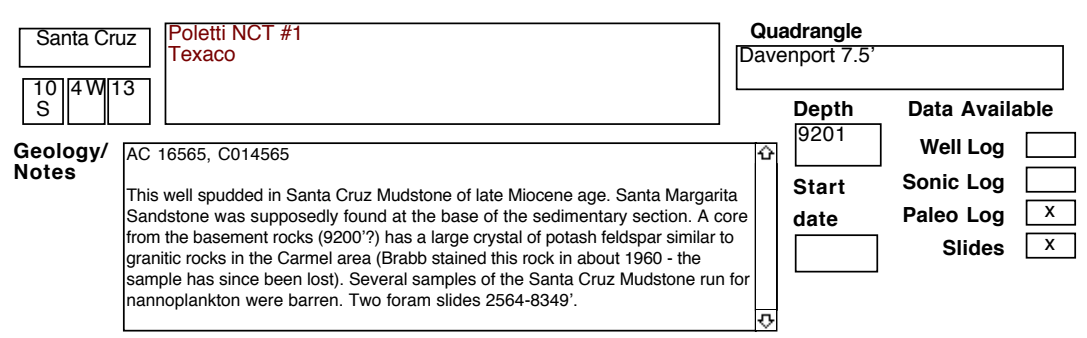

\begin{tabular}{l|l|l|l|l|l|l|}
\hline \multicolumn{1}{|c|}{ Sonoma } & $\begin{array}{l}\text { Bourke \#1 } \\
\text { Chevron-Petaluma Exploration Co. }\end{array}$ \\
\hline
\end{tabular}

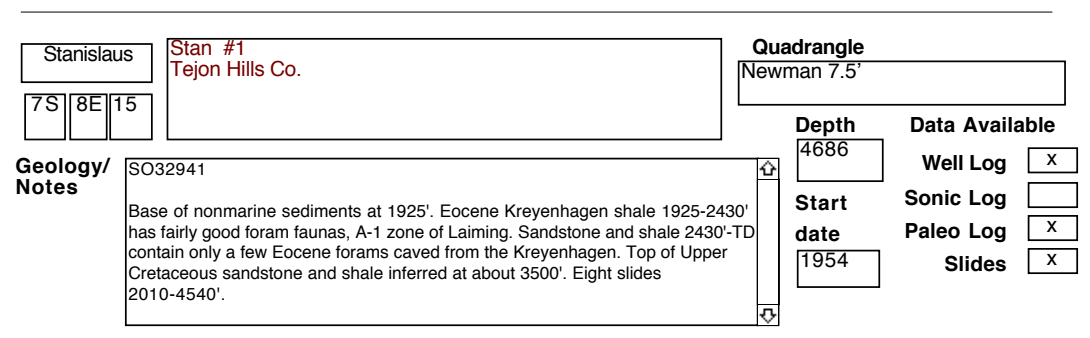


SELECTED OIL TEST WELLS IN CENTRAL CALIFORNIA DATABASE County

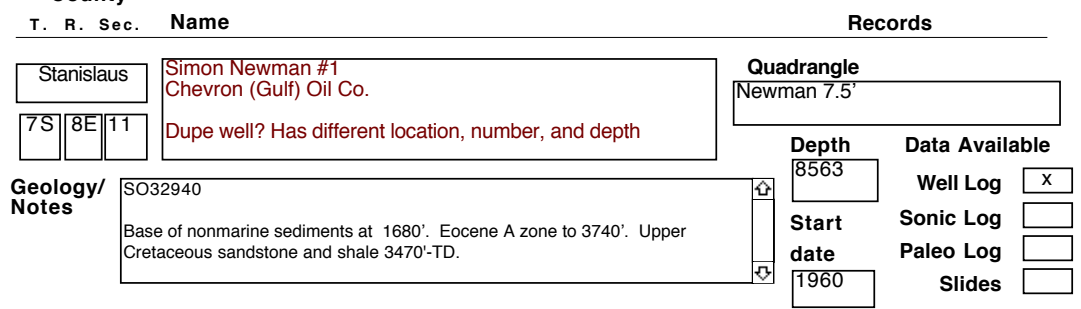

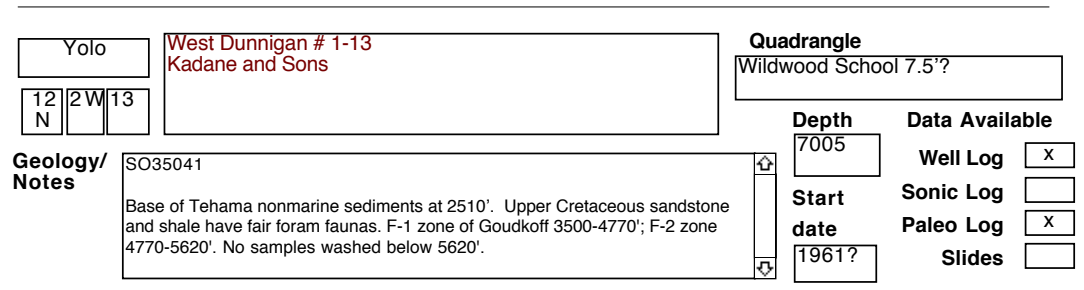

\begin{tabular}{l|l|l|l|l|l|}
\hline \multicolumn{1}{|c|}{ Yolo } & $\begin{array}{l}\text { Chapman \#1 } \\
\text { Marathon Oil Co. (formerly Ohio Oil Co) }\end{array}$ \\
\hline
\end{tabular}

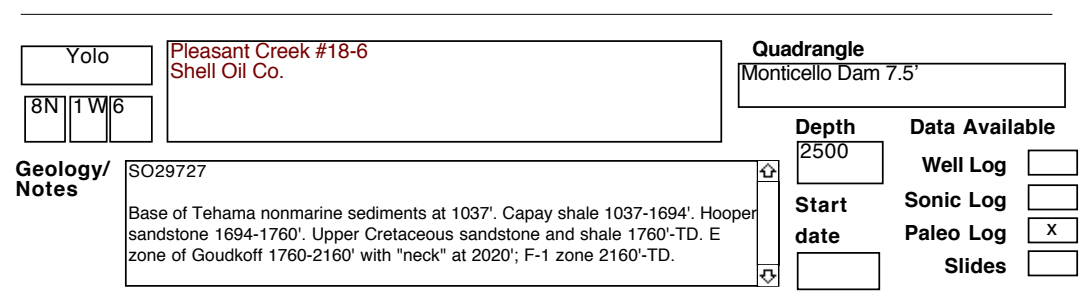

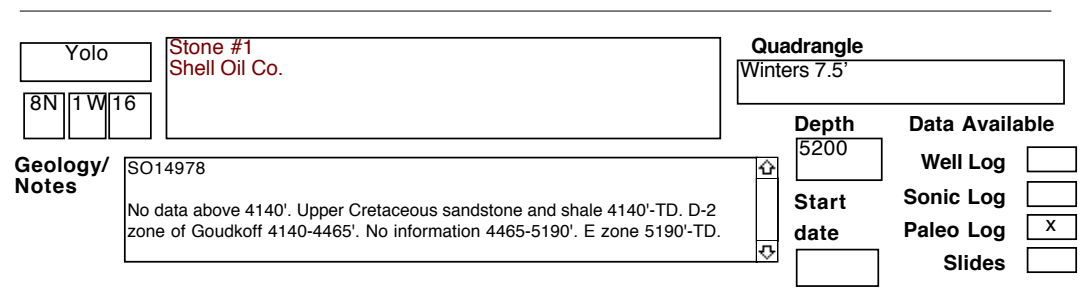


SELECTED OIL TEST WELLS IN CENTRAL CALIFORNIA DATABASE County

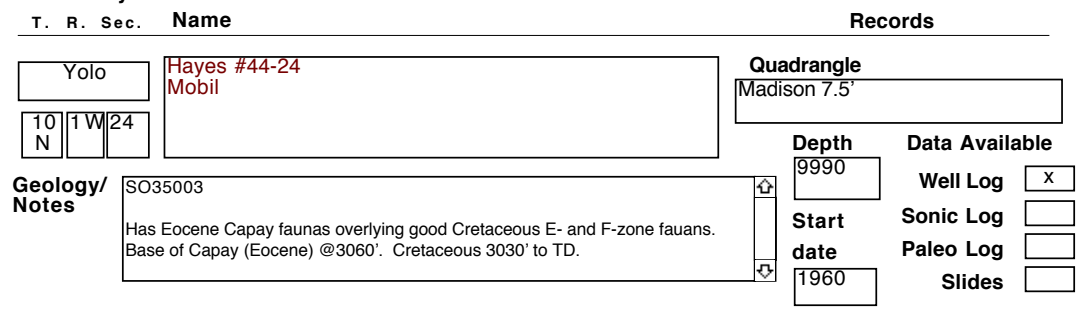

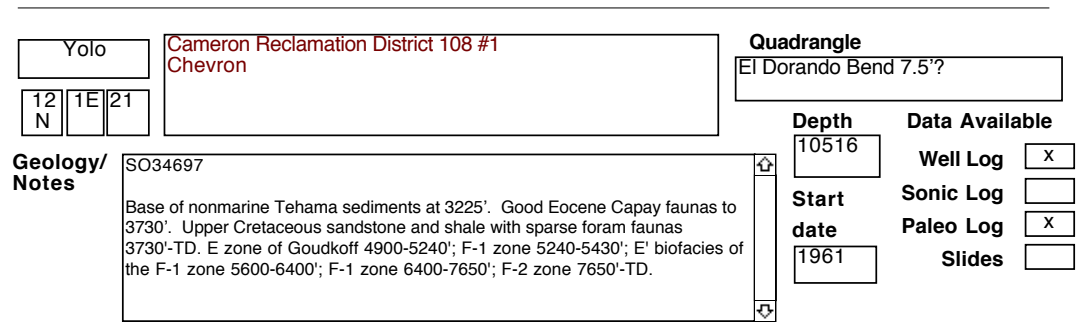

\begin{tabular}{l|l|l|l|l|l|l|}
\hline Yolo & $\begin{array}{l}\text { Clover \#1 } \\
\text { Monterey Oil Co. }\end{array}$ \\
\hline
\end{tabular}

\begin{tabular}{|l|l|l|l|l|}
\hline Yolo & $\begin{array}{l}\text { Klein \#1 } \\
\text { Chevron }\end{array}$ \\
Notes & $\begin{array}{l}\text { SO34616 } \\
\text { Base of Tehama nonmarine sediments at 2310'. Glauconitic sandstone } \\
2310-2840 ' \text { ' seems to be a mixture of marine and nonmarine beds. Eocene marine } \\
\text { shale 2840-4349' includes correlatives of the Markley and Nortonville formations, } \\
\text { 2840-3845', with good A-1 and A-2 zone of Laiming foram faunas; Domengine } \\
\text { sandstone, 3845-3586'; and Capay shale with excellent foram fannas correlative } \\
\text { with B-4 zone of Laiming. Upper Cretaceous sandstone and shale 4349'-TD has a } \\
\text { few good foram faunas in the lower part correlative with the D-2 zone of Goudkoff. } \\
\text { Have slides \#38 to 54 indicating that 37 are missing. }\end{array}$ \\
\hline
\end{tabular}

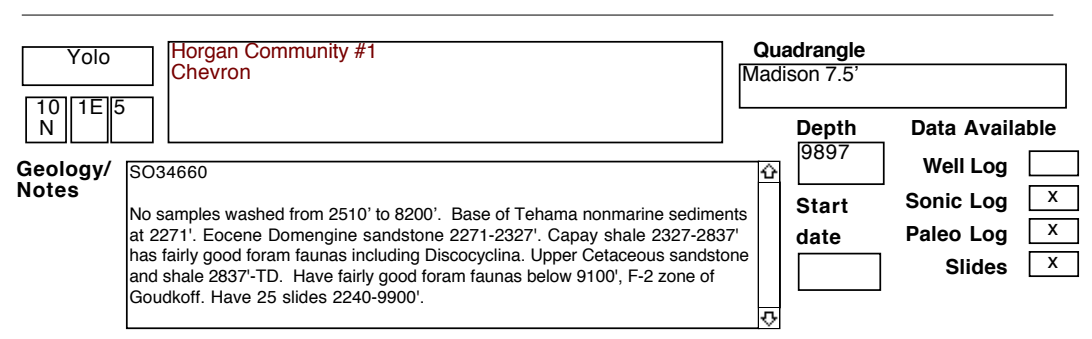




\section{SELECTED OIL TEST WELLS IN CENTRAL CALIFORNIA DATABASE}

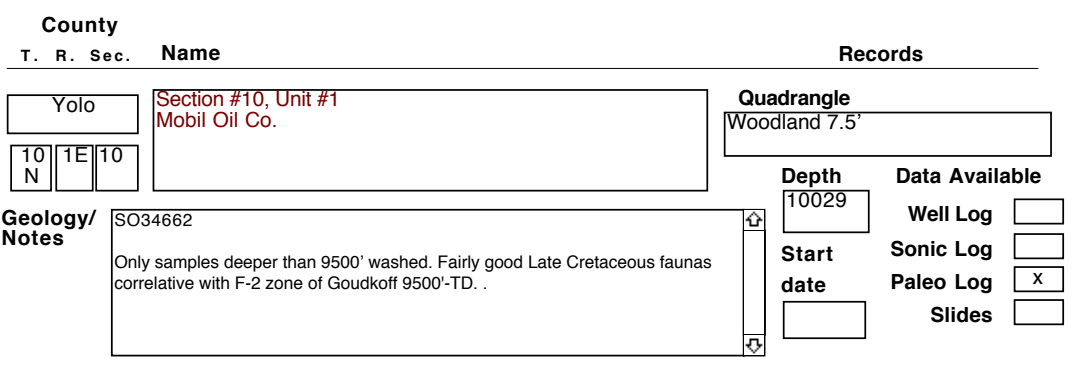

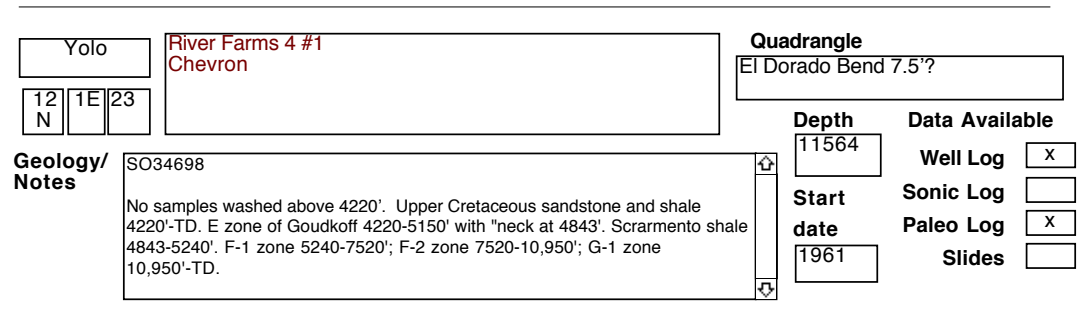

\begin{tabular}{|c|c|c|c|c|c|}
\hline \multicolumn{2}{|c|}{ Mendocino } & \multirow[t]{2}{*}{$\begin{array}{l}\text { Sun-Lepori \#1 } \\
\text { Chevron }\end{array}$} & \multicolumn{3}{|c|}{$\begin{array}{l}\text { Quadrangle } \\
\text { Point Arena 7.5 }\end{array}$} \\
\hline \begin{tabular}{|l|l|l|}
12 & 16 & 15 \\
$\mathrm{~N}$ & $\mathrm{~W}$ & \\
\end{tabular} & & & \multirow{2}{*}{$\begin{array}{l}\text { Depth } \\
6361\end{array}$} & \multicolumn{2}{|l|}{ Data Available } \\
\hline \multirow{4}{*}{$\begin{array}{l}\text { Geology/ } \\
\text { Notes }\end{array}$} & \multirow{4}{*}{\multicolumn{2}{|c|}{$\begin{array}{l}\text { C033854 } \\
\text { Siliceous shale 0-700'; siliceous shale with sand stlreaks } 700-3600 \text { '. Mostly shale } \\
\text { below } 3600^{\prime} \text {. All sands cored were poorly sorted, hard, and tight. At 6300', basic } \\
\text { igneous material, white chert, and mudstone were encountered, possible } \\
\text { sedimentary rubble at an erosion surface, or fault gouge. Foram fauna considered } \\
\text { all lower Zemorian by one paleontologist, and as Saucesian with a deep-water } \\
\text { Zemorrian character by another. Nannoplankton suggest that the upper } 1075^{\prime} \text { of } \\
\text { the well is Relizian, middle Miocene in age, with beds of Saucesian and Zemorrian } \\
\text { correlatives below. Have } 60 \text { foram slides } 540-6340^{\prime} \text {. }\end{array}$}} & & Well Log & $x$ \\
\hline & & & Start & Sonic Log & $x$ \\
\hline & & & date & Paleo Log & $x$ \\
\hline & & & 1962 & Slides & $x$ \\
\hline
\end{tabular}

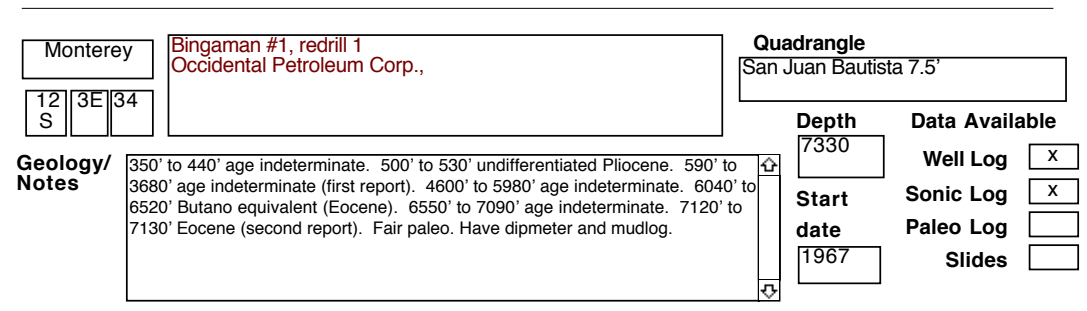

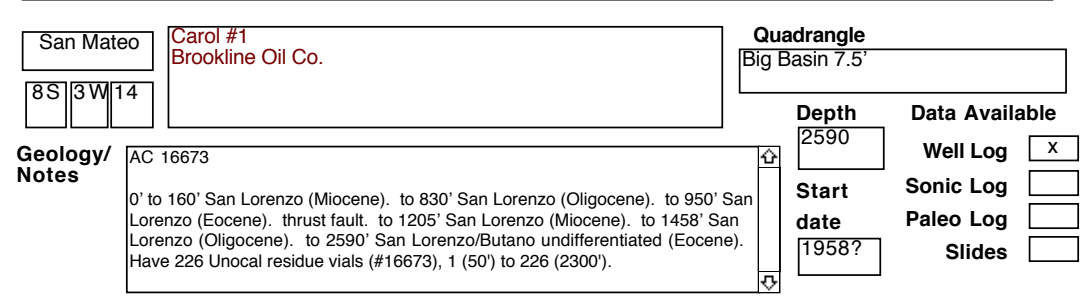


SELECTED OIL TEST WELLS IN CENTRAL CALIFORNIA DATABASE County

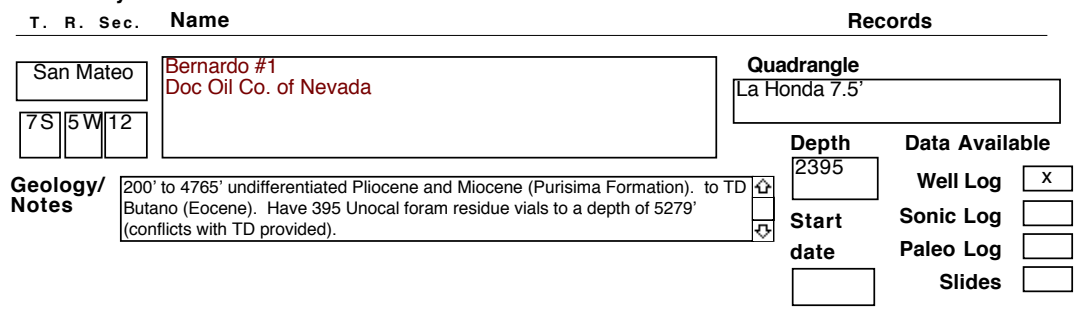

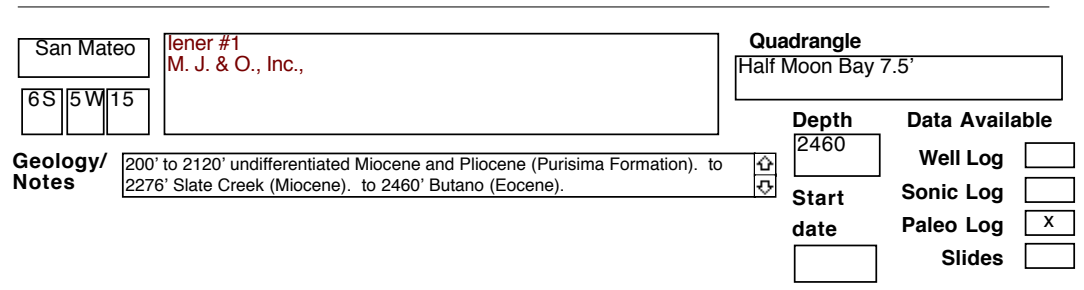

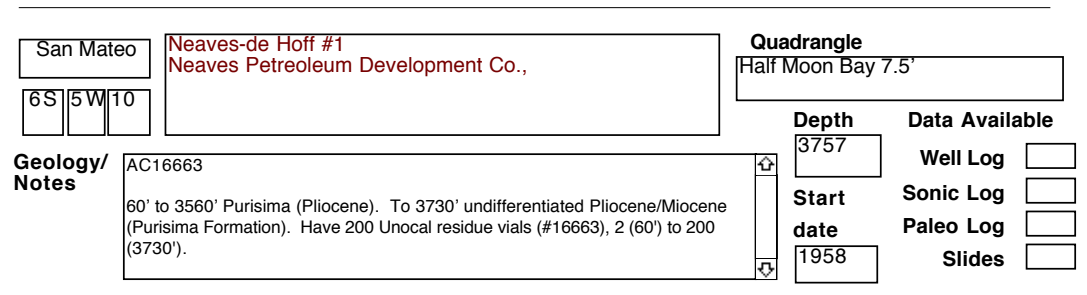

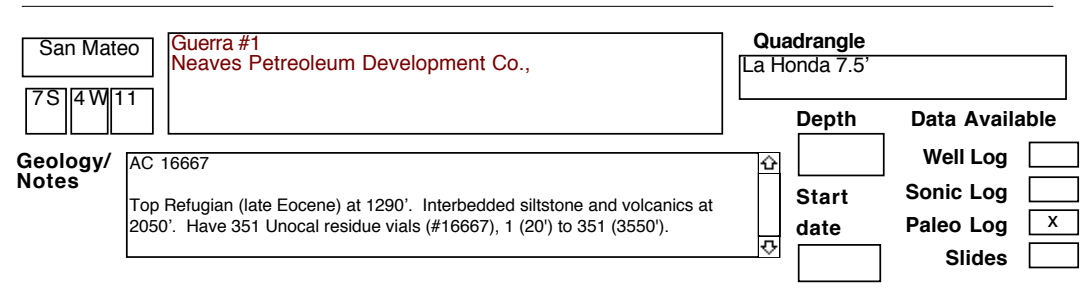

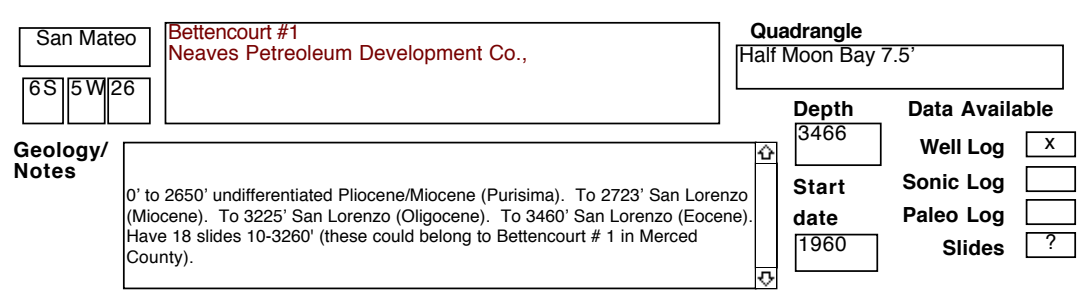


SELECTED OIL TEST WELLS IN CENTRAL CALIFORNIA DATABASE County

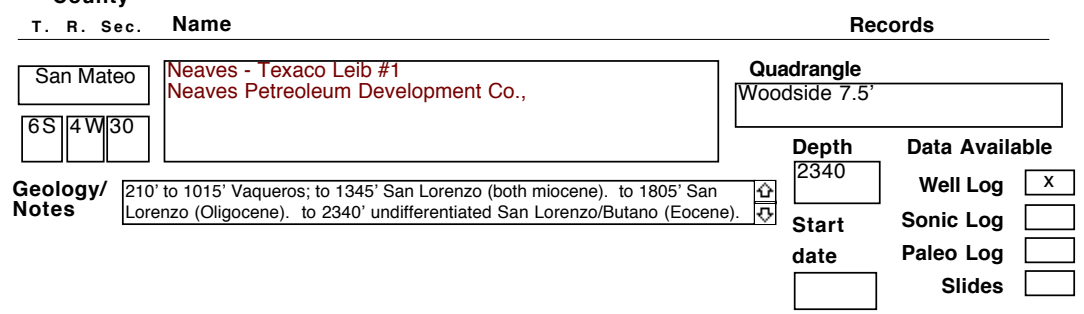

\begin{tabular}{|c|c|c|c|}
\hline San Mateo & \multirow[t]{2}{*}{$\begin{array}{l}\text { Butts \#1 } \\
\text { Shell Oil Co. }\end{array}$} & \multicolumn{2}{|c|}{$\begin{array}{l}\text { Quadrangle } \\
\text { Half Moon Bay 7.5' }\end{array}$} \\
\hline \begin{tabular}{|l|l|l|l|}
$6 S$ & $5 \mathrm{~W}$ & 21
\end{tabular} & & \multirow{2}{*}{$\begin{array}{l}\text { Depth } \\
3689\end{array}$} & Data Available \\
\hline \multirow{3}{*}{$\begin{array}{l}\text { Geology/ } \\
\text { Notes }\end{array}$} & & & Well Log \\
\hline & & Start & Sonic Log \\
\hline & & $\begin{array}{l}\text { date } \\
1928\end{array}$ & $\begin{array}{r}\text { Paleo Log } \\
\text { Slides }\end{array}$ \\
\hline
\end{tabular}

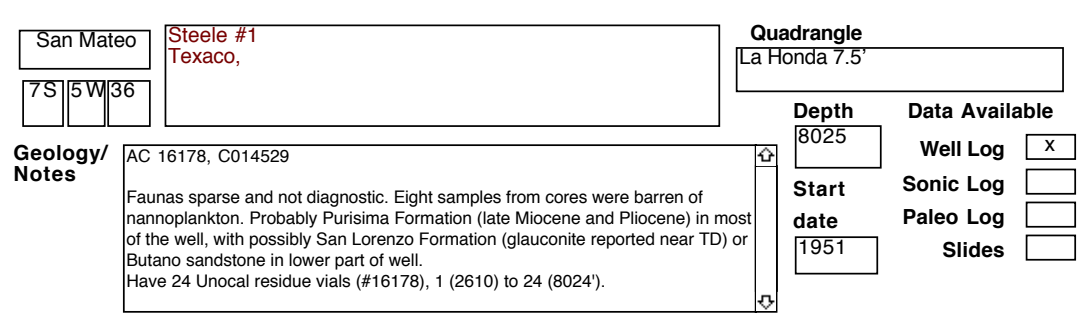

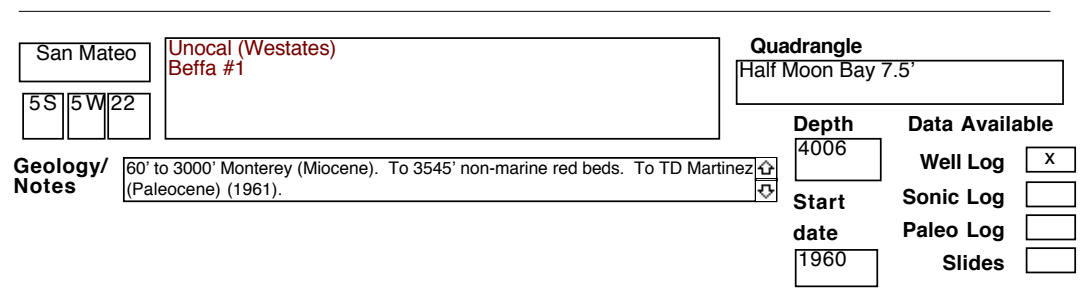

\begin{tabular}{|c|c|c|c|c|}
\hline \multirow{2}{*}{\begin{tabular}{|l|} 
San Mateo \\
\begin{tabular}{|l|l|l|}
$8 S$ & $3 \mathrm{~W}$ & 10 \\
\end{tabular} \\
\end{tabular}} & \multirow{2}{*}{$\begin{array}{l}\text { Union-Richfield-Eaton \#1 } \\
\text { Unocal /Arco, }\end{array}$} & \multicolumn{3}{|c|}{$\begin{array}{r}\text { Quadrangle } \\
\text { Big Basin 7.5' }\end{array}$} \\
\hline & & \multirow{2}{*}{$\begin{array}{l}\text { Depth } \\
3737\end{array}$} & \multicolumn{2}{|c|}{ Data Available } \\
\hline \multirow{3}{*}{$\begin{array}{l}\text { Geology/ } \\
\text { Notes }\end{array}$} & AC 16533 & & Well Log & $\mathrm{x}$ \\
\hline & ' to 1070 ' Vaqueros/San Lorenzo undifferentiated (Oligocene). San Lorenzo & Start & Sonic Log & \\
\hline & Eight slides from the interval $1370^{\prime}$ to $2260^{\prime}$. One slide from the interval $25^{\prime}$ to $80^{\prime}$ & date & Slides & $\overline{\mathrm{X}}$ \\
\hline
\end{tabular}


SELECTED OIL TEST WELLS IN CENTRAL CALIFORNIA DATABASE County

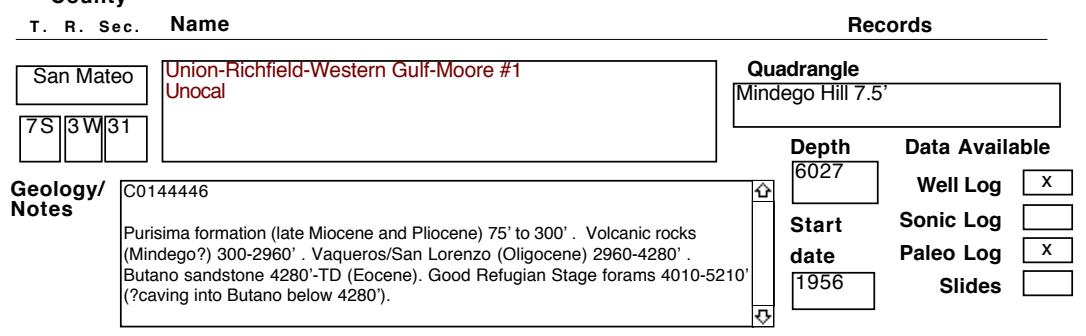

\begin{tabular}{|c|c|c|c|c|c|}
\hline San Mateo & \multirow[t]{2}{*}{$\begin{array}{l}\text { Union-Richfield-Western Gulf-Moore \#2 } \\
\text { Unocal, }\end{array}$} & \multicolumn{4}{|c|}{$\begin{array}{l}\text { Quadrangle } \\
\text { Mindego Hill 7.5, }\end{array}$} \\
\hline $8 S \mid 3 \mathrm{~W} 6$ & & \multirow{2}{*}{\multicolumn{2}{|c|}{$\begin{array}{l}\text { Depth } \\
4429\end{array}$}} & \multicolumn{2}{|c|}{ Data Available } \\
\hline \multirow{3}{*}{$\begin{array}{l}\text { Geology/ } \\
\text { Notes }\end{array}$} & \multirow{3}{*}{$\begin{array}{l}\text { Purisima Formation 30' TO 620', Miocene/Pliocene undifferentiated. } \\
\text { Vaqueros/San Lorenzo (Oligocene) } 620-3365^{5} \text {. San Lorenzo and Butano } \\
\text { Formations } 3365^{\prime} \text { to TD (Eocene). (Cited in California State well database as } \\
\text { having TD of } 4277^{\prime} \text {.) }\end{array}$} & & & Well Log & $\mathrm{x}$ \\
\hline & & \multirow[b]{2}{*}{0} & Start & Sonic Log & \\
\hline & & & 1960 & Slides & \\
\hline
\end{tabular}

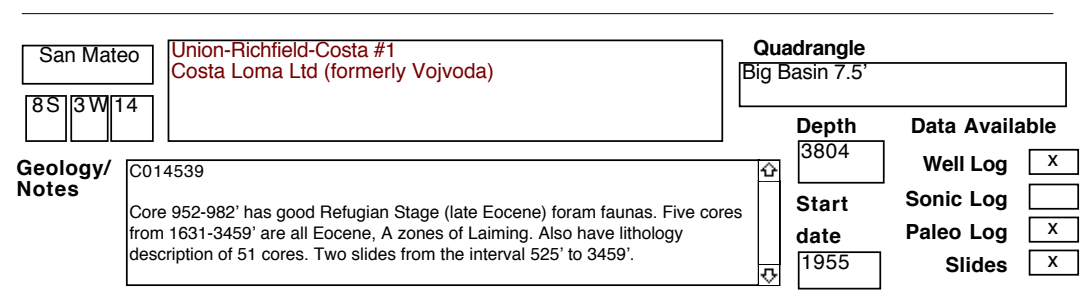

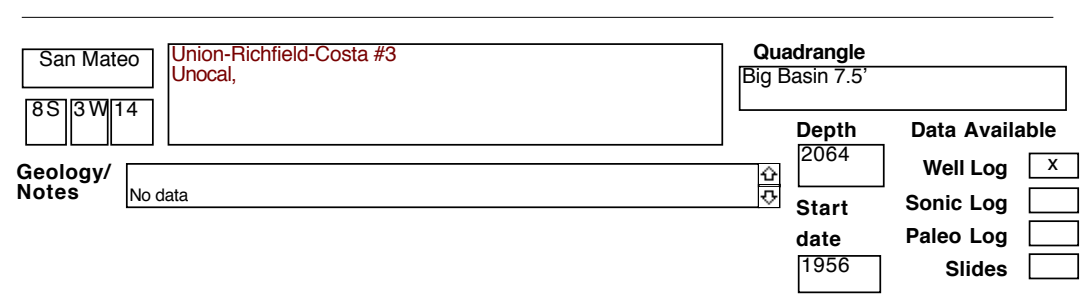

\begin{tabular}{|c|c|c|c|c|c|}
\hline San Matec & $\begin{array}{l}\text { SCL \# 1 (formerly Wells Fargo \#1) } \\
\text { Neaves Petroleum }\end{array}$ & $\begin{array}{r}\text { Qu } \\
\text { Minc }\end{array}$ & $\frac{\text { drangle }}{\text { ego Hill } 7}$ & & \\
\hline $8 \mathrm{~S} 3 \mathrm{~W} 7$ & & & \multirow{2}{*}{$\begin{array}{l}\text { Depth } \\
1028\end{array}$} & \multicolumn{2}{|c|}{ Data Available } \\
\hline \multirow{3}{*}{$\begin{array}{l}\text { Geology/ } \\
\text { Notes }\end{array}$} & C014535 & & & Well Log & $\mathrm{x}$ \\
\hline & $\begin{array}{l}\text { Pliocene Purisima Formation 80-906'. Mindego 1396' volcanics 906-1396'. } \\
\text { Vagueros sandstone (Oligocene) 1390-1755' Vagueros and/or San Lorenzo }\end{array}$ & & Start & $\begin{array}{l}\text { Sonic Log } \\
\text { Paleo Loq }\end{array}$ & $\bar{x}$ \\
\hline & $\begin{array}{l}\text { formation } 1755-3838^{\prime} \text {. Butano Sandstone (Eocene) } 3858^{\prime} \text {-TD. (1957). Two } \\
\text { slides from the interval } 340^{\prime} \text { to } 3150^{\prime} \text {. Four slides from the interval } 1639^{\prime} \text { to } 4430^{\prime} \\
\text { One slide from } 118^{\prime} \text {. }\end{array}$ & & 1956 & Slides & $x$ \\
\hline
\end{tabular}


SELECTED OIL TEST WELLS IN CENTRAL CALIFORNIA DATABASE County

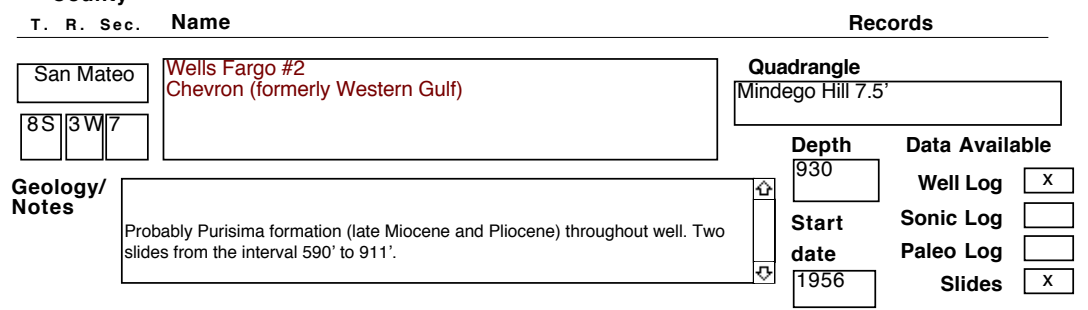

\begin{tabular}{l|l|l|l|l|l|}
\hline San Mateo & $\begin{array}{l}\text { Western Shore \#1 } \\
\text { Chevron (Gulf) Oil Co., }\end{array}$ \\
\hline
\end{tabular}

\begin{tabular}{|c|c|c|c|c|c|}
\hline San Benito & \multirow[t]{2}{*}{$\begin{array}{l}\text { Towle 1-12 } \\
\text { Holmes }\end{array}$} & \multicolumn{4}{|c|}{$\begin{array}{l}\text { Quadrangle } \\
\text { Cherry Peak7.5' }\end{array}$} \\
\hline \begin{tabular}{|c|c|c|}
15 & $7 \mathrm{E}$ & 12 \\
$\mathrm{~S}$ & & \\
\end{tabular} & & \multirow{2}{*}{\multicolumn{2}{|c|}{$\begin{array}{l}\text { Depth } \\
2395\end{array}$}} & \multicolumn{2}{|c|}{ Data Available } \\
\hline \multirow{3}{*}{$\begin{array}{l}\text { Geology/ } \\
\text { Notes }\end{array}$} & \multirow{3}{*}{ Need more information } & & & Well Log & \\
\hline & & 0 & Start & Sonic Log & $\mathrm{x}$ \\
\hline & & & $\begin{array}{l}\text { date } \\
1958\end{array}$ & $\begin{array}{r}\text { Paleo Log } \\
\text { Slides }\end{array}$ & \\
\hline
\end{tabular}

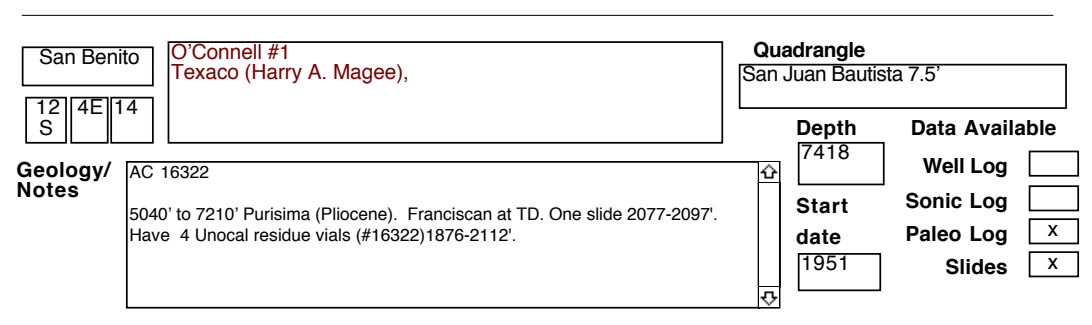

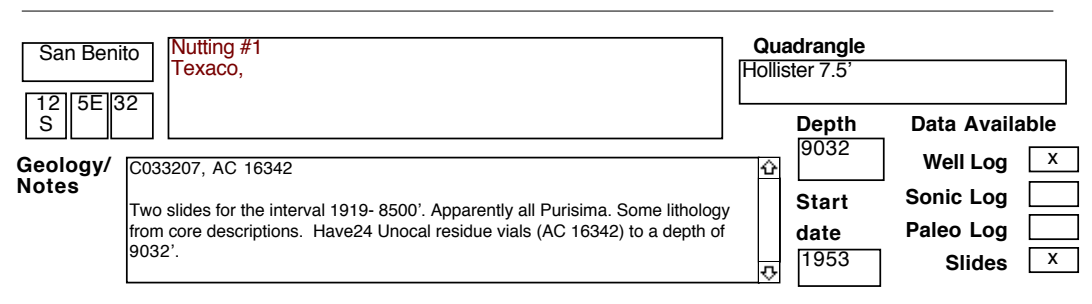


SELECTED OIL TEST WELLS IN CENTRAL CALIFORNIA DATABASE County

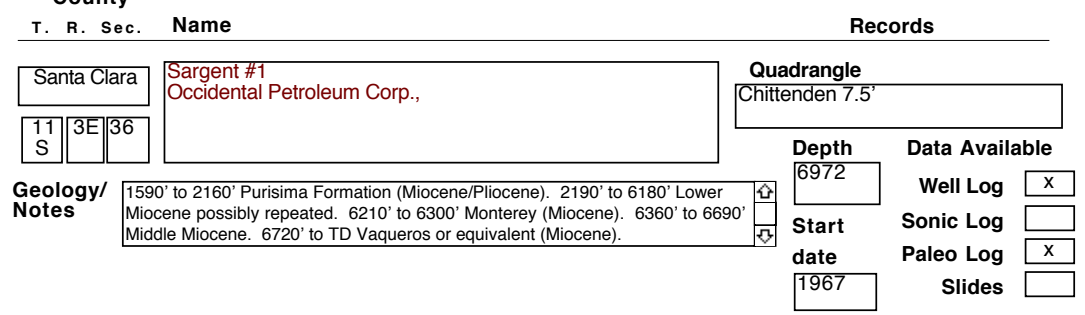

\begin{tabular}{|c|c|c|c|c|}
\hline Santa Clara & \multirow[t]{2}{*}{$\begin{array}{l}\text { Mayo-Hollister \#1 } \\
\text { Gene Reid Exploration Co. }\end{array}$} & \multicolumn{3}{|c|}{$\begin{array}{c}\text { Quadrangle } \\
\text { Chittenden } 7.5\end{array}$} \\
\hline \begin{tabular}{|c||c||c|}
12 & $4 \mathrm{E}$ & 16 \\
$\mathrm{~S}$ & & \\
\end{tabular} & & \multirow{2}{*}{\multicolumn{2}{|c|}{$\begin{array}{l}\text { Depth } \\
6079\end{array}$}} & Data Available \\
\hline \multirow{3}{*}{$\begin{array}{l}\text { Geology/ } \\
\text { Notes }\end{array}$} & \multirow{3}{*}{ o data. } & \multirow{3}{*}{ 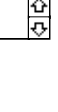 } & & Well Log \\
\hline & & & Start & Sonic Log \\
\hline & & & $\begin{array}{l}\text { date } \\
1948\end{array}$ & $\begin{array}{r}\text { Paleo Log } \\
\text { Slides }\end{array}$ \\
\hline
\end{tabular}

\begin{tabular}{|c|c|c|c|c|c|}
\hline \multirow{2}{*}{\begin{tabular}{|l} 
Santa Cruz \\
$\left.8 \mathrm{~S}\right|^{2 \mathrm{~W}} 27$
\end{tabular}} & \multirow[t]{2}{*}{$\begin{array}{l}\text { Janette \#1 } \\
\text { Grober }\end{array}$} & \multicolumn{4}{|c|}{$\begin{array}{c}\text { Quadrangle } \\
\text { Cupertino 7.5' }\end{array}$} \\
\hline & & \multirow{2}{*}{\multicolumn{2}{|c|}{$\begin{array}{l}\text { Depth } \\
3006\end{array}$}} & \multicolumn{2}{|c|}{ Data Available } \\
\hline \multirow{3}{*}{$\begin{array}{l}\text { Geology/ } \\
\text { Notes }\end{array}$} & ' to $1555^{\prime}$ Vaqueros/San Lorenzo (Miocene). to 1760 ' San Lorenzo & & & Well Log & $x$ \\
\hline & & & Start & Sonic Log & \\
\hline & & & date & Paleo Log & \\
\hline
\end{tabular}

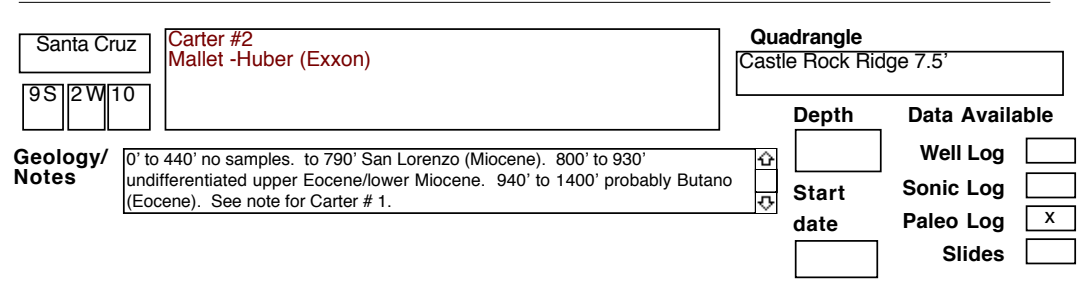

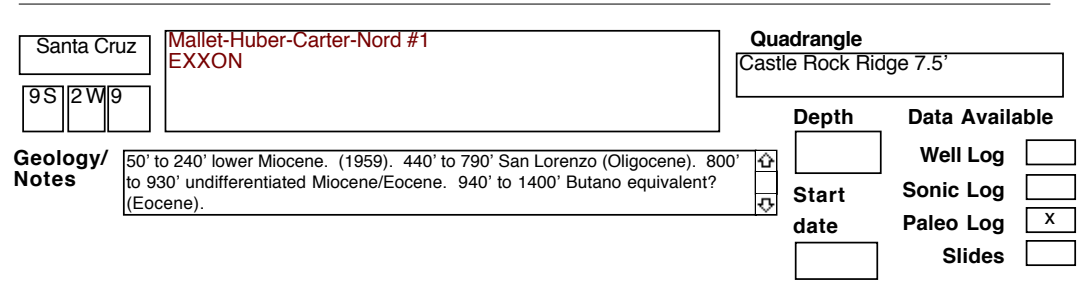


SELECTED OIL TEST WELLS IN CENTRAL CALIFORNIA DATABASE County

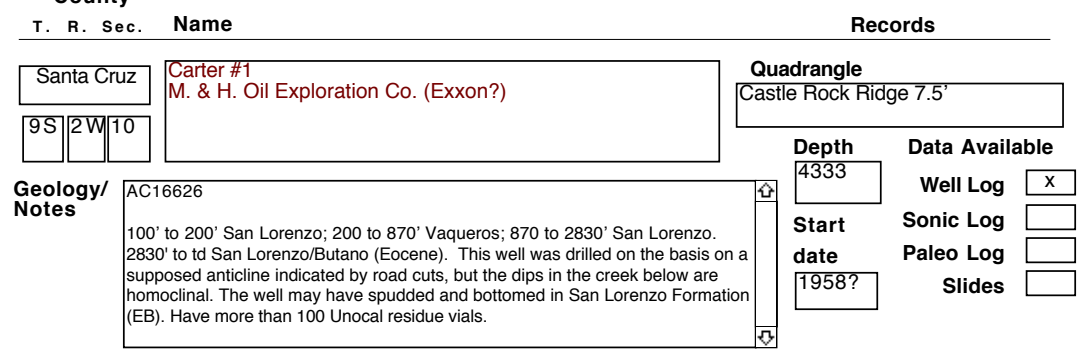

\begin{tabular}{|c|c|c|c|c|c|}
\hline Santa Cruz & \multirow[t]{2}{*}{$\begin{array}{l}\text { Hoffman \#1 } \\
\text { Royal Petroleum Co., }\end{array}$} & \multicolumn{4}{|c|}{ 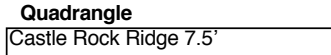 } \\
\hline $9 \mathrm{~S} 2 \mathrm{~V} 10$ & & \multirow{2}{*}{\multicolumn{2}{|c|}{$\begin{array}{l}\text { Depth } \\
4309\end{array}$}} & \multicolumn{2}{|c|}{ Data Available } \\
\hline \multirow{3}{*}{$\begin{array}{l}\text { Geology/ } \\
\text { Notes }\end{array}$} & ' to 1710' San Lorenzo (Miocene). to 2350' San Lorenzo (Oligocene). to & & & Well Log & $\mathrm{x}$ \\
\hline & 30' San Lorenzo/Butano undifferentiated (Eocene). & & Start & Sonic Log & \\
\hline & & & $\begin{array}{l}\text { date } \\
1960 ?\end{array}$ & $\begin{array}{r}\text { Paleo Log } \\
\text { Slides }\end{array}$ & \\
\hline
\end{tabular}

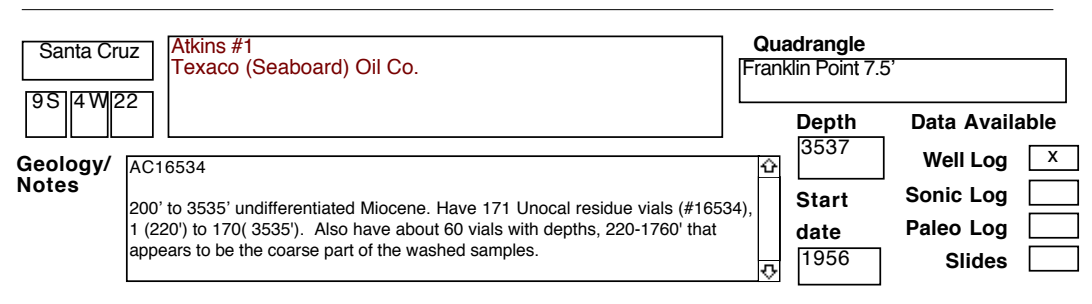

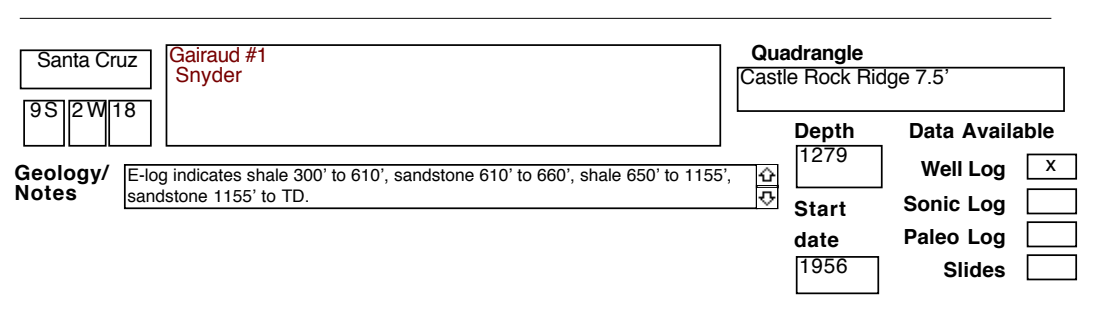

\begin{tabular}{l|l|l|l|l|l|}
\hline Santa Cruz & $\begin{array}{l}\text { Clement \#1 } \\
\text { Chevron (Gulf) Oil Co. }\end{array}$ \\
\hline
\end{tabular}


SELECTED OIL TEST WELLS IN CENTRAL CALIFORNIA DATABASE County

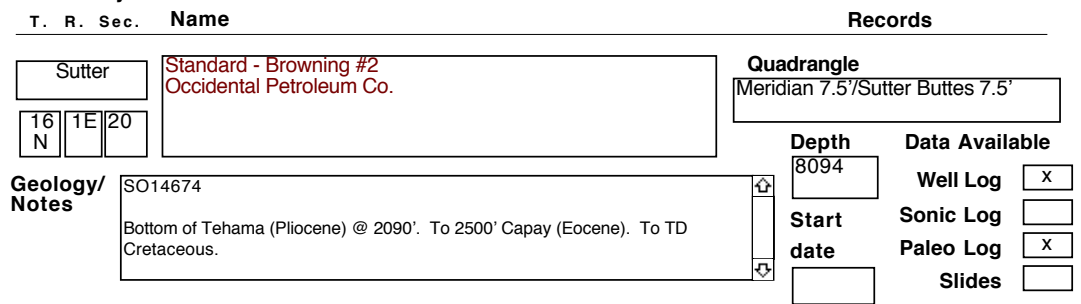

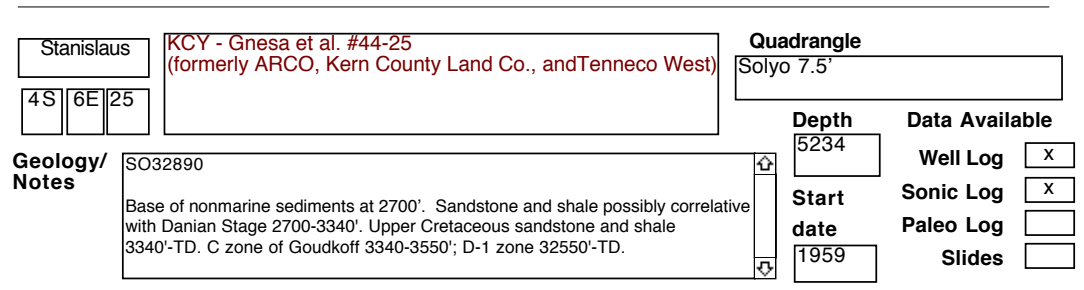

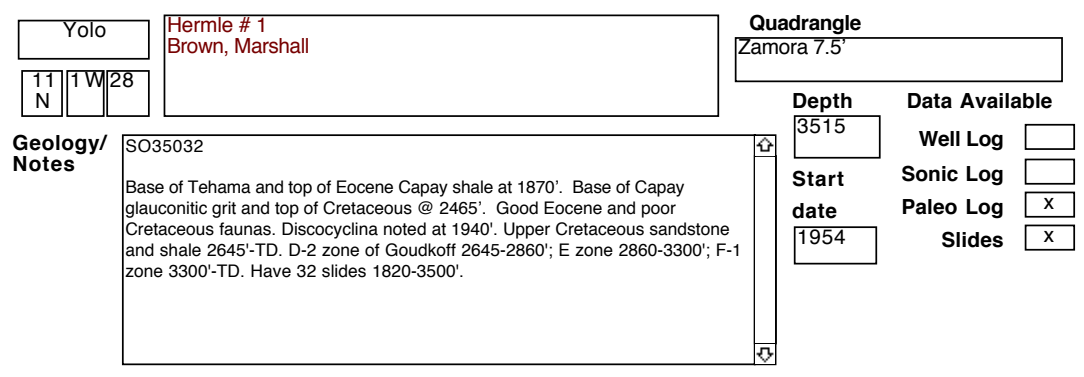

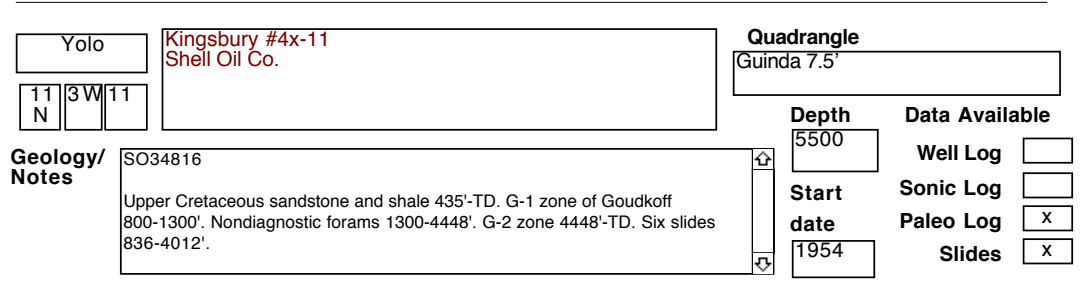

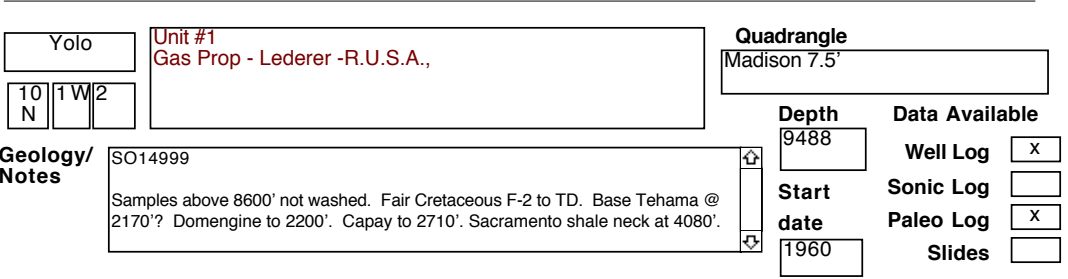


SELECTED OIL TEST WELLS IN CENTRAL CALIFORNIA DATABASE County

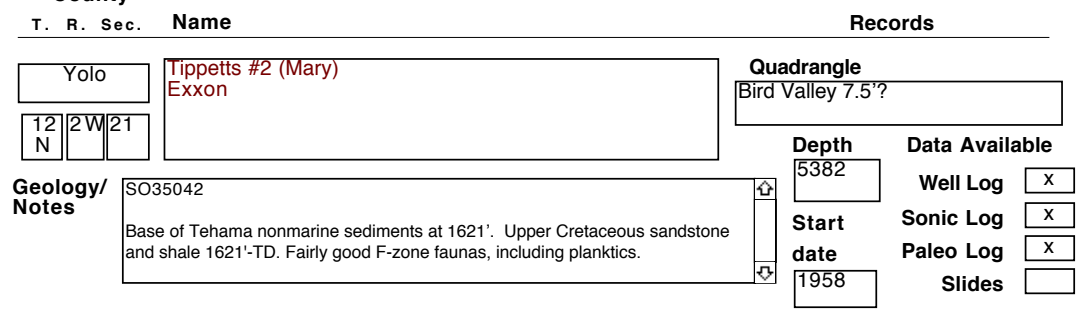

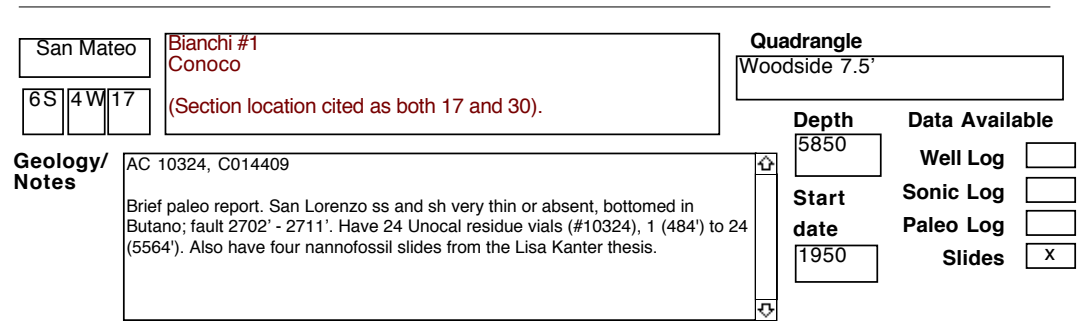

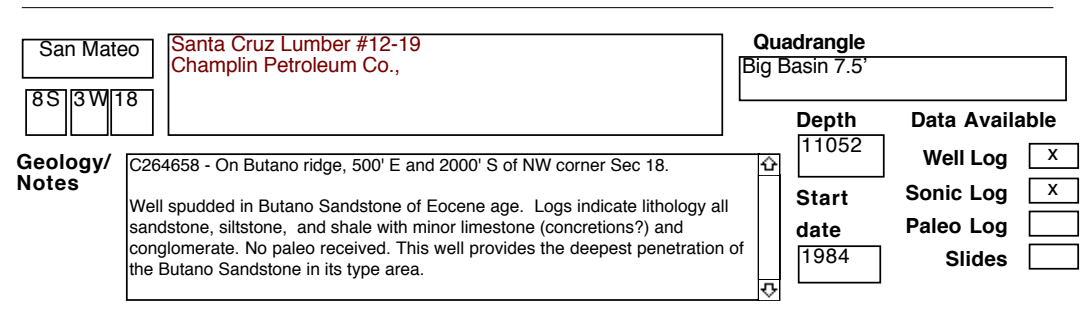

\begin{tabular}{|c|c|c|c|c|c|}
\hline Napa & \multirow[t]{2}{*}{$\begin{array}{l}\text { Tubbs-Elk \#1 } \\
\text { Elk Exploration Co. }\end{array}$} & \multicolumn{4}{|c|}{$\begin{array}{l}\text { Quadrangle } \\
\text { Sears Point 7.5' }\end{array}$} \\
\hline \begin{tabular}{|l|l|l|l|}
$4 \mathrm{~N}$ & 33 \\
\end{tabular} & & & Depth & \multicolumn{2}{|c|}{ Data Available } \\
\hline \multirow{3}{*}{$\begin{array}{l}\text { Geology/ } \\
\text { Notes }\end{array}$} & & \multirow{3}{*}{$\frac{6}{0}$} & & Well Log & \\
\hline & $\mathrm{d}$ additional information & & Start & Sonic Log & $x$ \\
\hline & & & $\begin{array}{l}\text { date } \\
1963\end{array}$ & $\begin{array}{r}\text { Paleo Log } \\
\text { Slides }\end{array}$ & \\
\hline
\end{tabular}

\begin{tabular}{|c|c|c|c|c|c|}
\hline San Benito & Law 80XX-1, redrill & \multicolumn{4}{|c|}{ Quadrangle } \\
\hline$S^{14}\left\|^{0-}\right\|^{\prime}$ & & \multirow{2}{*}{\multicolumn{2}{|c|}{$\begin{array}{l}\text { Depth } \\
6066\end{array}$}} & \multicolumn{2}{|c|}{ Data Available } \\
\hline \multirow{3}{*}{$\begin{array}{l}\text { Geology/ } \\
\text { Notes }\end{array}$} & \multirow{3}{*}{ Need additional information } & & & Well Log & \\
\hline & & \multirow[t]{2}{*}{ a } & Start & Sonic Log & $\mathrm{x}$ \\
\hline & & & $\begin{array}{l}\text { date } \\
1980\end{array}$ & $\begin{array}{r}\text { Paleo Log } \\
\text { Slides }\end{array}$ & \\
\hline
\end{tabular}


SELECTED OIL TEST WELLS IN CENTRAL CALIFORNIA DATABASE County

\begin{tabular}{|c|c|c|c|c|c|}
\hline T. R. Sec. & Name & \multicolumn{4}{|c|}{ Records } \\
\hline San Benito & \multirow[t]{2}{*}{$\begin{array}{l}\text { O'Connell \#1 } \\
\text { Getty (Tide Water) }\end{array}$} & \multicolumn{4}{|c|}{$\begin{array}{l}\text { Quadrangle } \\
\text { Chittenden }\end{array}$} \\
\hline \begin{tabular}{|l|l||l|}
12 & $4 \mathrm{E}$ & 4 \\
$\mathrm{~S}$ & & \\
\end{tabular} & & \multirow{2}{*}{\multicolumn{2}{|c|}{$\begin{aligned} & \text { Depth } \\
& 7234 \\
&\end{aligned}$}} & \multicolumn{2}{|c|}{ Data Available } \\
\hline \multirow{3}{*}{$\begin{array}{l}\text { Geology/ } \\
\text { Notes }\end{array}$} & isima 0-7148; Franciscan 7148-TD. Has dipmeter and several core & & & Well Log & $\mathrm{x}$ \\
\hline & criptions. & \multirow[t]{2}{*}{0} & Start & Sonic Log & \\
\hline & & & $\begin{array}{l}\text { date } \\
1952\end{array}$ & $\begin{array}{r}\text { Paleo Log } \\
\text { Slides }\end{array}$ & $\mathrm{x}$ \\
\hline
\end{tabular}

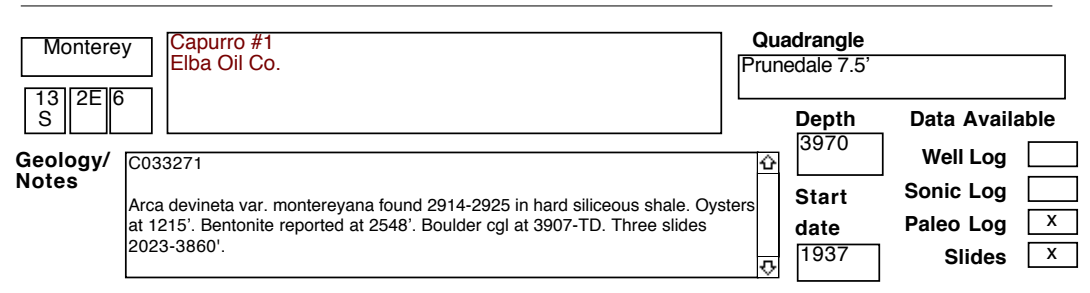

\begin{tabular}{|c|c|c|c|c|c|}
\hline Monterey & \multirow[t]{2}{*}{\begin{tabular}{|l|} 
Chesholm \#1 \\
Texaco (AGIP)
\end{tabular}} & \multicolumn{4}{|c|}{$\begin{array}{l}\text { Quadrangle } \\
\text { Gonzales 7.5' }\end{array}$} \\
\hline \begin{tabular}{|c|c|c|c|}
16 & $5 \mathrm{E}$ & 18 \\
$\mathrm{~S}$ & & & \\
\end{tabular} & & \multirow{2}{*}{\multicolumn{2}{|c|}{$\begin{array}{l}\text { Depth } \\
2306\end{array}$}} & \multicolumn{2}{|c|}{ Data Available } \\
\hline \multirow{4}{*}{$\begin{array}{l}\text { Geology/ } \\
\text { Notes }\end{array}$} & \multirow{4}{*}{ Need additional information } & & & Well Log & \\
\hline & & \multirow{3}{*}{$\theta$} & Start & Sonic Log & $x$ \\
\hline & & & date & Paleo Log & \\
\hline & & & 1000 & Slides & \\
\hline
\end{tabular}

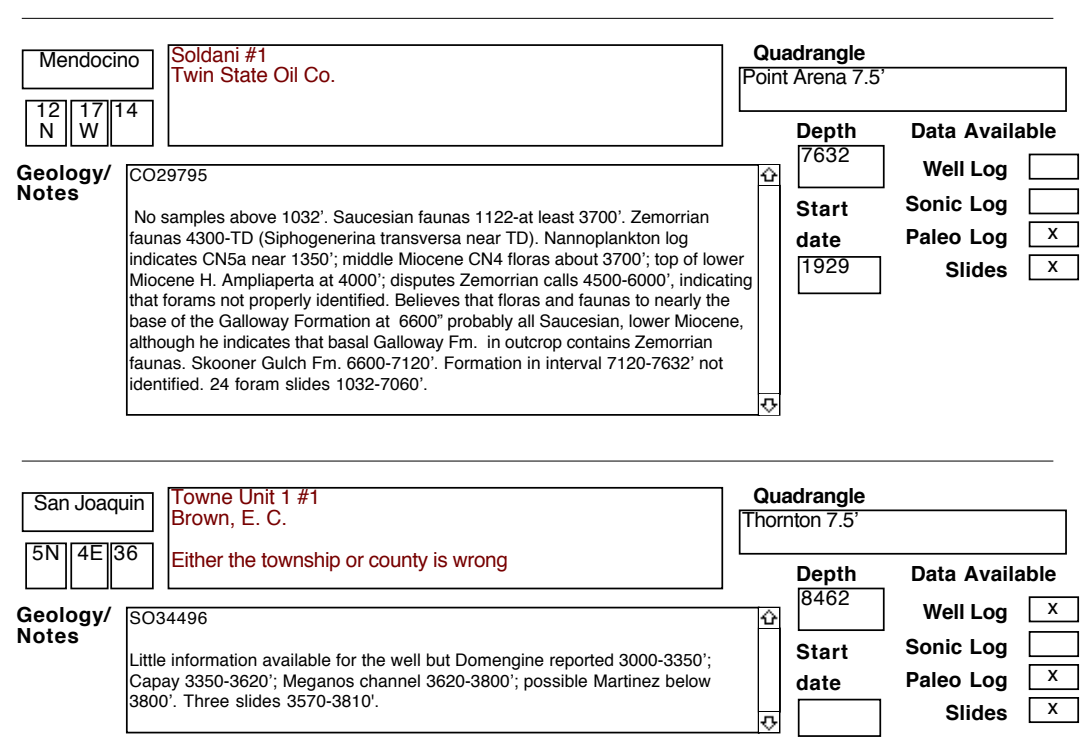


SELECTED OIL TEST WELLS IN CENTRAL CALIFORNIA DATABASE County

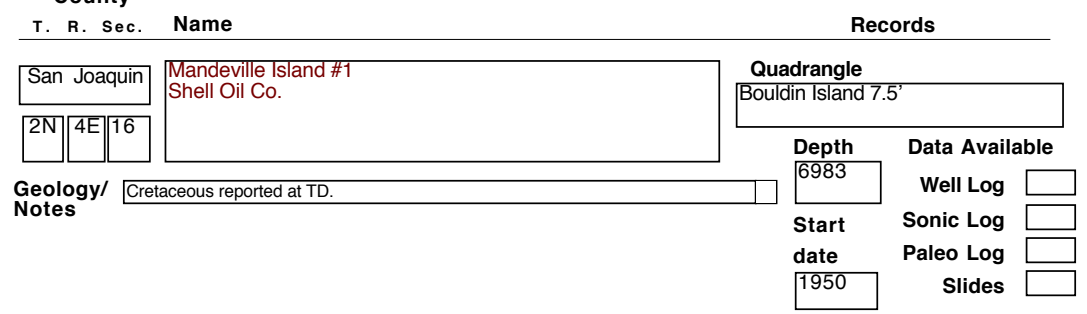

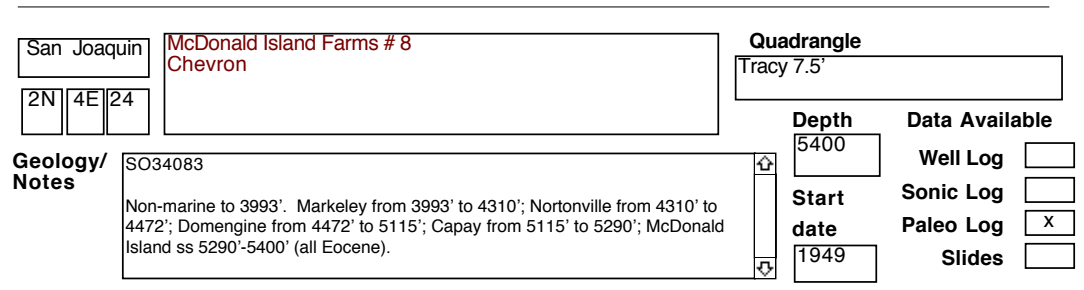

\begin{tabular}{l|l|l|l|l|l|}
\hline San Joaquin & $\begin{array}{l}\text { Holt Community \#1 } \\
\text { Chevron }\end{array}$ & \multicolumn{2}{l|}{ Quadrangle } \\
\hline
\end{tabular}

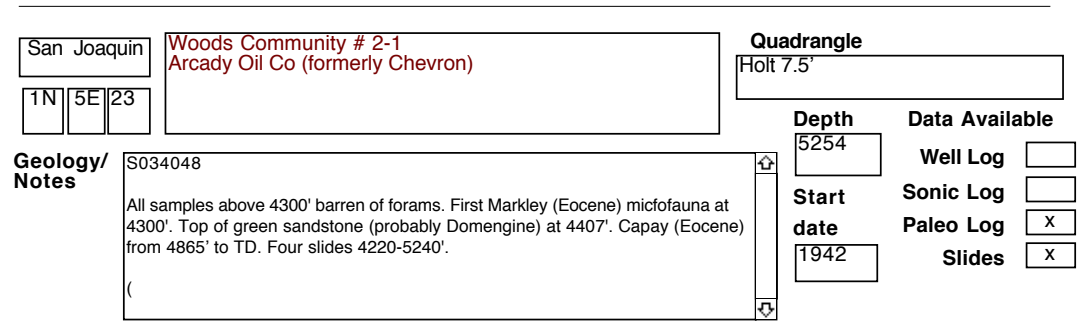

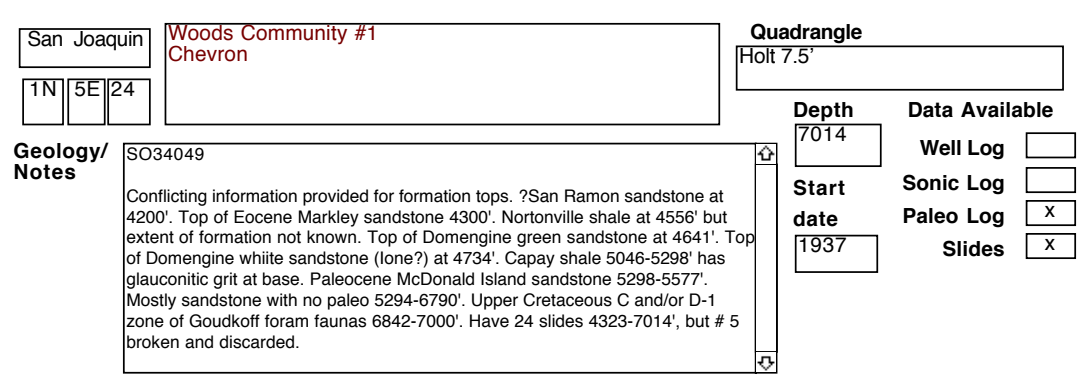




\section{SELECTED OIL TEST WELLS IN CENTRAL CALIFORNIA DATABASE}

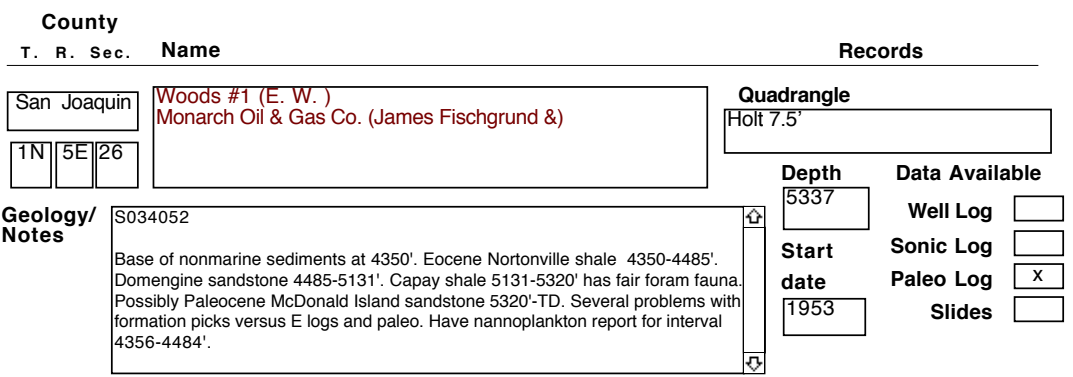

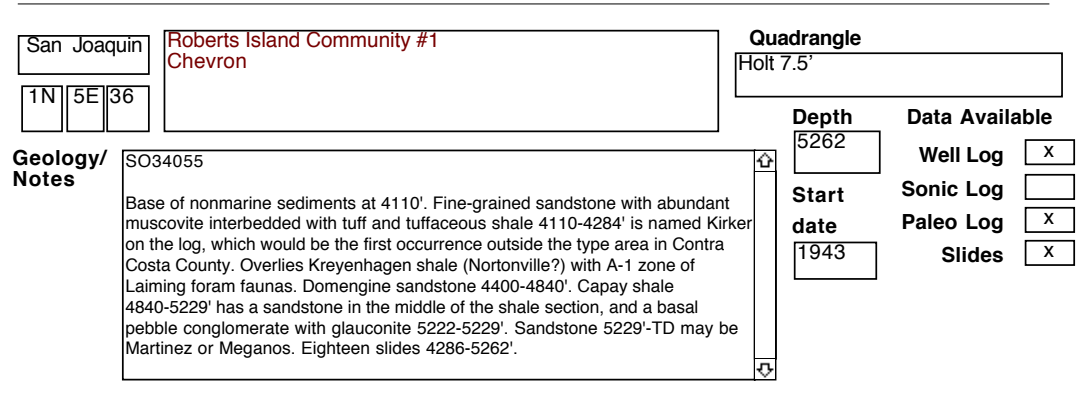

\begin{tabular}{l|l|l|l|l|}
\hline San Joaquin & $\begin{array}{l}\text { Wright \#1 } \\
\text { Shell Oil Co. }\end{array}$ & \multicolumn{2}{l|}{$\begin{array}{l}\text { Quadrangle } \\
\text { Vernalis } 7.5^{\prime}\end{array}$} \\
& & Depth & Data Available \\
\hline
\end{tabular}

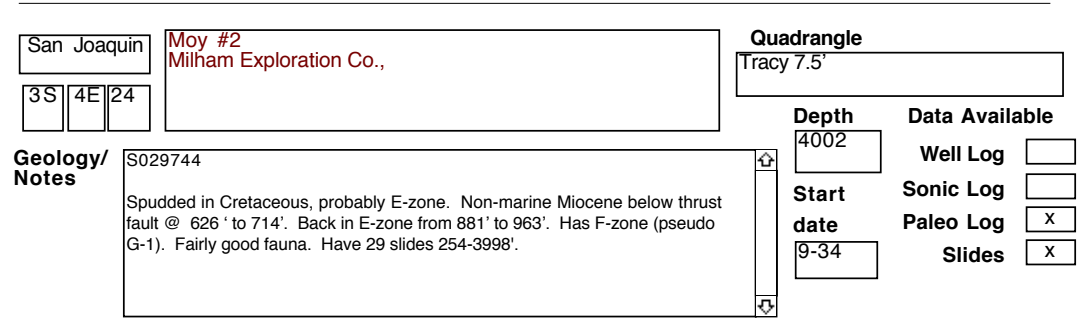

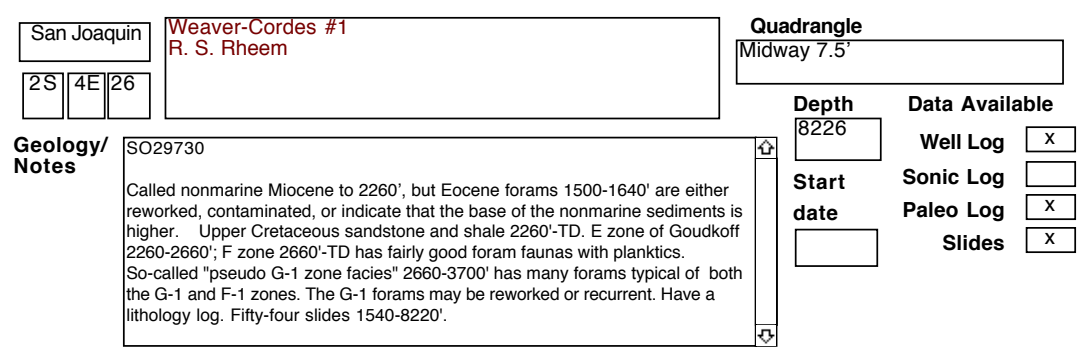


SELECTED OIL TEST WELLS IN CENTRAL CALIFORNIA DATABASE County

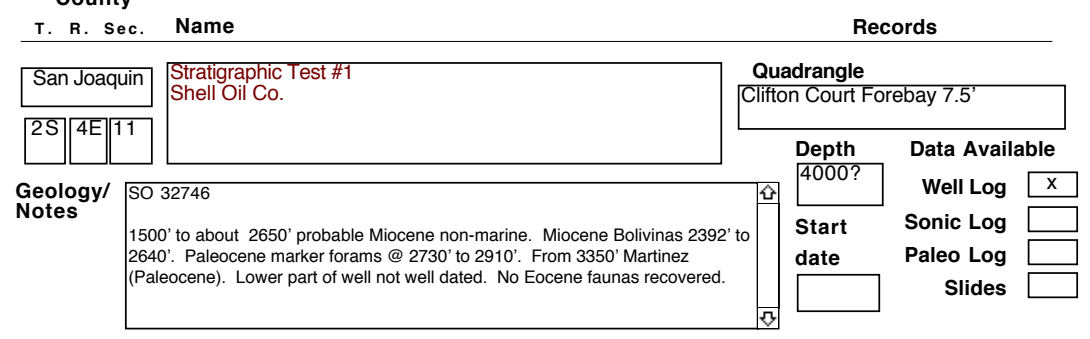

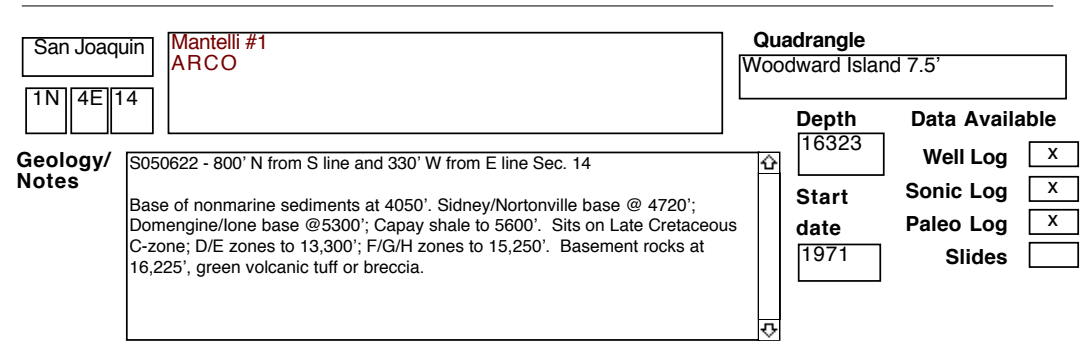

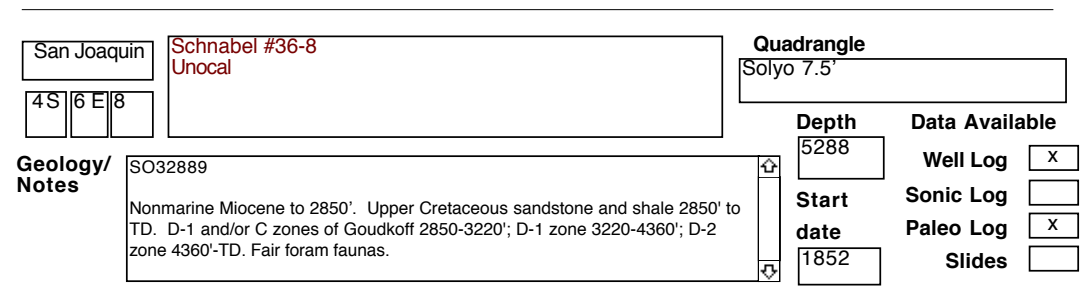

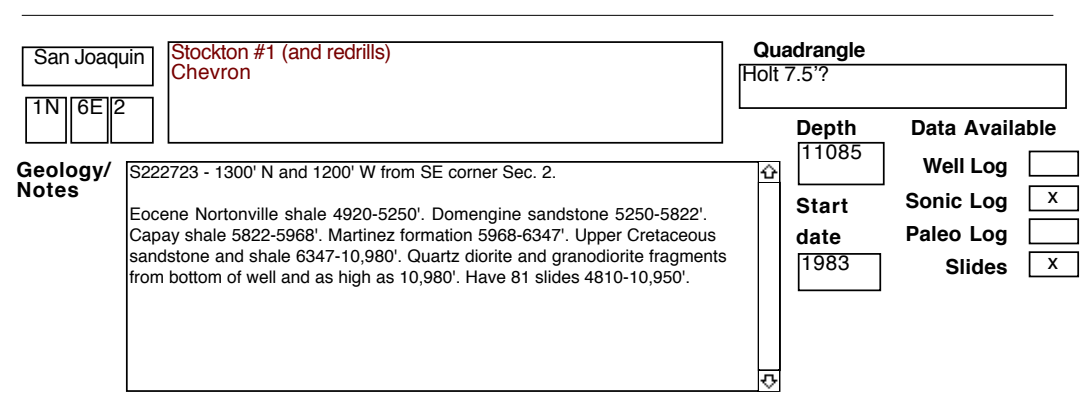

\begin{tabular}{l|l|l|l|l|l|l|}
\hline San Joaquin & $\begin{array}{l}\text { Blewett Community \#1 } \\
\text { Chevron }\end{array}$ & \multicolumn{2}{l|}{ Quadrangle } \\
\hline
\end{tabular}


SELECTED OIL TEST WELLS IN CENTRAL CALIFORNIA DATABASE County

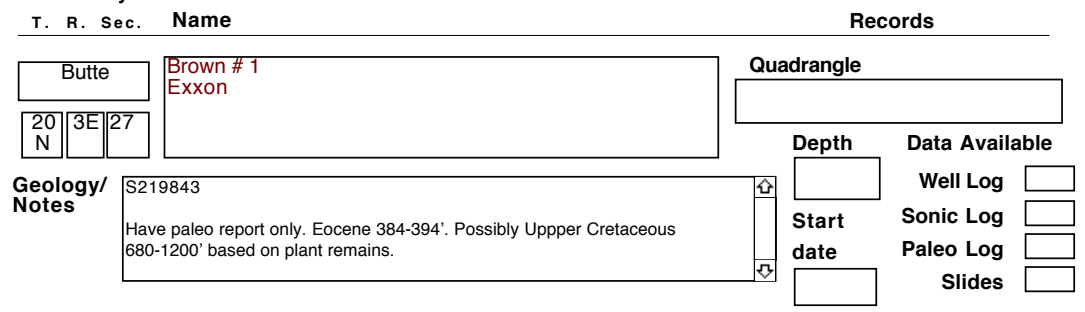

\begin{tabular}{l|l|l|l|l|l|}
\hline \multicolumn{1}{|c|}{$\begin{array}{l}\text { Butte } \\
\text { ARCO }\end{array}$} & \multicolumn{2}{l|}{ Quadrangle } \\
\hline
\end{tabular}

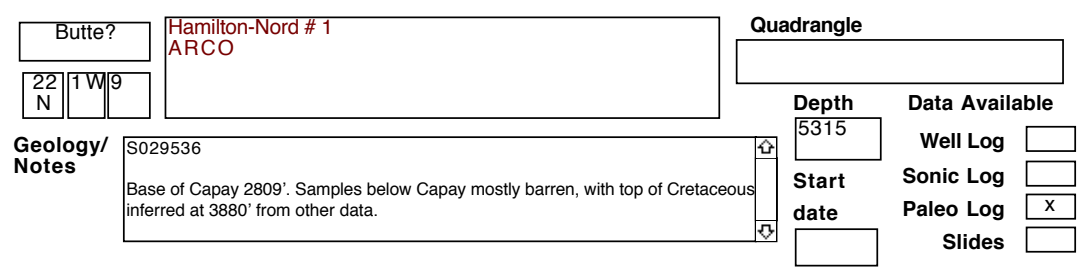

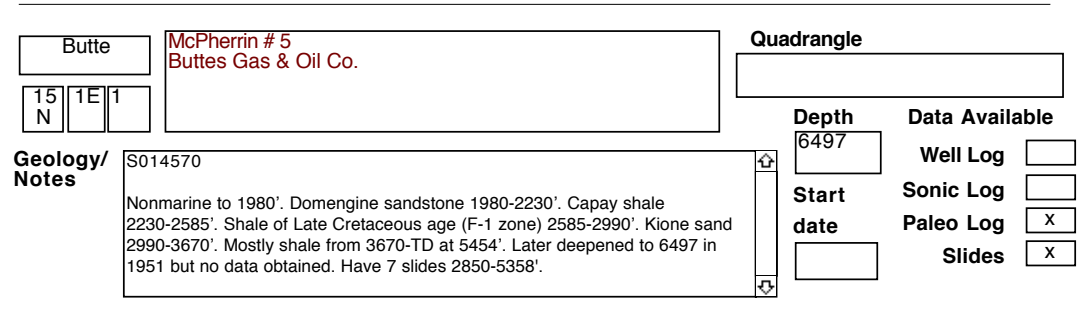

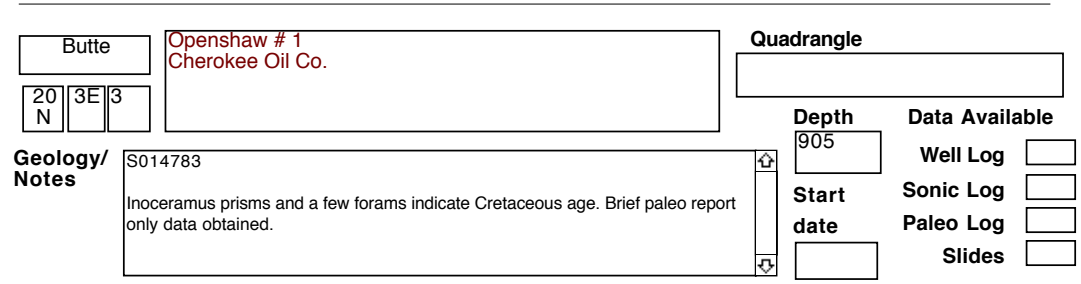




\section{SELECTED OIL TEST WELLS IN CENTRAL CALIFORNIA DATABASE}

County

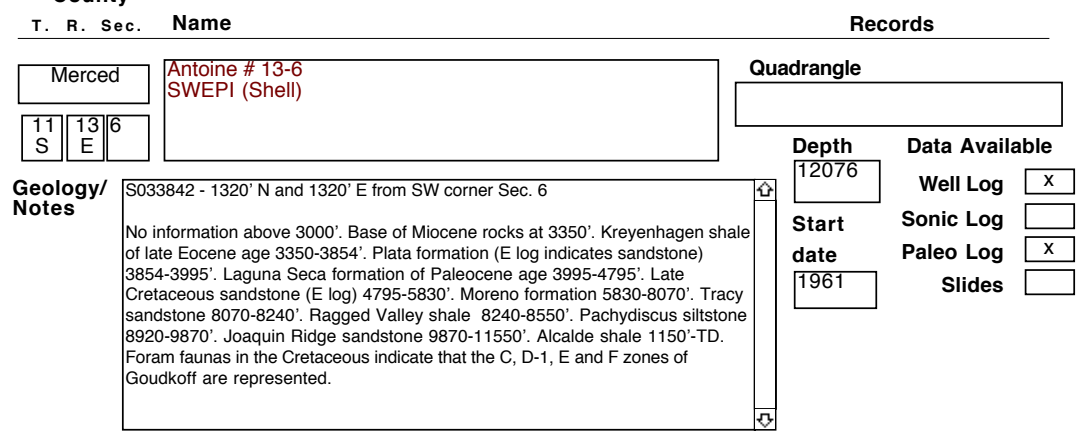

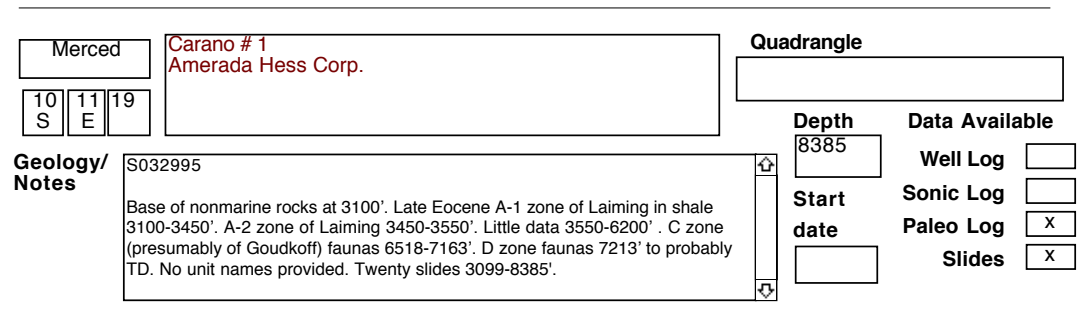

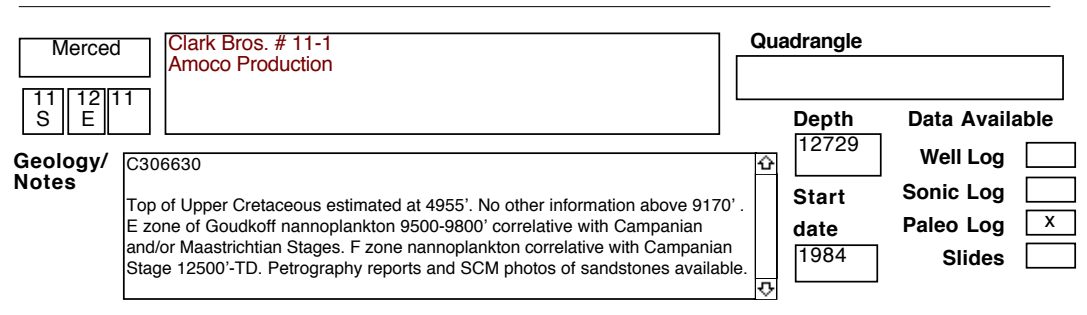

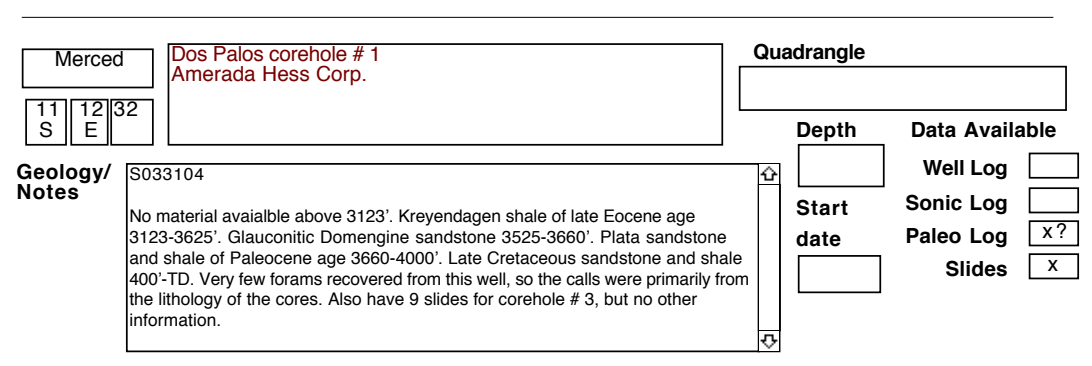

\begin{tabular}{|c|c|c|c|c|}
\hline Merced & Erreca \# 1 & Quadrangle & & \\
\hline \begin{tabular}{|l|l|l|l|}
$9 S$ & 11 & 1 \\
& & \\
& & \\
\end{tabular} & & \multirow{2}{*}{$\begin{array}{l}\text { Depth } \\
11448\end{array}$} & \multicolumn{2}{|c|}{ Data Available } \\
\hline \multirow{3}{*}{$\begin{array}{l}\text { Geology/ } \\
\text { Notes }\end{array}$} & S029742 & & Well Log & $x$ \\
\hline & Base of nonmarine sediments at $3775^{\prime}$. Kreyenhagen shale of late Eocene age & Start & Sonic Log & \\
\hline & $\begin{array}{l}\text { 3775-4250'. Rocks (E log suggests sandstone and shale) } 4250-7220^{\prime} \text { are } \\
\text { undated., but seismic logs suggest Moreno shale of Late Cretaceous age } \\
6370-7020^{\prime} \text {. Blewett sandstone with C and D-1 zone of Goodkoff foram faunas } \\
\text { 7020-8680'. E zone of Goudkoff } 9660-11040^{\prime} ; \text { F zone (Sacramento } \\
\text { shale)11040-11430'. Basement complex 11430-TD. }\end{array}$ & date & $\begin{array}{r}\text { Paleo Log } \\
\text { Slides }\end{array}$ & $x$ \\
\hline
\end{tabular}




\section{SELECTED OIL TEST WELLS IN CENTRAL CALIFORNIA DATABASE}

County

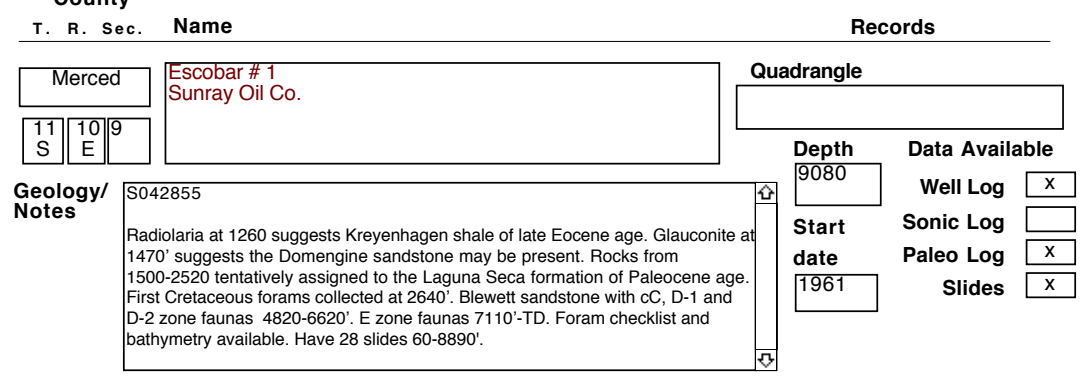

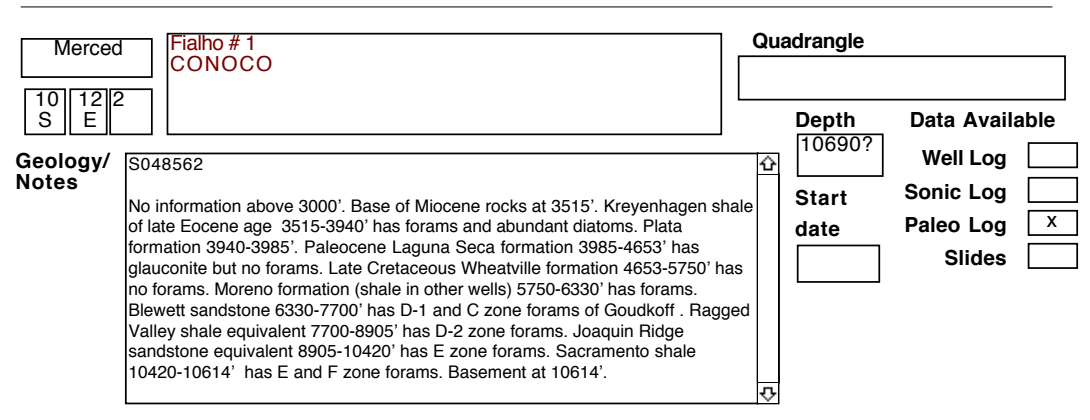

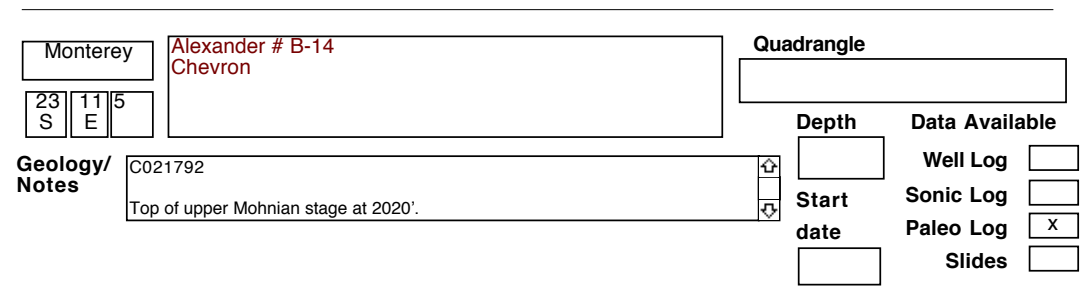

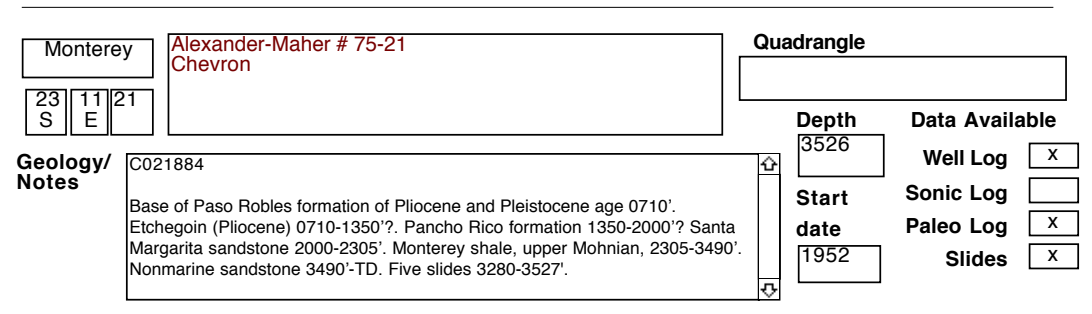

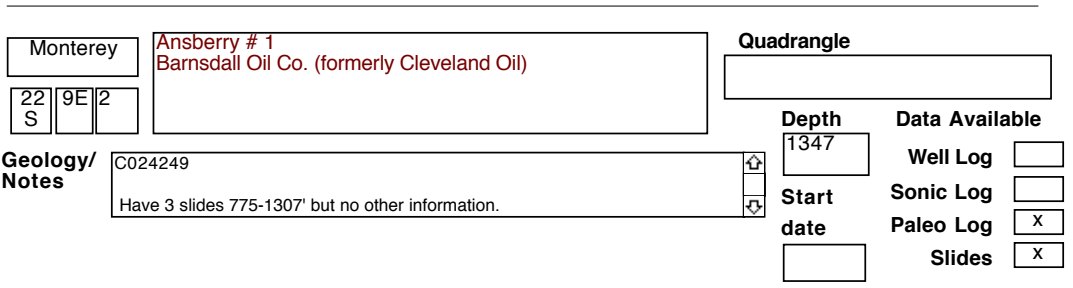


SELECTED OIL TEST WELLS IN CENTRAL CALIFORNIA DATABASE County

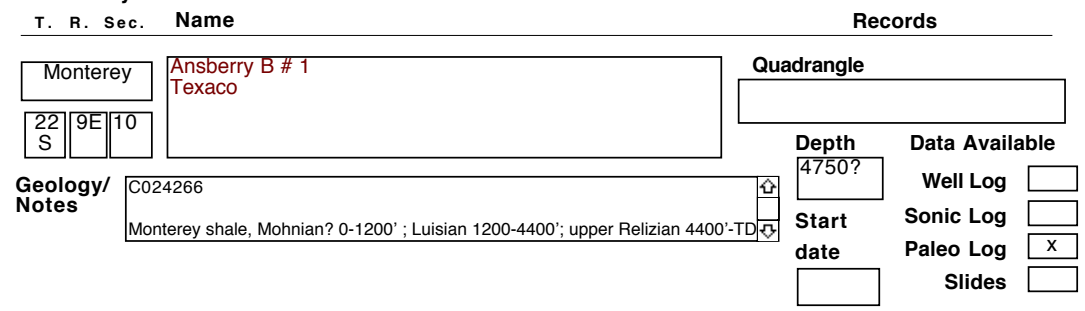

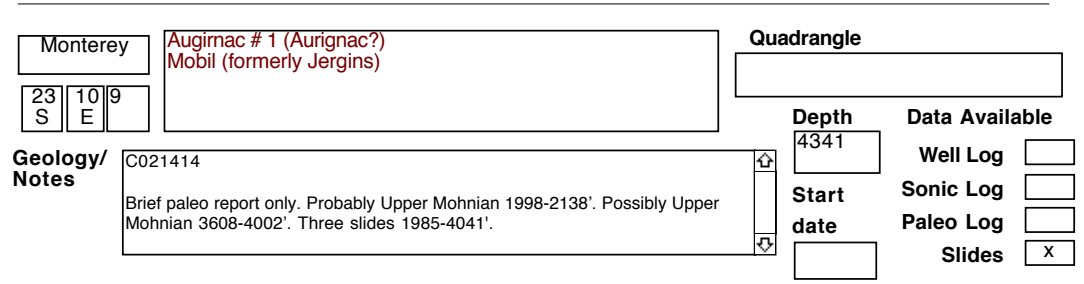

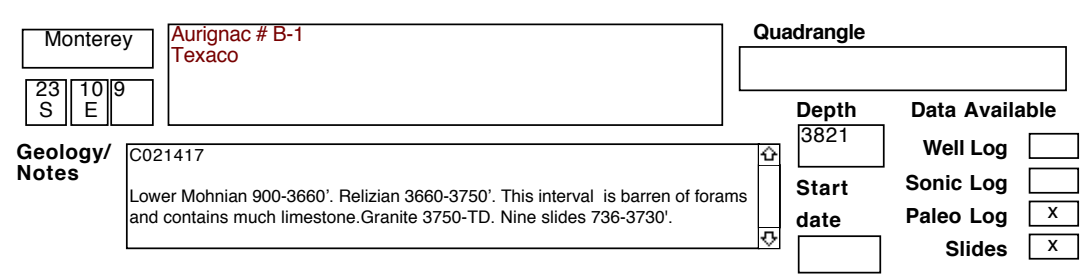

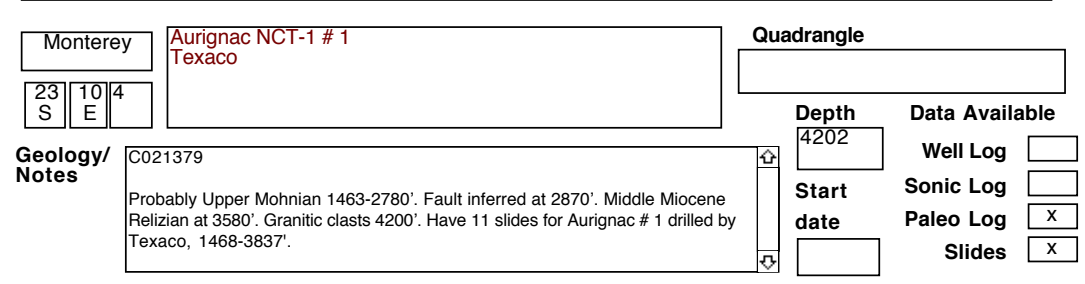

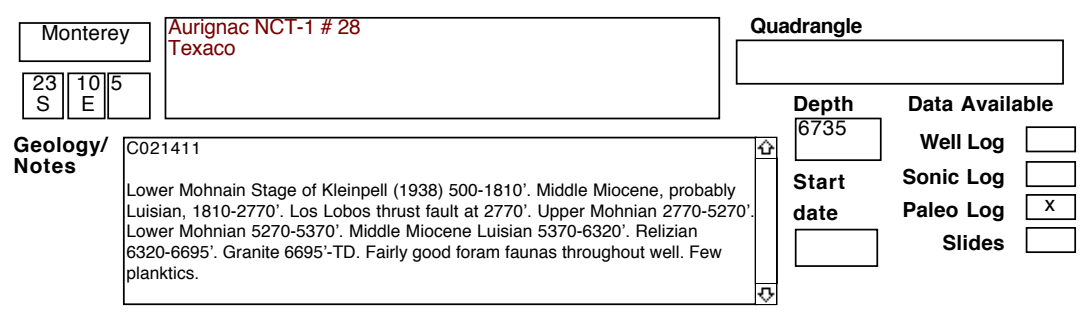


SELECTED OIL TEST WELLS IN CENTRAL CALIFORNIA DATABASE County

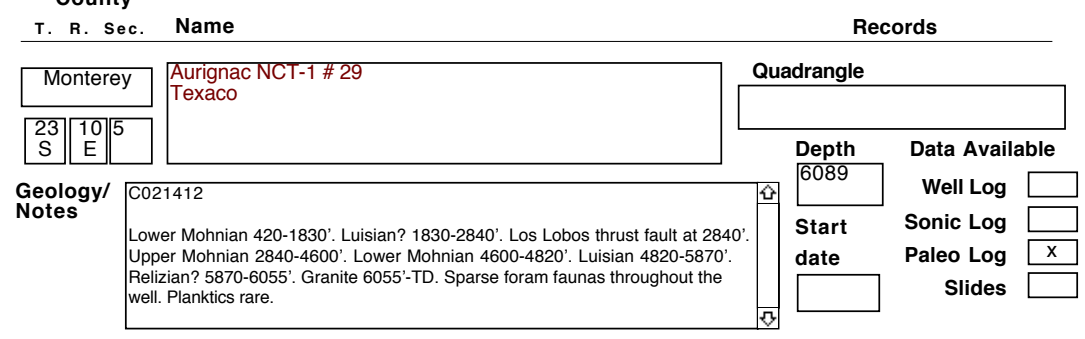

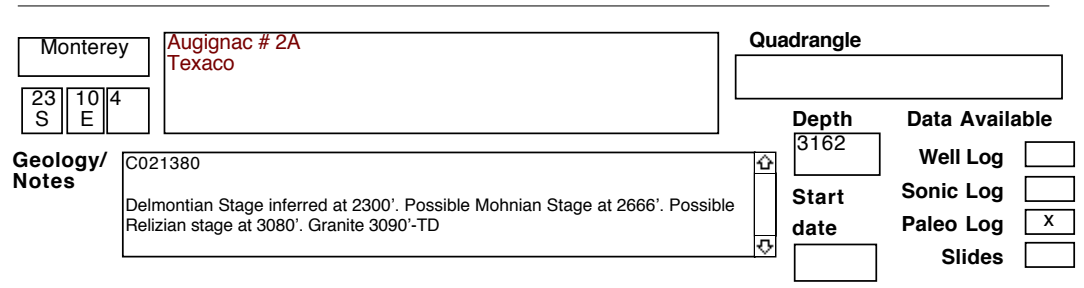

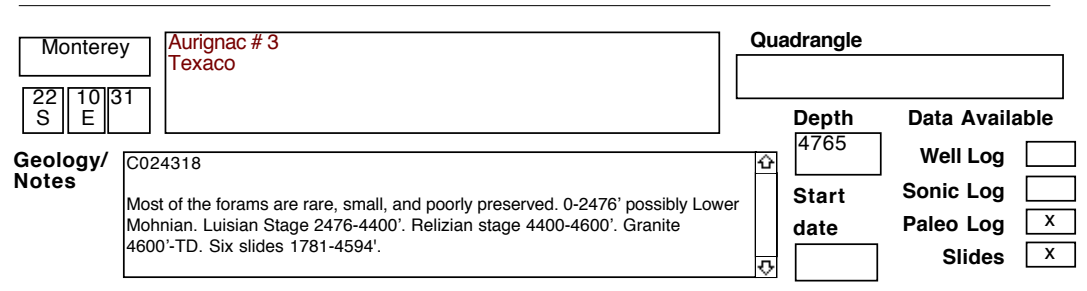

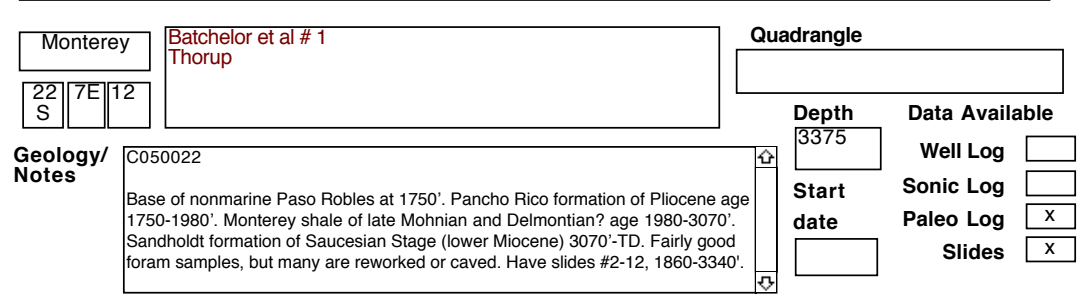

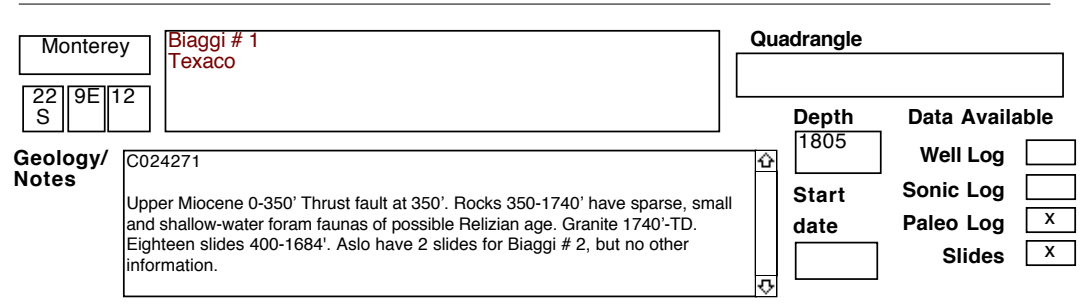


SELECTED OIL TEST WELLS IN CENTRAL CALIFORNIA DATABASE County

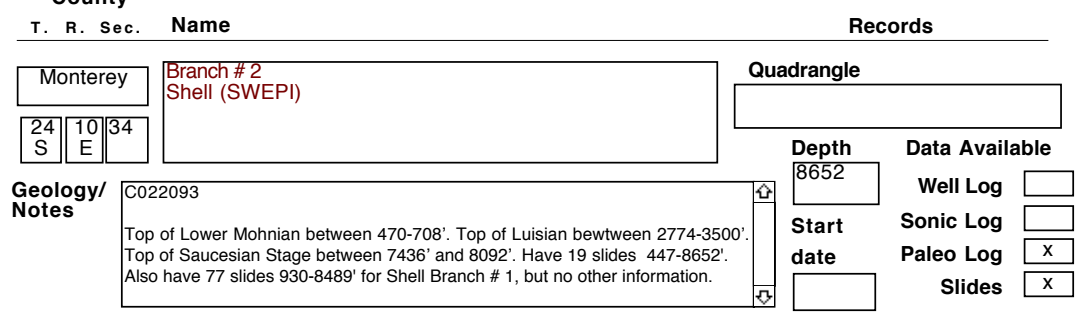

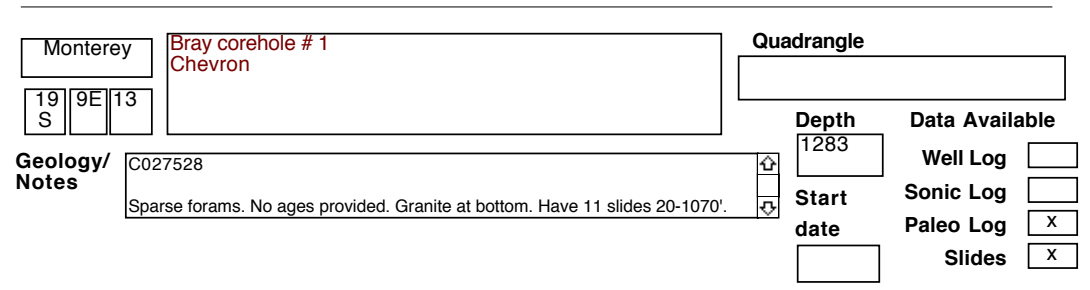

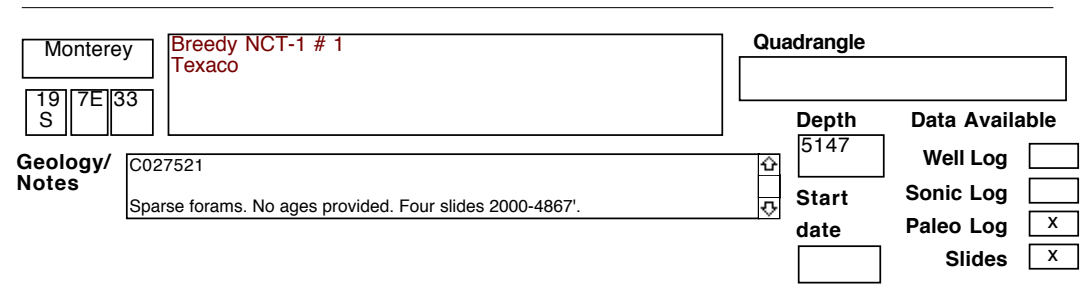

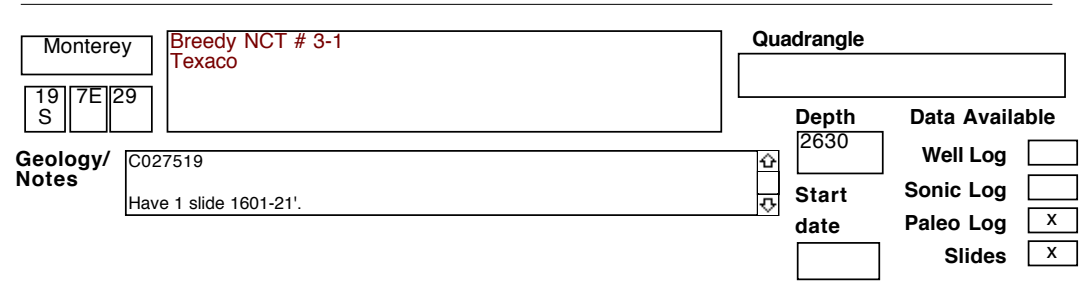

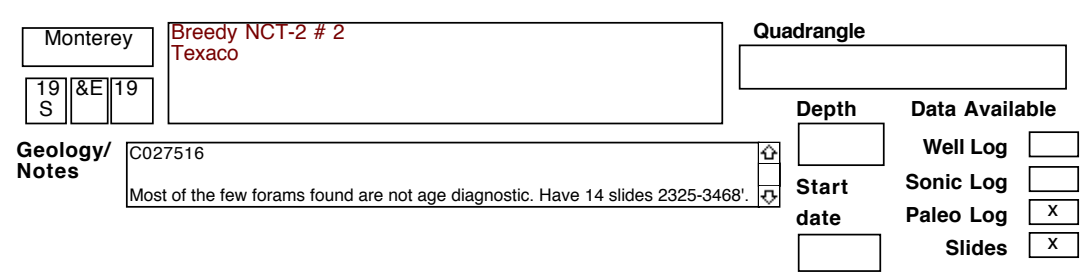


SELECTED OIL TEST WELLS IN CENTRAL CALIFORNIA DATABASE County

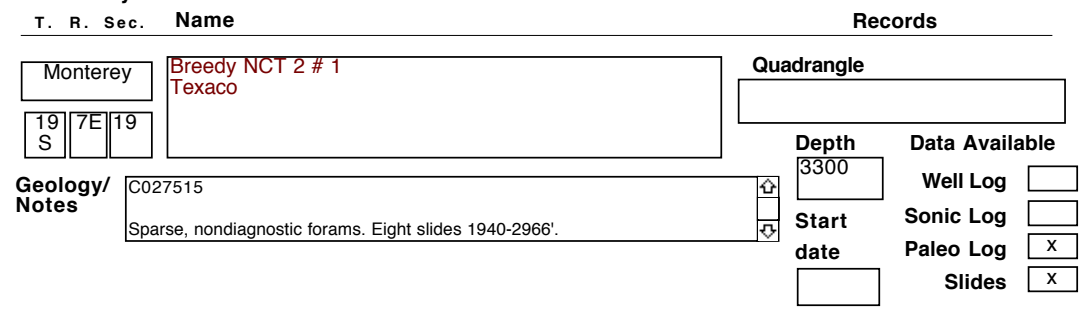

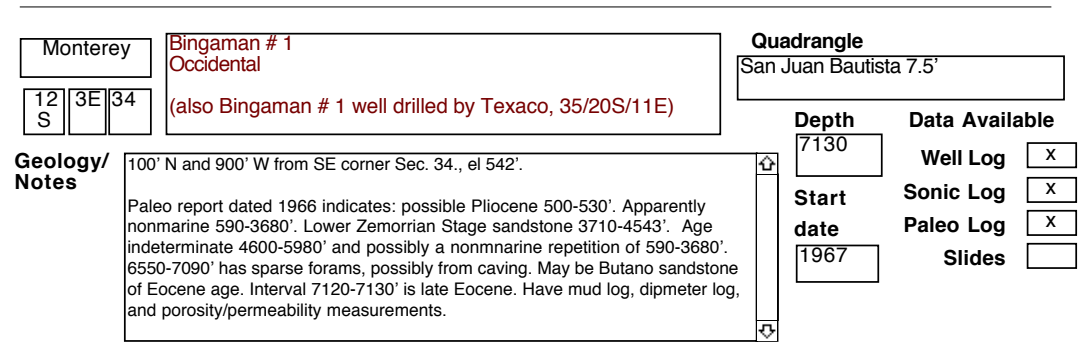

\begin{tabular}{|l||l|l|l|l|l|}
\hline Monterey & $\begin{array}{l}\text { Buttes-Work \# 1 } \\
\text { Butte Resources }\end{array}$ \\
\hline Geology/ \\
Notes
\end{tabular}

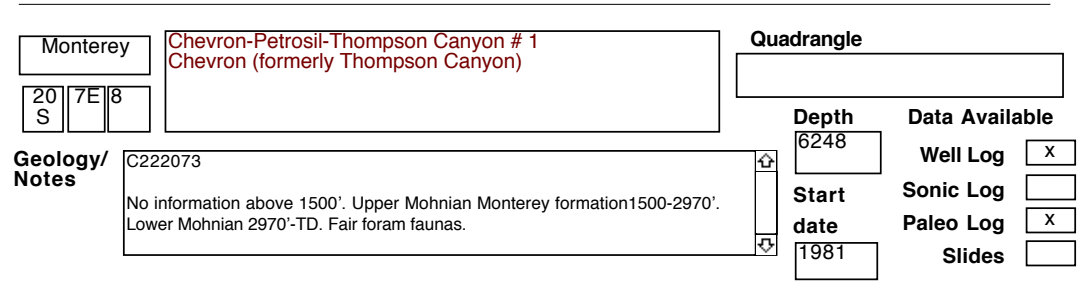

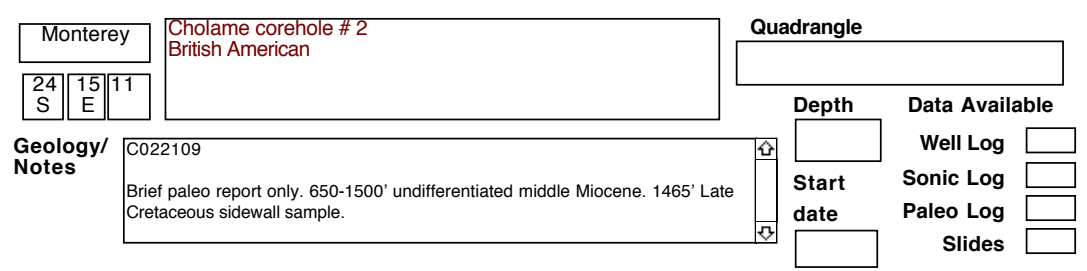


SELECTED OIL TEST WELLS IN CENTRAL CALIFORNIA DATABASE County

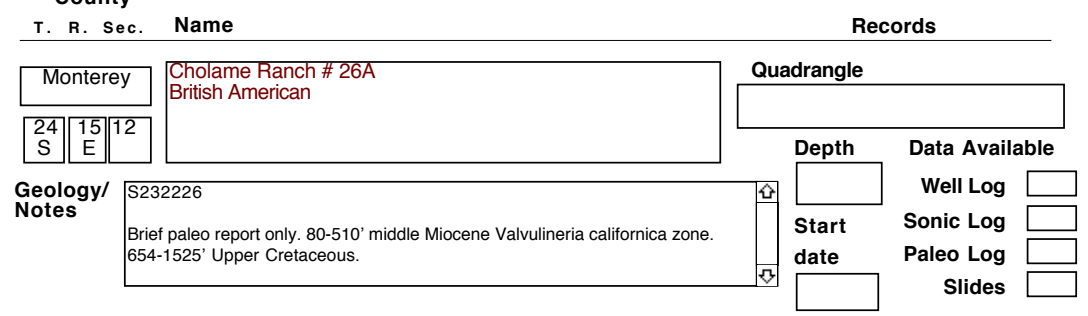

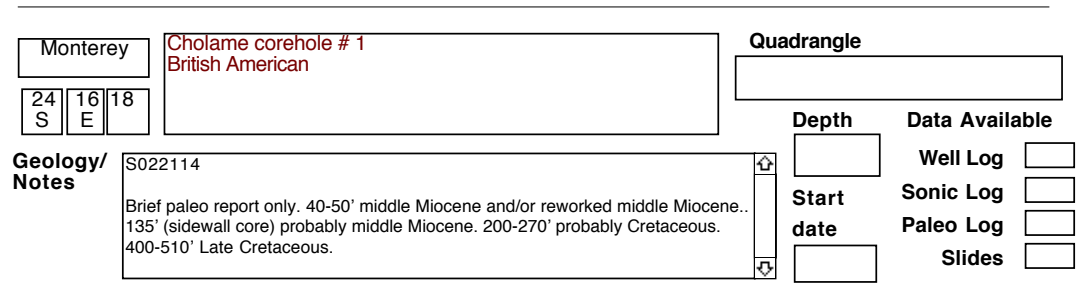

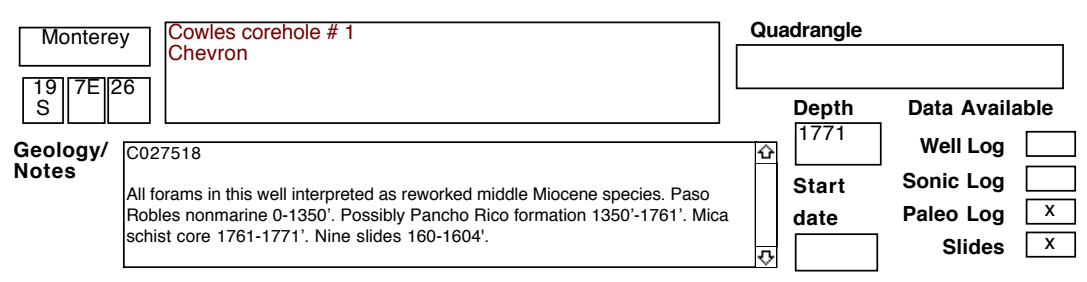

\begin{tabular}{l|l|l|l|l|l|l|}
\hline Monterey & $\begin{array}{l}\text { Davis \# 14 } \\
\text { Mobil (formerly Superior) }\end{array}$ & \multicolumn{2}{l|}{ Quadrangle } \\
\hline
\end{tabular}

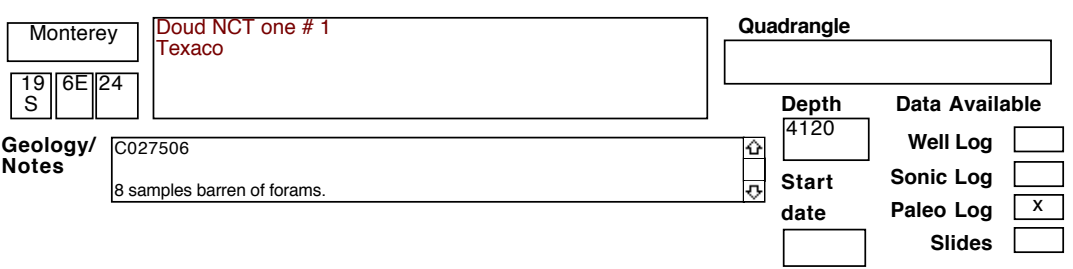


SELECTED OIL TEST WELLS IN CENTRAL CALIFORNIA DATABASE County

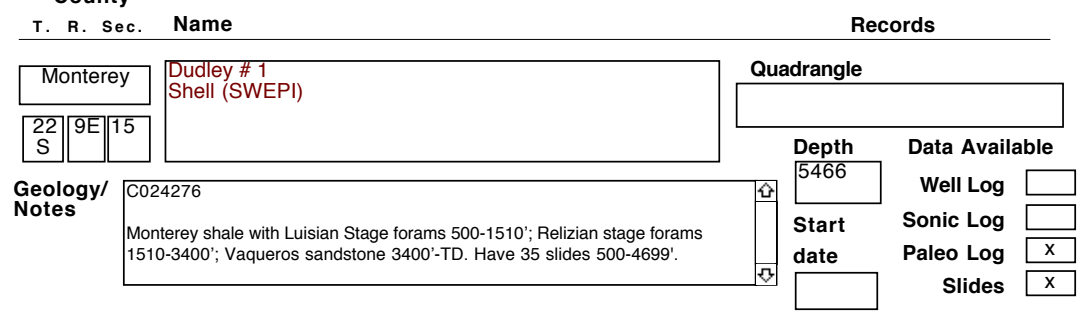

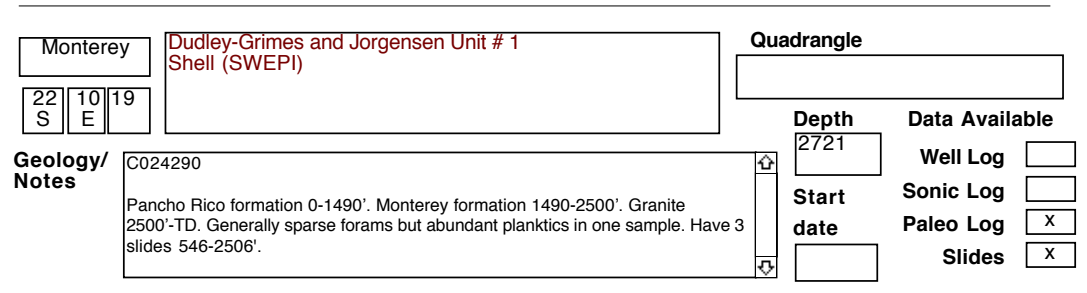

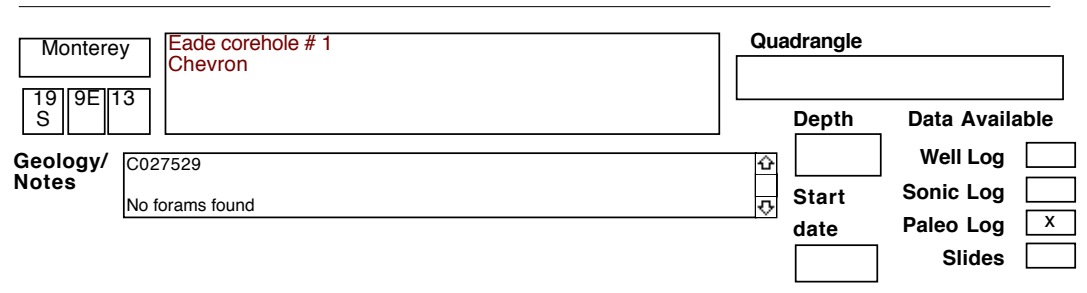

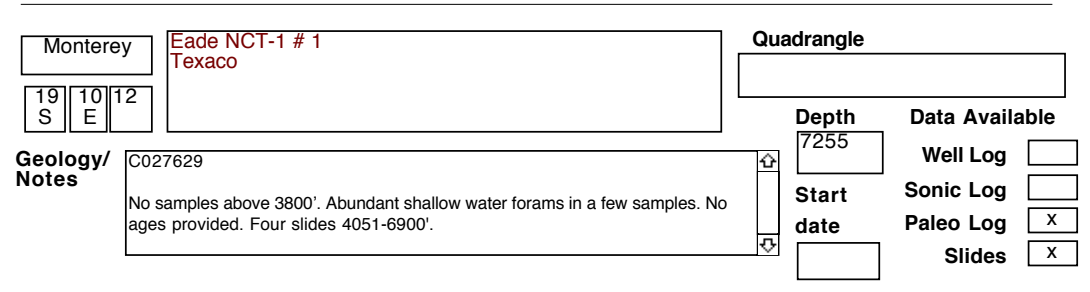

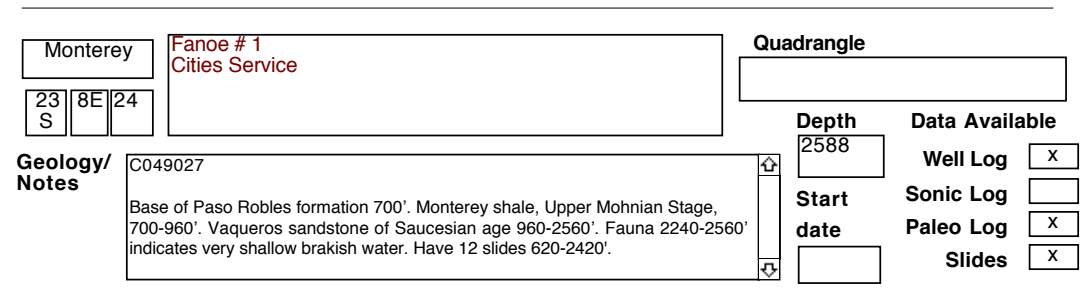


SELECTED OIL TEST WELLS IN CENTRAL CALIFORNIA DATABASE County

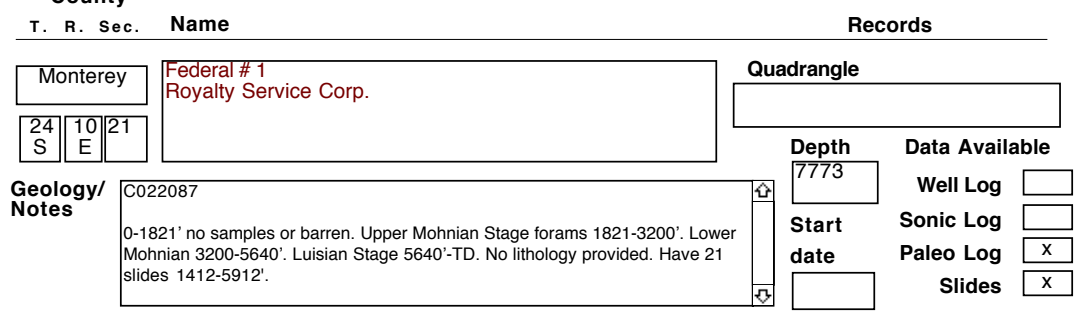

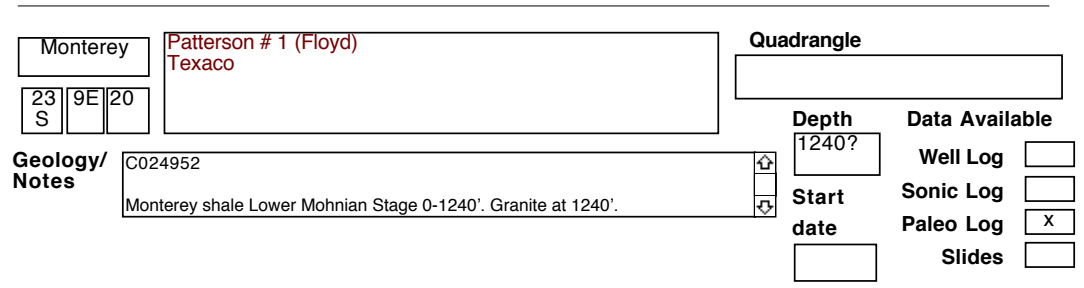

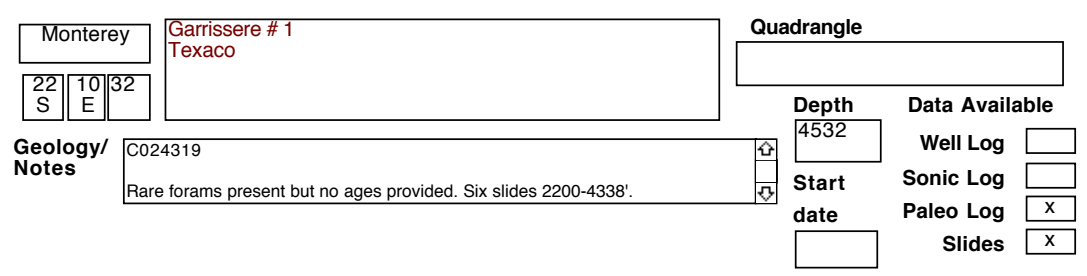

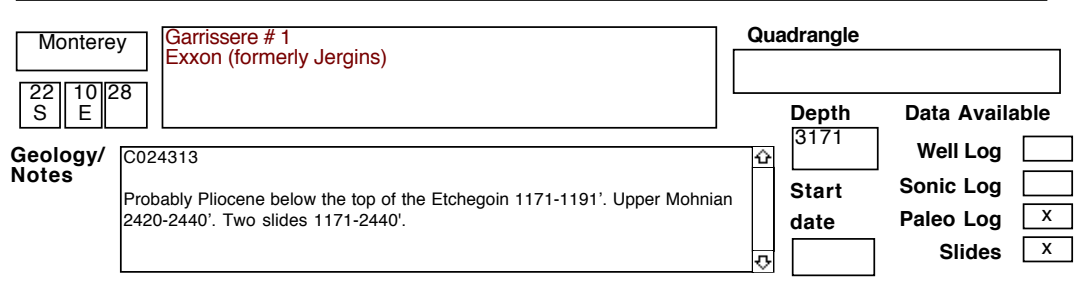

\begin{tabular}{|c|c|c|c|c|c|}
\hline Monterey & \multirow{2}{*}{$\begin{array}{l}\text { Garrissere \#1 (Alice) } \\
\text { Texaco }\end{array}$} & \multicolumn{4}{|c|}{ Quadrangle } \\
\hline \begin{tabular}{|l|l|l|}
22 & $9 \mathrm{E}$ & 24 \\
$\mathrm{~S}$ & & \\
\end{tabular} & & \multirow{2}{*}{\multicolumn{2}{|c|}{$\begin{array}{l}\text { Depth } \\
4262\end{array}$}} & \multicolumn{2}{|c|}{ Data Available } \\
\hline \multirow{3}{*}{$\begin{array}{l}\text { Geology/ } \\
\text { Notes }\end{array}$} & \multirow{3}{*}{$\begin{array}{l}\text { C024278 } \\
\text { Monterey formation Mohnian stage 0-1450'; Luisian 1450-4220'. Basement at } \\
\text { 4220'. Have } 23 \text { slides 1050-3900'. }\end{array}$} & & & Well Log & $\mathrm{x}$ \\
\hline & & & Start & Sonic Log & \\
\hline & & $\pi$ & 1952? & Slides & $\mathrm{X}$ \\
\hline
\end{tabular}


SELECTED OIL TEST WELLS IN CENTRAL CALIFORNIA DATABASE County

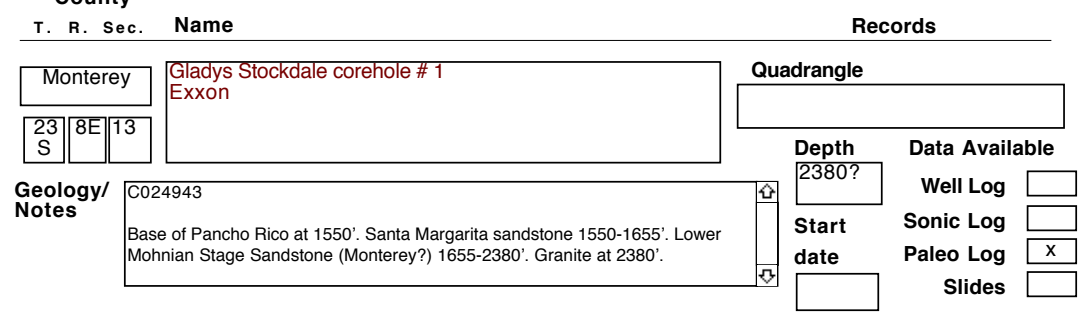

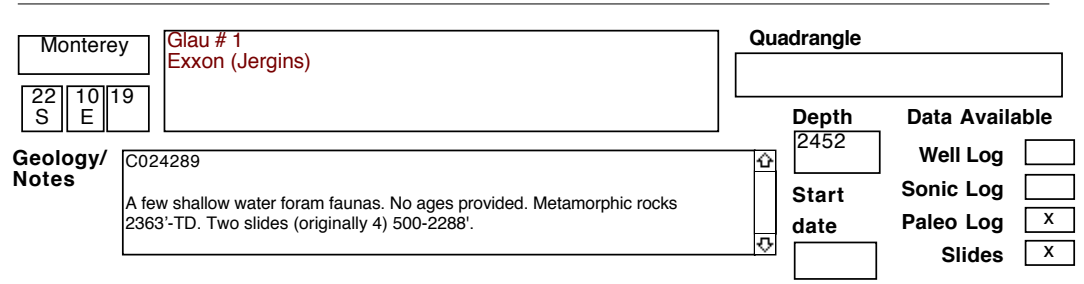

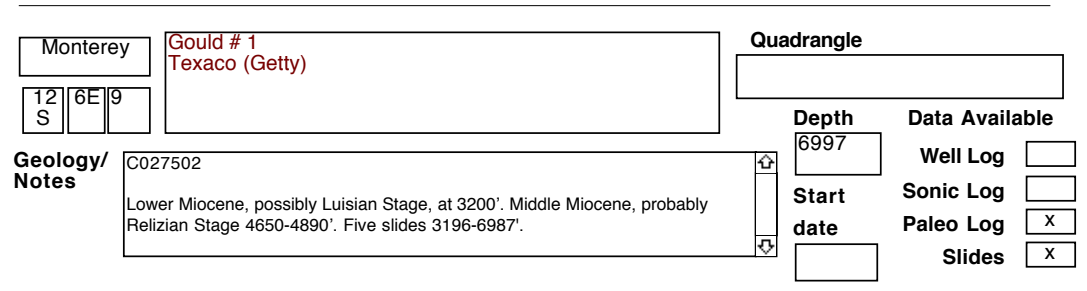

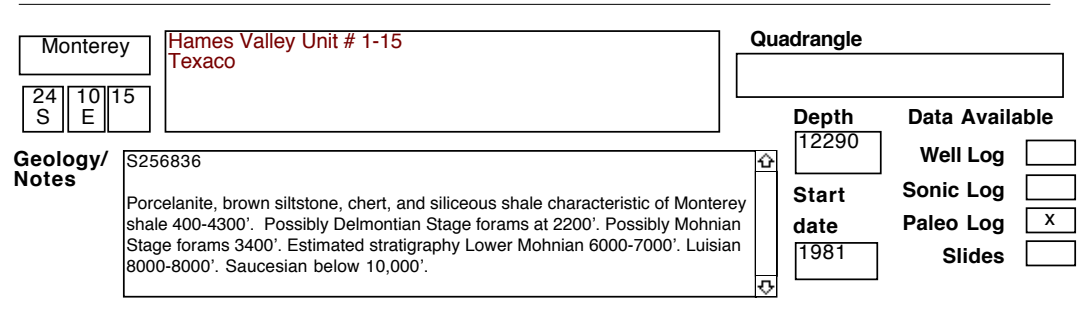

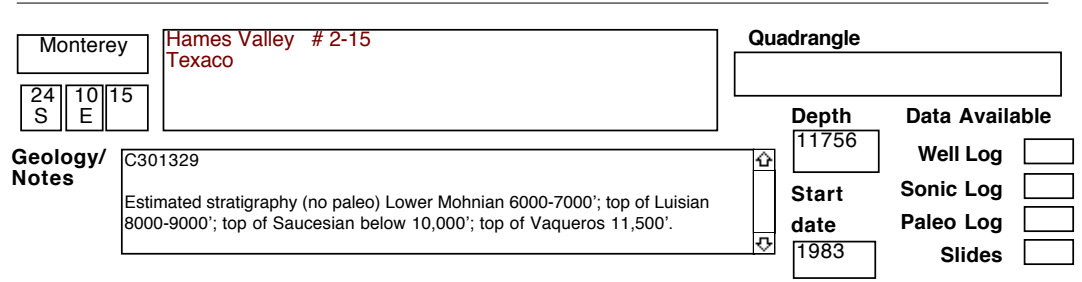


SELECTED OIL TEST WELLS IN CENTRAL CALIFORNIA DATABASE County

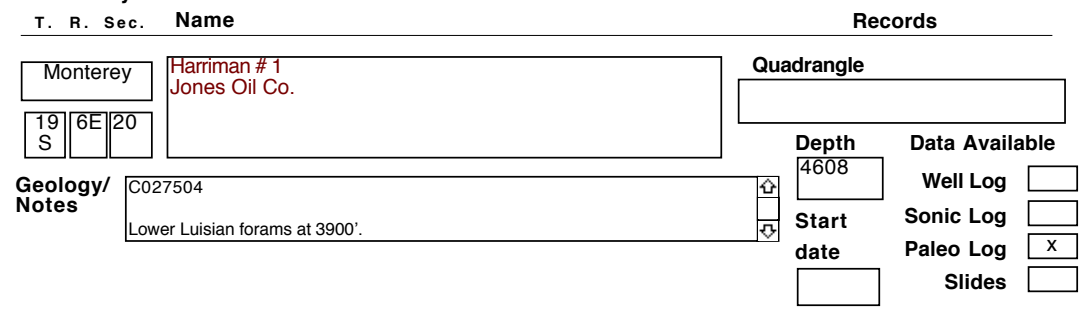

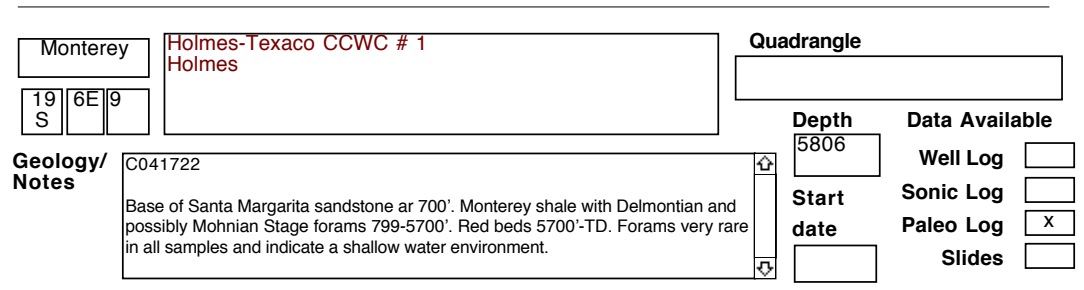

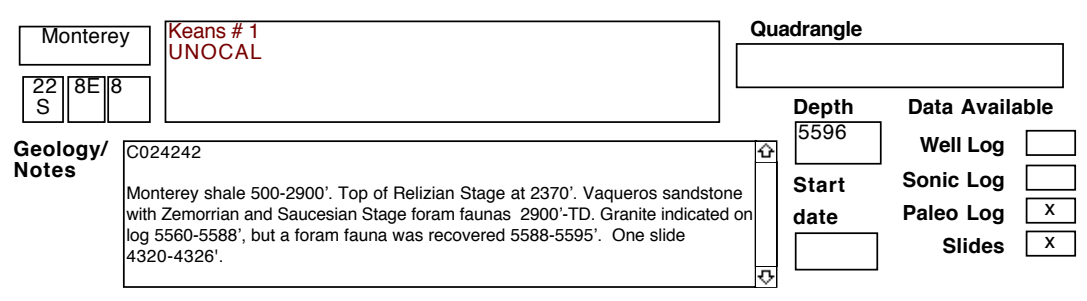

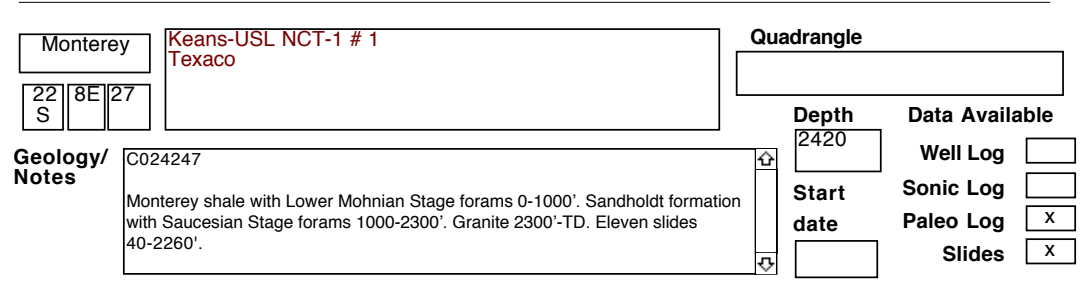

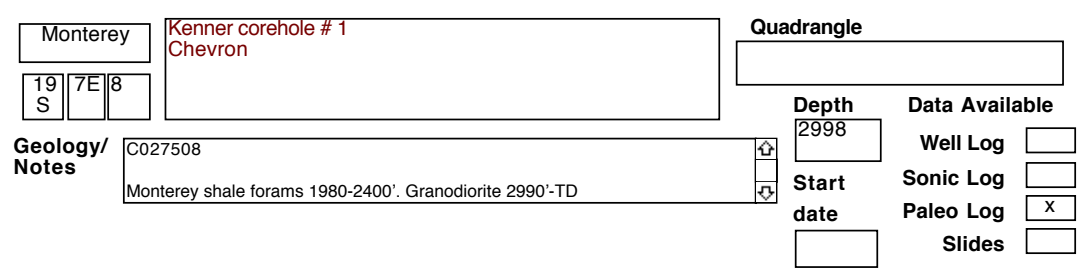


SELECTED OIL TEST WELLS IN CENTRAL CALIFORNIA DATABASE County

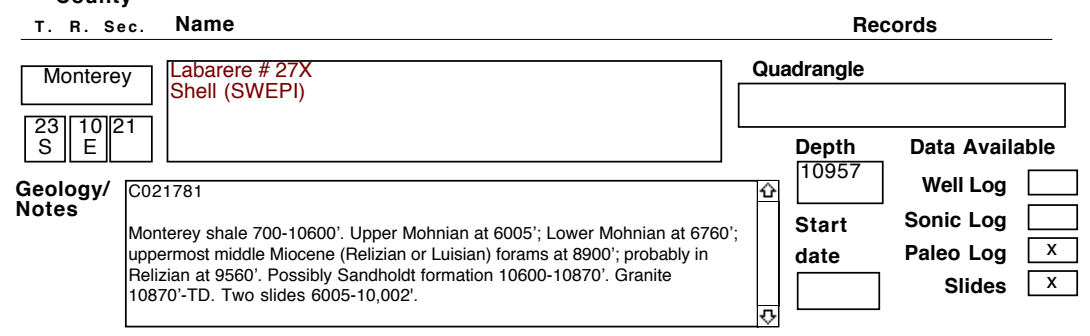

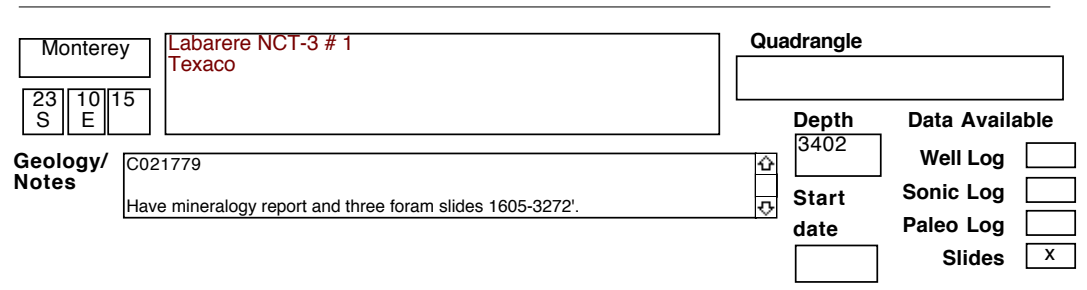

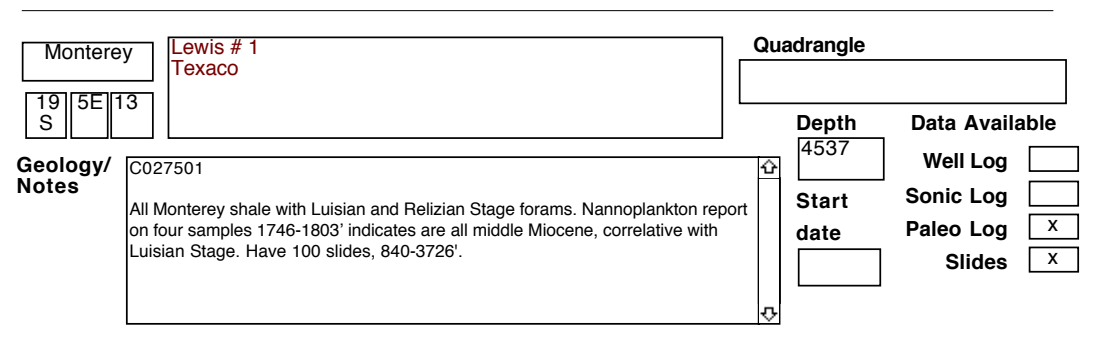

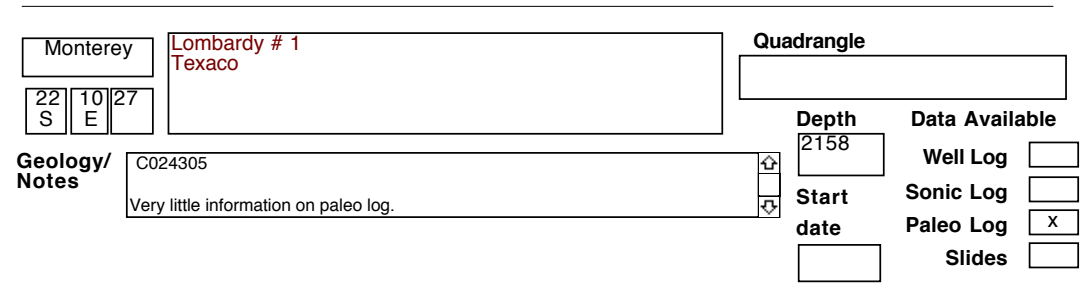

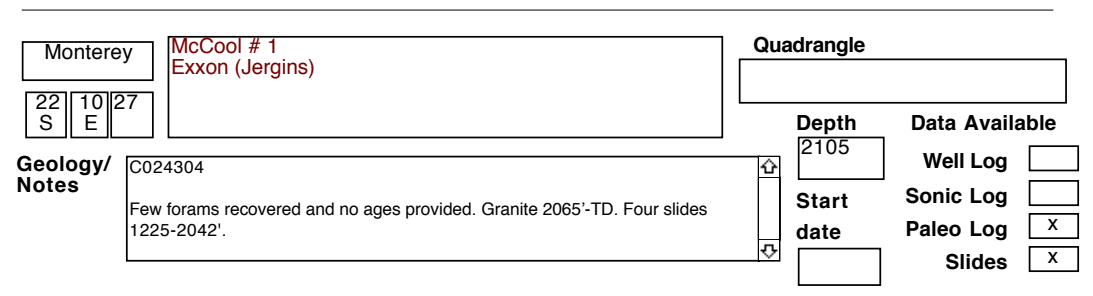


SELECTED OIL TEST WELLS IN CENTRAL CALIFORNIA DATABASE County

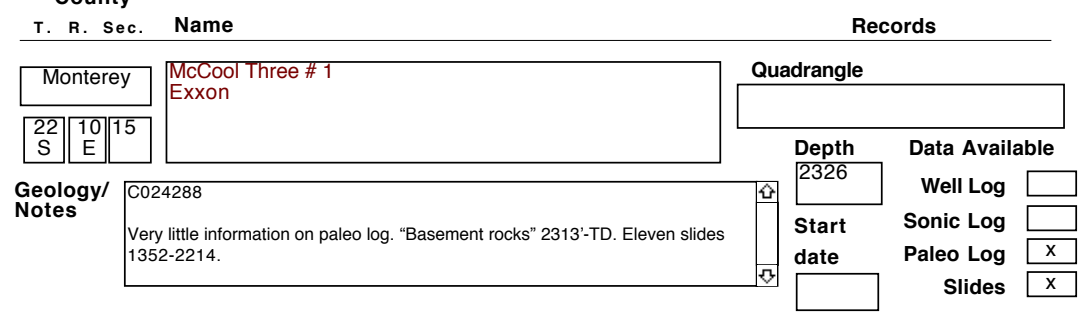

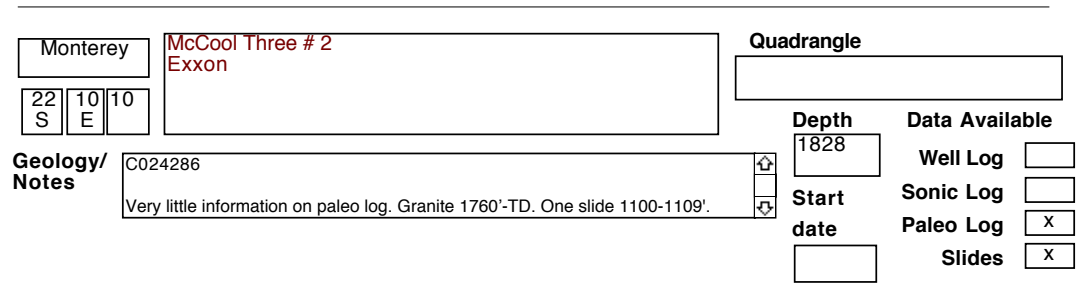

\begin{tabular}{l|l|l|l|l|l|l|}
\hline Monterey & Mee \# 3 & \\
\hline UNOCAL & & \\
\hline
\end{tabular}

\begin{tabular}{l|l|l|l|l|l|l|}
\hline Monterey & $\begin{array}{l}\text { Mohawk-MJM and M \# 9G-1 } \\
\text { Taliaferro and Turne }\end{array}$ \\
\hline
\end{tabular}

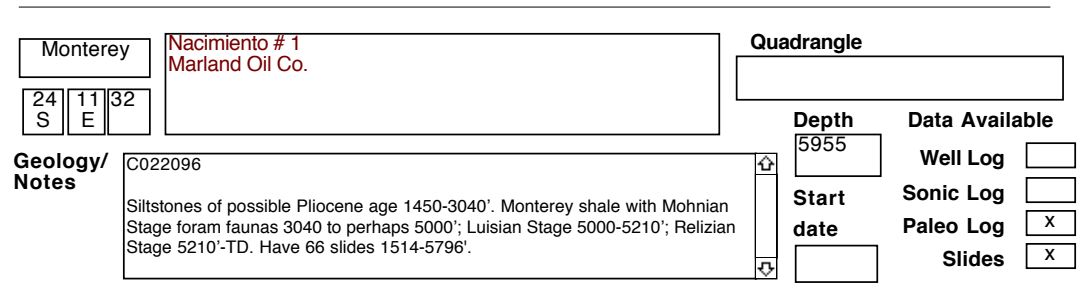


SELECTED OIL TEST WELLS IN CENTRAL CALIFORNIA DATABASE County

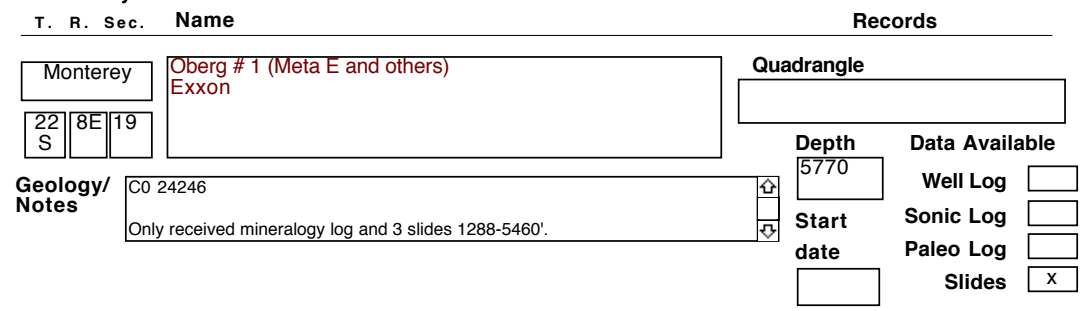

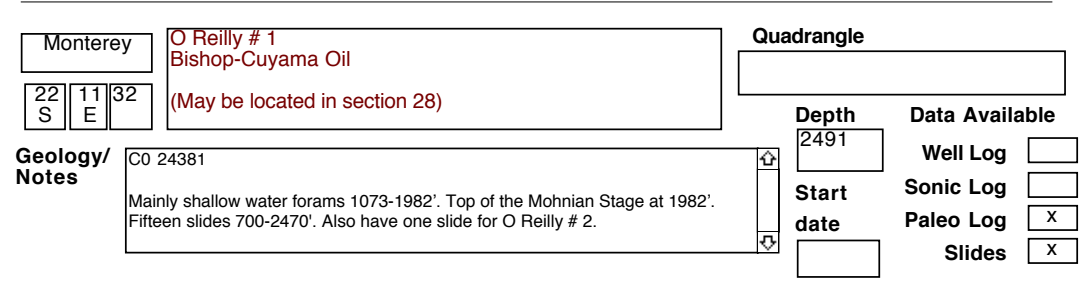

\begin{tabular}{|c|c|c|c|c|}
\hline Monterey & Orradre \# 1 & Quadrangle & & \\
\hline \begin{tabular}{|c|c|c|}
23 & 11 & 31 \\
$\mathrm{~S}$ & $\mathrm{E}$ & \\
\end{tabular} & & $\begin{array}{l}\text { Depth } \\
3795\end{array}$ & \multicolumn{2}{|c|}{ Data Available } \\
\hline $\begin{array}{l}\text { Geology/ } \\
\text { Notes }\end{array}$ & $\begin{array}{l}21889 \\
\text { obably Upper Mohnian Stage forams 2100-2500'. Top of a sandstone at 3158'. } \\
\text { rams very sparse below 2500'. Six slides 2040-3390'. Also have two slides for } \\
\text { radre \# } 1 \text { drilled by Superior Oil Co., but no location given on slide. }\end{array}$ & $\begin{array}{l}\text { Start } \\
\text { date }\end{array}$ & $\begin{array}{l}\text { Well Log } \\
\text { Sonic Log } \\
\text { Paleo Log } \\
\text { Slides }\end{array}$ & $\frac{x}{x}$ \\
\hline
\end{tabular}

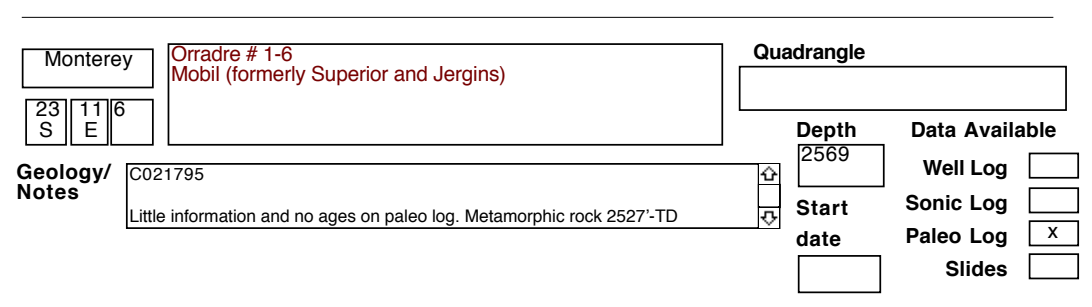

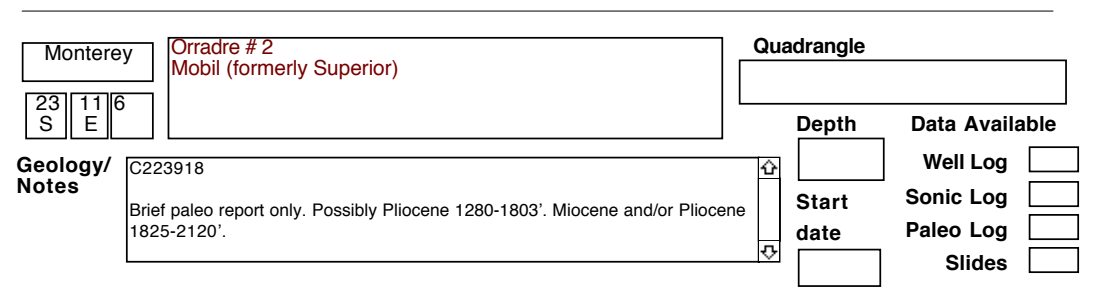


SELECTED OIL TEST WELLS IN CENTRAL CALIFORNIA DATABASE County

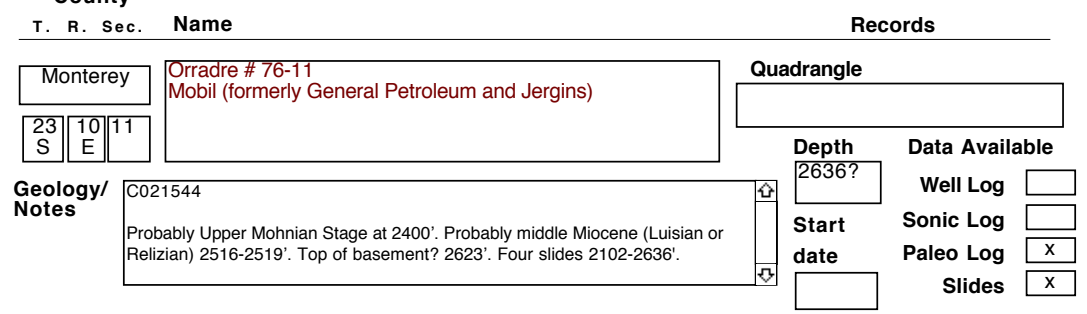

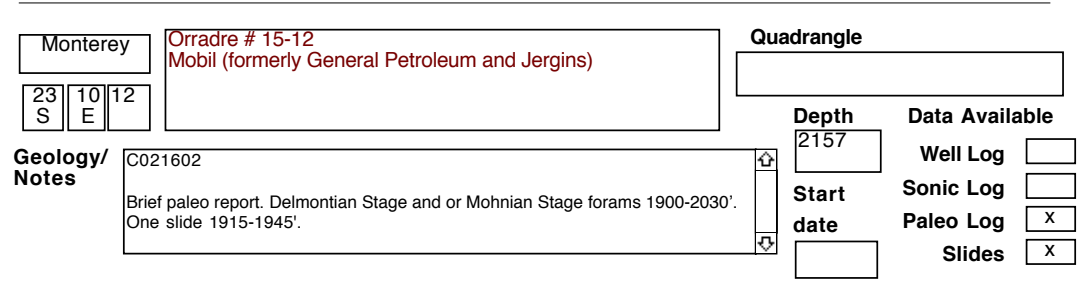

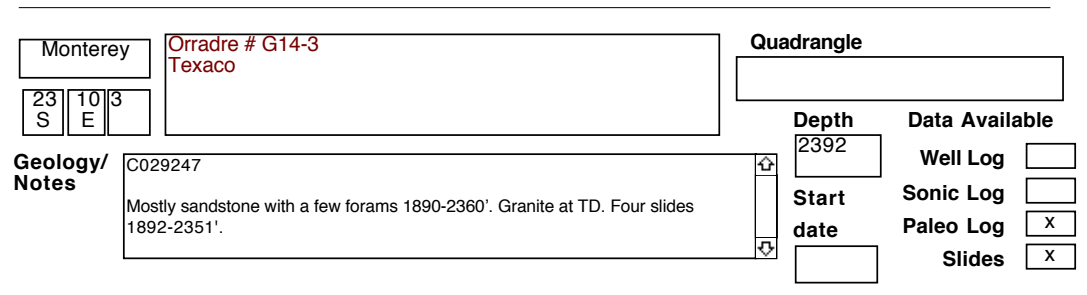

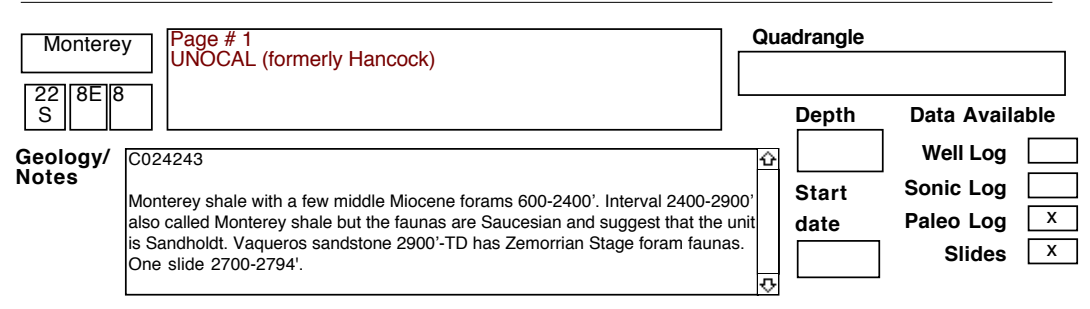

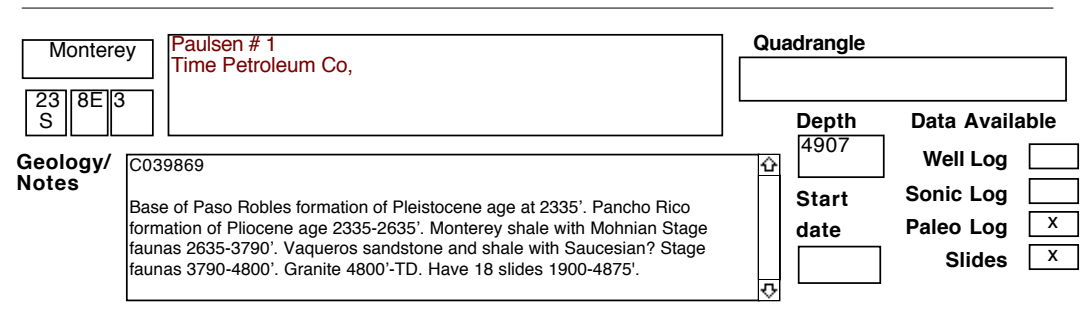




\section{SELECTED OIL TEST WELLS IN CENTRAL CALIFORNIA DATABASE}

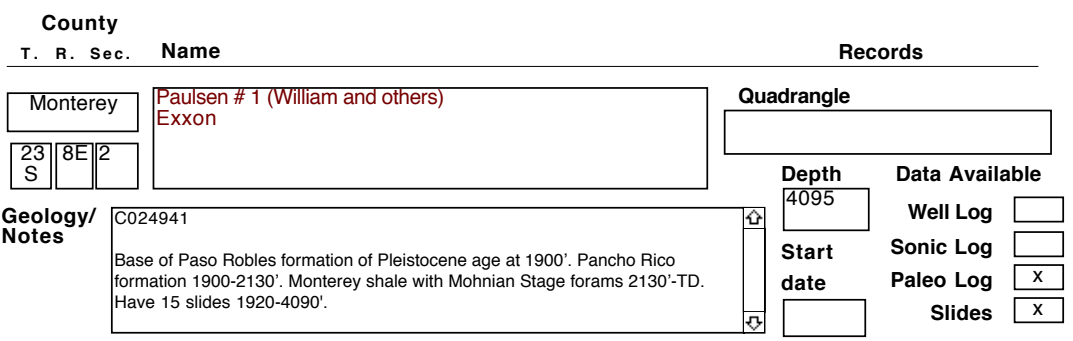

\begin{tabular}{|c|c|c|c|c|c|}
\hline Monterey & Peidmont Land and Cattle Co \# 1 & \multicolumn{4}{|c|}{ Quadrangle } \\
\hline 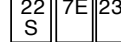 & & \multirow{2}{*}{\multicolumn{2}{|c|}{$\begin{array}{l}\text { Depth } \\
6093\end{array}$}} & \multicolumn{2}{|c|}{ Data Available } \\
\hline \multirow{2}{*}{$\begin{array}{l}\text { Geology/ } \\
\text { Notes }\end{array}$} & 24241 & & & Well Log & \\
\hline & $\begin{array}{l}\text { se of Paso Robles formation at 510'. Santa Margarita sandstone 510-956'. } \\
\text { nterey shale with Mohnian Stage forams 956-2500'. Shale and sandstone } \\
\text { indholdt?) withSaucesian Stage and possibly Zemorrian Stage forams } \\
0^{\prime}-\text { TD. Also have figure with aluminum-silica ratios and a mineral log. }\end{array}$ & & $\begin{array}{l}\text { Start } \\
\text { date }\end{array}$ & $\begin{array}{r}\text { Sonic Log } \\
\text { Paleo Log } \\
\text { Slides }\end{array}$ & $x$ \\
\hline
\end{tabular}

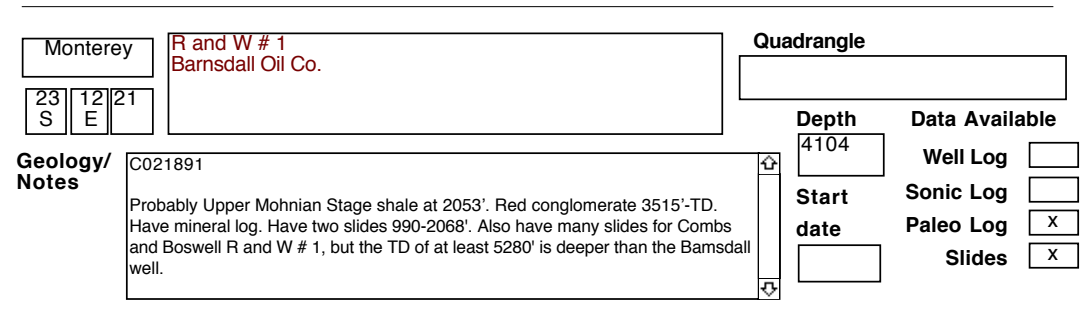

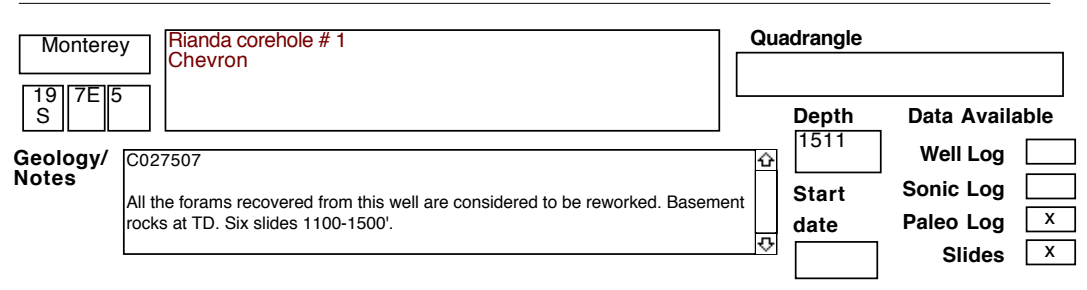

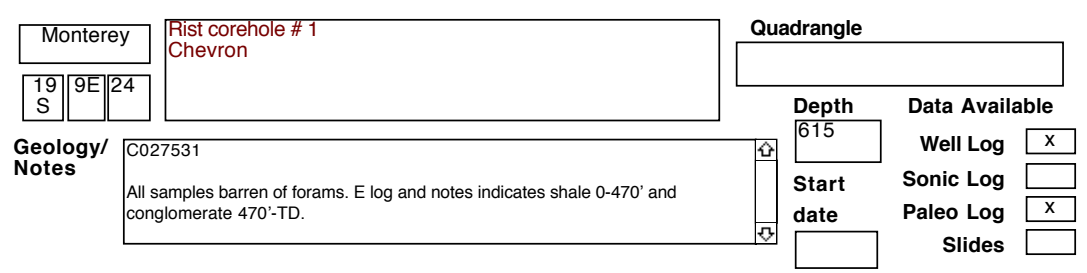


SELECTED OIL TEST WELLS IN CENTRAL CALIFORNIA DATABASE County

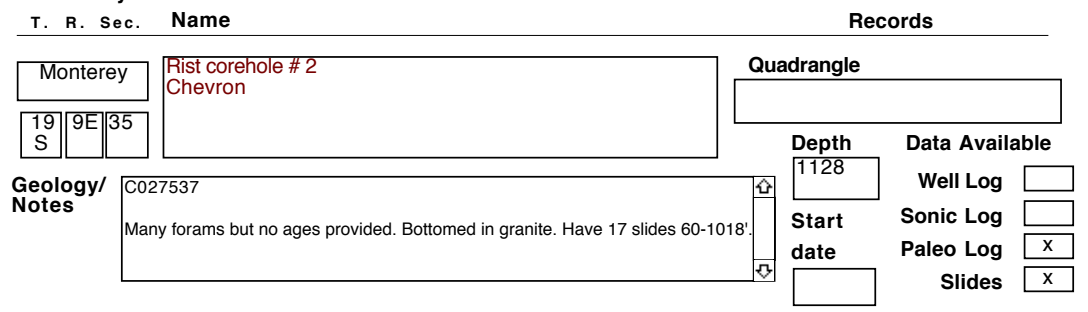

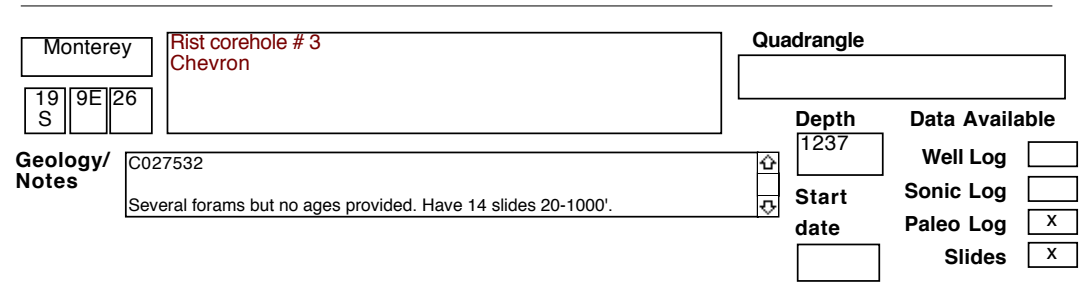

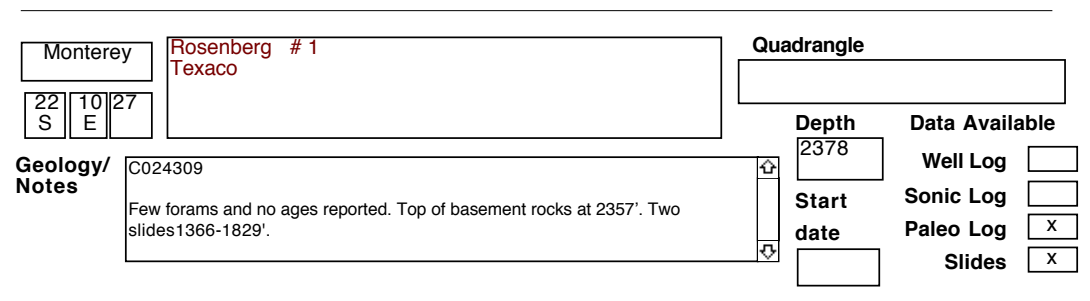

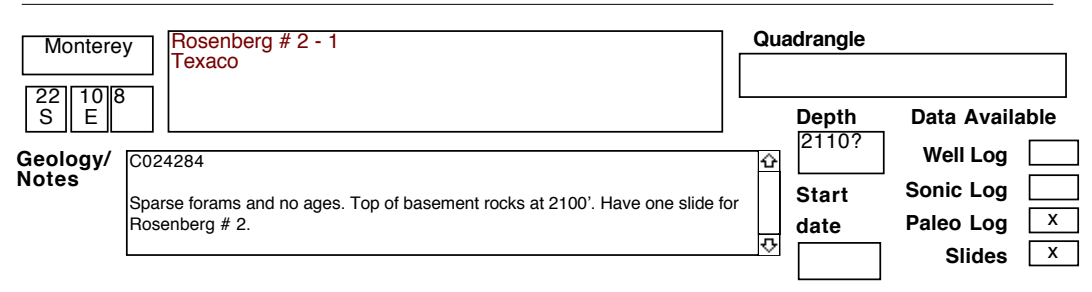

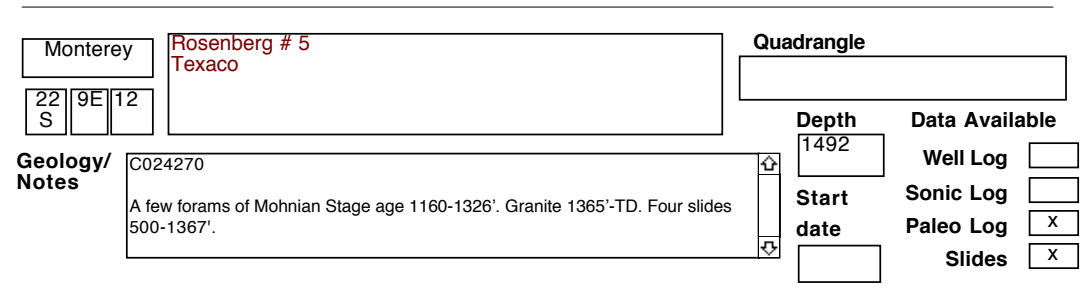


SELECTED OIL TEST WELLS IN CENTRAL CALIFORNIA DATABASE County

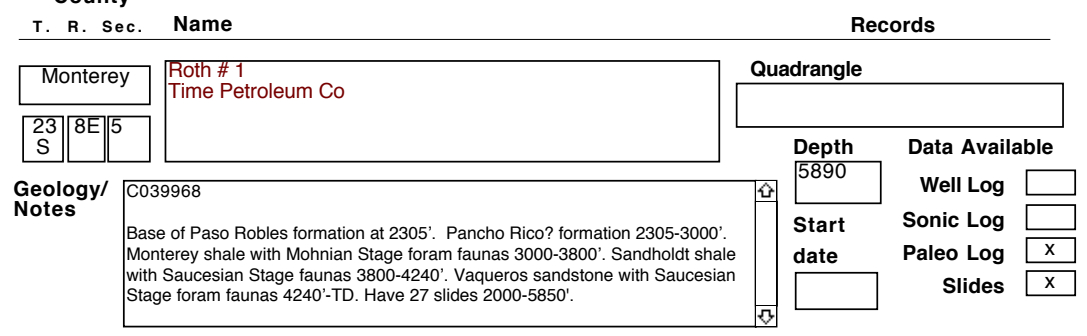

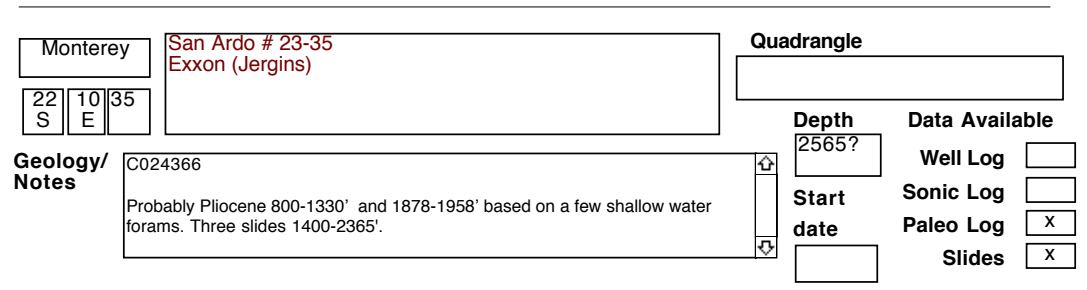

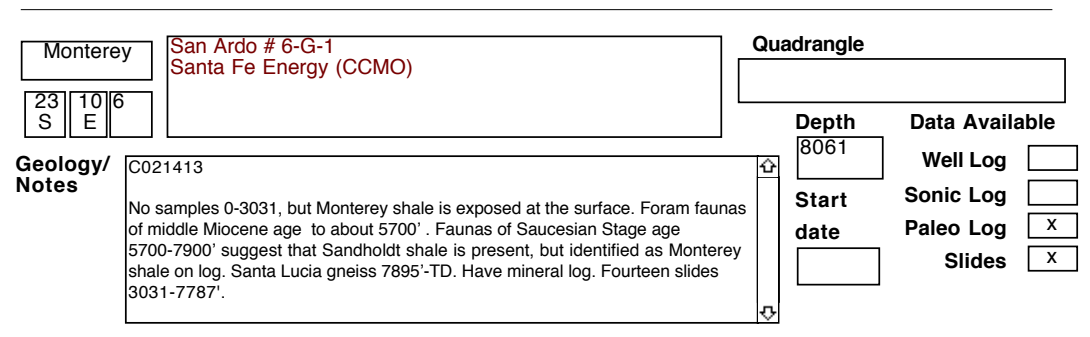

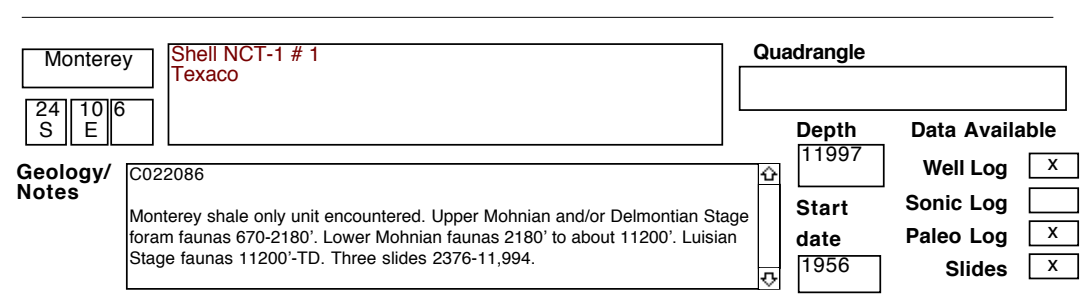

\begin{tabular}{l|l|l|l|l|l|}
\hline Monterey & $\begin{array}{l}\text { Shell-Texas-Beedy \# } \\
\text { Shell (SWEPI) }\end{array}$ \\
\hline
\end{tabular}


SELECTED OIL TEST WELLS IN CENTRAL CALIFORNIA DATABASE County

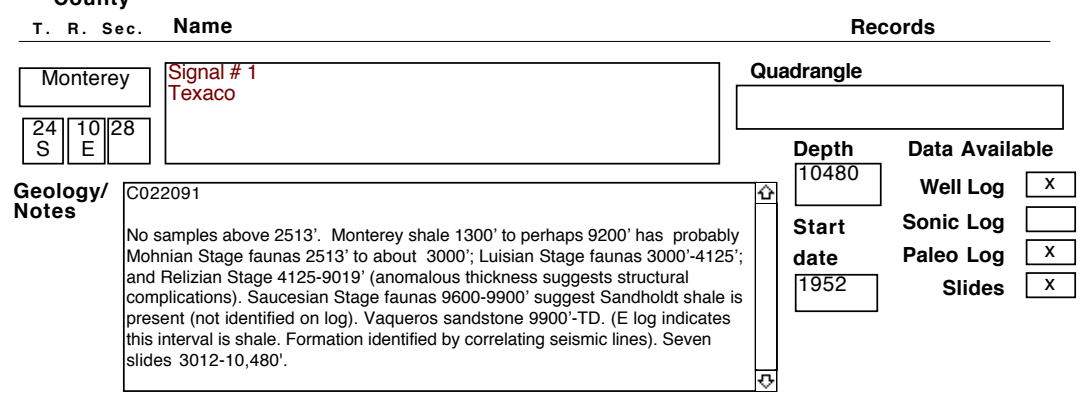

\begin{tabular}{l|l|l|l|l|l|}
\hline Monterey & $\begin{array}{l}\text { Silva \# 1 } \\
\text { Santa Fe Energy }\end{array}$ \\
\hline
\end{tabular}

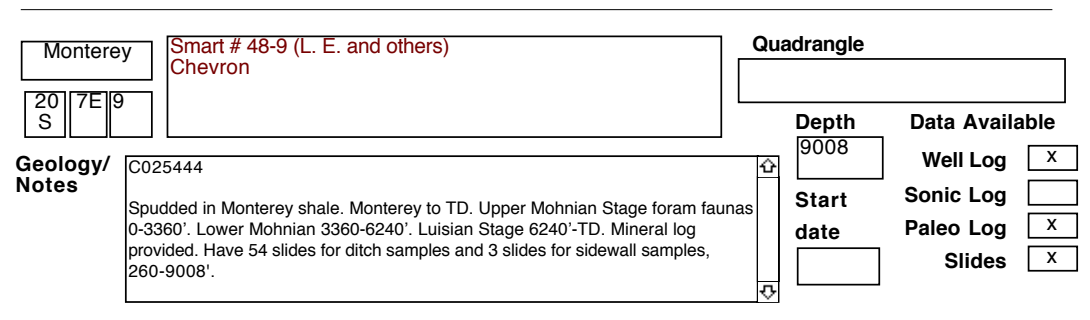

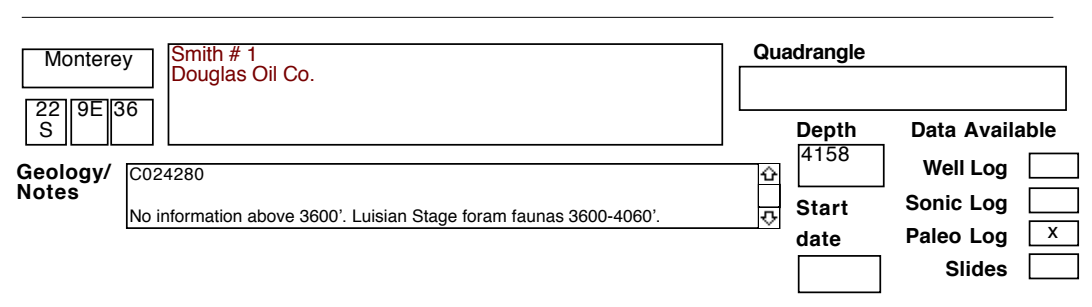

\begin{tabular}{l|l|l|l|l|l|l|}
\hline Monterey & $\begin{array}{l}\text { Sumner-Gould \# 18-14 } \\
\text { Chevron }\end{array}$ \\
\hline
\end{tabular}


SELECTED OIL TEST WELLS IN CENTRAL CALIFORNIA DATABASE

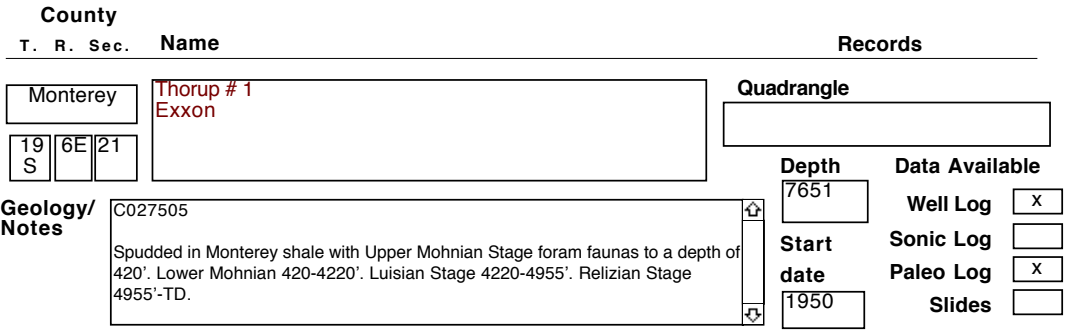

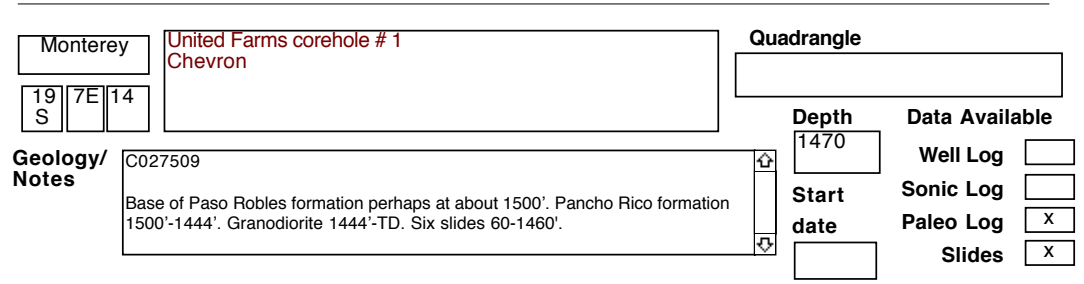

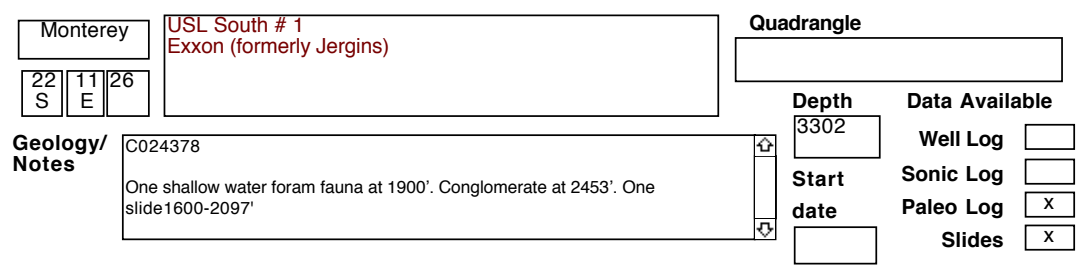

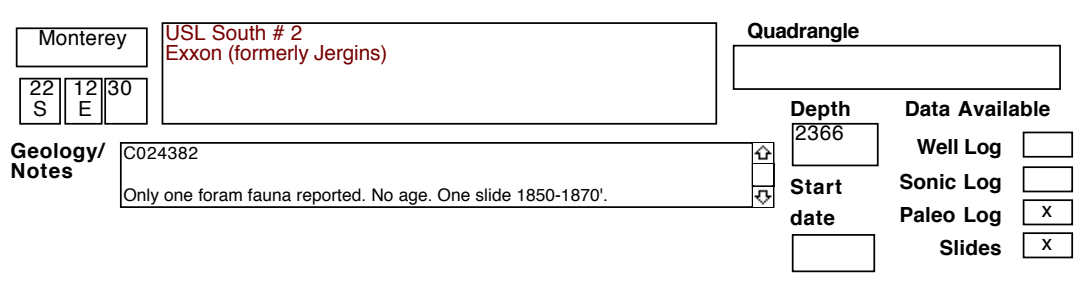

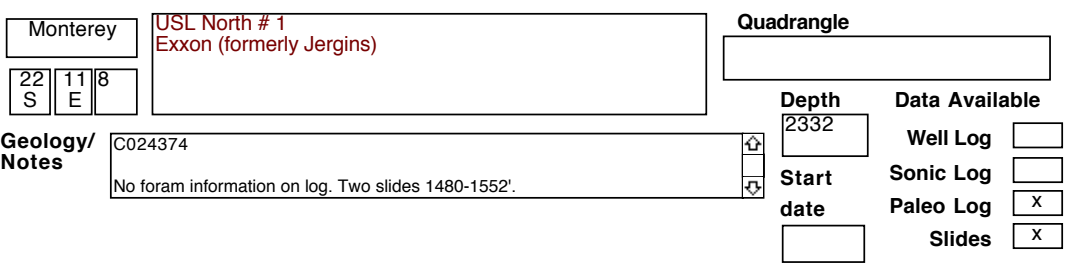


SELECTED OIL TEST WELLS IN CENTRAL CALIFORNIA DATABASE County

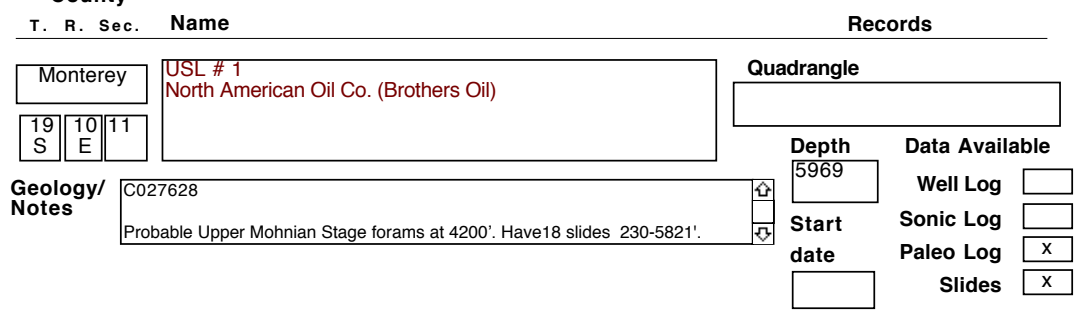

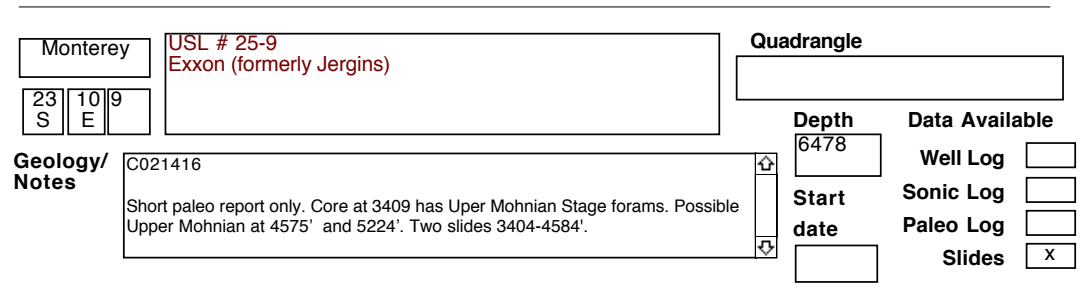

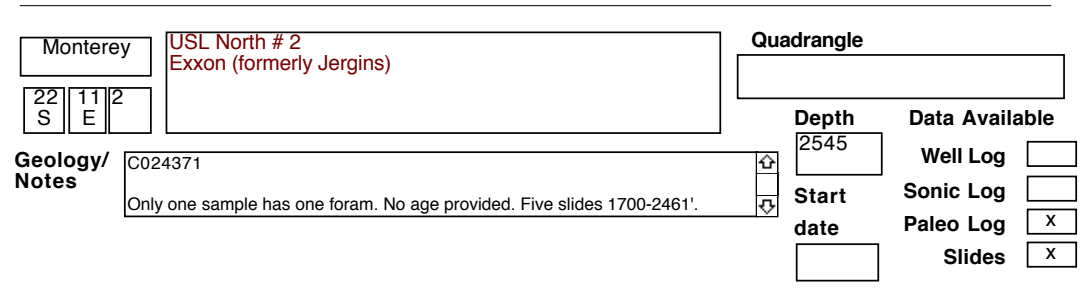

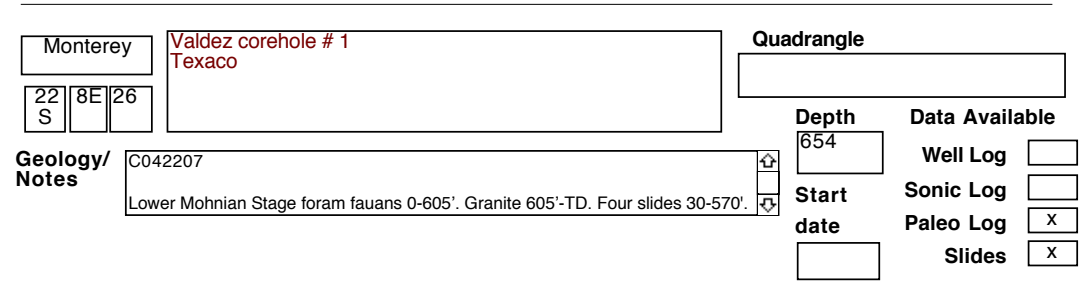

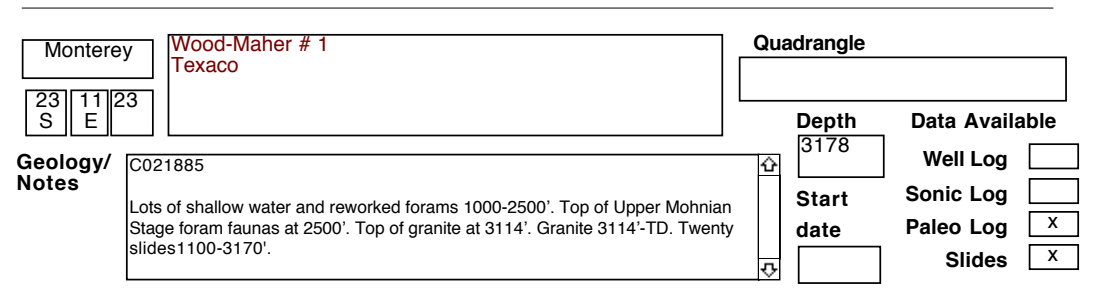


SELECTED OIL TEST WELLS IN CENTRAL CALIFORNIA DATABASE County

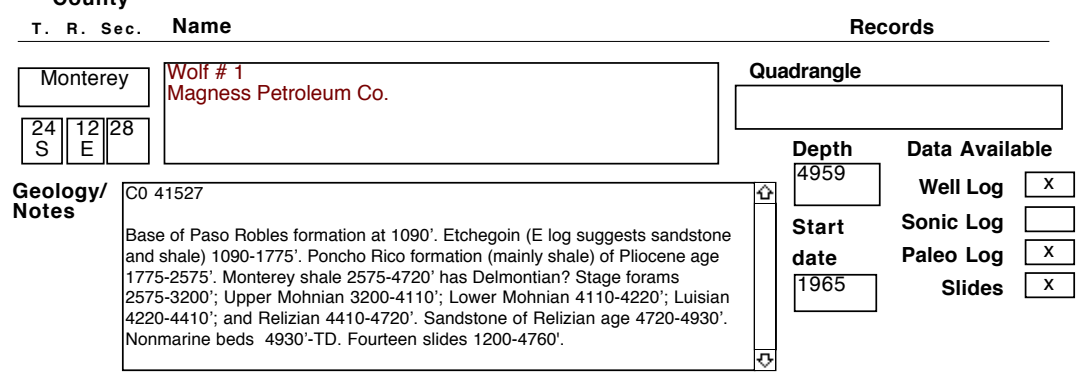

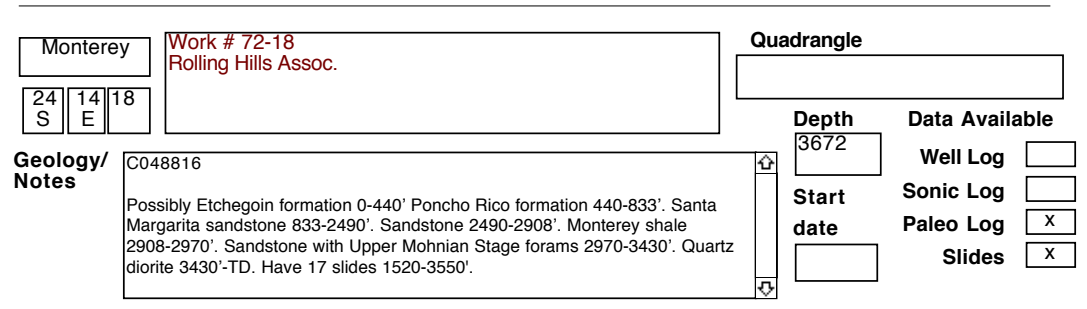

\begin{tabular}{l|l|l|l|l|l|l|l|}
\hline Monterey & $\begin{array}{l}\text { Wright-Texas \# 25 } \\
\text { Mobil (General Petroleum) }\end{array}$ & \multicolumn{2}{l|}{ Quadrangle } \\
\hline
\end{tabular}

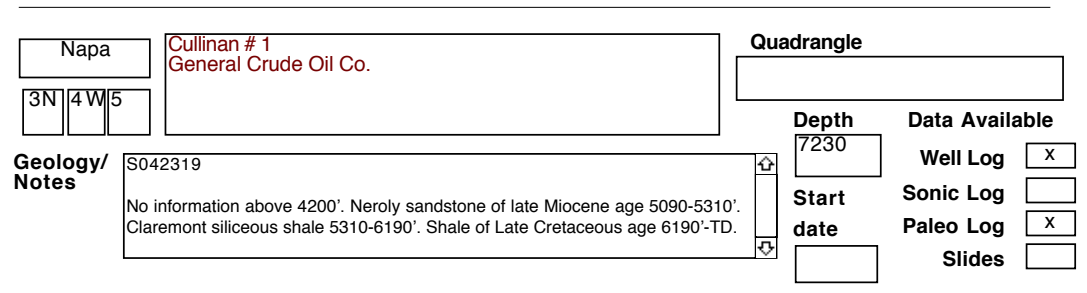

\begin{tabular}{|c|c|c|c|c|c|}
\hline Placer & Van Dyke corehole \# 1 & \multicolumn{4}{|c|}{ Quadrangle } \\
\hline \begin{tabular}{|l|||l|}
13 & $5 E$ \\
$N$ & \\
\end{tabular} & & \multirow{2}{*}{\multicolumn{2}{|c|}{$\begin{array}{l}\text { Depth } \\
2676\end{array}$}} & \multicolumn{2}{|c|}{ Data Available } \\
\hline \multirow{3}{*}{$\begin{array}{l}\text { Geology/ } \\
\text { Notes }\end{array}$} & S034746 & & & Well Log & $\mathrm{x}$ \\
\hline & Base of nonmarineTehama formation at 540'. Late Eocene Markley sandstone & & Start & Sonic Log & \\
\hline & & (3) & 1954 & Slides & \\
\hline
\end{tabular}


SELECTED OIL TEST WELLS IN CENTRAL CALIFORNIA DATABASE County

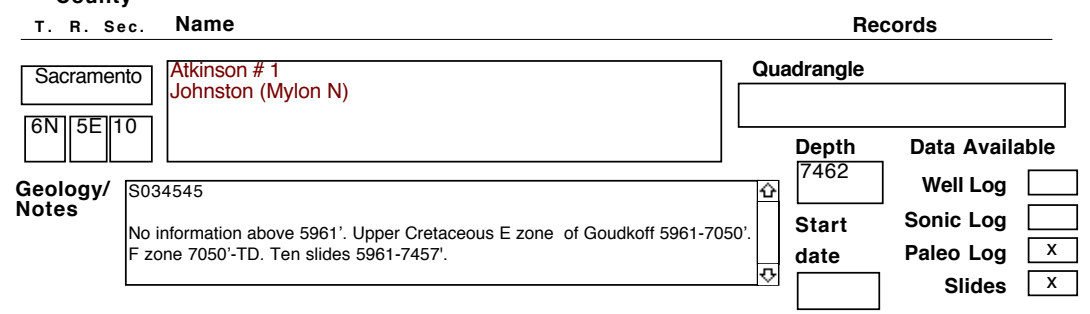

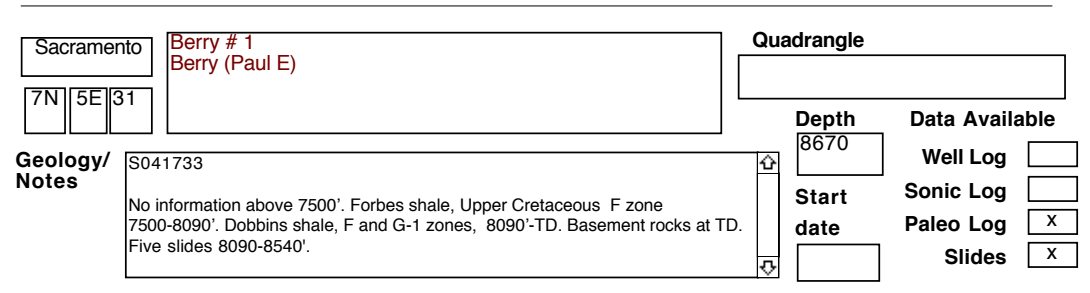

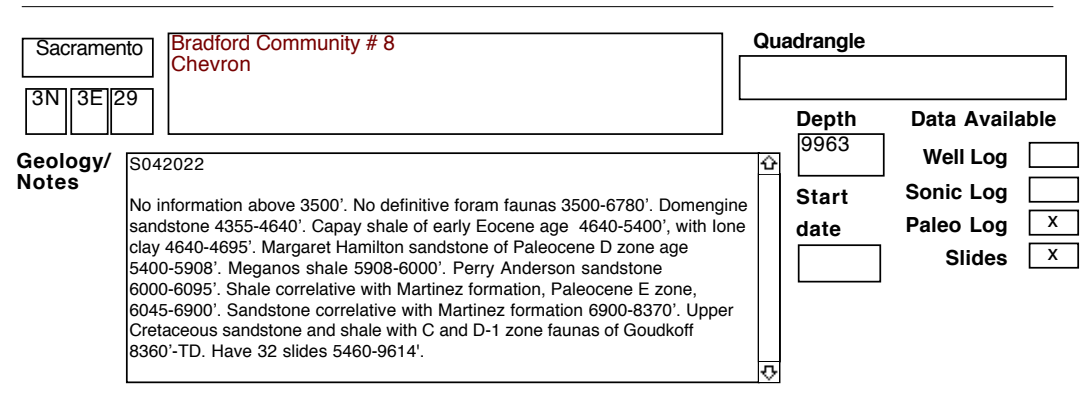

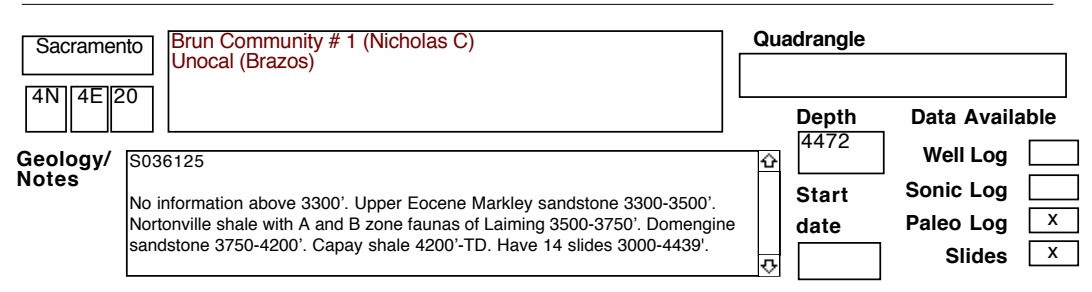

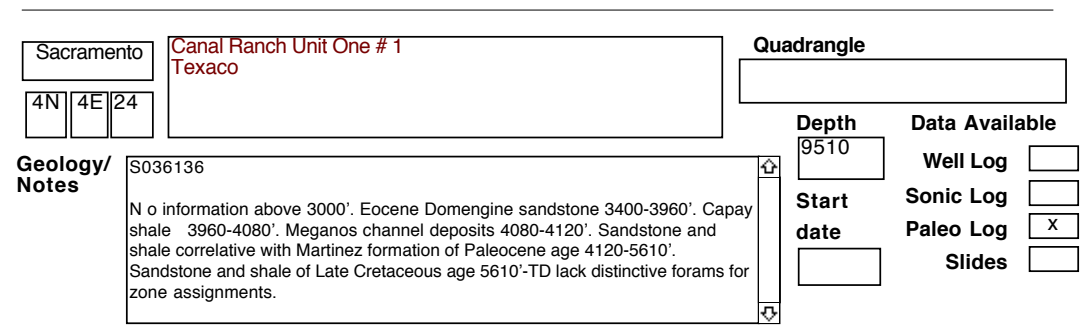


SELECTED OIL TEST WELLS IN CENTRAL CALIFORNIA DATABASE County

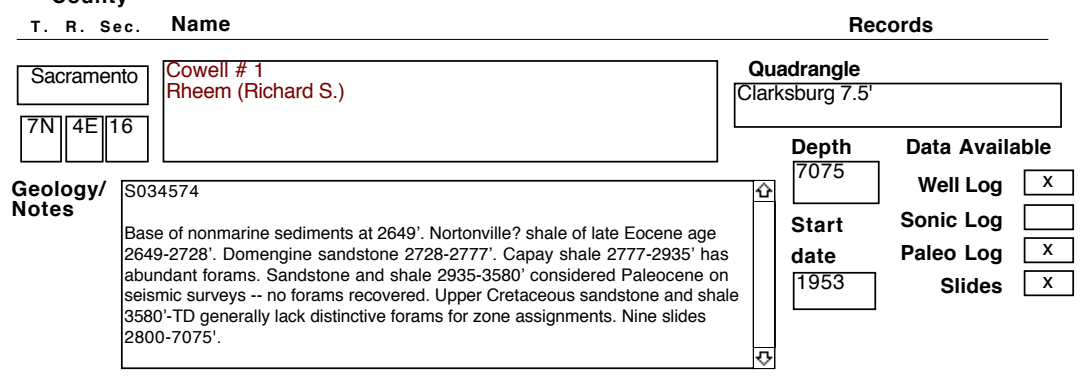

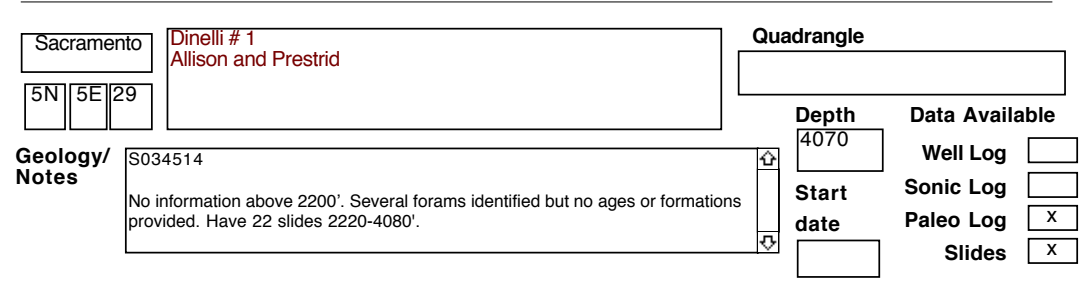

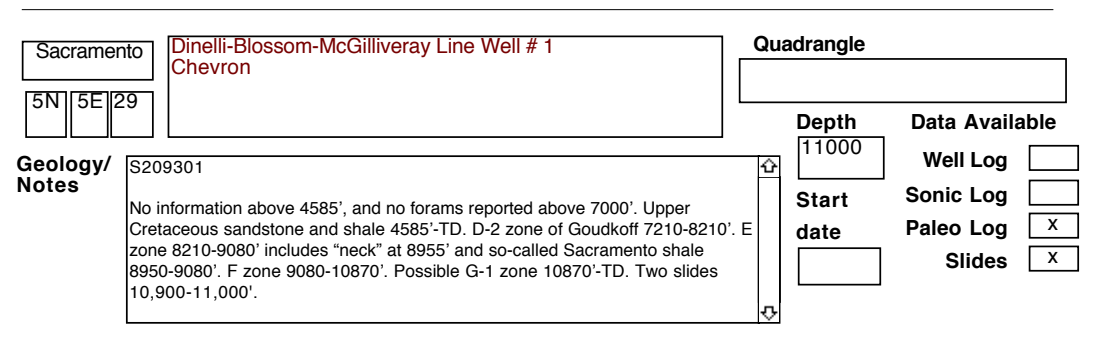

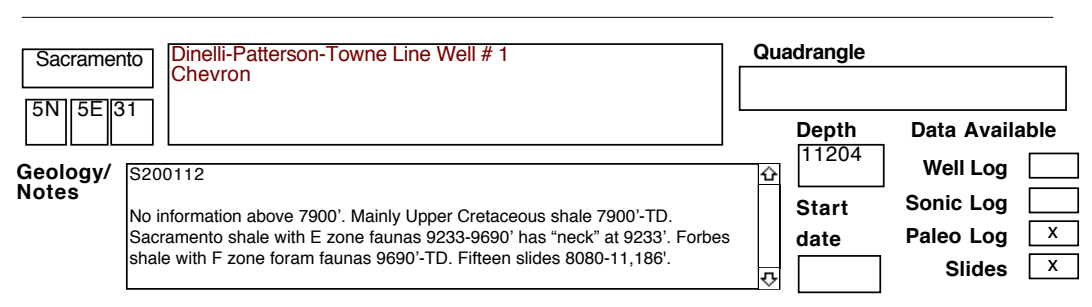

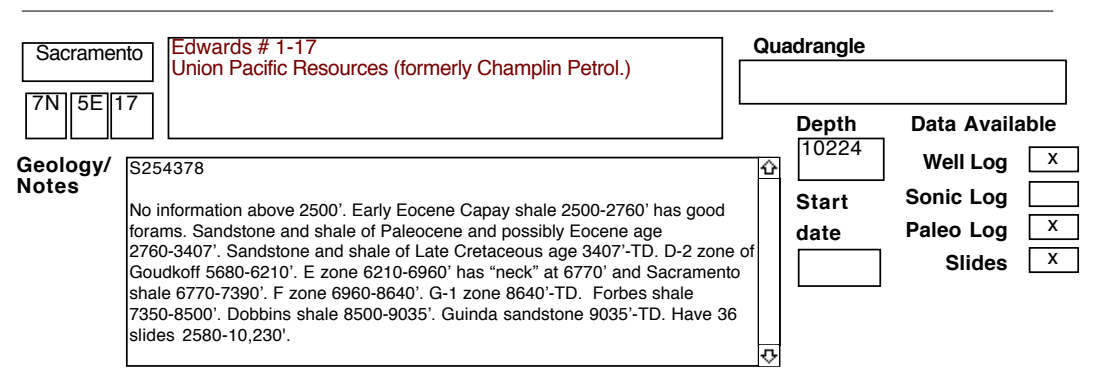


SELECTED OIL TEST WELLS IN CENTRAL CALIFORNIA DATABASE County

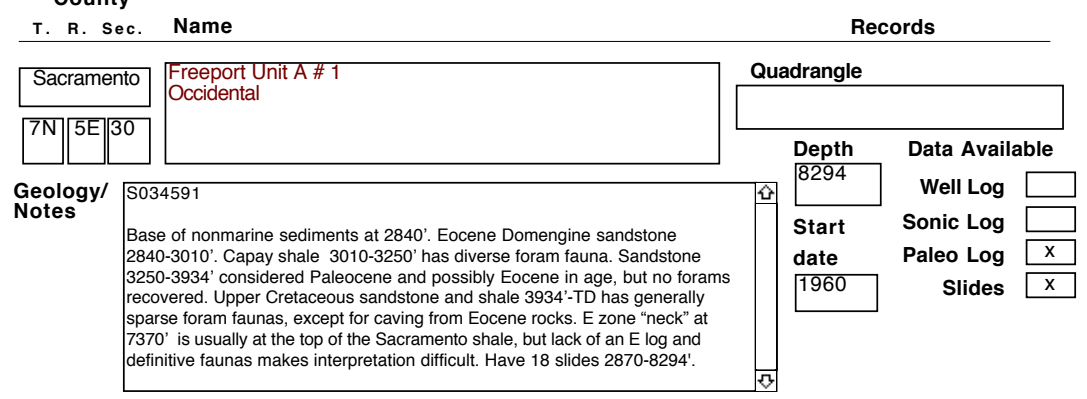

\begin{tabular}{l|l|l|l|l|l|}
\hline Sacramento & $\begin{array}{l}\text { Hansen \$1 } \\
\text { Artnell Oil \& Gas }\end{array}$ \\
\hline
\end{tabular}

\begin{tabular}{l|l|l|l|l|l|l|}
\hline Sacramento & $\begin{array}{l}\text { Kramer \#1 } \\
\text { Bender (E. A.) }\end{array}$ \\
\hline
\end{tabular}

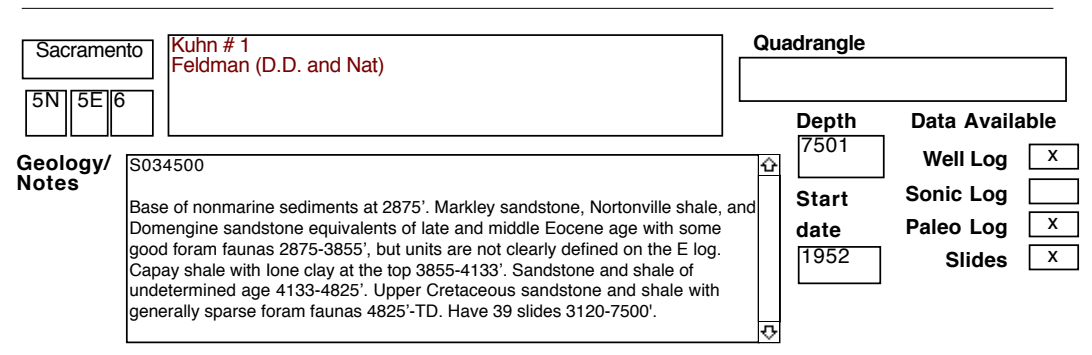

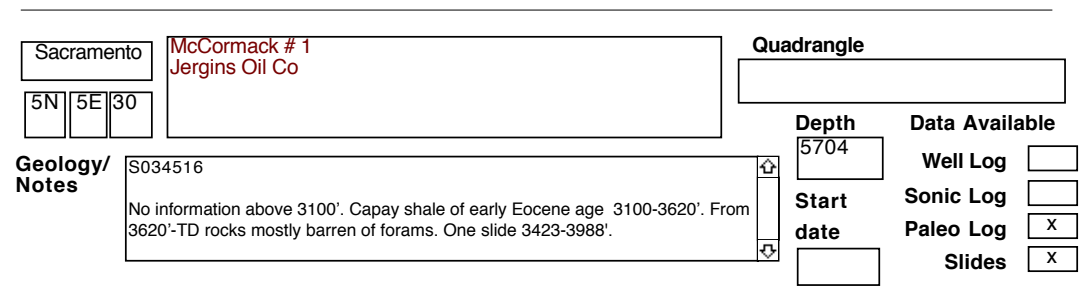




\section{SELECTED OIL TEST WELLS IN CENTRAL CALIFORNIA DATABASE}

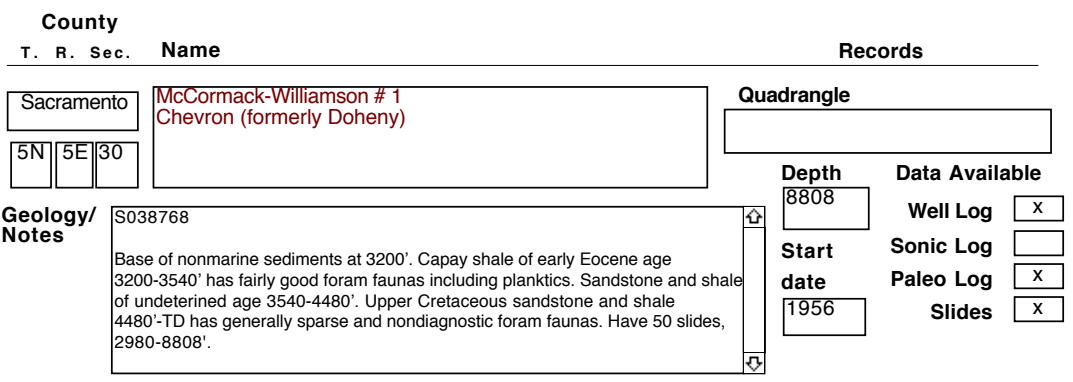

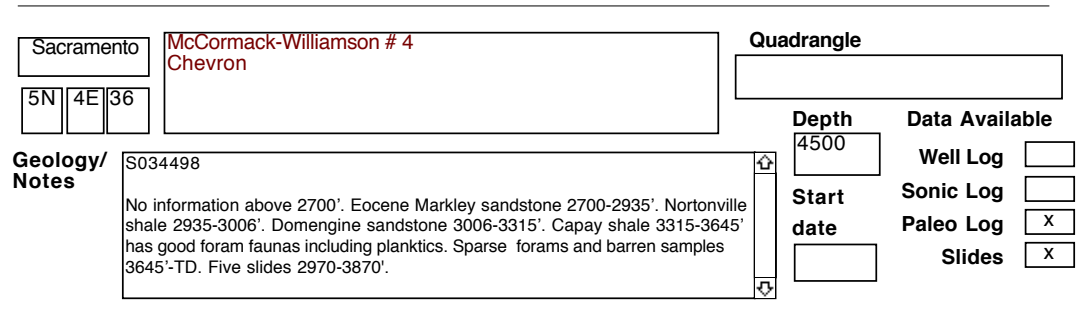

\begin{tabular}{|c|c|c|c|c|c|}
\hline Sacramento & \multirow{2}{*}{$\begin{array}{l}\text { McCormack-Williamson \# } 5 \\
\text { Chevron }\end{array}$} & \multicolumn{4}{|c|}{ Quadrangle } \\
\hline \begin{tabular}{|l|l|l|}
$5 \mathrm{~N}$ & $4 \mathrm{E}$ \\
\end{tabular} & & \multirow{2}{*}{\multicolumn{2}{|c|}{$\begin{array}{l}\text { Depth } \\
8301\end{array}$}} & \multicolumn{2}{|c|}{ Data Available } \\
\hline \multirow{3}{*}{$\begin{array}{l}\text { Geology/ } \\
\text { Notes }\end{array}$} & 34477 & & & Well Log & \\
\hline & 'y samples $7830-8250$ ' were washed for forams. Base of Delta shale of Late & & Start & Sonic Log & \\
\hline & 'taceous age, D-2 zone of Goudkoff, at 7710'. Three slides 7830-8250'. & & date & Paleo Log & $\frac{x}{x}$ \\
\hline
\end{tabular}

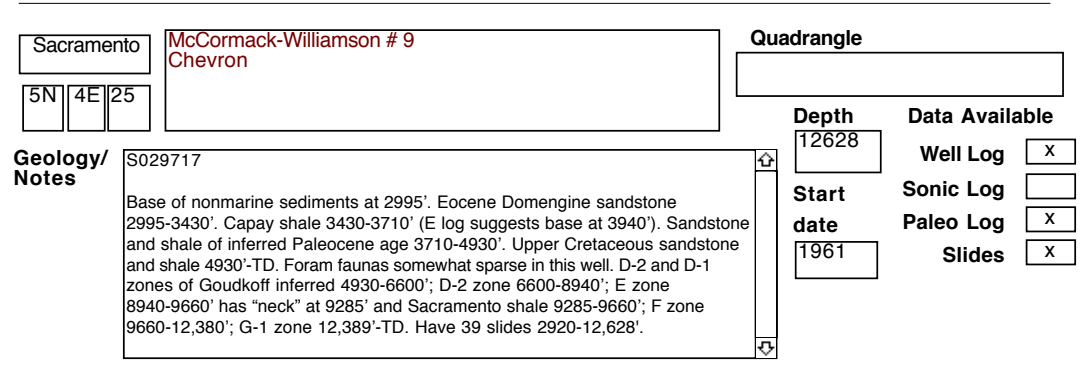

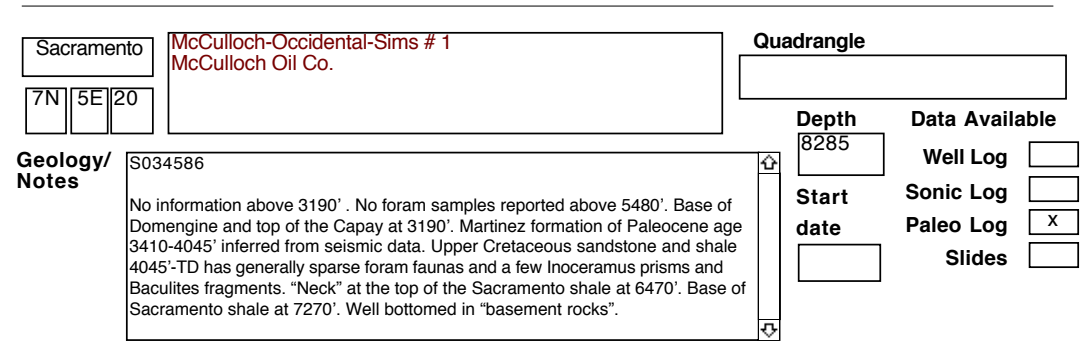


SELECTED OIL TEST WELLS IN CENTRAL CALIFORNIA DATABASE County

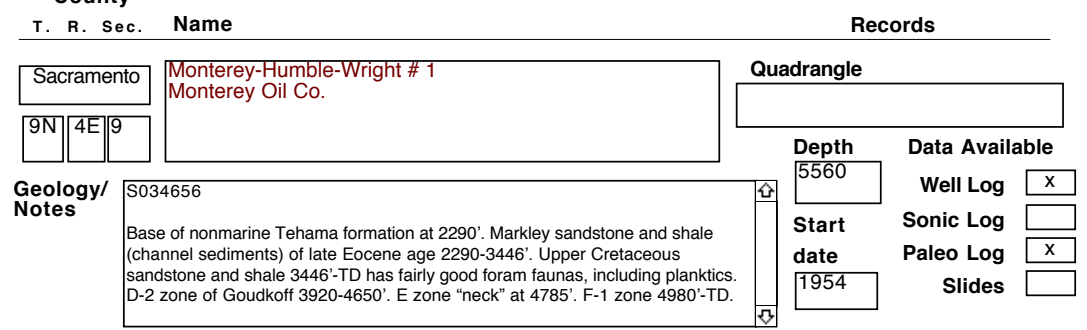

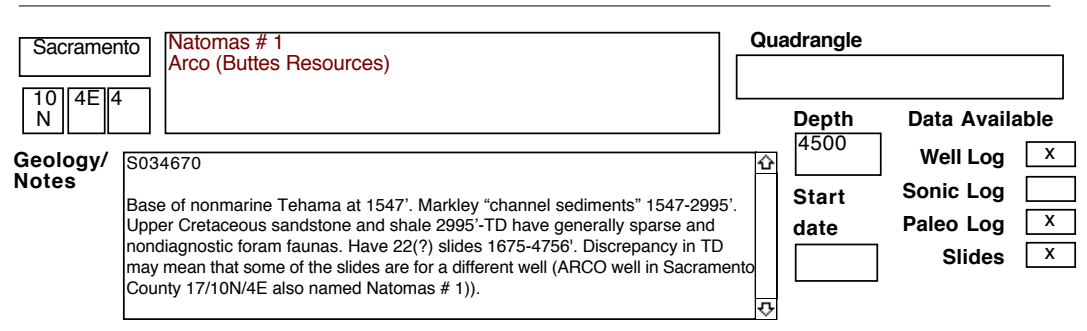

\begin{tabular}{l|l|l|l|l|l|l|l|}
\hline Sacramento & $\begin{array}{l}\text { Natomas \# 1 } \\
\text { ARCO }\end{array}$ \\
(note well with same name but S034670) \\
Notes
\end{tabular}

\begin{tabular}{l|l|l|l|l|l|l|}
\hline Sacramento & $\begin{array}{l}\text { Natomas \# } 2 \\
\text { ARCO }\end{array}$ \\
\hline
\end{tabular}

\begin{tabular}{l|l|l|l|l|l|}
\hline Sacramento & $\begin{array}{l}\text { Oulton Land Co \# 2 } \\
\text { Chevron }\end{array}$ & \multicolumn{2}{l|}{ Quadrangle } \\
\hline
\end{tabular}


SELECTED OIL TEST WELLS IN CENTRAL CALIFORNIA DATABASE

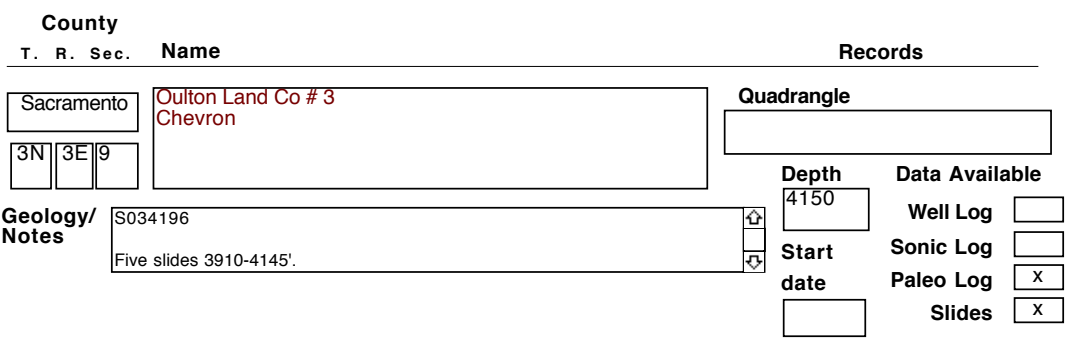

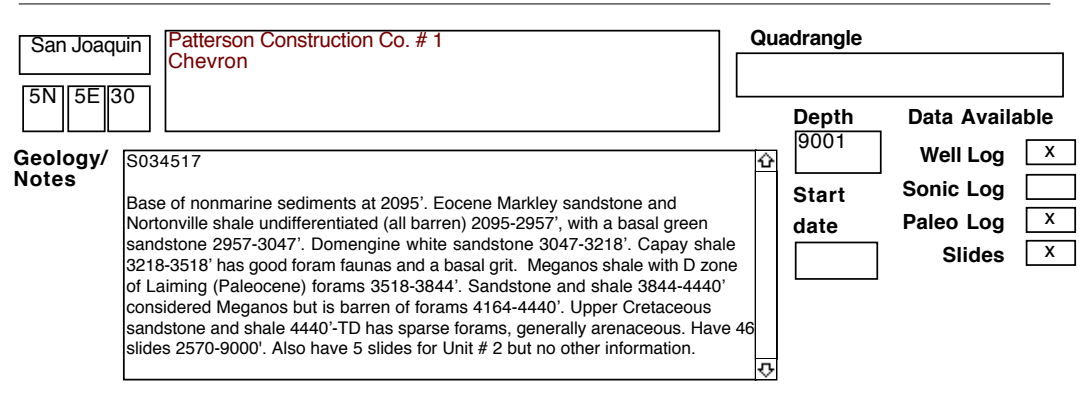

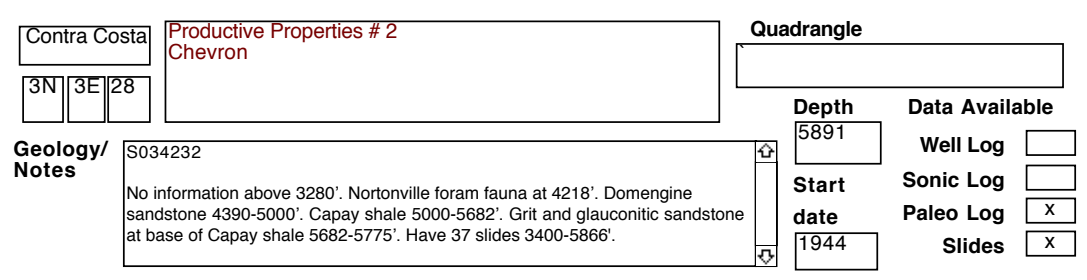

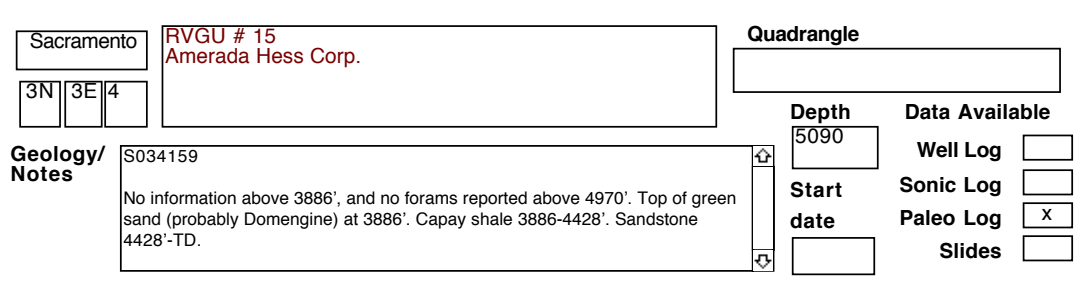

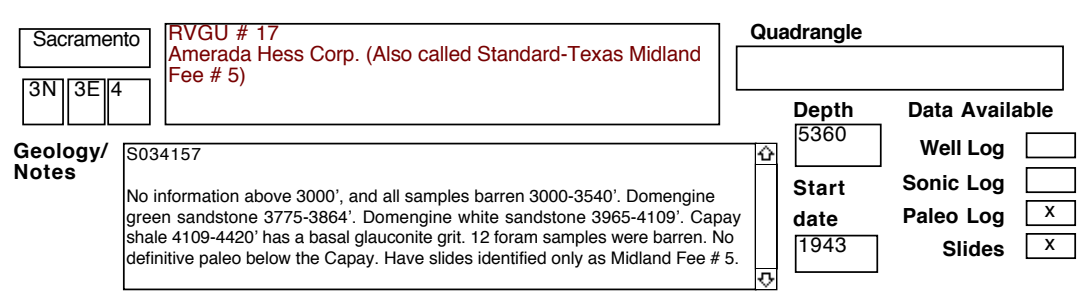




\section{SELECTED OIL TEST WELLS IN CENTRAL CALIFORNIA DATABASE}

\begin{tabular}{l}
$\begin{array}{l}\text { County } \\
\text { T. R. Sec. Name }\end{array}$ \\
$\begin{array}{l}\text { Sacramento } \\
\text { Notes }\end{array}$ \\
$\begin{array}{l}\text { RVGU \# 19 } \\
\text { Amerada Hess Corp. (formerly Midland Fee ) }\end{array}$ \\
$\begin{array}{l}\text { Base of pre-Eocene sediments at 2970'. Late Eocene Markley sandstone and } \\
\text { shale 2970-3532' has forams correlative with A-1 zone of Laiming . Nortonville } \\
\text { shale 3532-3752' has only rare arenaceous forams (Anomalous. Another unit?). } \\
\text { Domengine sandstone (including glauconitic sandstone) and shale with poor } \\
\text { foram faunas 3752-4153'. Capay? shale 4153-4414' also has poor or no forams. } \\
\text { Upper Cretaceous sandstone and shale 4414'-TD have rare forams, Inoceramus } \\
\text { prisms, and Baculites fragments. }\end{array}$ \\
\hline
\end{tabular}

Records

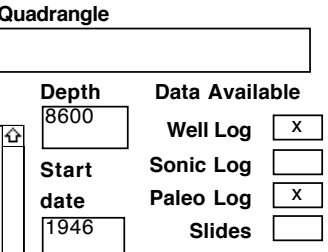

\begin{tabular}{|l|l|l|}
\hline Sacramento & \multicolumn{2}{|l|}{$\begin{array}{l}\text { RVGU \# 145 (formerly Midland Fee \#-6) } \\
\text { Amerada Hess }\end{array}$} \\
\hline $8 \mathrm{~N}$ & $4 \mathrm{E}$ & 23 \\
\end{tabular}

Geology/ 5034181

Notes

(1)

Samples not washed above 4303'. Base of post-Eocene nonmarine sediments

1990'. Markley sandstone and shale of late Eocene age 1990-3500'. Nortonvil

shale 3500-3867. Green sandstone (probably glaucontic) $3867-3940$ put in

by others. Domengine white sandstone 3940-4393'. Capay shale 4393-5310'

Meganos sandstone and shale 5310'-TD has Discocyclina at 5441' and a flood of

glauconite at 5707'

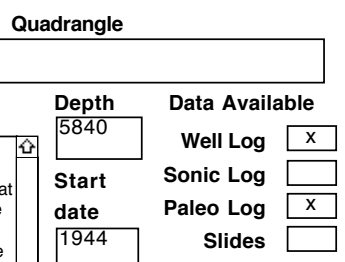

\begin{tabular}{|c|c|c|}
\hline Sac & amento & $\begin{array}{l}\text { Sacramento \# } 1 \\
\text { Jergins Oil Co }\end{array}$ \\
\hline & $4 \mathrm{E} \mid 23$ & \\
\hline
\end{tabular}

\section{Geology/ $\$ 034635$}

Notes

No information above 2500'. Base of Tehama nonmarine sediments at 2600'.

Glauconite 2621-2631'. Laiming's B-1 zone (Eocene) 2641-2661'. Little

information 2661-5600'. Upper Cretaceous E zone foram faunas of Goudkoff at

$5900^{\prime}$ along with Inoceramus prisms. F-1 zone at 6430'. Three slides 3085-4375'.

\begin{tabular}{|c|c|c|}
\hline uadrangle & & \\
\hline Depth & Data Availabl & \\
\hline 1460 & Well Log & \\
\hline Start & Sonic Log & \\
\hline date & Paleo Log & $x$ \\
\hline & Slides & $\mathrm{x}$ \\
\hline
\end{tabular}

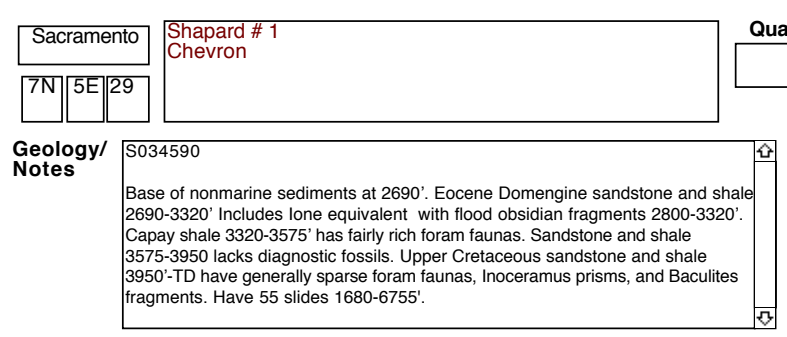

Quadrangle

Depth Data Available

$6755 \quad$ Well Log $x$

start

Star

Sonic Log

date

Paleo Log $x$

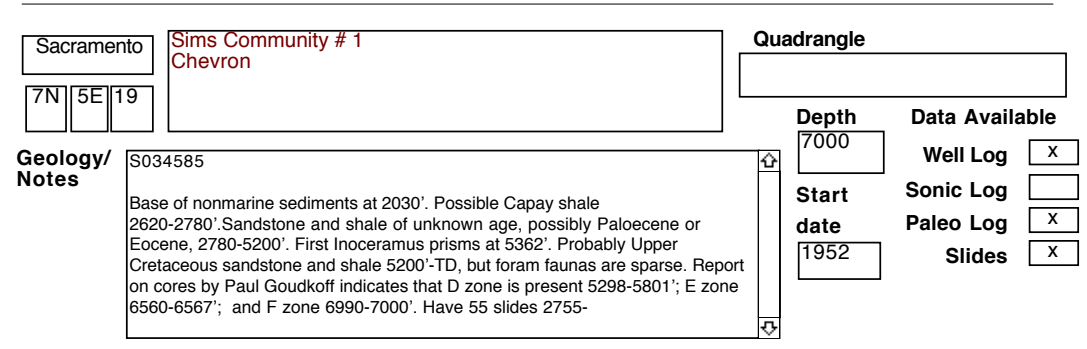


SELECTED OIL TEST WELLS IN CENTRAL CALIFORNIA DATABASE County

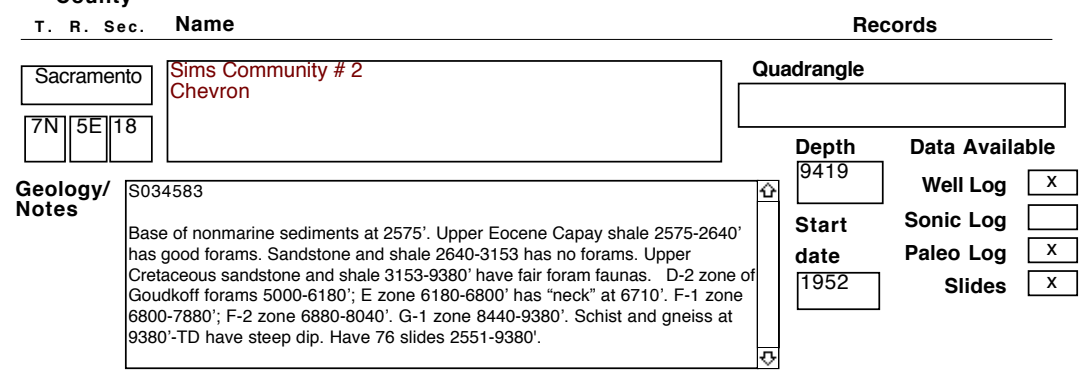

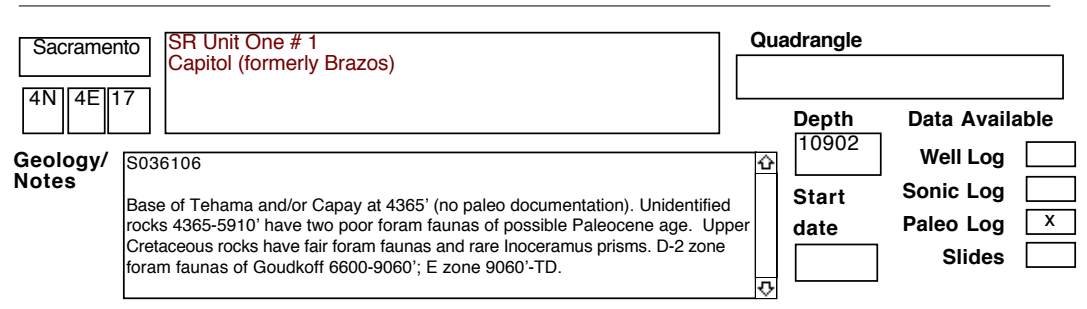

\begin{tabular}{|c|c|c|c|c|c|}
\hline Sacramento & Staten Island Land Co. \# 1 & \multicolumn{4}{|c|}{ Quadrangle } \\
\hline \begin{tabular}{|l|l|l|l|l|}
$3 \mathrm{~N}$ & $4 \mathrm{E}$ & 4
\end{tabular} & & \multirow{2}{*}{\multicolumn{2}{|c|}{$\begin{array}{l}\text { Depth } \\
4653\end{array}$}} & \multicolumn{2}{|c|}{ Data Available } \\
\hline \multirow{4}{*}{$\begin{array}{l}\text { Geology/ } \\
\text { Notes }\end{array}$} & 36046 & & & Well Log & \\
\hline & foram faunas hut no aces or formations nrovided One slide & & Start & Sonic Log & \\
\hline & & & date & Paleo Log & $\mathrm{x}$ \\
\hline & & & & Slides & $\mathrm{x}$ \\
\hline
\end{tabular}

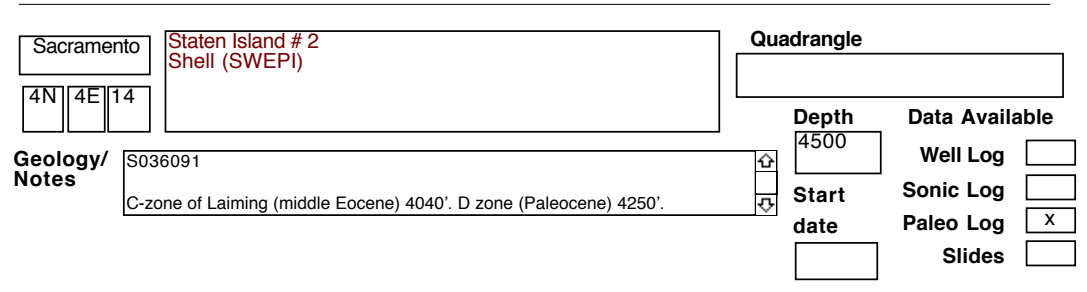

\begin{tabular}{|c|c|c|c|c|c|}
\hline Sacramente & \multirow{2}{*}{$\begin{array}{l}\text { Stone Lake Unit \# } 1 \\
\text { BP Exploration (formerly Kern Oil Co.) }\end{array}$} & \multicolumn{4}{|c|}{ Quadrangle } \\
\hline $6 \mathrm{~N}\|5 \mathrm{E}\|^{32}$ & & \multirow{2}{*}{\multicolumn{2}{|c|}{$\begin{array}{l}\text { Depth } \\
8636\end{array}$}} & \multicolumn{2}{|c|}{ Data Available } \\
\hline \multirow{3}{*}{$\begin{array}{l}\text { Geology/ } \\
\text { Notes }\end{array}$} & 34547 & & & Well Log & \\
\hline & samples washed above $6600^{\prime}$. Probably Upper Cretaceous sandstone and & & Start & Sonic Log & \\
\hline & les 6600-8600'. & & & Slides & $\mathrm{x}$ \\
\hline
\end{tabular}


SELECTED OIL TEST WELLS IN CENTRAL CALIFORNIA DATABASE County

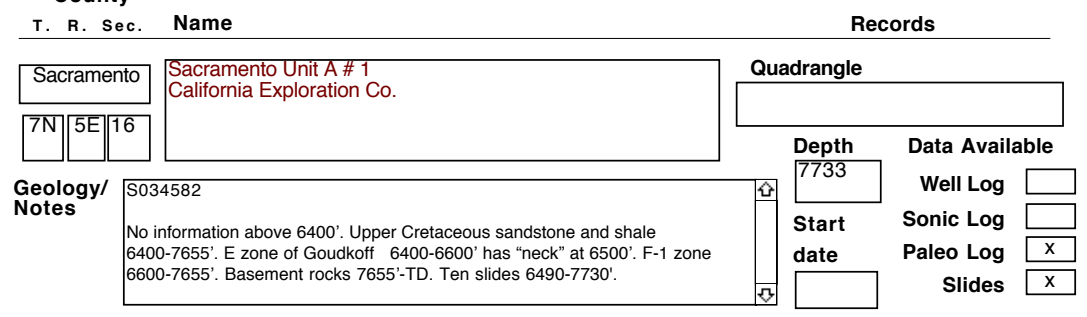

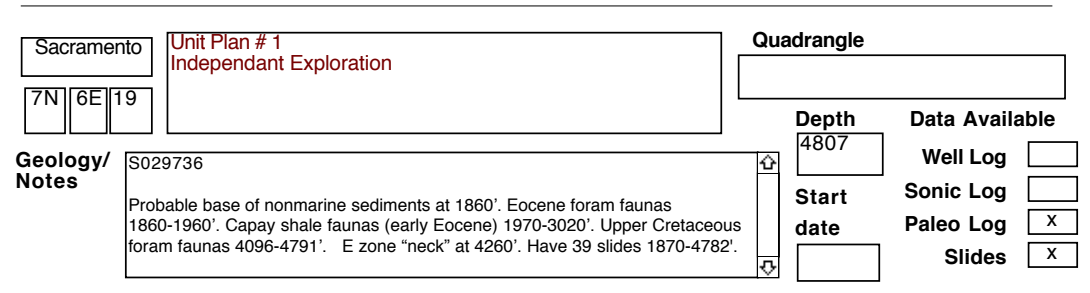

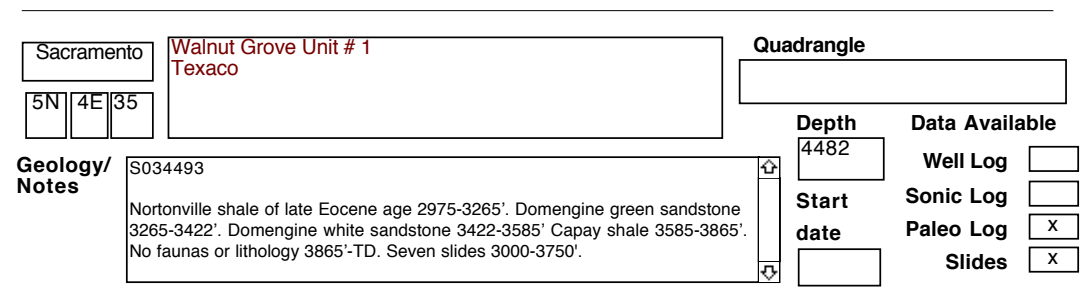

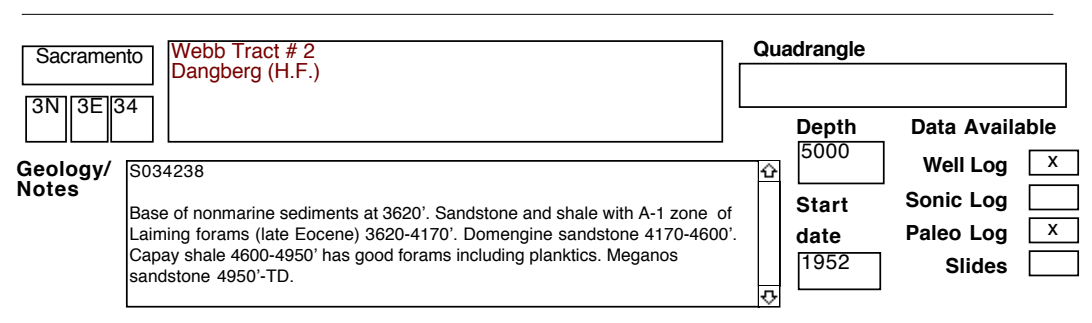

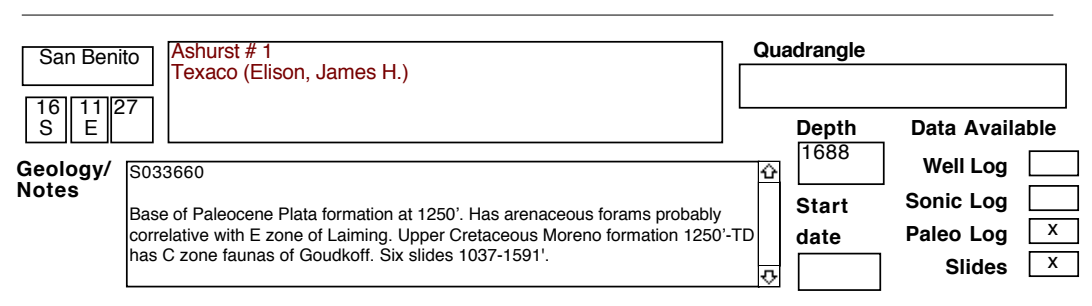


SELECTED OIL TEST WELLS IN CENTRAL CALIFORNIA DATABASE County

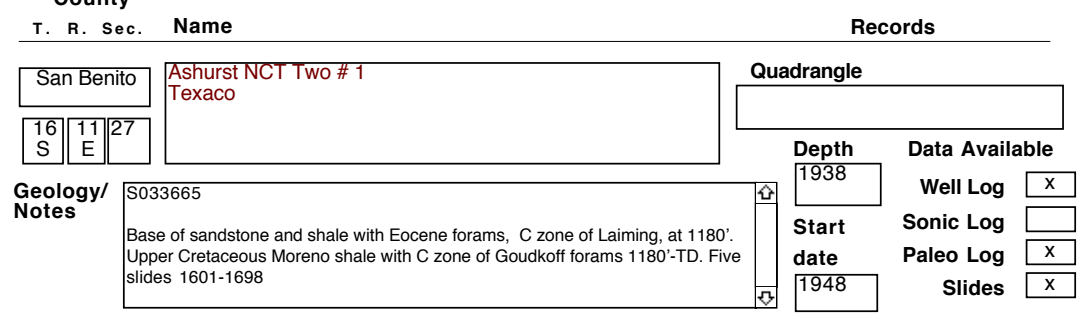

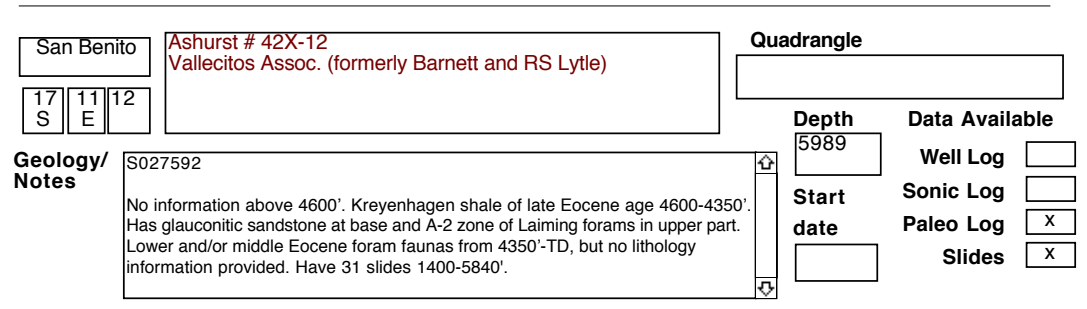

\begin{tabular}{l|l|l|l|l|l|l|}
\hline San Benito & $\begin{array}{l}\text { Ashurst \# 52A-33 } \\
\text { Oil California Corp. (formerly McKnight and Franco Western) }\end{array}$ \\
\hline
\end{tabular}

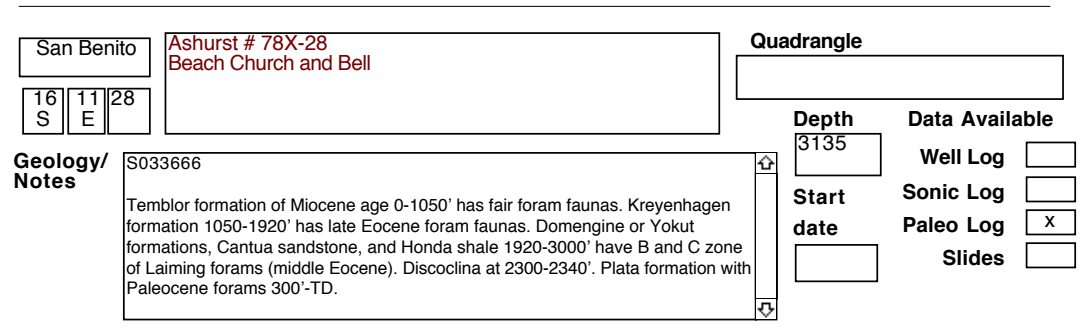

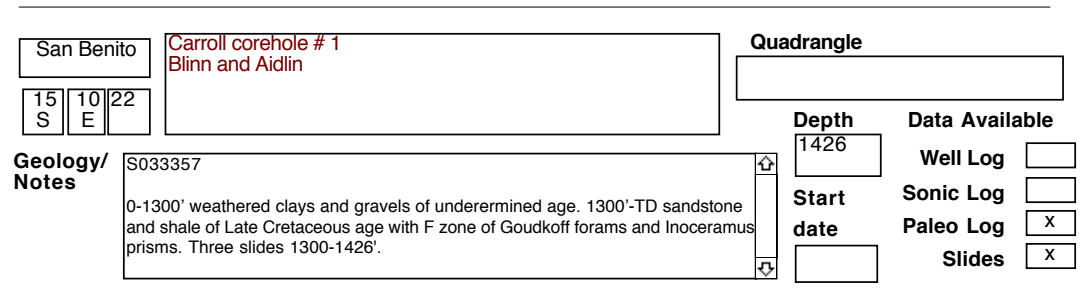


SELECTED OIL TEST WELLS IN CENTRAL CALIFORNIA DATABASE County

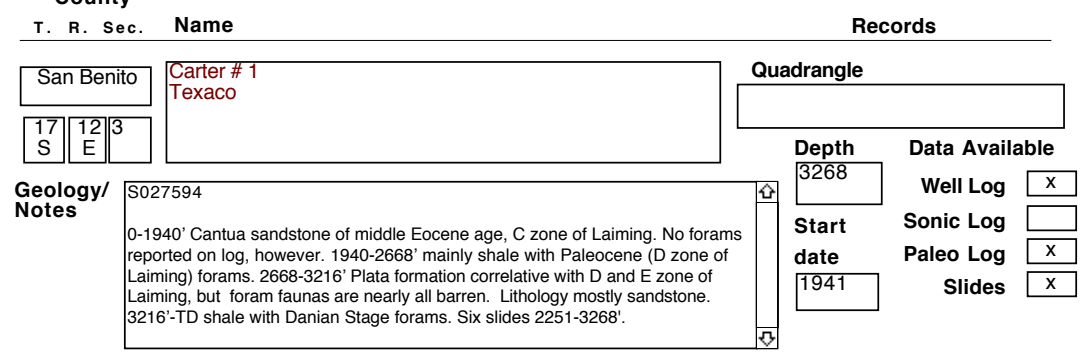

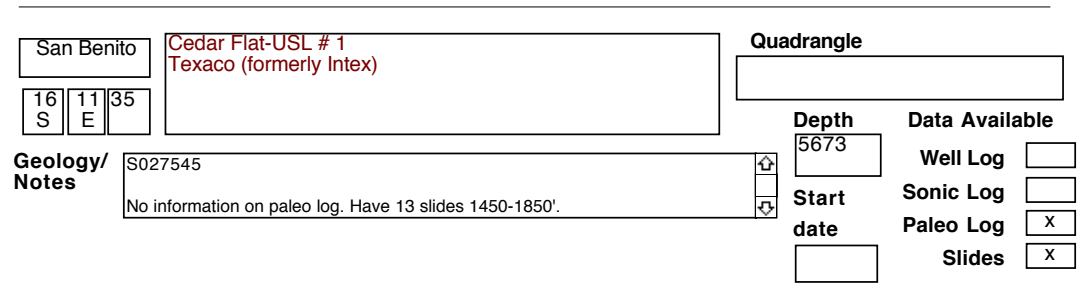

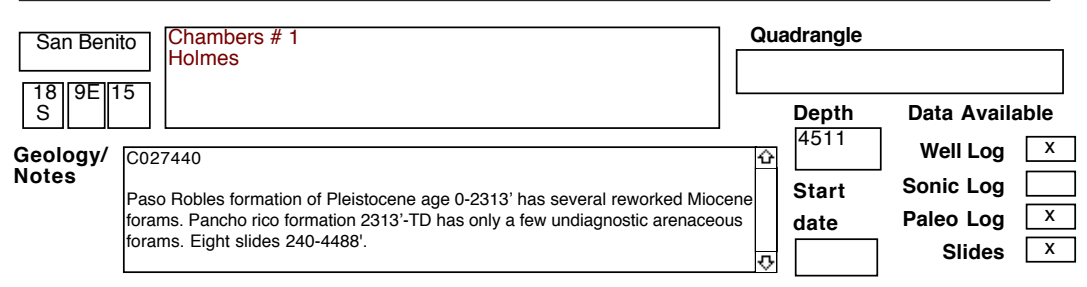

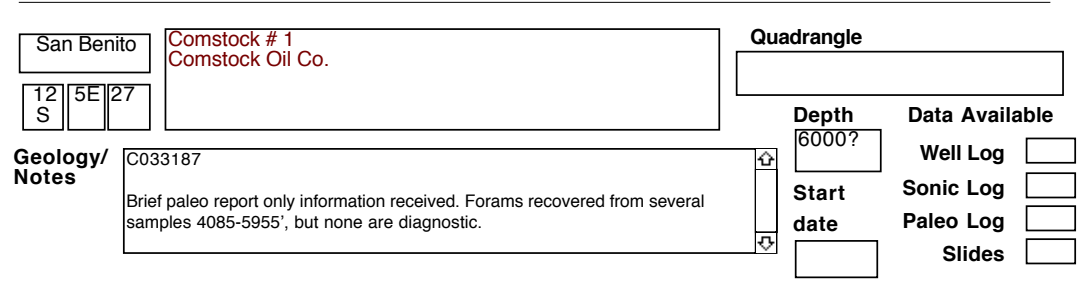

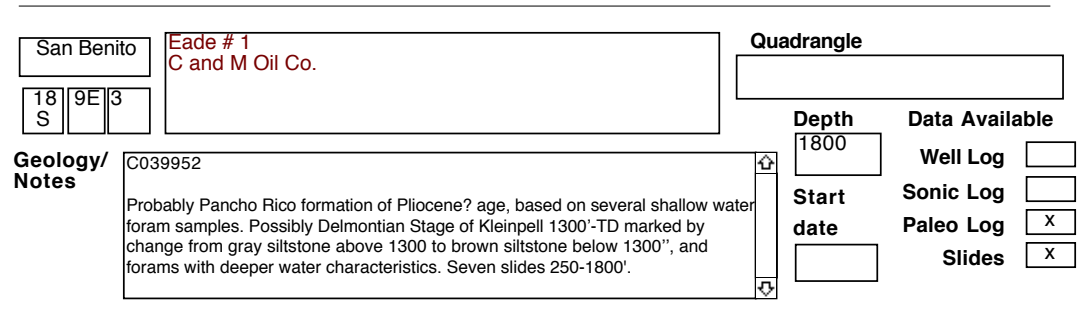


SELECTED OIL TEST WELLS IN CENTRAL CALIFORNIA DATABASE County

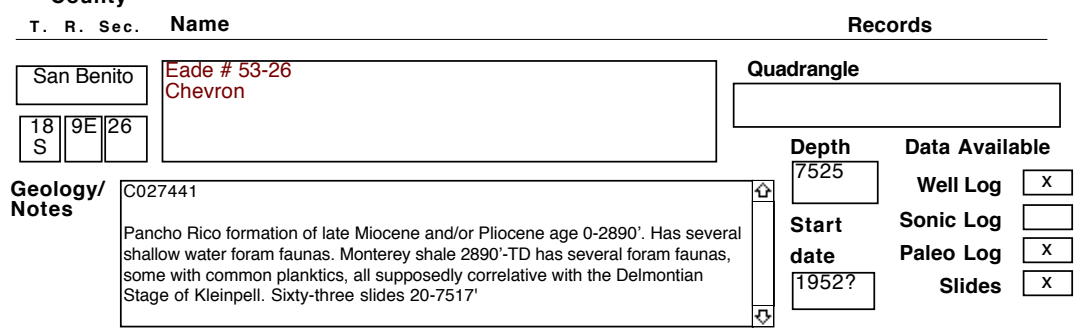

\begin{tabular}{l|l|l|l|l|l|l|}
\hline San Benito & $\begin{array}{l}\text { Fortier \# 1 } \\
\text { Fortier and Associates }\end{array}$ \\
\hline
\end{tabular}

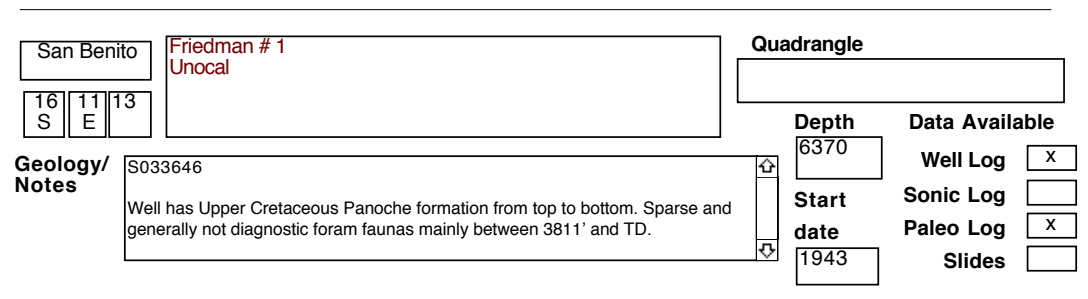

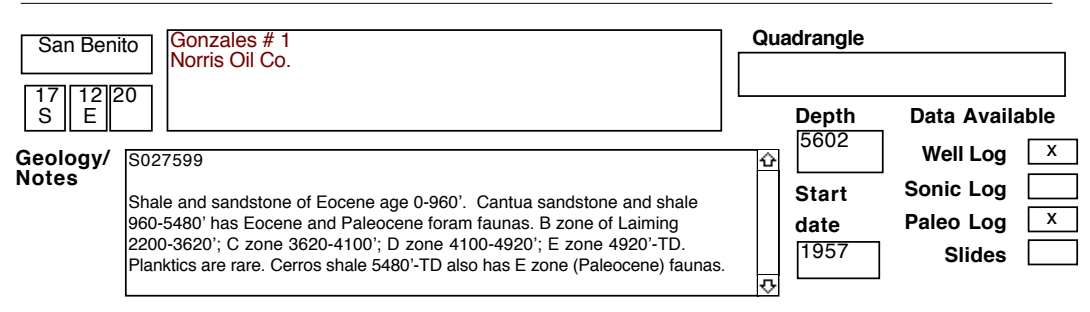

\begin{tabular}{|c|c|c|c|c|c|}
\hline San Benito & Hansen corehole \#1 & \multicolumn{4}{|c|}{ Quadrangle } \\
\hline \begin{tabular}{|l|l|l|}
17 & $8 \mathrm{E}$ & 34 \\
$\mathrm{~S}$ & & \\
\end{tabular} & & & Depth & Data Availab & \\
\hline $\begin{array}{l}\text { Geology/ } \\
\text { Notes }\end{array}$ & $\begin{array}{l}27033 \\
\text { ainly shallow water foram faunas } 0-800 \text { '. No lithology or formation information } \\
\text { vided. No samples below } 1400^{\prime} \text {. Granite at TD. Six slides 0-900'. Also have } \\
\text { other set of } 10 \text { slides } 20-840^{\prime} \text {. }\end{array}$ & 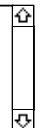 & $\begin{array}{l}\text { Start } \\
\text { date }\end{array}$ & $\begin{array}{r}\text { Well Log } \\
\text { Sonic Log } \\
\text { Paleo Log } \\
\text { Slides }\end{array}$ & $\frac{x}{x}$ \\
\hline
\end{tabular}


SELECTED OIL TEST WELLS IN CENTRAL CALIFORNIA DATABASE County

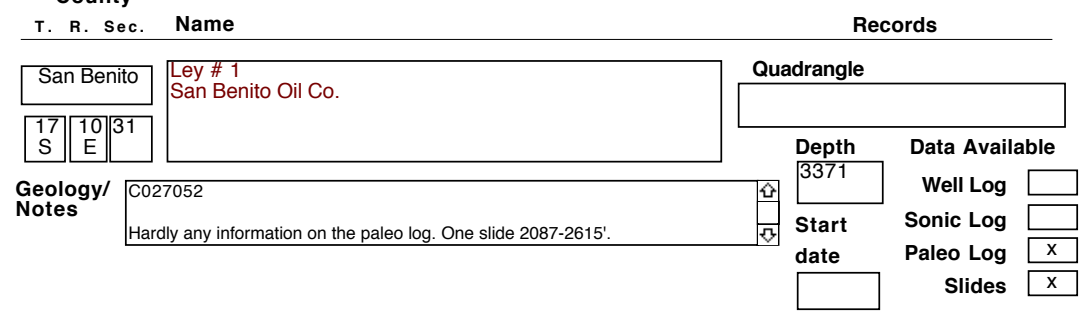

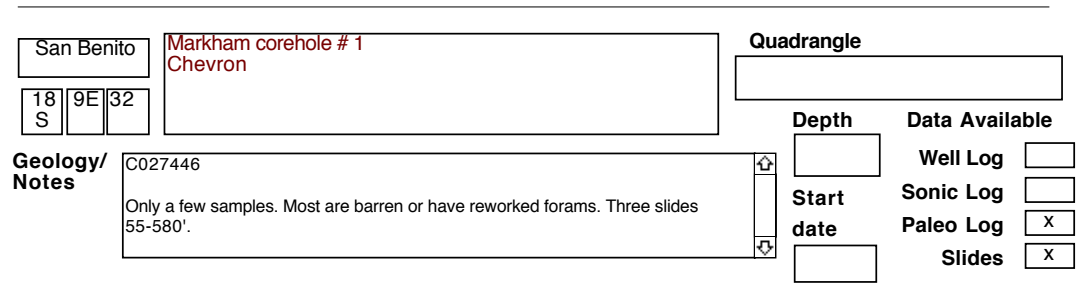

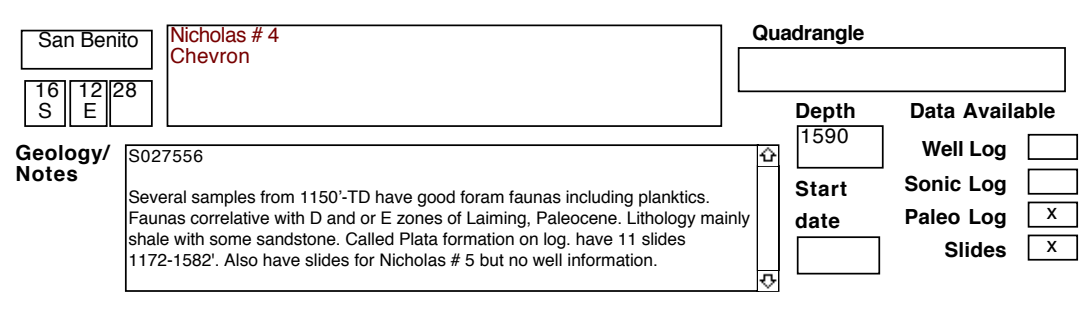

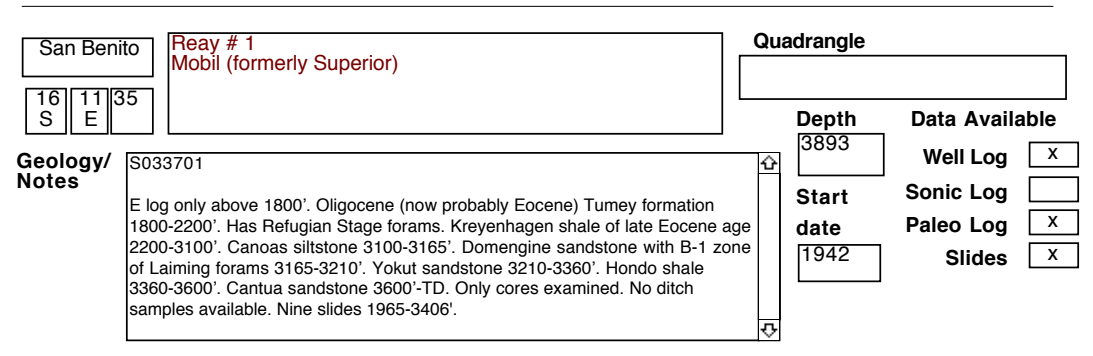

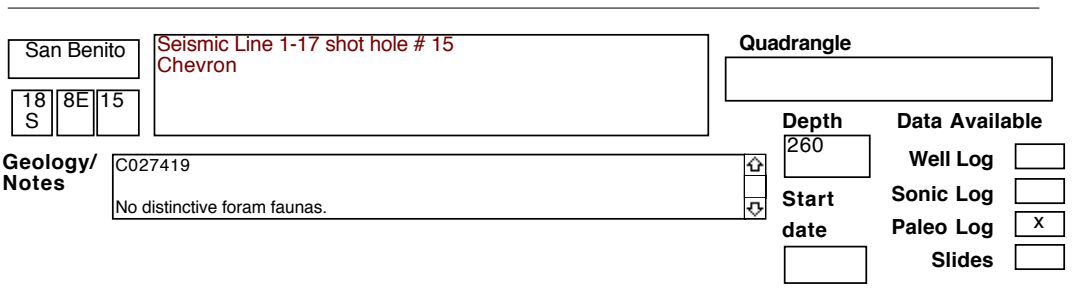


SELECTED OIL TEST WELLS IN CENTRAL CALIFORNIA DATABASE County

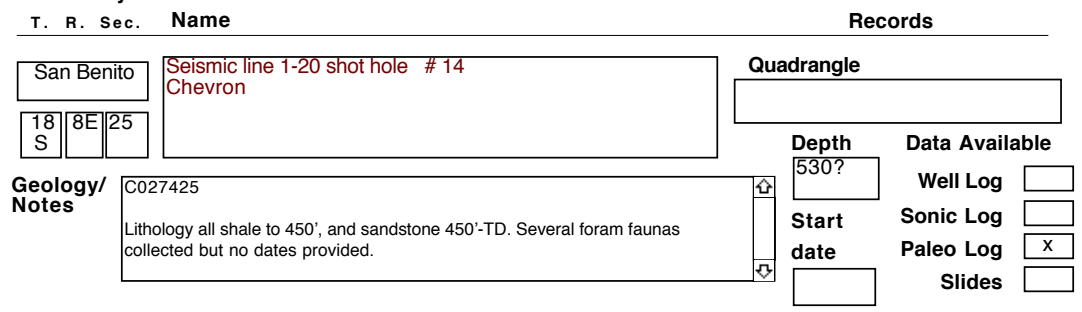

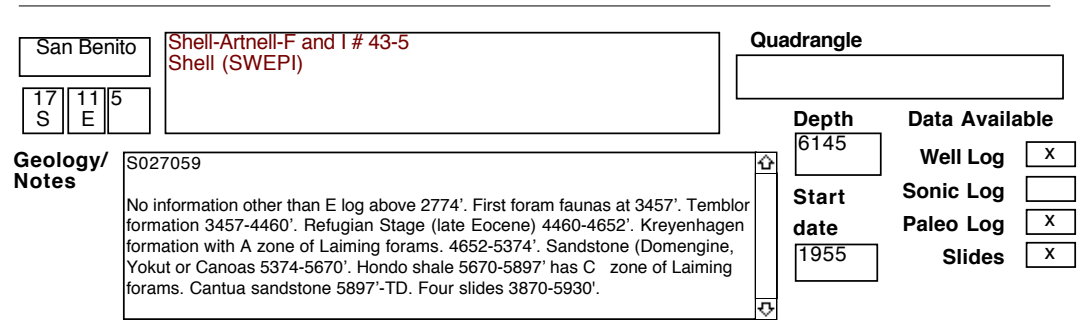

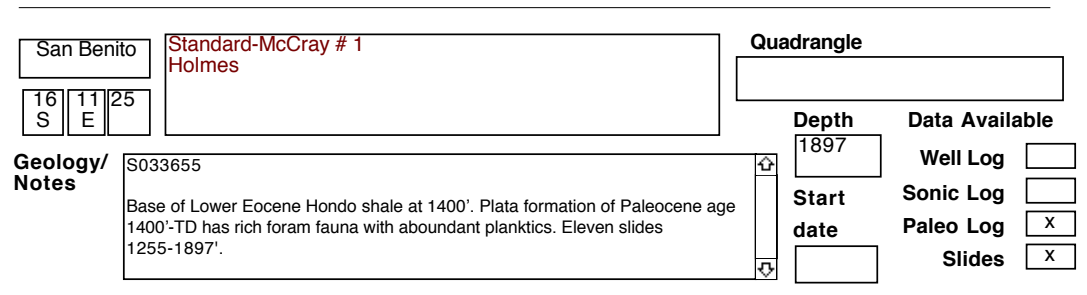

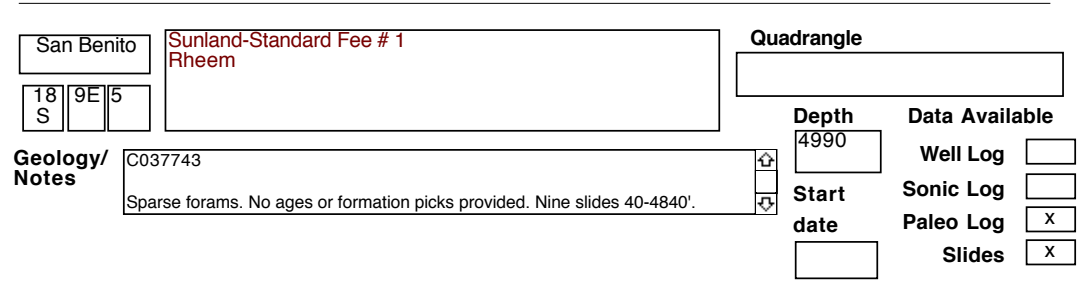

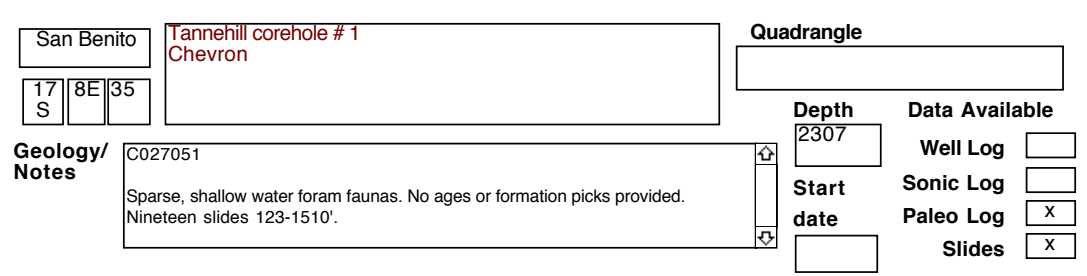


SELECTED OIL TEST WELLS IN CENTRAL CALIFORNIA DATABASE County

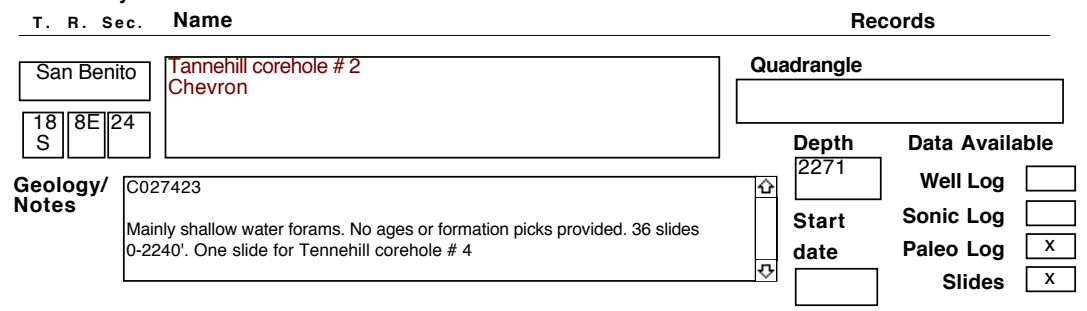

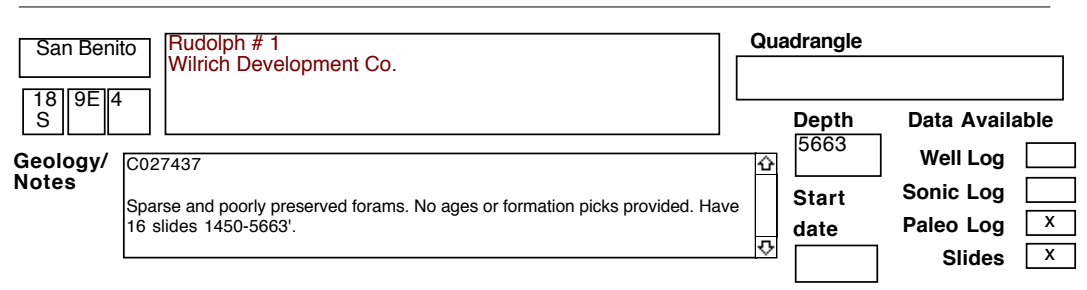

\begin{tabular}{l|l|l|l|l|l|}
\hline San Benito & Wright-USL \# 4-35 & \multicolumn{2}{l|}{ Quadrangle } \\
\hline & & & \\
\hline
\end{tabular}

\begin{tabular}{|c|c|c|c|c|}
\hline San Mateo & \multirow[t]{2}{*}{$\begin{array}{l}\text { C and V Exploration \# } 1 \text { (formerly Transsierra-Muller \# 2) } \\
\text { La Honda Petrol. Dev. }\end{array}$} & \multicolumn{3}{|c|}{$\begin{array}{r}\text { Quadrangle } \\
\text { Lahonda 7.5' }\end{array}$} \\
\hline \multicolumn{4}{|c|}{\begin{tabular}{|l|l|l|}
$7 S$ & 47
\end{tabular}} & \\
\hline \multirow{3}{*}{$\begin{array}{l}\text { Geology/ } \\
\text { Notes }\end{array}$} & 27188 & $\theta$ & & Well Log \\
\hline & scription of 4 cores only material received. & & Start & Sonic Log \\
\hline & & $\pi$ & & Slides \\
\hline
\end{tabular}

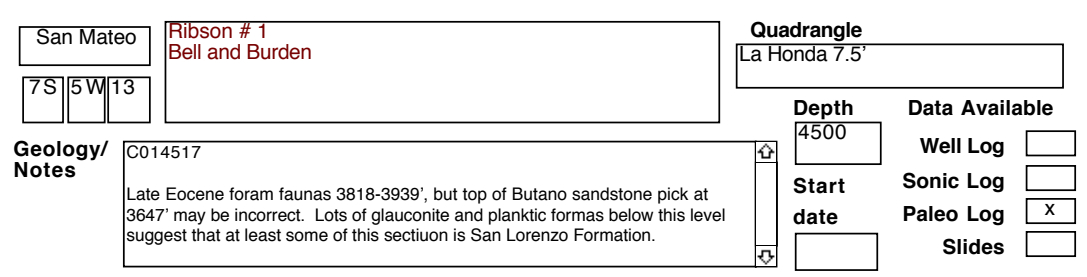




\section{SELECTED OIL TEST WELLS IN CENTRAL CALIFORNIA DATABASE}

County

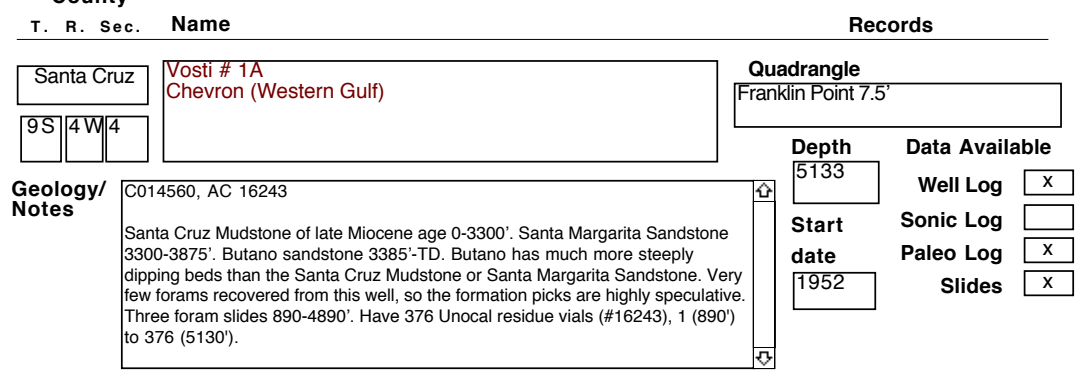

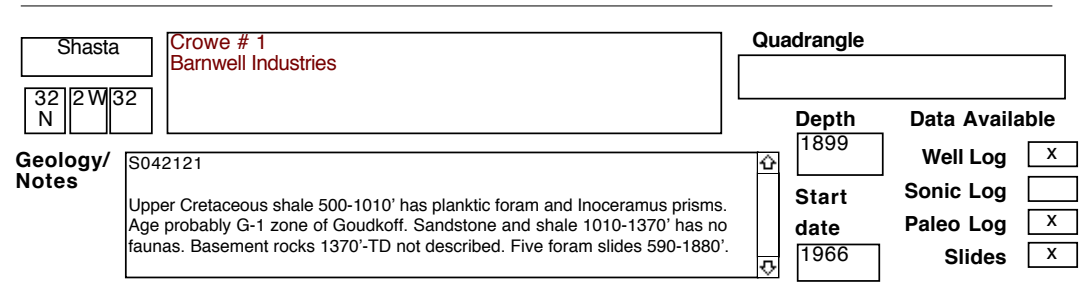

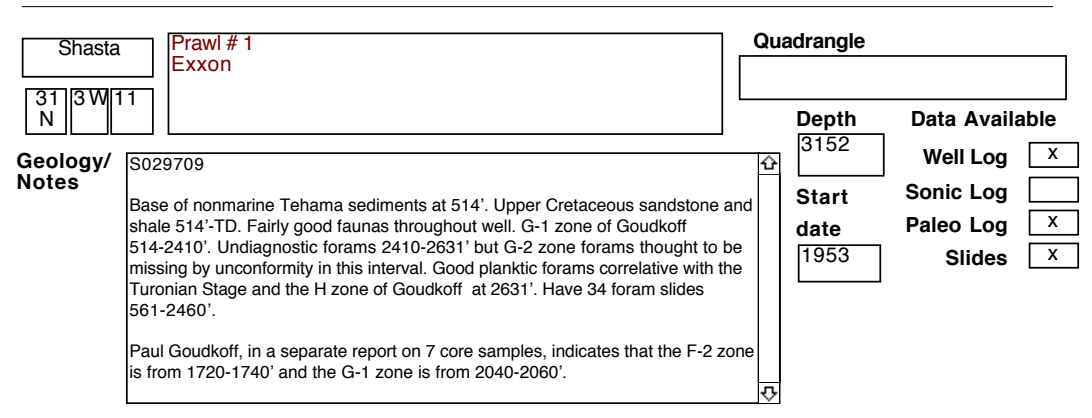

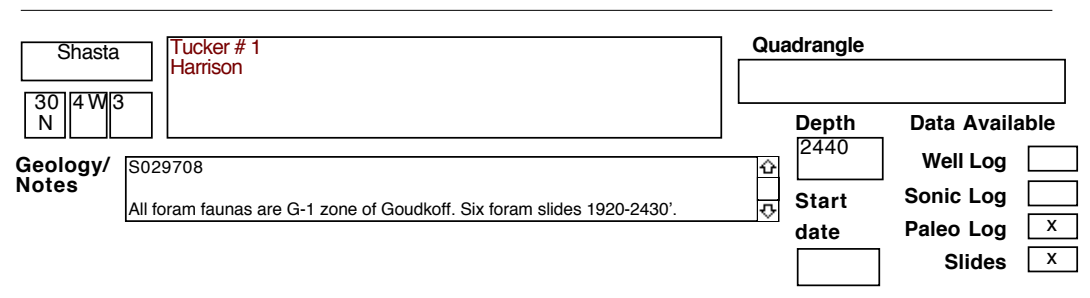

\begin{tabular}{|l|l|l|l|l|}
\hline Siskiyou & $\begin{array}{l}\text { Kuck \# 1-26 } \\
\text { Klamath Exploration }\end{array}$ \\
Geology/ & C301400 \\
Notes & $\begin{array}{l}\text { Petrography report indicates either lithic arenites 2930-5270' with grains of schist } \\
\text { and volcanics or feldspathic sandstones with fewer lithic clasts. In the interval } \\
\text { 4040-5120', the sandstones are interbedded with various altered volcanic rocks. } \\
\text { Schist was encountered first at 5360'. The sandstones are highly indurated. } \\
\text { Photomicrographs of the sandstones and volcanic rocks are provided. }\end{array}$ \\
\hline
\end{tabular}


SELECTED OIL TEST WELLS IN CENTRAL CALIFORNIA DATABASE County

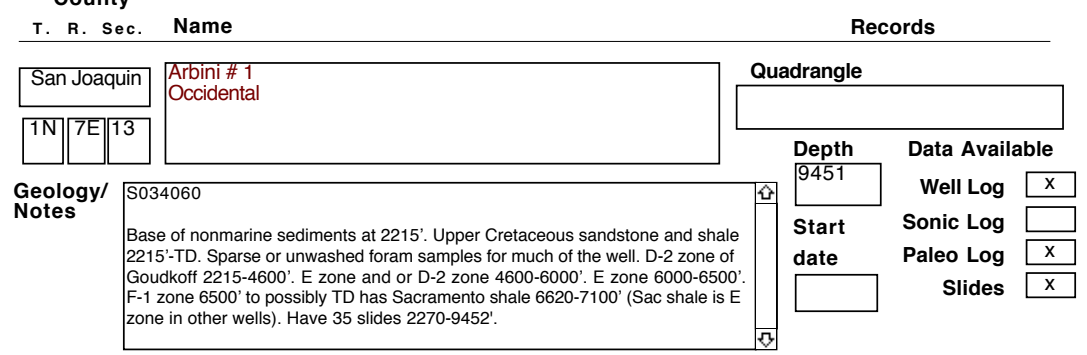

\begin{tabular}{|c|c|c|c|c|c|}
\hline San Joaquin & \multirow{2}{*}{$\begin{array}{l}\text { Airport Western \# } 1 \\
\text { McFarland Energy }\end{array}$} & \multicolumn{4}{|c|}{ Quadrangle } \\
\hline \begin{tabular}{|l|l|l|}
$1 \mathrm{~N}$ & $6 \mathrm{E}$ & 24 \\
\end{tabular} & & \multirow{2}{*}{\multicolumn{2}{|c|}{$\begin{array}{l}\text { Depth } \\
8790\end{array}$}} & \multicolumn{2}{|c|}{ Data Available } \\
\hline \multirow{3}{*}{$\begin{array}{l}\text { Geology/ } \\
\text { Notes }\end{array}$} & 43596 & & & Well Log & \\
\hline & rmation available only for the interval $7700-8700^{\prime}$. Sparse foram fauna and & & Start & Sonic Log & \\
\hline & 0-8790'. & $\pi$ & & Slides & $\mathrm{X}$ \\
\hline
\end{tabular}

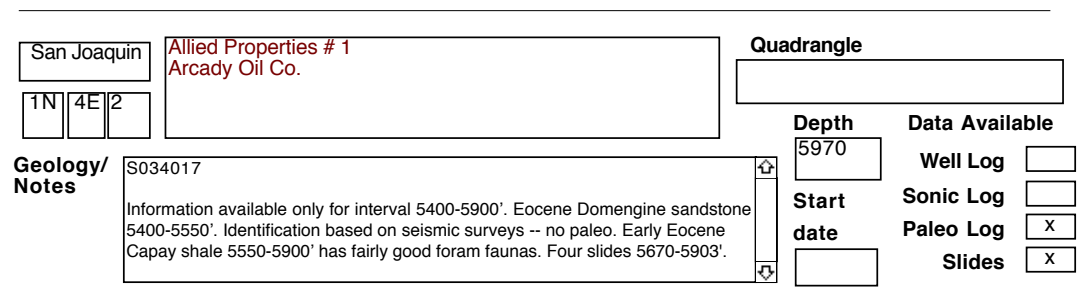

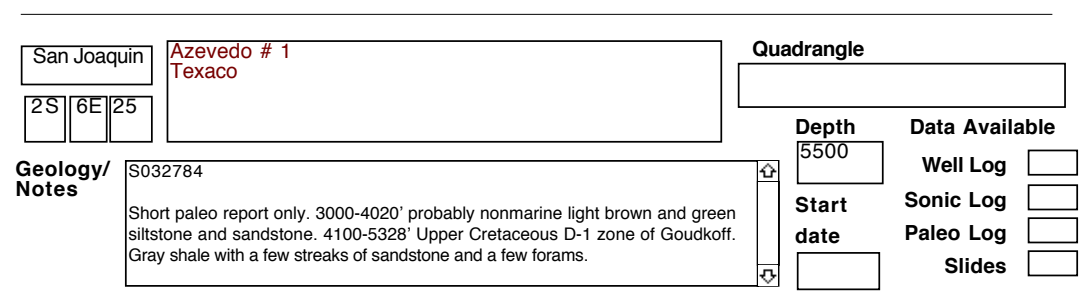

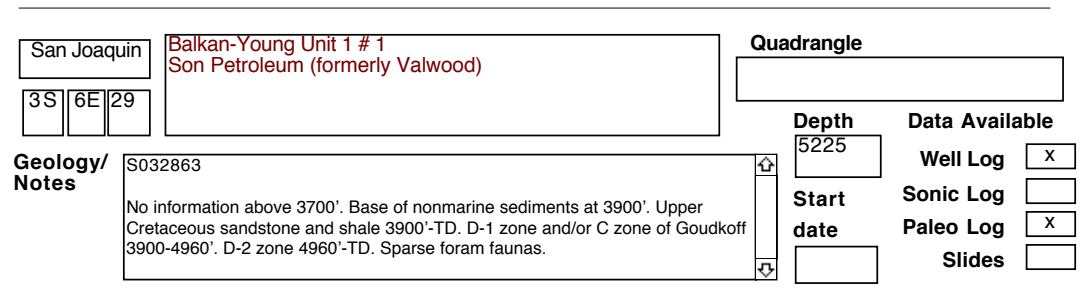


SELECTED OIL TEST WELLS IN CENTRAL CALIFORNIA DATABASE

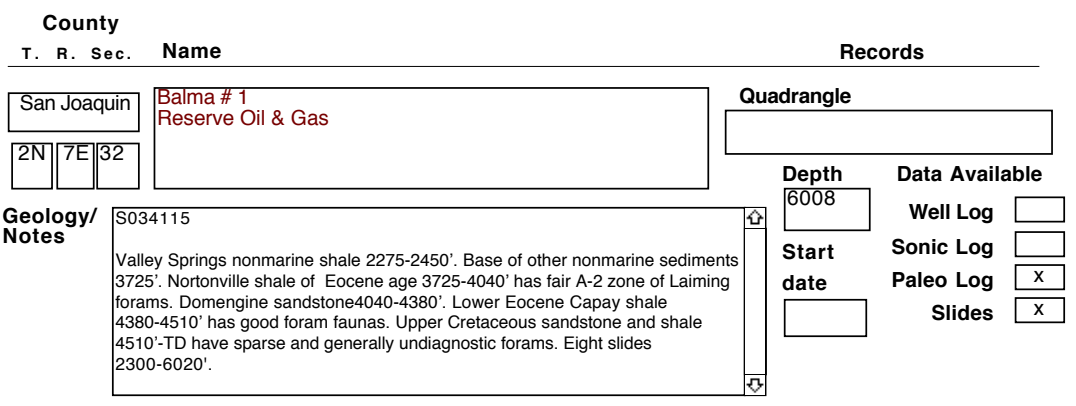

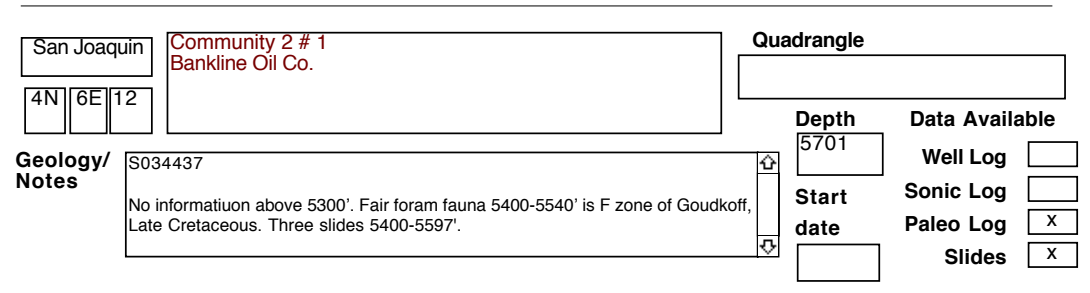

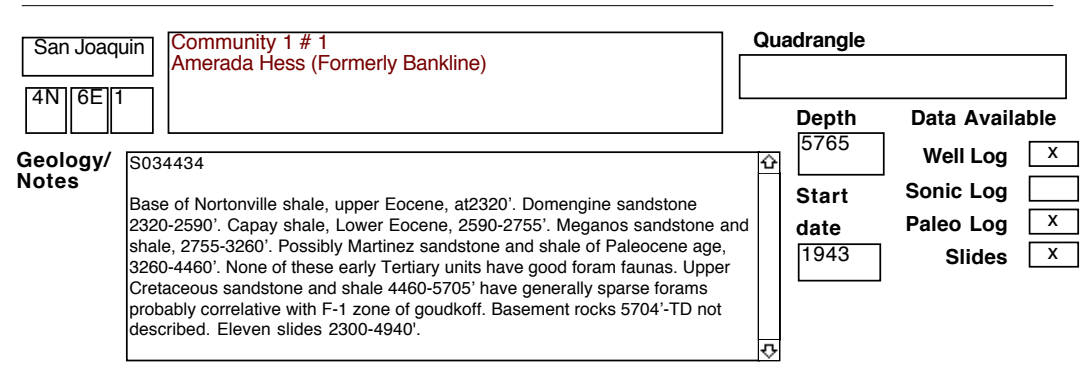

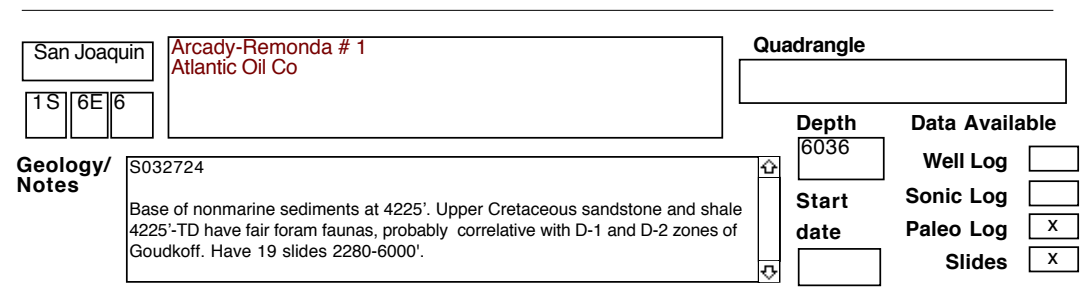

\begin{tabular}{l|l|l|l|l|l|l|}
\hline San Joaquin & $\begin{array}{l}\text { Bastian \#1 } \\
\text { Sunray }\end{array}$ \\
\hline
\end{tabular}


SELECTED OIL TEST WELLS IN CENTRAL CALIFORNIA DATABASE

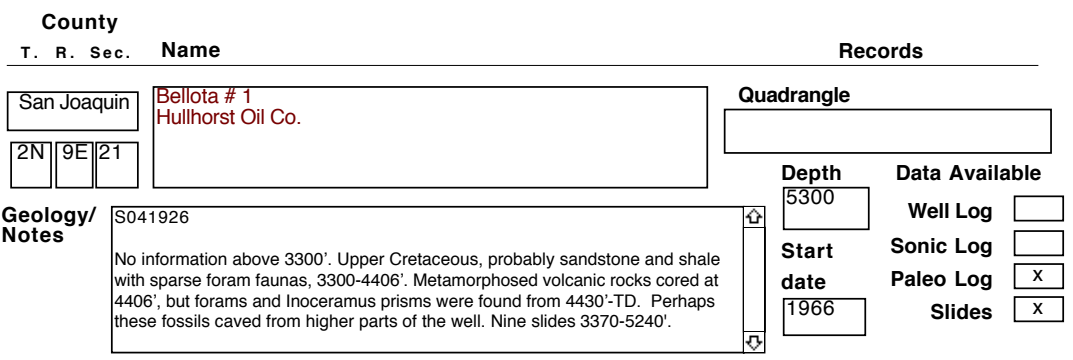

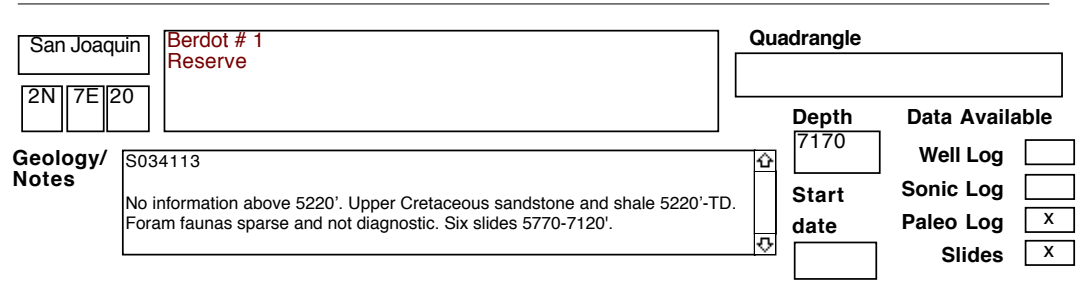

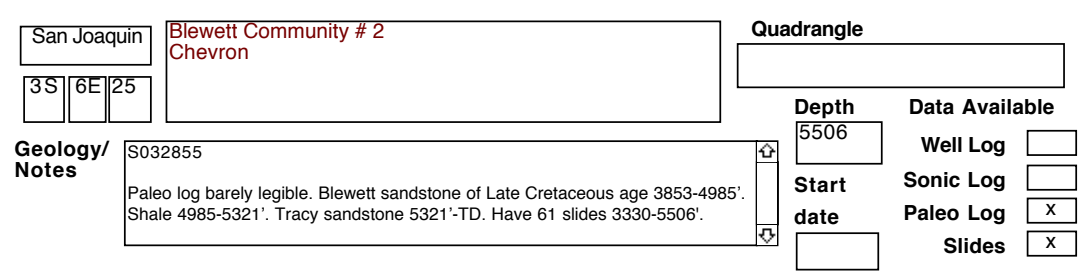

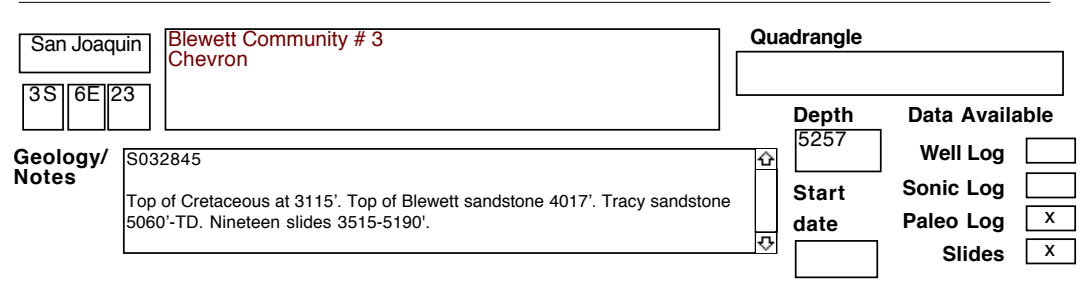

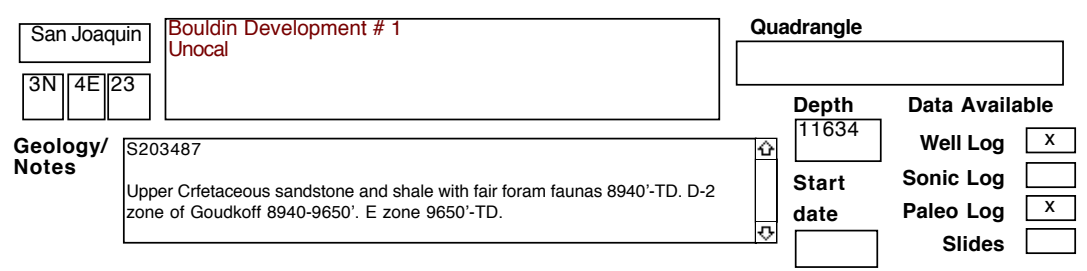


SELECTED OIL TEST WELLS IN CENTRAL CALIFORNIA DATABASE County

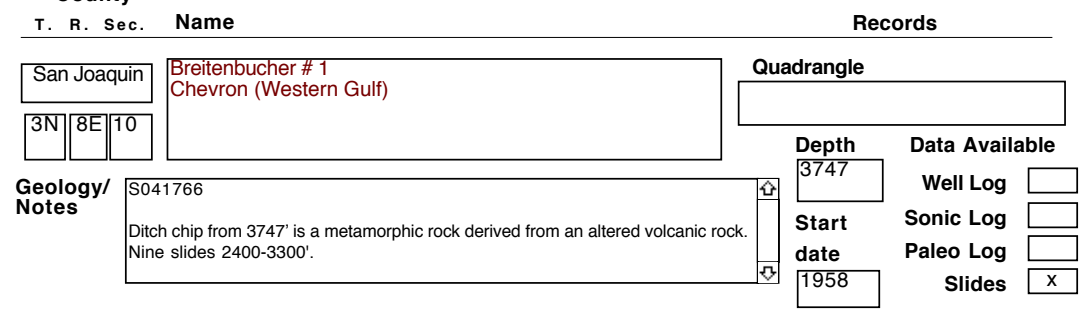

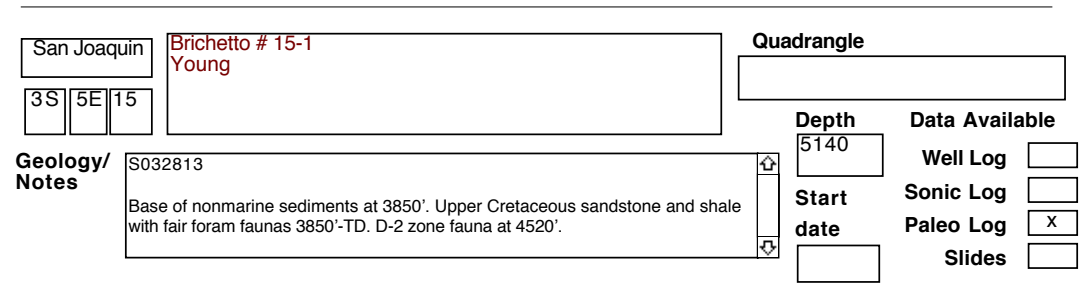

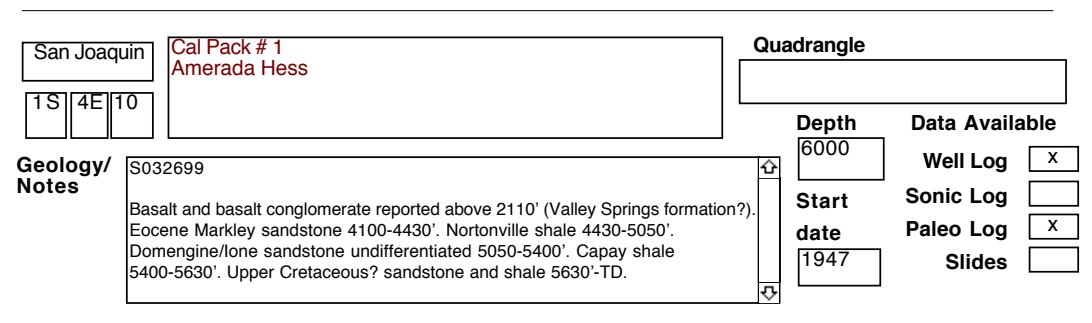

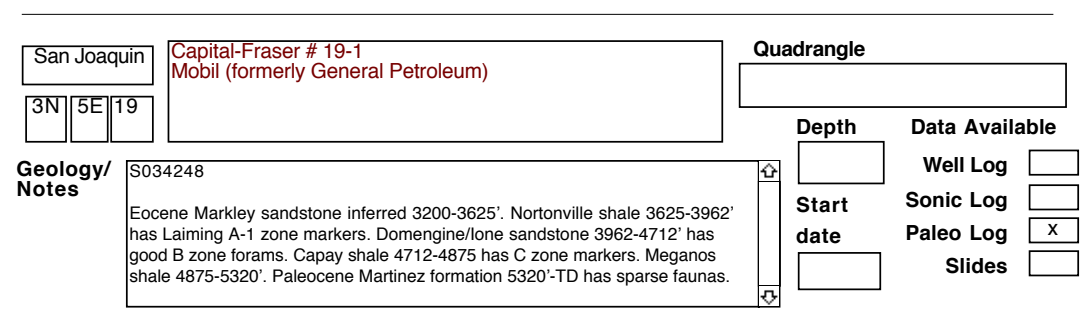

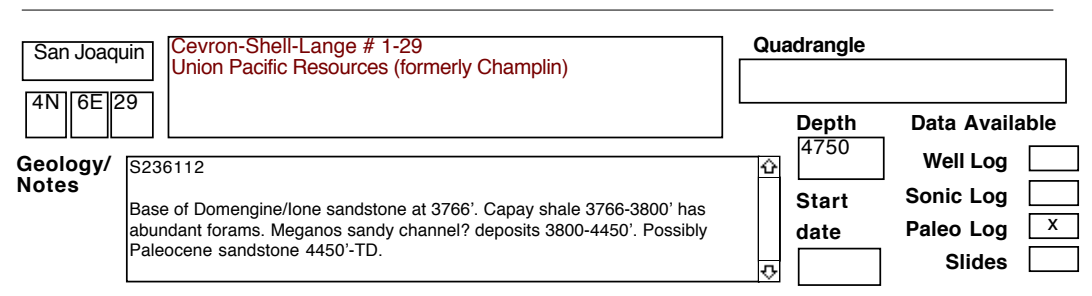




\section{SELECTED OIL TEST WELLS IN CENTRAL CALIFORNIA DATABASE}

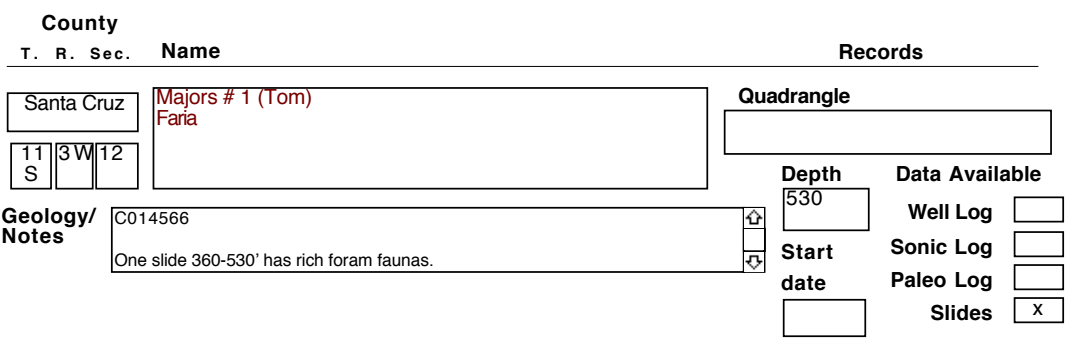

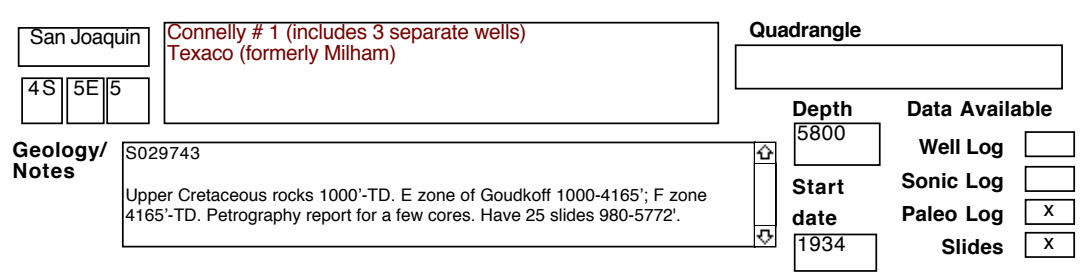

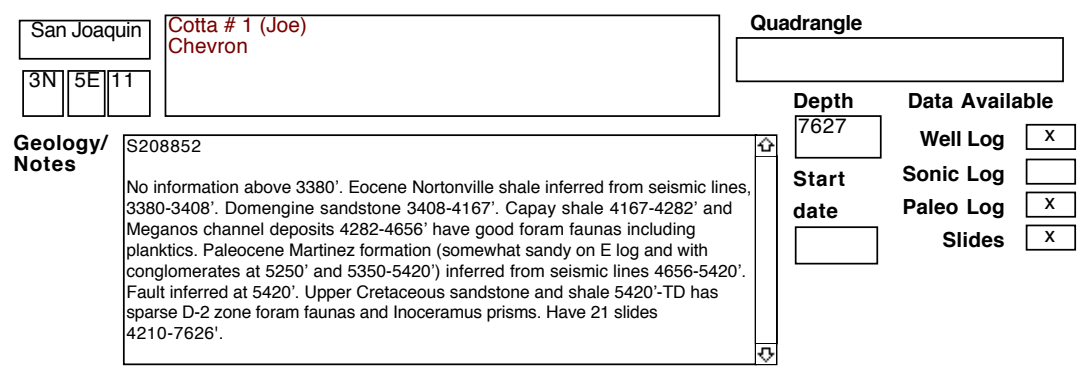

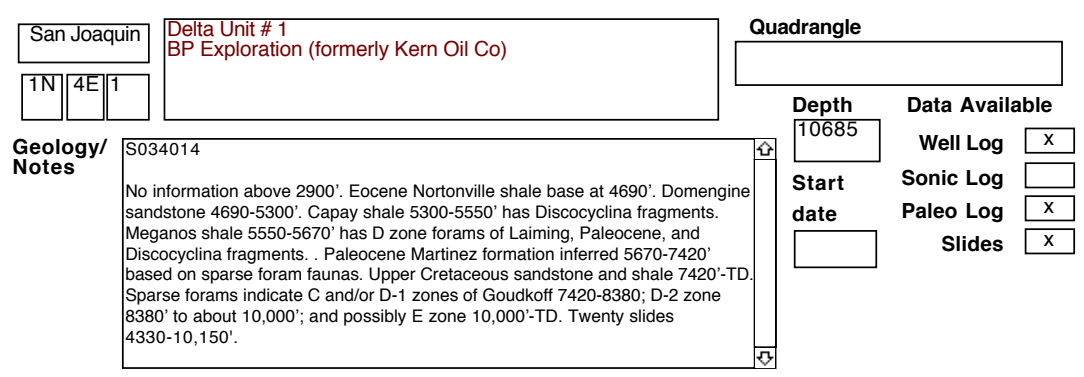

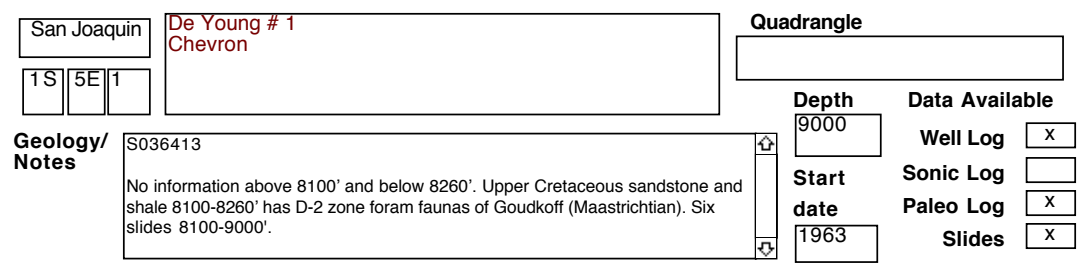


SELECTED OIL TEST WELLS IN CENTRAL CALIFORNIA DATABASE County

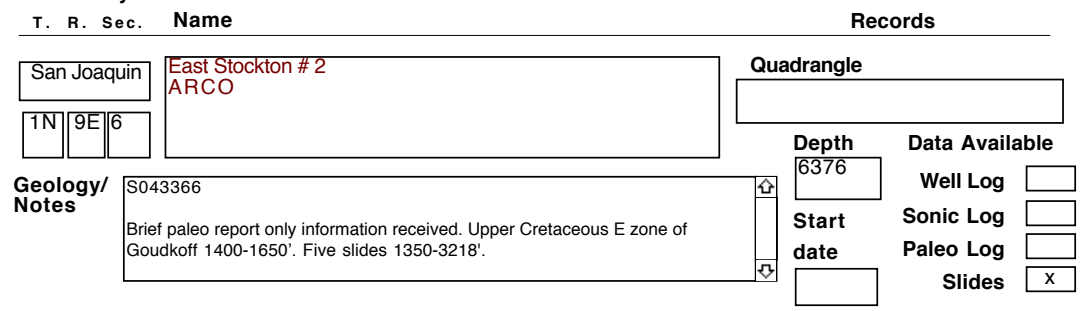

\begin{tabular}{|c|c|c|c|c|}
\hline San Joaqu & East Stockton \#1 & \multicolumn{3}{|l|}{ Quadrangle } \\
\hline $1 \mathrm{~N} 8 \mathrm{E}$ & & \multirow{2}{*}{$\begin{array}{l}\text { Depth } \\
8544\end{array}$} & \multicolumn{2}{|c|}{ Data Available } \\
\hline \multirow{4}{*}{$\begin{array}{l}\text { Geology/ } \\
\text { Notes }\end{array}$} & S034064 & & \multirow{4}{*}{$\begin{array}{r}\text { Well Log } \\
\text { Sonic Log } \\
\text { Paleo Log } \\
\text { Slides }\end{array}$} & $\mathrm{x}$ \\
\hline & Base of nonmarine sediments at 1760'. Upper Cretaceous sandstone and shale & Start & & \\
\hline & 1760 '-8533'. Quartz diorite $8533^{\prime}$-TD. Most of the foram samples are not & date & & $\mathrm{x}$ \\
\hline & $\begin{array}{l}\text { lage-diagnostic, but faunas correlative with the D-2, E, and F-1 zones of Goudkoff } \\
\text { are present. } 88 \text { cores and } 56 \text { picked slides are reported for this well. } \\
\text { Nannoplankton samples from several cores were either barren or not diagnostic. } \\
\text { Have foram check list with } 62 \text { species. Thirty-two slides } 1832-8532 \text { '. }\end{array}$ & 1944 & & $\mathrm{x}$ \\
\hline
\end{tabular}

\begin{tabular}{|c|c|c|c|c|c|}
\hline San Joaquir & Holly Sugar \# B-1 & Qu & adrangle & & \\
\hline \begin{tabular}{|l|l|l|}
$2 S$ & \\
\end{tabular} & & \multirow{2}{*}{\multicolumn{2}{|c|}{$\begin{array}{l}\text { Depth } \\
5707\end{array}$}} & \multicolumn{2}{|c|}{ Data Available } \\
\hline \multirow{4}{*}{$\begin{array}{l}\text { Geology/ } \\
\text { Notes }\end{array}$} & 32748 & & & Well Log & $x$ \\
\hline & se of nonmarine sediments at $3840^{\prime}$. Sandstone and shale of Paleocene & \multirow{3}{*}{\multicolumn{2}{|c|}{$\begin{array}{l}\begin{array}{l}\text { Start } \\
\text { date }\end{array} \\
1961 ?\end{array}$}} & Sonic Log & \\
\hline & t/or Late Cretaceous age $3940^{\prime}-\mathrm{TD}$ have poor foram faunas. Five slides & & & Paleo Log & $x$ \\
\hline & & & & Slides & $\mathrm{x}$ \\
\hline
\end{tabular}

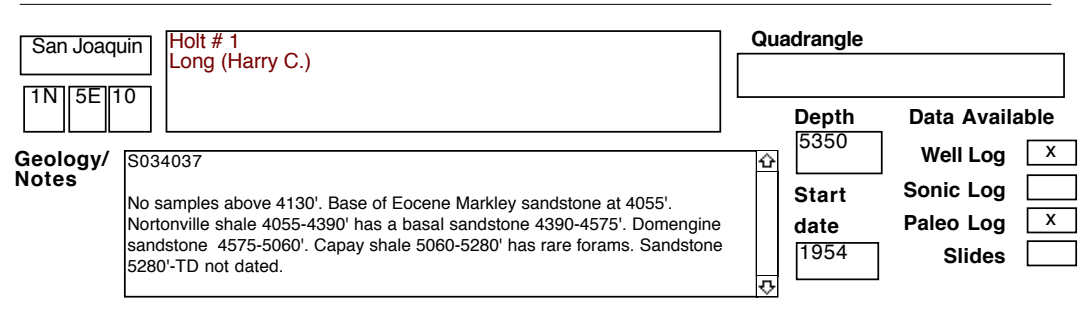

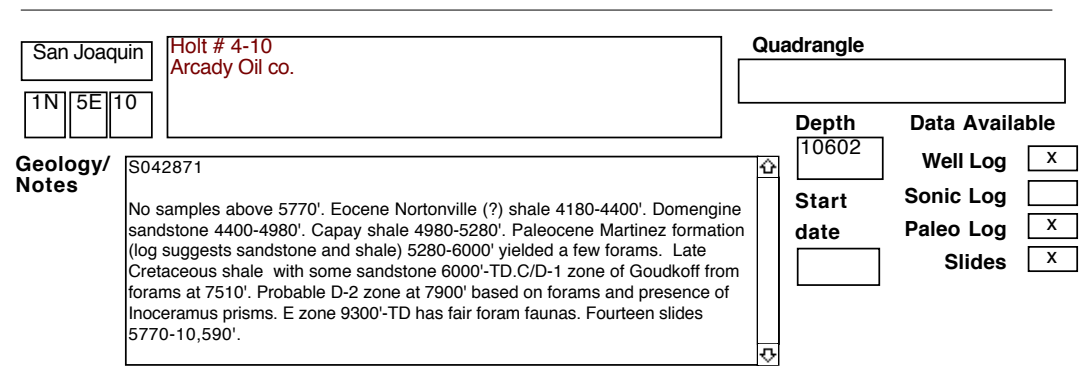


SELECTED OIL TEST WELLS IN CENTRAL CALIFORNIA DATABASE County

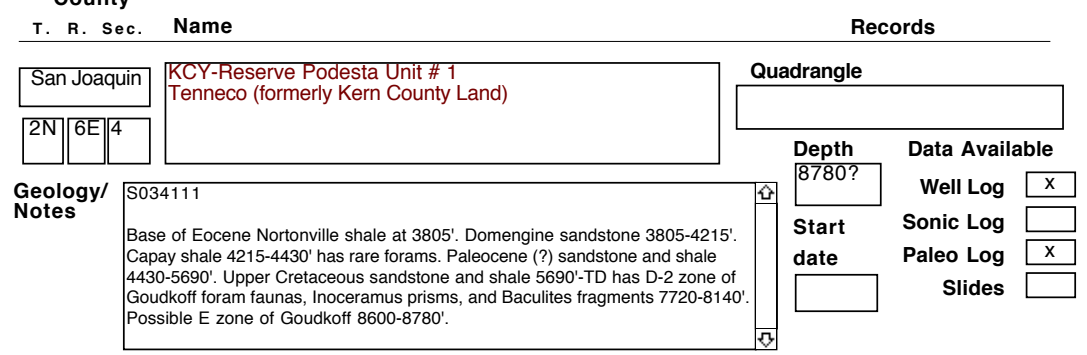

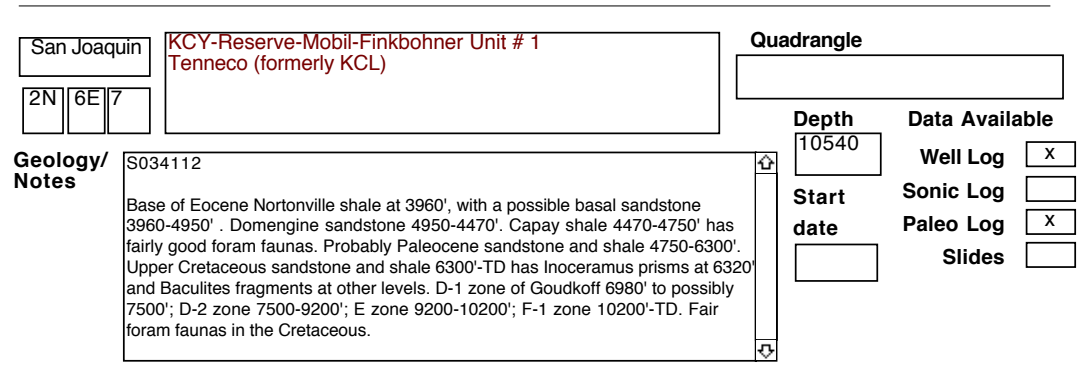

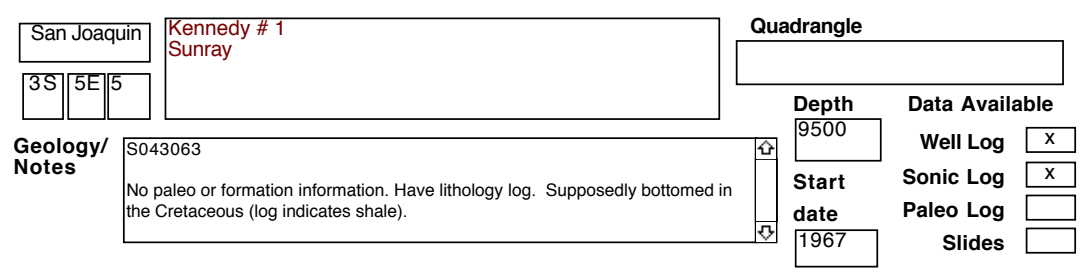

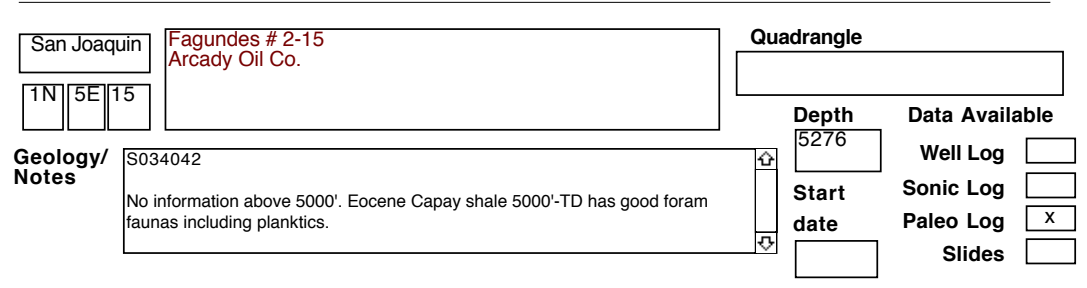

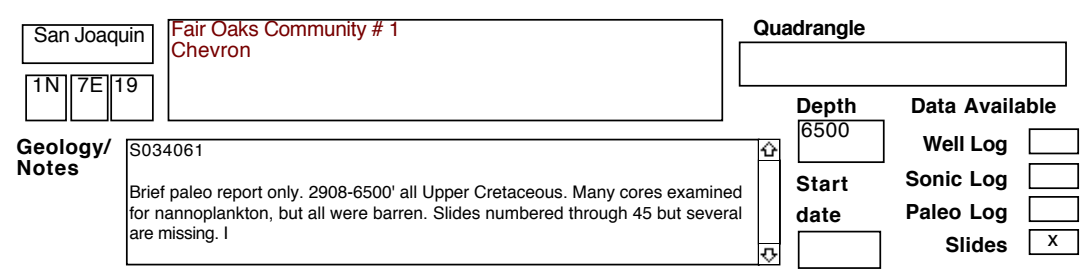




\section{SELECTED OIL TEST WELLS IN CENTRAL CALIFORNIA DATABASE}

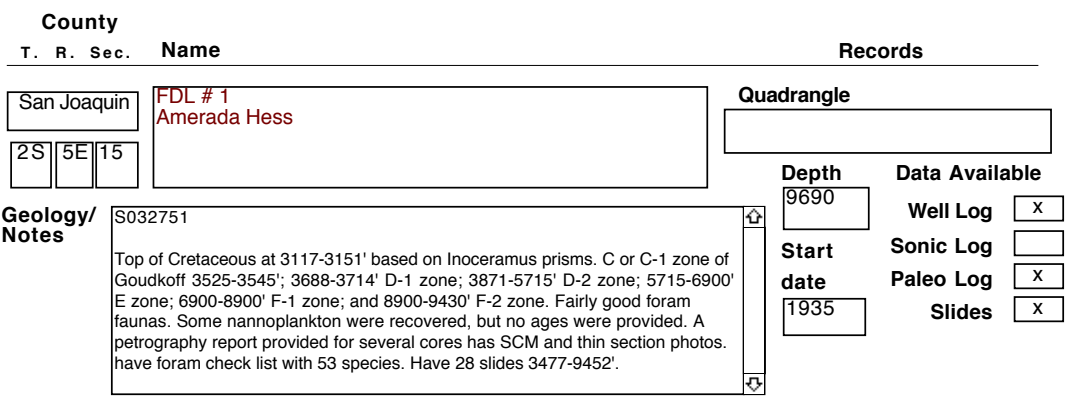

\begin{tabular}{l|l|l|l|l|l|}
\hline San Joaquin & $\begin{array}{l}\text { Ferguson \# 1 (W. C.) } \\
\text { Marathon (formerly Ohio Oil Co.) }\end{array}$ & \multicolumn{2}{l|}{ Quadrangle } \\
\hline
\end{tabular}

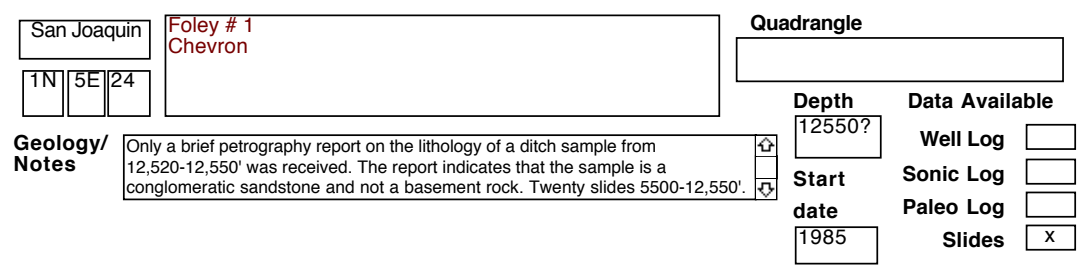

\begin{tabular}{l|l|l|l|l|}
\hline San Joaquin & $\begin{array}{l}\text { G andP corehole \# 1 } \\
\text { Amerada Hess }\end{array}$ \\
\hline
\end{tabular}

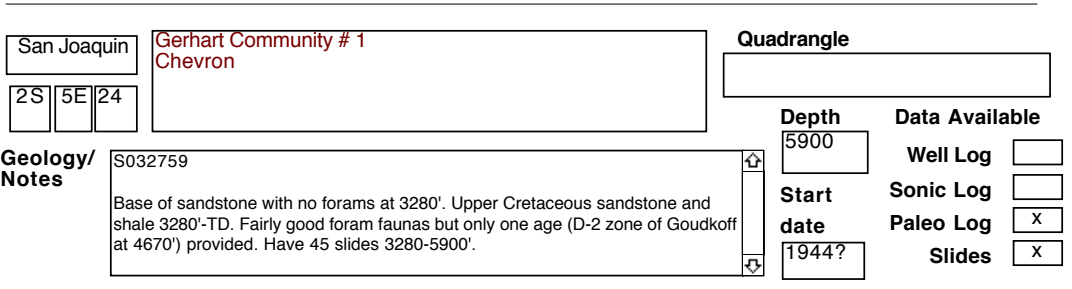


SELECTED OIL TEST WELLS IN CENTRAL CALIFORNIA DATABASE County

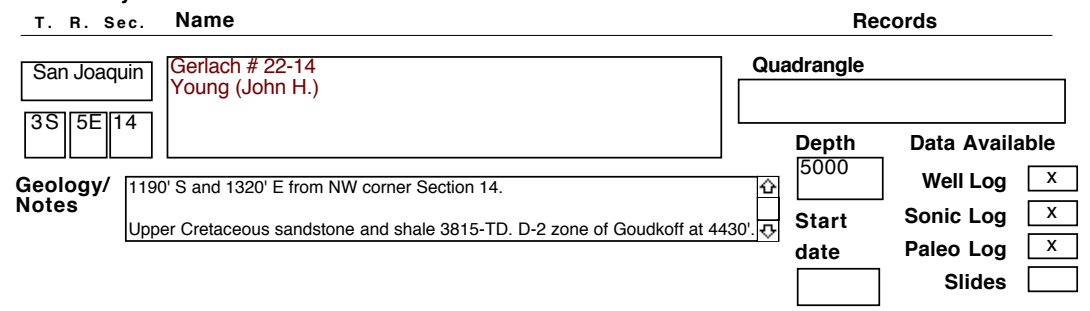

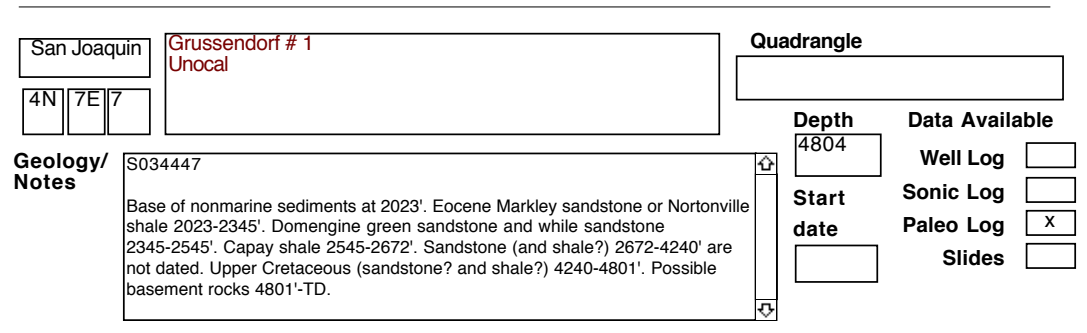

\begin{tabular}{|c|c|c|c|c|}
\hline San Joaquil & in Lathrop Uniot B \# 5 & \multirow{3}{*}{$\begin{array}{l}\text { Depth } \\
12787\end{array}$} & & \\
\hline \begin{tabular}{|l|l|l|}
$1 S$ & $6 E$ \\
\end{tabular} & & & \multicolumn{2}{|c|}{ Data Available } \\
\hline \multirow{4}{*}{$\begin{array}{l}\text { Geology/ } \\
\text { Notes }\end{array}$} & \multirow{4}{*}{$\begin{array}{l}\text { S032727 } \\
\text { No information above 6370'. Upper Cretaceous shale and sandstone 6430'-TD. E } \\
\text { zone of Goudkoff foram faunas 6370-9950'. F and G-1 zones 9950'-TD. Fair foram } \\
\text { faunas. Have } 29 \text { slides } 6370-12,610^{\prime} \text {. }\end{array}$} & & Well Log & $x$ \\
\hline & & \multirow{3}{*}{$\begin{array}{l}\text { Start } \\
\text { date }\end{array}$} & Sonic Log & \\
\hline & & & Paleo Log & $\mathrm{x}$ \\
\hline & & & Slides & $\mathrm{x}$ \\
\hline
\end{tabular}

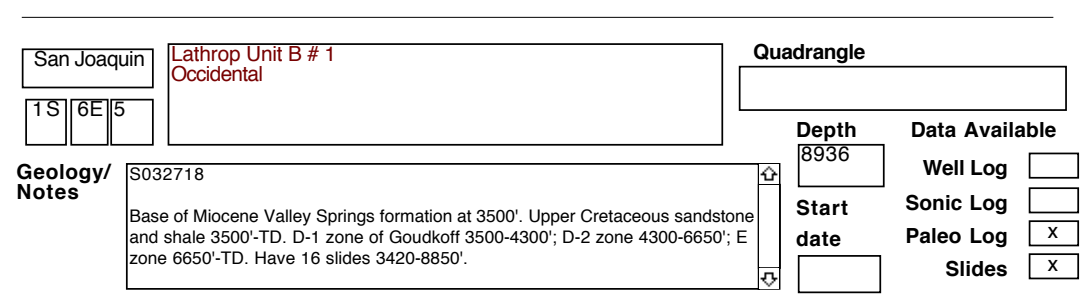

\begin{tabular}{l|l|l|l|l|l|}
\hline San Joaquin & $\begin{array}{l}\text { Lawrence and Stevens \# 1 } \\
\text { Texaco }\end{array}$ & \multicolumn{2}{|l|}{ Quadrangle } \\
\hline
\end{tabular}


SELECTED OIL TEST WELLS IN CENTRAL CALIFORNIA DATABASE County

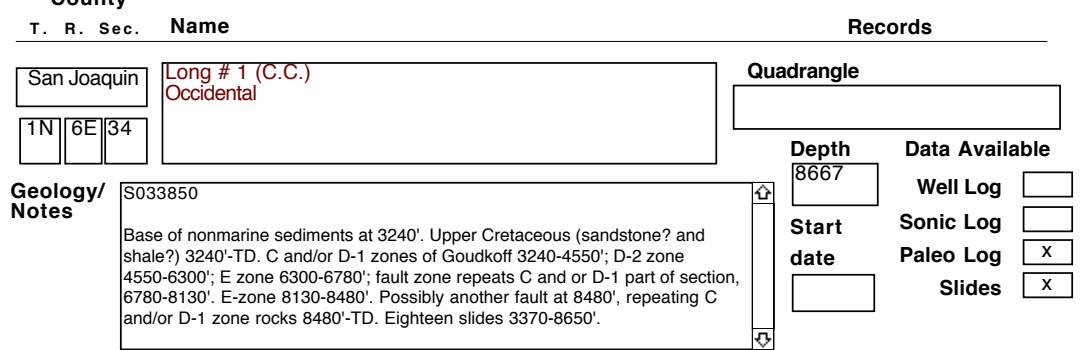

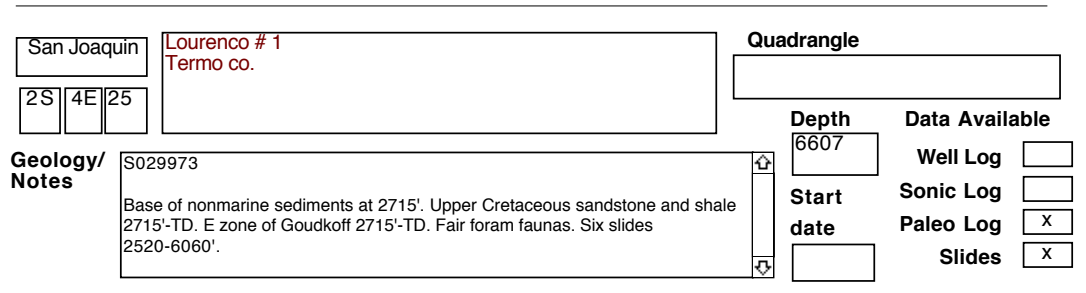

\begin{tabular}{l|l|l|l|l|l|l|l|}
\hline San Joaquin & $\begin{array}{l}\text { Martenet \# 25-2 } \\
\text { Amerada Hess }\end{array}$ \\
\hline
\end{tabular}

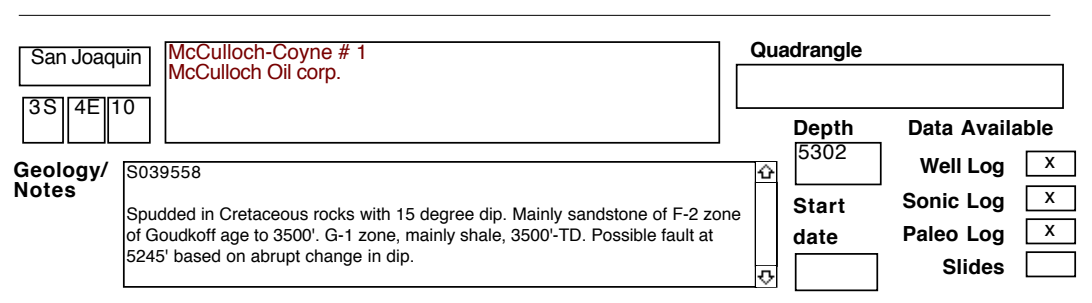

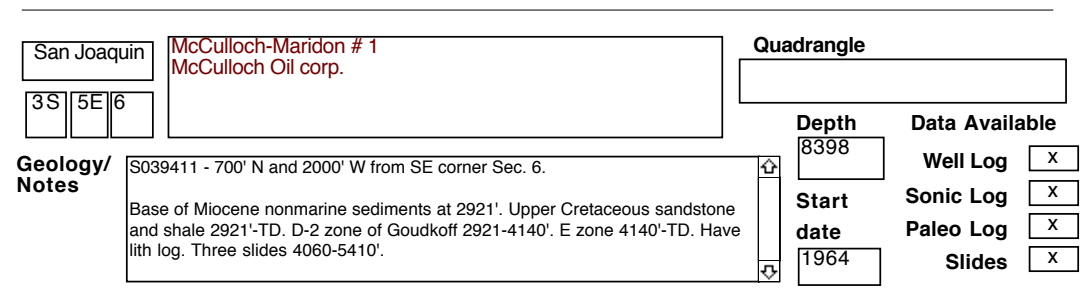


SELECTED OIL TEST WELLS IN CENTRAL CALIFORNIA DATABASE County

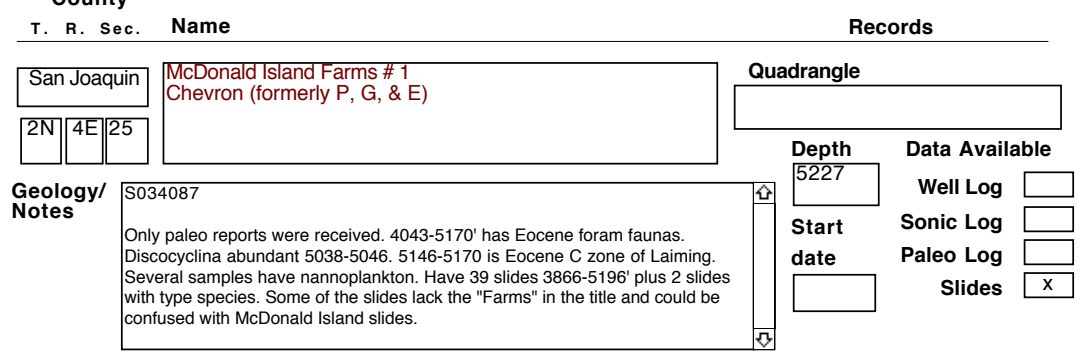

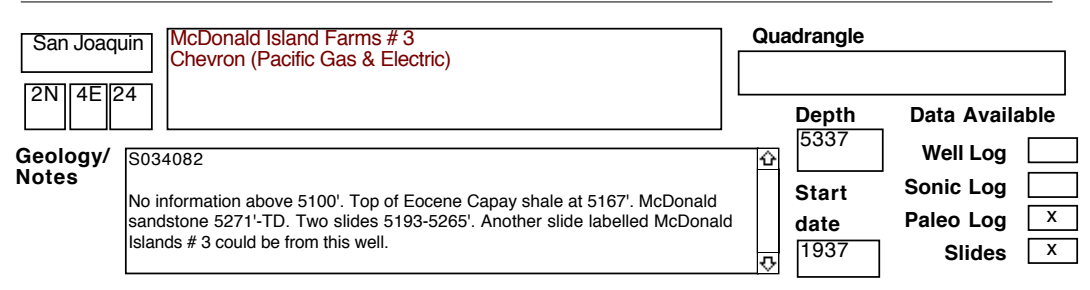

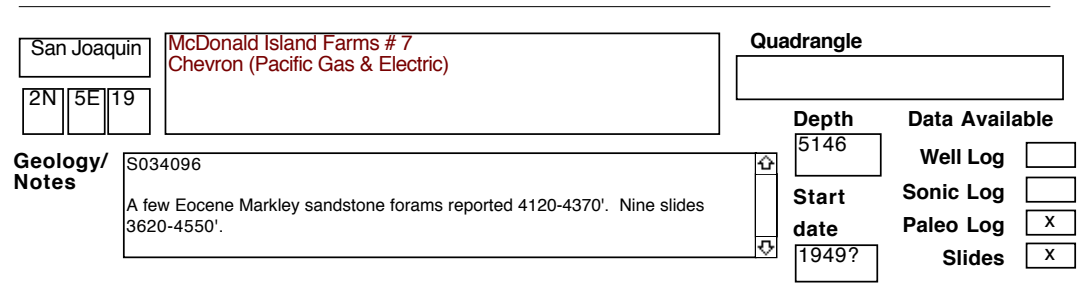

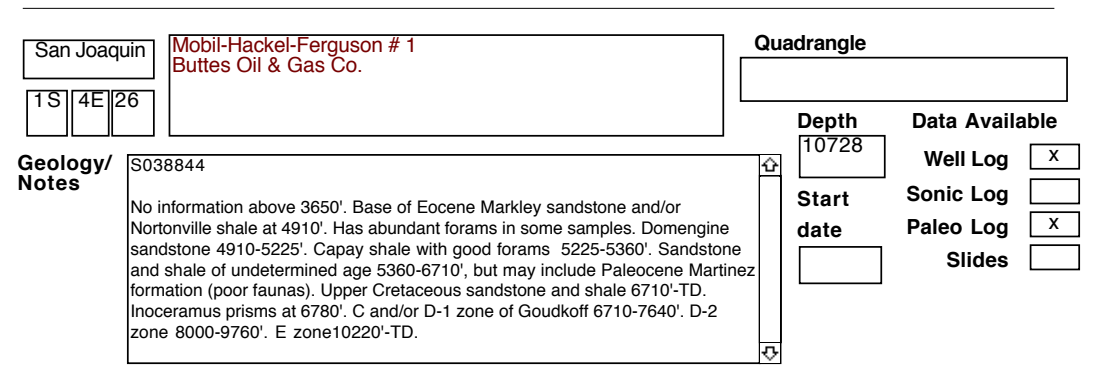

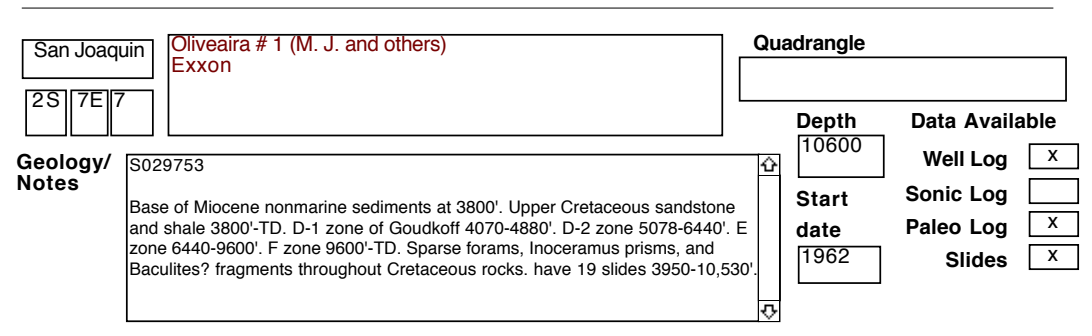


SELECTED OIL TEST WELLS IN CENTRAL CALIFORNIA DATABASE County

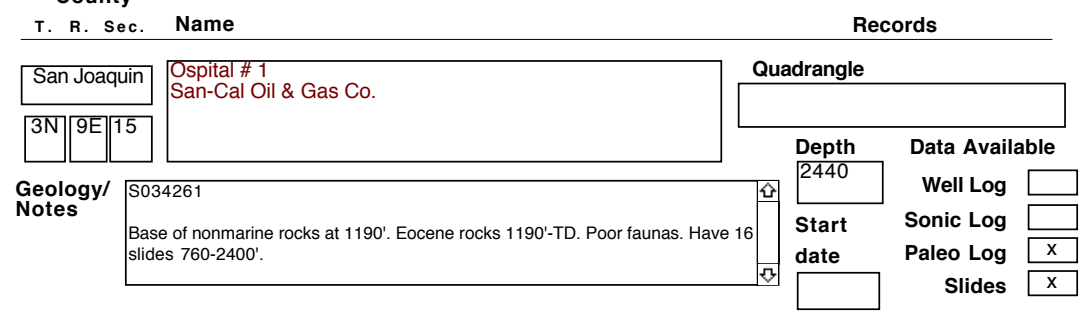

\begin{tabular}{|c|c|c|c|c|}
\hline San Joaquin & Peterson Fee \# 43-17 & Quadrangle & & \\
\hline \begin{tabular}{|l||l|}
$3 S$ & $5 E$ \\
\end{tabular} & & \multirow{3}{*}{$\begin{array}{l}\text { Depth } \\
9890\end{array}$} & \multirow{2}{*}{\multicolumn{2}{|c|}{ Data Available }} \\
\hline & & & & \\
\hline \multirow{3}{*}{\multicolumn{2}{|c|}{$\begin{array}{l}\text { Geology/ No paleo or lithology information available. } \\
\text { Notes }\end{array}$}} & & \multirow{3}{*}{$\begin{array}{r}\text { Well Log } \\
\text { Sonic Log } \\
\text { Paleo Log } \\
\text { Slides }\end{array}$} & \\
\hline & & Start & & $x$ \\
\hline & & $\begin{array}{l}\text { date } \\
1982\end{array}$ & & \\
\hline
\end{tabular}

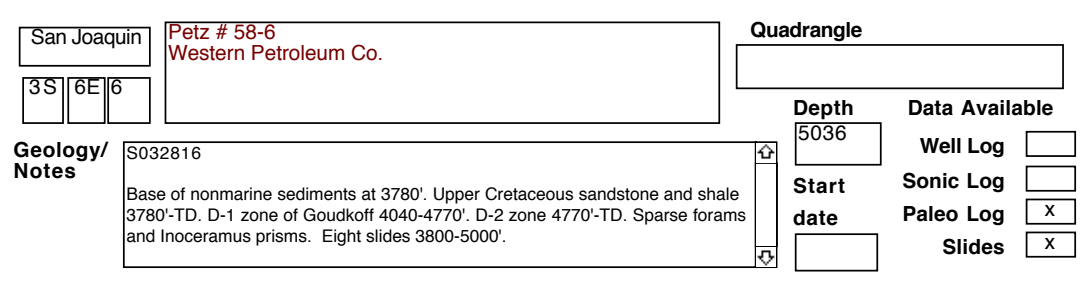

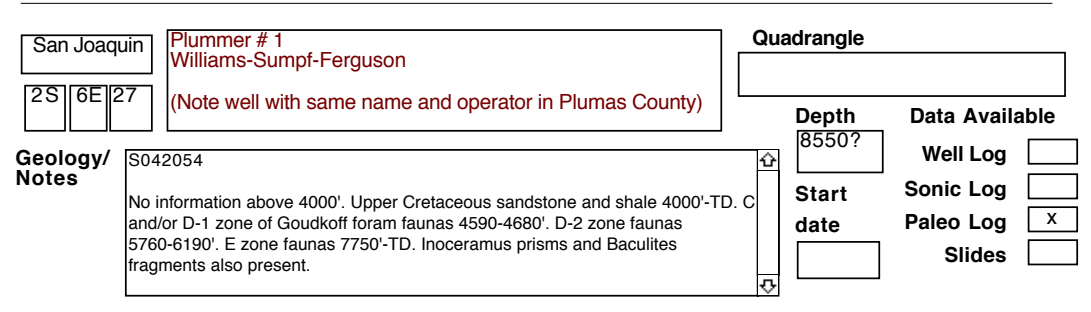

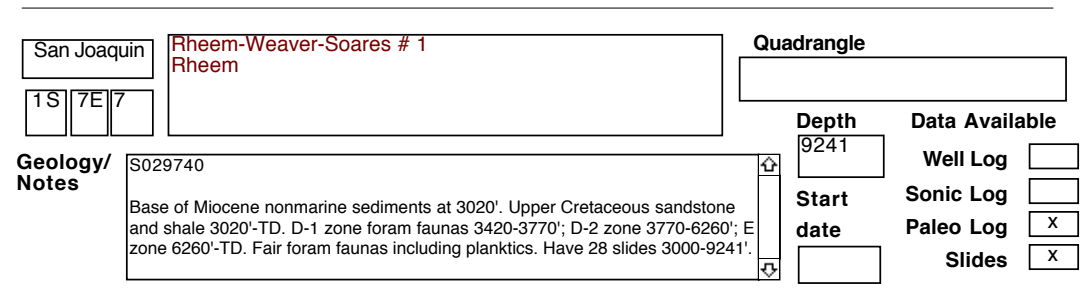


SELECTED OIL TEST WELLS IN CENTRAL CALIFORNIA DATABASE County

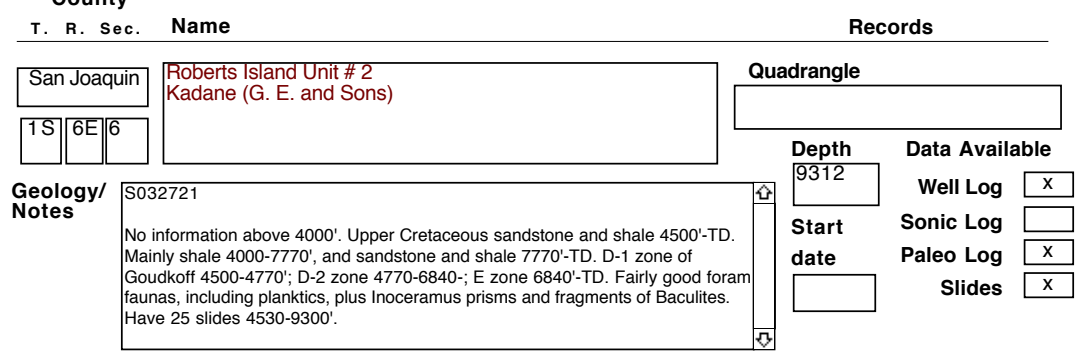

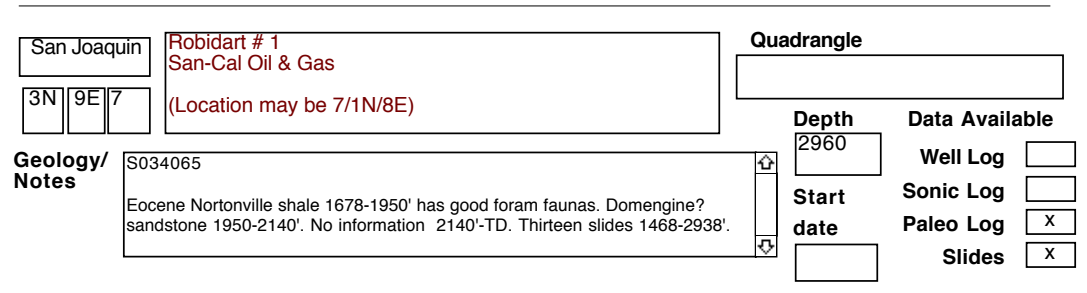

\begin{tabular}{l|l|l|l|l|l|l|}
\hline San Joaquin & $\begin{array}{l}\text { Rodgers \# 1 } \\
\text { Occidental }\end{array}$ \\
\hline
\end{tabular}

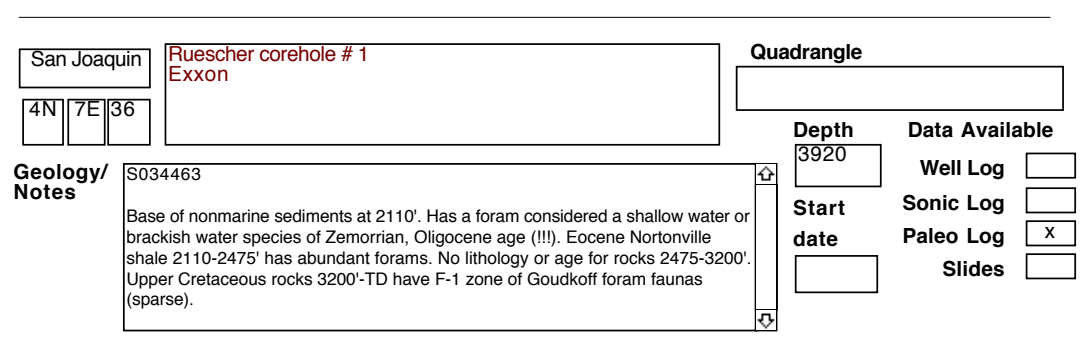

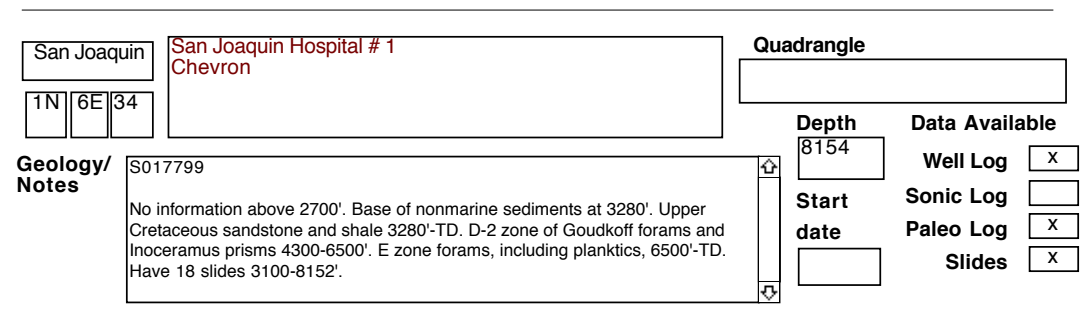


SELECTED OIL TEST WELLS IN CENTRAL CALIFORNIA DATABASE County

T. R. Sec. Name
\begin{tabular}{|l|l|l|l|l|}
\hline San Joaquin & $\begin{array}{l}\text { Santos \# 1-15 } \\
\text { Arcady Oil Co. }\end{array}$ \\
Geology/ \\
Notes
\end{tabular}
$\begin{aligned} & \text { S034043 information above 4840'. lone formation (sandstone?) 4840-5130'. The lone } \\
& \text { in this and other wells is identified by the lack of forams, the presence of siderite } \\
& \text { pellets, and a generally sandy character. Capay shale 5130-5255' has a fair foram } \\
& \text { fauna including planktics. McDonald Island sandstone 5255'-TD. }\end{aligned}$

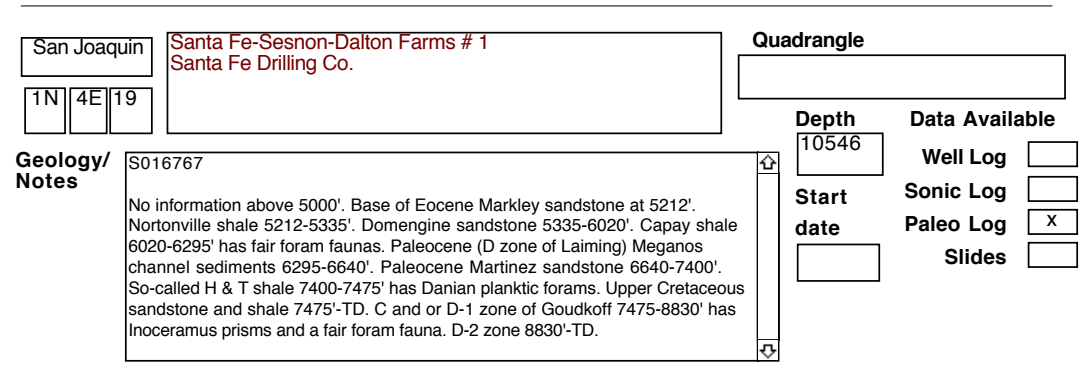

\begin{tabular}{l|l|l|l|l|l|l|}
\hline San Joaquin & $\begin{array}{l}\text { Porter-Sesnon-Rheem \# 63-27 } \\
\text { Sesnon (Porter and others) }\end{array}$ \\
\hline
\end{tabular}

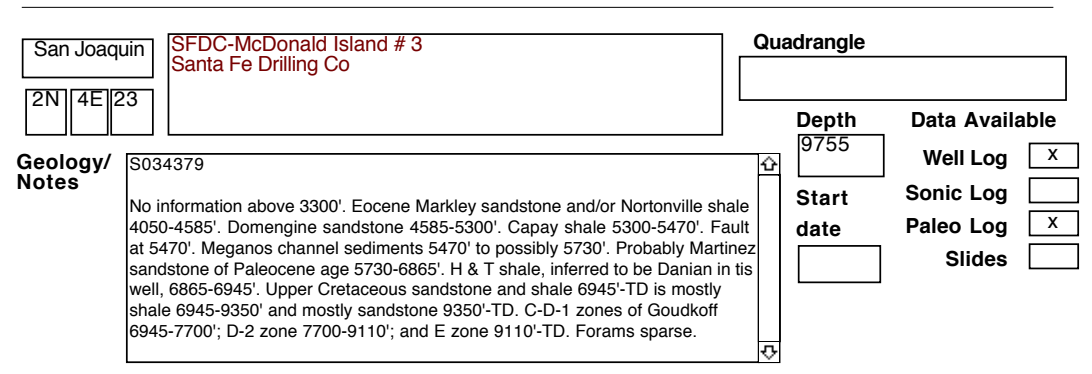

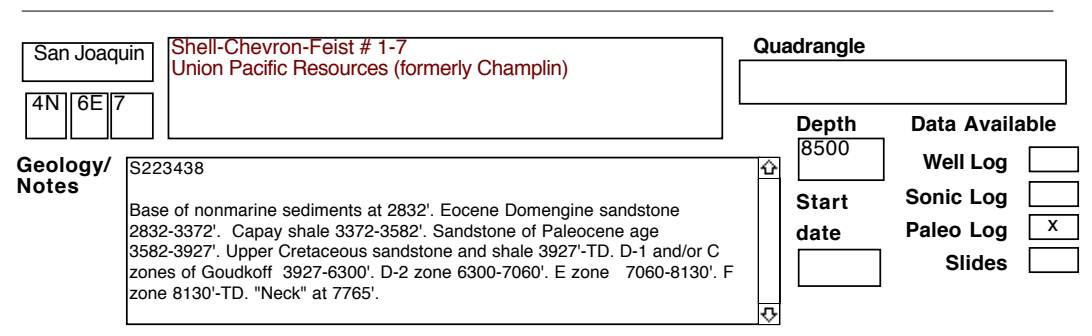


SELECTED OIL TEST WELLS IN CENTRAL CALIFORNIA DATABASE County

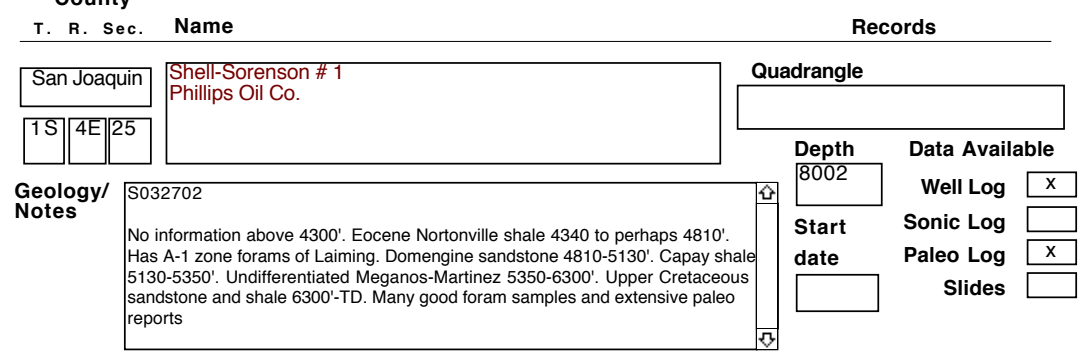

\begin{tabular}{l|l|l|l|l|}
\hline San Joaquin & $\begin{array}{l}\text { Shell-Brovelli \# 1 } \\
\text { Sunray }\end{array}$ \\
\hline & $\begin{array}{l}\text { Geology/ } \\
\text { Notes }\end{array}$ & $\begin{array}{l}\text { S034436 } \\
\text { Base of nonmarine sediments inferred at 1870', but may be lower. Sandstone and } \\
\text { shale1870-3050' inferred to be Eocene, but they lack forams and may be much } \\
\text { younger. Eocene Capay shale with a distinctive foram fauna 3050-3180'. } \\
\text { Sandstone 3180-3662' may be Paleocene but contains mostly forams caved from } \\
\text { the Capay. Upper Cretaceous sandstone and shale 3662-7292'. E zone of } \\
\text { Goudkoff 5420-6000' has "neck" at 5902'. F zone 6150-7242'. Hornblende } \\
\text { diorite 7292'-TD. }\end{array}$ \\
\hline
\end{tabular}

\begin{tabular}{|c|c|c|c|c|}
\hline San Joaquil & Signal-Von Sosten \# 1 & Zuadrangle & & \\
\hline \begin{tabular}{|l|l|l|l}
$2 S$ & $6 \mathrm{E}$ & 9
\end{tabular} & & \multirow{2}{*}{$\begin{array}{l}\text { Depth } \\
9021\end{array}$} & \multicolumn{2}{|c|}{ Data Available } \\
\hline \multirow{3}{*}{$\begin{array}{l}\text { Geology/ } \\
\text { Notes }\end{array}$} & $3787-100^{\prime} \mathrm{N}$ and $2400^{\prime} \mathrm{W}$ from SE corner Sec. 9. & & Well Log & $\mathrm{x}$ \\
\hline & se of nonmarine sediments at $3480^{\prime}$. Upper Cretaceous sandstone and shale & Start & Sonic Log & \\
\hline & $\begin{array}{l}\text { 180'-TD. C and/or D-1 zones of Goudkoff 3480-5950'. D-2 zone 5950-7150'. } \\
\text { רcertain zone 7150-8830'. E zone 8830'-TD. Mainly sparse, arenaceous forams. }\end{array}$ & $\begin{array}{l}\text { date } \\
1969\end{array}$ & $\begin{array}{r}\text { Paleo Log } \\
\text { Slides }\end{array}$ & $\mathrm{x}$ \\
\hline
\end{tabular}

\begin{tabular}{l|l|l|l|l|l|}
\hline San Joaquin & $\begin{array}{l}\text { Silva \# 1 } \\
\text { Arcady Oil Co }\end{array}$ \\
\hline
\end{tabular}

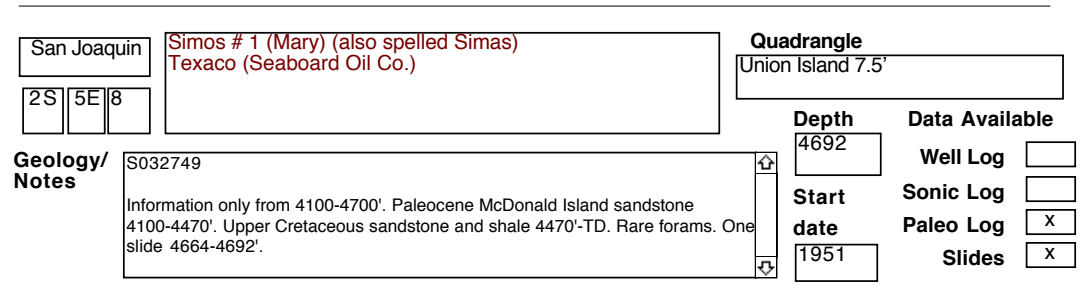


SELECTED OIL TEST WELLS IN CENTRAL CALIFORNIA DATABASE County

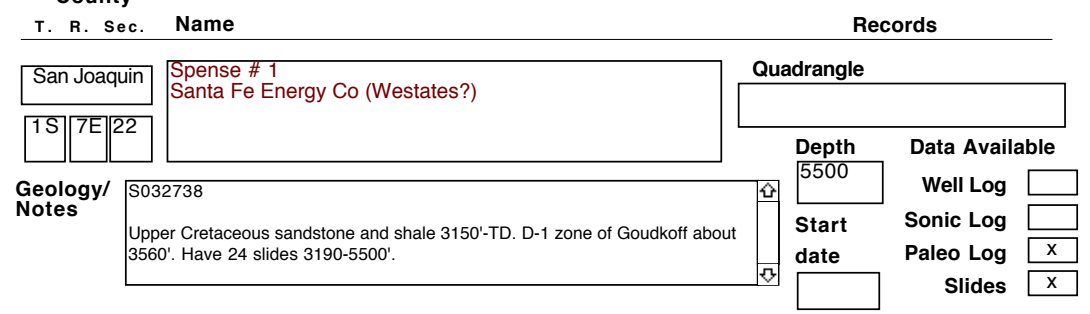

\begin{tabular}{|c|c|c|c|c|c|}
\hline San Joaquin & \multirow{3}{*}{$\begin{array}{l}\text { Steele \# 47-19 } \\
\text { Steele (Horace) }\end{array}$} & \multicolumn{4}{|c|}{ Quadrangle } \\
\hline \multirow[t]{2}{*}{\begin{tabular}{|l|l|l|}
$3 S$ & $5 E$ & 19
\end{tabular}} & & \multirow{2}{*}{\multicolumn{2}{|c|}{ Depth }} & \multirow{2}{*}{\multicolumn{2}{|c|}{ Data Available }} \\
\hline & & & & & \\
\hline \multirow{3}{*}{$\begin{array}{l}\text { Geology/ } \\
\text { Notes }\end{array}$} & 55' N and 2850' W from SE corner Sec. 19. & 目 & & Well Log & \\
\hline & ve lithology log, but no paleo provided. & 0 & \multirow{2}{*}{$\begin{array}{l}\text { Start } \\
\text { date }\end{array}$} & Sonic Log & $\mathrm{x}$ \\
\hline & & & & Slides & \\
\hline
\end{tabular}

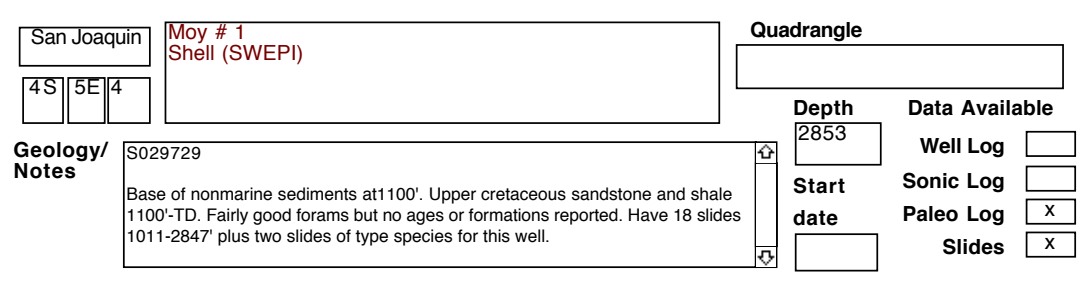

\begin{tabular}{|l|l|l|l|}
\hline San Joaquin & $\begin{array}{l}\text { Sunol Securities \# } 4 \\
\text { Unocal }\end{array}$ & \multicolumn{2}{l|}{ Quadrangle } \\
\hline
\end{tabular}

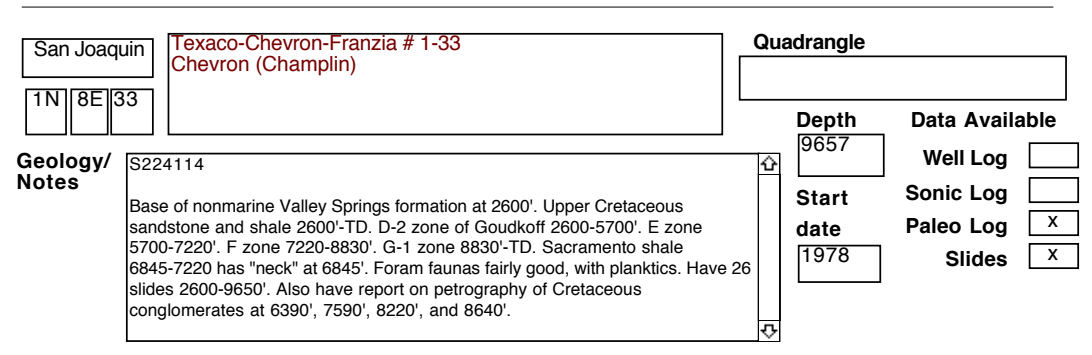


SELECTED OIL TEST WELLS IN CENTRAL CALIFORNIA DATABASE County

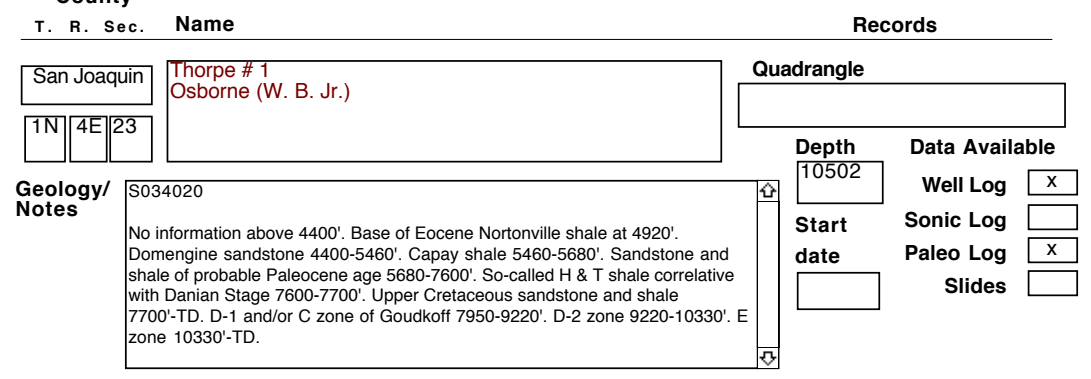

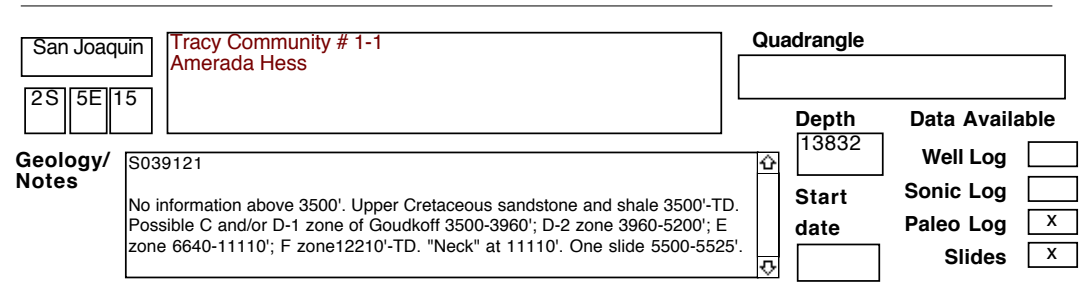

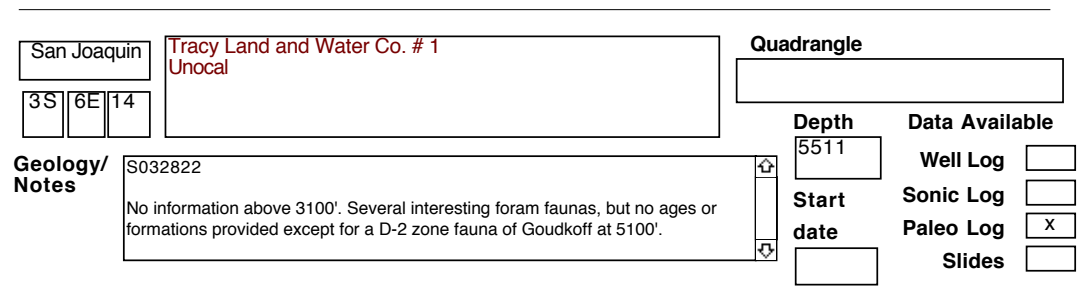

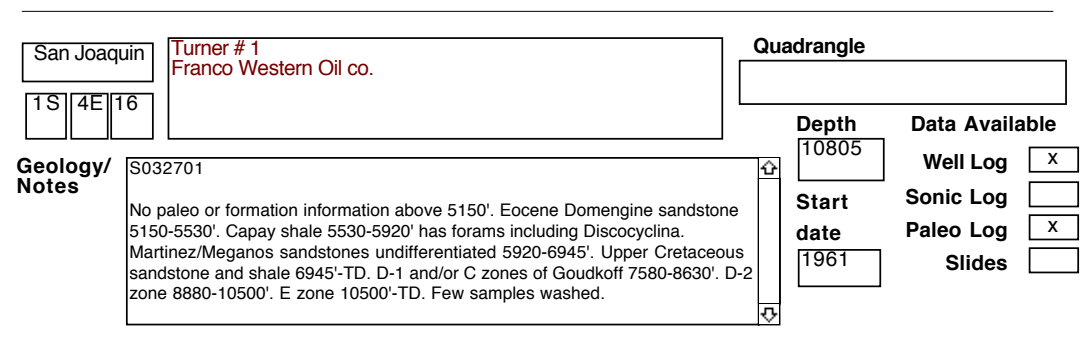

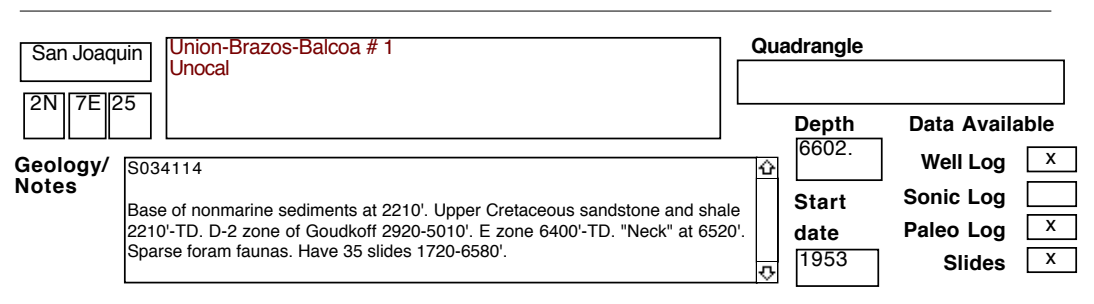




\section{SELECTED OIL TEST WELLS IN CENTRAL CALIFORNIA DATABASE}

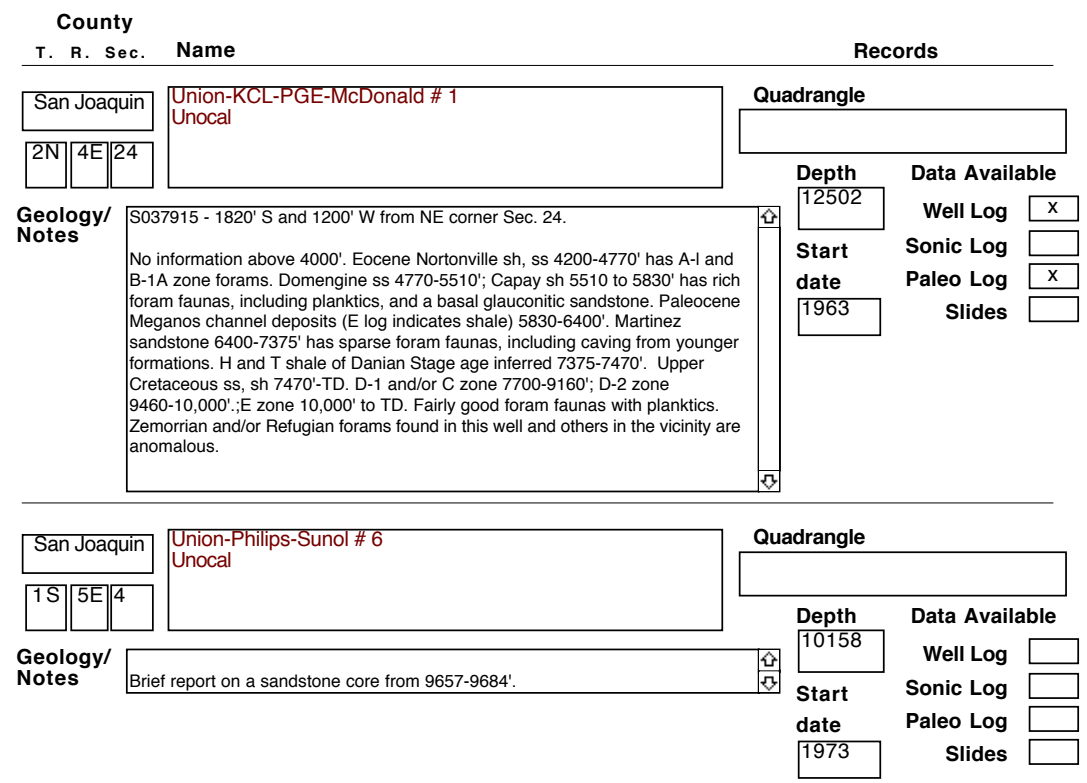

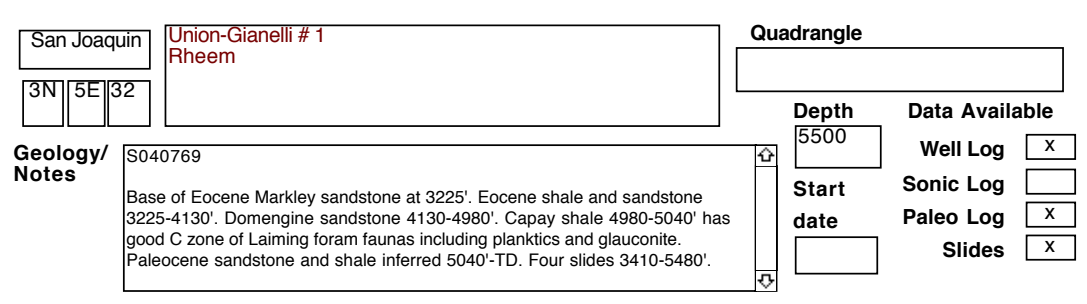

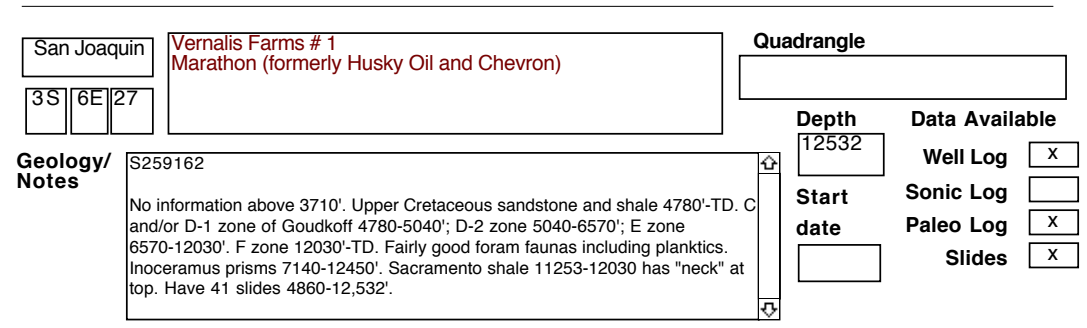

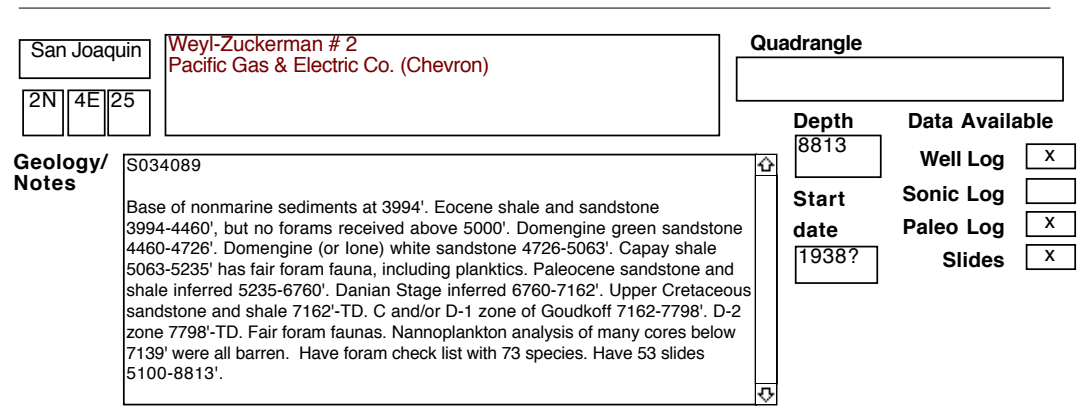




\section{SELECTED OIL TEST WELLS IN CENTRAL CALIFORNIA DATABASE}

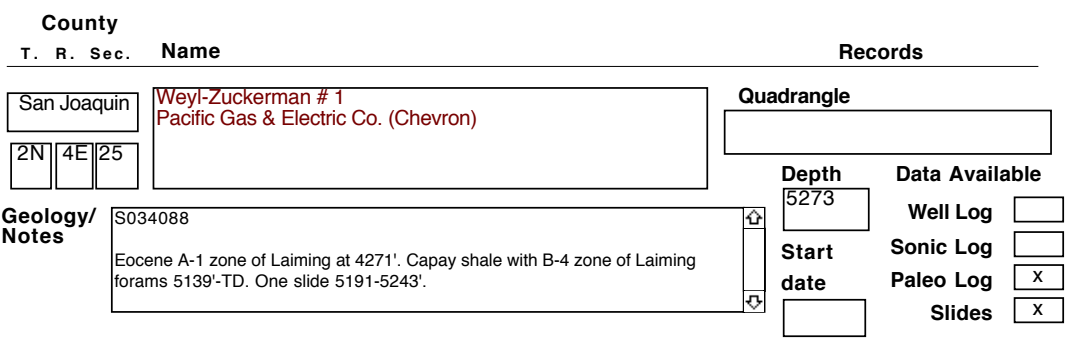

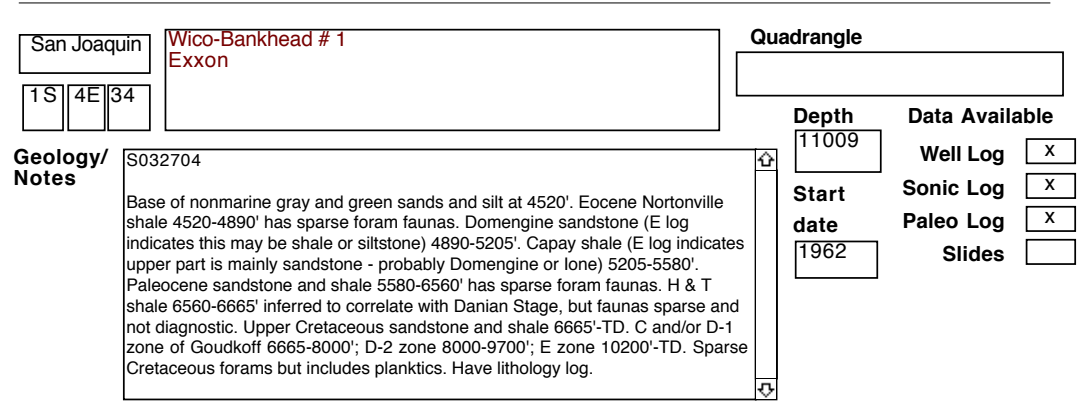

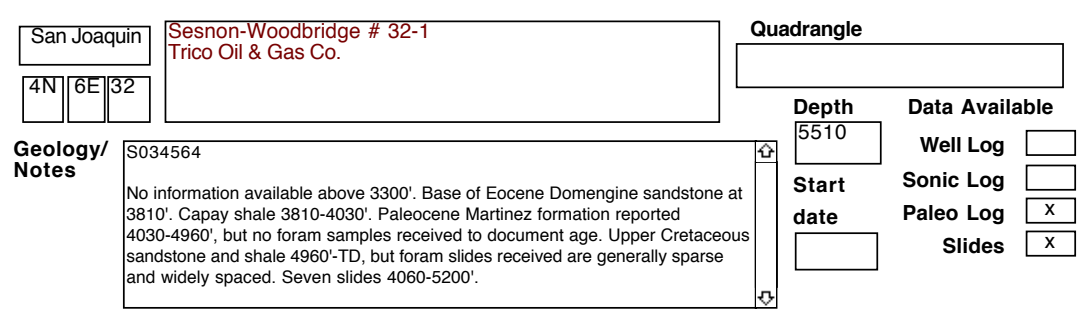

\begin{tabular}{l|l|l|l|l|l|}
\hline San Joaquin & $\begin{array}{l}\text { Woods Community \# 2-5 } \\
\text { Chevron }\end{array}$ \\
\hline
\end{tabular}

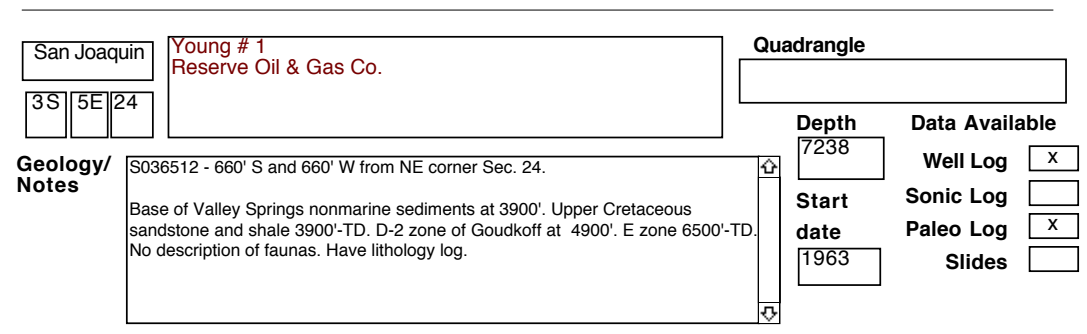


SELECTED OIL TEST WELLS IN CENTRAL CALIFORNIA DATABASE County

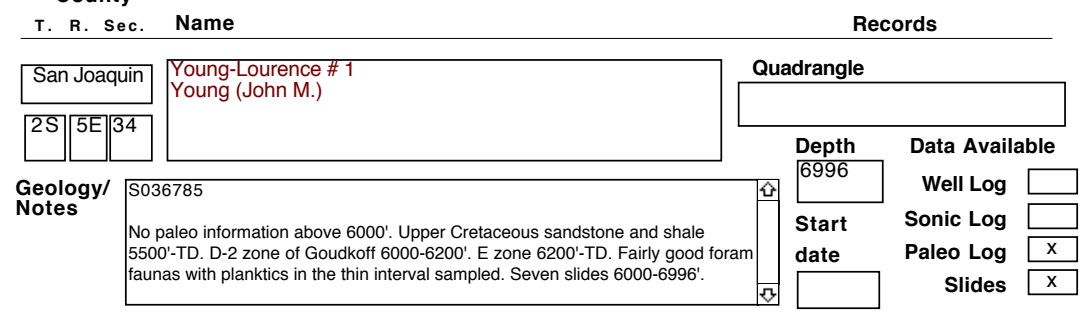

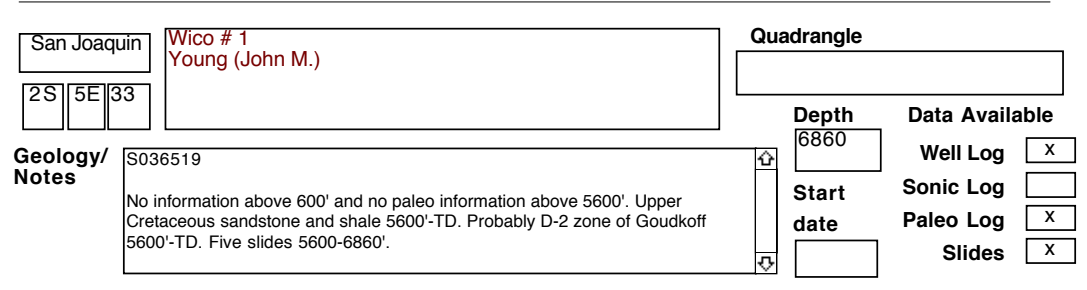

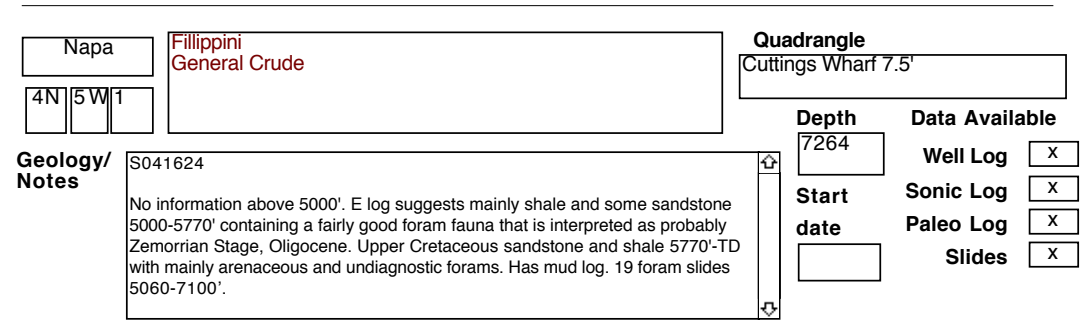

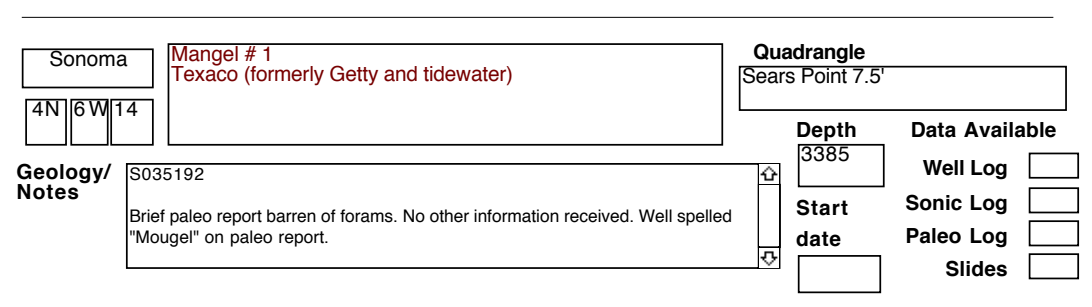

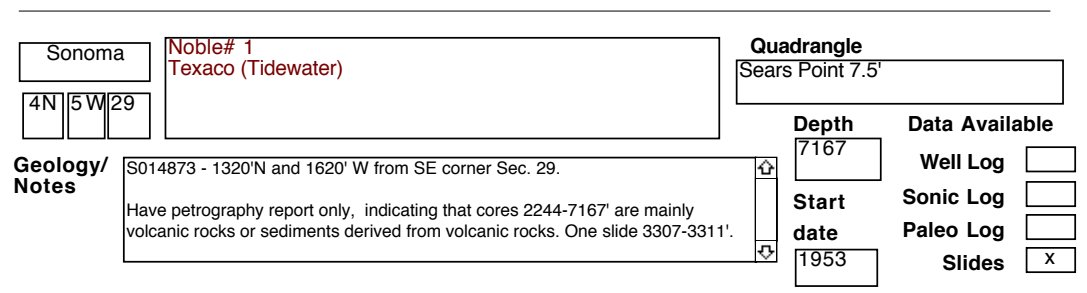


SELECTED OIL TEST WELLS IN CENTRAL CALIFORNIA DATABASE County

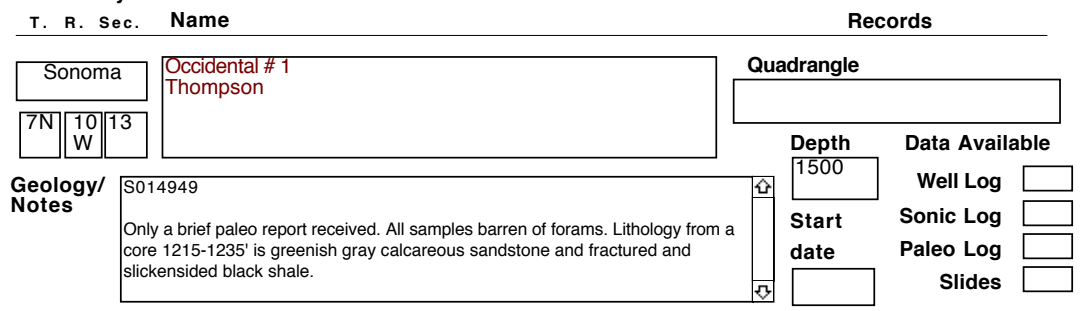

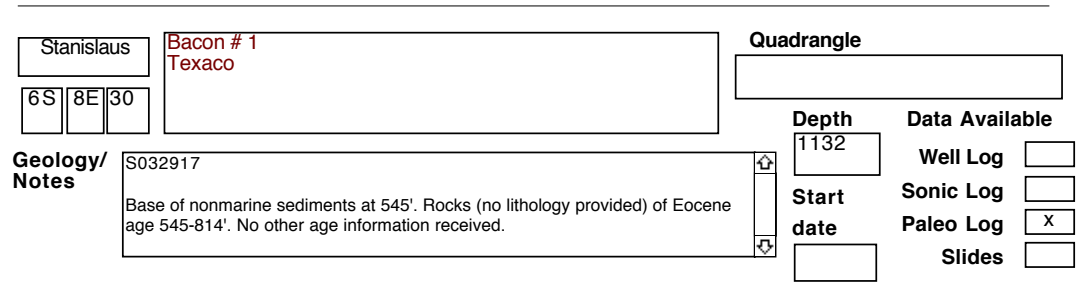

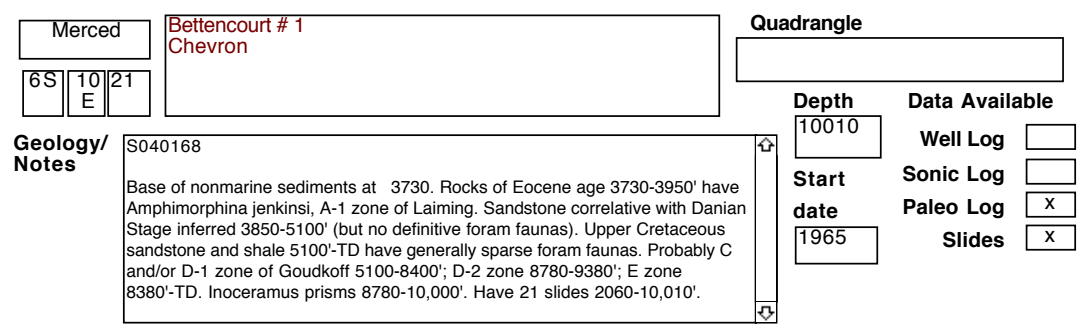

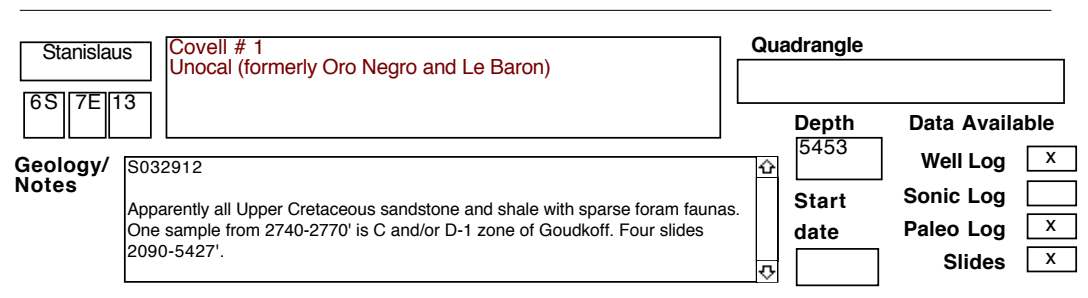

\begin{tabular}{l|l|l|l|l|l|}
\hline Stanislaus & $\begin{array}{l}\text { Covell \# 28-1 } \\
\text { Unocal (formerly Los Nietos) }\end{array}$ & \multicolumn{2}{l|}{ Quadrangle } \\
\hline
\end{tabular}


SELECTED OIL TEST WELLS IN CENTRAL CALIFORNIA DATABASE County

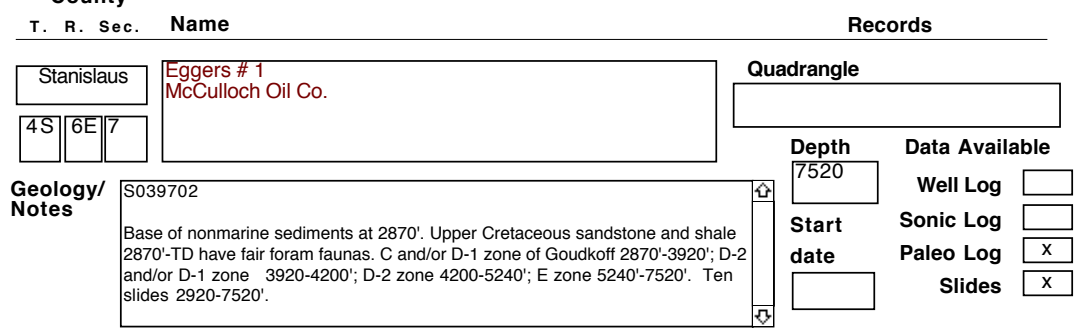

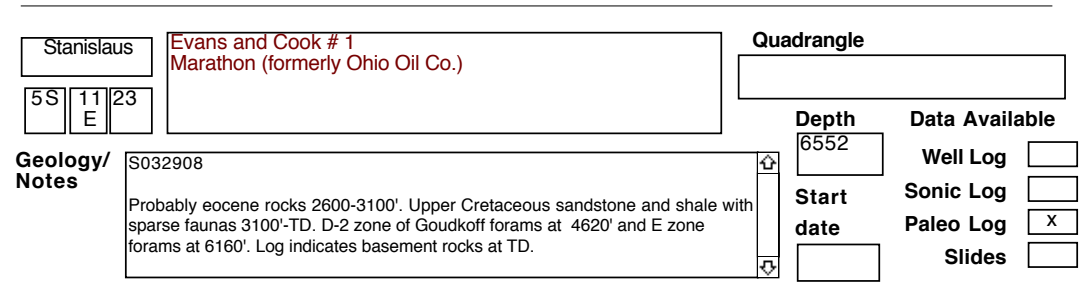

\begin{tabular}{l|l|l|l|l|l|l|l|}
\hline Stanislaus & $\begin{array}{l}\text { Ferreira \# 1 } \\
\text { ARCO }\end{array}$ \\
\hline
\end{tabular}

\begin{tabular}{l|l|l|l|l|l|}
\hline Stanislaus & $\begin{array}{l}\text { Forni \#1 } \\
\text { Occidental }\end{array}$ \\
\hline
\end{tabular}

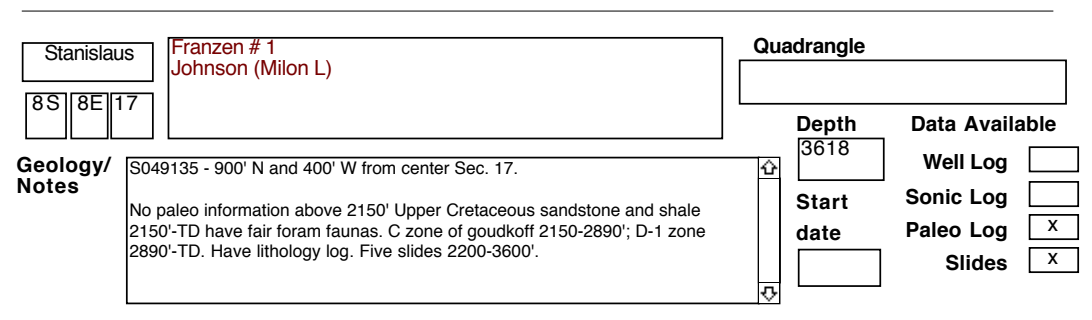


SELECTED OIL TEST WELLS IN CENTRAL CALIFORNIA DATABASE County

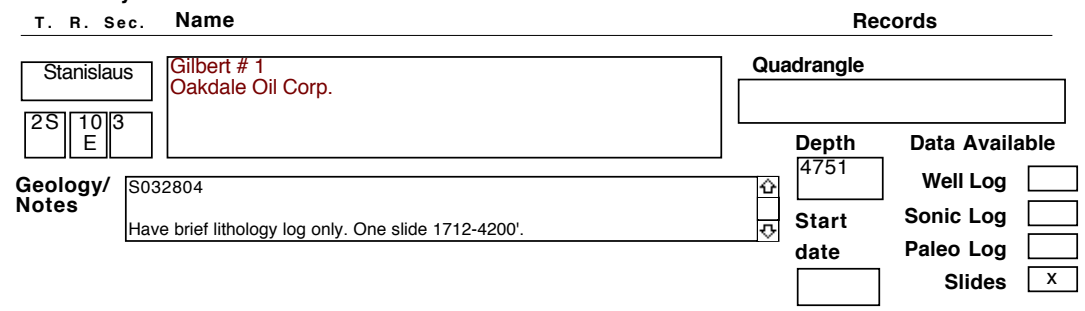

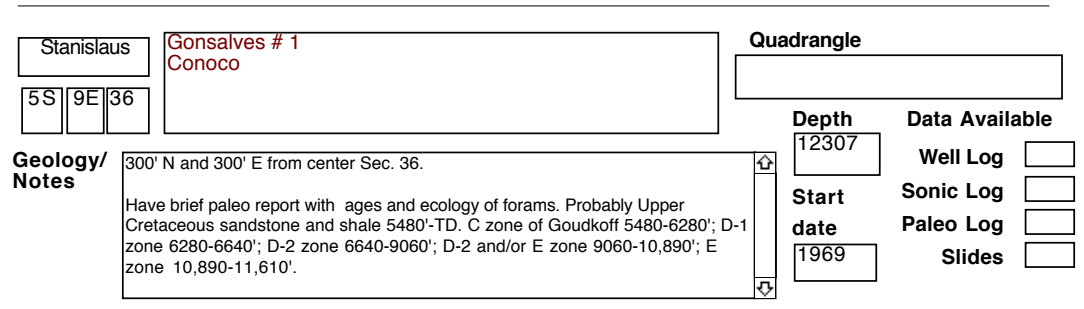

\begin{tabular}{l|l|l|l|l|l|l|}
\hline Stanislaus & $\begin{array}{l}\text { Lemos Unit \# 1 } \\
\text { Chevron (Gulf) }\end{array}$ \\
\hline
\end{tabular}

\begin{tabular}{l|l|l|l|l|l|}
\hline Stanislaus & $\begin{array}{l}\text { KCL-Santa Fe-Thompson \# 1-6 } \\
\text { Kern County Land Co }\end{array}$ \\
\hline
\end{tabular}

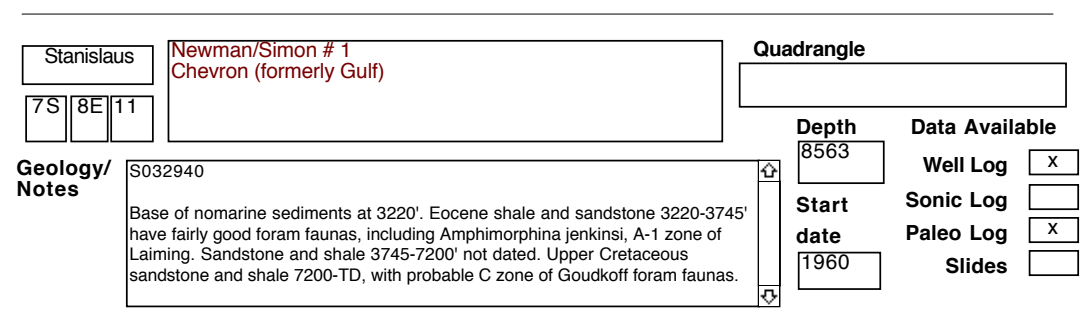


SELECTED OIL TEST WELLS IN CENTRAL CALIFORNIA DATABASE County

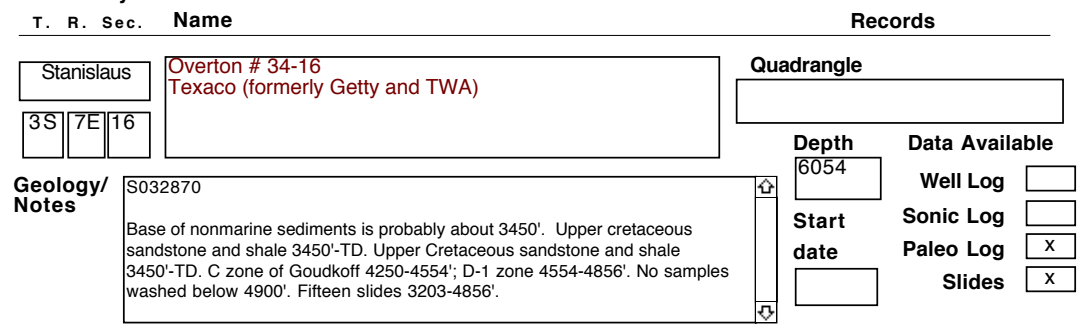

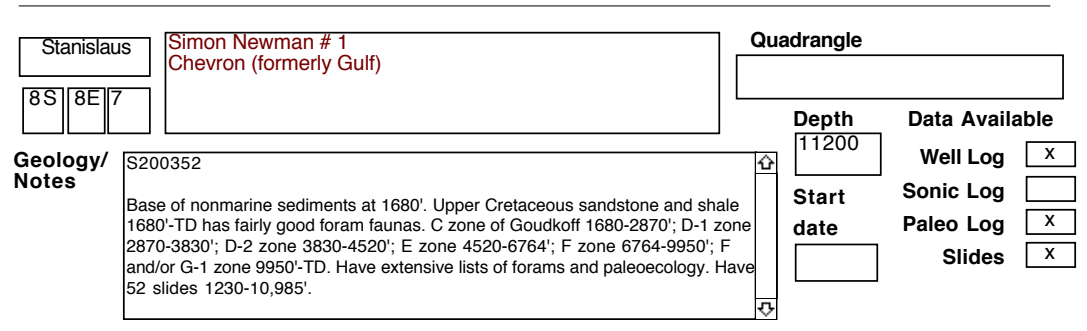

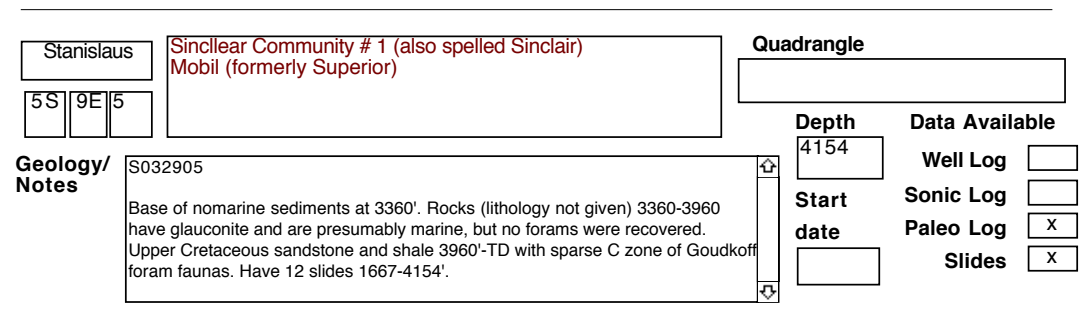

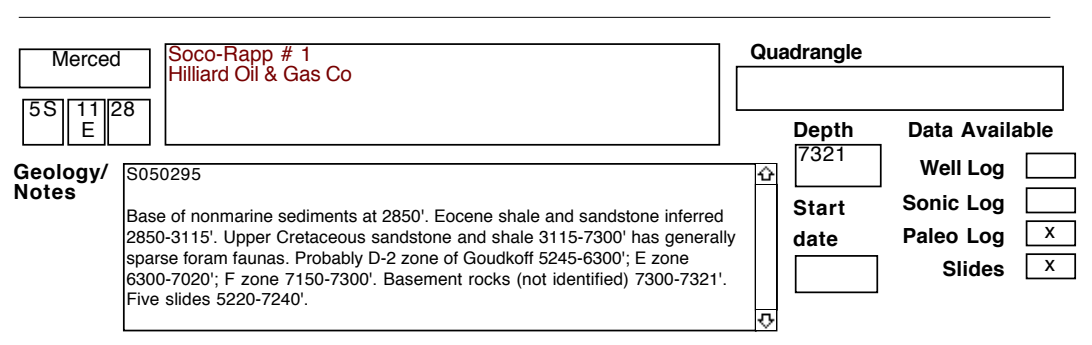

\begin{tabular}{l|l|l|l|l|l|}
\hline Stanislaus & $\begin{array}{l}\text { SouthlandRoyalty-Vaughn \# 27-6 } \\
\text { Great Basins Petroleum Co. }\end{array}$ \\
\hline
\end{tabular}


SELECTED OIL TEST WELLS IN CENTRAL CALIFORNIA DATABASE County

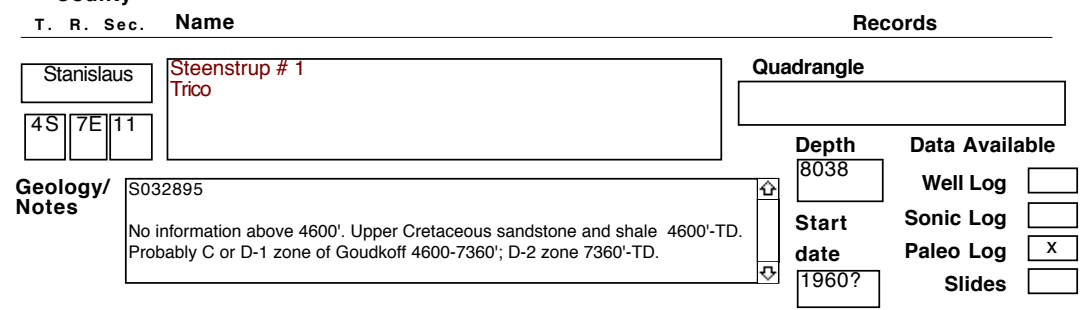

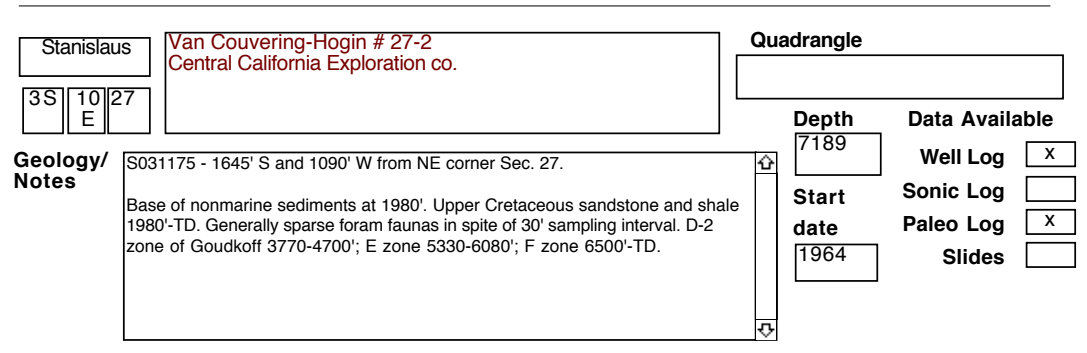

\begin{tabular}{l|l|l|l|l|l|l|}
\hline \multicolumn{1}{|c|}{ Sutter } & $\begin{array}{l}\text { Atlantic-Pogue \# 1 } \\
\text { Texkan Oil and R. R. Herring }\end{array}$ \\
\hline
\end{tabular}

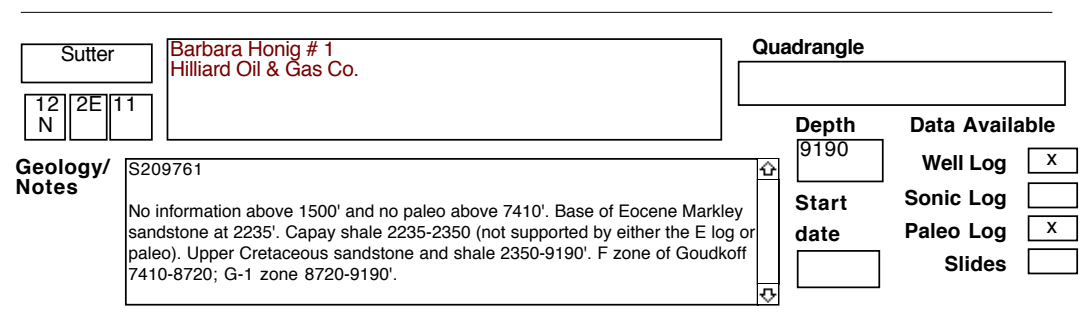

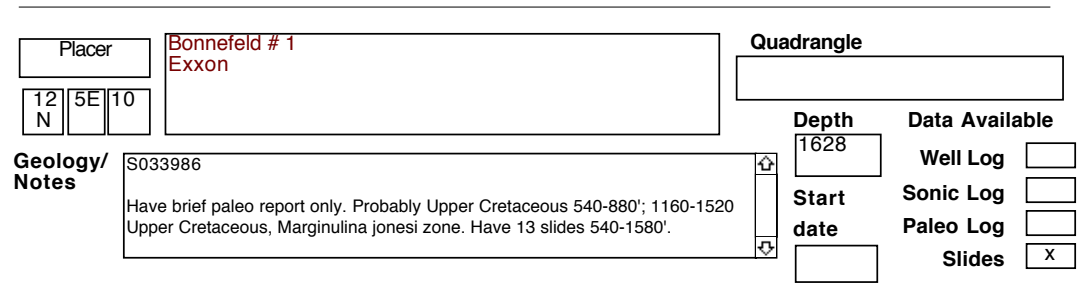


SELECTED OIL TEST WELLS IN CENTRAL CALIFORNIA DATABASE County

\begin{tabular}{|c|c|c|c|c|c|}
\hline T. R. Sec & Name & \multicolumn{4}{|c|}{ Records } \\
\hline Sutter & Browning \# 1 & Qu & drangle & & \\
\hline \begin{tabular}{|c||c|c|}
16 & $1 \mathrm{E}$ & 19 \\
$\mathrm{~N}$ & & \\
\end{tabular} & & & Depth & Data Availab & \\
\hline $\begin{array}{l}\text { Geology/ } \\
\text { Notes }\end{array}$ & $\begin{array}{l}14854 \\
\text { information above 7100'. Upper Cretaceous shale, G-1 zone of Goudkoff } \\
\text { 30-7664'. Seven slides 7090-7664'. }\end{array}$ & $\varphi$ & $\begin{array}{l}\text { Start } \\
\text { date } \\
1961 ?\end{array}$ & $\begin{array}{r}\text { Well Log } \\
\text { Sonic Log } \\
\text { Paleo Log } \\
\text { Slides }\end{array}$ & $\mathrm{x}$ \\
\hline
\end{tabular}

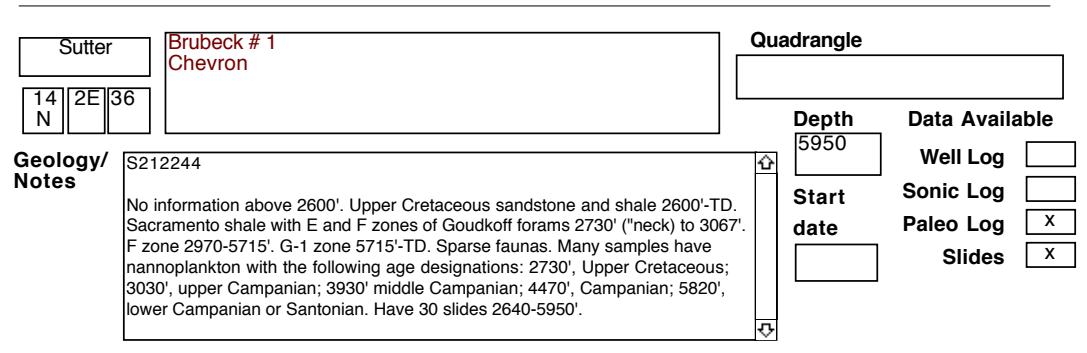

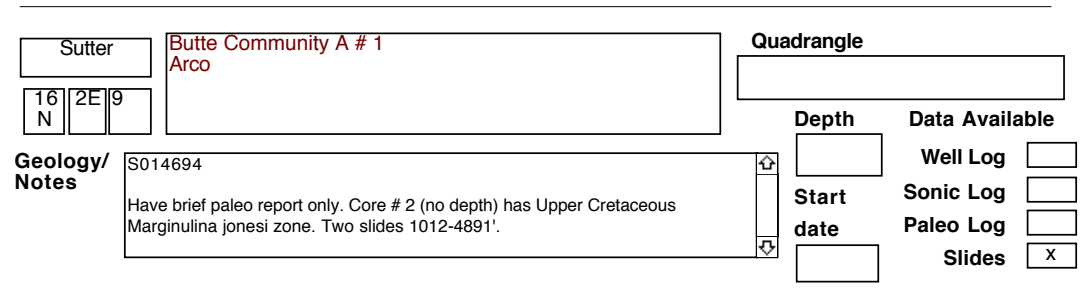

\begin{tabular}{l|l|l|l|l|l|}
\hline \multicolumn{1}{|c|}{ Sutter } & $\begin{array}{l}\text { Buttes \# 12 } \\
\text { Buttes Oil \& Gas Co. }\end{array}$ \\
\hline
\end{tabular}

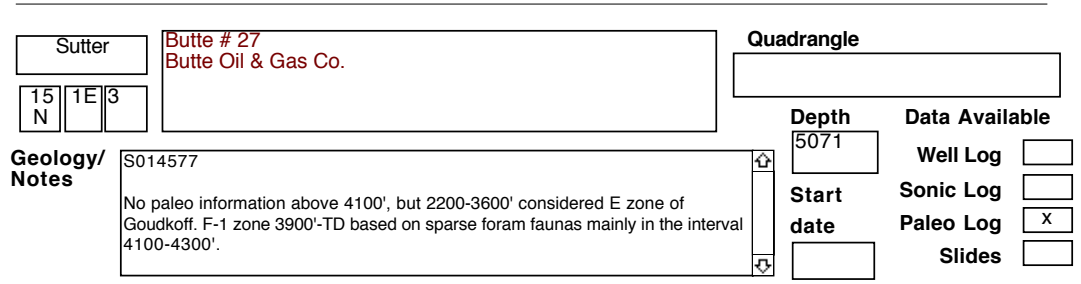




\section{SELECTED OIL TEST WELLS IN CENTRAL CALIFORNIA DATABASE}

County

\begin{tabular}{ll|l|}
\hline T. & R. Sec. & Name \\
\hline \multicolumn{2}{|c|}{ Sutter } & Buttes \# 14 \\
Buttes Oil \& Gas Co \\
\hline 15 & $1 \mathrm{E}$ & 12 \\
\hline
\end{tabular}

\section{Geology/ S014587}

Notes

Base of Tehama nonmarine sediments at $1980^{\prime}$. Eocene Domengine or lone sandstone 1980-2085'. Capay shale with fair foram faunas 2085-2460'. Upper Cretaceous sandstone and shale 2460-7770' has generally sparse foram faunas. F-1- zone of Goudkoff 5460-6260'; G-1 zone 6260-6920'; G-2 zone 6920-7770'. Granodiorite basement rocks 7770'-TD. Have 32 slides 2080-7800'.

\begin{tabular}{|c|c|c|c|}
\hline \multicolumn{4}{|c|}{ Records } \\
\hline \multicolumn{4}{|c|}{ Quadrangle } \\
\hline & Depth & Data Availa & \\
\hline 0 & & Well Log & $\mathrm{x}$ \\
\hline & Start & Sonic Log & \\
\hline & date & Paleo Log & $x$ \\
\hline & & Slides & $\mathrm{x}$ \\
\hline
\end{tabular}

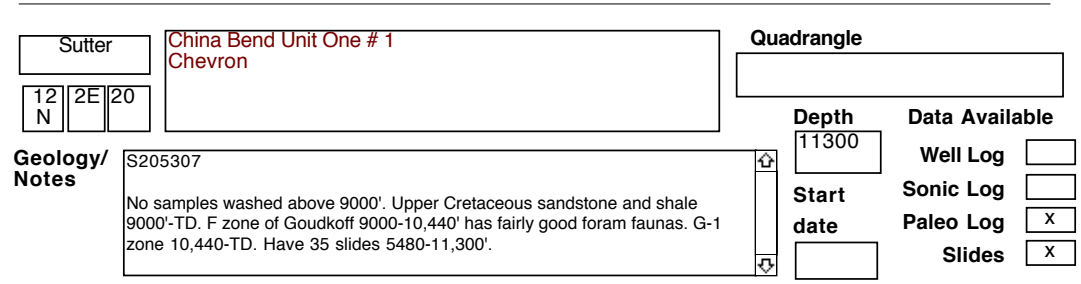

\begin{tabular}{l|l|l|l|l|l|l|}
\hline \multicolumn{1}{|c|}{ Sutter } & $\begin{array}{l}\text { Crawford\# } \\
\text { Seaboard Oil }\end{array}$ \\
\hline
\end{tabular}

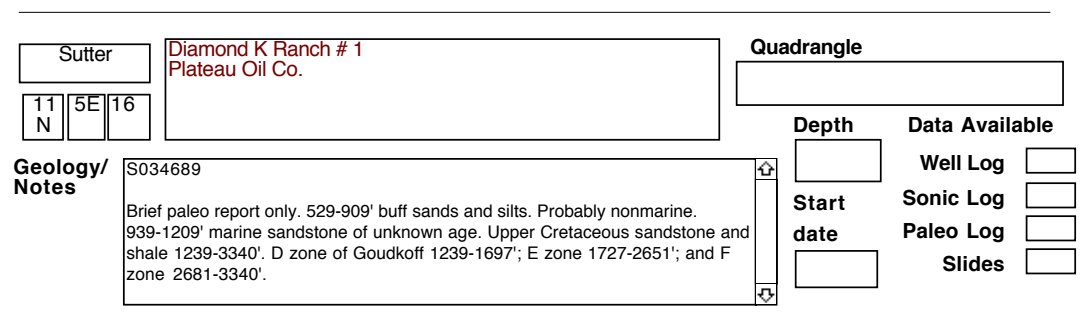

\begin{tabular}{l|l|l|l|l|l|}
\hline \multicolumn{1}{|c|}{ Sutter } & $\begin{array}{l}\text { Epperson \# 1 } \\
\text { Atlantic Oil Co. }\end{array}$ \\
\hline
\end{tabular}




\section{SELECTED OIL TEST WELLS IN CENTRAL CALIFORNIA DATABASE}

County

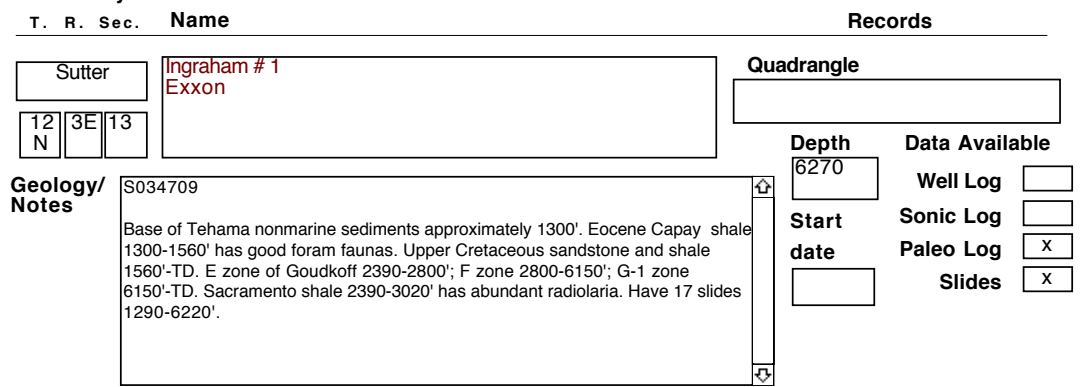

\begin{tabular}{|l|l|l|l|l|l|}
\hline \multicolumn{1}{|c|}{ Sutter } & $\begin{array}{l}\text { Justeson \# 1 } \\
\text { Sunray }\end{array}$ \\
\hline
\end{tabular}

\begin{tabular}{|c|c|}
\hline Sutter & $\begin{array}{l}\text { Lamb \# } 1-30 \\
\text { Kadane (GE and sons) }\end{array}$ \\
\hline \begin{tabular}{l|l|l}
14 & $2 \mathrm{E}$ & 30 \\
$\mathrm{~N}$ & &
\end{tabular} & \\
\hline
\end{tabular}

\section{Geology/ 5034000}

Notes

Base of nonmarine sediments at 2265'. Eocene Nortonville shale reported

2265-2580', but no samples received. Domengine sandstone 2580-2760'.

Capay shale $2760-3050$ ' has good foram faunas including planktics. Upper

Cretaceous sandstone and shale $3050^{\prime}$-TD has fair foram faunas, including

planktics. D-2 zone of Goudkoff 3050-3400'; E zone 3400-3750'; F-1 zone

3750-6830' includes E zone biofacies; F-2 zone 6830-7460'; G-1 zone 7460'-TD,

Sacramento shale $3500-3860$ ' has "neck" at 3600 '. Have 21 slides $2700-7470$ '.

\begin{tabular}{|c|c|c|c|}
\hline \multicolumn{4}{|c|}{ Quadrangle } \\
\hline & \multirow{2}{*}{$\begin{array}{l}\text { Depth } \\
7505\end{array}$} & \multicolumn{2}{|c|}{ Data Available } \\
\hline & & Well Log & $x$ \\
\hline & Start & Sonic Log & \\
\hline & date & Paleo Log & $x$ \\
\hline & & Slides & $x$ \\
\hline
\end{tabular}

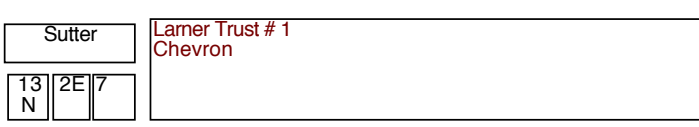

Geology/ S206016

Notes

Points determined by seismic electrical surveys: top of Capay shale at 2924'; top

of Upper Cretaceous sandstone at 3198'; and "neck" in Sacramento shale at

3875'. Normal fault at 7595' believed to cut out part of section. F zone of Goudkoff

rocks $8010^{\prime}$-TD. Have 19 slides $4740-8040^{\prime}$

\begin{tabular}{|c|c|c|}
\hline Quadrangle & & \\
\hline Depth & Data Availab & \\
\hline (0.5 & Well Log & \\
\hline Start & Sonic Log & \\
\hline date & Paleo Log & $x$ \\
\hline 1974 & Slides & $x$ \\
\hline
\end{tabular}

\section{列}




\section{SELECTED OIL TEST WELLS IN CENTRAL CALIFORNIA DATABASE}

County

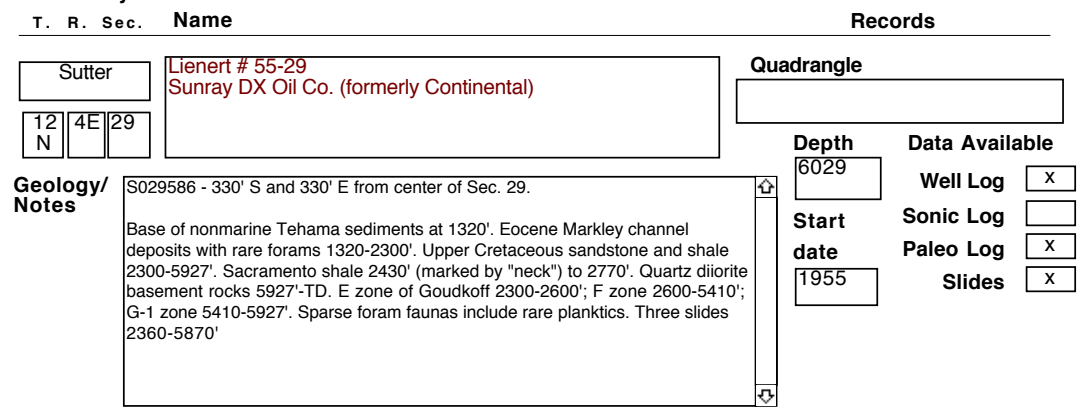

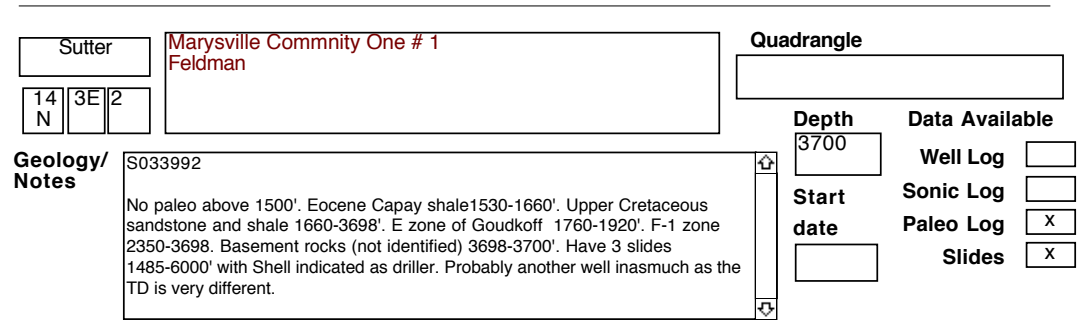

\begin{tabular}{l|l|l|l|l|l|}
\hline \multicolumn{1}{|c|}{ Sutter } & $\begin{array}{l}\text { McPherrin \# 3 } \\
\text { Buttes Oil \& Gas Co. }\end{array}$ \\
\hline
\end{tabular}

\begin{tabular}{l|l|l|l|l|l|l|}
\hline \multicolumn{1}{|c|}{ Sutter } & $\begin{array}{l}\text { Montna \# 1 } \\
\text { Brown (Marshall) }\end{array}$ \\
\hline
\end{tabular}

\begin{tabular}{l|l|l|l|l|l|}
\hline \multicolumn{1}{|c|}{ Sutter } & $\begin{array}{l}\text { Rossi \# 1 } \\
\text { Chevron }\end{array}$ \\
\hline
\end{tabular}


SELECTED OIL TEST WELLS IN CENTRAL CALIFORNIA DATABASE County

\begin{tabular}{|c|c|}
\hline Sutter & $\begin{array}{l}\text { Sanborn and Sons \# } 1 \\
\text { Mobil (formerly Cameron Oil) }\end{array}$ \\
\hline \begin{tabular}{l|l|l|l|l}
15 & $1 \mathrm{E}$ & 32 \\
$\mathrm{~N}$ & &
\end{tabular} & \\
\hline
\end{tabular}

\section{Geology/ 5014606}

Notes

No information above $700^{\prime}$ and no paleo above $3370^{\prime}$. Base of nonmarine
sediments at $2800^{\prime}$. Eocene Capay shale $2800-3010^{\prime}$. Upper Cretaceous

sandstone and shale 3010'-TD. E zone of Goudkoff 3200-3500' has sparse foram

faunas. Sacramento shale3300-3740' has "neck" at3420'. F-1 zone inferred 3550-3800' and 6000-6390'. F-2 zone 6390'-TD. Have 18 slides 3400-8100'

\begin{tabular}{|c|c|c|c|}
\hline \multicolumn{4}{|c|}{ Records } \\
\hline \multicolumn{4}{|c|}{ Quadrangle } \\
\hline & Depth & \multicolumn{2}{|c|}{ Data Available } \\
\hline s & & Well Log & $x$ \\
\hline & Start & Sonic Log & \\
\hline & date & Paleo Log & $\mathrm{x}$ \\
\hline & & Slides & $\mathrm{x}$ \\
\hline
\end{tabular}

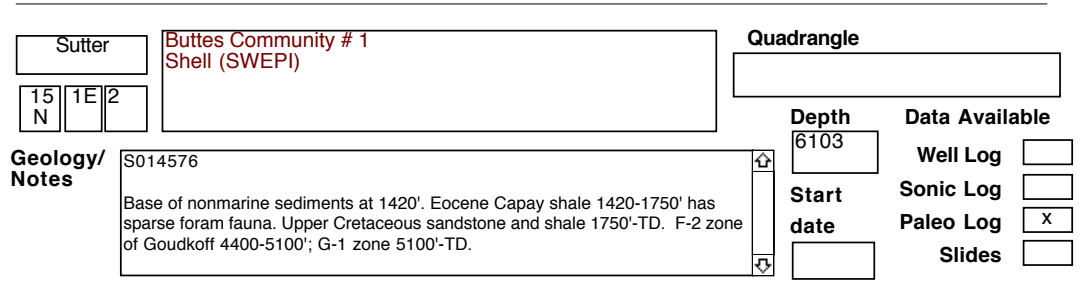

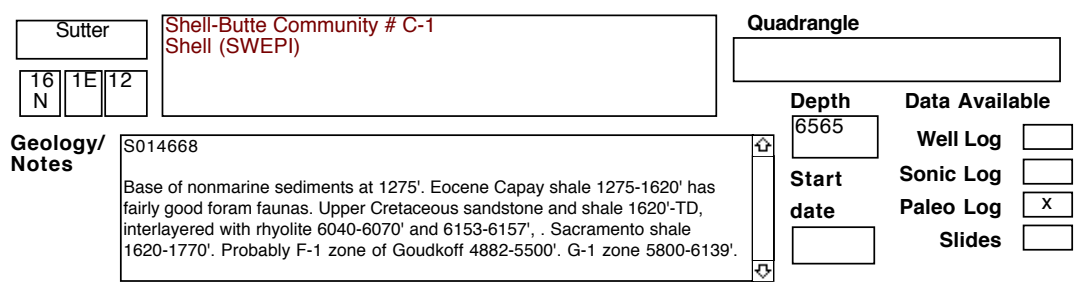

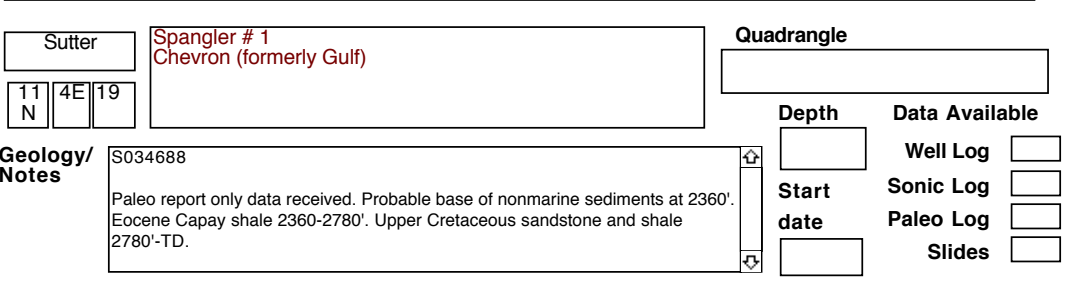

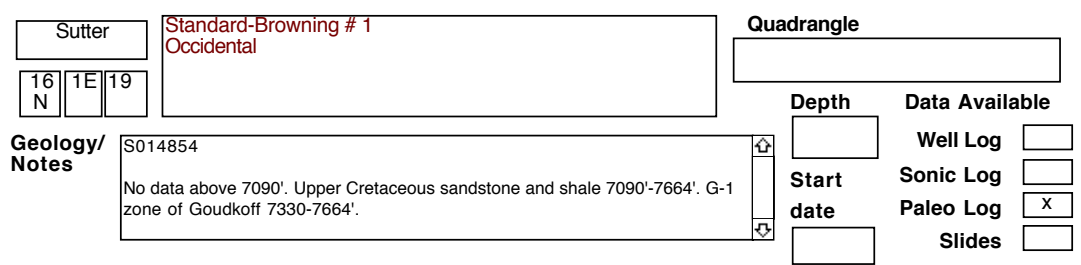


SELECTED OIL TEST WELLS IN CENTRAL CALIFORNIA DATABASE County

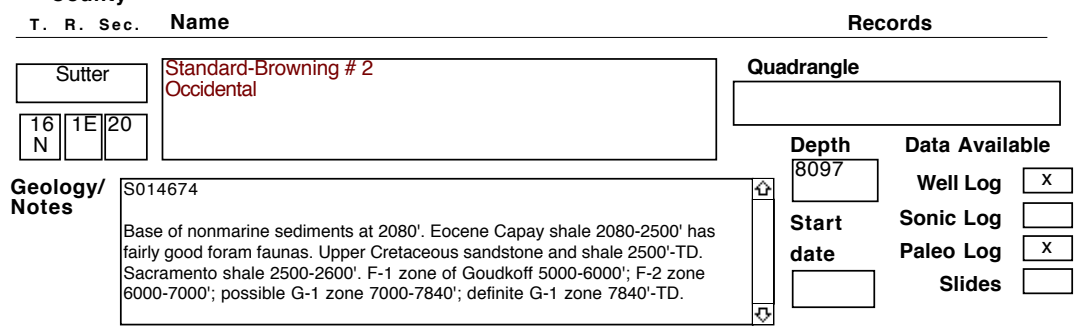

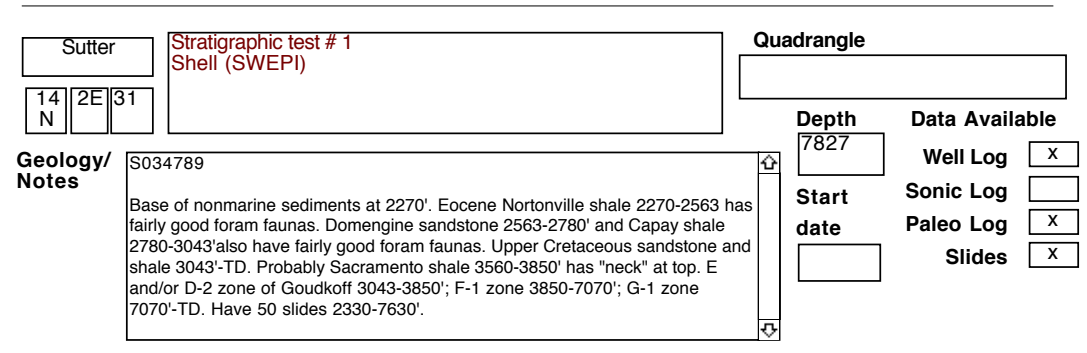

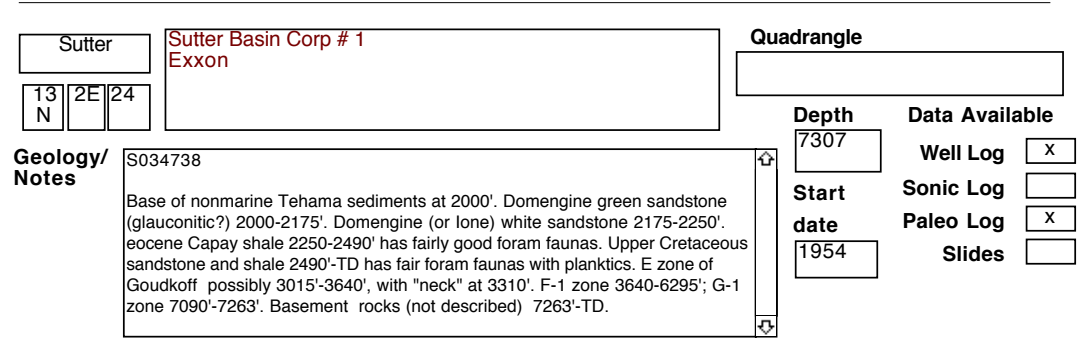

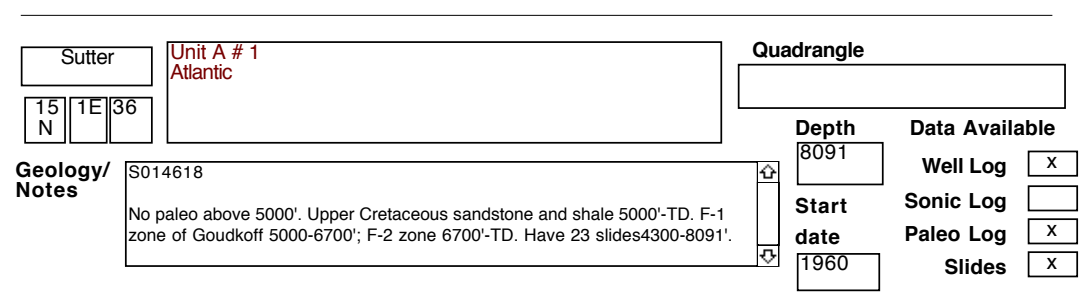

\begin{tabular}{l|l|l|l|l|l|l|}
\hline Sutter & $\begin{array}{l}\text { Zall corehole \#1 } \\
\text { Exxon }\end{array}$ \\
\hline
\end{tabular}


SELECTED OIL TEST WELLS IN CENTRAL CALIFORNIA DATABASE County

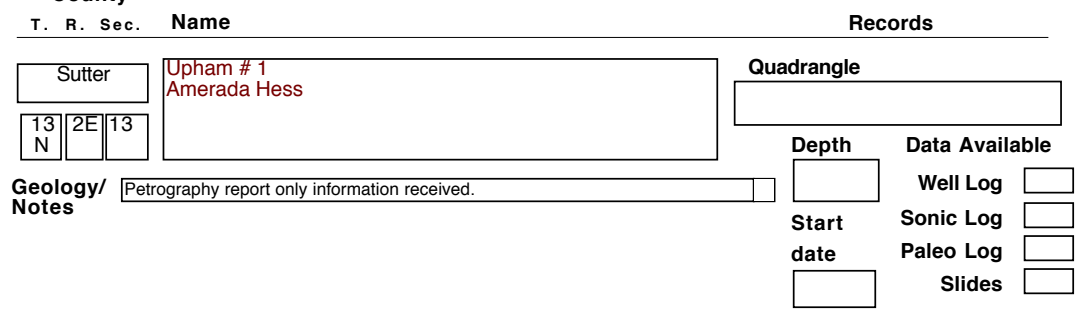

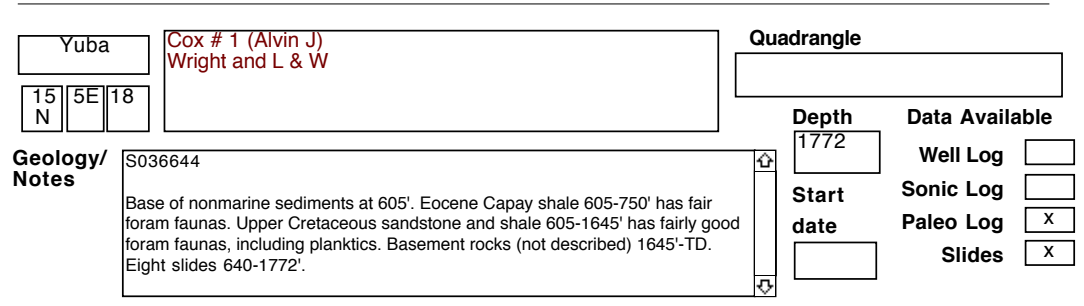

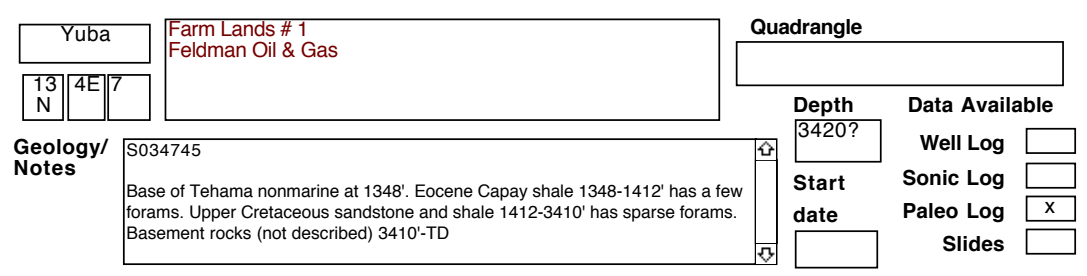

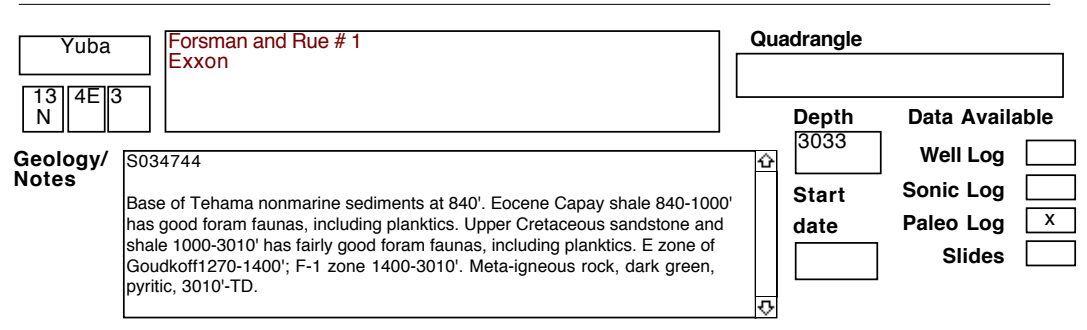

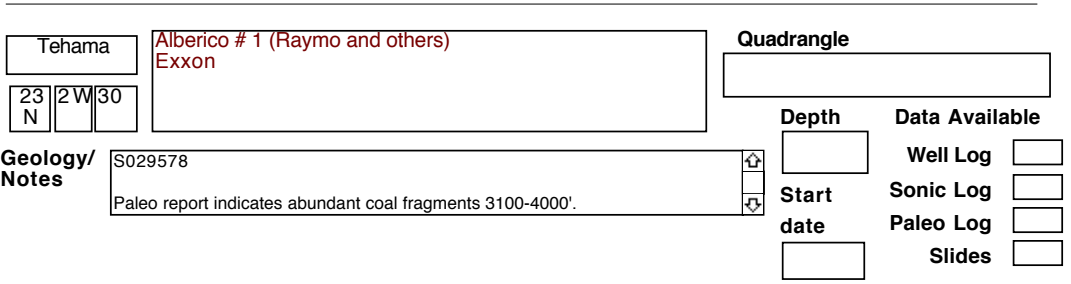




\section{SELECTED OIL TEST WELLS IN CENTRAL CALIFORNIA DATABASE}

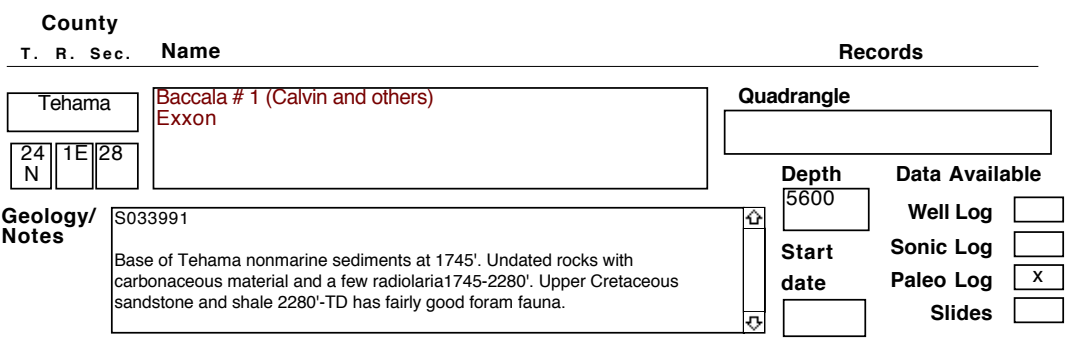

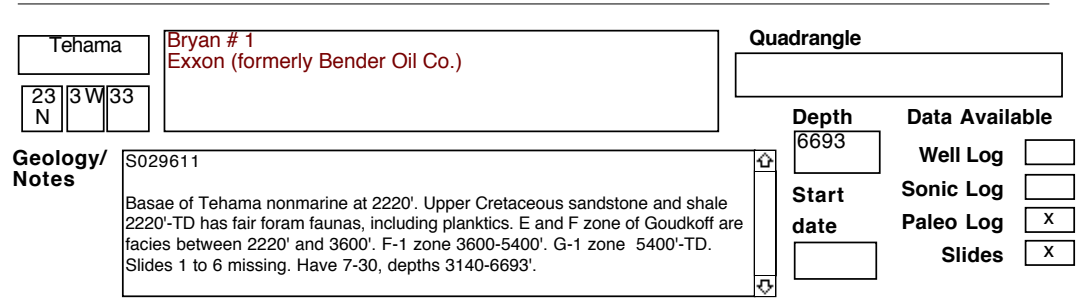

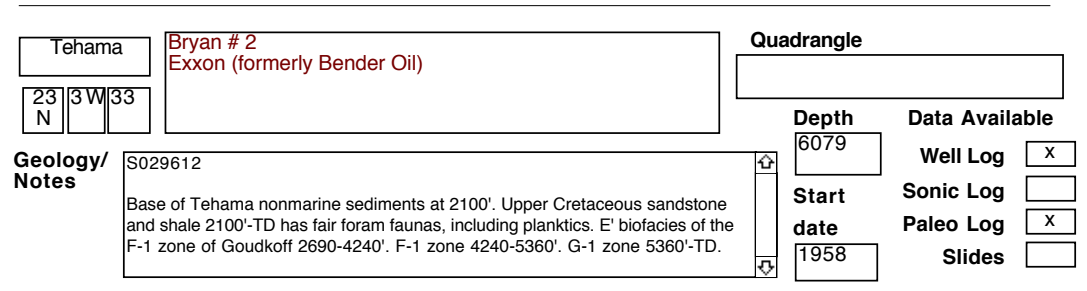

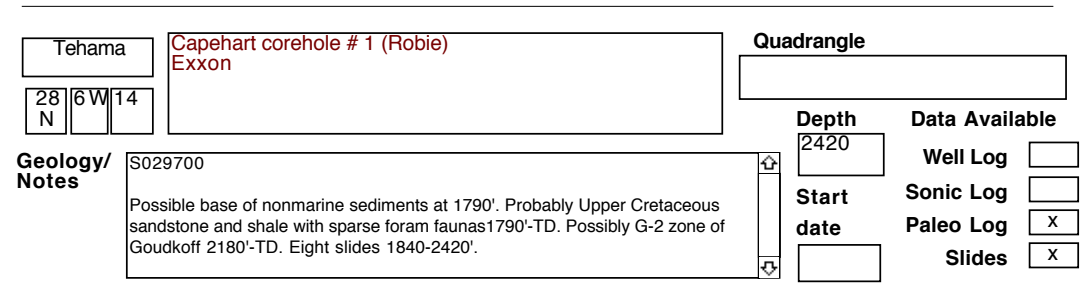

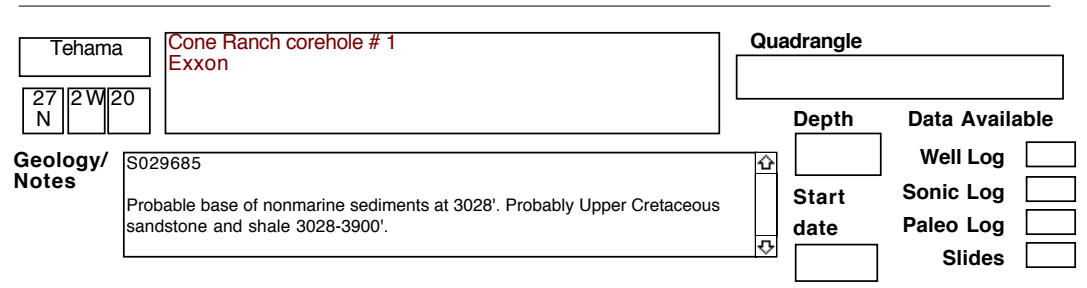


SELECTED OIL TEST WELLS IN CENTRAL CALIFORNIA DATABASE County

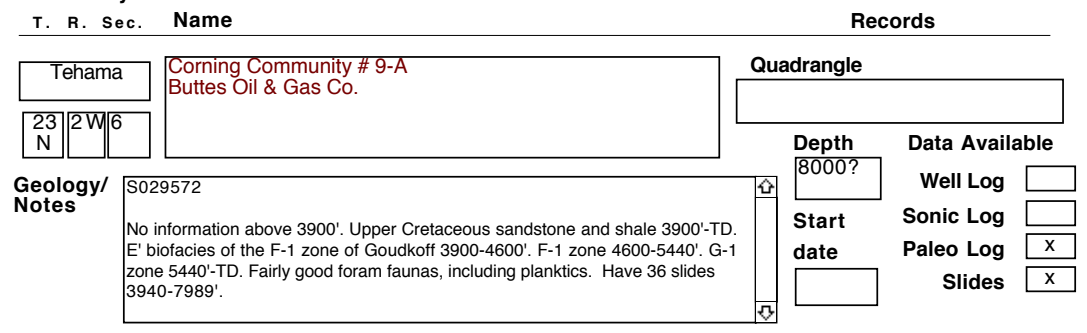

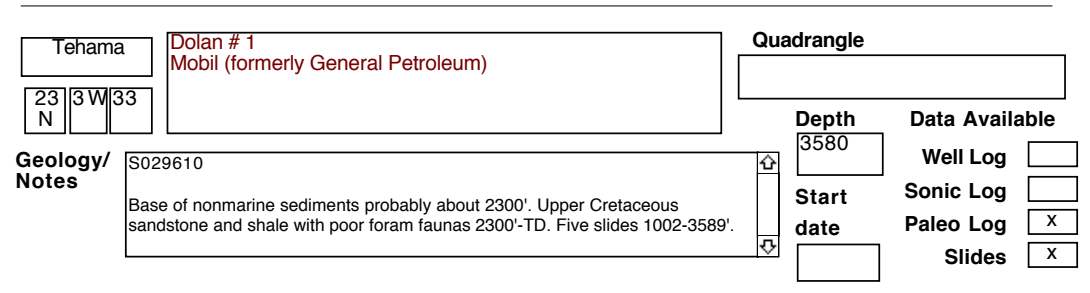

\begin{tabular}{l|l|l|l|l|l|l|l|l|}
\hline Tehama & $\begin{array}{l}\text { Durrer corehole \# 1 } \\
\text { Exxon }\end{array}$ \\
\hline
\end{tabular}

\begin{tabular}{|l|l|l|l|l|l|}
\hline Tehama & $\begin{array}{l}\text { Ewers-Mooney \# 1 } \\
\text { Northeastern Counties Petroleum }\end{array}$ \\
\hline
\end{tabular}

\begin{tabular}{l|l|l|l|l|l|l|l|l|}
\hline Tehama & $\begin{array}{l}\text { Flournoy \# 1-A } \\
\text { Exxon }\end{array}$ \\
\hline
\end{tabular}


SELECTED OIL TEST WELLS IN CENTRAL CALIFORNIA DATABASE County

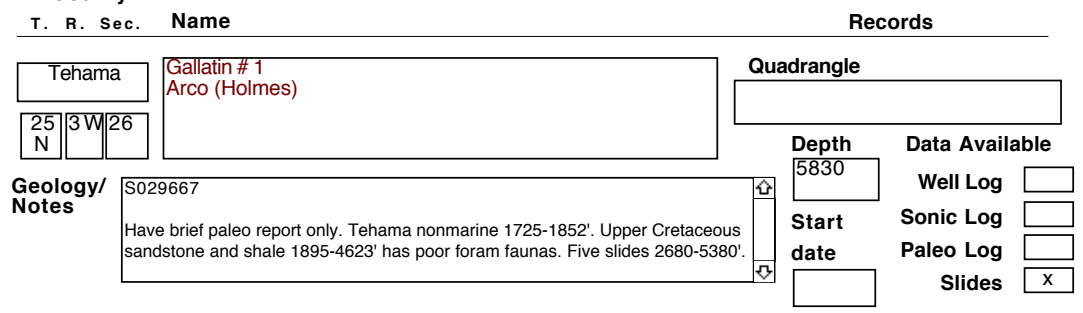

\begin{tabular}{l|l|l|l|l|l|}
\hline Tehama & $\begin{array}{l}\text { Hall \# 1 (AM) } \\
\text { Exxon }\end{array}$ & \multicolumn{2}{|l|}{ Quadrangle } \\
\hline
\end{tabular}

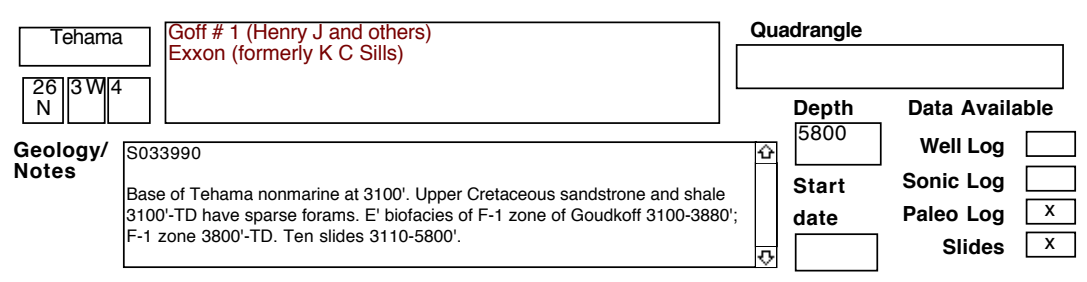

\begin{tabular}{|l|l|l|l|l|l|}
\hline Tehama & $\begin{array}{l}\text { Hancock-Shell-Anchordoguy \# 1 } \\
\text { Phillips Oil Co (formerly Aminoil) }\end{array}$ \\
\hline
\end{tabular}

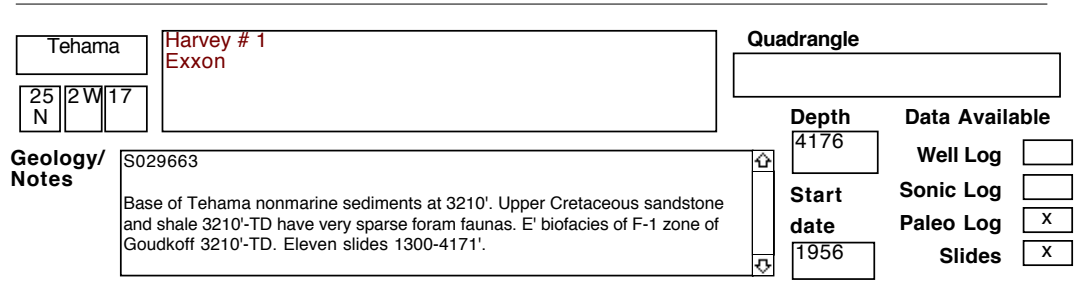


SELECTED OIL TEST WELLS IN CENTRAL CALIFORNIA DATABASE County

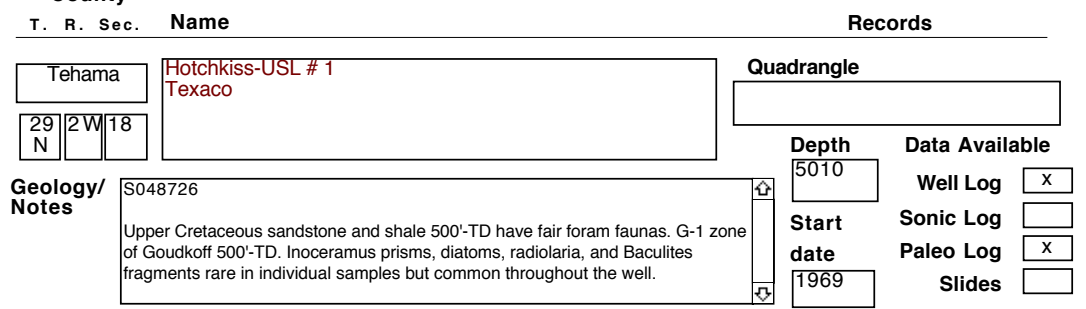

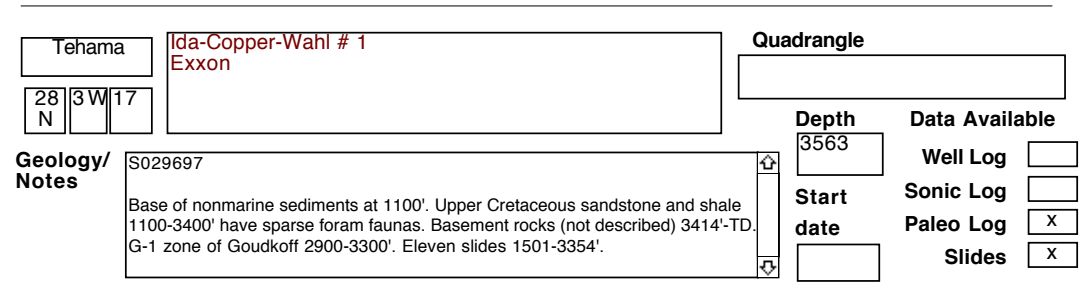

\begin{tabular}{l|l|l|l|l|l|l|}
\hline Tehama & $\begin{array}{l}\text { Jelly Bend \# 18-8 } \\
\text { Texaco }\end{array}$ \\
Geology/ & S0 29703 \\
Notes & $\begin{array}{l}\text { Base of Tehama nonmarine sediments at 522'. Upper Cretaceous sandstone and } \\
\text { shale 522-7110' have fairly good foram faunas, including planktics. Fairly good } \\
\text { spore and pollen range chart for 26 species. Serpentinite indicated on log } \\
\text { 7110'-TD, but paleo report indicates that the interval 7094-7128' is a massive, } \\
\text { fine-grained gray rock and that the washed residue is crystalline schist. Probably } \\
\text { G-1 zone of Goudkoff 522-6530'. G-2 zone 6530-6740'. Possibly H zone } \\
\text { 6470-7110'. Have 19 slides 610-7106. }\end{array}$ \\
\hline
\end{tabular}

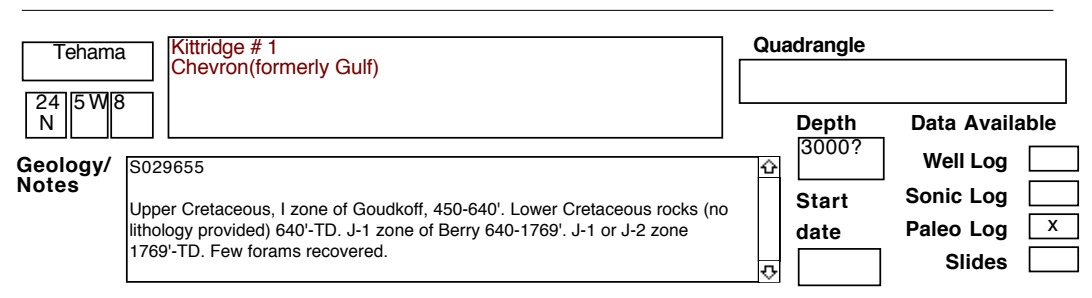

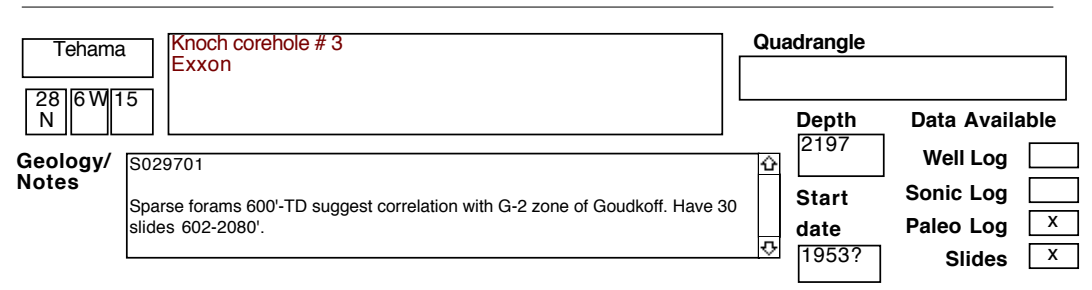


SELECTED OIL TEST WELLS IN CENTRAL CALIFORNIA DATABASE County

\begin{tabular}{l|l|l|l|l|}
\hline T. R. Sec. & Name & \multicolumn{2}{c|}{ Records } \\
\hline Tehama & $\begin{array}{l}\text { Lyon Cattle co \# 1 } \\
\text { Exxon }\end{array}$ \\
\hline
\end{tabular}

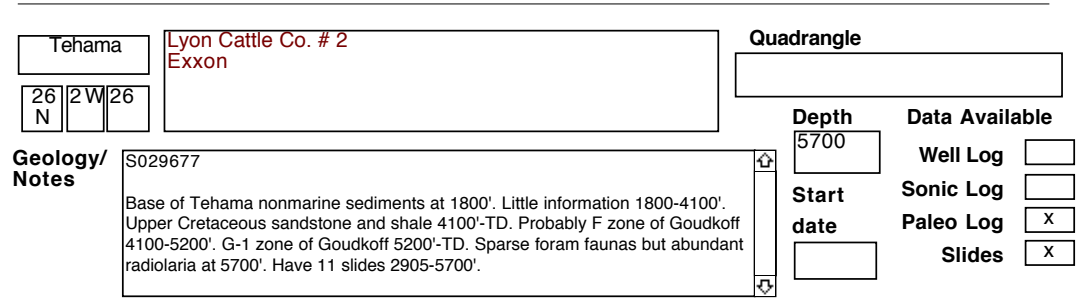

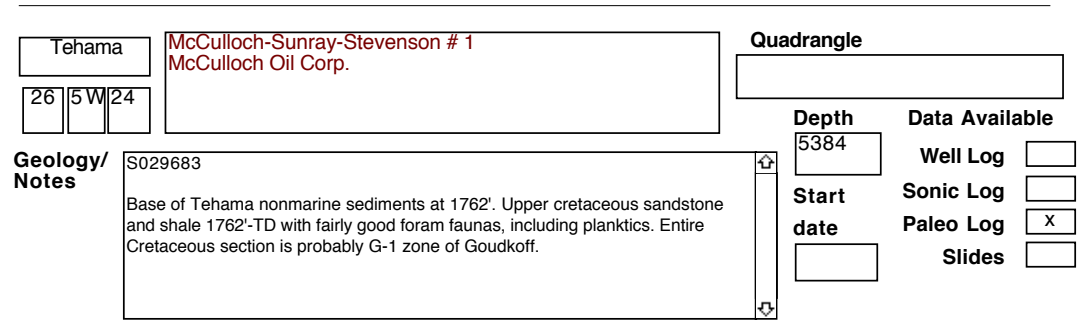

\begin{tabular}{l|l|l|l|l|l|}
\hline Tehama & $\begin{array}{l}\text { McCulloch-Superior-Victor Ranch \# 4 } \\
\text { Sunray DX Oil Co }\end{array}$ \\
\hline
\end{tabular}

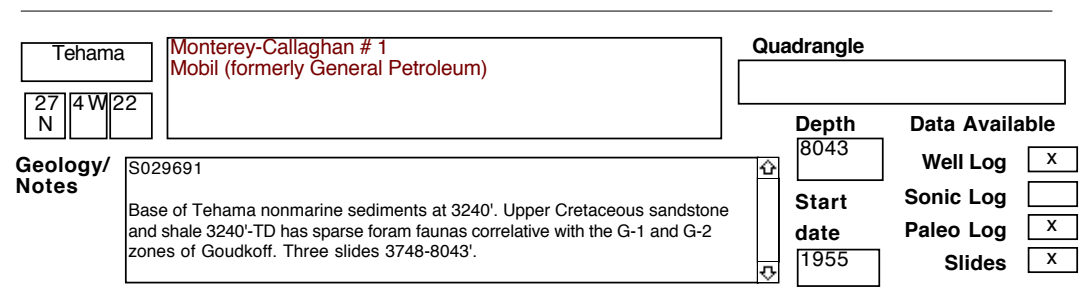


SELECTED OIL TEST WELLS IN CENTRAL CALIFORNIA DATABASE County

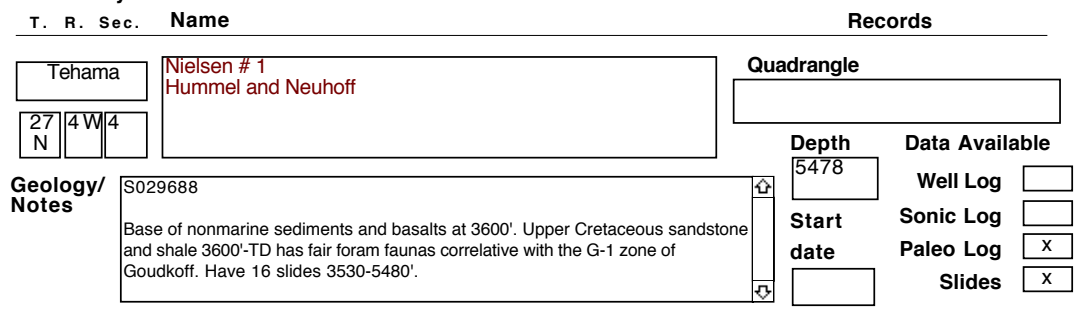

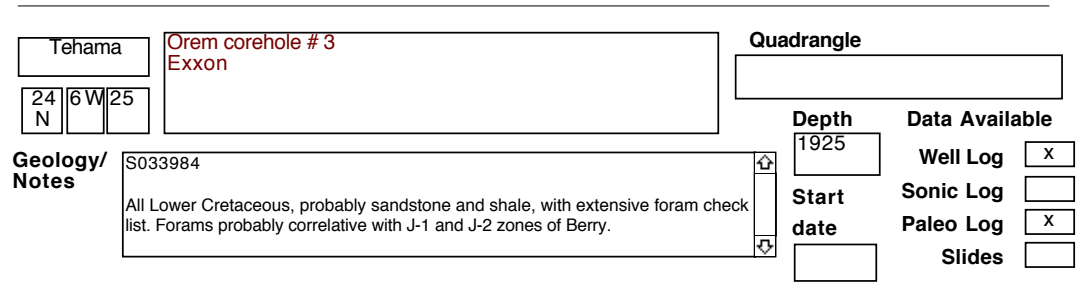

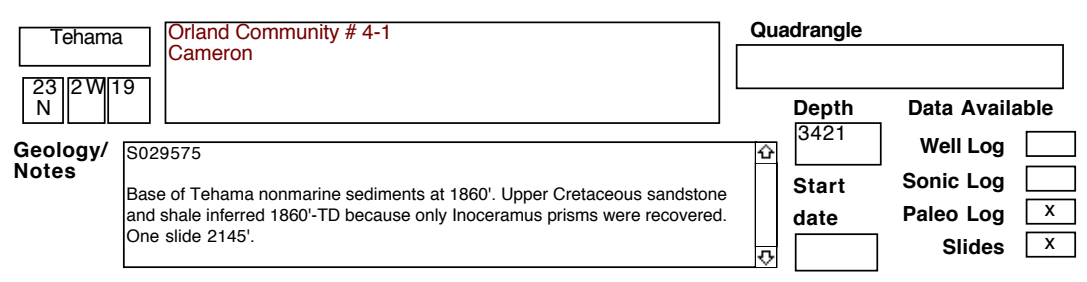

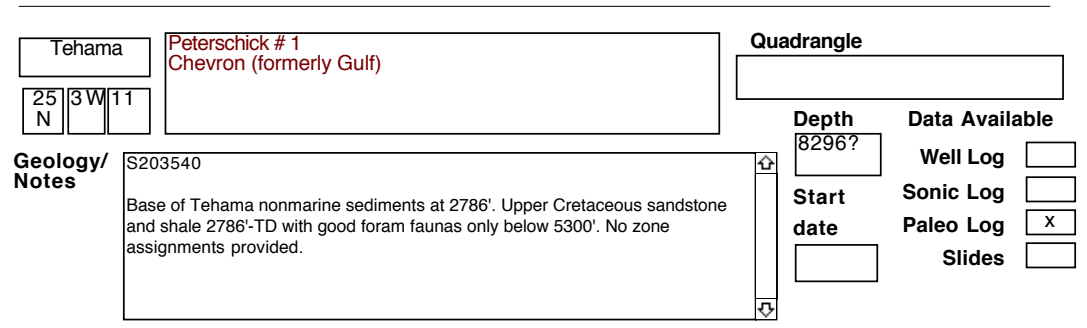

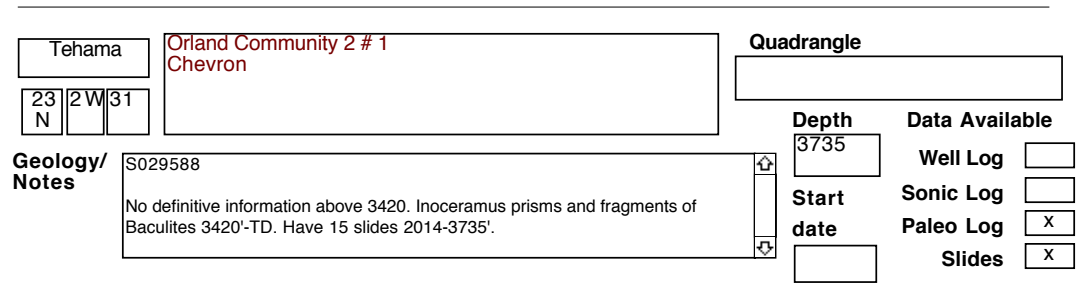




\section{SELECTED OIL TEST WELLS IN CENTRAL CALIFORNIA DATABASE}

County

\begin{tabular}{l|l|l|l|l|}
\hline T. R. Sec. & Name & Records \\
\hline Tehama & $\begin{array}{l}\text { Saldubehere \# 1 } \\
\text { Dewitt (formerly Superior) }\end{array}$ \\
\hline
\end{tabular}

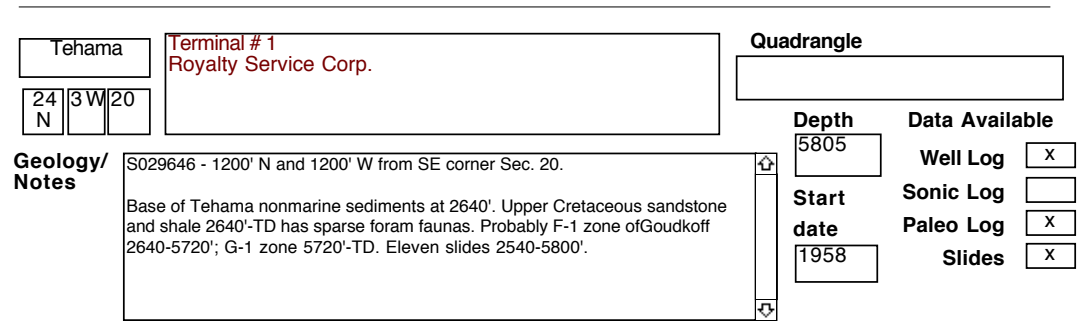

\begin{tabular}{l|l|l|l|l|l|l|l|}
\hline Tehama & Tuscan Springs \# A-1 \\
GEC Oil Co.
\end{tabular}

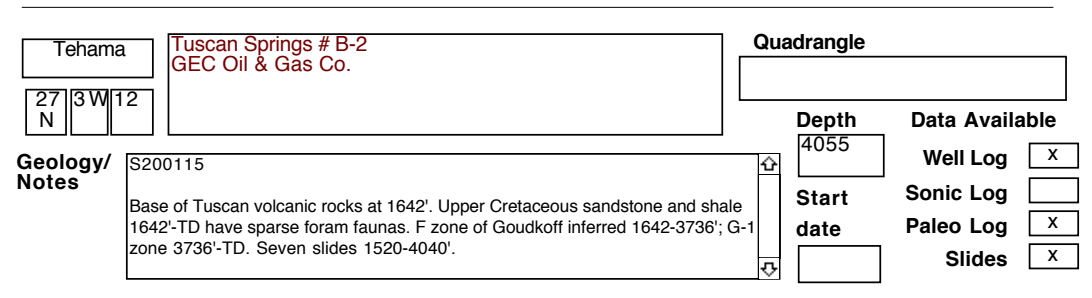

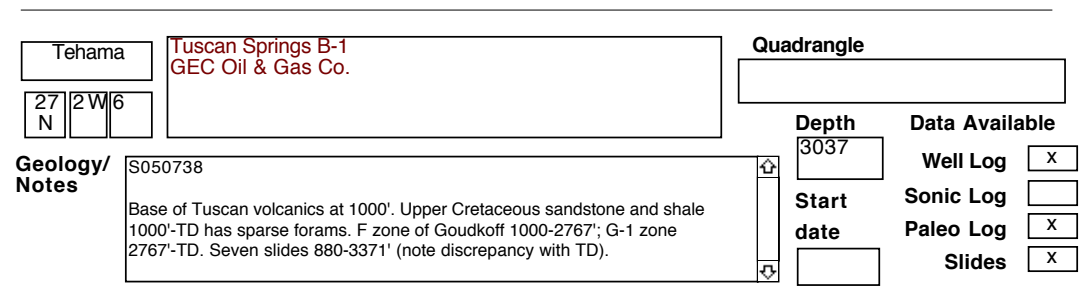


SELECTED OIL TEST WELLS IN CENTRAL CALIFORNIA DATABASE County

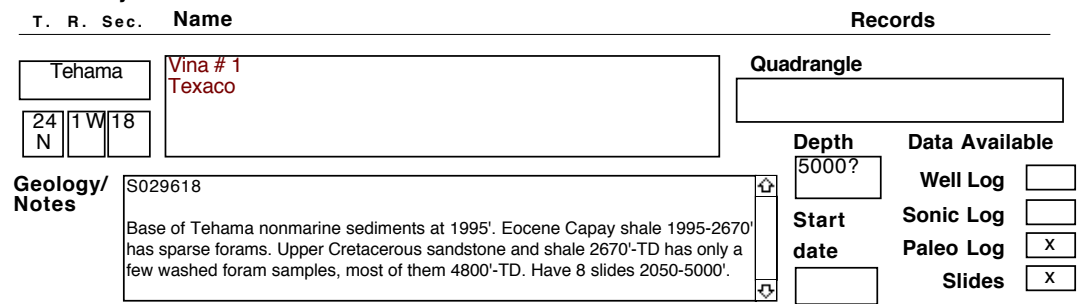

\begin{tabular}{l|l|l|l|l|l|l|}
\hline Tehama & $\begin{array}{l}\text { Walbridge \# } 1 \mathrm{~A} \\
\text { Texaco }\end{array}$ & \multicolumn{2}{|l|}{ Quadrangle } \\
\hline
\end{tabular}

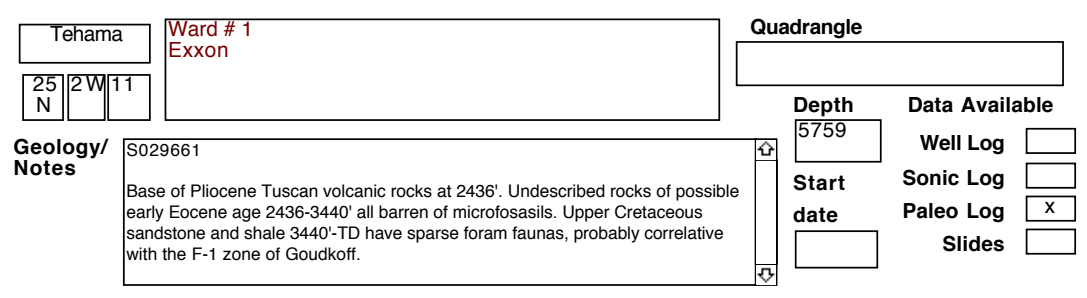

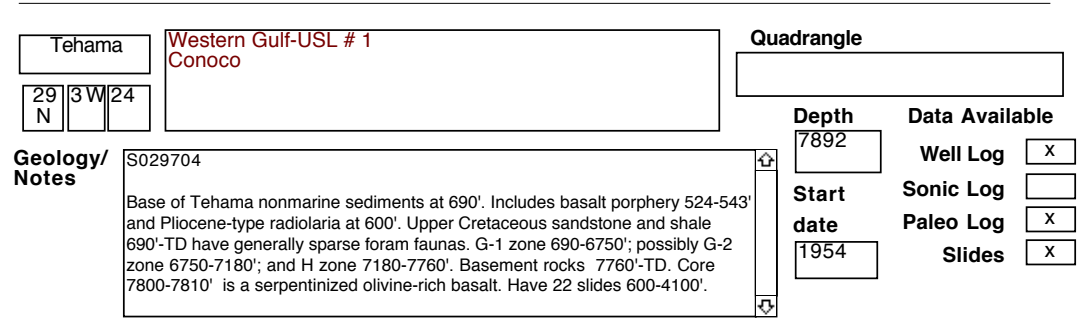

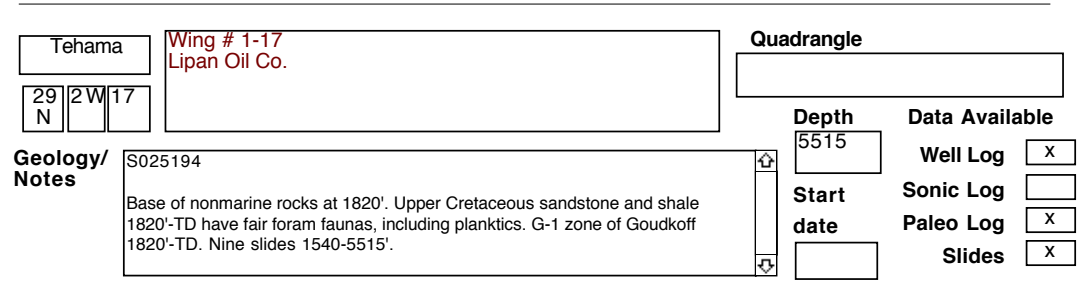


SELECTED OIL TEST WELLS IN CENTRAL CALIFORNIA DATABASE County

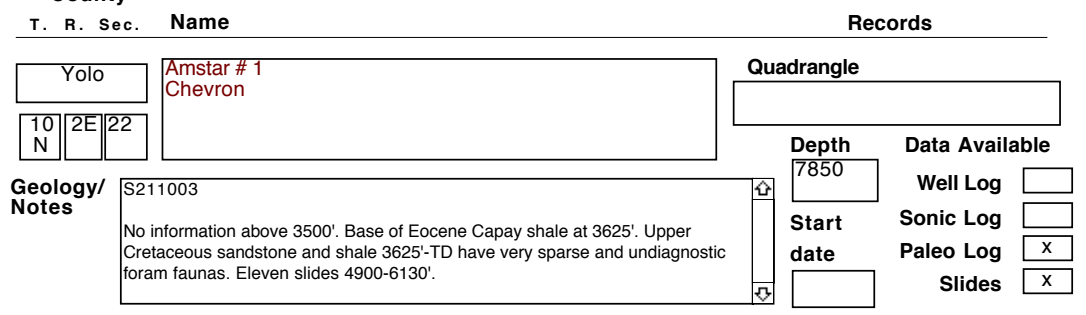

\begin{tabular}{l|l|l|l|l|l|l|}
\hline Yolo & $\begin{array}{l}\text { Amstar \# 2 } \\
\text { Chevron }\end{array}$ \\
\hline
\end{tabular}

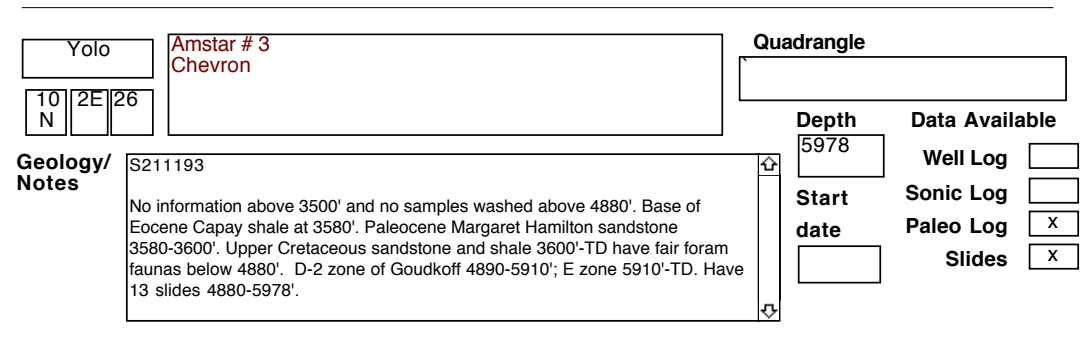

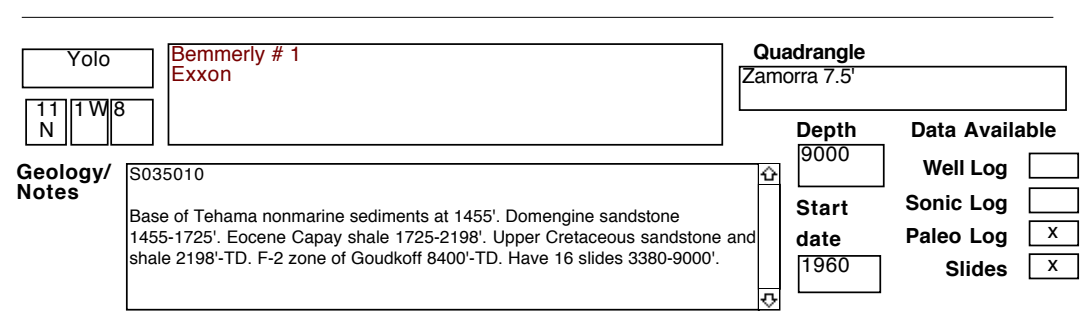

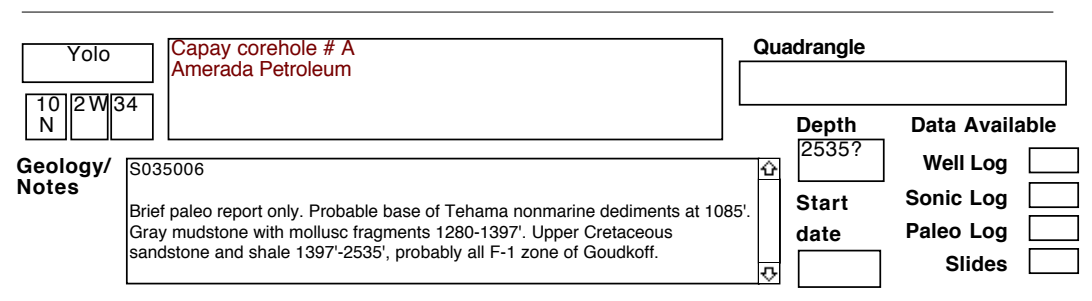


SELECTED OIL TEST WELLS IN CENTRAL CALIFORNIA DATABASE County

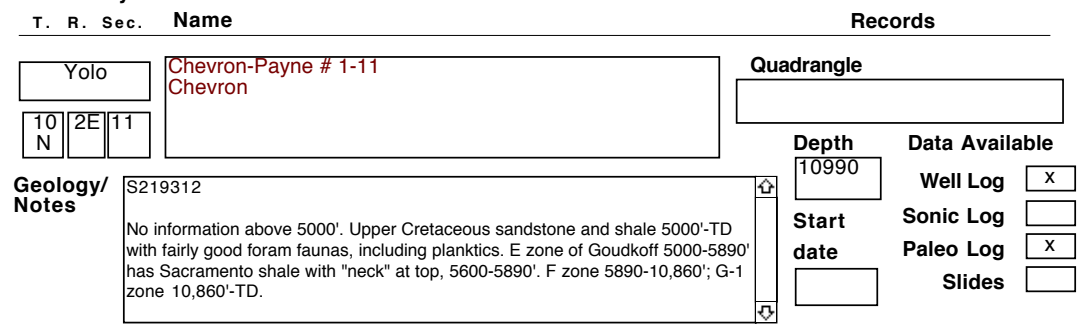

\begin{tabular}{|c|c|c|c|c|}
\hline Yolo & \multirow{2}{*}{\begin{tabular}{|l|} 
Correa \# 1 \\
Chevron
\end{tabular}} & \multicolumn{3}{|l|}{ Quadrangle } \\
\hline $7 \mathrm{~N} /\left.4 \mathrm{E}\right|^{2}$ & & \multirow{2}{*}{$\begin{array}{l}\text { Depth } \\
7490\end{array}$} & \multicolumn{2}{|c|}{ Data Available } \\
\hline \multirow{4}{*}{$\begin{array}{l}\text { Geology/ } \\
\text { Notes }\end{array}$} & S034577 & & \multirow{4}{*}{$\begin{array}{r}\text { Well Log } \\
\text { Sonic Log } \\
\text { Paleo Log } \\
\text { Slides }\end{array}$} & $\bar{x}$ \\
\hline & Base of Tehama nonmarine sediments at $2670^{\prime}$. Eocene Capay shale $2670-2890$ & Start & & \\
\hline & has good foram faunas. Sandstone and shale $2890-3435$ ' has no diagnostic & date & & $\mathrm{x}$ \\
\hline & 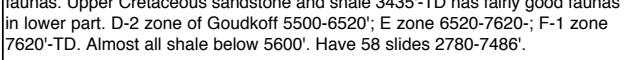 & 1952 & & $\mathrm{x}$ \\
\hline
\end{tabular}

\begin{tabular}{|c|c|c|c|c|}
\hline Yolo & Cook (J.E.) & Quadrangle & & \\
\hline \begin{tabular}{|l|l|l|l|}
12 & $1 \mathrm{~W}$ & 31 \\
$\mathrm{~N}$ & & \\
\end{tabular} & & \multirow{2}{*}{$\begin{array}{l}\text { Depth } \\
10066\end{array}$} & \multicolumn{2}{|c|}{ Data Available } \\
\hline \multirow{3}{*}{\multicolumn{2}{|c|}{$\begin{array}{l}\text { Geology/ Only have an E log. } \\
\text { Notes }\end{array}$}} & & Well Log & $x$ \\
\hline & & Start & Sonic Log & \\
\hline & & $\begin{array}{l}\text { date } \\
1974\end{array}$ & $\begin{array}{r}\text { Paleo Log } \\
\text { Slides }\end{array}$ & \\
\hline
\end{tabular}

\begin{tabular}{|l|l|l|l|}
\hline Yolo & \multicolumn{2}{|l|}{$\begin{array}{l}\text { Deland and Ferris \# } 4 \\
\text { Getty }\end{array}$} & \multicolumn{2}{l|}{ Quadrangle } \\
\hline
\end{tabular}

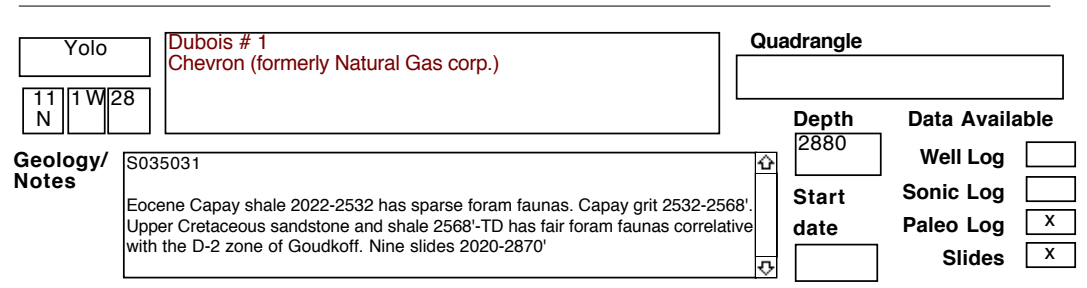




\section{SELECTED OIL TEST WELLS IN CENTRAL CALIFORNIA DATABASE}

County

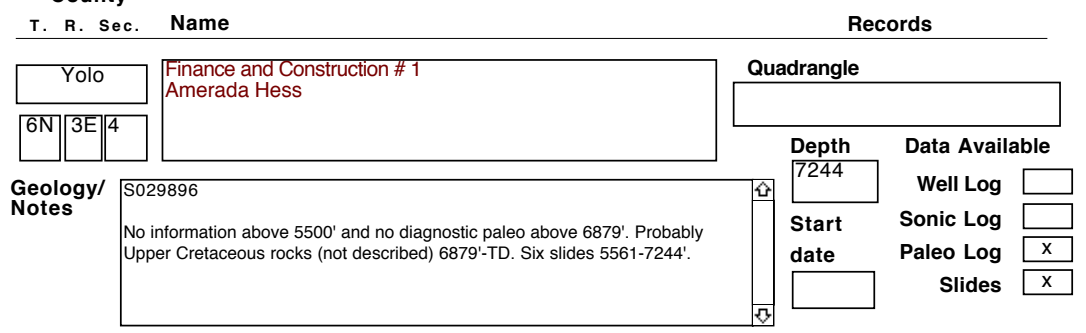

\begin{tabular}{l|l|l|l|l|l|}
\hline Yolo & $\begin{array}{l}\text { Gallen Camp \# 1 } \\
\text { Mobil (General Petroleum) }\end{array}$ \\
\hline
\end{tabular}

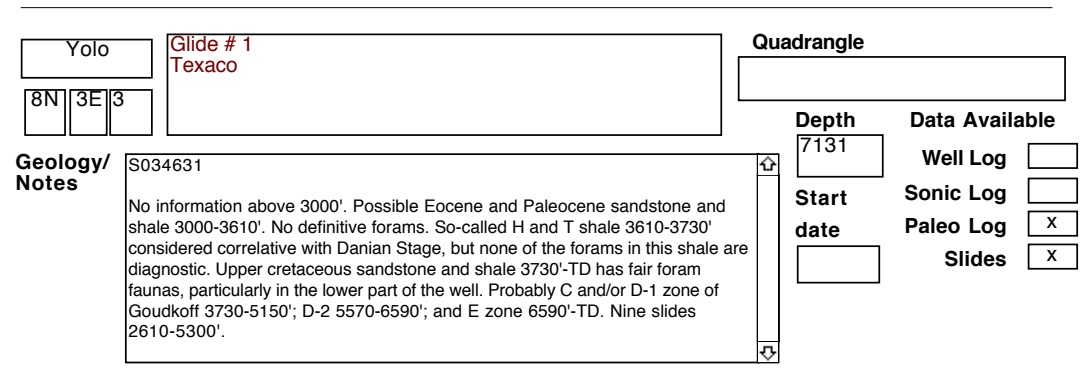

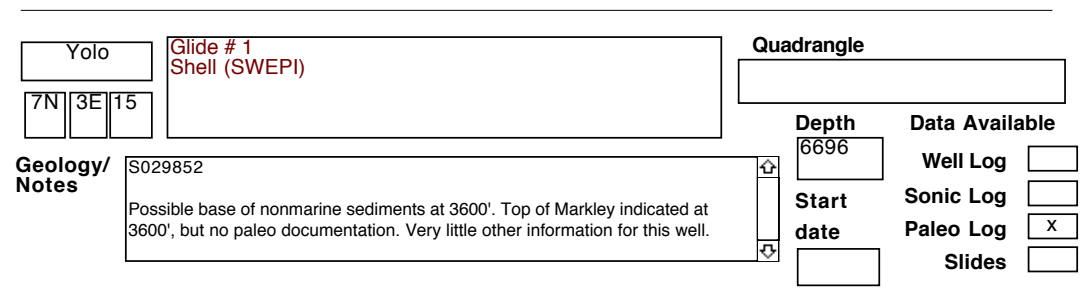

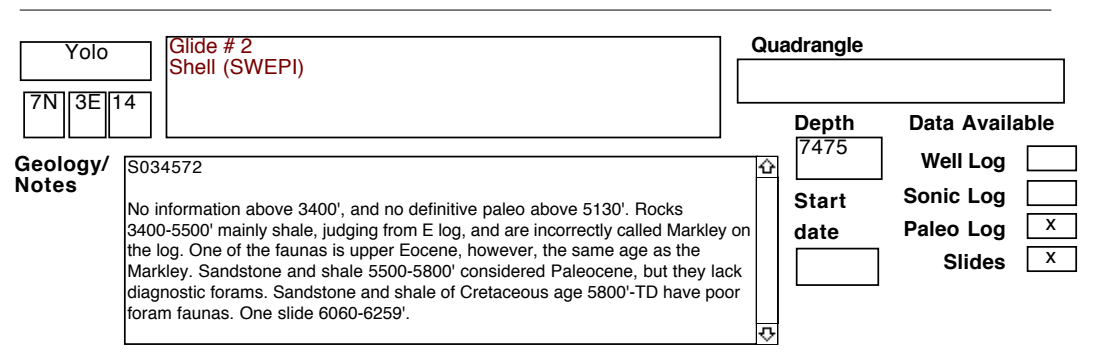




\section{SELECTED OIL TEST WELLS IN CENTRAL CALIFORNIA DATABASE}

County

\begin{tabular}{|c|c|}
\hline Yolo & $\begin{array}{l}\text { Glide-Court \# 65-10 } \\
\text { Mobil (formerly General Petroleum) }\end{array}$ \\
\hline \begin{tabular}{|l||l||l|}
$7 \mathrm{~N}$ & $3 \mathrm{E}$ &
\end{tabular} & \\
\hline
\end{tabular}

\section{Geology/ S034571}

Notes

No paleo samples available above 12,020 '. Base of nonmarine Tehama sediments inferred at about 3550'. Eocene Markley channel deposits inferred 3550-4410'. Upper Cretaceous sandstone and shale 4410-15,100' have fairly good samples in the interval examined below 12,020'. Basement rocks 15,100-TD described as quartzite and metamorphic rocks. Goudkoff's zones established by correlation above 12,020'. G-1 zone 12,600-14,930'; G-2 zone 14,930-15,050'; H zone $15,050-15,100^{\prime}$. Have 36 slides 12,040-15,280

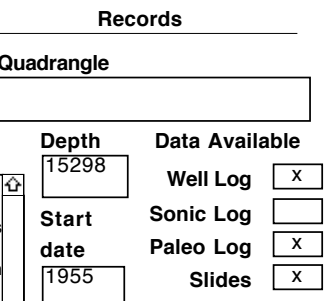

\begin{tabular}{|c|c|c|}
\hline Yolo & \multicolumn{2}{|l|}{$\begin{array}{l}\text { Glide Court \# 1 } \\
\text { Mobil (General Petroleum) }\end{array}$} \\
\hline $7 \mathrm{~N}$ & $3 \mathrm{E}$ & 10 \\
\hline
\end{tabular}

Geology/ S034570

Notes

No samples examined above $7640^{\prime}$. Base of nonmarine sediments at about 3100'. Eocene Markley channel deposits $3100-4235^{\prime}$ are considered correlative with A-1 zone of Laiming and Refugian Stage of Schenck and Kleinpel.

Paleocene sandstone inferred $4235-4550$. H andT shale correlative with Danian

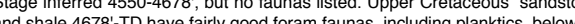

7640'. Base of D-2 zone of Goudkoff at 7920'. E zone 7920-9010'. F-2 zon

9010-13,050': G-1 zone 13,050'-TD. Sacramento shale with "neck" at top

8880-9220'. Have 61 slides 7660-13,321'.

\begin{tabular}{|c|c|c|c|}
\hline \multicolumn{4}{|c|}{ Quadrangle } \\
\hline \multirow{5}{*}{ 요 } & \multirow{2}{*}{$\begin{array}{l}\text { Depth } \\
13321\end{array}$} & \multicolumn{2}{|c|}{ Data Available } \\
\hline & & Well Log & $x$ \\
\hline & Start & Sonic Log & \\
\hline & date & Paleo Log & $x$ \\
\hline & 1953 & Slides & $\mathrm{x}$ \\
\hline
\end{tabular}

\begin{tabular}{|c|c|}
\hline Yolo & $\begin{array}{l}\text { Dunnigan Unit \# 1-1 (also called Hermle \# 1) } \\
\text { Texaco }\end{array}$ \\
\hline $\begin{array}{ll}11 \mathrm{~W} \\
\mathrm{~N}\end{array}$ & \\
\hline
\end{tabular}

\section{Geology/ 5035016}

Notes

Eocene Capay shale 1914-2380' Glauconitic grit at base of Capay 2380-2417'. Six slides 2300-4022

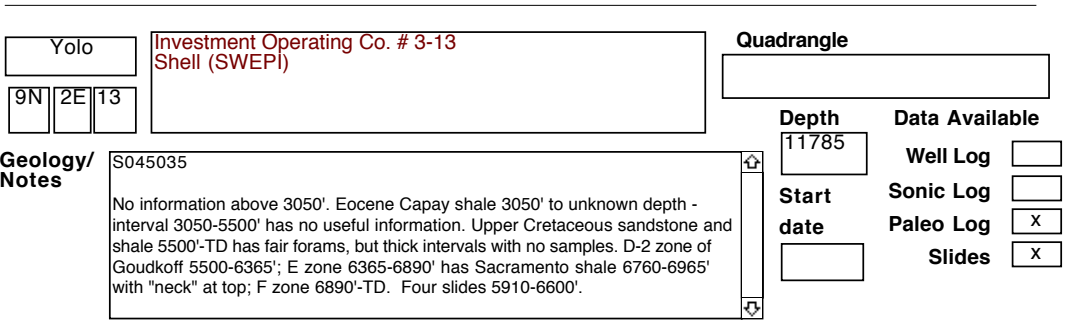

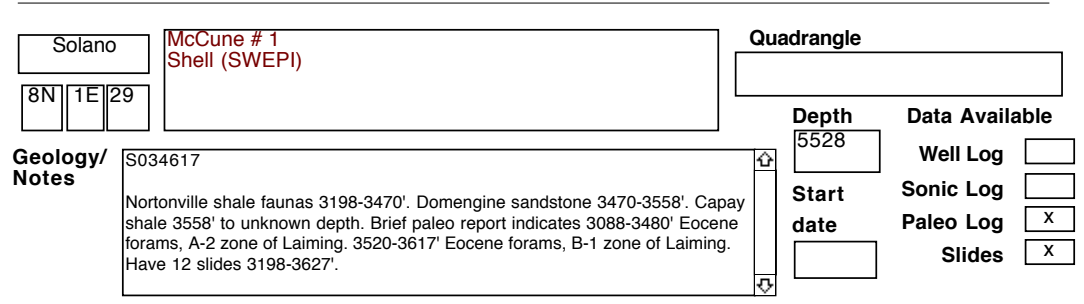


SELECTED OIL TEST WELLS IN CENTRAL CALIFORNIA DATABASE County

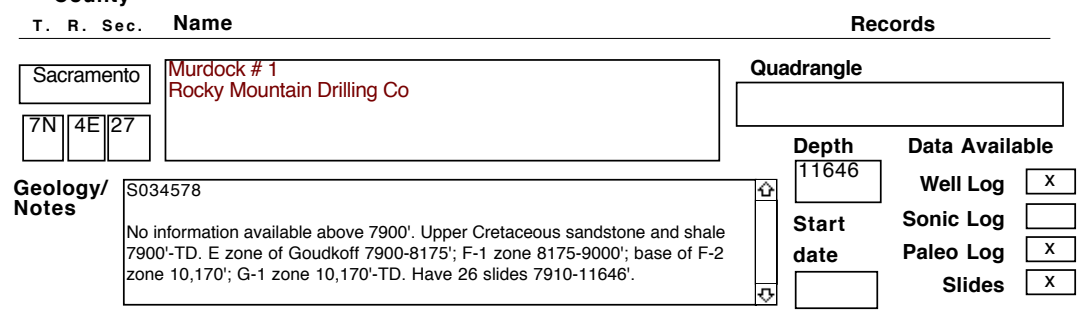

\begin{tabular}{l|l|l|l|l|l|l|}
\hline Yolo & $\begin{array}{l}\text { Lee Bow \# 1 } \\
\text { Dome Oil \& Gas Co. }\end{array}$ \\
\hline
\end{tabular}

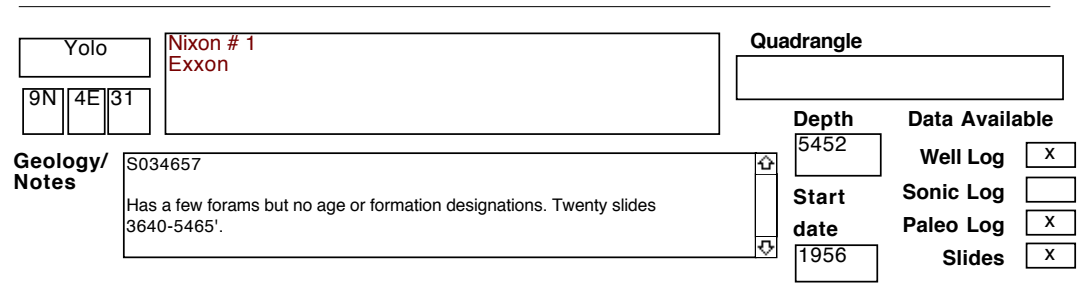

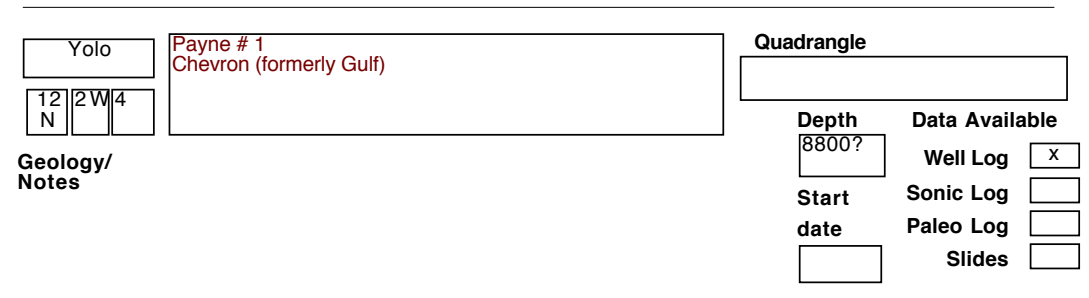

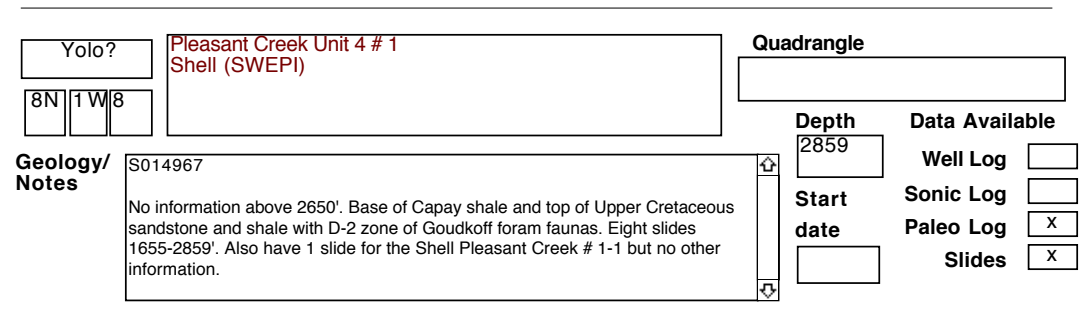


SELECTED OIL TEST WELLS IN CENTRAL CALIFORNIA DATABASE County

\begin{tabular}{|c|c|c|c|c|}
\hline T. R. Sec. & Name & \multicolumn{3}{|c|}{ Records } \\
\hline Yolo & \multirow{2}{*}{$\begin{array}{l}\text { Rieff \# } 1 \\
\text { Arco }\end{array}$} & \multicolumn{3}{|l|}{ Quadrangle } \\
\hline \begin{tabular}{|l|l|l|}
11 & $1 \mathrm{E}$ & 29 \\
$\mathrm{~N}$ & & \\
\end{tabular} & & \multirow{2}{*}{$\begin{array}{l}\text { Depth } \\
15893 ?\end{array}$} & \multicolumn{2}{|c|}{ Data Available } \\
\hline \multirow{4}{*}{$\begin{array}{l}\text { Geology/ } \\
\text { Notes }\end{array}$} & & & Well Log & $\mathrm{x}$ \\
\hline & & Start & Sonic Log & \\
\hline & & $\begin{array}{l}\text { date } \\
1972\end{array}$ & Paleo Log & \\
\hline & & & & \\
\hline
\end{tabular}

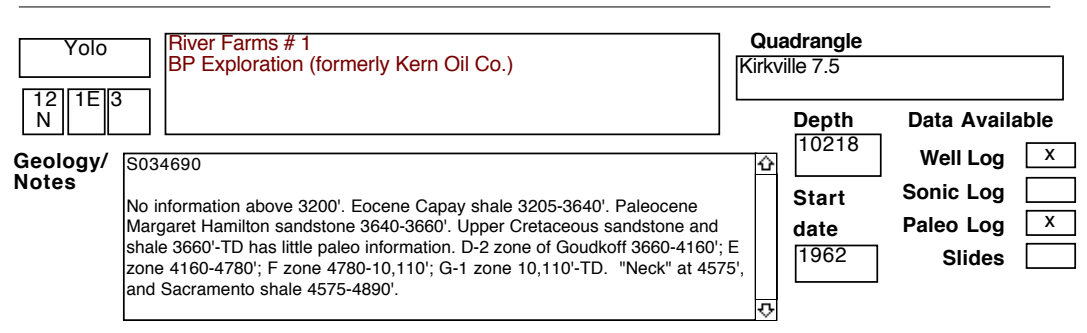

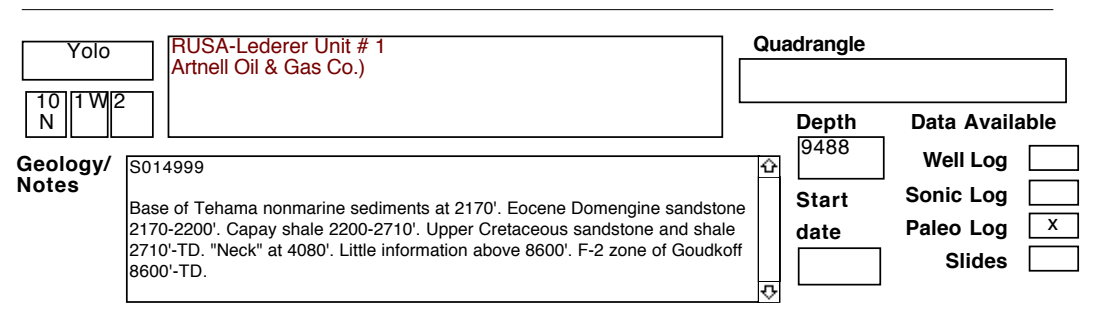

\begin{tabular}{|l|l|l|l|l|l|}
\hline \multicolumn{1}{|c|}{$\begin{array}{l}\text { Salisbury \#1 } \\
\text { Chevron }\end{array}$} \\
Geology/ \\
Notes
\end{tabular}

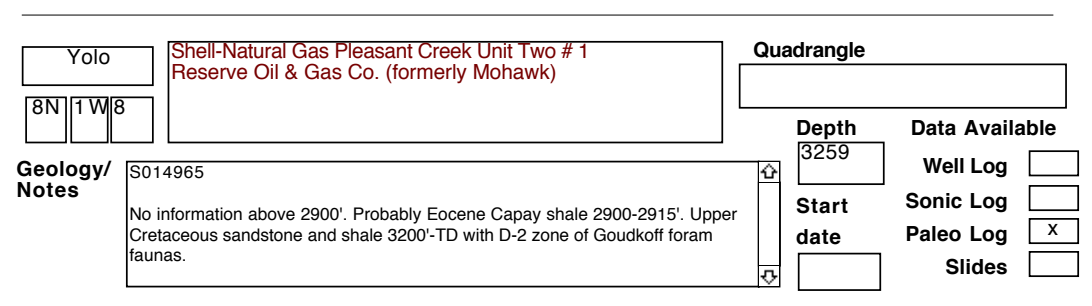


SELECTED OIL TEST WELLS IN CENTRAL CALIFORNIA DATABASE County

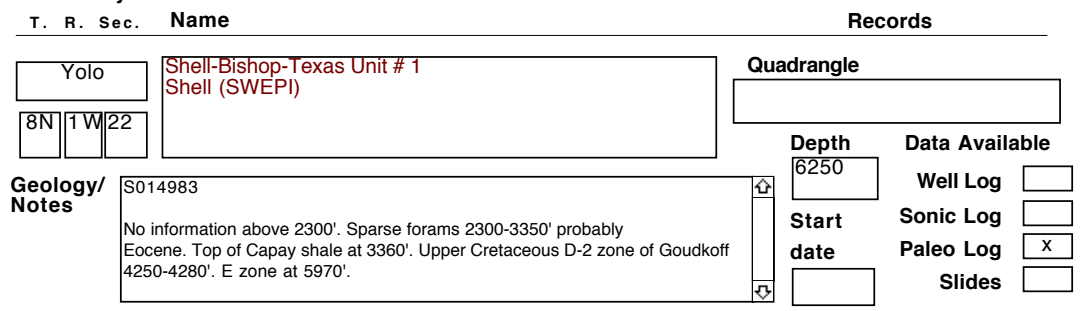

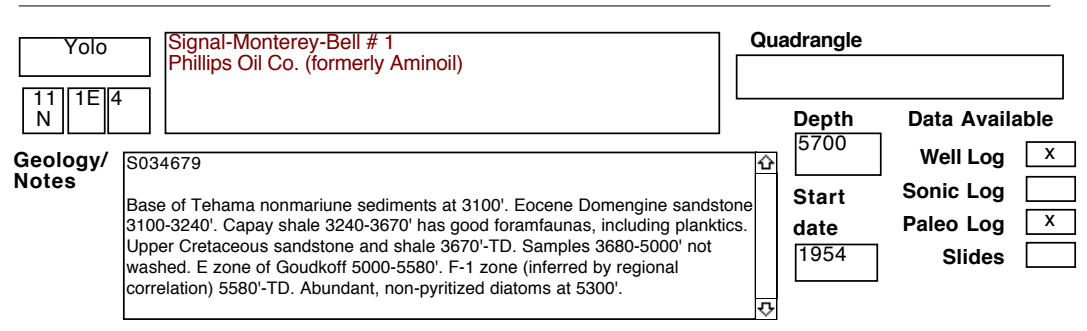

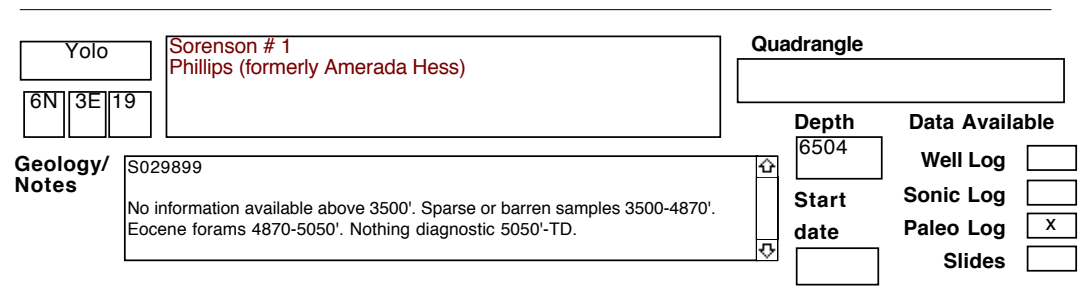

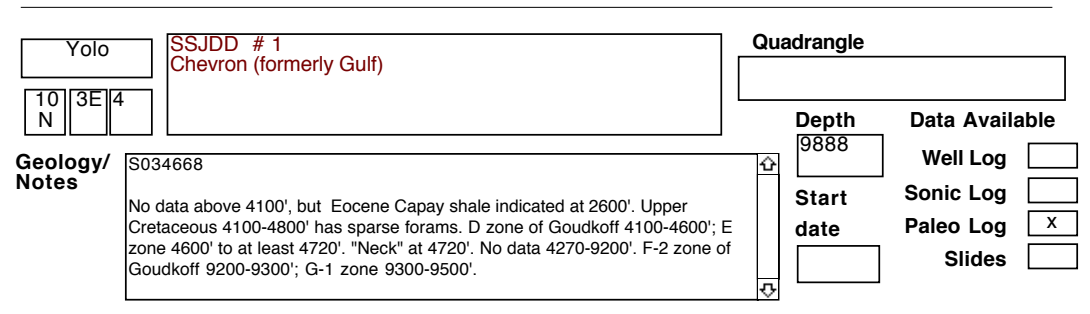

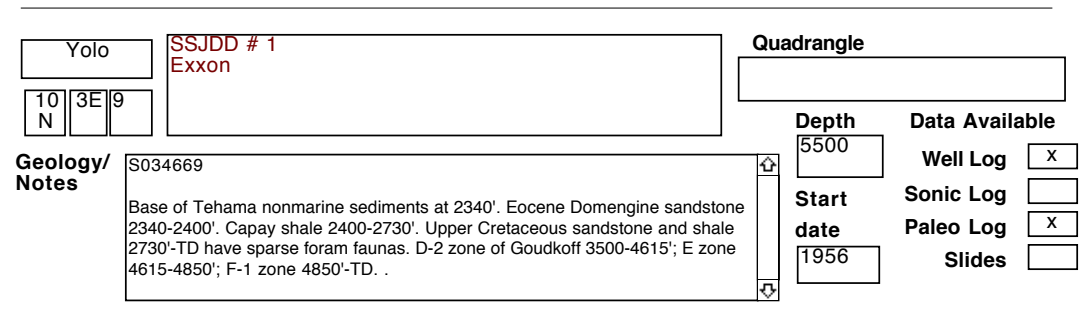


SELECTED OIL TEST WELLS IN CENTRAL CALIFORNIA DATABASE County

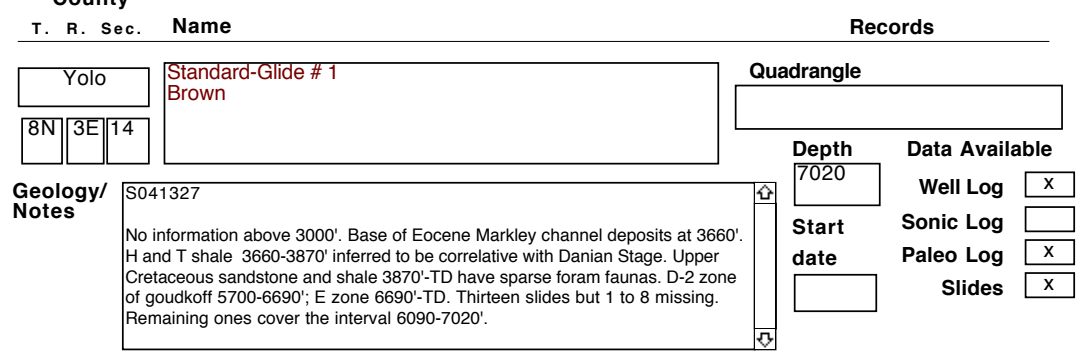

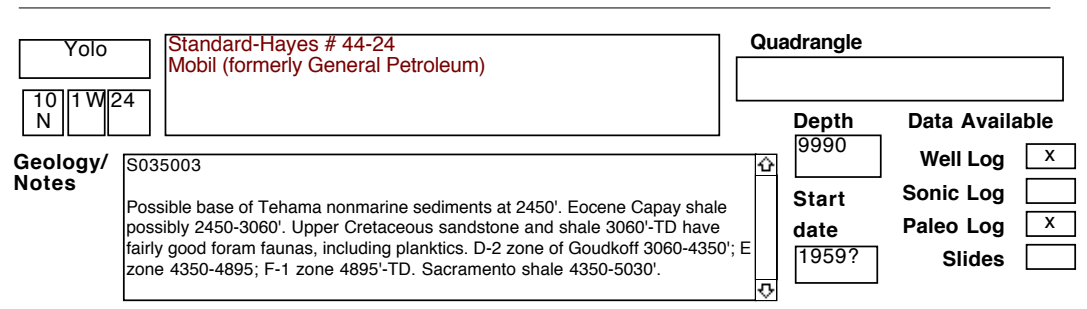

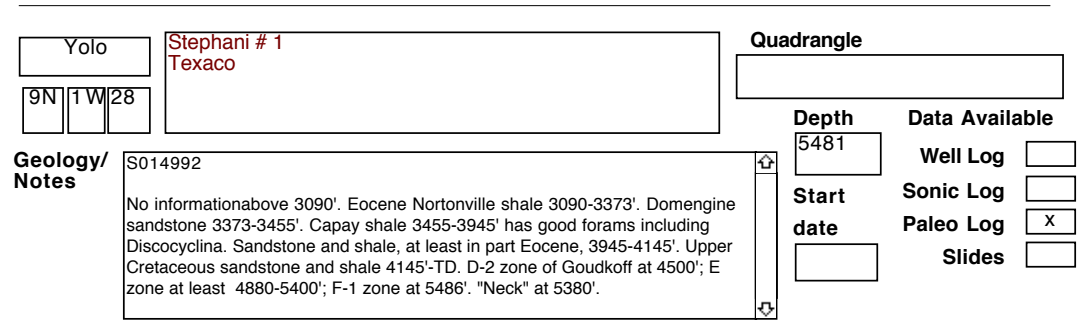

\begin{tabular}{|l|l|l|l|l|l|}
\hline Solo & $\begin{array}{l}\text { Swan \# 1 } \\
\text { Chevron }\end{array}$ \\
\hline
\end{tabular}

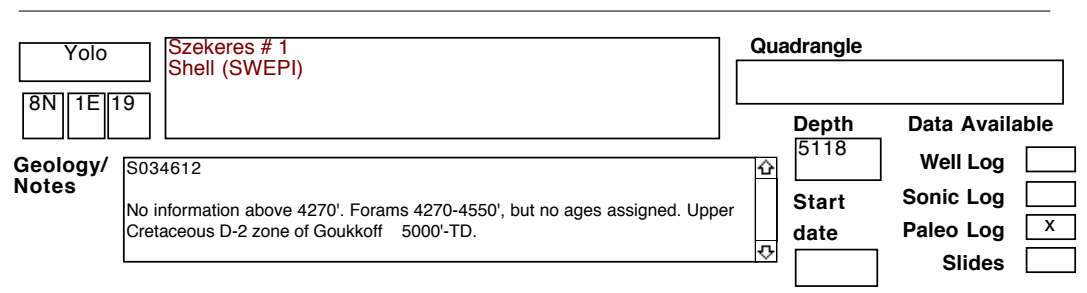




\section{SELECTED OIL TEST WELLS IN CENTRAL CALIFORNIA DATABASE}

County

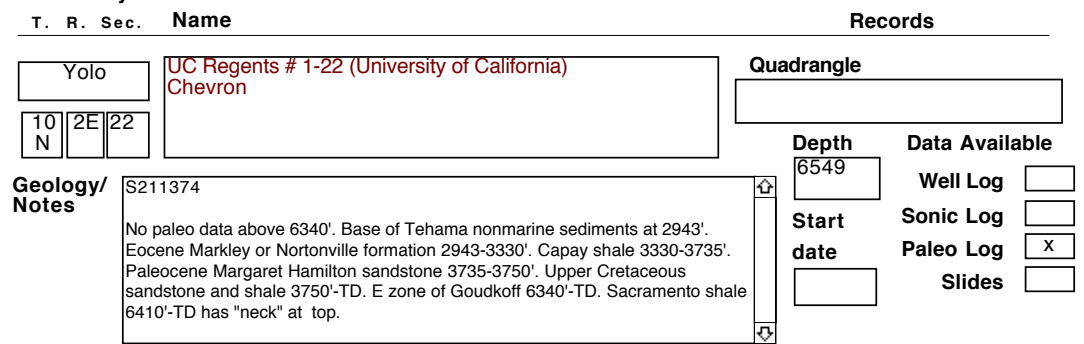

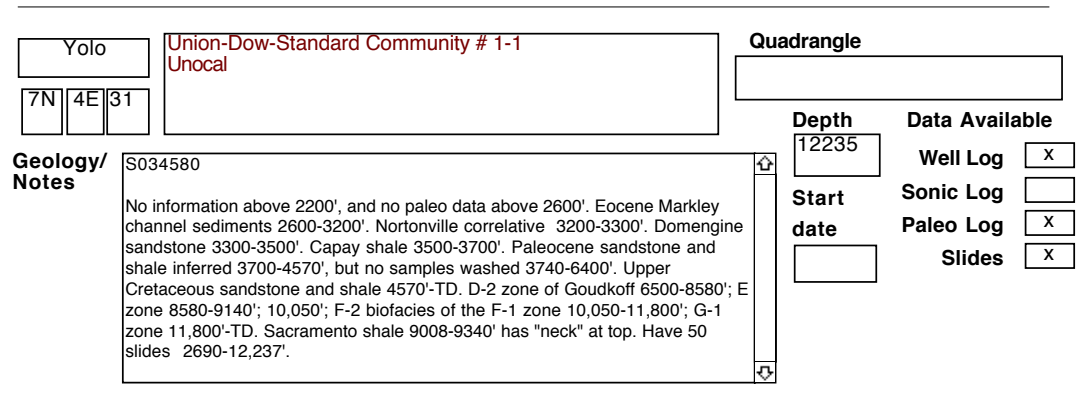

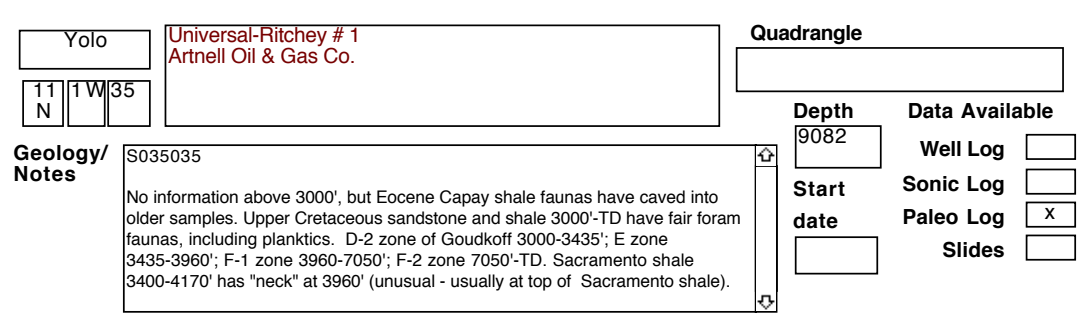

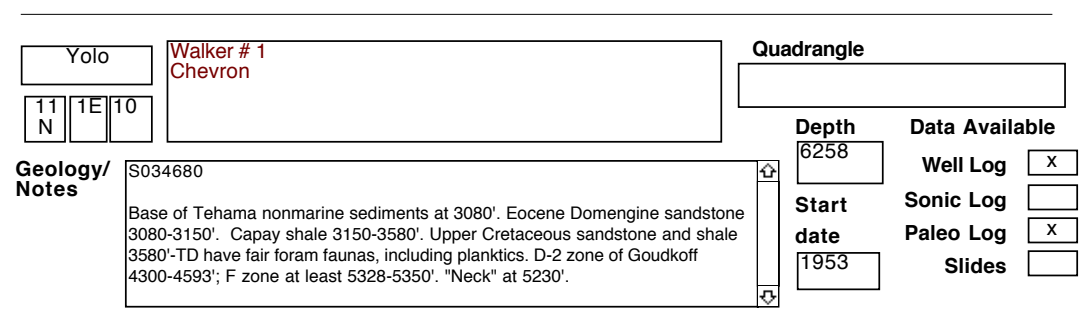

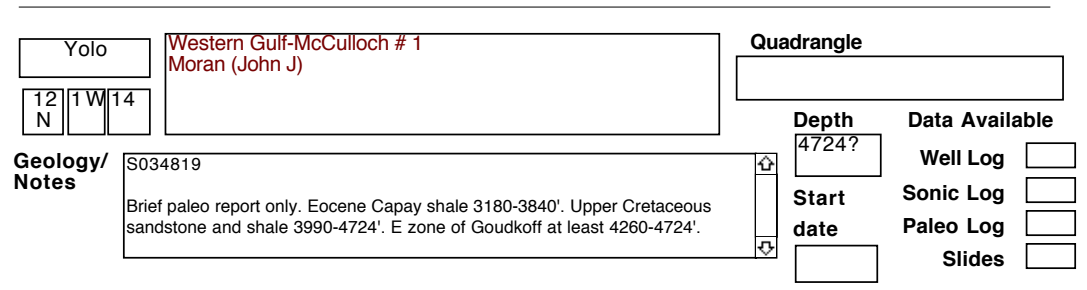


SELECTED OIL TEST WELLS IN CENTRAL CALIFORNIA DATABASE County

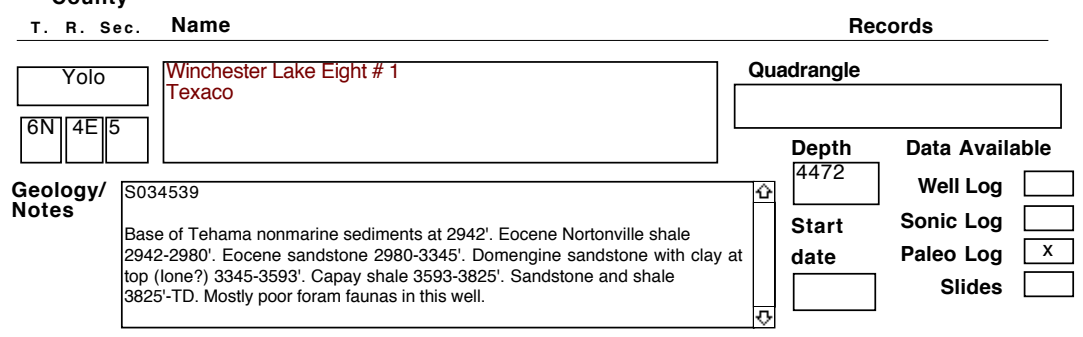

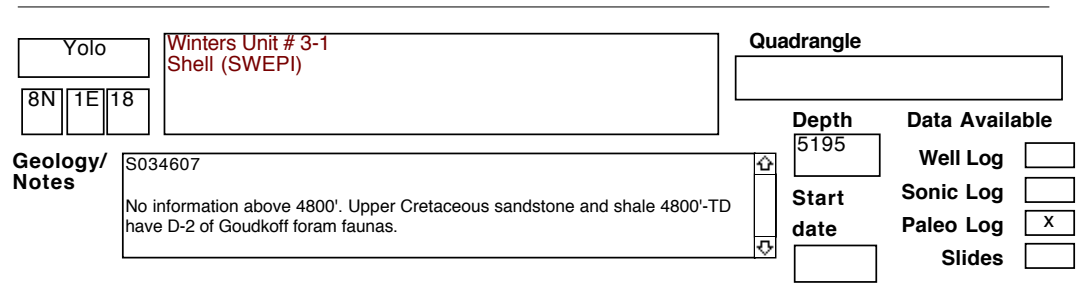

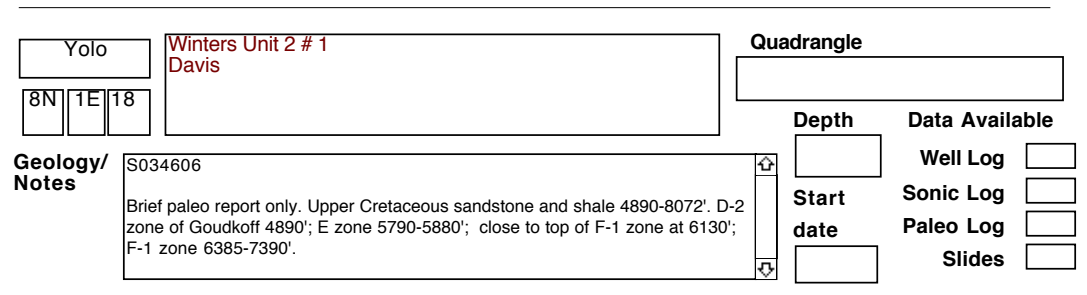

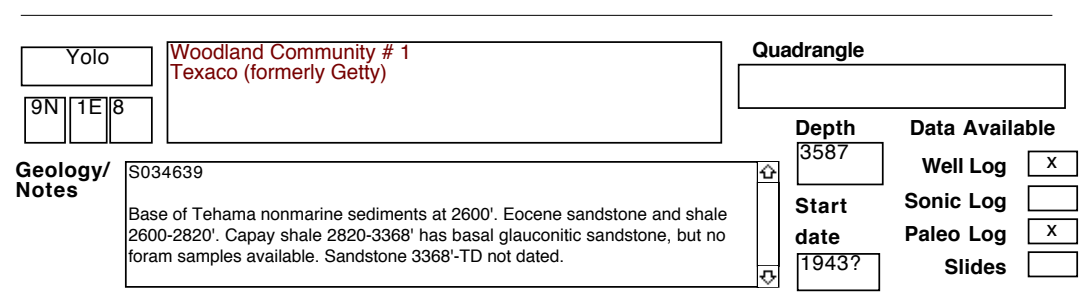

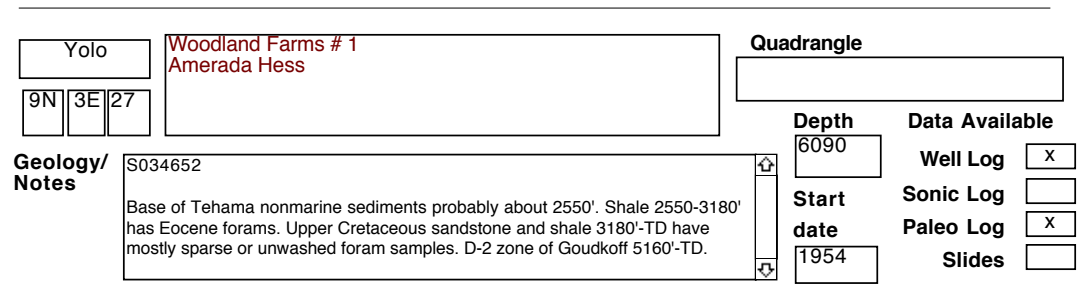


SELECTED OIL TEST WELLS IN CENTRAL CALIFORNIA DATABASE County

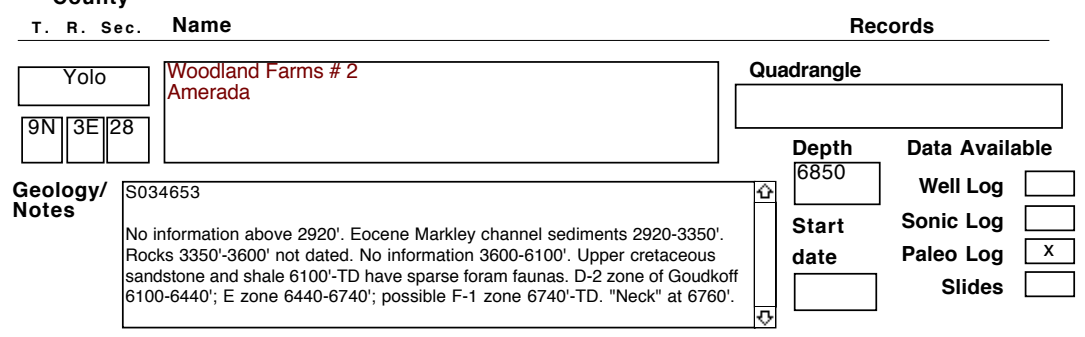

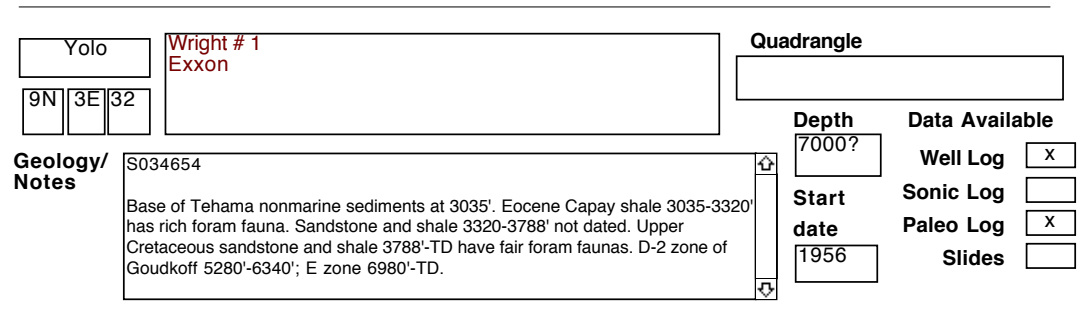

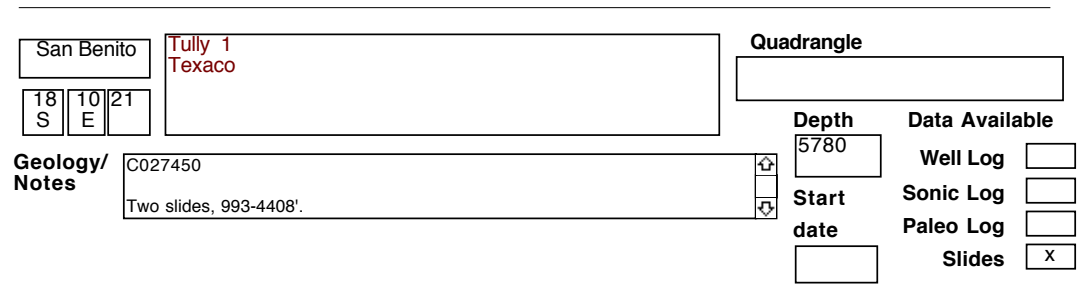

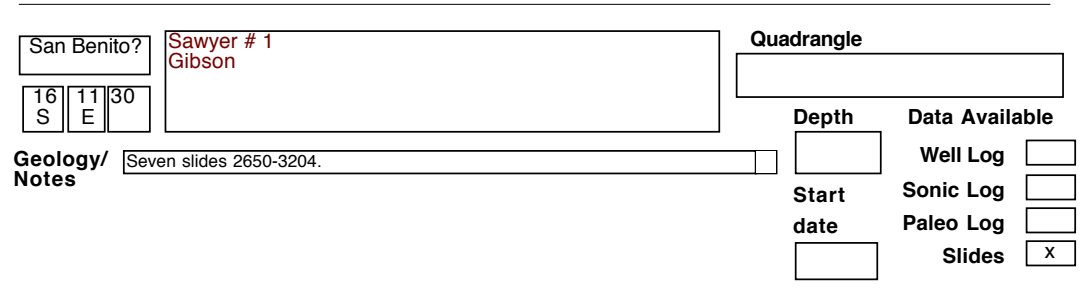

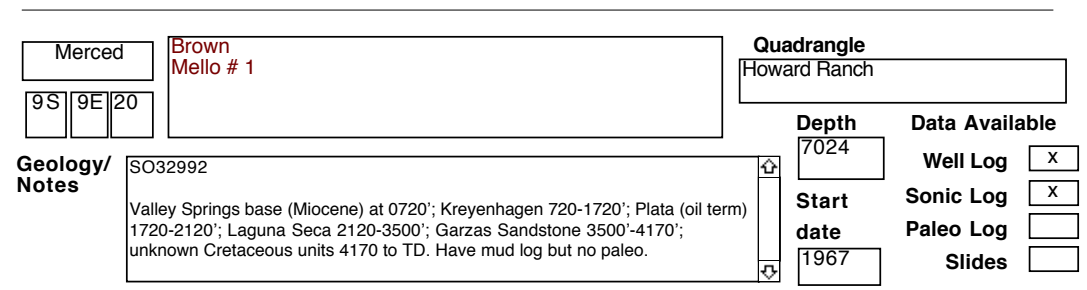


SELECTED OIL TEST WELLS IN CENTRAL CALIFORNIA DATABASE County

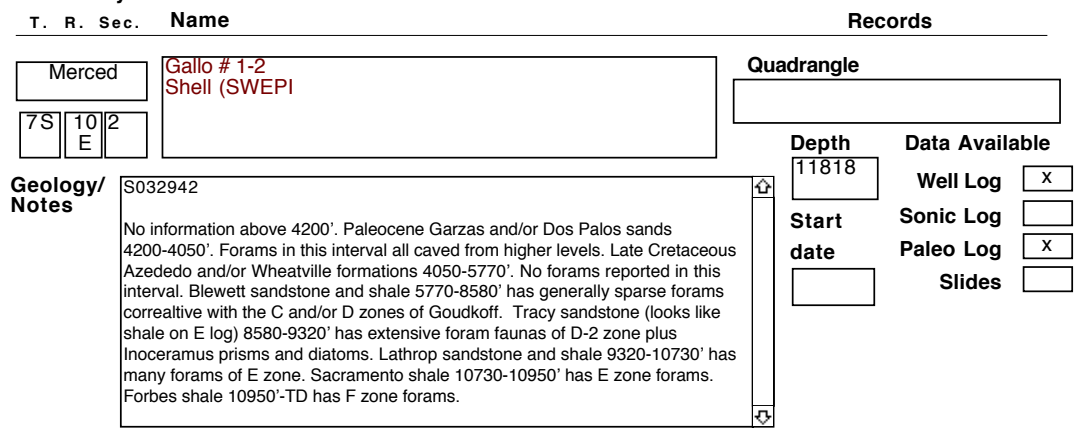

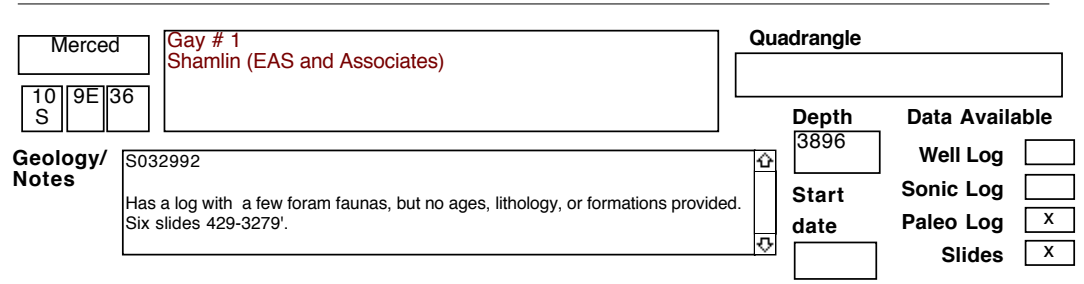

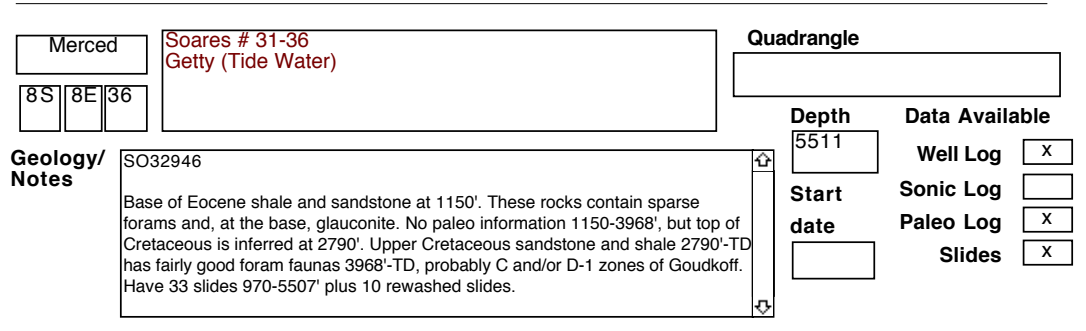

\begin{tabular}{l|l|l|l|l|l|}
\hline Merced & $\begin{array}{l}\text { Howard \# 1 } \\
\text { Texaco (formerly Seaboard-Tidewater) }\end{array}$ \\
\hline
\end{tabular}

\begin{tabular}{l|l|l|l|l|l|}
\hline Merced & $\begin{array}{l}\text { Ora Loma \# 1 } \\
\text { Bergman Oil Co. }\end{array}$ \\
\hline
\end{tabular}


SELECTED OIL TEST WELLS IN CENTRAL CALIFORNIA DATABASE County

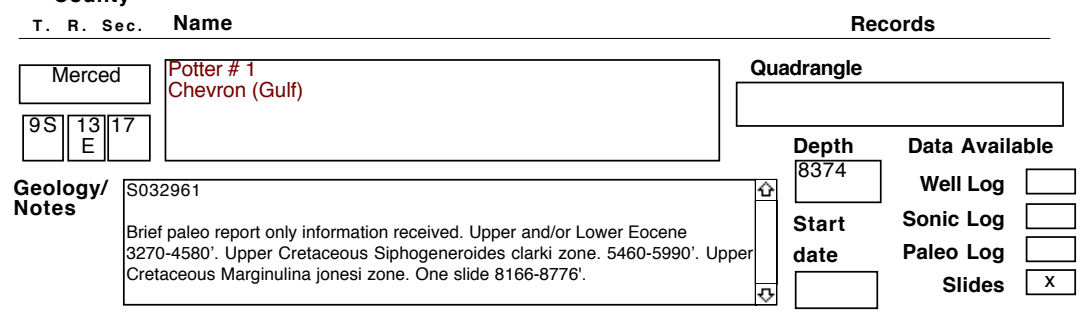

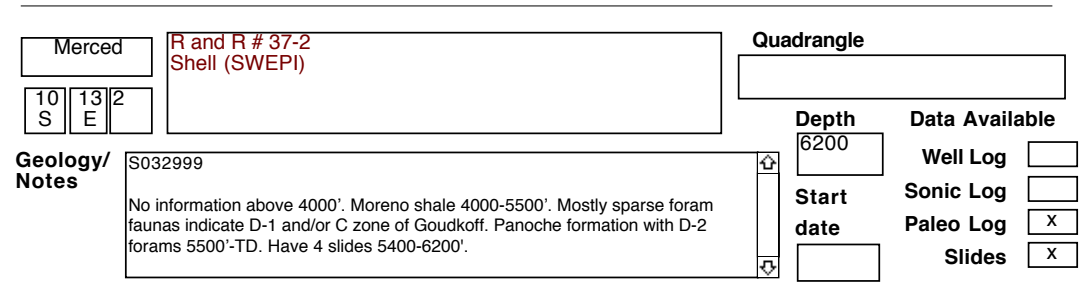

\begin{tabular}{l|l|l|l|l|l|l|}
\hline Merced & $\begin{array}{l}\text { Redfern \# 51 } \\
\text { Chevron }\end{array}$ \\
\hline
\end{tabular}

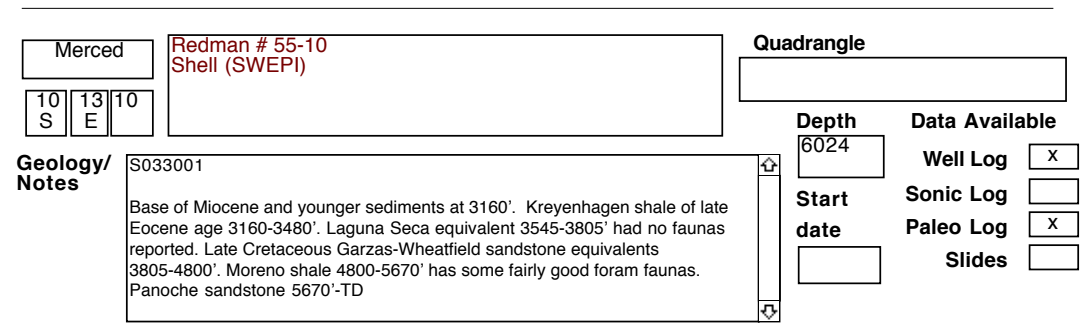

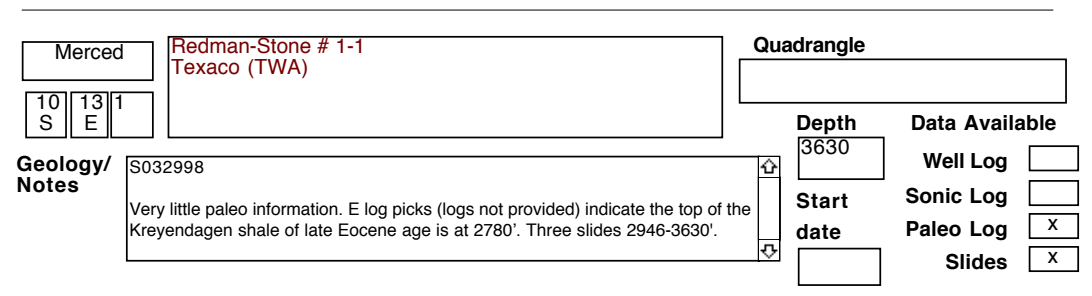




\section{SELECTED OIL TEST WELLS IN CENTRAL CALIFORNIA DATABASE}

County

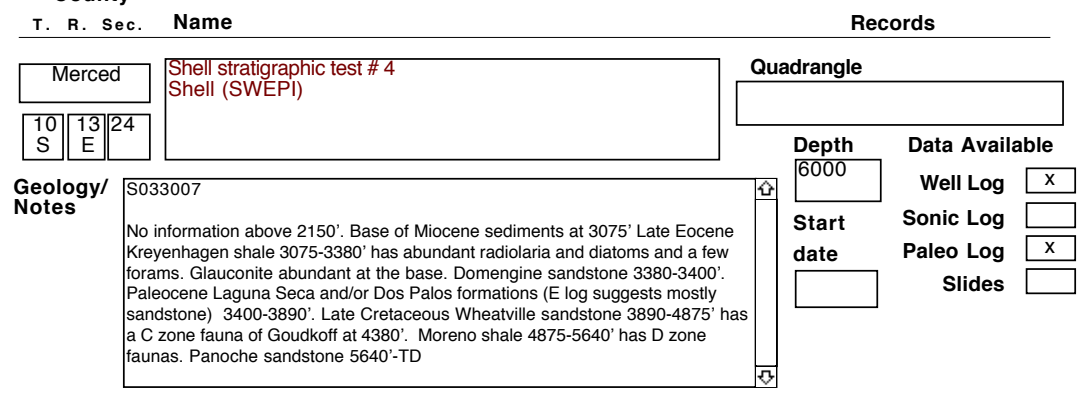

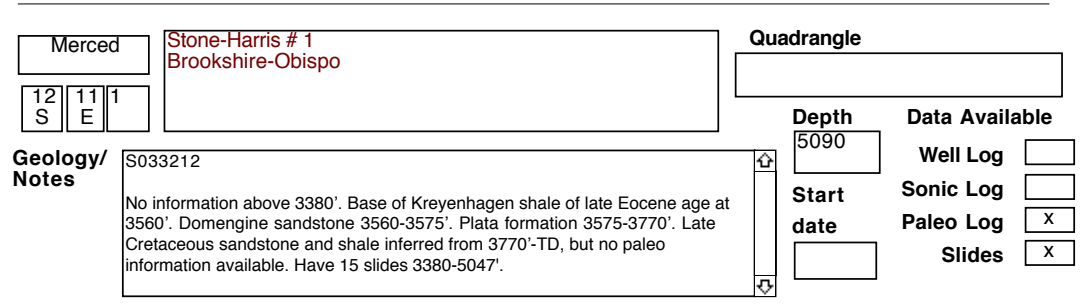

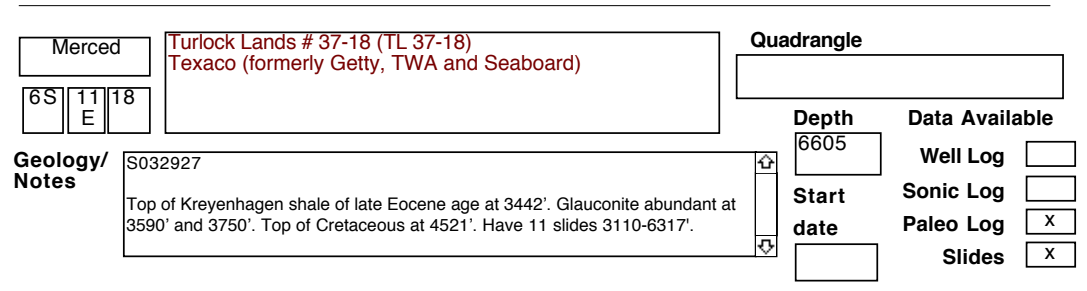

\begin{tabular}{l|l|l|l|l|l|}
\hline Merced & $\begin{array}{l}\text { West Chowchilla stratigraphic test \#3 } \\
\text { Shell (SWEPI) }\end{array}$ \\
\hline
\end{tabular}

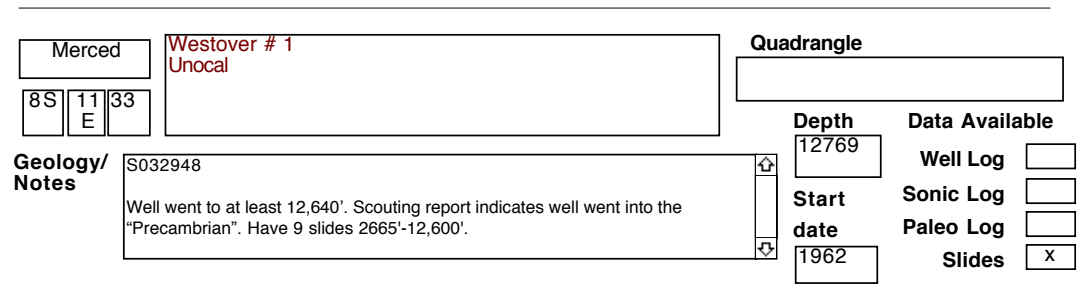


SELECTED OIL TEST WELLS IN CENTRAL CALIFORNIA DATABASE County

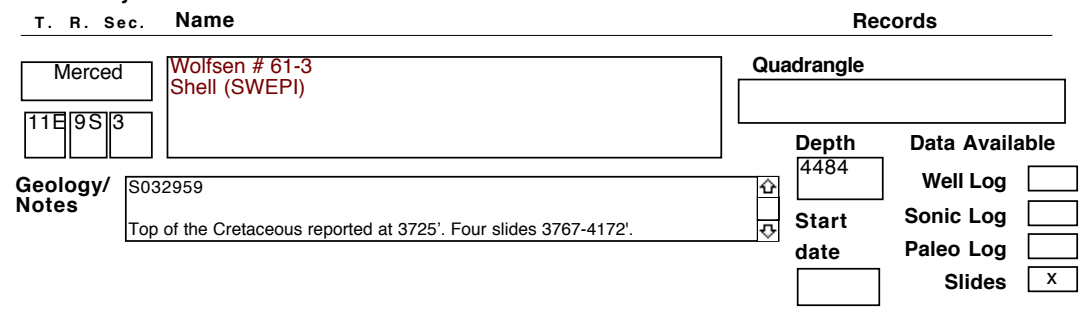

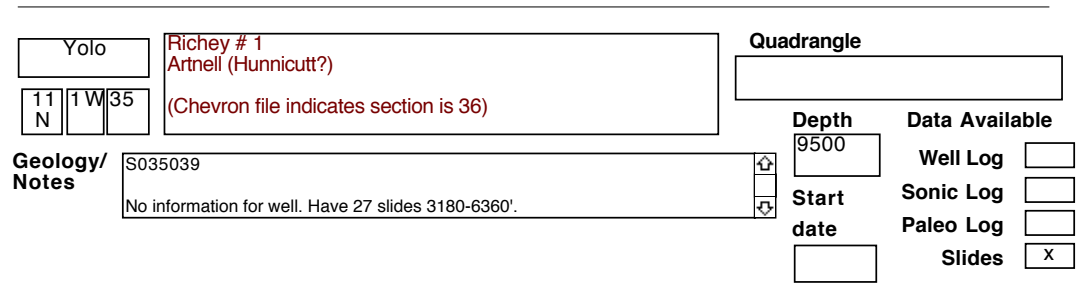

\begin{tabular}{|c|c|c|c|c|c|}
\hline San Benito & \multirow{2}{*}{$\begin{array}{l}\text { Eade\# 27-35 } \\
\text { Chevron }\end{array}$} & \multicolumn{4}{|c|}{ Quadrangle } \\
\hline \begin{tabular}{|c|c|c|}
18 & $9 \mathrm{E}$ & 35 \\
$\mathrm{~S}$ & & \\
\end{tabular} & & & & & \\
\hline \multirow{4}{*}{$\begin{array}{l}\text { Geology/ } \\
\text { Notes }\end{array}$} & slides $1000-2950$ '. No other information. Also have one slide for Chevron & 今 & & Well Log & \\
\hline & & & Start & Sonic Log & \\
\hline & & & date & Paleo Log & \\
\hline & & & & Slides & $\mathrm{x}$ \\
\hline
\end{tabular}

\begin{tabular}{l|l|l|l|l|l|}
\hline San Benito & $\begin{array}{l}\text { Ashurst \# 3-5 (Number probably should be 5-5) } \\
\text { Artnell }\end{array}$ & \multicolumn{2}{l|}{ Quadrangle } \\
\hline
\end{tabular}

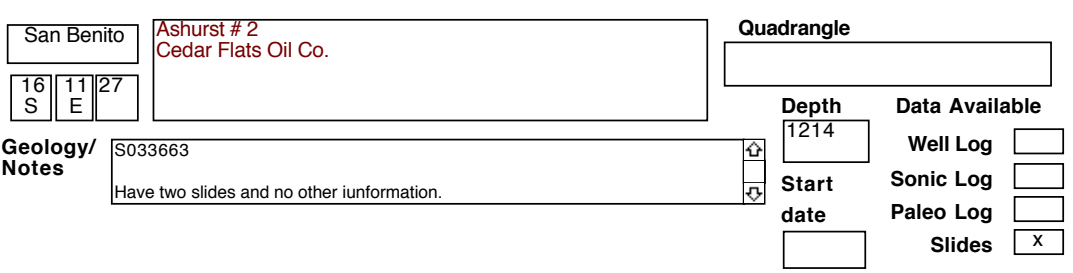


SELECTED OIL TEST WELLS IN CENTRAL CALIFORNIA DATABASE County

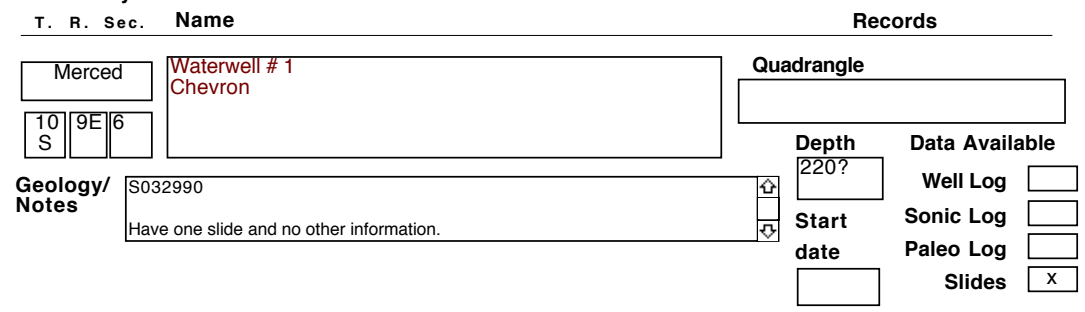

\begin{tabular}{|c|c|c|c|c|}
\hline Merced & San Joaquin \# 1 & Quadrangle & & \\
\hline \begin{tabular}{|c||c||c|}
12 & 11 \\
$\mathrm{~S}$ & $\mathrm{E}$ & 1 \\
\end{tabular} & & Depth & \multicolumn{2}{|c|}{ Data Available } \\
\hline \multirow{3}{*}{$\begin{array}{l}\text { Geology/ } \\
\text { Notes }\end{array}$} & slides $3402-4840$ ' but no other information. & & Well Log & \\
\hline & & Start & Sonic Log & \\
\hline & & date & $\begin{array}{r}\text { Paleo Log } \\
\text { Slides }\end{array}$ & $\mathrm{x}$ \\
\hline
\end{tabular}

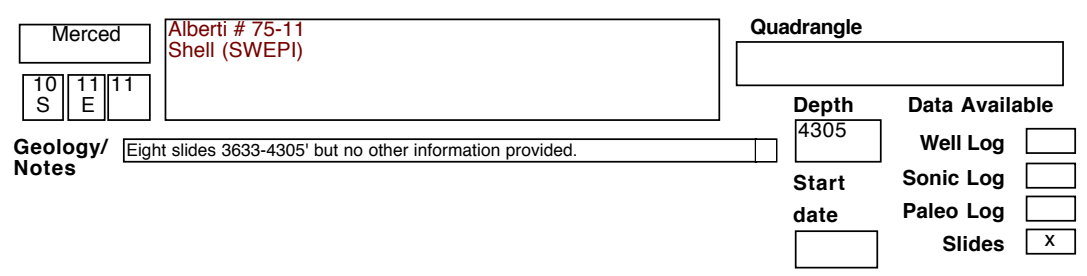

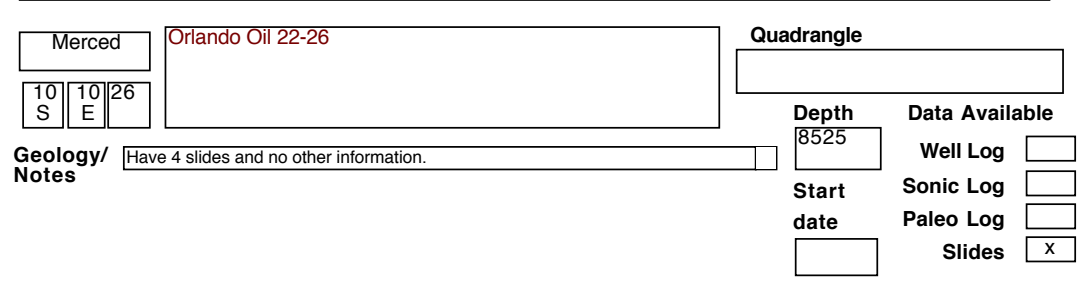

\begin{tabular}{l|l|l|l|l|l|l|}
\hline Monterey & $\begin{array}{l}\text { Wolf } 51 \mathrm{X}-15 \\
\text { Brown-Standard }\end{array}$ & \multicolumn{2}{l|}{ Quadrangle } \\
\hline
\end{tabular}


SELECTED OIL TEST WELLS IN CENTRAL CALIFORNIA DATABASE County

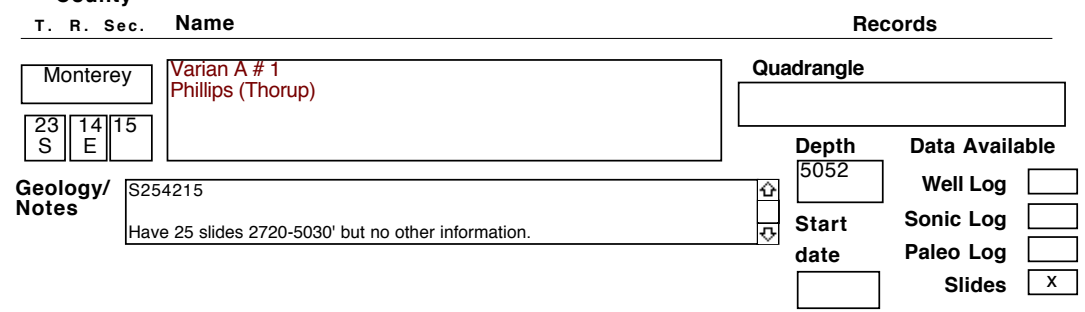

\begin{tabular}{|c|c|c|c|c|}
\hline Monterey & Stockdale \# 1 & Quadrangle & & \\
\hline \begin{tabular}{|c|c|c|}
23 & $8 \mathrm{E}$ & 13 \\
$\mathrm{~S}$ & & \\
\end{tabular} & & Depth & \multicolumn{2}{|c|}{ Data Available } \\
\hline \multirow{4}{*}{\multicolumn{2}{|c|}{$\begin{array}{l}\text { Geology/ Six slides } 1202-2345 \text { ' but no other information. } \\
\text { Notes }\end{array}$}} & & \multirow{4}{*}{\multicolumn{2}{|c|}{$\begin{aligned} \text { Well Log } & \\
\text { Sonic Log } & \\
\text { Paleo Log } & \\
\text { Slides } & \mathrm{X}\end{aligned}$}} \\
\hline & & Start & & \\
\hline & & date & & \\
\hline & & & & \\
\hline
\end{tabular}

\begin{tabular}{l|l|l|l|l|}
\hline Monterey & BCB-Kent-Basham \# 1-31 & \\
\hline
\end{tabular}

\begin{tabular}{|c|c|c|c|c|c|}
\hline Monterey & Aurignac NCT-1 \# 1-4 & \multicolumn{4}{|c|}{ Quadrangle } \\
\hline \begin{tabular}{|c|c||c|}
23 & 10 & 4 \\
$S$ & $E$ & \\
\end{tabular} & & & Depth & Data Availab & \\
\hline $\begin{array}{l}\text { Geology/ } \\
\text { Notes }\end{array}$ & $\begin{array}{l}\text { C021381 } \\
\text { Have } 4 \text { slides 1730-2715' but no other information. Also have one slide for } \\
\text { Aurignac \# 1-5. }\end{array}$ & 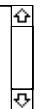 & $\begin{array}{l}\text { Start } \\
\text { date }\end{array}$ & $\begin{array}{l}\text { Well Log } \\
\text { Sonic Log } \\
\text { Paleo Log }\end{array}$ & $\bar{x}$ \\
\hline
\end{tabular}

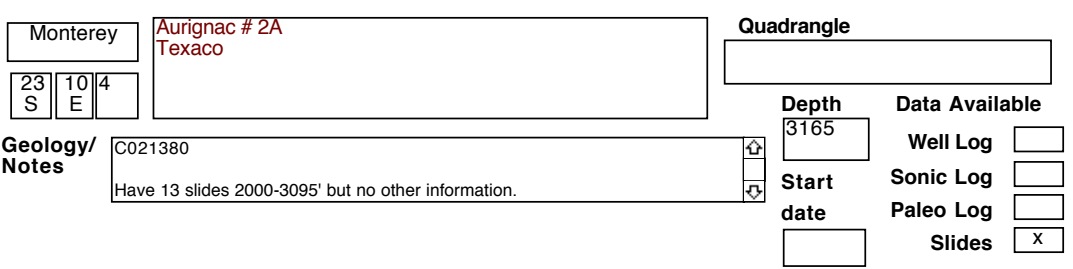


SELECTED OIL TEST WELLS IN CENTRAL CALIFORNIA DATABASE County

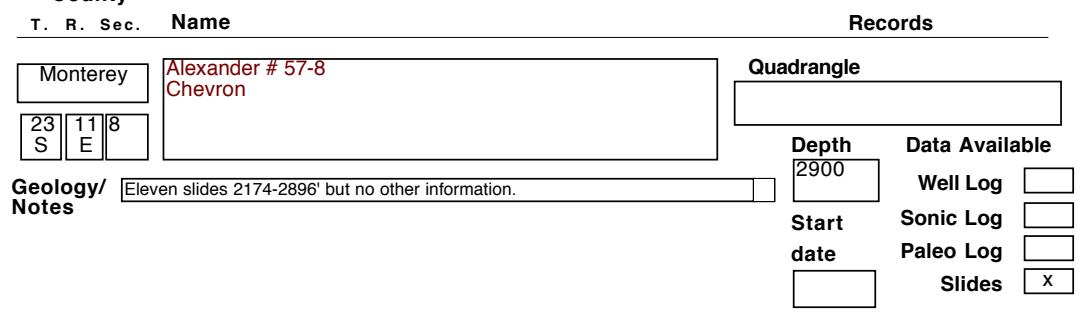

\begin{tabular}{|l|l|l|l|l|}
\hline Monterey & $\begin{array}{l}\text { Arroyo Seco \# 1 } \\
\text { Texaco }\end{array}$ \\
Geology/ \\
Notes
\end{tabular}

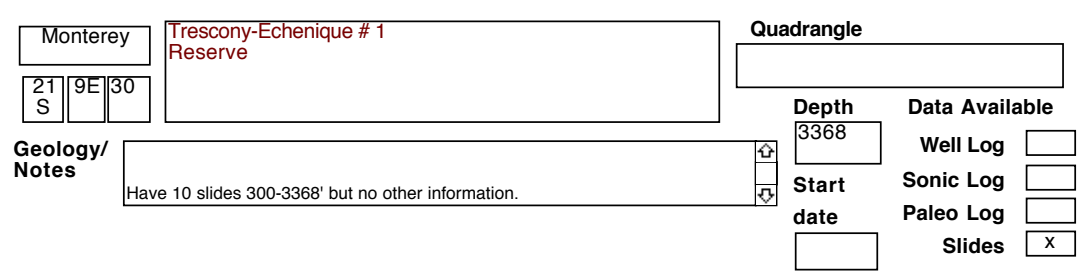

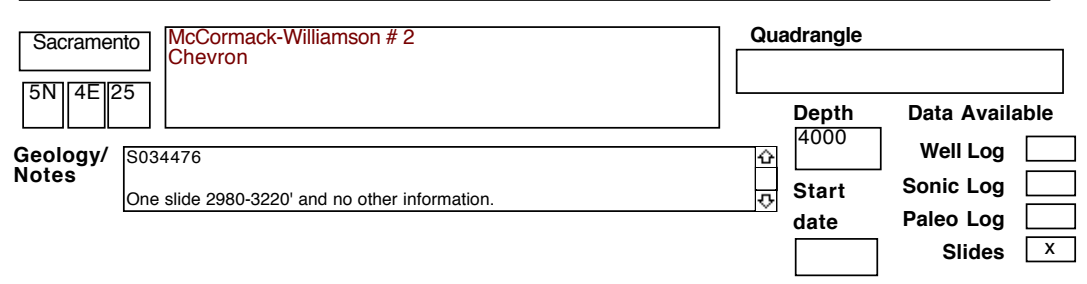

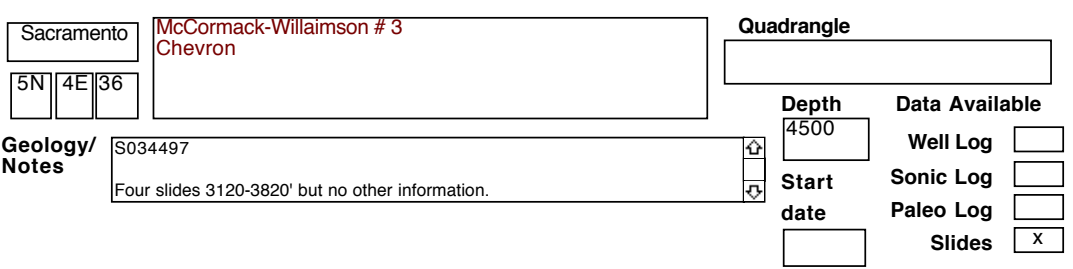


SELECTED OIL TEST WELLS IN CENTRAL CALIFORNIA DATABASE County

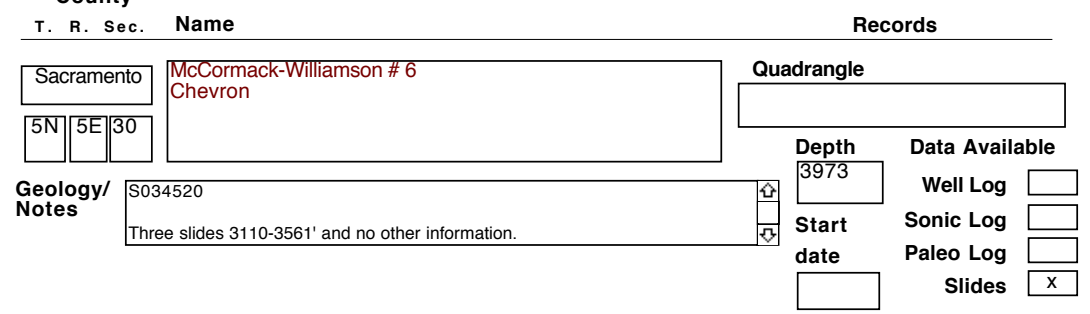

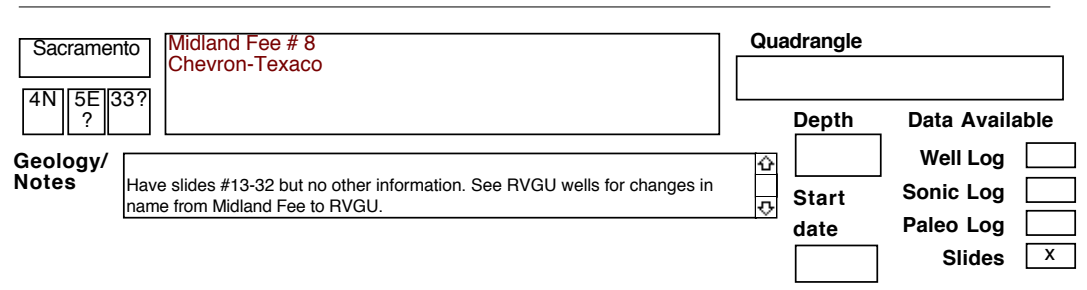

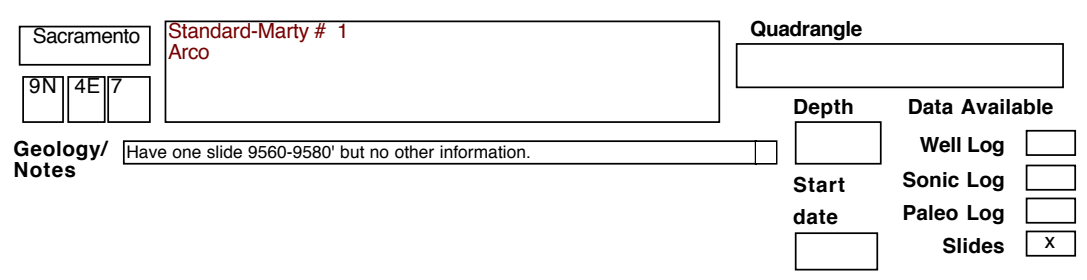

\begin{tabular}{|c|c|c|c|c|c|}
\hline Sacramento & \multirow{2}{*}{$\begin{array}{l}\text { Welch Fee \# } 2 \\
\text { Texaco }\end{array}$} & \multicolumn{4}{|c|}{ Quadrangle } \\
\hline${ }^{310}\left\|^{3 E}\right\|^{3}$ & & \multicolumn{2}{|r|}{ Depth } & \multicolumn{2}{|c|}{ Data Available } \\
\hline \multirow{4}{*}{$\begin{array}{l}\text { Geology/ } \\
\text { Notes }\end{array}$} & & \multirow{4}{*}{$\underline{0}$} & & Well Log & \\
\hline & slides $3750-4300^{\prime}$ but no other information. & & Start & Sonic Log & \\
\hline & & & date & Paleo Log & \\
\hline & & & & & \\
\hline
\end{tabular}

\begin{tabular}{|c|c|c|c|c|c|}
\hline Sacramento & \multirow{2}{*}{$\begin{array}{l}\text { Welch Fee \# H-5 } \\
\text { Texaco }\end{array}$} & \multicolumn{4}{|c|}{ Quadrangle } \\
\hline \begin{tabular}{|l|l|l|}
4 & $3 \mathrm{E}$ & 31 \\
$\mathrm{~N}$ & & \\
\end{tabular} & & \multicolumn{2}{|r|}{ Depth } & \multicolumn{2}{|c|}{ Data Available } \\
\hline \multirow{3}{*}{$\begin{array}{l}\text { Geology/ } \\
\text { Notes }\end{array}$} & & \multirow{3}{*}{ 电 } & & Well Log & \\
\hline & & & $\begin{array}{l}\text { Start } \\
\text { date }\end{array}$ & $\begin{array}{l}\text { Sonic Log } \\
\text { Paleo Log }\end{array}$ & \\
\hline & & & & Slides & $\mathrm{x}$ \\
\hline
\end{tabular}


SELECTED OIL TEST WELLS IN CENTRAL CALIFORNIA DATABASE

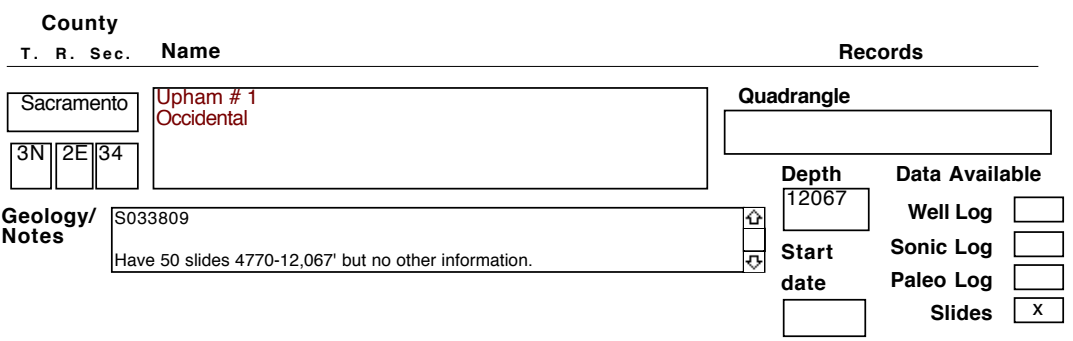

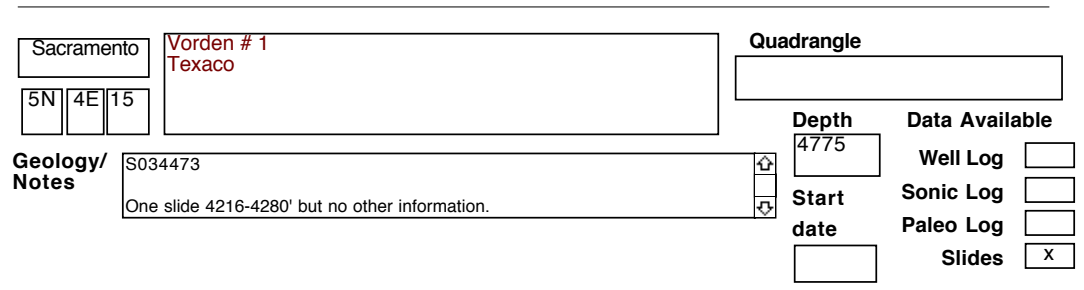

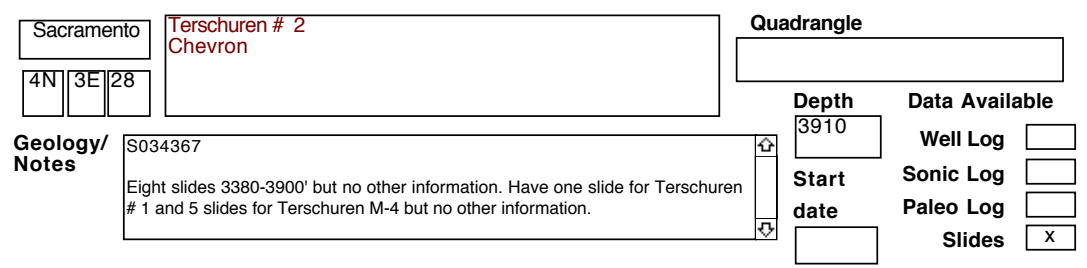

\begin{tabular}{|c|c|c|c|c|c|}
\hline Sacramento & \multirow{2}{*}{\begin{tabular}{|l} 
Locke \# 1-1 \\
Unocal (Brazos)
\end{tabular}} & \multicolumn{4}{|c|}{ Quadrangle } \\
\hline \begin{tabular}{|l|l|l|l|}
$5 N$ & $4 E$ \\
\end{tabular} & & \multirow{2}{*}{\multicolumn{2}{|c|}{$\begin{array}{l}\text { Depth } \\
8500\end{array}$}} & \multicolumn{2}{|c|}{ Data Available } \\
\hline \multirow{3}{*}{$\begin{array}{l}\text { Geology/ } \\
\text { Notes }\end{array}$} & 4480 & & & Well Log & \\
\hline & slide $2900-3260^{\prime}$ but no other information. & \multirow[t]{2}{*}{3} & Start & Sonic Log & \\
\hline & & & & Slides & $\mathrm{x}$ \\
\hline
\end{tabular}

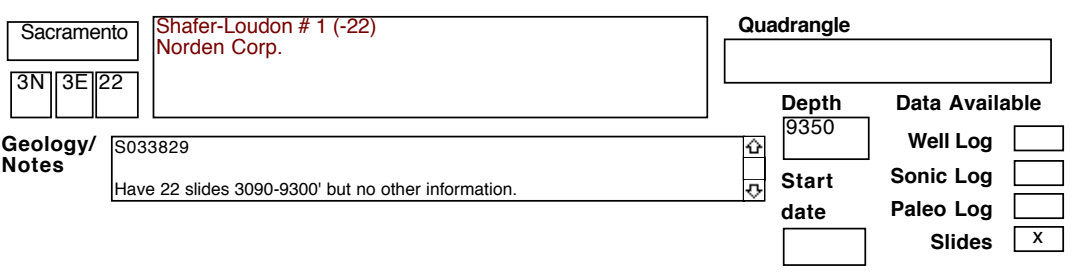


SELECTED OIL TEST WELLS IN CENTRAL CALIFORNIA DATABASE County

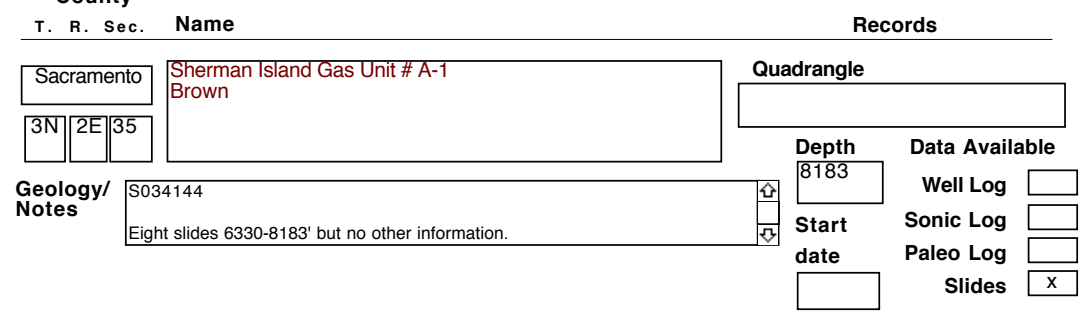

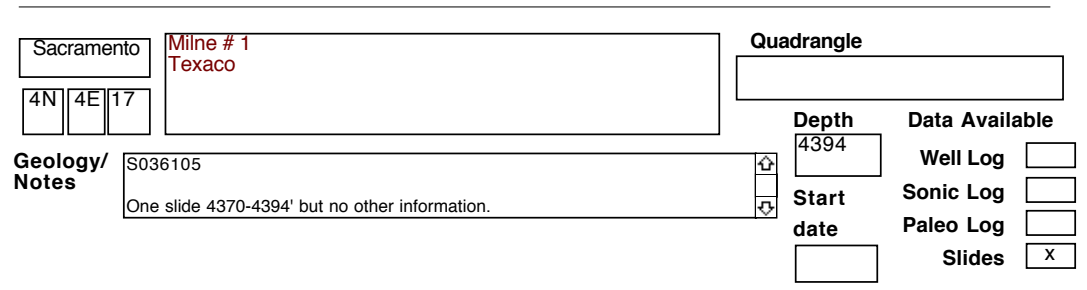

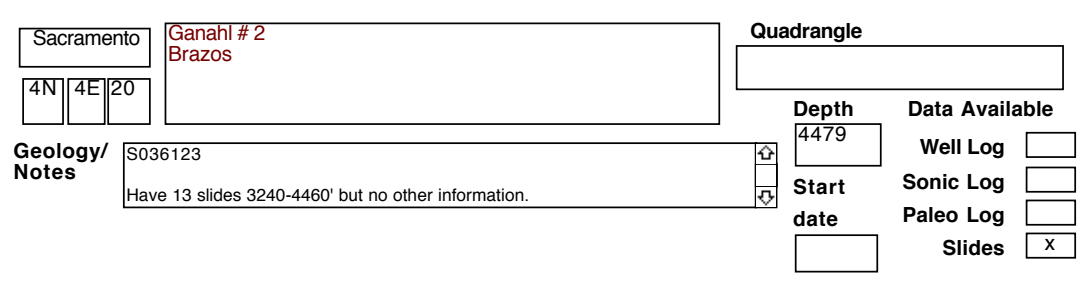

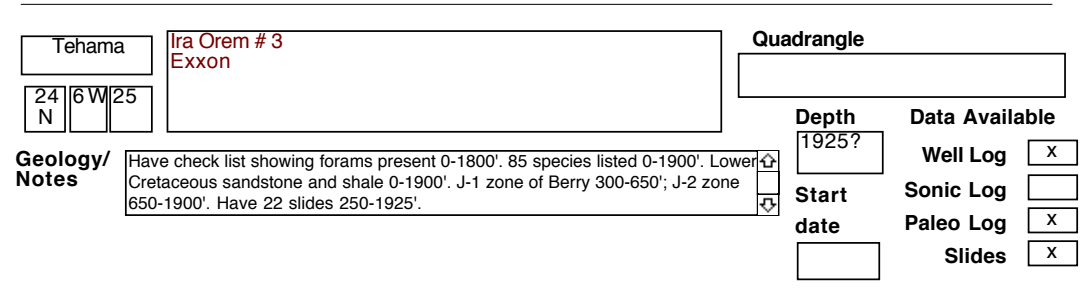

\begin{tabular}{|c|c|c|c|c|c|}
\hline Sacramento & \multirow{2}{*}{$\begin{array}{l}\text { Dee Community \# } 1 \\
\text { Rio Hondo }\end{array}$} & \multicolumn{4}{|c|}{ Quadrangle } \\
\hline $7 \mathrm{~N}|5 \mathrm{E}| 20$ & & \multirow{2}{*}{\multicolumn{2}{|c|}{ 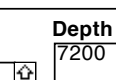 }} & \multicolumn{2}{|c|}{ Data Available } \\
\hline \multirow{3}{*}{$\begin{array}{l}\text { Geology/ } \\
\text { Notes }\end{array}$} & 34587 & & & Well Log & \\
\hline & e 22 slides $2335-7200^{\prime}$ but no other information. & $\pi$ & Start & Sonic Log & \\
\hline & & & & Slides & $\mathrm{x}$ \\
\hline
\end{tabular}


SELECTED OIL TEST WELLS IN CENTRAL CALIFORNIA DATABASE

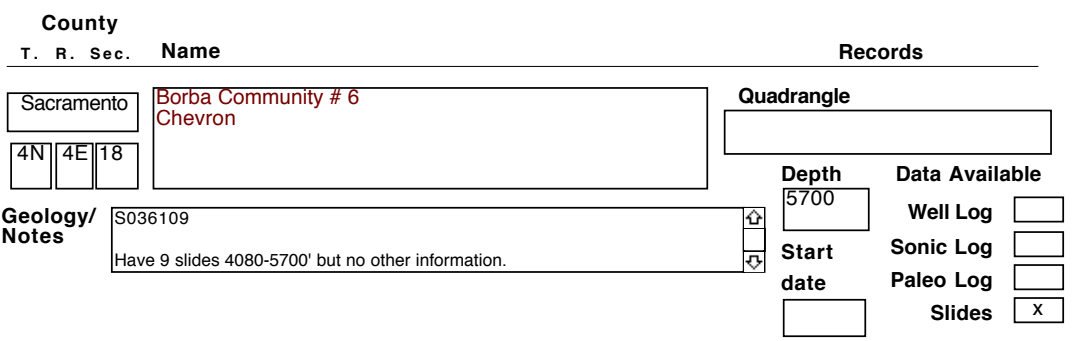

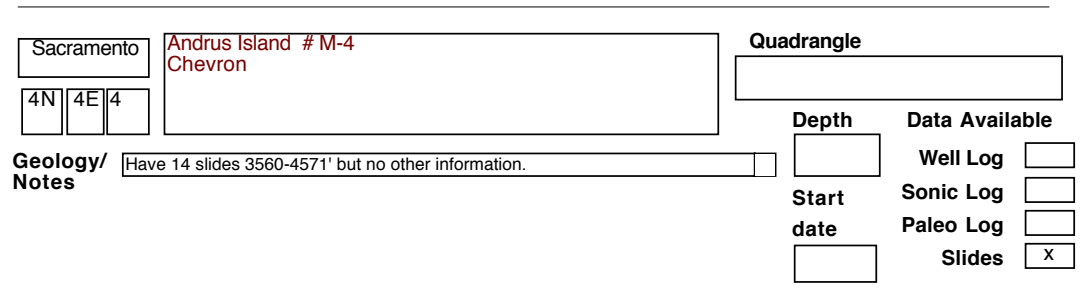

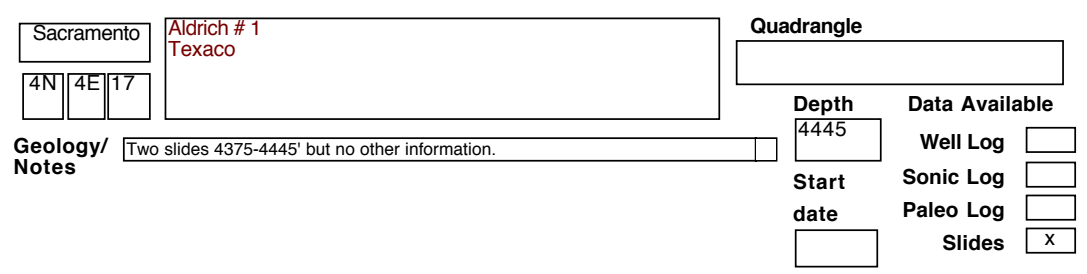

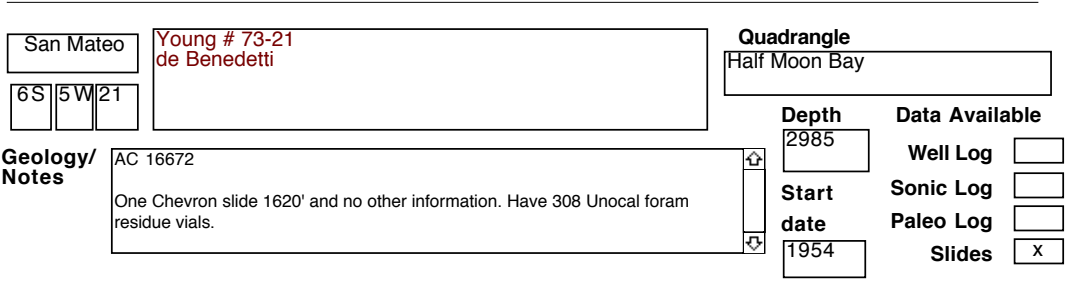

\begin{tabular}{|c|c|c|c|c|}
\hline San Mateo & Mannell \#1 & Quadrangle & & \\
\hline $3 \mathrm{~S} 6 \mathrm{~W} 25$ & & & & \\
\hline$D^{-\infty}$ & & Depth & Data Availabl & \\
\hline Geology/ N & r Colma. One slide 2084'? and no other information. & & Well Log & \\
\hline & & Start & Sonic Log & \\
\hline & & date & Paleo Log & \\
\hline & & 1931 & Slides & $x$ \\
\hline
\end{tabular}


SELECTED OIL TEST WELLS IN CENTRAL CALIFORNIA DATABASE County

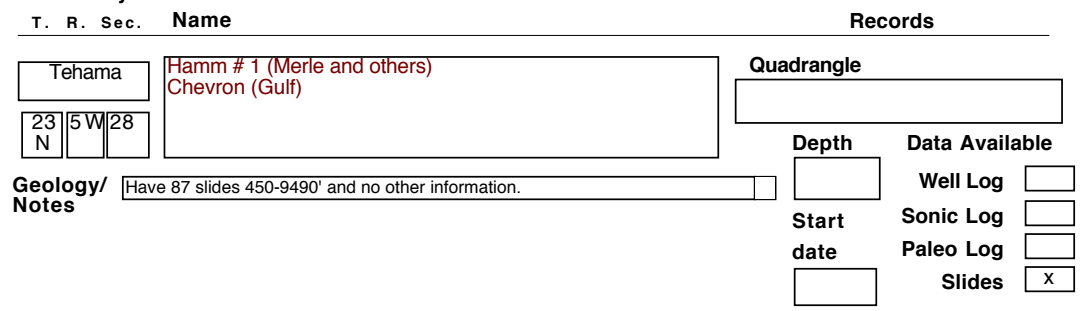

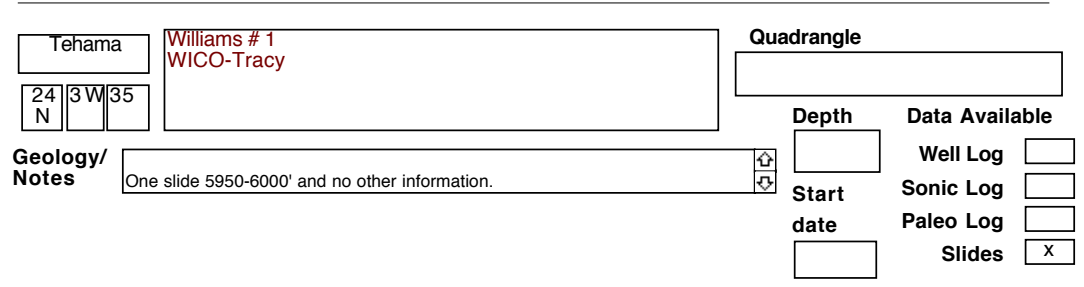

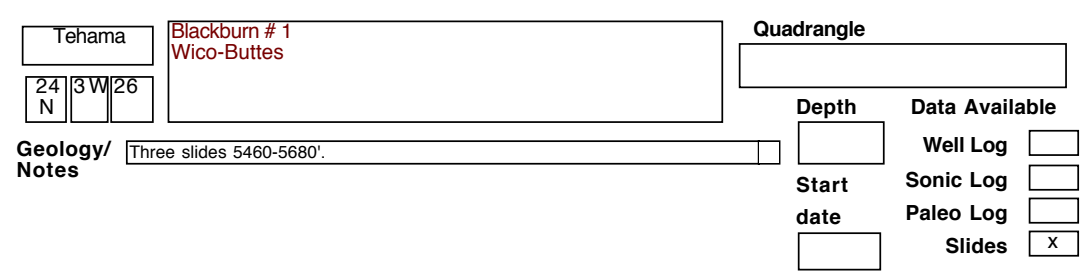

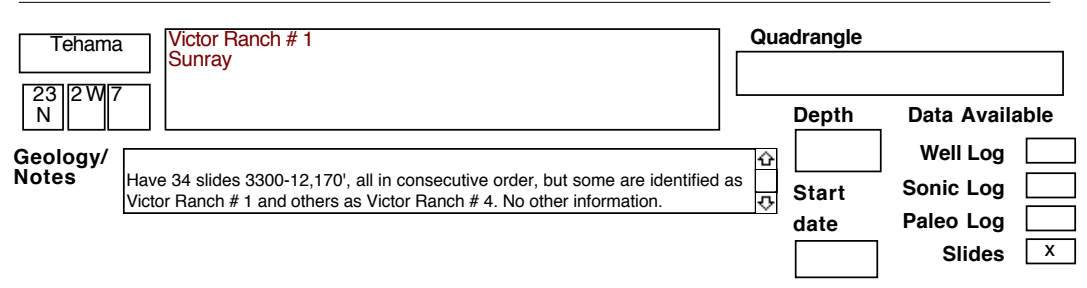

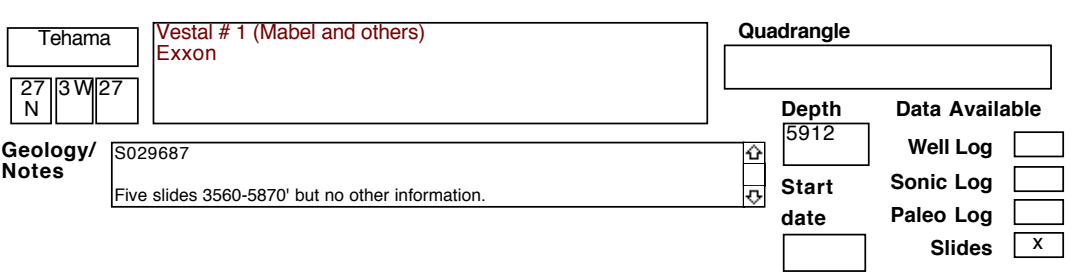


SELECTED OIL TEST WELLS IN CENTRAL CALIFORNIA DATABASE County

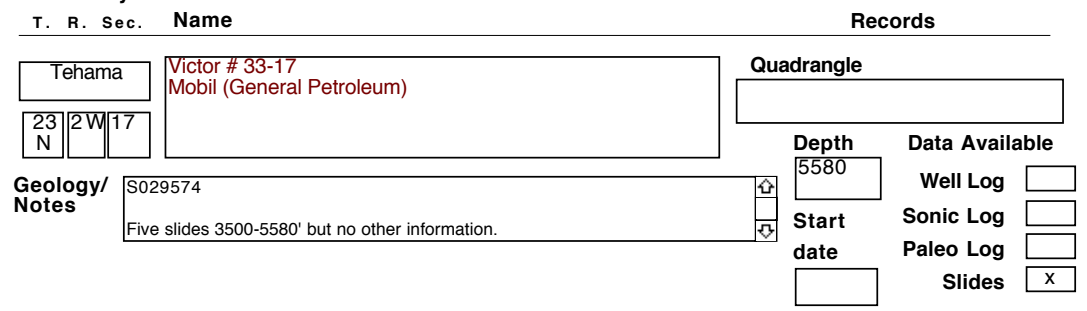

\begin{tabular}{l|l|l|l|}
\hline Tehama & $\begin{array}{l}\text { Stevenson \# 1 } \\
\text { McCulloch-Sunray }\end{array}$ & \multicolumn{2}{l|}{ Quadrangle } \\
\hline
\end{tabular}

\begin{tabular}{l|l|l|l|l|l|}
\hline Tehama & $\begin{array}{l}\text { Roberts \# 1 (Elizabeth and others) } \\
\text { Exxon }\end{array}$ & \multicolumn{2}{l|}{ Quadrangle } \\
& & & \\
\hline
\end{tabular}

\begin{tabular}{l|l|l|l|l|l|}
\hline Tehama & $\begin{array}{l}\text { McKenzie \#1-14 } \\
\text { Lipan }\end{array}$ & \multicolumn{2}{l|}{ Quadrangle } \\
\hline
\end{tabular}

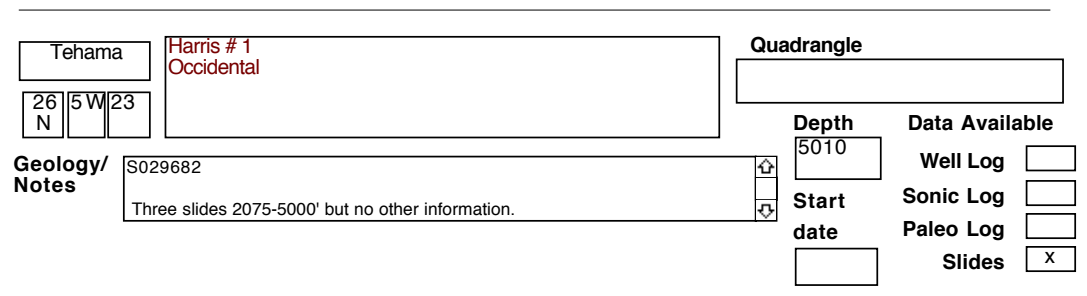


SELECTED OIL TEST WELLS IN CENTRAL CALIFORNIA DATABASE County

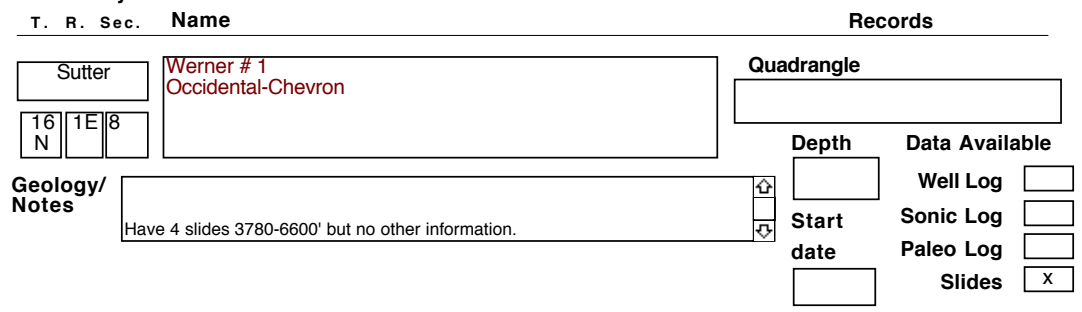

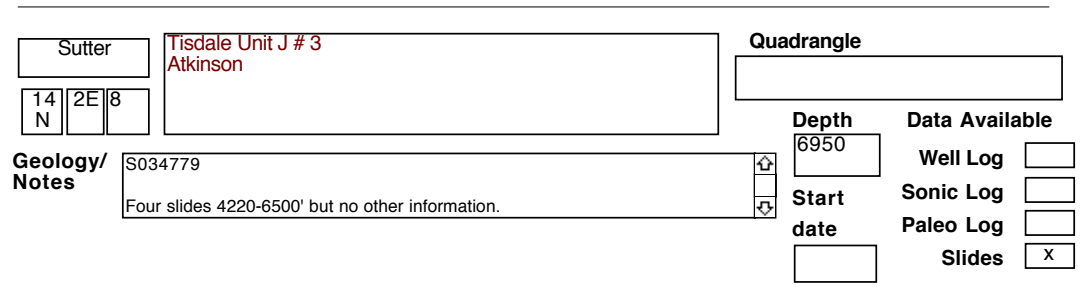

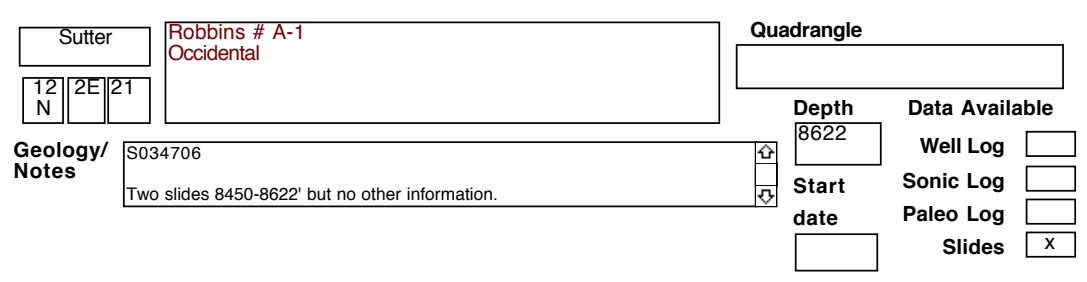

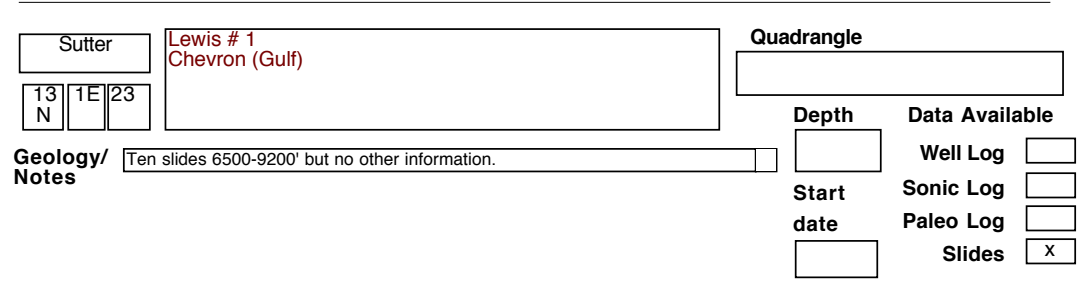

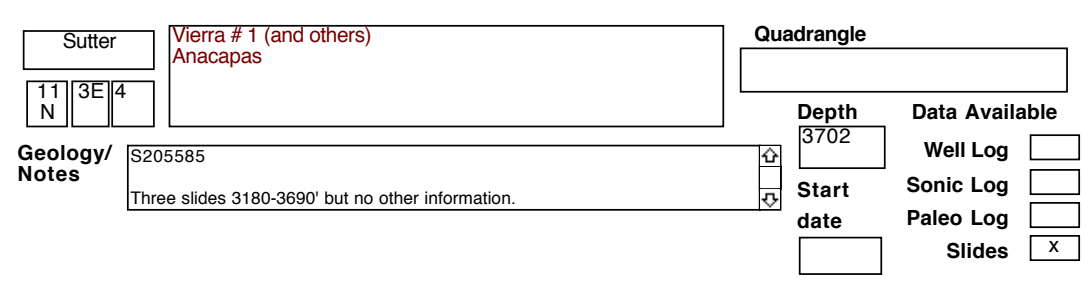


SELECTED OIL TEST WELLS IN CENTRAL CALIFORNIA DATABASE County

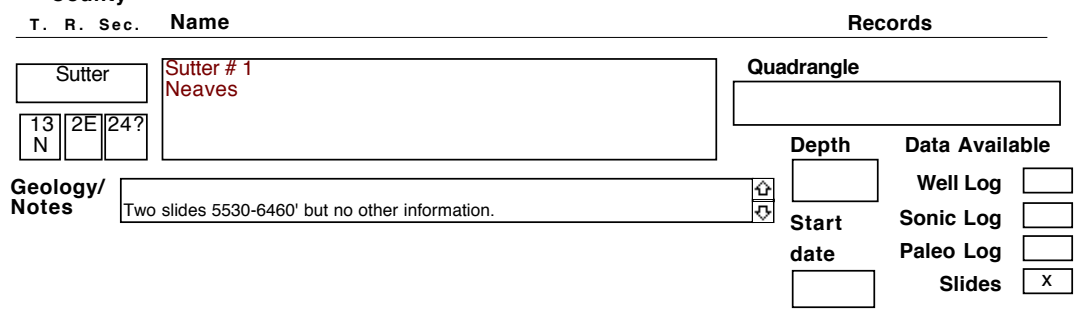

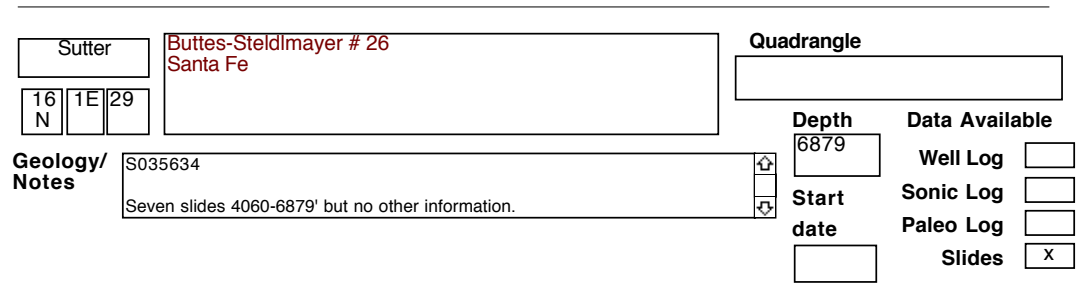

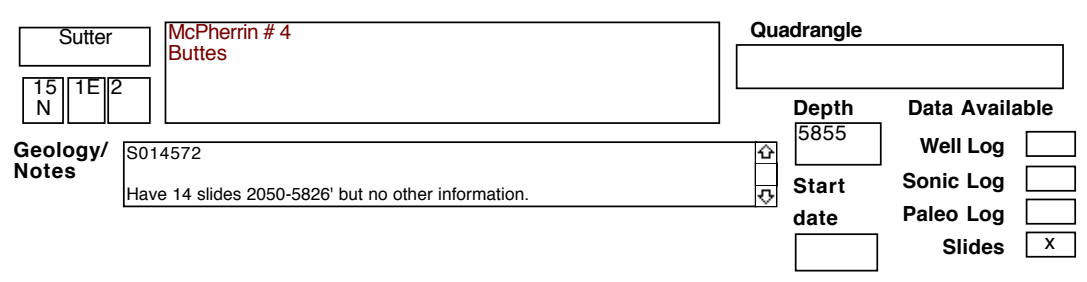

\begin{tabular}{l|l|l|l|l|l|}
\hline \multicolumn{1}{|c|}{ Sutter } & $\begin{array}{l}\text { McPherrin \#6 } \\
\text { Buttes }\end{array}$ & \multicolumn{2}{l|}{ Quadrangle } \\
\hline
\end{tabular}

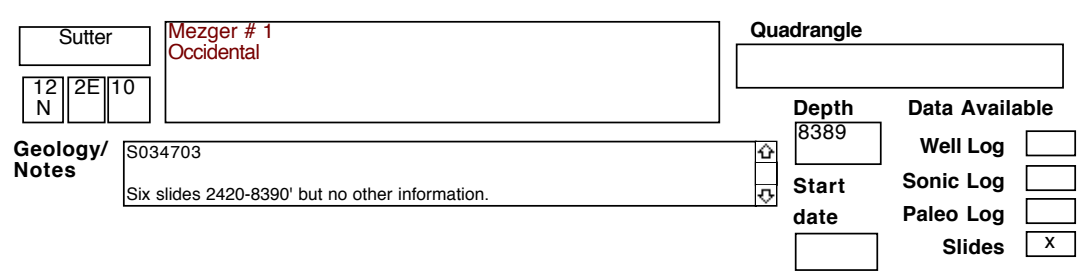


SELECTED OIL TEST WELLS IN CENTRAL CALIFORNIA DATABASE County

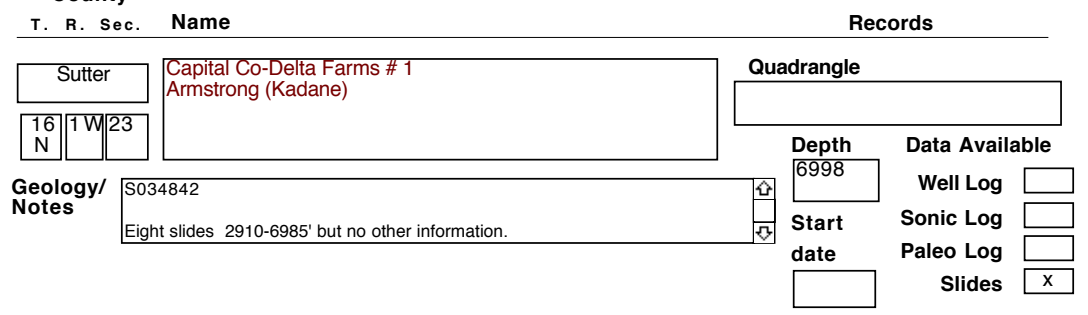

\begin{tabular}{l|l|l|l|}
\hline Sutter & $\begin{array}{l}\text { Browning \# 2 } \\
\text { Occidental }\end{array}$ & \multicolumn{2}{l|}{ Quadrangle } \\
\hline
\end{tabular}

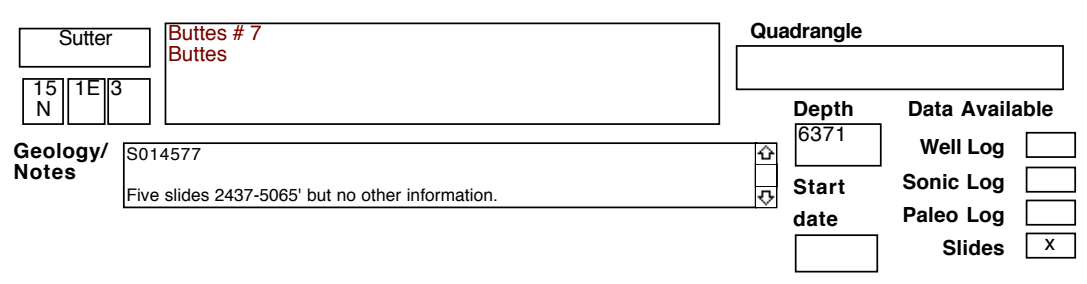

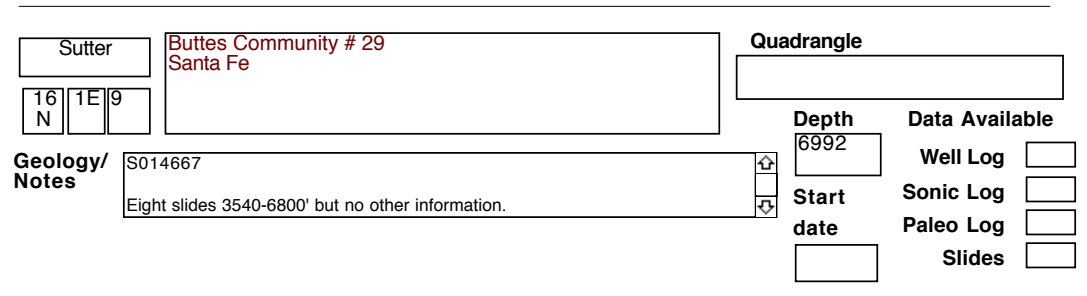

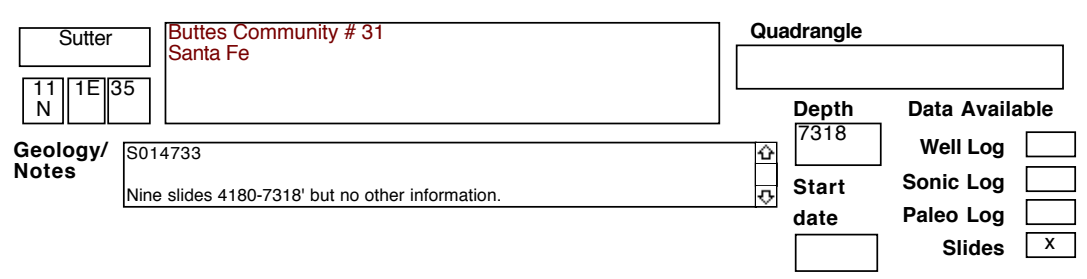


SELECTED OIL TEST WELLS IN CENTRAL CALIFORNIA DATABASE

County

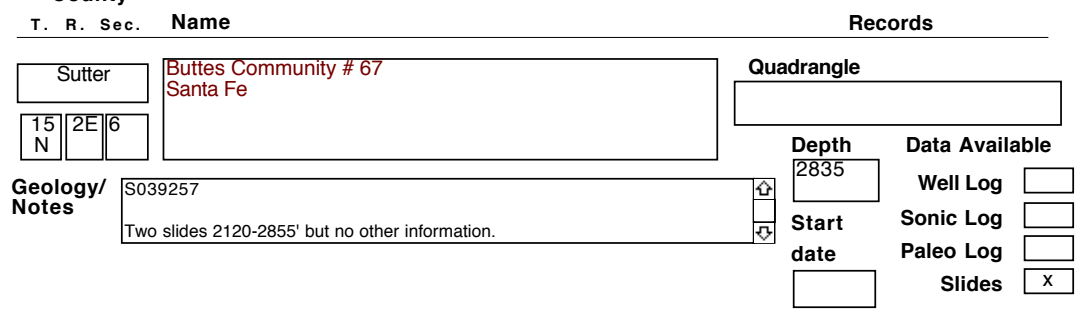

\begin{tabular}{l|l|l|l|l|l|}
\hline Stanislaus & Wishire \# 88-26E & \\
\hline
\end{tabular}

\begin{tabular}{|c|c|c|c|c|c|}
\hline Stanislaus & \multirow{2}{*}{$\begin{array}{l}\text { Sesnon-Vernalis Unit } 8 \text { \# 14-33 } \\
\text { Porter Sesnon }\end{array}$} & \multicolumn{4}{|c|}{ Quadrangle } \\
\hline \begin{tabular}{|l|l|l|l|}
$3 \mathrm{~S}$ & $6 \mathrm{E}$ \\
\end{tabular} & & \multicolumn{2}{|r|}{ Depth } & \multicolumn{2}{|l|}{ Data Available } \\
\hline \multirow{4}{*}{$\begin{array}{l}\text { Geology/ } \\
\text { Notes }\end{array}$} & \multirow{4}{*}{ Have 6 slides $3760-5300$ ' but no other information. } & \multirow{4}{*}{$\frac{6}{n}$} & & Well Log & \\
\hline & & & Start & Sonic Log & \\
\hline & & & date & Paleo Log & \\
\hline & & & & Slides & $\mathrm{x}$ \\
\hline
\end{tabular}

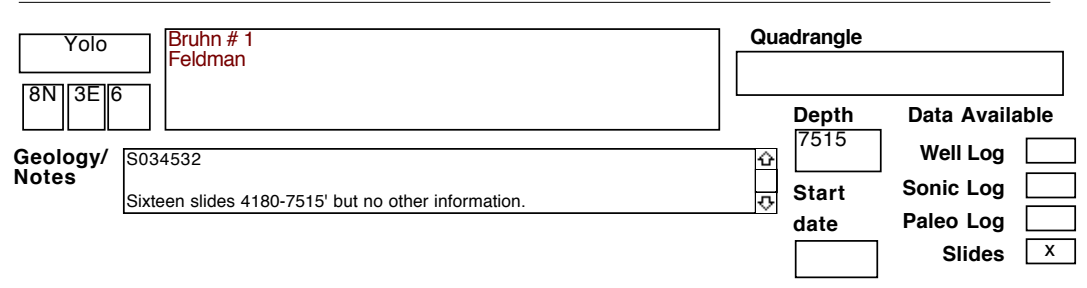

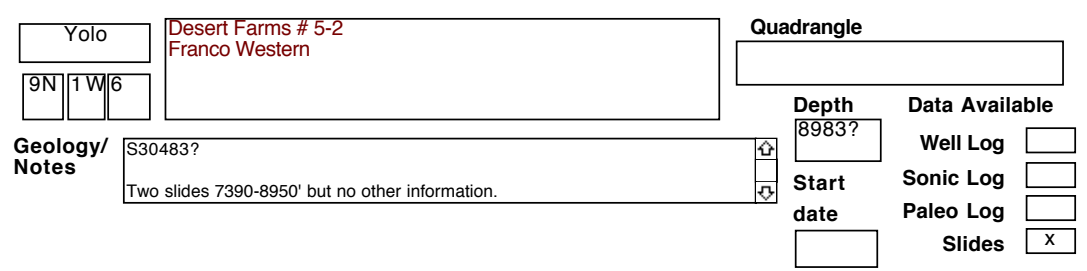


SELECTED OIL TEST WELLS IN CENTRAL CALIFORNIA DATABASE County

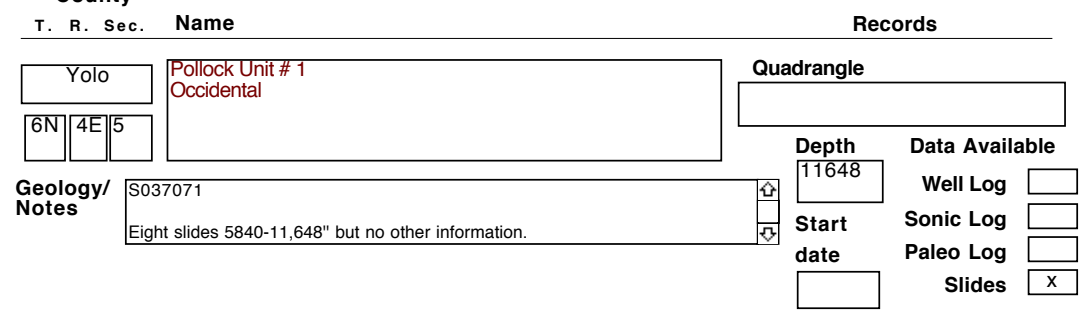

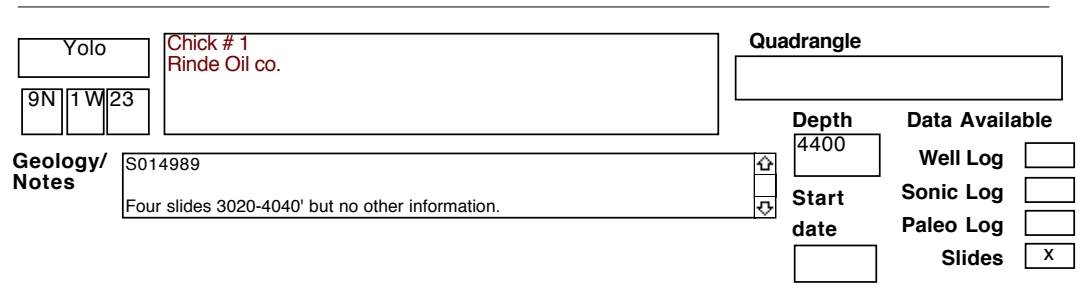

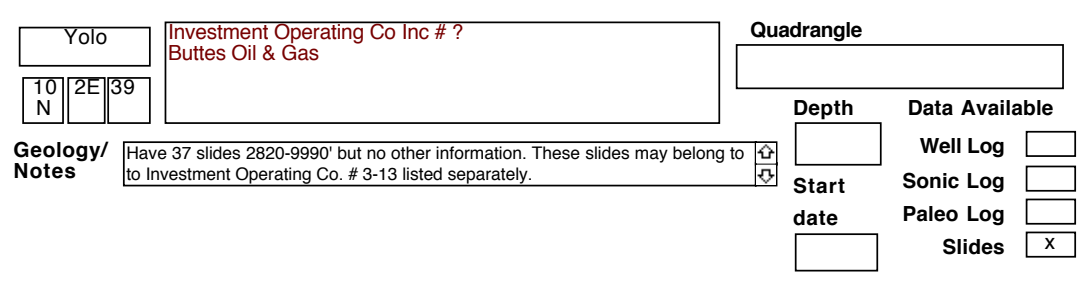

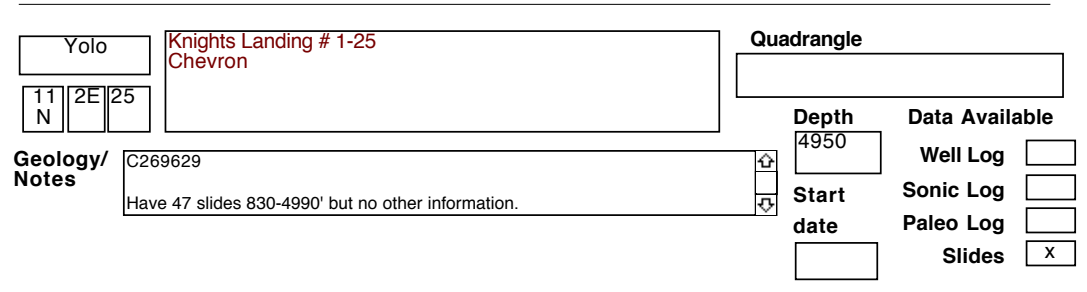

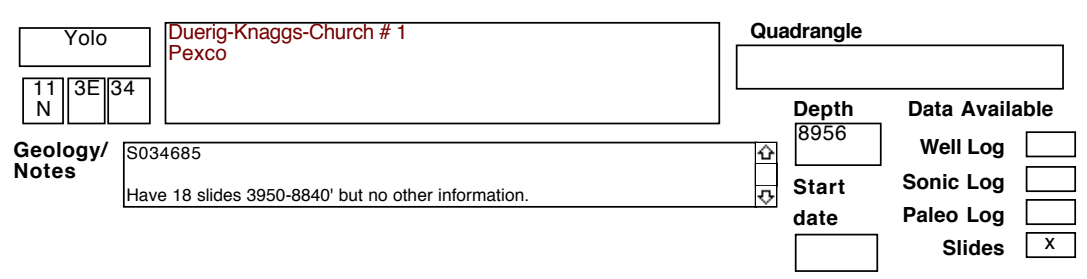


SELECTED OIL TEST WELLS IN CENTRAL CALIFORNIA DATABASE County

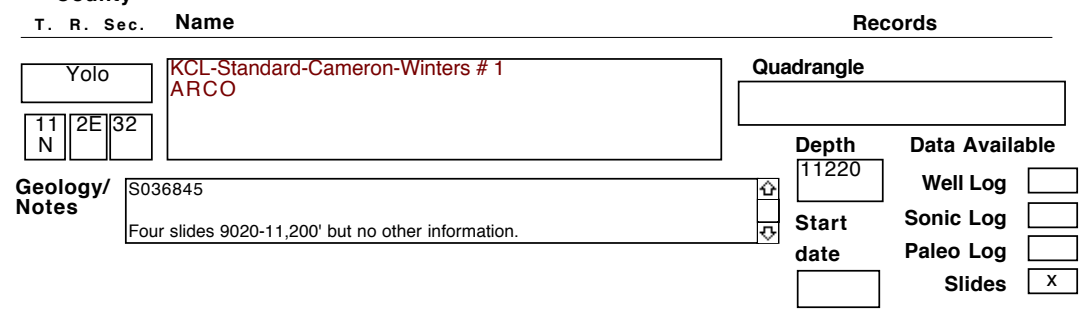

\begin{tabular}{l|l|l|l|}
\hline \multicolumn{1}{|c|}{$\begin{array}{l}\text { Payne \# 1 } \\
\text { Champlin }\end{array}$} & \multicolumn{2}{l|}{ Quadrangle } \\
\hline
\end{tabular}

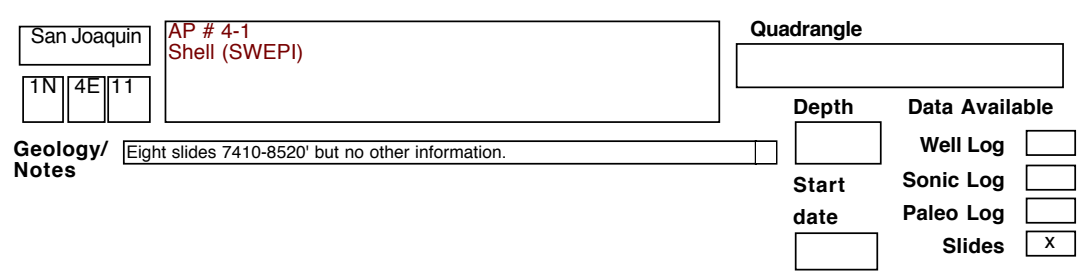

\begin{tabular}{|c|c|c|c|c|c|}
\hline San Joaquin & Blewett Community \# 5 & \multicolumn{4}{|c|}{ Quadrangle } \\
\hline \begin{tabular}{|l|l|l|}
$3 S$ & $6 E$ & 23 \\
\end{tabular} & & \multirow{2}{*}{\multicolumn{2}{|c|}{$\begin{array}{l}\text { Depth } \\
3950\end{array}$}} & \multicolumn{2}{|c|}{ Data Available } \\
\hline \multirow{3}{*}{$\begin{array}{l}\text { Geology/ } \\
\text { Notes }\end{array}$} & 2847 & & & Well Log & \\
\hline & slides $3400-3490^{\prime}$ but no other information. & 田 & Start & Sonic Log & \\
\hline & & & & Slides & $\mathrm{X}$ \\
\hline
\end{tabular}

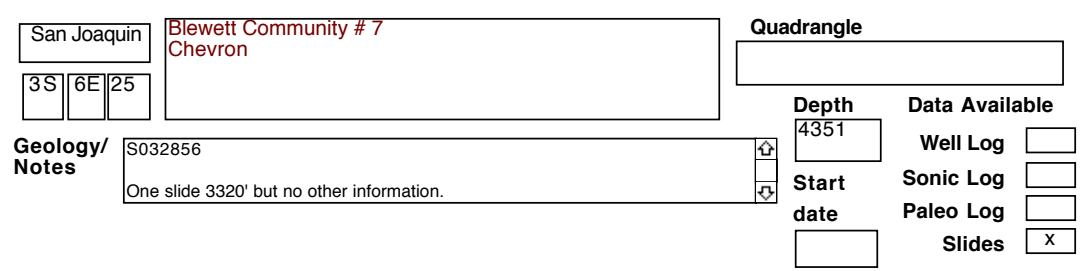


SELECTED OIL TEST WELLS IN CENTRAL CALIFORNIA DATABASE County

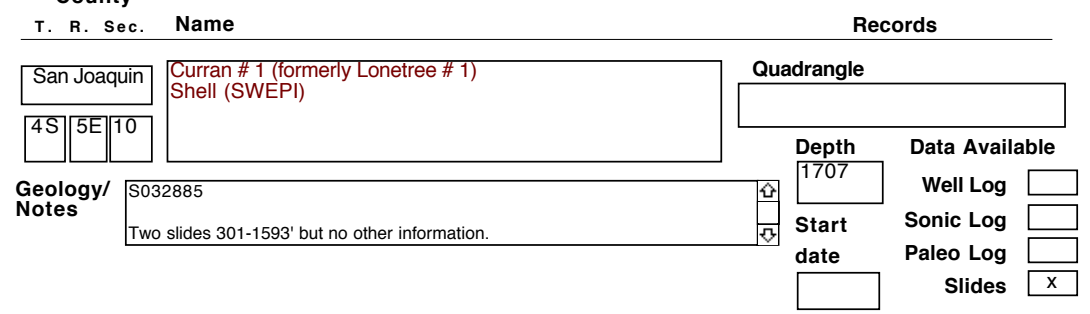

\begin{tabular}{|c|c|c|c|c|}
\hline San Joaquin & \multirow{3}{*}{$\begin{array}{l}\text { Feist \# 1-7 } \\
\text { Champlin }\end{array}$} & \multicolumn{3}{|l|}{ Quadrangle } \\
\hline \multirow[t]{2}{*}{\begin{tabular}{|l|l|l|}
$4 \mathrm{~N}$ & $6 \mathrm{E}$ & 7
\end{tabular}} & & & \multirow{2}{*}{\multicolumn{2}{|c|}{ Data Available }} \\
\hline & & Depth & & \\
\hline \multirow{3}{*}{\multicolumn{2}{|c|}{$\begin{array}{l}\text { Geology/ Have } 18 \text { slides } 3750-8410^{\prime} \text { but no other information. } \\
\text { Notes }\end{array}$}} & & Well Log & \\
\hline & & Start & Sonic Log & \\
\hline & & date & $\begin{array}{r}\text { Paleo Log } \\
\text { Slides }\end{array}$ & $\mathrm{x}$ \\
\hline
\end{tabular}

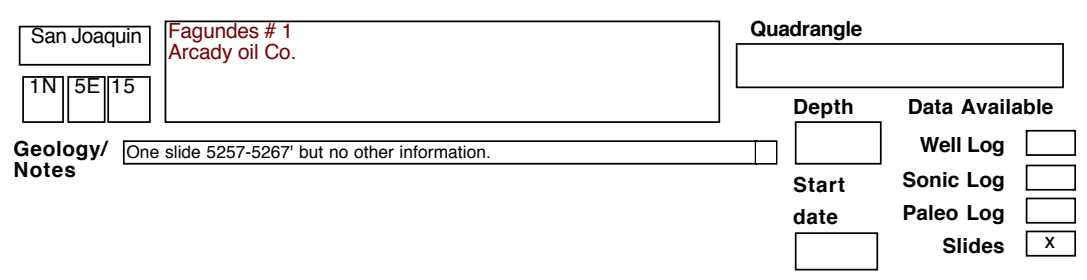

\begin{tabular}{l|l|l|l|l|}
\hline San Joaquin & $\begin{array}{l}\text { Kuckuk \#1-26 } \\
\text { Nordon }\end{array}$ & \multicolumn{2}{l|}{ Quadrangle } \\
\hline
\end{tabular}

\begin{tabular}{|c|c|c|c|c|}
\hline San Joaquin & Holt \# 1 & Quadrangle & & \\
\hline \begin{tabular}{|l|l|l|}
$1 \mathrm{~N}$ & $5 \mathrm{E}$ & 9 \\
\end{tabular} & & Depth & Data Availab & \\
\hline eology/ Se & n slides $4310-5350$ ' but no other information. & & Well Log & \\
\hline & & Start & Sonic Log & \\
\hline & & date & Paleo Log & $\bar{x}$ \\
\hline
\end{tabular}


SELECTED OIL TEST WELLS IN CENTRAL CALIFORNIA DATABASE County

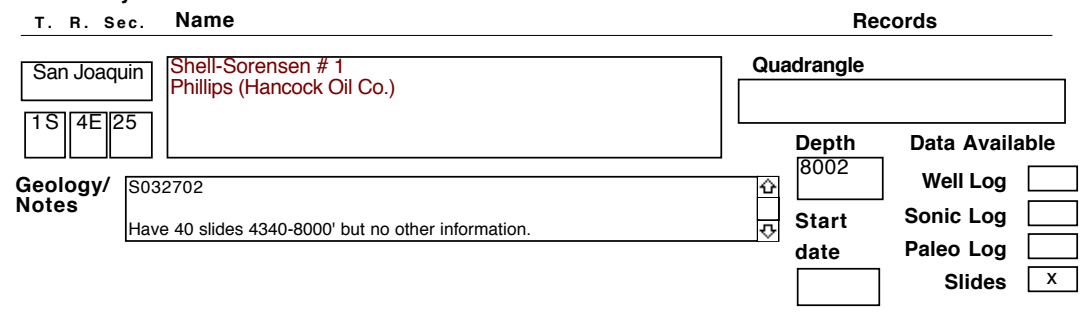

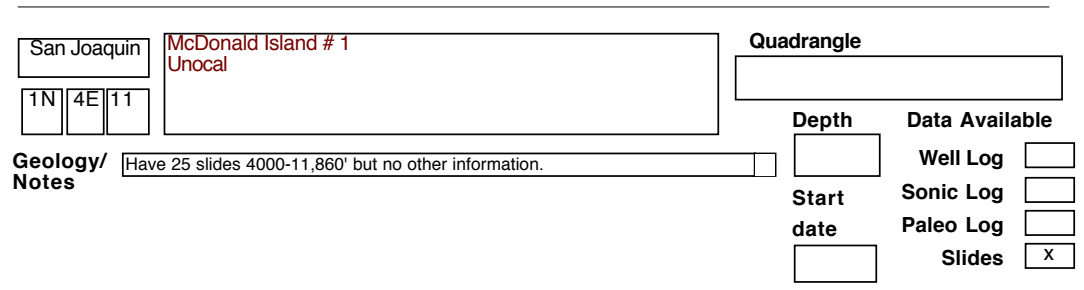

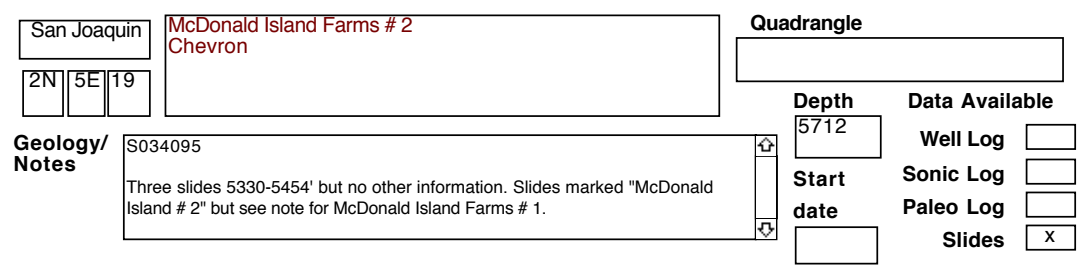

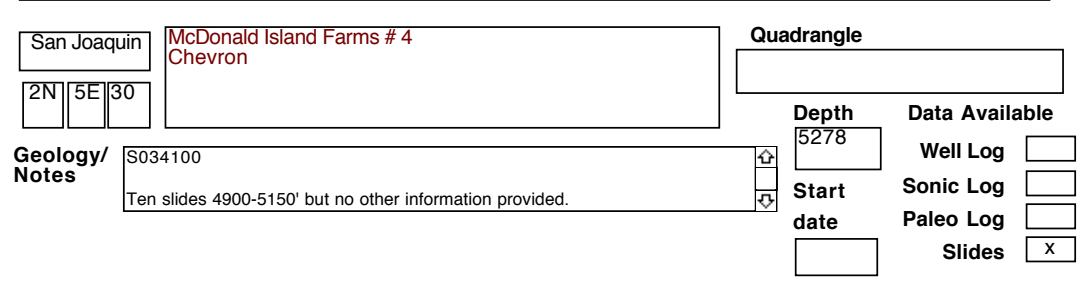

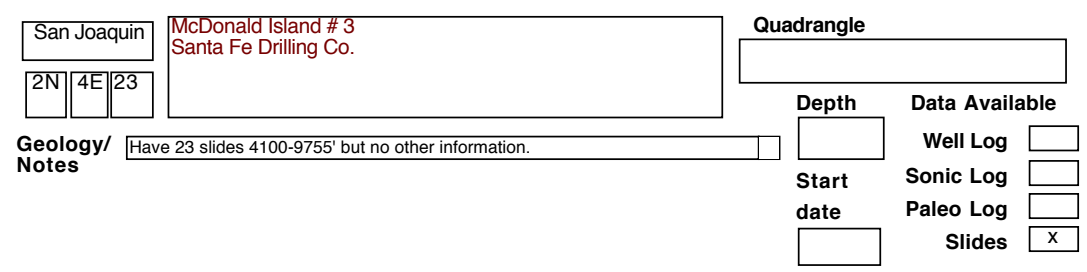


SELECTED OIL TEST WELLS IN CENTRAL CALIFORNIA DATABASE

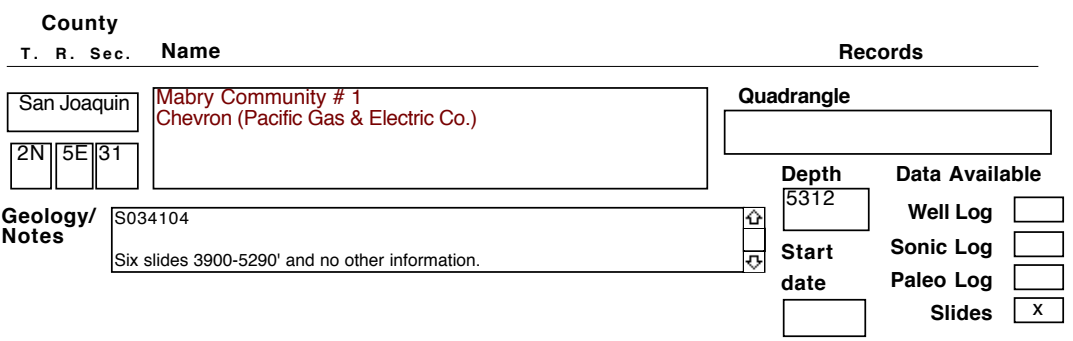

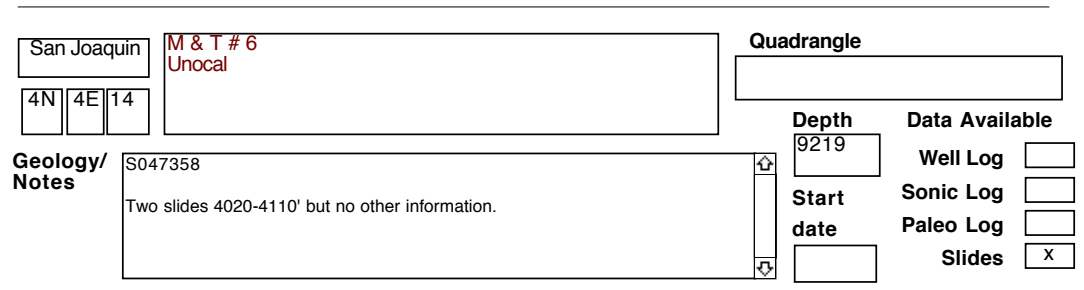

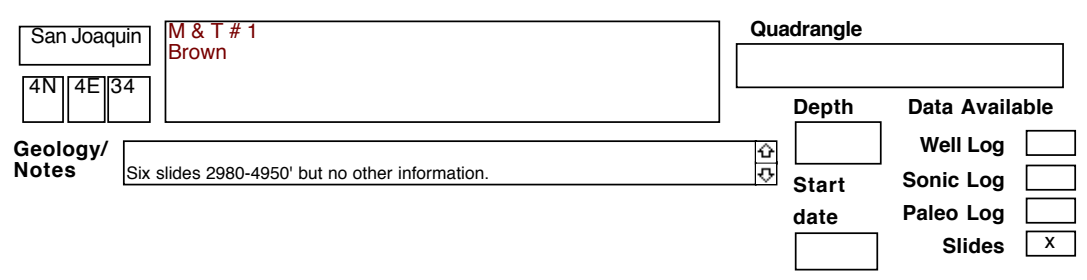

\begin{tabular}{|c|c|c|c|c|c|}
\hline San Joaquin & Brazos-McGillivray \#1 & \multicolumn{4}{|c|}{ Quadrangle } \\
\hline \multicolumn{6}{|l|}{\begin{tabular}{|l||l|l|}
$5 S$ & $5 \mathrm{E}$ & 29 \\
\end{tabular}} \\
\hline \multirow{3}{*}{$\begin{array}{l}\text { Geology/ } \\
\text { Notes }\end{array}$} & 29513 & \multicolumn{2}{|r|}{$\begin{array}{l}\text { Depth } \\
4510\end{array}$} & Well Log & \\
\hline & ee slides $3020-3480^{\prime}$ but no other information. & \multirow[t]{2}{*}{ 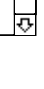 } & Start & Sonic Log & \\
\hline & & & date & Paleo Log & $\bar{x}$ \\
\hline
\end{tabular}

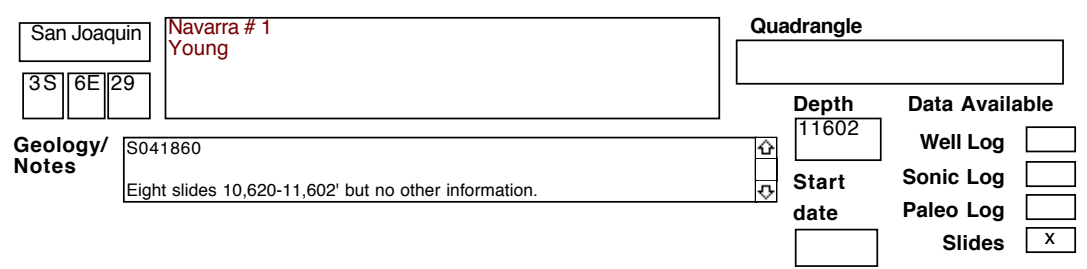


SELECTED OIL TEST WELLS IN CENTRAL CALIFORNIA DATABASE

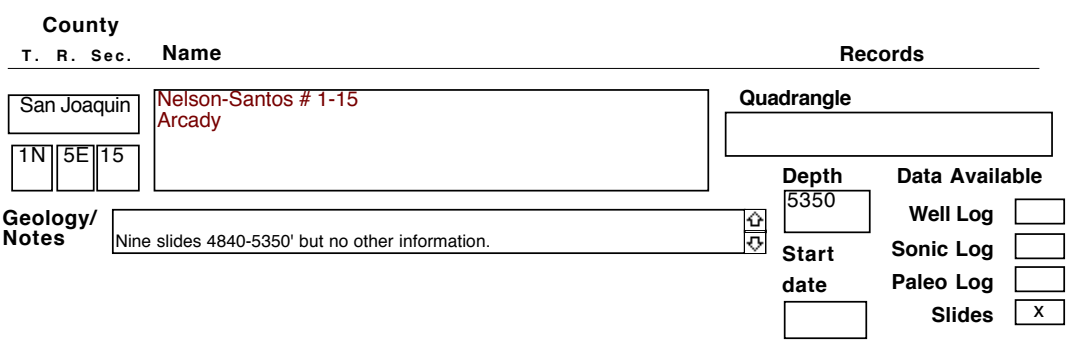

\begin{tabular}{l|l|l|l|l|l|}
\hline San Joaquin & $\begin{array}{l}\text { Pacific States \# 1 } \\
\text { Ohio Oil Co. }\end{array}$ & \multicolumn{2}{l|}{ Quadrangle } \\
\hline
\end{tabular}
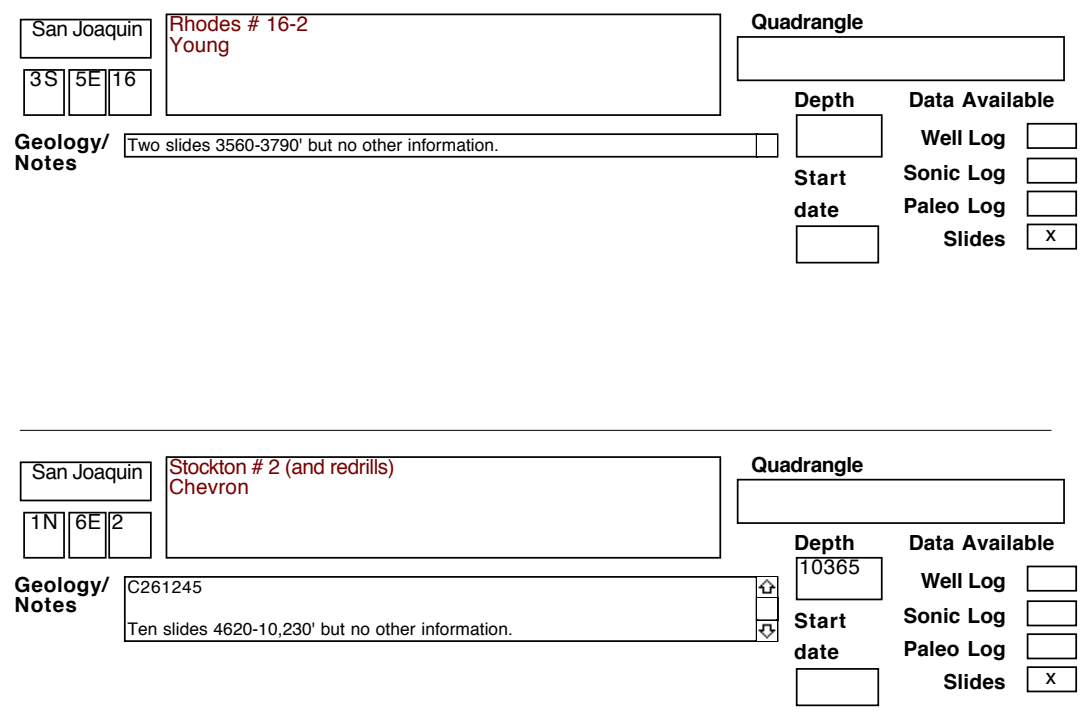

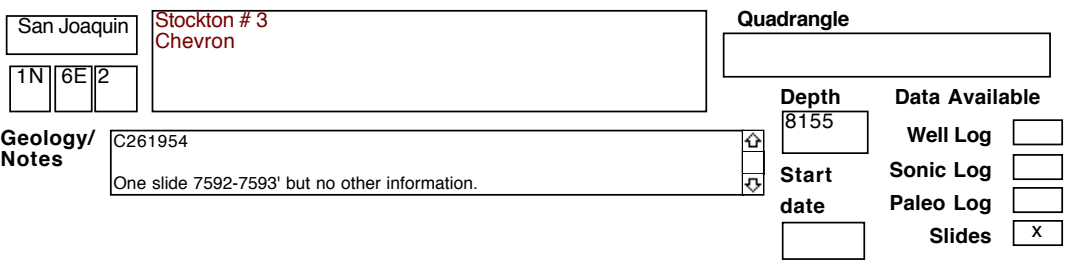


SELECTED OIL TEST WELLS IN CENTRAL CALIFORNIA DATABASE County

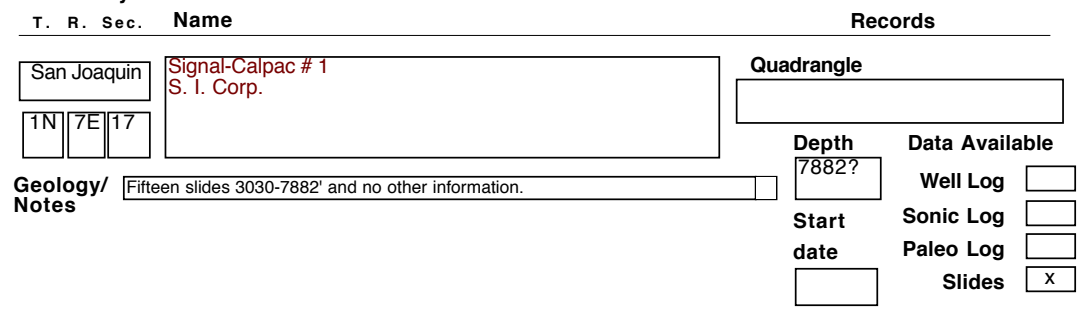

\begin{tabular}{l|l|l|l|l|}
\hline San Joaquin & $\begin{array}{l}\text { Speckman \# 3-9 } \\
\text { Arcady }\end{array}$ & \multicolumn{2}{|l|}{ Quadrangle } \\
\hline
\end{tabular}

\begin{tabular}{|c|c|c|c|c|}
\hline San Joaquin & \multirow{2}{*}{$\begin{array}{l}\text { Best \# } 1 \\
\text { Signal }\end{array}$} & \multicolumn{3}{|l|}{ Quadrangle } \\
\hline $20\left\|^{0 \mathrm{C}}\right\|^{3}$ & & \multirow{2}{*}{ Depth } & \multicolumn{2}{|c|}{ Data Available } \\
\hline \multirow{4}{*}{\multicolumn{2}{|c|}{ Geology/ Twelve slides 5990-9450' but no other information. }} & & Well Log & \\
\hline & & \multirow{3}{*}{$\begin{array}{l}\text { Start } \\
\text { date }\end{array}$} & Sonic Log & \\
\hline & & & Paleo Log & \\
\hline & & & Slides & $\mathrm{x}$ \\
\hline
\end{tabular}

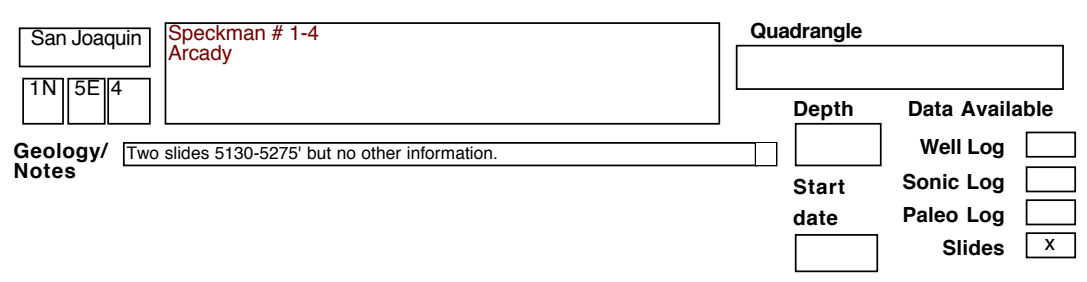

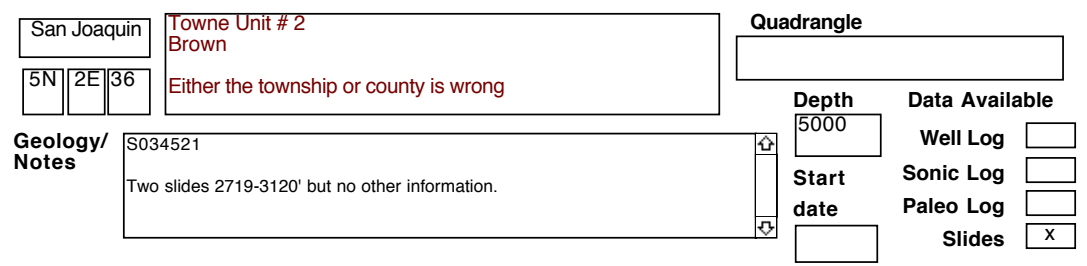


SELECTED OIL TEST WELLS IN CENTRAL CALIFORNIA DATABASE

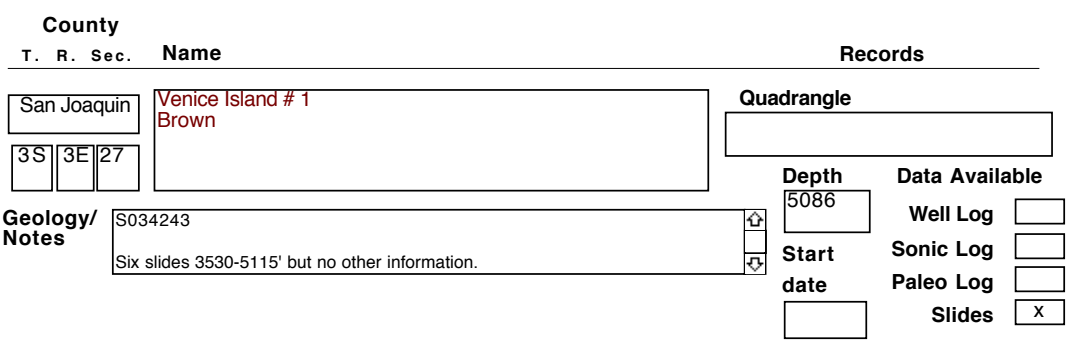

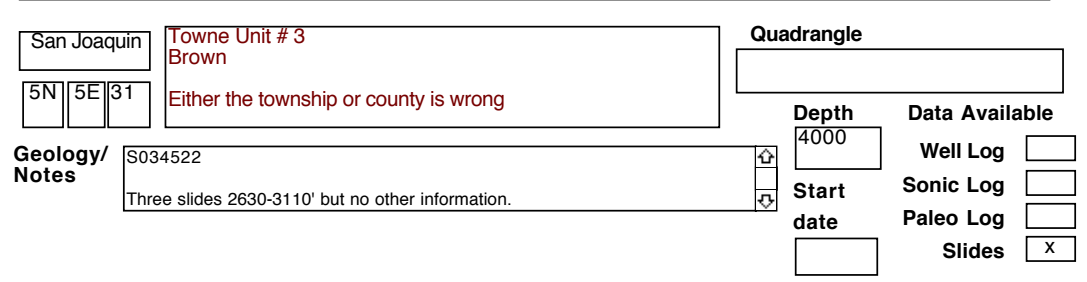

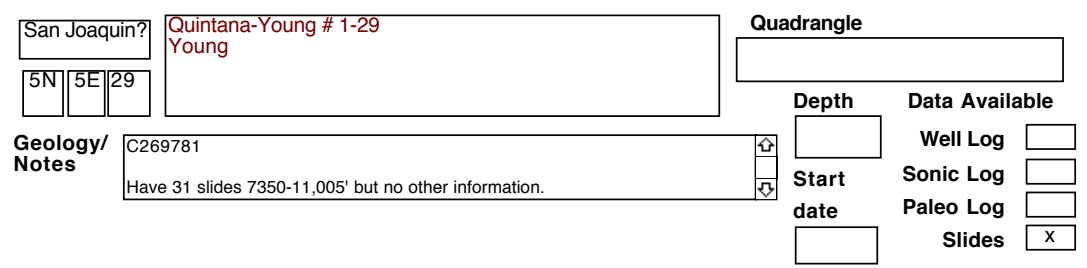

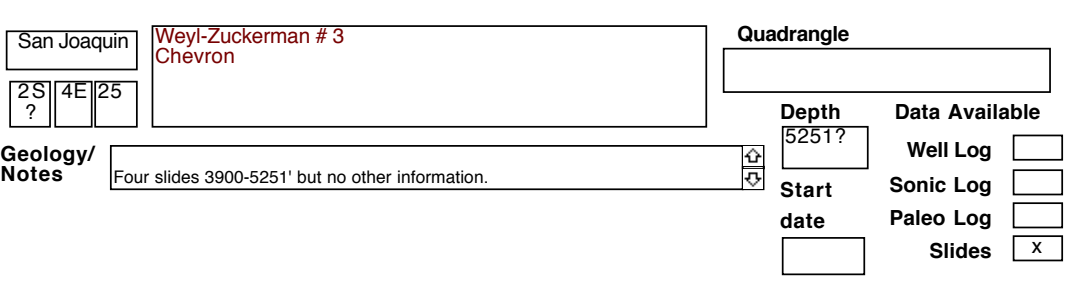

\begin{tabular}{l|l|l|l|l|l|l|}
\hline San Joaquin & $\begin{array}{l}\text { Young Unit 2 \# 3 } \\
\text { Balkan Corp. }\end{array}$ & \multicolumn{2}{|l|}{ Quadrangle } \\
\hline
\end{tabular}


SELECTED OIL TEST WELLS IN CENTRAL CALIFORNIA DATABASE

\begin{tabular}{|c|c|c|c|c|c|}
\hline $\begin{array}{l}\text { County } \\
\text { T. R. Sec. }\end{array}$ & Name & \multicolumn{4}{|c|}{ Records } \\
\hline Sacramento & \multirow{2}{*}{$\begin{array}{l}\text { Anderson \# } 2 \text { (Neal) } \\
\text { Amerada }\end{array}$} & \multicolumn{4}{|c|}{ Quadrangle } \\
\hline \begin{tabular}{|l|l||l}
$3 \mathrm{~N}$ & $3 \mathrm{E}$ & 19 \\
\end{tabular} & & \multirow{2}{*}{\multicolumn{2}{|c|}{$\begin{array}{l}\text { Depth } \\
6218\end{array}$}} & \multicolumn{2}{|c|}{ Data Available } \\
\hline \multirow{3}{*}{$\begin{array}{l}\text { Geology/ } \\
\text { Notes }\end{array}$} & 4215 & & & Well Log & \\
\hline & e 14 slides $5400-6218^{\prime}$ & 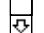 & Start & Sonic Log & \\
\hline & & & & Slides & $\bar{x}$ \\
\hline
\end{tabular}

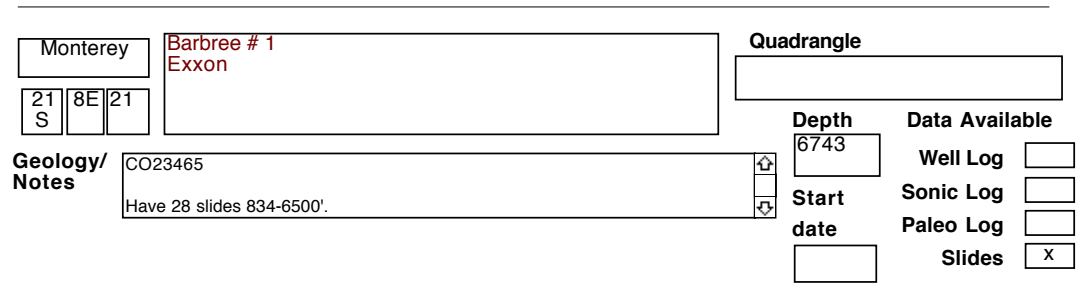

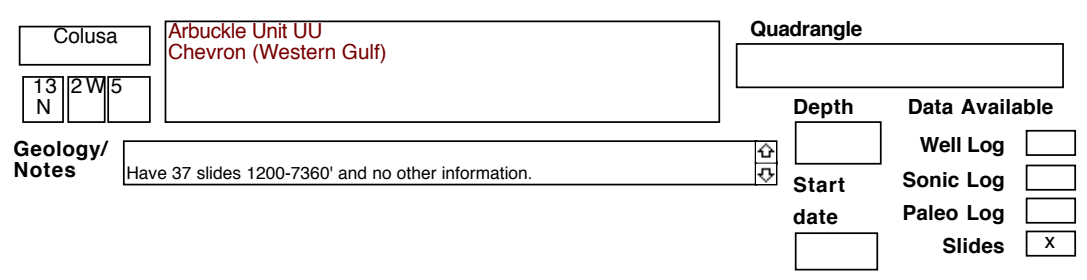

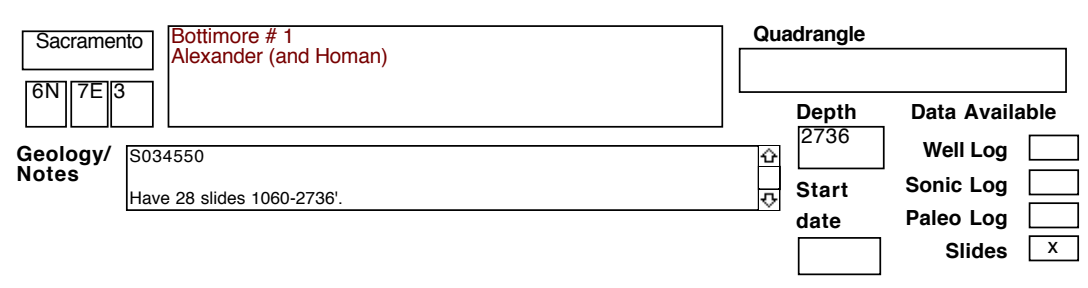

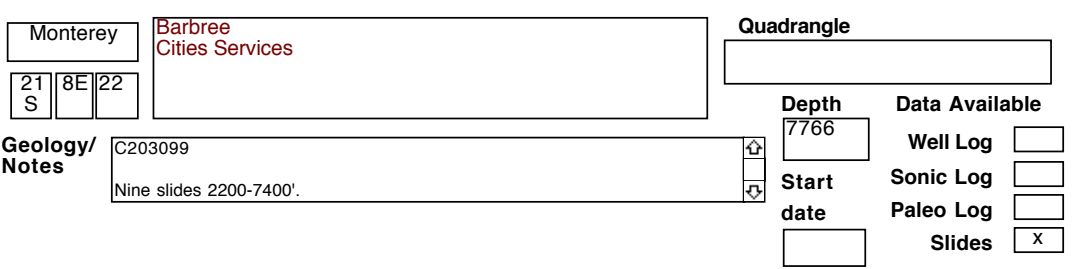


SELECTED OIL TEST WELLS IN CENTRAL CALIFORNIA DATABASE

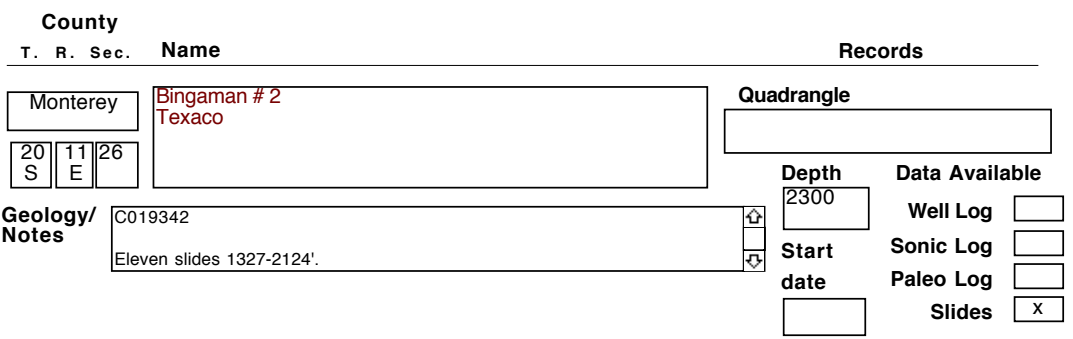

\begin{tabular}{|c|c|c|c|c|c|}
\hline Monterey & \multirow{2}{*}{$\begin{array}{l}\text { Basham \#1 } \\
\text { Texaco }\end{array}$} & \multicolumn{4}{|c|}{ Quadrangle } \\
\hline \begin{tabular}{|c|c|c|}
20 & $7 \mathrm{E}$ & 36 \\
$\mathrm{~S}$ & & \\
\end{tabular} & & \multirow{2}{*}{\multicolumn{2}{|c|}{$\begin{array}{l}\text { Depth } \\
5477\end{array}$}} & \multicolumn{2}{|l|}{ Data Available } \\
\hline \multirow{3}{*}{$\begin{array}{l}\text { Geology/ } \\
\text { Notes }\end{array}$} & 6977 & & & Well Log & \\
\hline & 'en slides 1176-4796'. & 0 & Start & Sonic Log & \\
\hline & & & & Slides & $\mathrm{x}$ \\
\hline
\end{tabular}

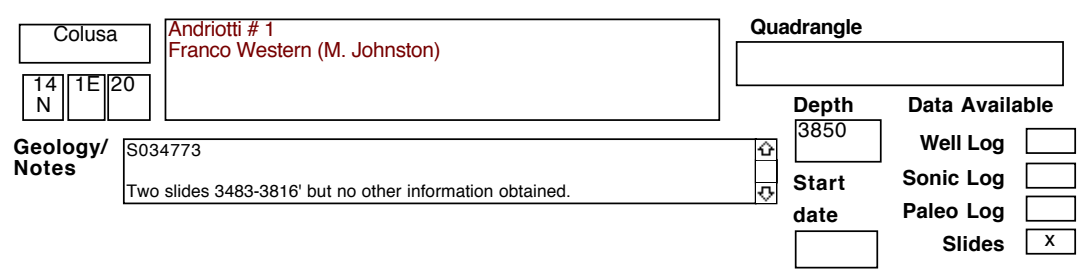

\begin{tabular}{l|l|l|l|l|l|}
\hline San Joaquin & $\begin{array}{l}\text { Avila \# 1 } \\
\text { Ohio (Marathon) }\end{array}$ & \multicolumn{2}{l|}{ Quadrangle } \\
\hline
\end{tabular}

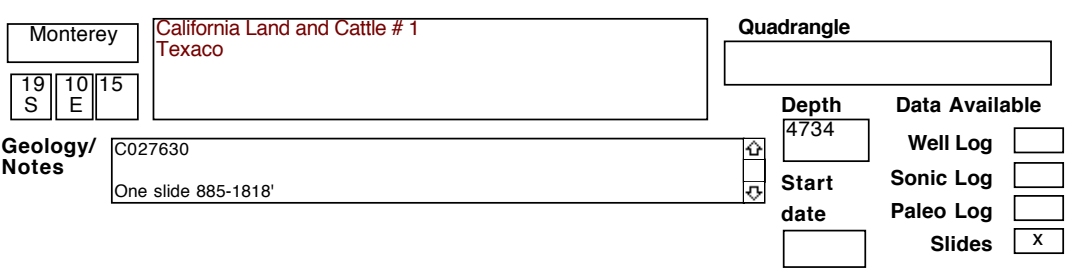


SELECTED OIL TEST WELLS IN CENTRAL CALIFORNIA DATABASE County

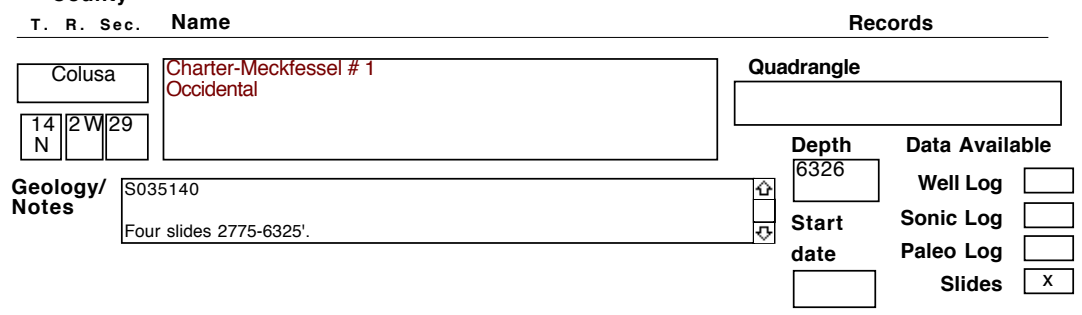

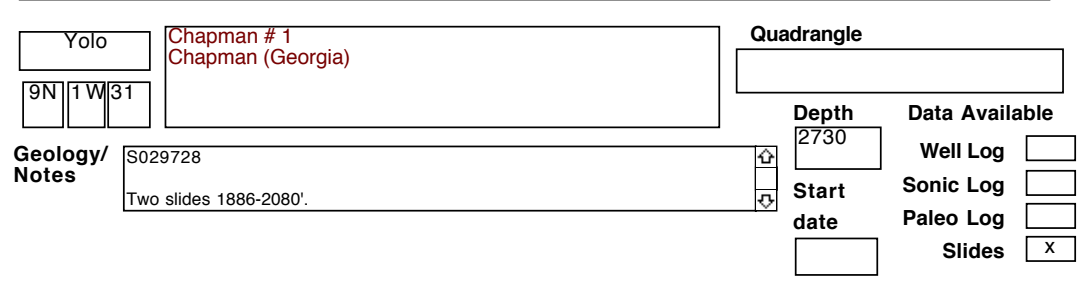

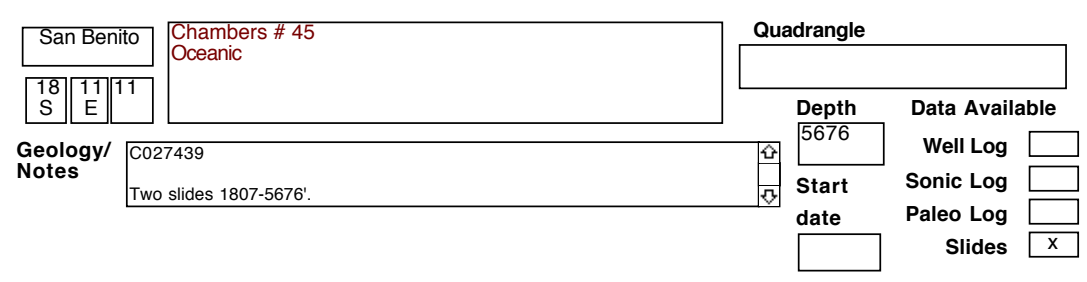

\begin{tabular}{l|l|l|l|l|l|}
\hline Monterey & $\begin{array}{l}\text { Cooper Corehole \# } \\
\text { Chevron }\end{array}$ & \multicolumn{2}{l|}{ Quadrangle } \\
\hline
\end{tabular}

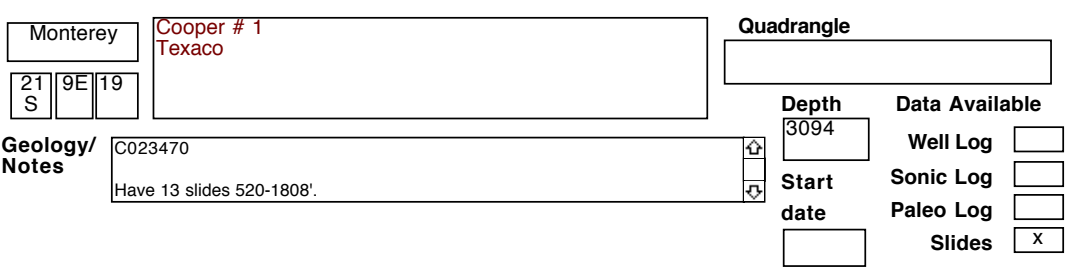


SELECTED OIL TEST WELLS IN CENTRAL CALIFORNIA DATABASE

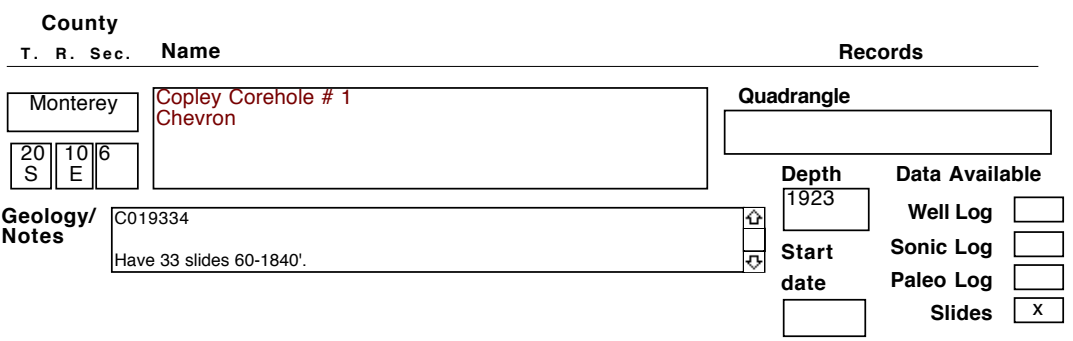

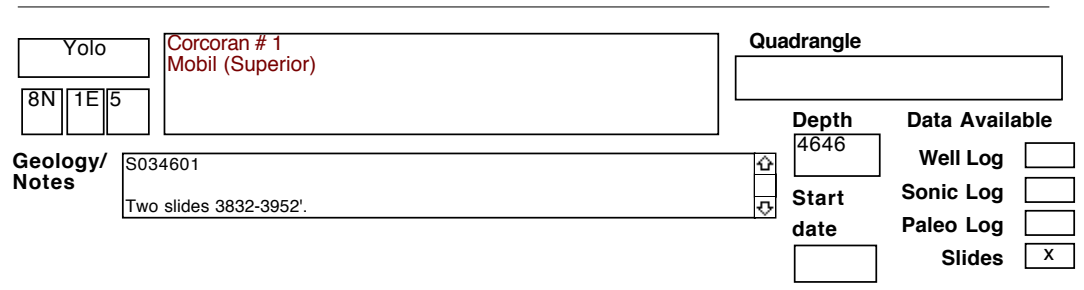

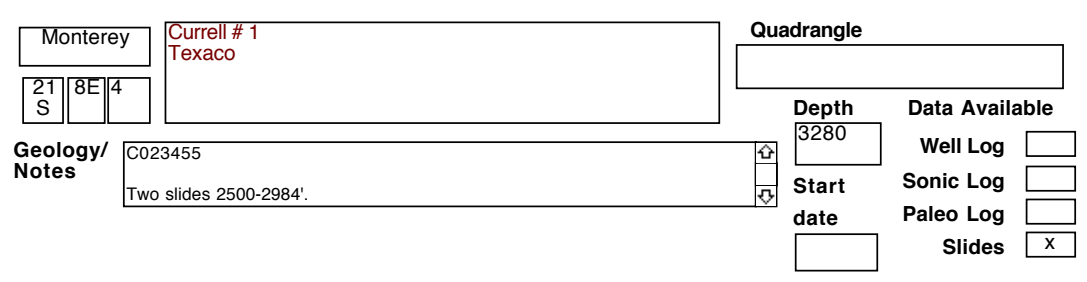

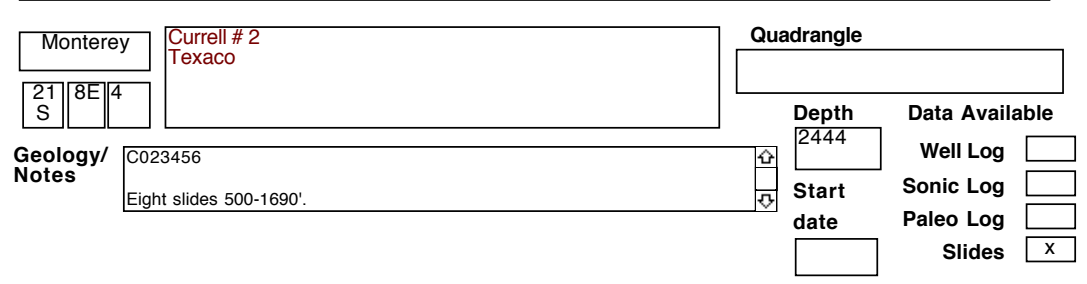

\begin{tabular}{|c|c|c|c|c|c|}
\hline Colusa & \multirow{2}{*}{\begin{tabular}{|l|} 
Davis \# 1 \\
Tiger Oil
\end{tabular}} & \multicolumn{4}{|c|}{ Quadrangle } \\
\hline \begin{tabular}{|l|l|l|}
18 & \\
$\mathrm{~N}$
\end{tabular} & & \multirow{2}{*}{\multicolumn{2}{|c|}{$\begin{array}{l}\text { Depth } \\
3990\end{array}$}} & \multicolumn{2}{|c|}{ Data Available } \\
\hline \multirow{3}{*}{$\begin{array}{l}\text { Geology/ } \\
\text { Notes }\end{array}$} & 24365 & & & Well Log & \\
\hline & ree slides 2160-2610'. & B & Start & Sonic Log & \\
\hline & & & & Slides & $\mathrm{x}$ \\
\hline
\end{tabular}


SELECTED OIL TEST WELLS IN CENTRAL CALIFORNIA DATABASE County

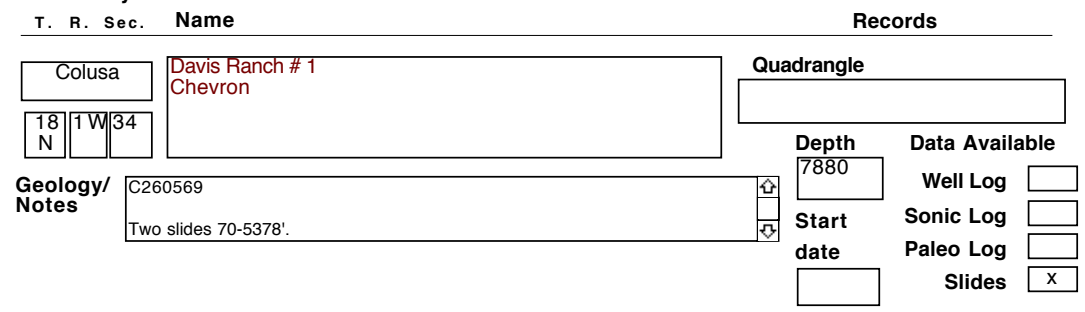

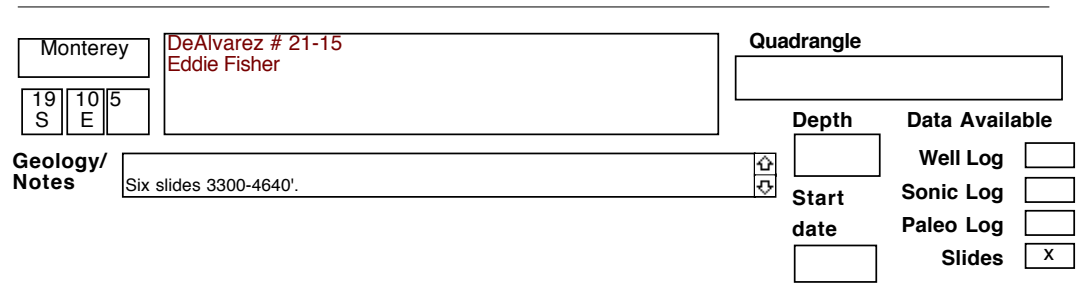

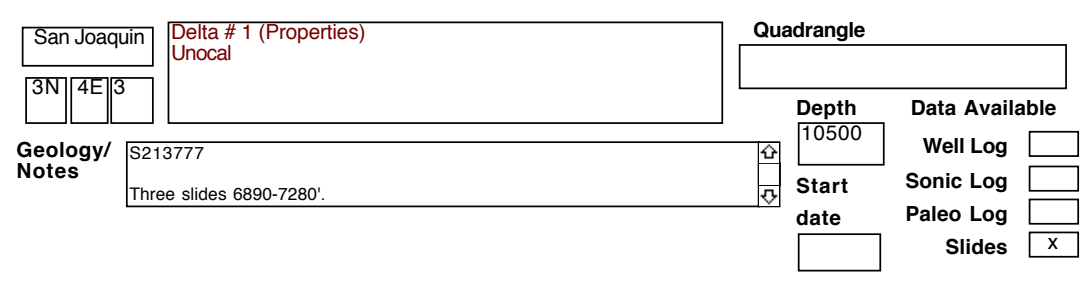

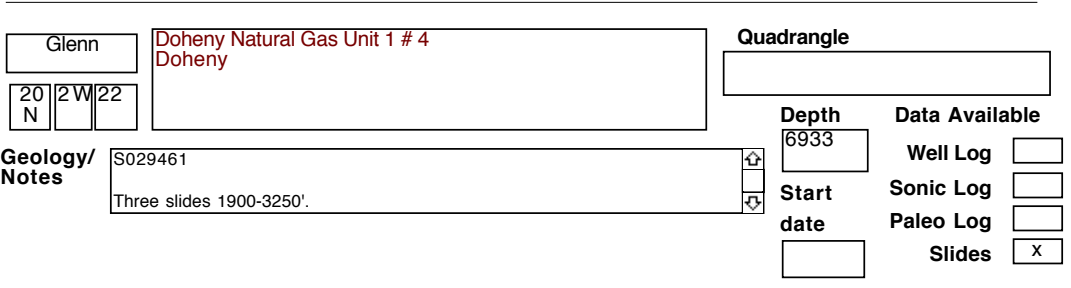

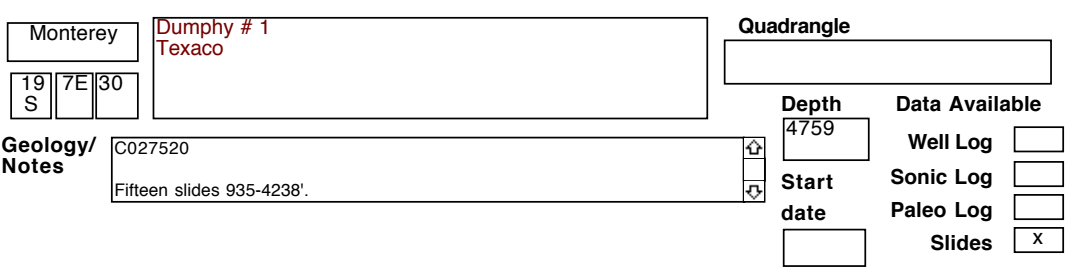


SELECTED OIL TEST WELLS IN CENTRAL CALIFORNIA DATABASE

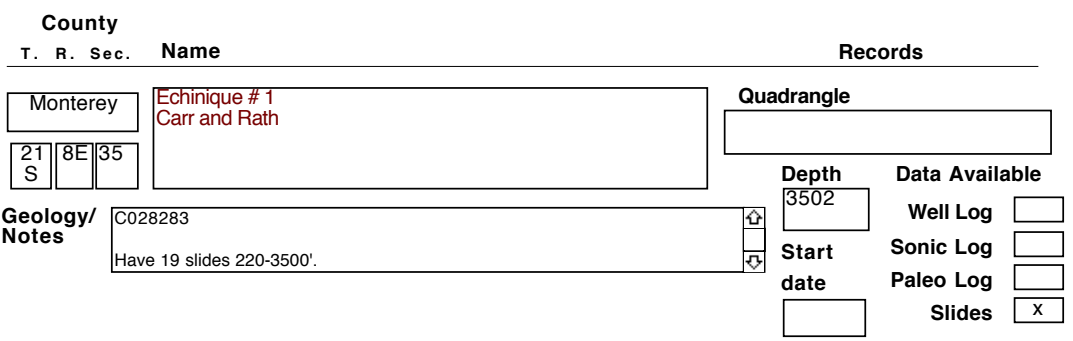

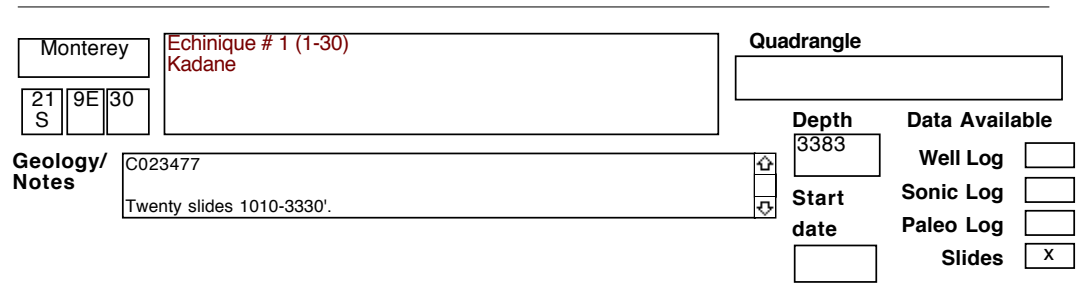

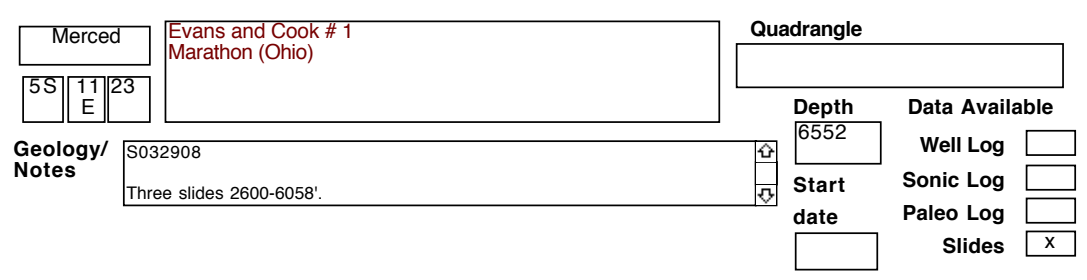

\begin{tabular}{|c|c|c|c|c|c|}
\hline Sacramento & Elk Grove Community \# 3-1 & \multicolumn{4}{|c|}{ Quadrangle } \\
\hline${ }^{7 \mathrm{~N}}\left\|^{6 \mathrm{E}}\right\|^{9}$ & & \multirow{2}{*}{\multicolumn{2}{|c|}{$\begin{array}{l}\text { Depth } \\
1687\end{array}$}} & \multicolumn{2}{|c|}{ Data Available } \\
\hline \multirow{3}{*}{$\begin{array}{l}\text { Geology/ } \\
\text { Notes }\end{array}$} & 4593 & & & Well Log & \\
\hline & slide $1400-1530^{\prime}$. & \multirow[t]{2}{*}{ 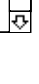 } & Start & Sonic Log & \\
\hline & & & & Slides & $\mathrm{x}$ \\
\hline
\end{tabular}

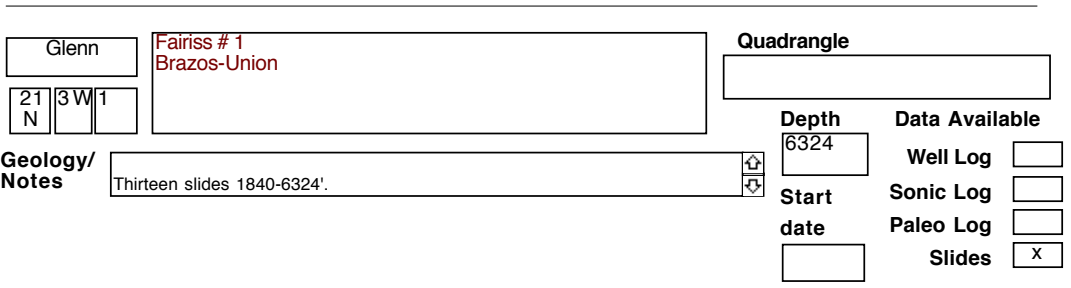


SELECTED OIL TEST WELLS IN CENTRAL CALIFORNIA DATABASE County

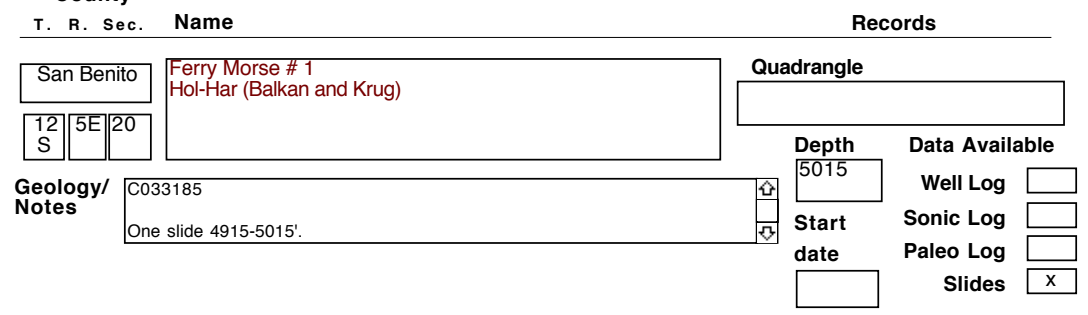

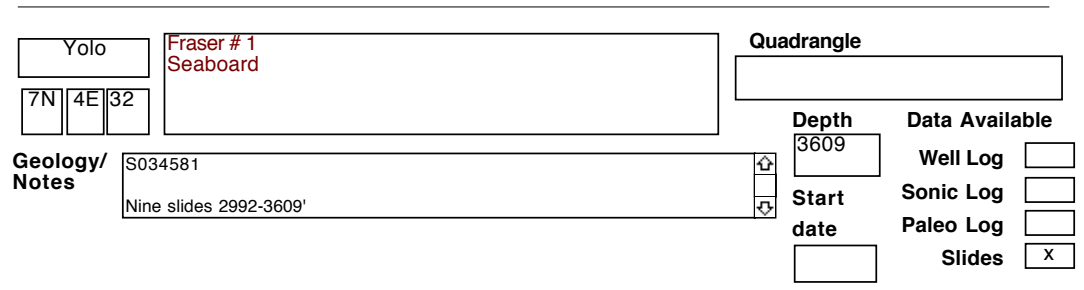

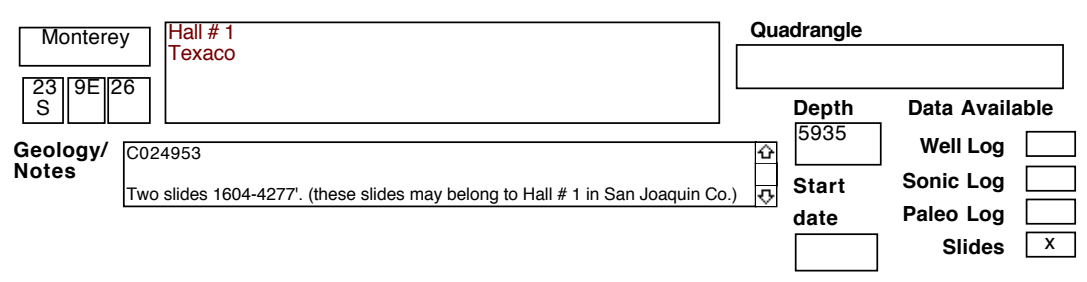

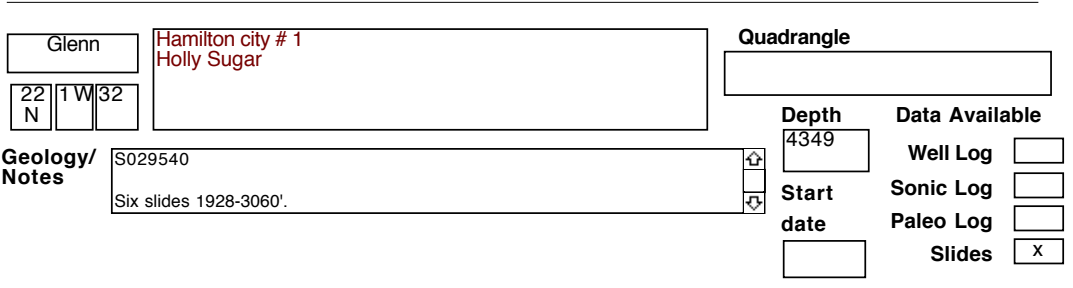

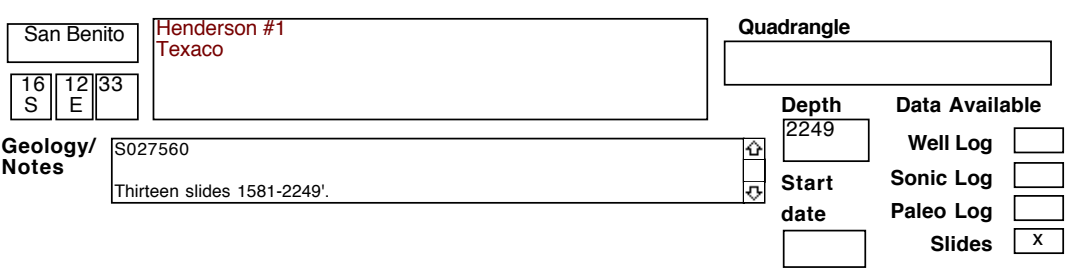


SELECTED OIL TEST WELLS IN CENTRAL CALIFORNIA DATABASE County

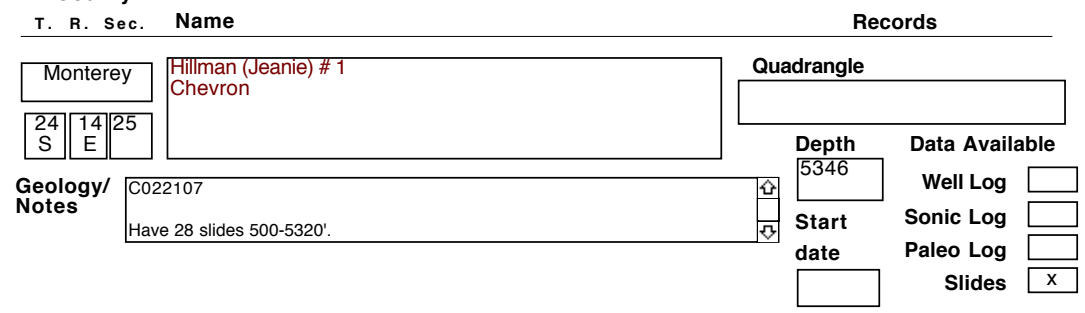

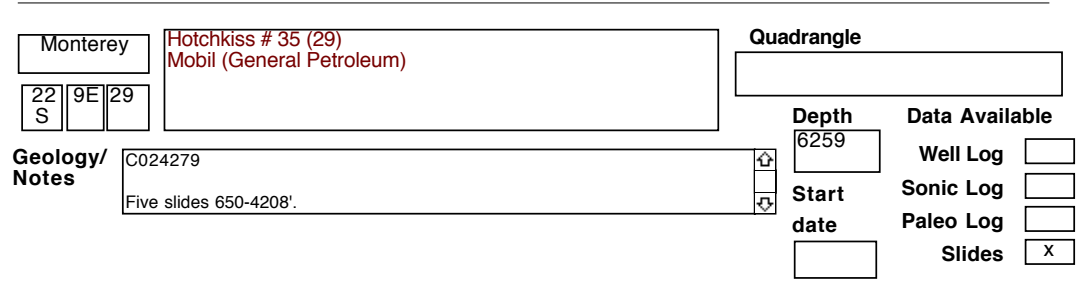

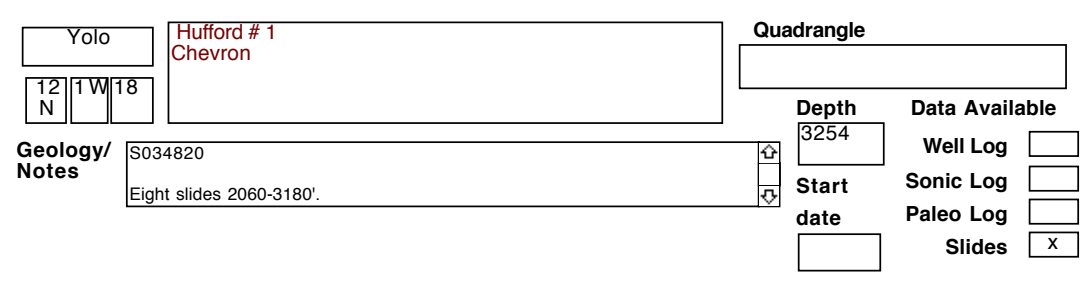

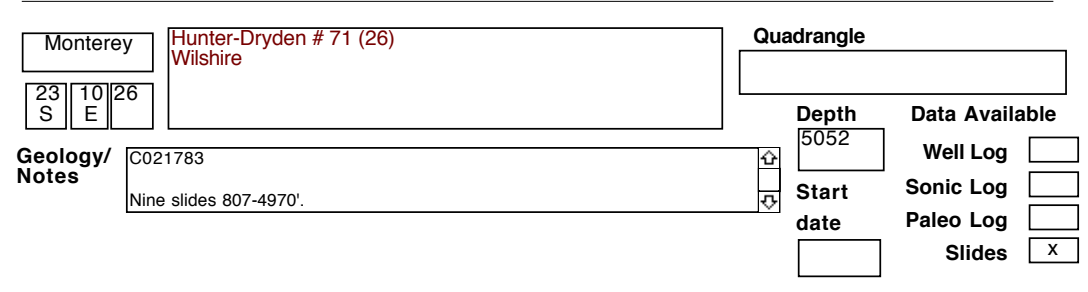

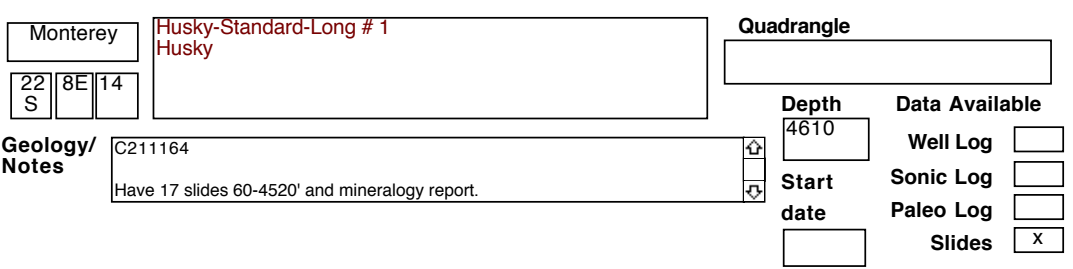


SELECTED OIL TEST WELLS IN CENTRAL CALIFORNIA DATABASE County

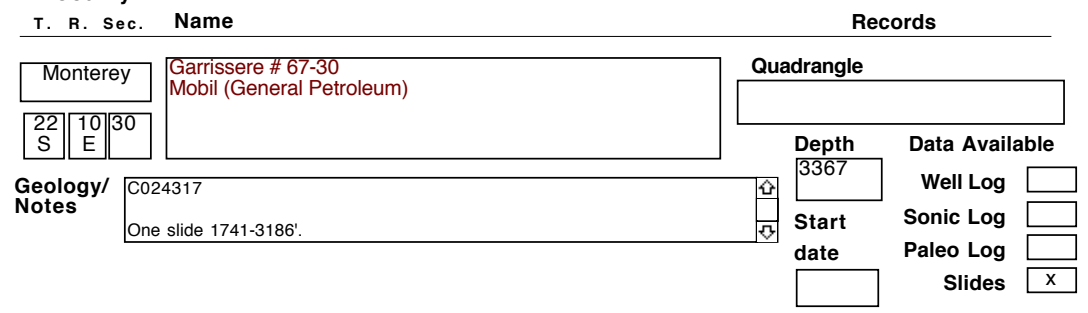

\begin{tabular}{l|l|l|l|l|l|}
\hline \multicolumn{1}{|c|}{$\begin{array}{l}\text { Colusa } \\
\text { Mobil (Cameron) }\end{array}$} & \multicolumn{2}{l|}{ Quadrangle } \\
\hline
\end{tabular}

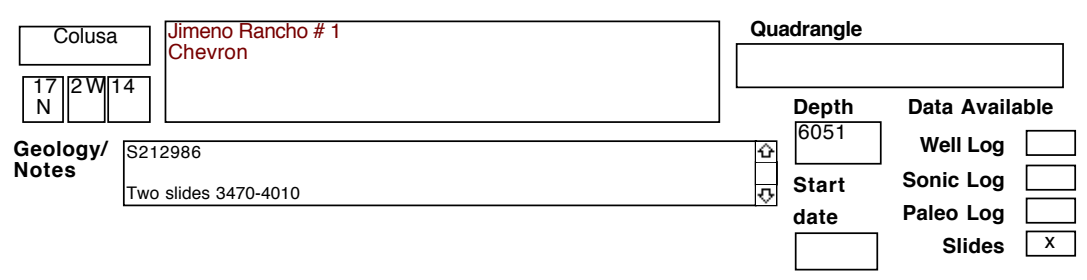

\begin{tabular}{l|l|l|l|l|l|}
\hline Monterey & $\begin{array}{l}\text { Johnson \#1-24 } \\
\text { Salinas Valley }\end{array}$ & \multicolumn{2}{l|}{ Quadrangle } \\
\hline
\end{tabular}

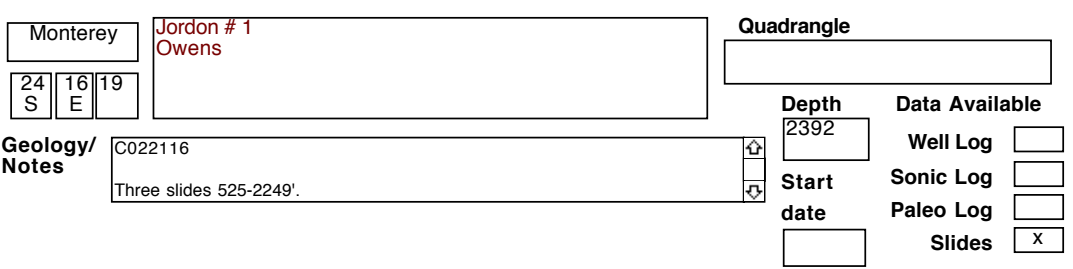


SELECTED OIL TEST WELLS IN CENTRAL CALIFORNIA DATABASE County

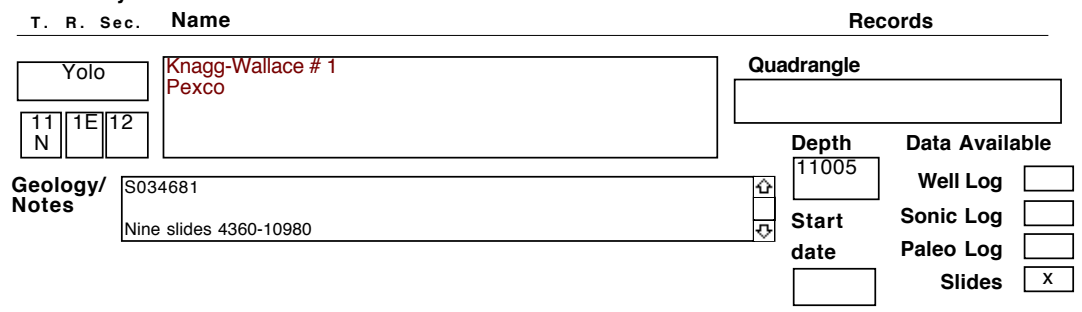

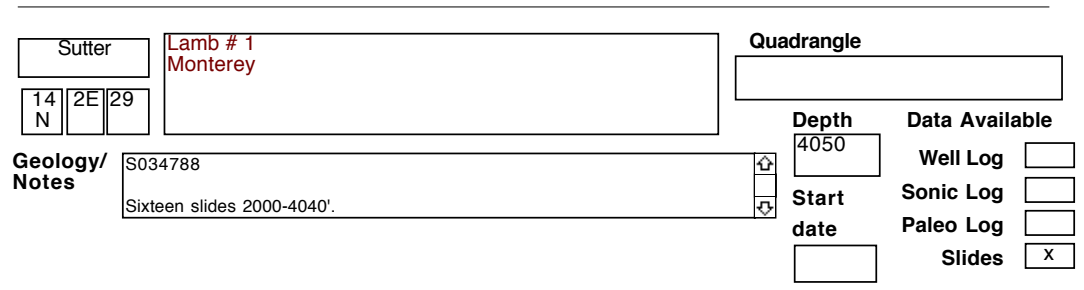

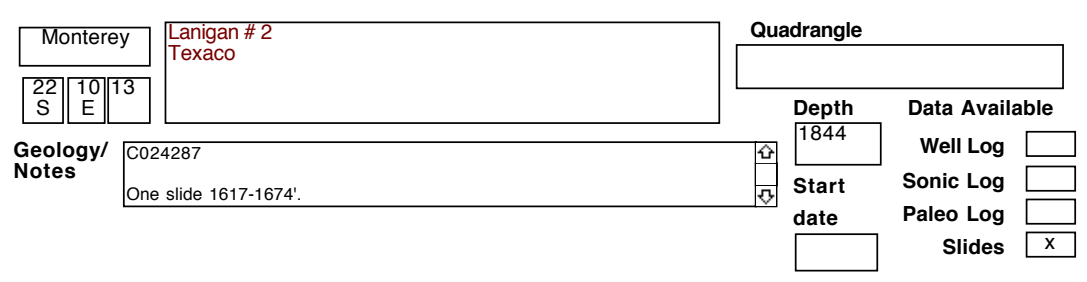

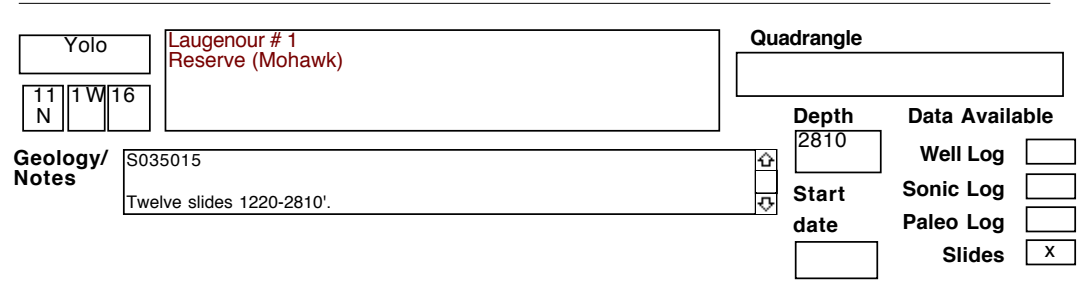

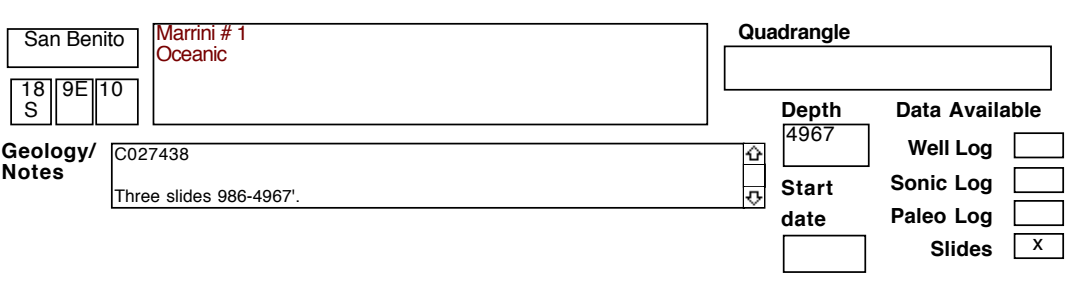


SELECTED OIL TEST WELLS IN CENTRAL CALIFORNIA DATABASE County

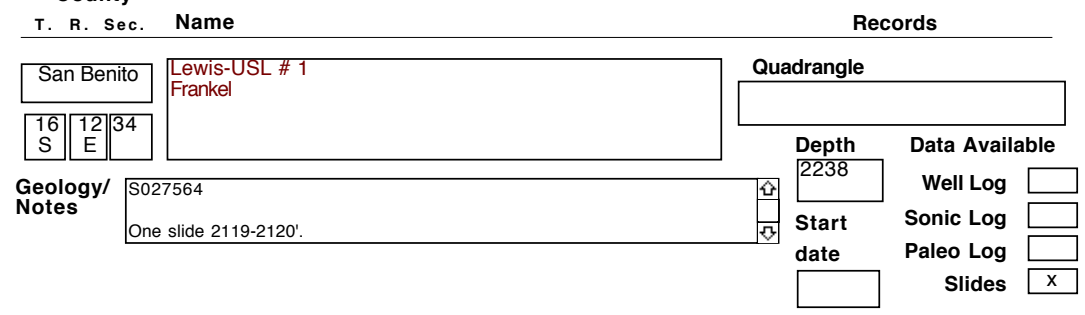

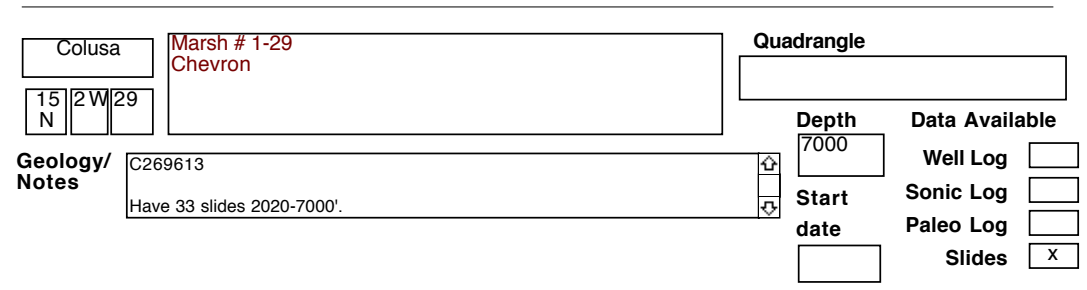

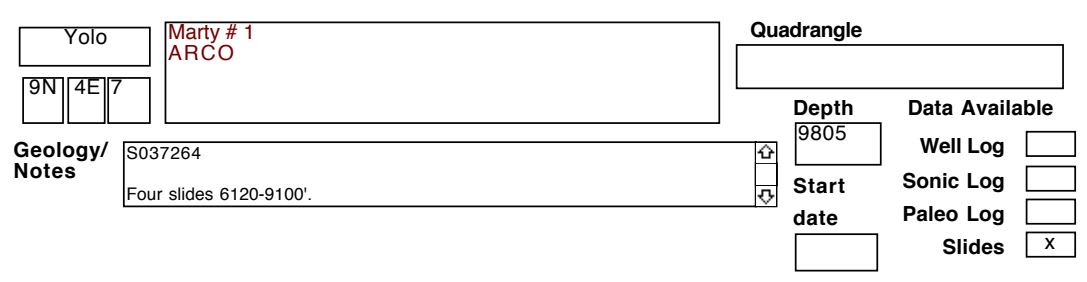

\begin{tabular}{l|l|l|l|l|l|}
\hline San Benito & $\begin{array}{l}\text { Murphy \# 1 } \\
\text { Texaco (TWA) }\end{array}$ & \multicolumn{2}{l|}{ Quadrangle } \\
\hline
\end{tabular}

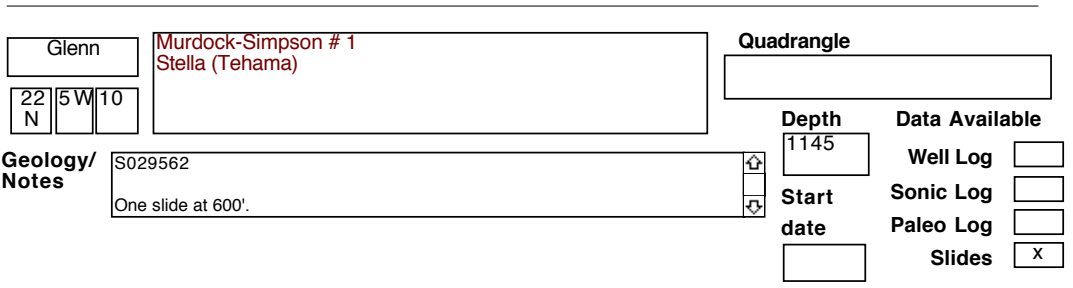


SELECTED OIL TEST WELLS IN CENTRAL CALIFORNIA DATABASE

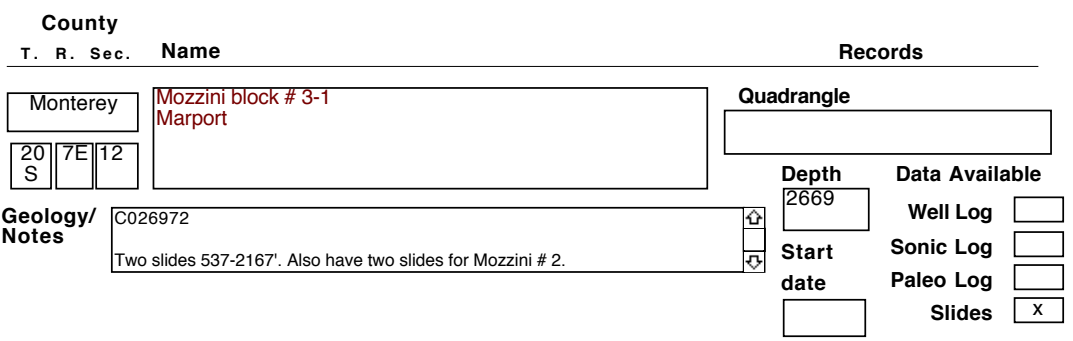

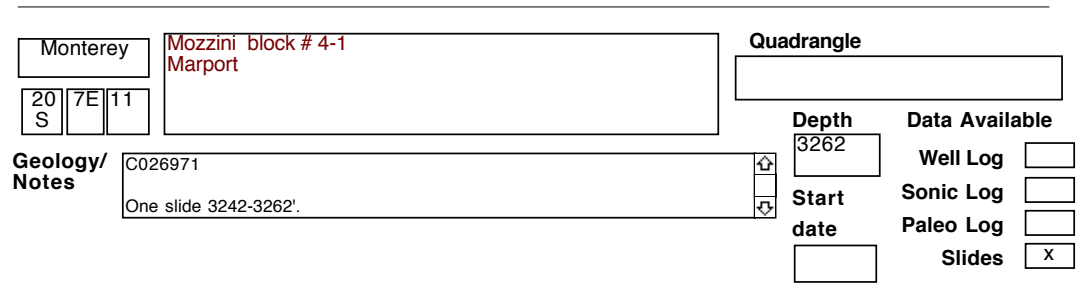

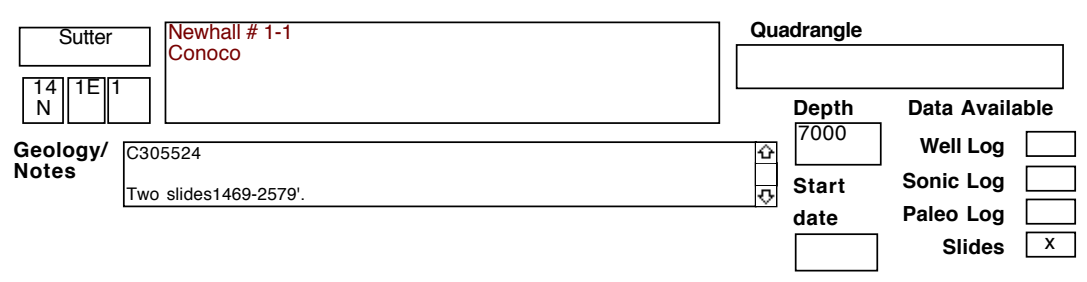

\begin{tabular}{l|l|l|l|l|l|}
\hline San Benito & $\begin{array}{l}\text { Nicholas \# 2 } \\
\text { E and B NA (Ciervo) }\end{array}$ & \multicolumn{2}{l|}{ Quadrangle } \\
\hline
\end{tabular}

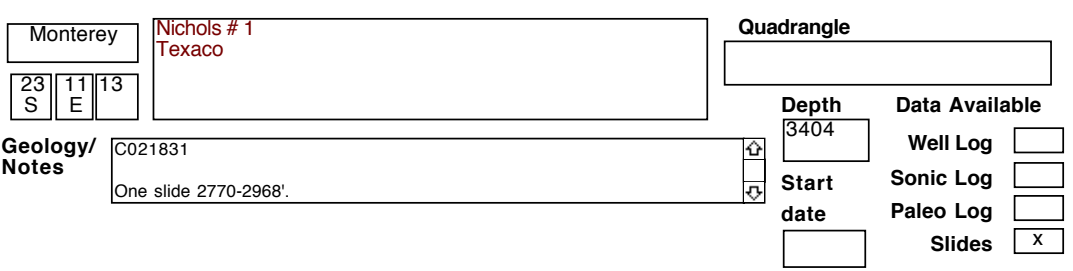


SELECTED OIL TEST WELLS IN CENTRAL CALIFORNIA DATABASE County

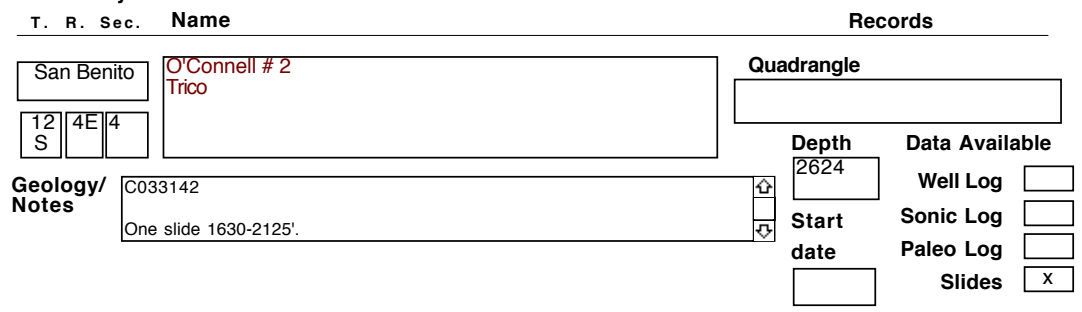

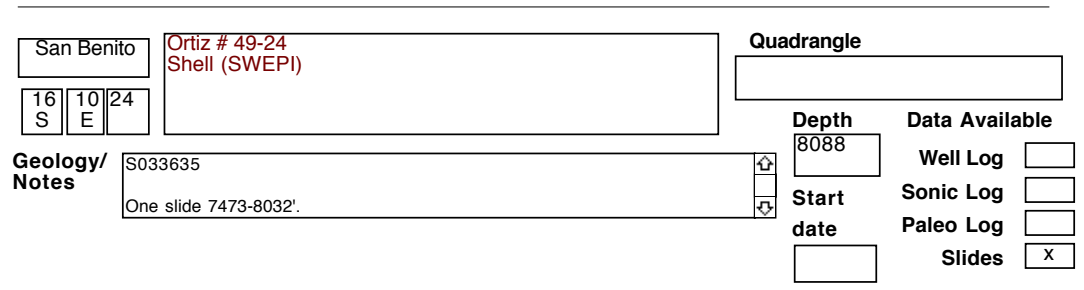

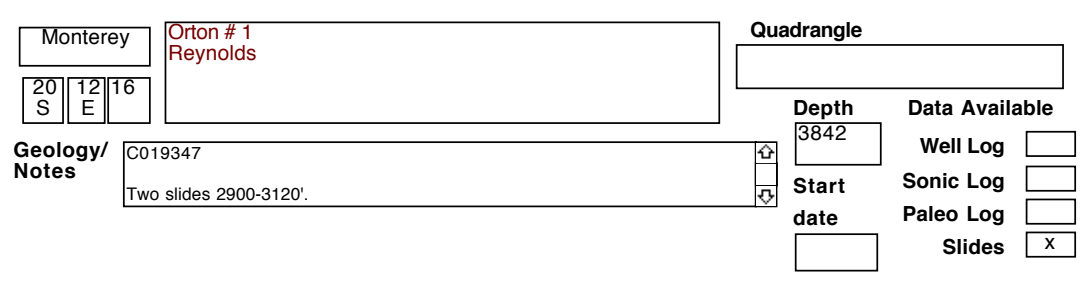

\begin{tabular}{l|l|l|l|l|l|}
\hline Monterey & $\begin{array}{l}\text { Quinn corehole \# 1 } \\
\text { Chevron }\end{array}$ & \multicolumn{2}{l|}{ Quadrangle } \\
\hline
\end{tabular}

\begin{tabular}{l|l|l|l|l|l|}
\hline \multicolumn{1}{|c|}{ Sutter } & $\begin{array}{l}\text { Otterson-Wibur \#1 } \\
\text { Occidental }\end{array}$ & \multicolumn{2}{l|}{ Quadrangle } \\
\hline
\end{tabular}


SELECTED OIL TEST WELLS IN CENTRAL CALIFORNIA DATABASE

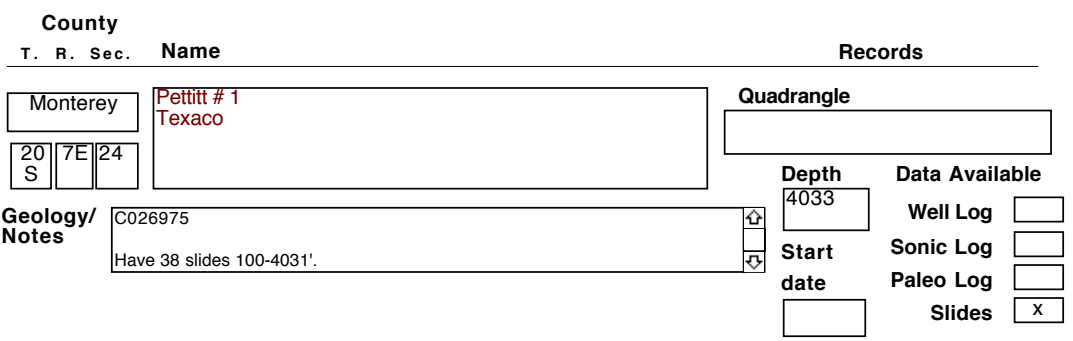

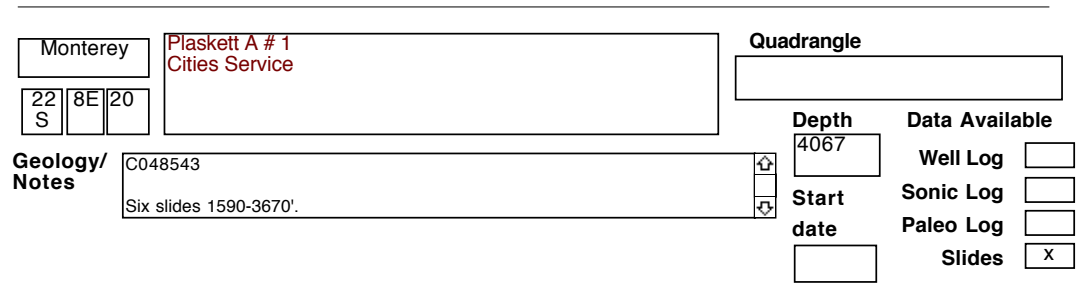

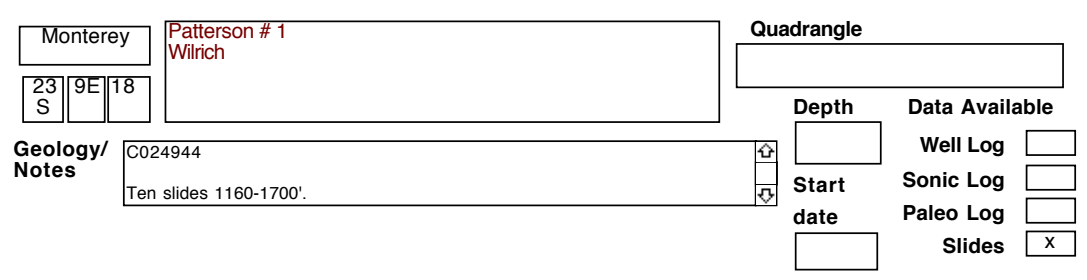

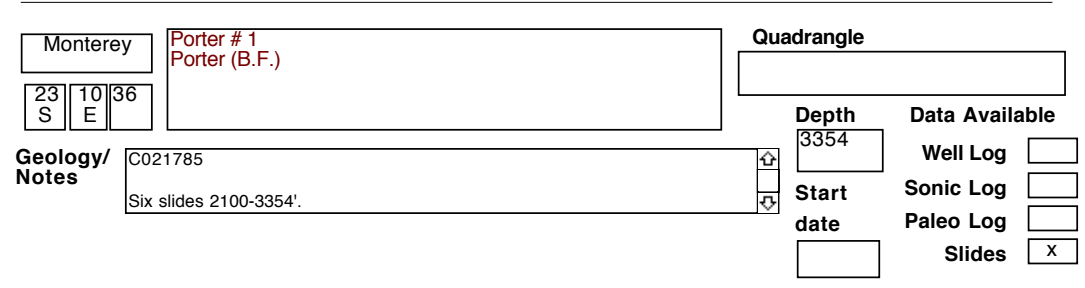

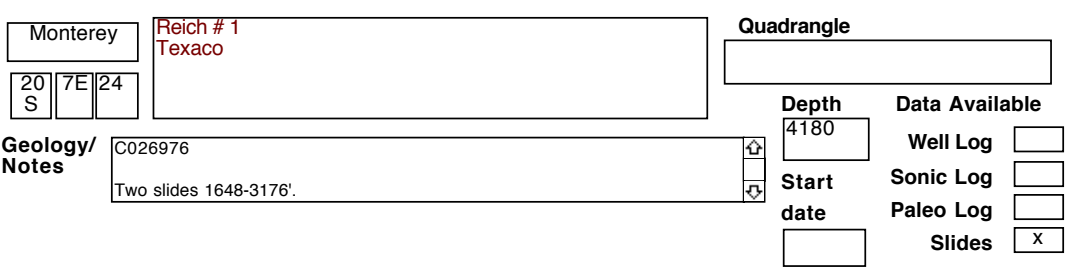


SELECTED OIL TEST WELLS IN CENTRAL CALIFORNIA DATABASE County

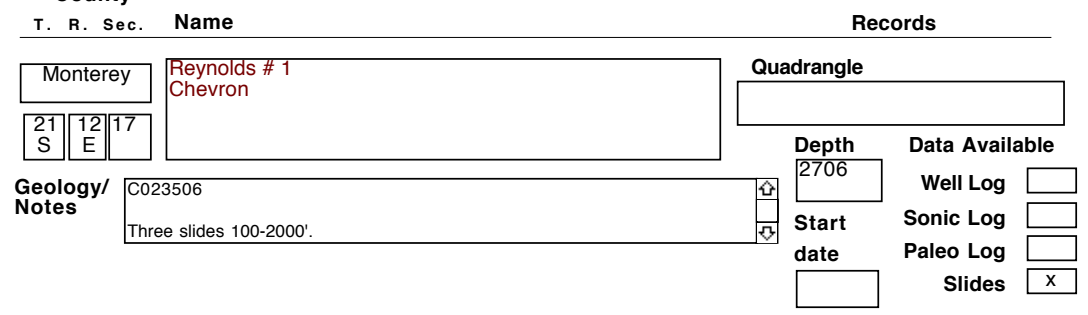

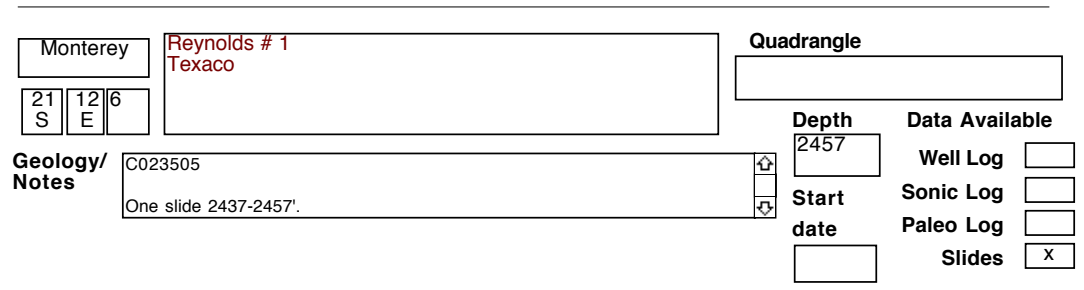

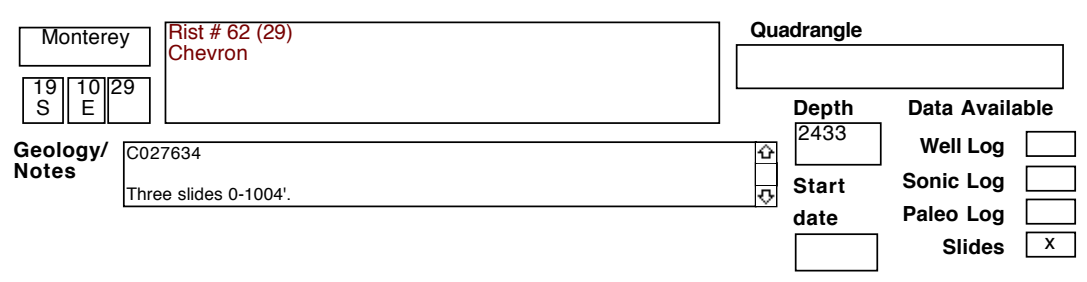

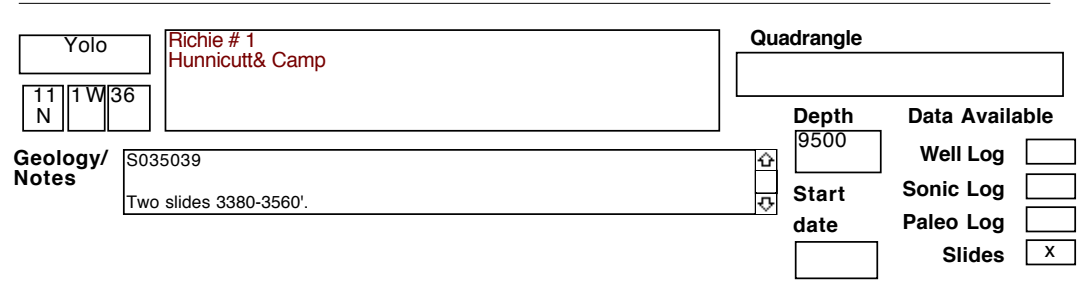

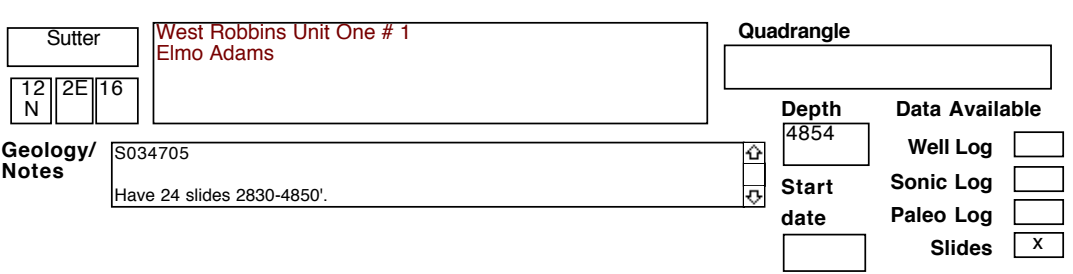


SELECTED OIL TEST WELLS IN CENTRAL CALIFORNIA DATABASE County

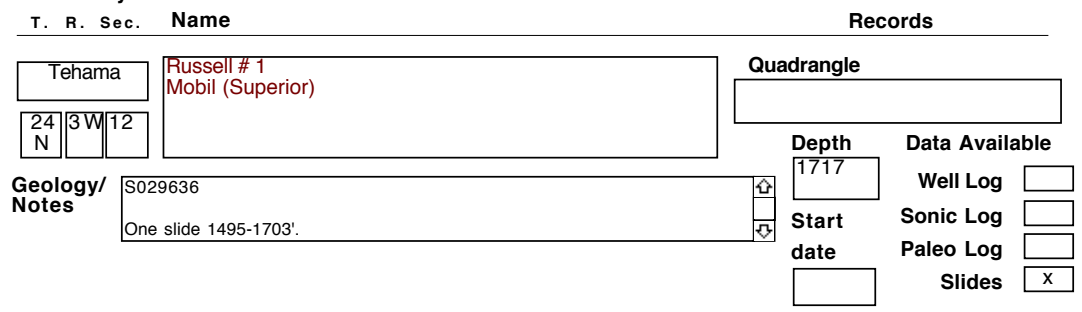

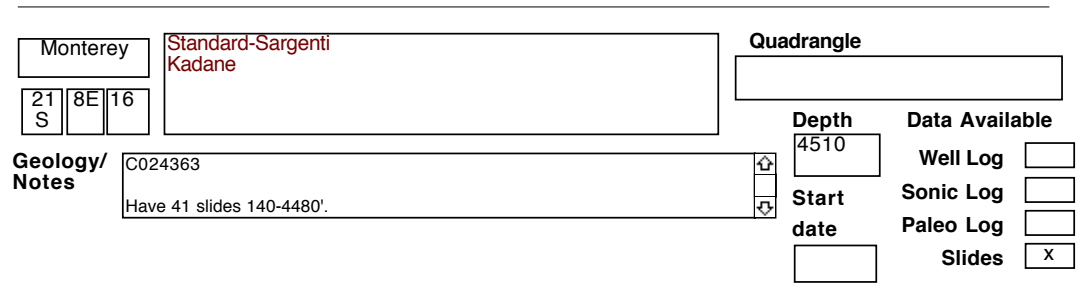

\begin{tabular}{l|l|l|l|l|l|}
\hline \multicolumn{1}{|c|}{ Colusa } & $\begin{array}{l}\text { Sachreiter \#3 } \\
\text { Occidental }\end{array}$ & \multicolumn{2}{l|}{ Quadrangle } \\
\hline
\end{tabular}

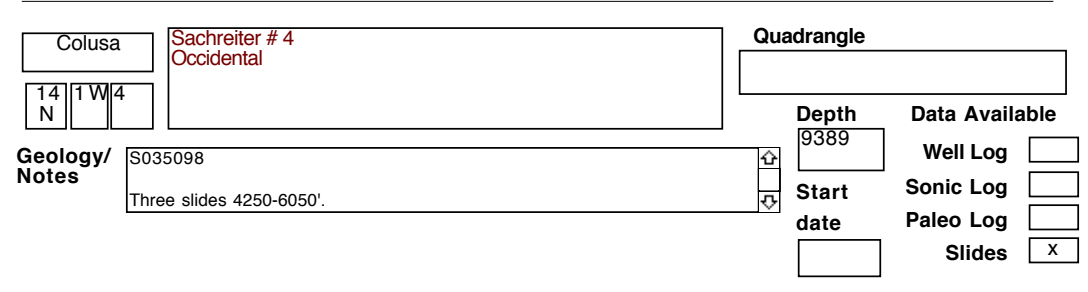

\begin{tabular}{l|l|l|l|l|l|}
\hline \multicolumn{1}{|c|}{ Sutter } & $\begin{array}{l}\text { Shannon \#1 } \\
\text { Monterey }\end{array}$ & \multicolumn{2}{l|}{ Quadrangle } \\
\hline
\end{tabular}


SELECTED OIL TEST WELLS IN CENTRAL CALIFORNIA DATABASE County

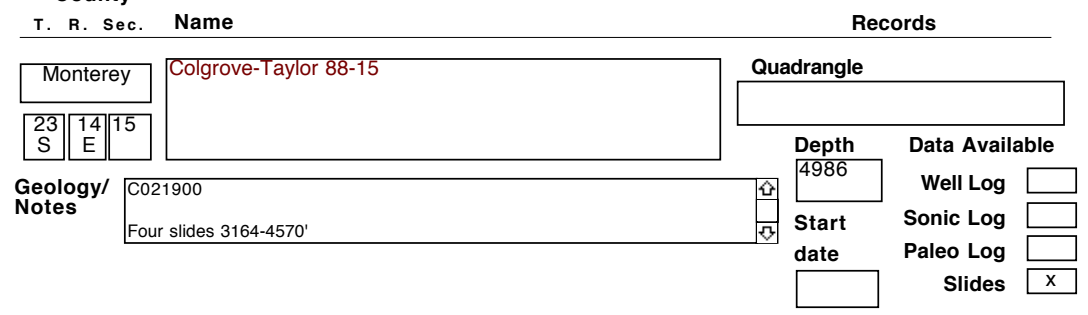

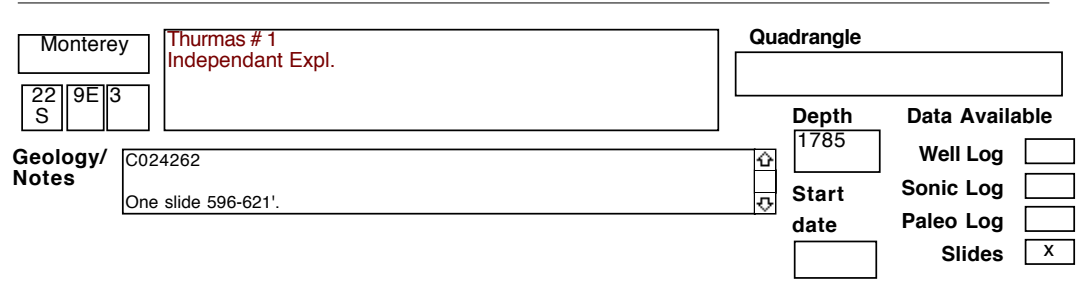

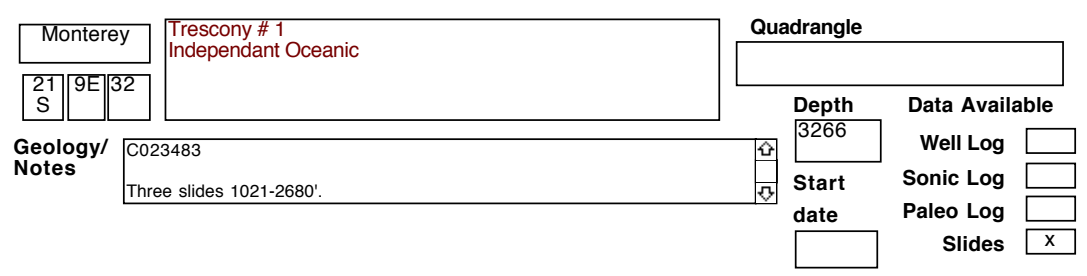

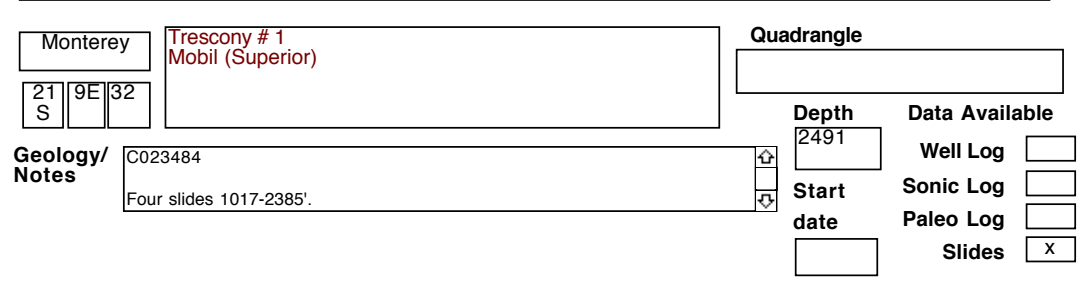

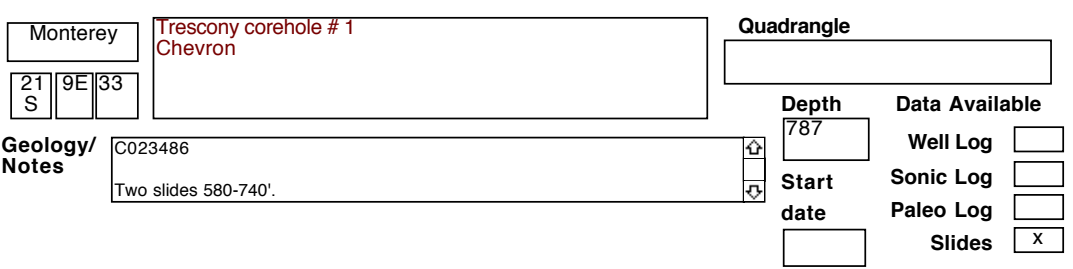


SELECTED OIL TEST WELLS IN CENTRAL CALIFORNIA DATABASE

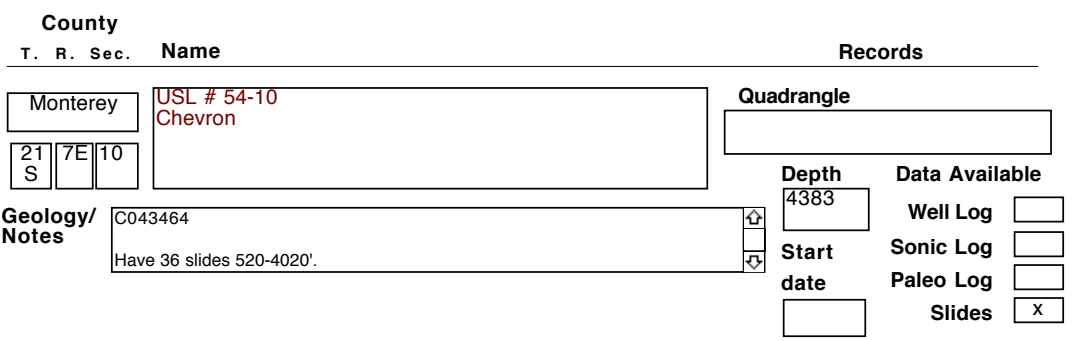

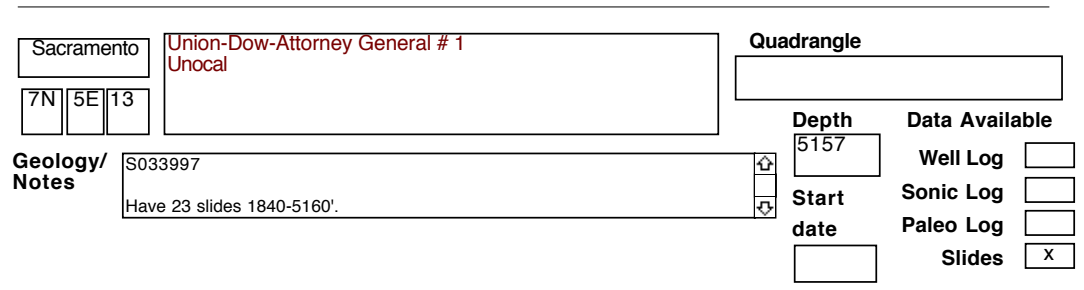

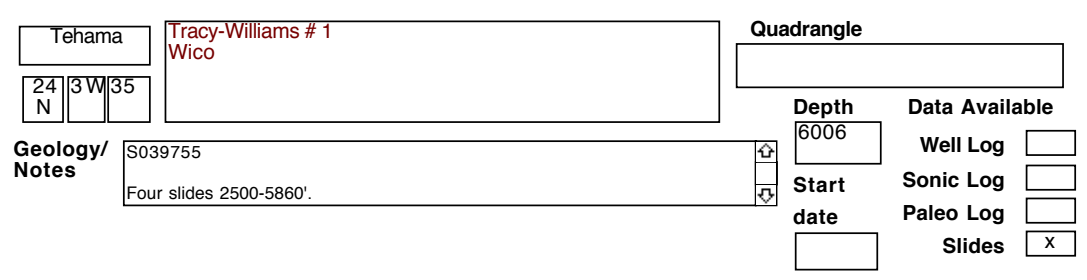

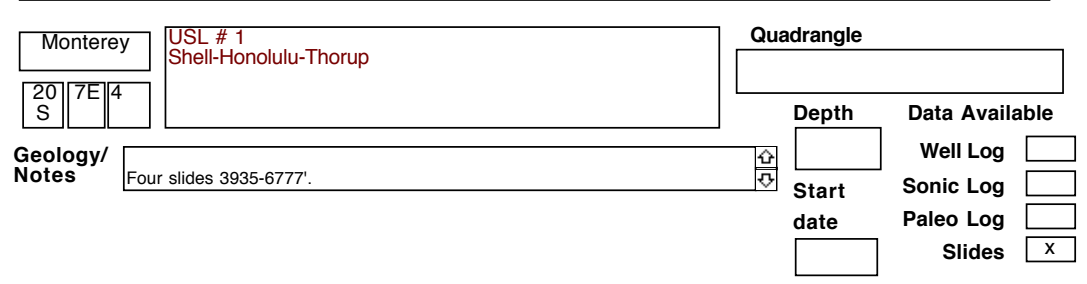

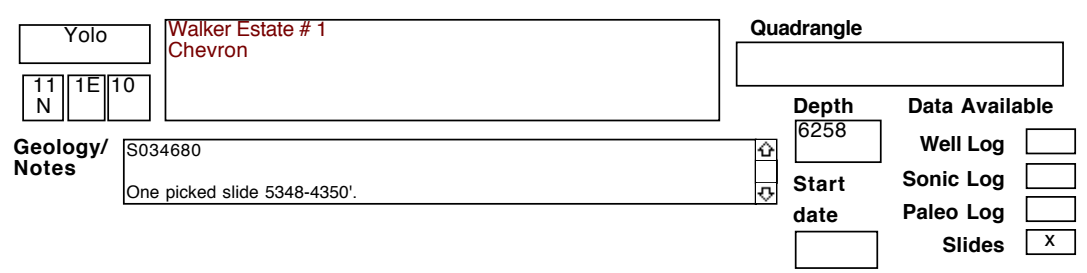


SELECTED OIL TEST WELLS IN CENTRAL CALIFORNIA DATABASE

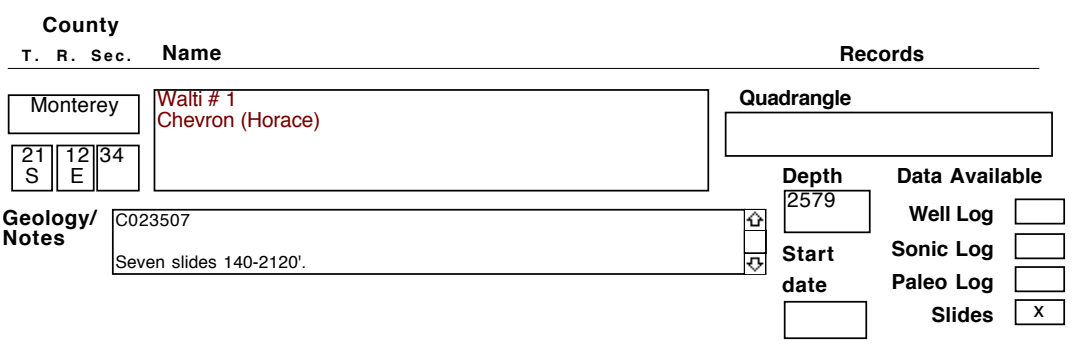

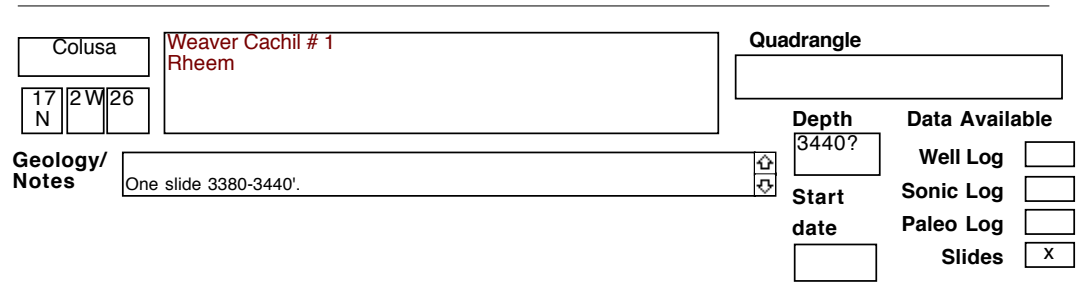

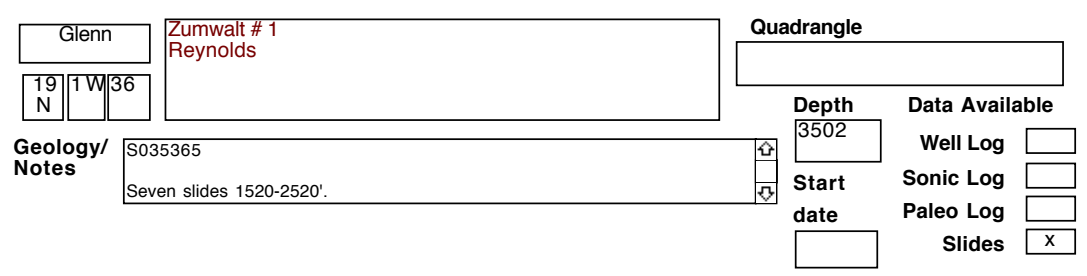

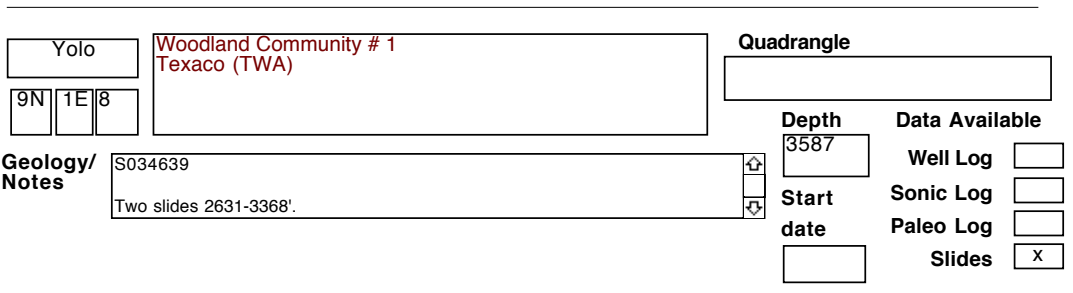

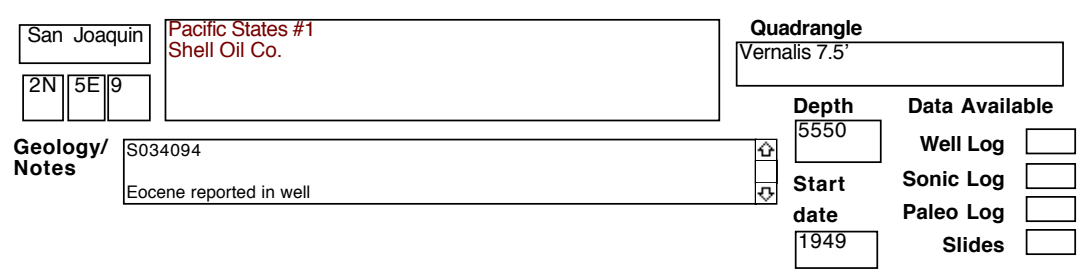


SELECTED OIL TEST WELLS IN CENTRAL CALIFORNIA DATABASE

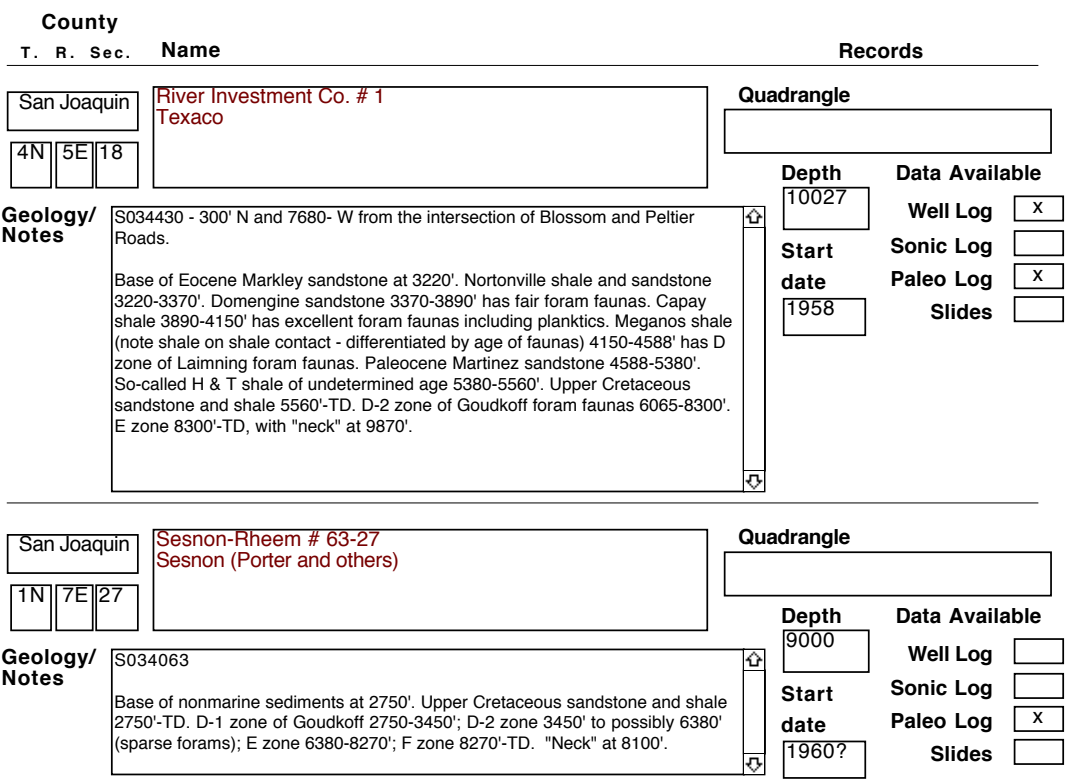

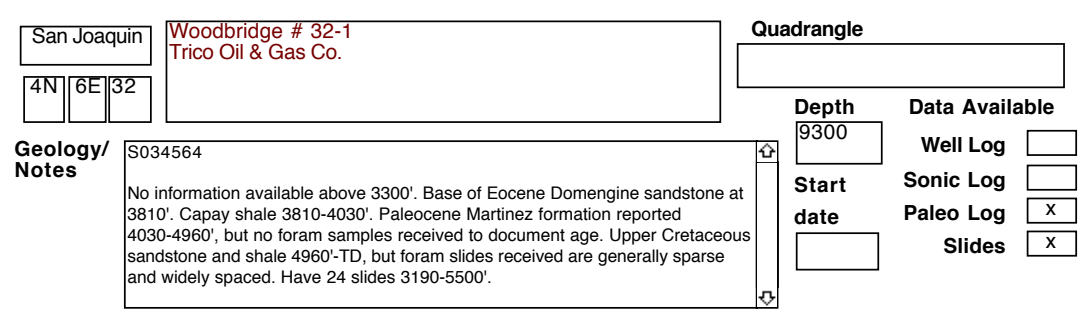

\begin{tabular}{|c|c|c|c|c|c|}
\hline San Joaquin & \multirow{2}{*}{$\begin{array}{l}\text { Young-Wico \# } 1 \\
\text { Young (John M.) }\end{array}$} & \multicolumn{4}{|c|}{ Quadrangle } \\
\hline \begin{tabular}{|l|l|}
$2 S$ & $5 E$
\end{tabular} & & \multirow{2}{*}{\multicolumn{2}{|c|}{$\begin{array}{l}\text { Depth } \\
6860\end{array}$}} & \multicolumn{2}{|c|}{ Data Available } \\
\hline \multirow{2}{*}{$\begin{array}{l}\text { Geology/ } \\
\text { Notes }\end{array}$} & 36519 & & & Well Log & $\mathrm{x}$ \\
\hline & $\begin{array}{l}\text { information above } 600 \text { ' and no paleo information above } 5600^{\prime} \text {. Upper } \\
\text { taceous sandstone and shale } 5600^{\prime}-\text { TD. Probably D-2 zone of Goudkoff } \\
0^{\prime}-\text { TD. }\end{array}$ & & $\begin{array}{l}\text { Start } \\
\text { date }\end{array}$ & $\begin{array}{r}\text { Sonic Log } \\
\text { Paleo Log } \\
\text { Slides }\end{array}$ & $\mathrm{x}$ \\
\hline
\end{tabular}

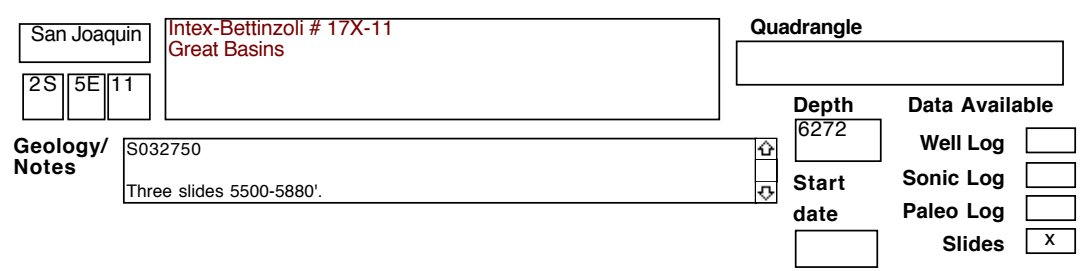


SELECTED OIL TEST WELLS IN CENTRAL CALIFORNIA DATABASE

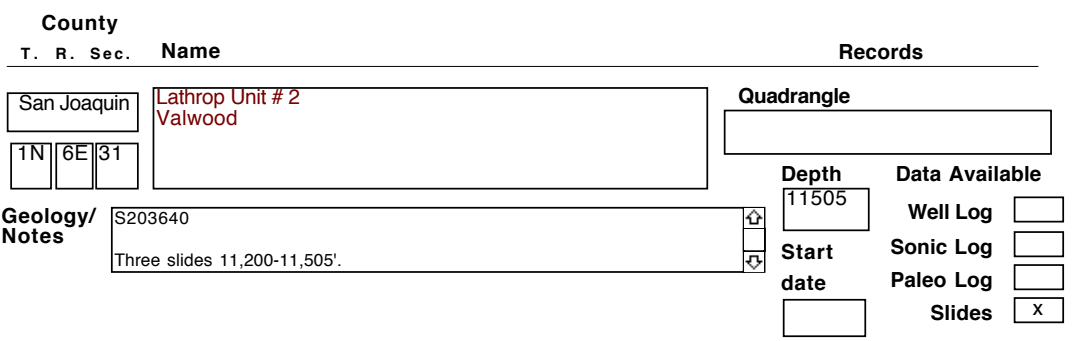

\begin{tabular}{l|l|l|l|l|l|}
\hline San Joaquin & $\begin{array}{l}\text { McMullin \# 1 } \\
\text { Signet }\end{array}$ & \multicolumn{2}{l|}{ Quadrangle } \\
\hline
\end{tabular}

\begin{tabular}{l|l|l|l|l|l|}
\hline San Joaquin & $\begin{array}{l}\text { Paradise Cut Unit A \#1 } \\
\text { Occidental }\end{array}$ & \multicolumn{2}{l|}{ Quadrangle } \\
\hline
\end{tabular}

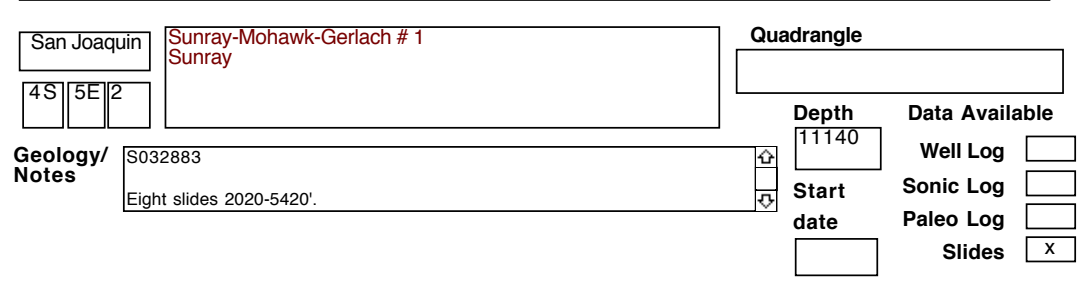

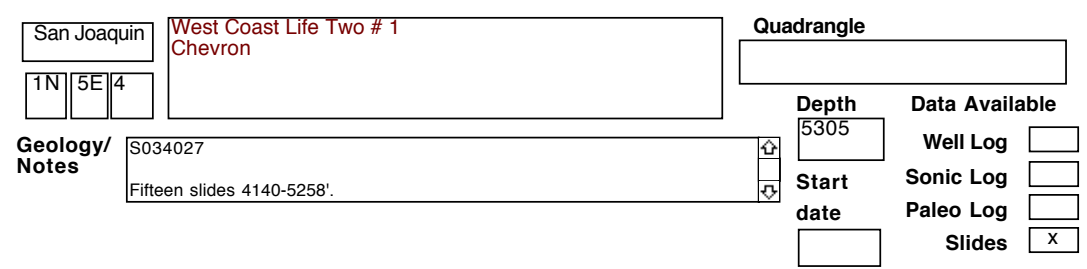


SELECTED OIL TEST WELLS IN CENTRAL CALIFORNIA DATABASE County

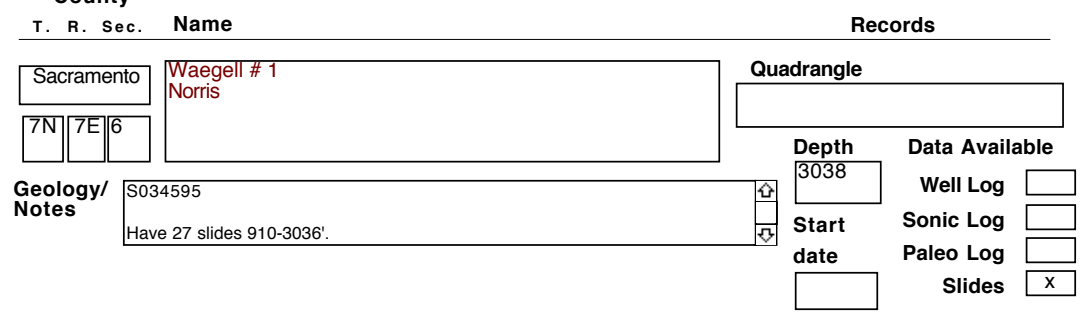

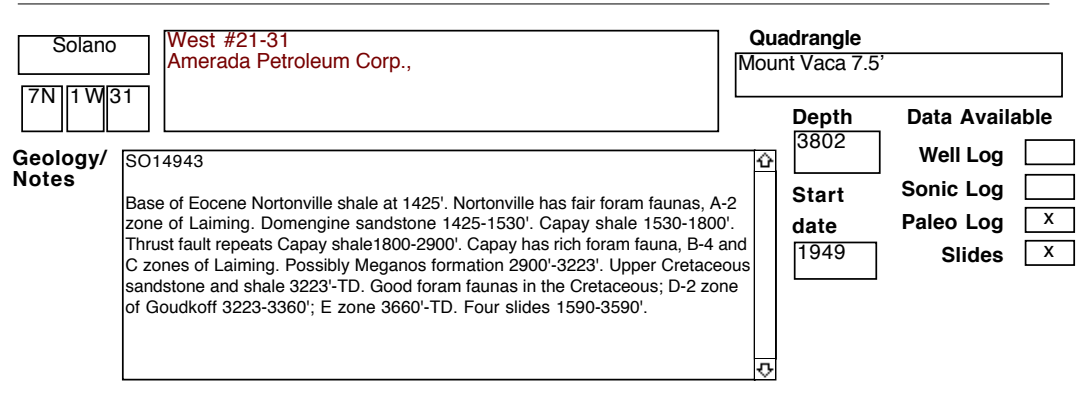

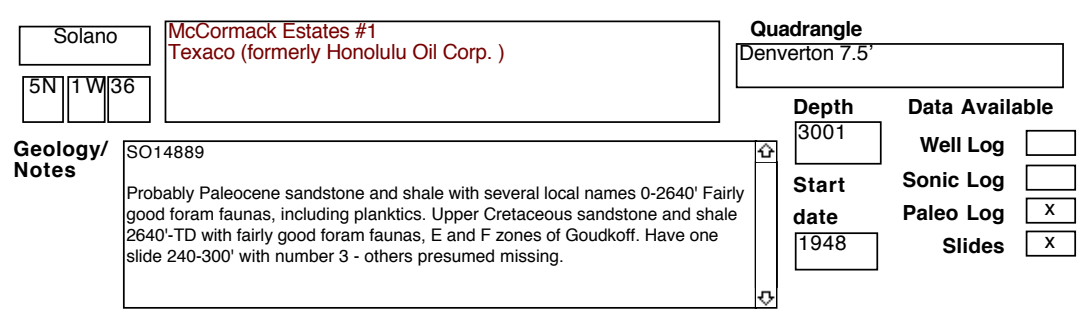

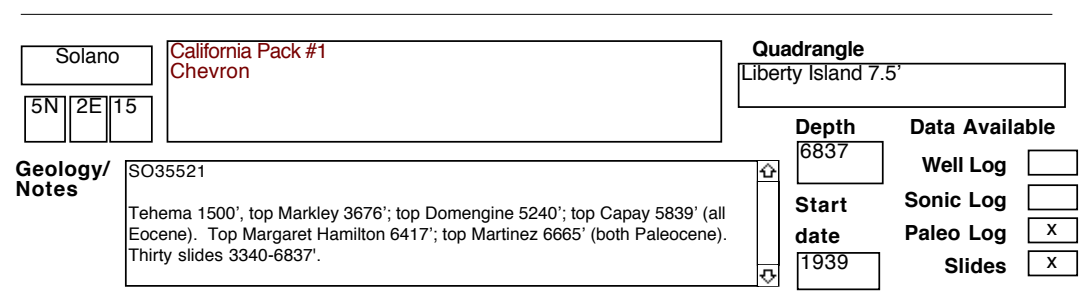

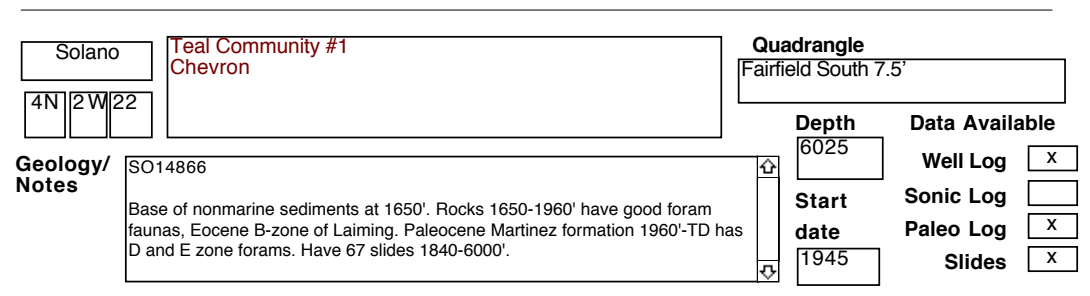


SELECTED OIL TEST WELLS IN CENTRAL CALIFORNIA DATABASE County

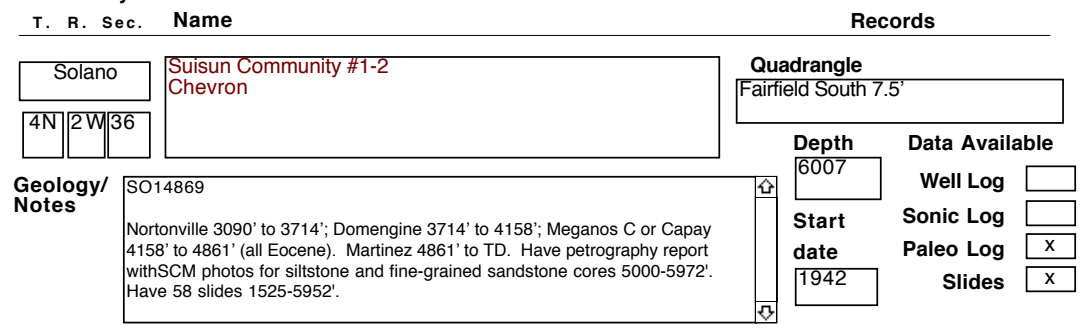

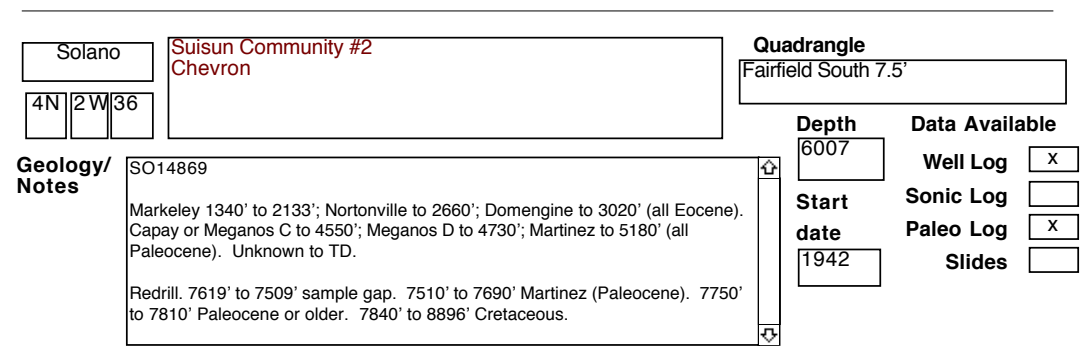

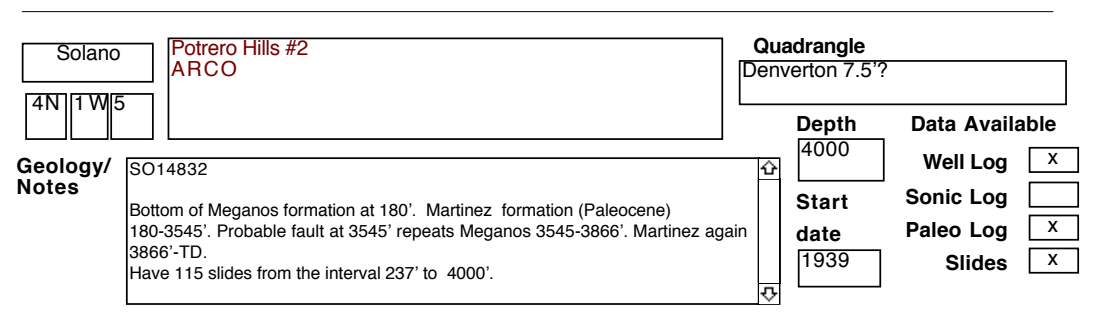

\begin{tabular}{|l|l|l|l|l|l|}
\hline Solano & $\begin{array}{l}\text { Potrero Hills \#1 } \\
\text { ARCO }\end{array}$ \\
\hline
\end{tabular}

\begin{tabular}{|c|c|c|c|c|}
\hline Solano & $\begin{array}{l}\text { Potrero Hills \#3 } \\
\text { ARCO }\end{array}$ & $\begin{array}{r}\text { Quadrangle } \\
\text { Denverton } 7.5\end{array}$ & & \\
\hline \begin{tabular}{ll|l}
$4 \mathrm{~N}$ & $1 \mathrm{~W}$ & 10
\end{tabular} & & \multirow{2}{*}{$\begin{array}{l}\text { Depth } \\
3800\end{array}$} & \multicolumn{2}{|c|}{ Data Available } \\
\hline \multirow{3}{*}{\multicolumn{2}{|c|}{$\begin{array}{l}\text { Geology/ Have } 50 \text { slides } 669-3800^{\prime} \text { and no other information. } \\
\text { Notes }\end{array}$}} & & Well Log & \\
\hline & & Start & Sonic Log & \\
\hline & & $\begin{array}{l}\text { date } \\
1940\end{array}$ & $\begin{array}{r}\text { Paleo Log } \\
\text { Slides }\end{array}$ & $\mathrm{x}$ \\
\hline
\end{tabular}


SELECTED OIL TEST WELLS IN CENTRAL CALIFORNIA DATABASE County

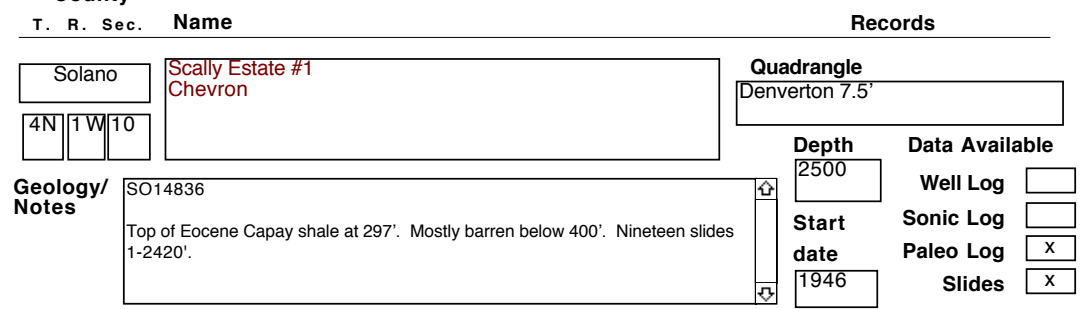

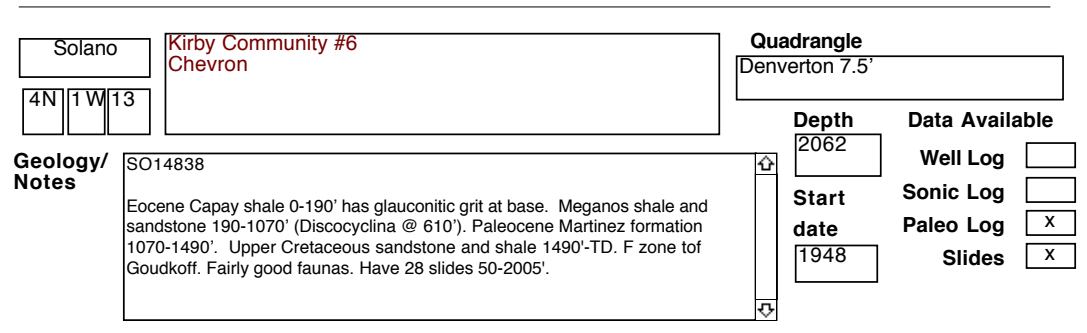

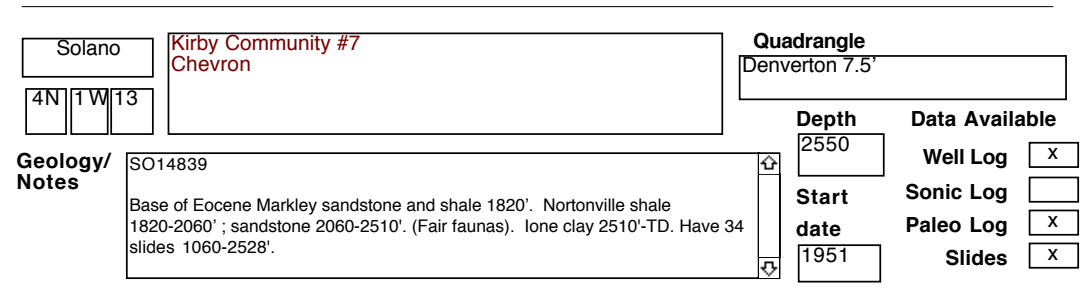

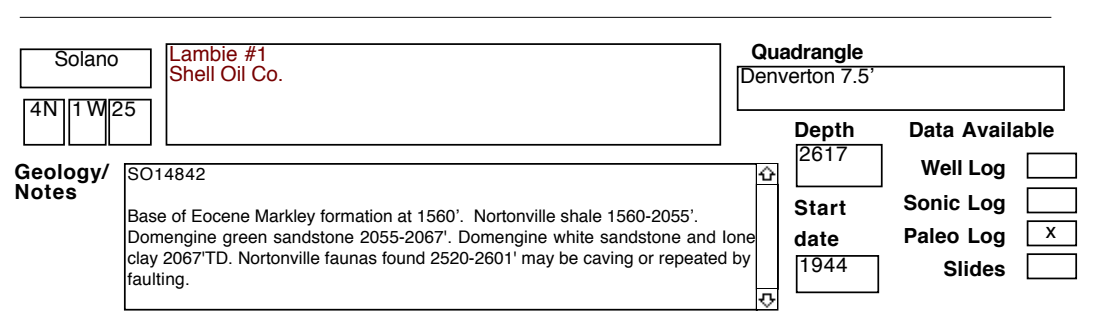

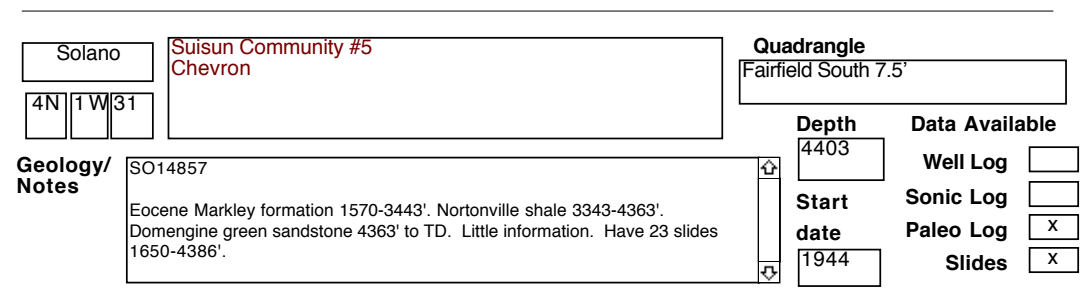


SELECTED OIL TEST WELLS IN CENTRAL CALIFORNIA DATABASE County

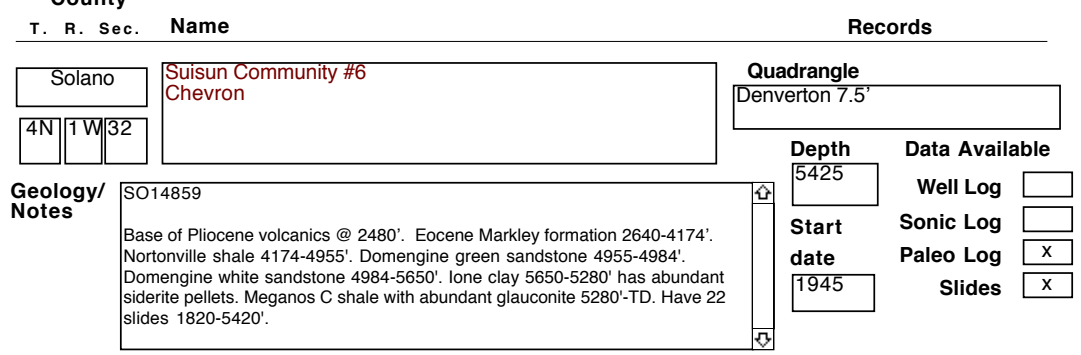

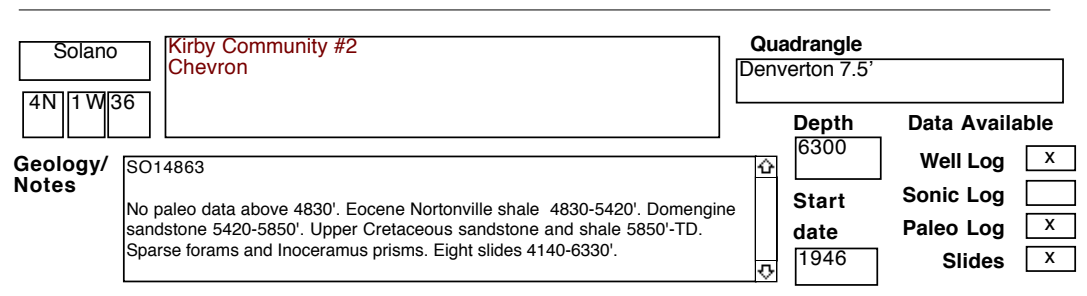

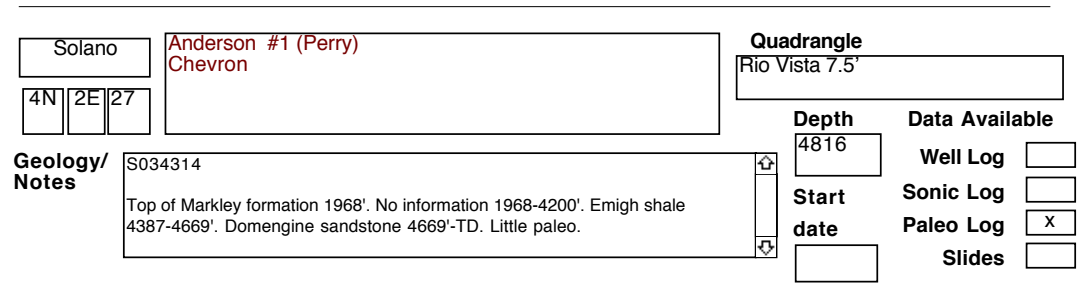

\begin{tabular}{l|l|l|l|l|}
\hline \multicolumn{1}{|c|}{ Solano } & $\begin{array}{l}\text { Donohue et al \#1 } \\
\text { Texaco (formerly Getty and Tide Water) }\end{array}$ \\
\hline
\end{tabular}

\begin{tabular}{|c|c|c|c|c|c|}
\hline Solano & \multirow[t]{2}{*}{$\begin{array}{l}\text { Griffin \#1 } \\
\text { Cameron, (George T.) }\end{array}$} & \multicolumn{4}{|c|}{$\begin{array}{l}\text { Quadrangle } \\
\text { Vine Hill 7.5' }\end{array}$} \\
\hline \multicolumn{2}{|c|}{$3 \mathrm{~N}|2 \mathrm{~V}| 16$} & & Depth & Data Availab & \\
\hline \multirow{3}{*}{$\begin{array}{l}\text { Geology/ } \\
\text { Notes }\end{array}$} & \multirow{3}{*}{$\begin{array}{l}\text { Volcanics 200-1060'. Neroly brackish water silts } 1060^{\prime}-2390^{\prime} \text {. Upper Cretaceous } \\
\text { sandstone and shale } 2390^{\prime}-\text { TD. }\end{array}$} & 0 & & Well Log & \\
\hline & & & Start & Sonic Log & \\
\hline & & & $\begin{array}{l}\text { date } \\
1950\end{array}$ & $\begin{array}{r}\text { Paleo Log } \\
\text { Slides }\end{array}$ & $\mathrm{x}$ \\
\hline
\end{tabular}




\section{SELECTED OIL TEST WELLS IN CENTRAL CALIFORNIA DATABASE}

County

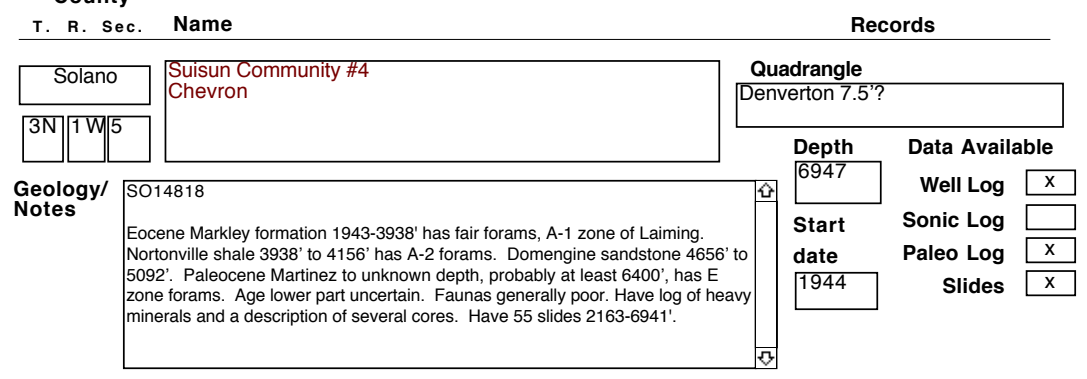

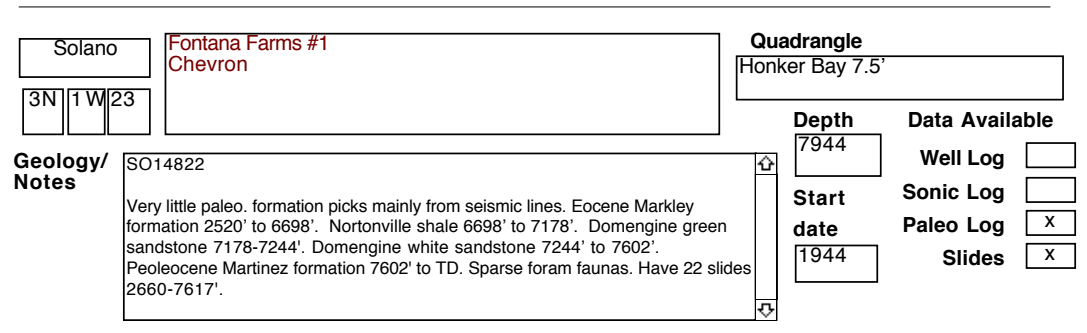

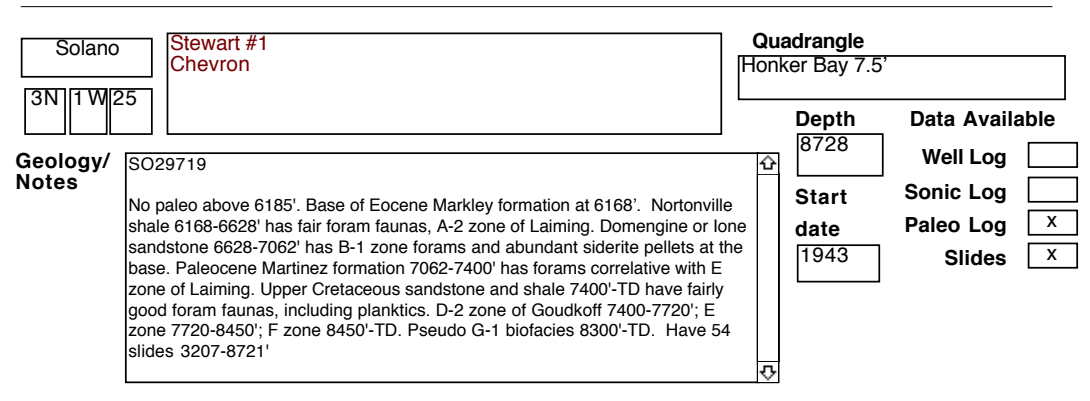

\begin{tabular}{l|l|l|l|l|l|}
\hline Solano & $\begin{array}{l}\text { Honker Community \#1 } \\
\text { Chevron }\end{array}$ & \multicolumn{2}{|c|}{ Quadrangle } \\
\hline
\end{tabular}

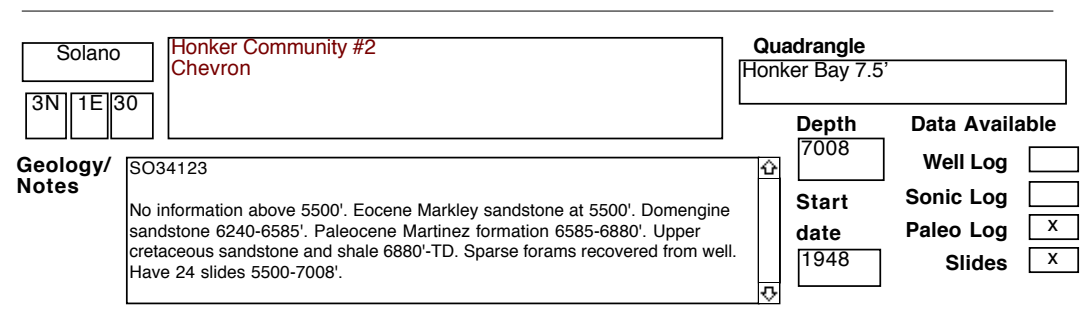


SELECTED OIL TEST WELLS IN CENTRAL CALIFORNIA DATABASE County

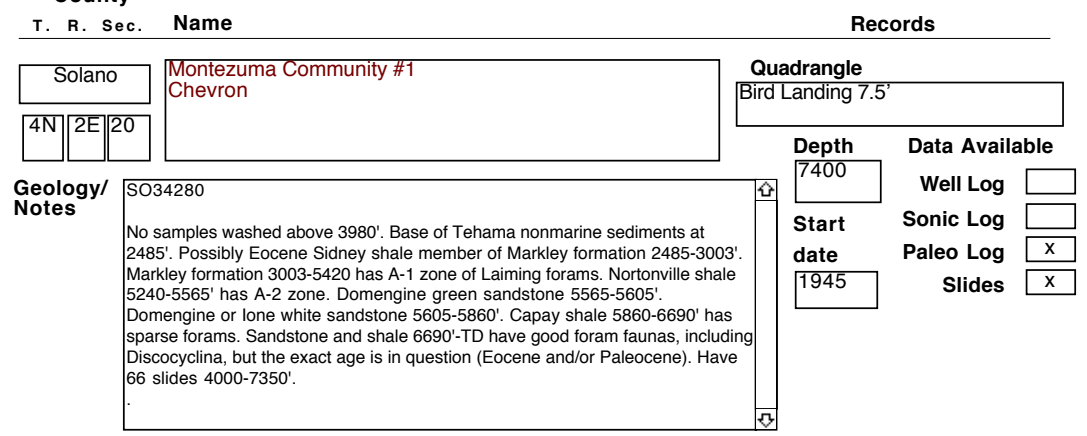

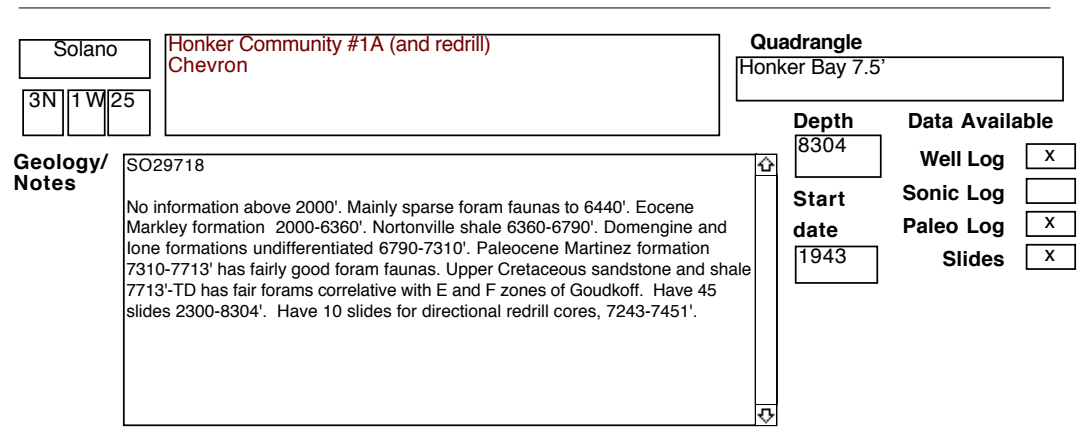

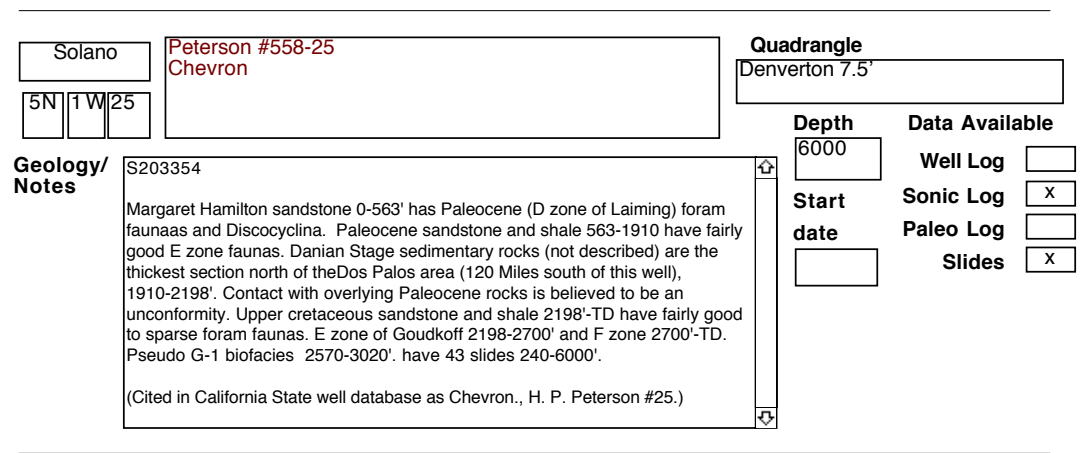

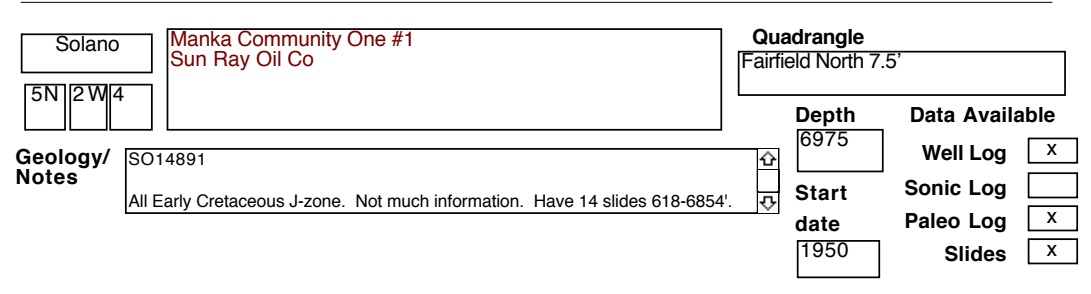

\begin{tabular}{|l|l|l|l|l|l|}
\hline Solano & $\begin{array}{l}\text { Peterson \#556-25 } \\
\text { Chevron }\end{array}$ (note other well with same location and name but different \\
depth. Redrill?)
\end{tabular}


SELECTED OIL TEST WELLS IN CENTRAL CALIFORNIA DATABASE County

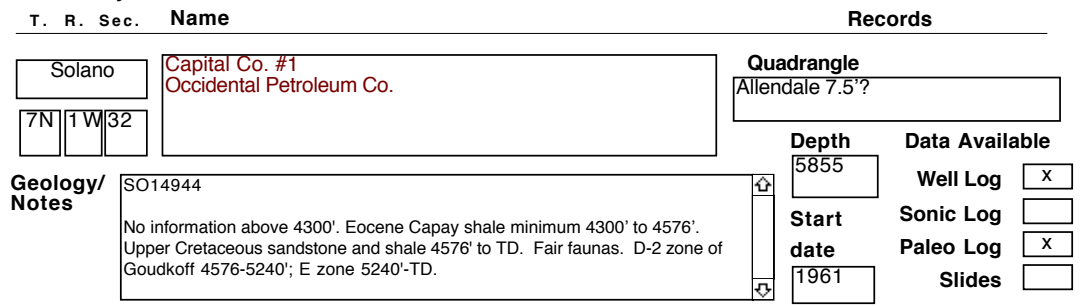

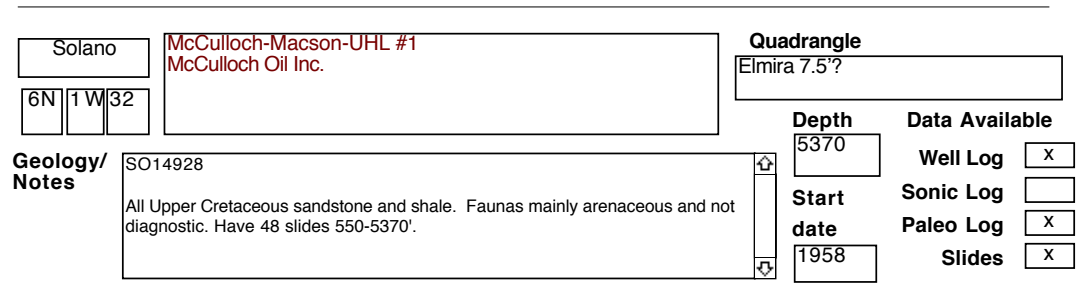

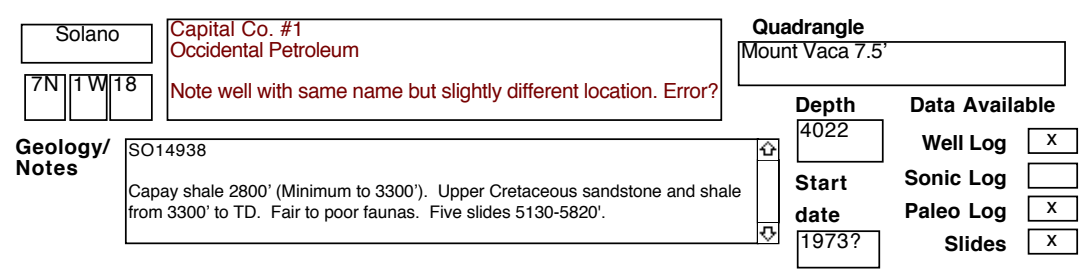

\begin{tabular}{|c|c|c|c|c|c|}
\hline Solano & \multirow[t]{2}{*}{$\begin{array}{l}\text { McCulloch - Macson Scally Unit \#1 } \\
\text { McCulloch Oil Exploration Co., }\end{array}$} & \multicolumn{4}{|c|}{$\begin{array}{l}\text { Quadrangle } \\
\text { Denverson 7.5' }\end{array}$} \\
\hline \begin{tabular}{ll|l|l|l|}
$4 \mathrm{~N}$ & 10
\end{tabular} & & \multirow{2}{*}{\multicolumn{2}{|c|}{$\begin{array}{l}\text { Depth } \\
9011\end{array}$}} & \multicolumn{2}{|c|}{ Data Available } \\
\hline \multirow{3}{*}{$\begin{array}{l}\text { Geology/ } \\
\text { Notes }\end{array}$} & 29725 & & & Well Log & $\mathrm{x}$ \\
\hline & oocene Meganos sandstone and shale $0-1320$ & B & Start & Sonic Log & \\
\hline & & & $\begin{array}{l}\text { date } \\
1959\end{array}$ & Slides & \\
\hline
\end{tabular}

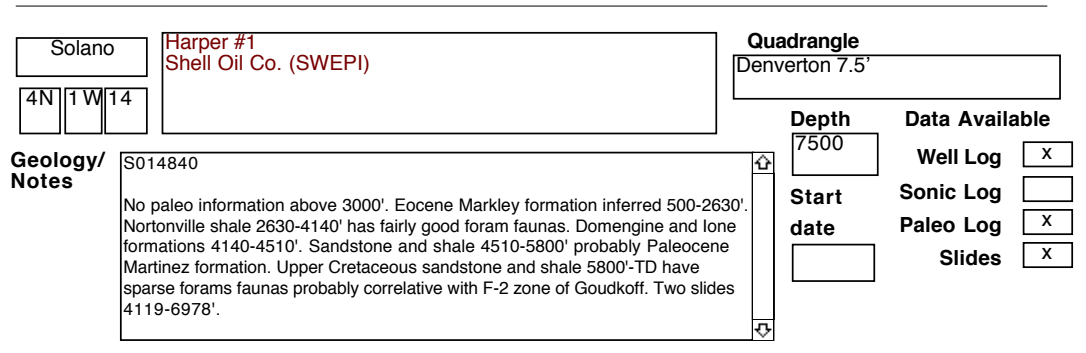


SELECTED OIL TEST WELLS IN CENTRAL CALIFORNIA DATABASE County

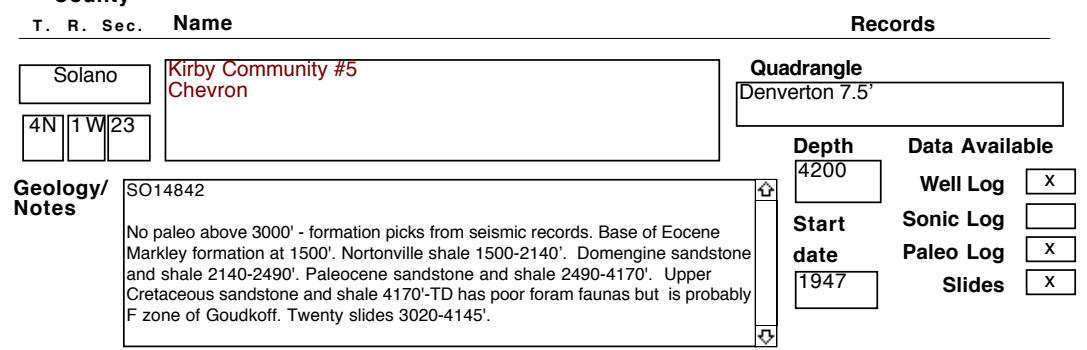

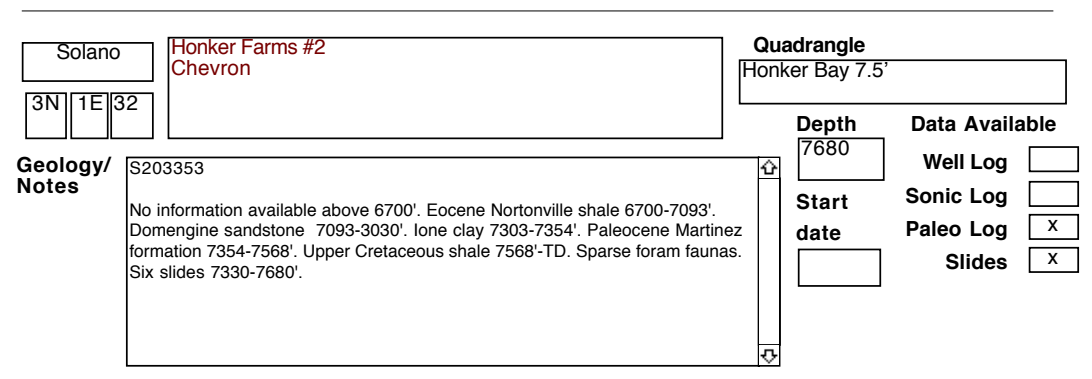

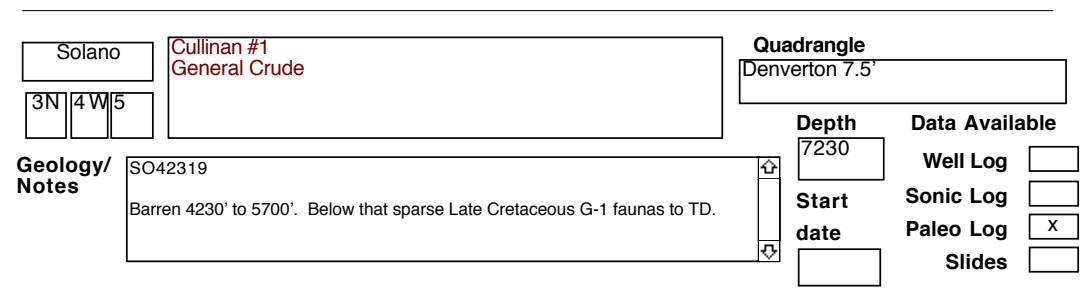

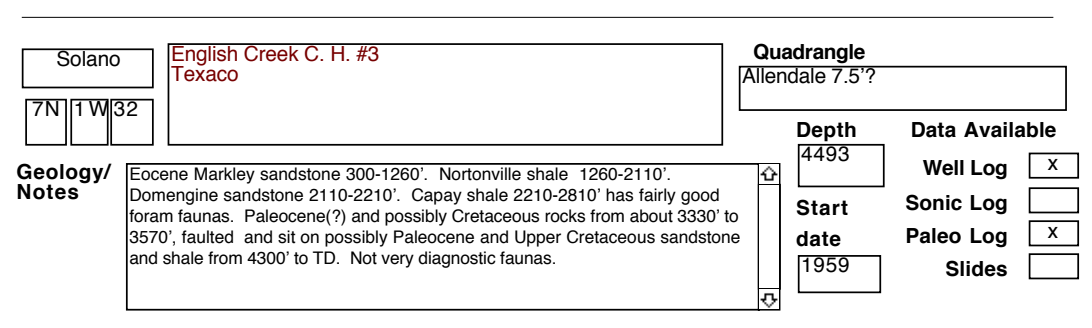

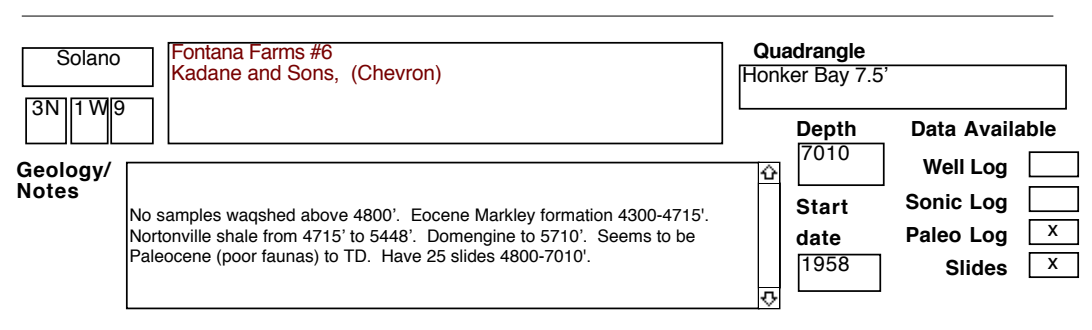




\section{SELECTED OIL TEST WELLS IN CENTRAL CALIFORNIA DATABASE}

County

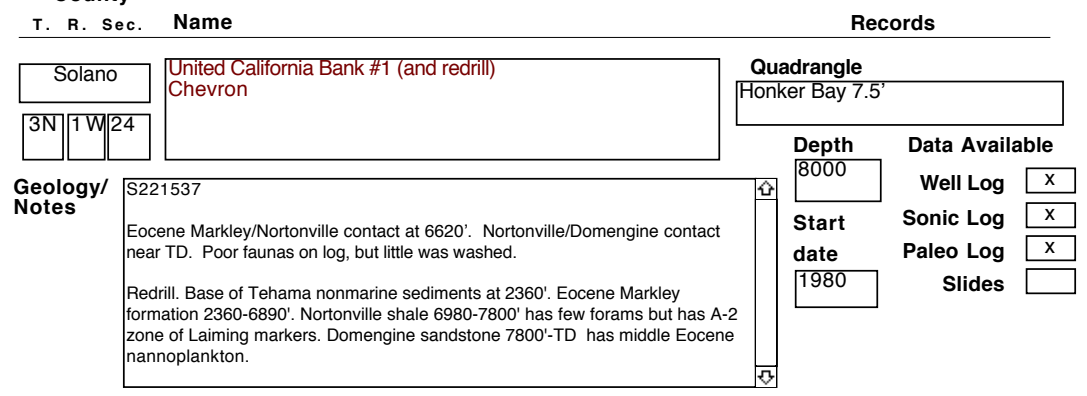

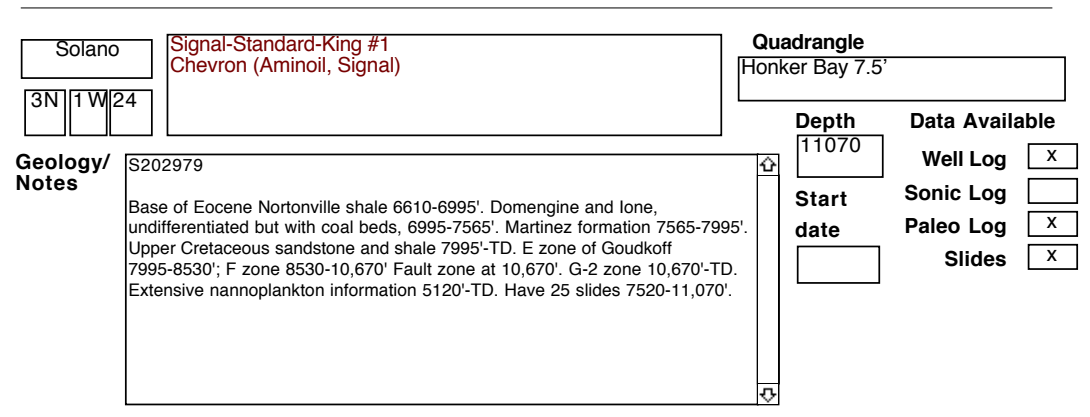

\begin{tabular}{l|l|l|l|l|l|l|l|}
\hline Solano & $\begin{array}{l}\text { Suisun Community \#15 } \\
\text { Chevron }\end{array}$ \\
\hline
\end{tabular}

\begin{tabular}{|l|l|l|l|l|l|l|}
\hline Solano & $\begin{array}{l}\text { Fontana Farms \#7 } \\
\text { Chevron }\end{array}$ \\
\hline
\end{tabular}

\begin{tabular}{|c|c|c|c|c|}
\hline Solano & \multirow[t]{2}{*}{$\begin{array}{l}\text { Tolenas Farms \#1 } \\
\text { Chevron (Gulf) Oil Corp. }\end{array}$} & \multicolumn{3}{|c|}{$\begin{array}{l}\text { Quadrangle } \\
\text { Elmira 7.5'? }\end{array}$} \\
\hline $5 \mathrm{~N} / \mathrm{W} 20$ & & Depth & \multicolumn{2}{|c|}{ Data Available } \\
\hline \multirow{3}{*}{$\begin{array}{l}\text { Geology/ } \\
\text { Notes }\end{array}$} & 14886 & & Well Log & $\mathrm{x}$ \\
\hline & leocene Martinez formation 0-1190' has fair to good foram faunas, E zone of & Start & Sonic Log & \\
\hline & $\begin{array}{l}\text { arse, arenaceous forams. Probably F-1 zone of Goudkoff 1320-2060'; F-2 zone } \\
\text { 40-500'; G-1 5000'-TD. }\end{array}$ & $\begin{array}{l}\text { date } \\
1961\end{array}$ & Slides & \\
\hline
\end{tabular}


SELECTED OIL TEST WELLS IN CENTRAL CALIFORNIA DATABASE County

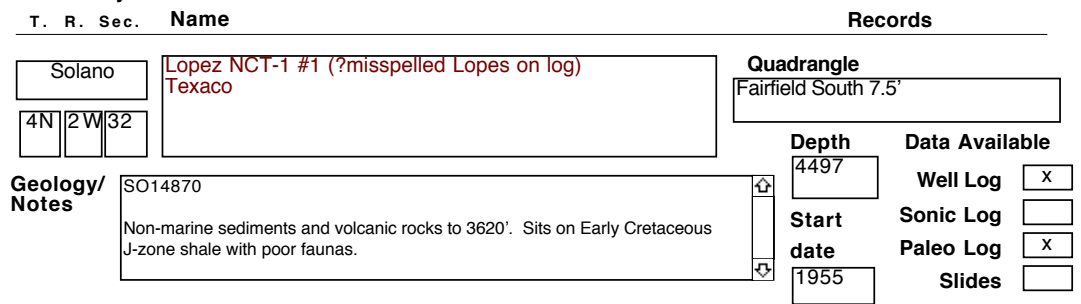

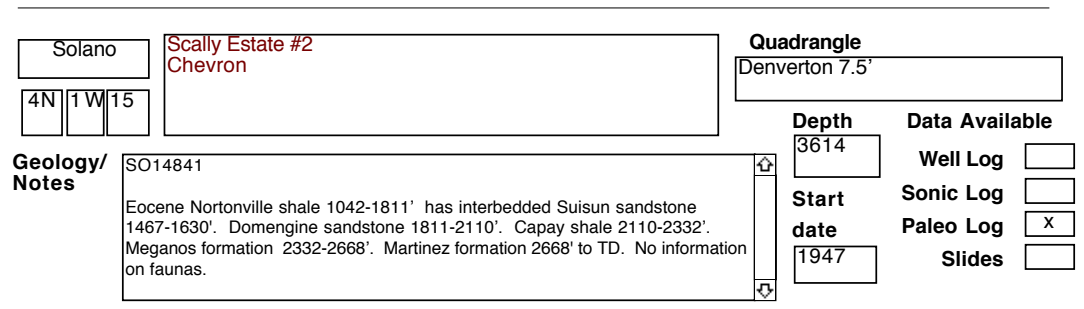

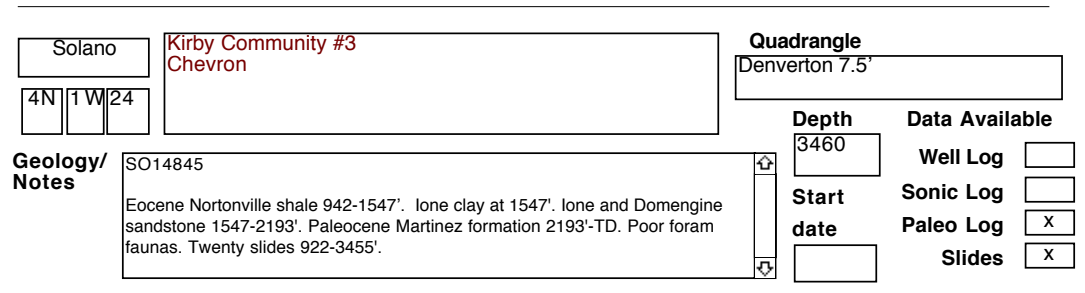

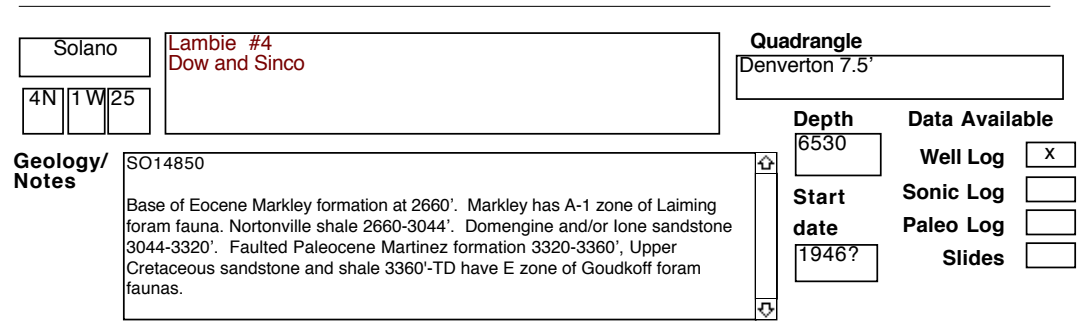

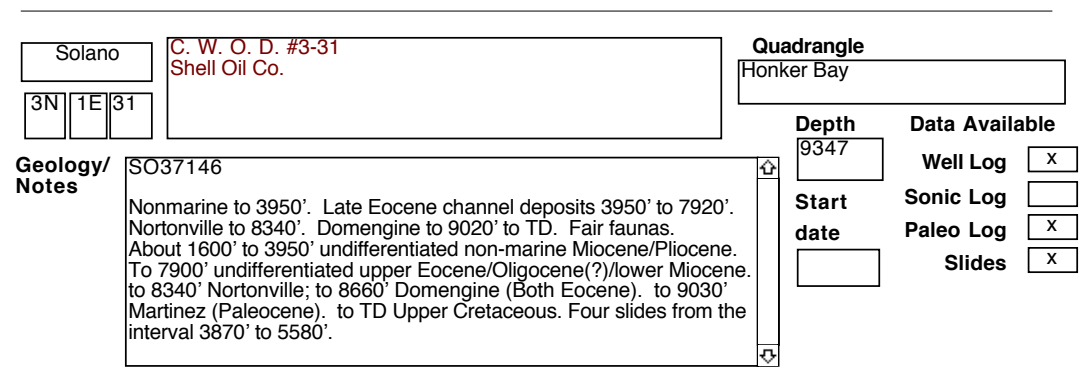


SELECTED OIL TEST WELLS IN CENTRAL CALIFORNIA DATABASE County

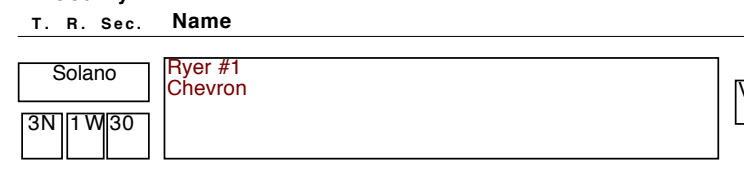

\section{Geology/ $\$ 033640$}

Notes

Base of nonmarine sediments at 1940 '. Sediments have some reworked forams. Sandstone derived from volcanic rocks 1940-2200'. Age and name not known.

Eocene Markley formation 2200-3929' has very sparse foram faunas.

Nortonville shale 3929-4530' has fair foram faunas, including planktics.

Domengine sandstone $4530-5140$ ' may have lone clay 5030-5070'. Paleocene Martinez formation (log indicates sandstone and shale) 5140'-TD has mainly arenaceous forams. Have 33 slides 1390-8940.

Records

\begin{tabular}{|c|c|c|c|}
\hline \multicolumn{4}{|c|}{$\begin{array}{c}\text { Quadrangle } \\
\text { Vine Hill 7.5'? }\end{array}$} \\
\hline & Depth & \multicolumn{2}{|c|}{ Data Available } \\
\hline 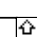 & 8941 & Well Log & $\mathrm{x}$ \\
\hline & Start & Sonic Log & \\
\hline & date & Paleo Log & $\mathrm{x}$ \\
\hline & $1968 ?$ & Slides & $x$ \\
\hline
\end{tabular}

\begin{tabular}{|c|l|l|}
\hline Solano & $\begin{array}{l}\text { Van Sickle Gas Unit \#3 } \\
\text { Chevron }\end{array}$ \\
\hline $3 \mathrm{~N}$ & $1 \mathrm{E}$ & 32 \\
\hline
\end{tabular}

Geology/ S200950

Notes

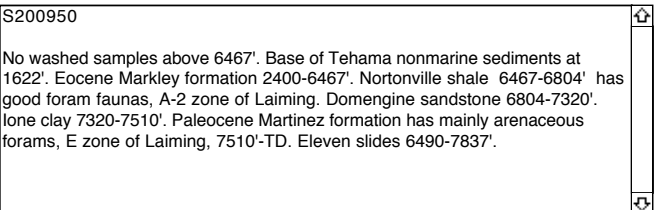

\begin{tabular}{|c|c|c|c|}
\hline \multicolumn{4}{|c|}{$\begin{array}{c}\text { Quadrangle } \\
\text { Honker Bay 7.5 }\end{array}$} \\
\hline & \multirow{2}{*}{$\begin{array}{l}\text { Depth } \\
7837\end{array}$} & \multicolumn{2}{|c|}{ Data Available } \\
\hline 0 & & Well Log & \\
\hline & Start & Sonic Log & \\
\hline & date & Paleo Log & $\mathrm{x}$ \\
\hline & & Slides & $\mathrm{x}$ \\
\hline
\end{tabular}

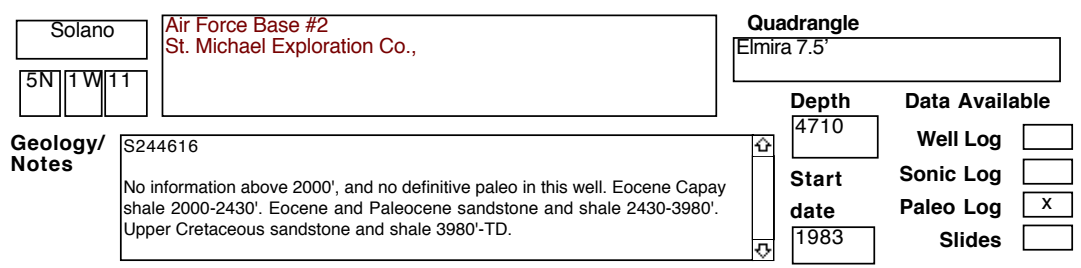

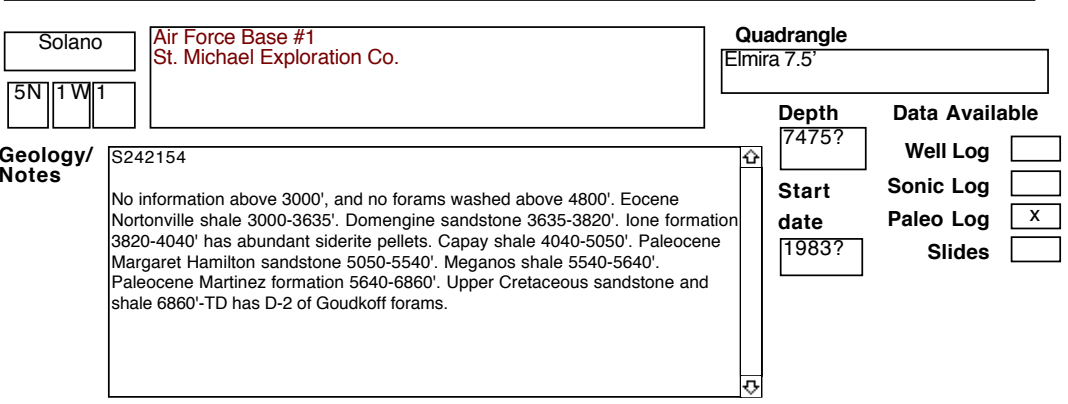

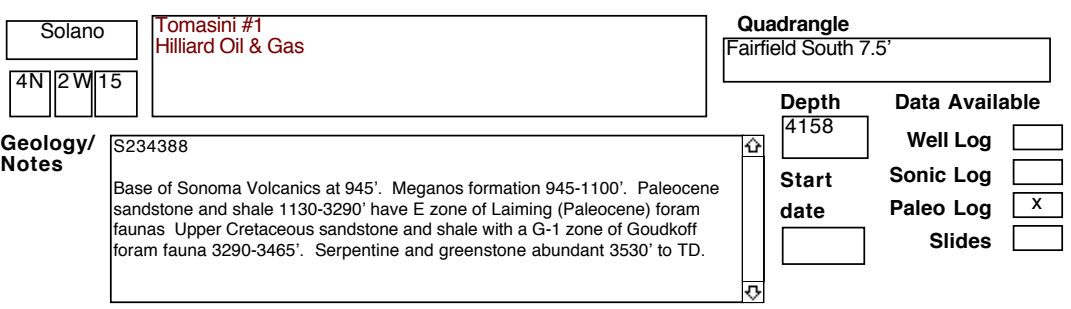




\section{SELECTED OIL TEST WELLS IN CENTRAL CALIFORNIA DATABASE}

County

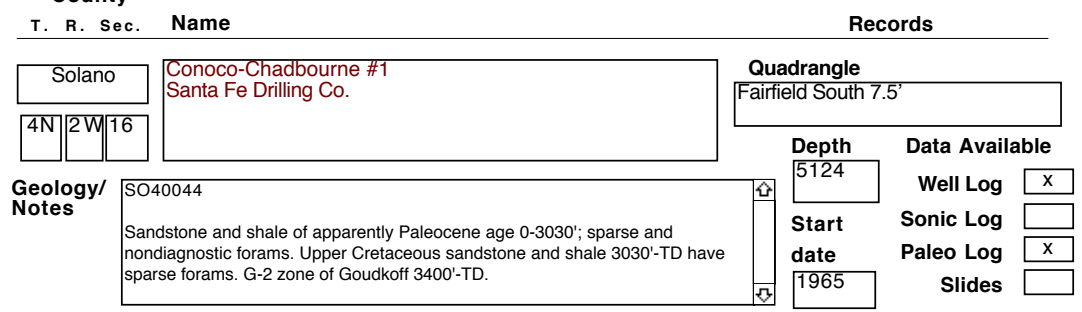

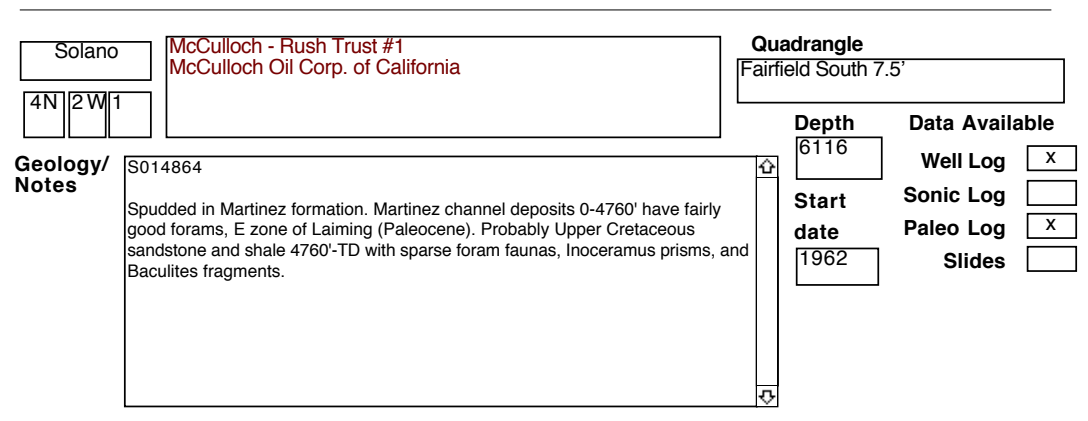

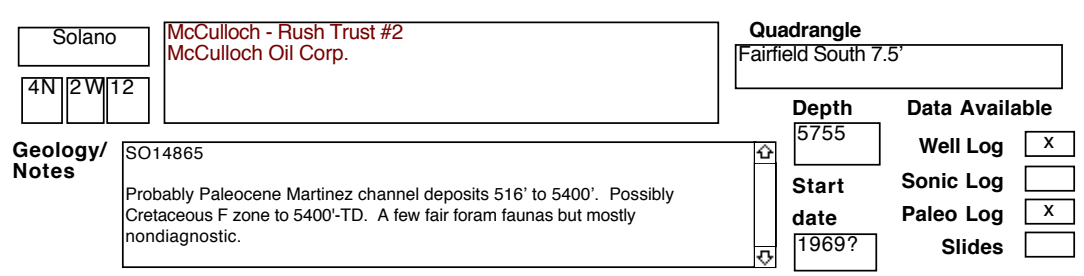

\begin{tabular}{l|l|l|l|l|l|l|}
\hline Solano & $\begin{array}{l}\text { Suisun Community \#13 } \\
\text { Chevron }\end{array}$ \\
\hline
\end{tabular}

\begin{tabular}{|c|c|c|c|c|c|}
\hline Solano & \multirow[t]{2}{*}{ ] $\begin{array}{l}\text { Fontana Farms \#10 } \\
\text { Chevron }\end{array}$} & \multicolumn{4}{|c|}{$\begin{array}{c}\text { Quadrangle } \\
\text { Denverton 7.5'? }\end{array}$} \\
\hline \multicolumn{2}{|c|}{\begin{tabular}{|l|l|l|}
$4 \mathrm{~N}$ & $1 \mathrm{~W}$ & 32 \\
\end{tabular}} & & Depth & Data Availab & \\
\hline \multirow{3}{*}{$\begin{array}{l}\text { Geology/ } \\
\text { Notes }\end{array}$} & S222187 & 0 & & Well Log & \\
\hline & Eocene Domengine sandstone $4920-5225$ '. Ione clay 5225-5296'. Paleocene & & Start & Sonic Log & \\
\hline & 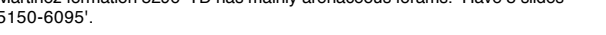 & & & Slides & $\overline{\bar{x}}$ \\
\hline
\end{tabular}


SELECTED OIL TEST WELLS IN CENTRAL CALIFORNIA DATABASE County

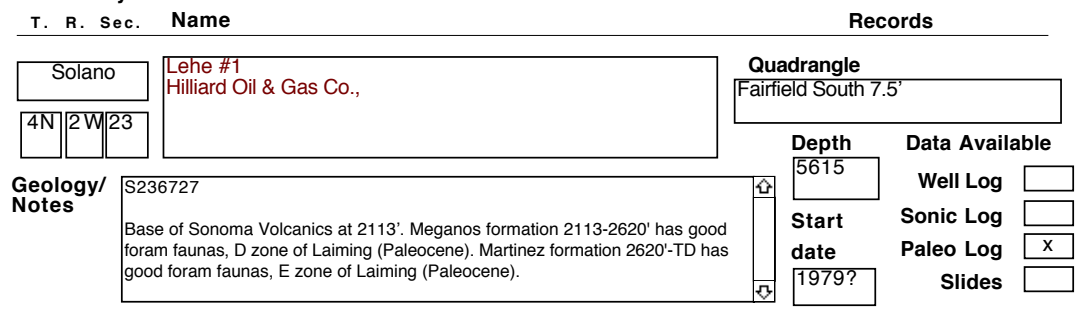

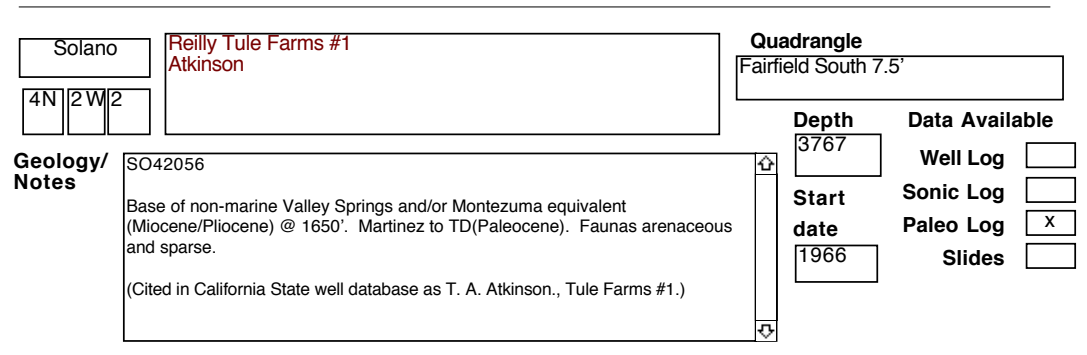

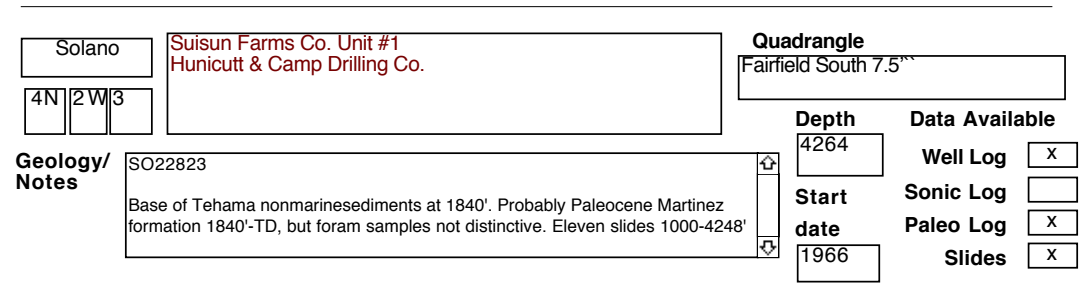

\begin{tabular}{|l|l|l|l|l|l|}
\hline Solano & $\begin{array}{l}\text { Suisun Community \#8 } \\
\text { Chevron }\end{array}$ \\
\hline
\end{tabular}

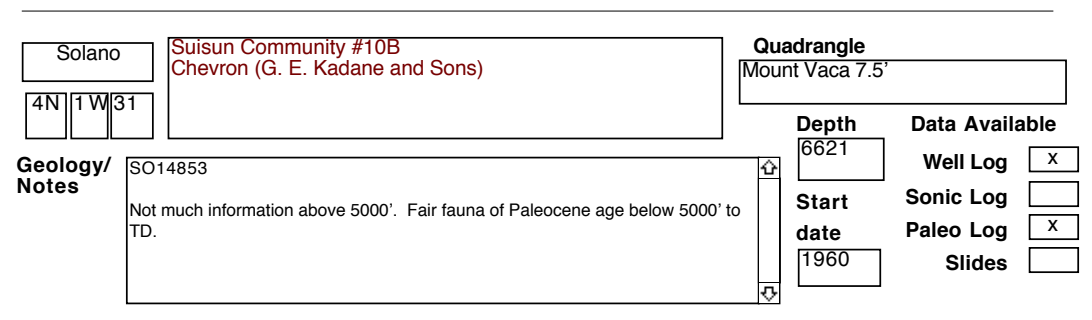


SELECTED OIL TEST WELLS IN CENTRAL CALIFORNIA DATABASE County

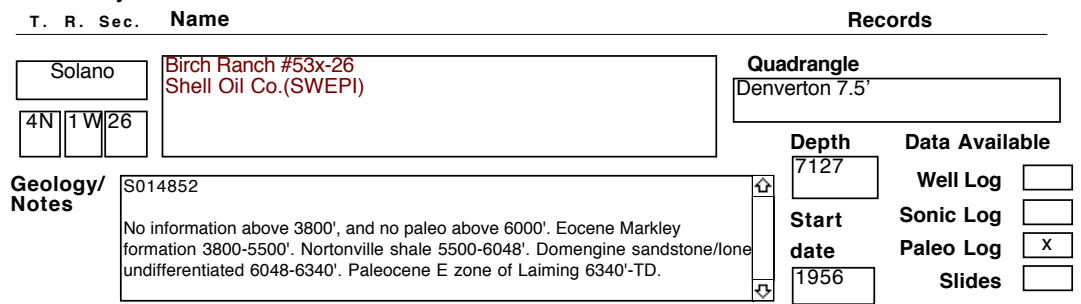

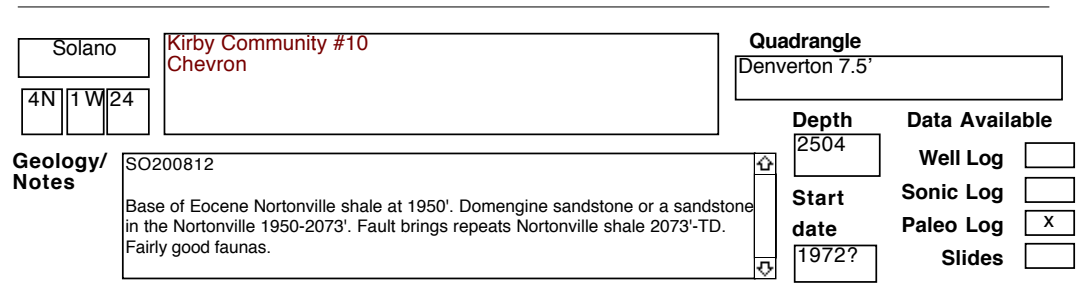

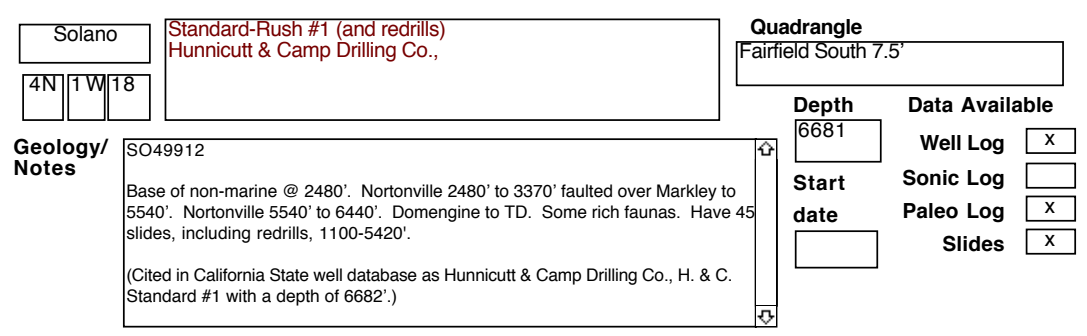

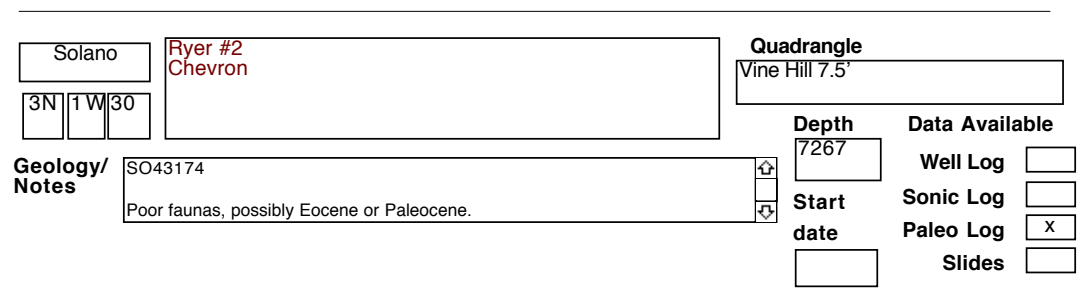

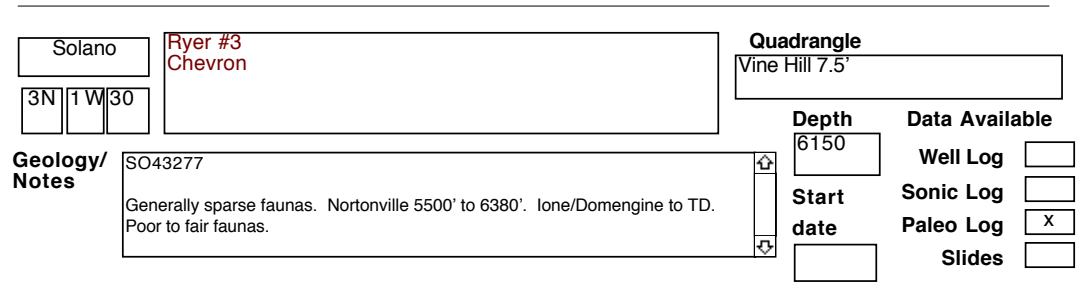




\section{SELECTED OIL TEST WELLS IN CENTRAL CALIFORNIA DATABASE}

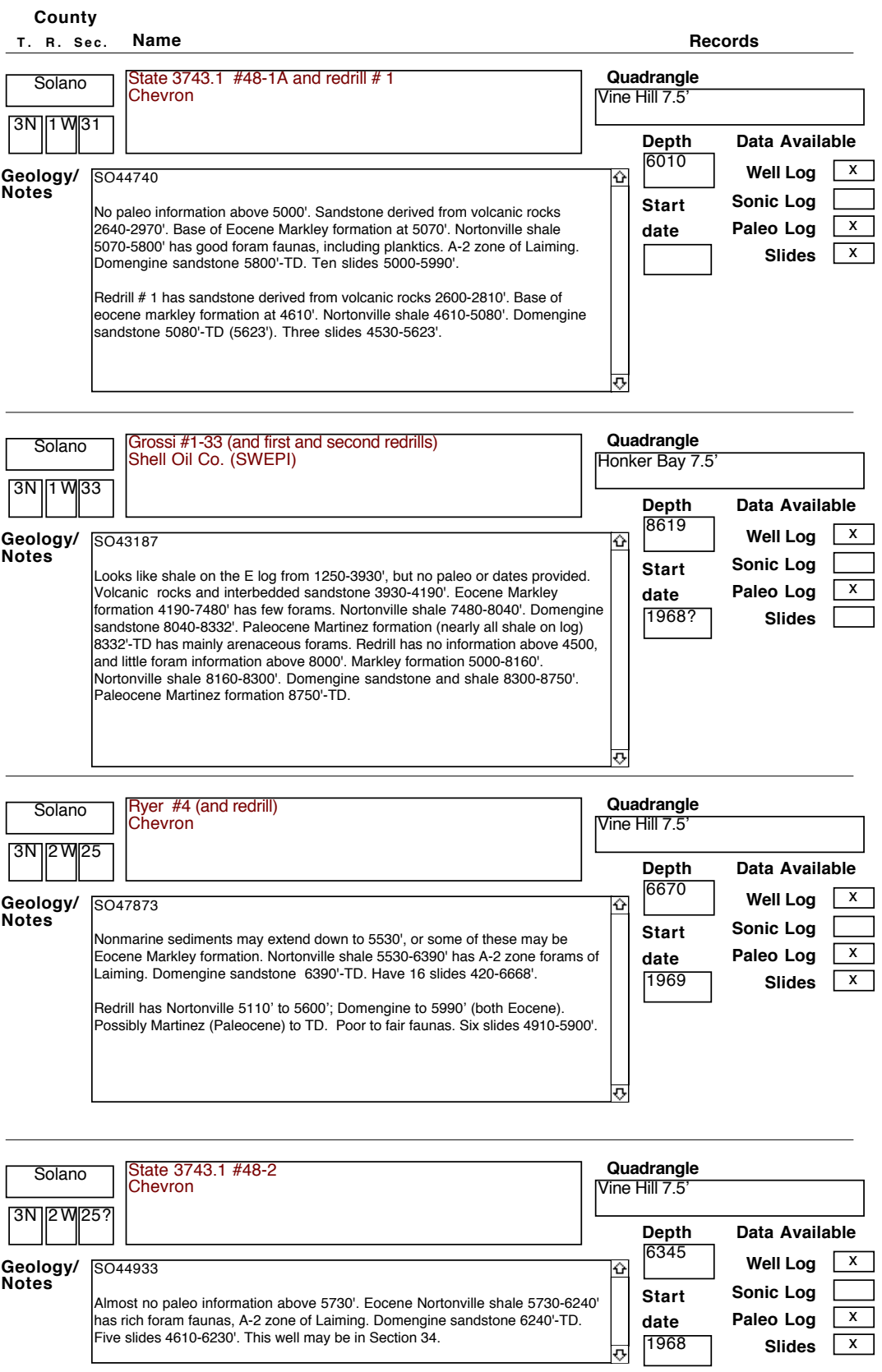

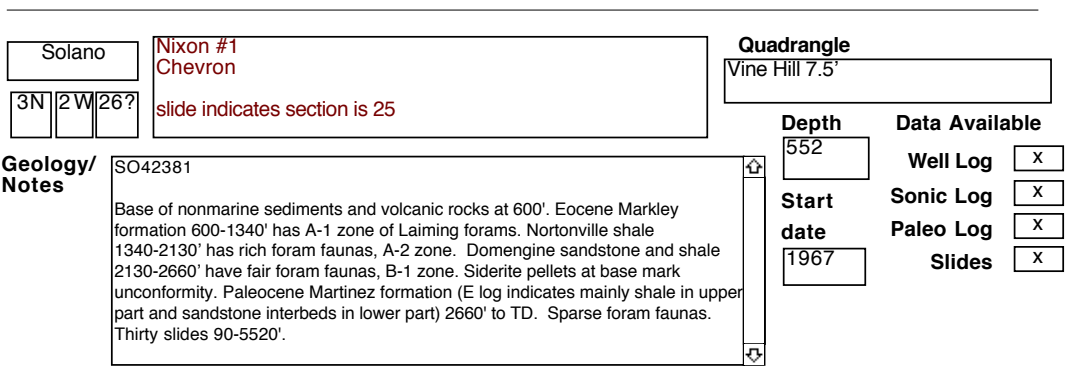


SELECTED OIL TEST WELLS IN CENTRAL CALIFORNIA DATABASE County

\begin{tabular}{|c|c|c|c|c|c|}
\hline T. R. Se & Name & \multicolumn{4}{|c|}{ Records } \\
\hline Solano & \multirow[t]{2}{*}{$\begin{array}{l}\text { State 3998.1 \#53-1 } \\
\text { Chevron }\end{array}$} & \multicolumn{4}{|c|}{$\begin{array}{l}\text { Quadrangle } \\
\text { Vine Hill 7.5' }\end{array}$} \\
\hline $3 \mathrm{~N} 2 \mathrm{~W} / 3$ & & & Depth & \multicolumn{2}{|c|}{ Data Available } \\
\hline \multirow{3}{*}{$\begin{array}{l}\text { Geology/ } \\
\text { Notes }\end{array}$} & 47792 & 6 & 1096 & Well Log & $x$ \\
\hline & $\begin{array}{l}\text { arse faunas, mainly Elphidium. Shallow water faunas similar to those in the } \\
\text { mbre formation, upper Miocene, San Pablo Basin. Ten slides } 1180-6880^{\prime}\end{array}$ & & $\begin{array}{l}\text { Start } \\
\text { date }\end{array}$ & $\begin{array}{l}\text { Sonic Log } \\
\text { Paleo Log }\end{array}$ & $\mathrm{x}$ \\
\hline & & & 1969 & Slides & $\mathrm{x}$ \\
\hline
\end{tabular}

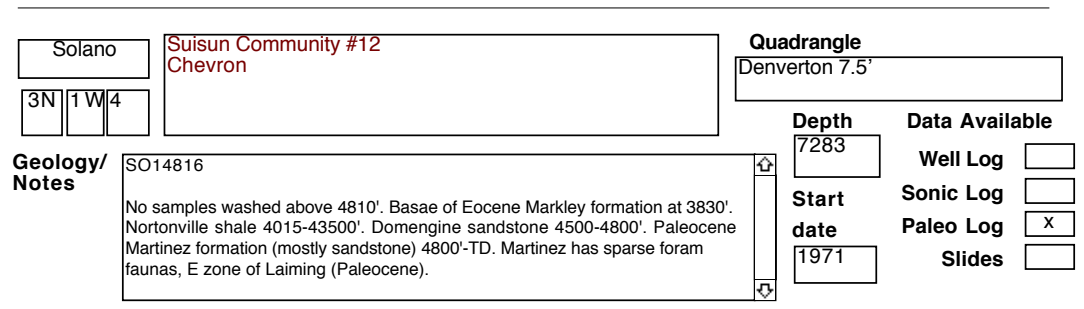

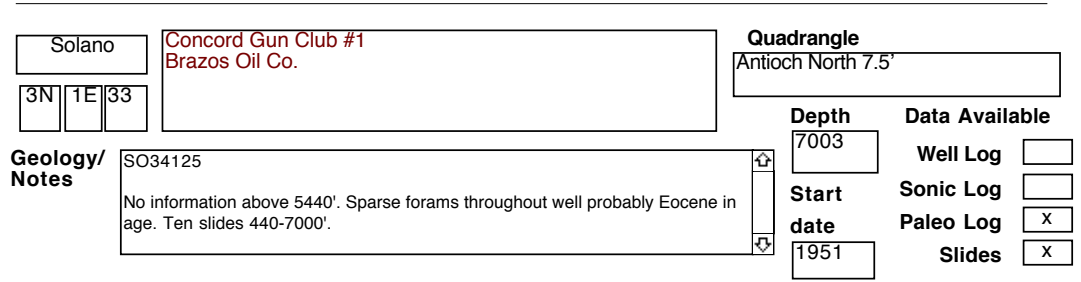

\begin{tabular}{l|l|l|l|l|}
\hline Solano & $\begin{array}{l}\text { Feykert \#1 (and redrill) } \\
\text { Chevron }\end{array}$ \\
\hline
\end{tabular}

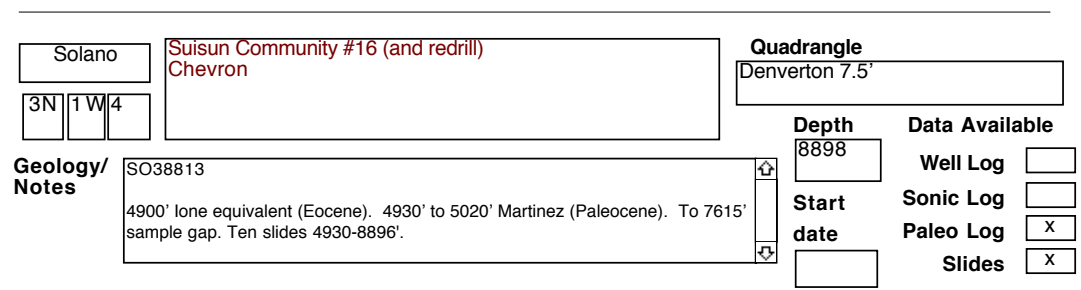




\section{SELECTED OIL TEST WELLS IN CENTRAL CALIFORNIA DATABASE}

County

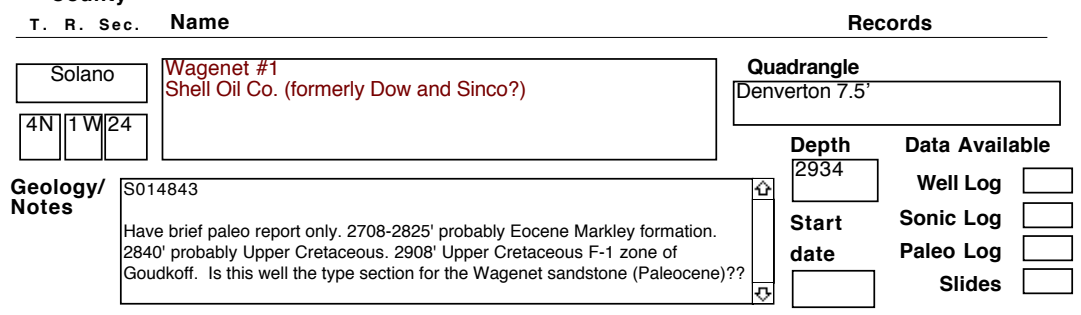

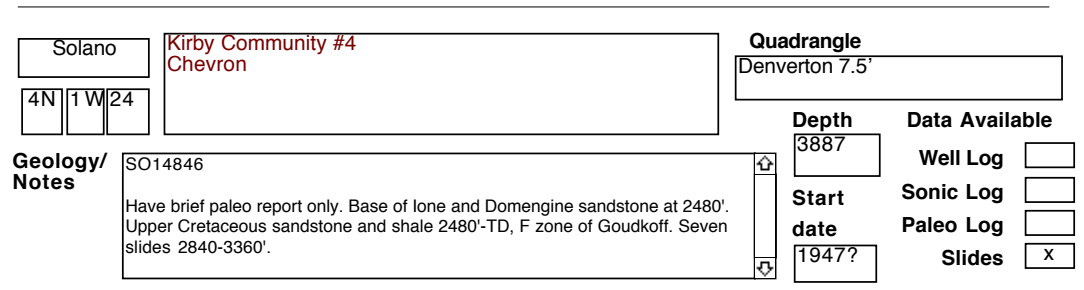

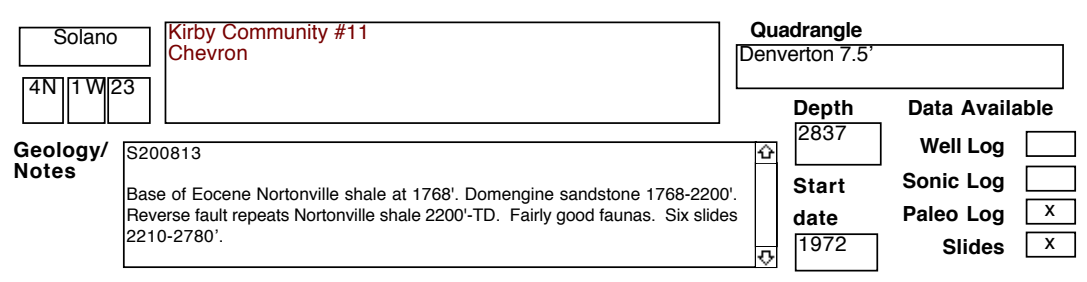

\begin{tabular}{|c|c|c|c|c|}
\hline Solano & ] $\begin{array}{l}\text { Suisun Community \#3 } \\
\text { Chevron }\end{array}$ & $\begin{array}{l}\text { Quadrangle } \\
\text { Honker Bay } 7\end{array}$ & & \\
\hline${ }^{3 N}\left\|^{1 \mathrm{~V}}\right\|^{5}$ & & \multirow{2}{*}{$\begin{array}{l}\text { Depth } \\
5645\end{array}$} & \multicolumn{2}{|c|}{ Data Available } \\
\hline \multirow{4}{*}{$\begin{array}{l}\text { Geology/ } \\
\text { Notes }\end{array}$} & SO14817 & & \multirow{4}{*}{$\begin{array}{r}\text { Well Log } \\
\text { Sonic Log } \\
\text { Paleo Log } \\
\text { Slides }\end{array}$} & $\mathrm{x}$ \\
\hline & Base of Eocene Markley formation at 3273'. Fortonville shale $3273-3830^{\prime}$. & Start & & \\
\hline & Domengine sandstone 3830-4130'. Meganos formation (log indicates almost all & date & & $\mathrm{X}$ \\
\hline & $\begin{array}{l}\text { part of Nortonville has nannoplankton belonging to the Chiphrag. quadratus } \\
\text { zone. Meganos formation has Discoaster tribranchiatus zone. Have } 58 \text { slides } \\
3552-5627^{\prime} \text {. }\end{array}$ & $1965 ?$ & & $\mathrm{x}$ \\
\hline
\end{tabular}

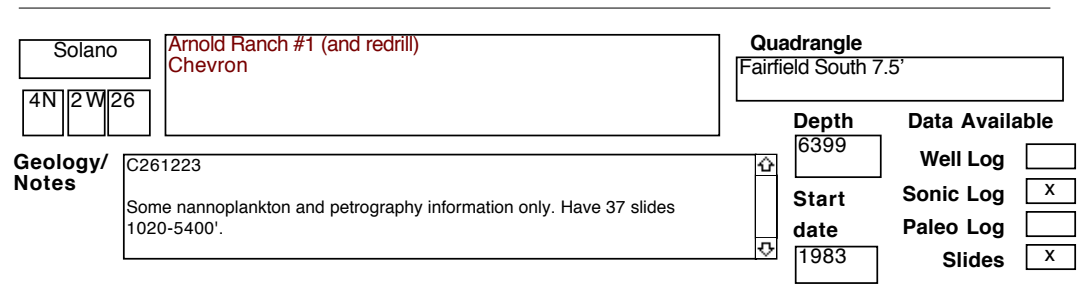


SELECTED OIL TEST WELLS IN CENTRAL CALIFORNIA DATABASE County

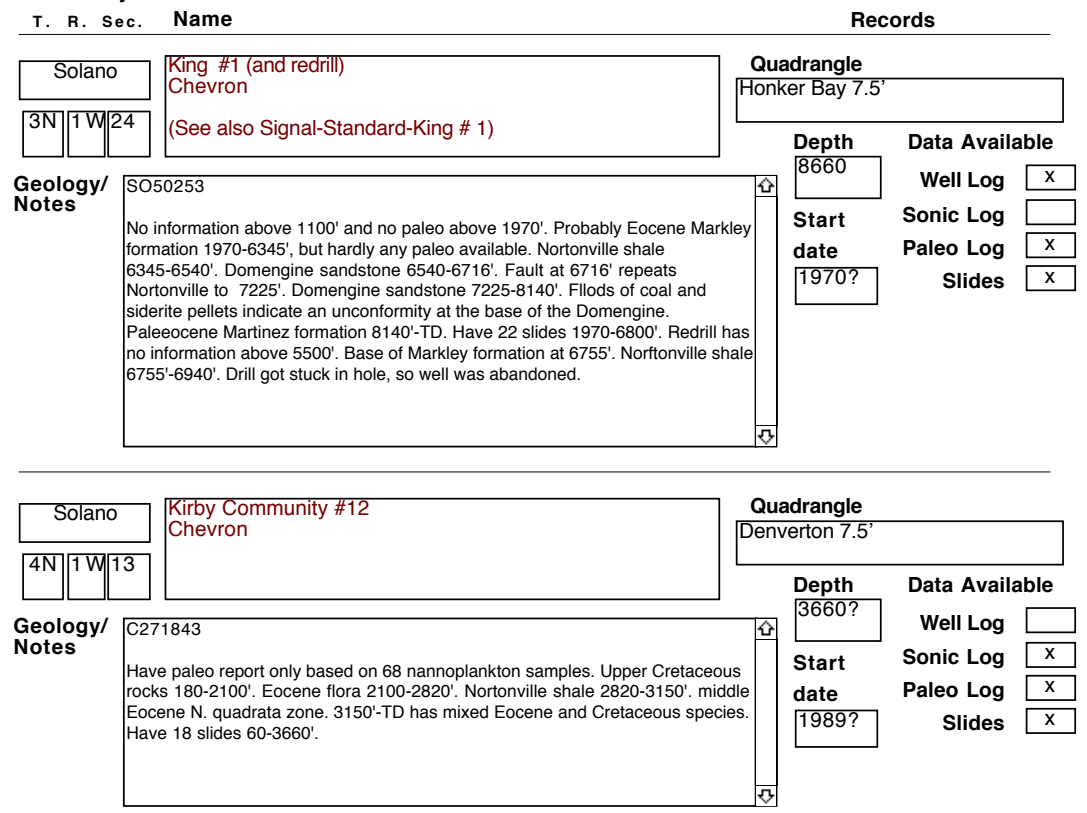

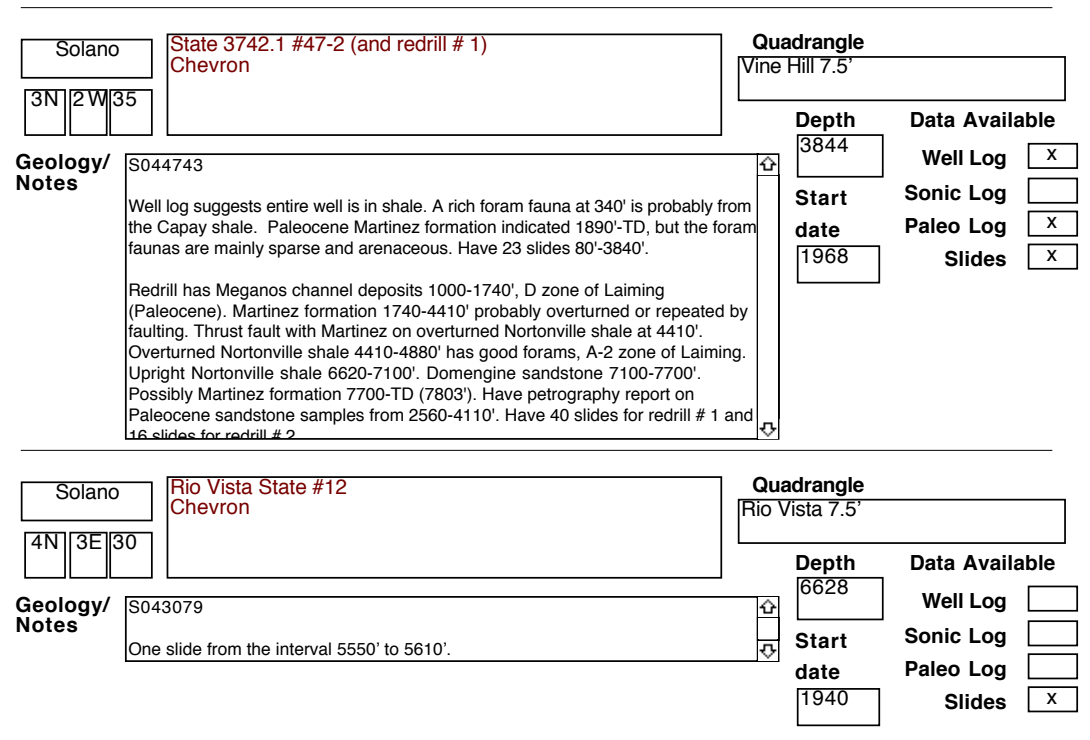

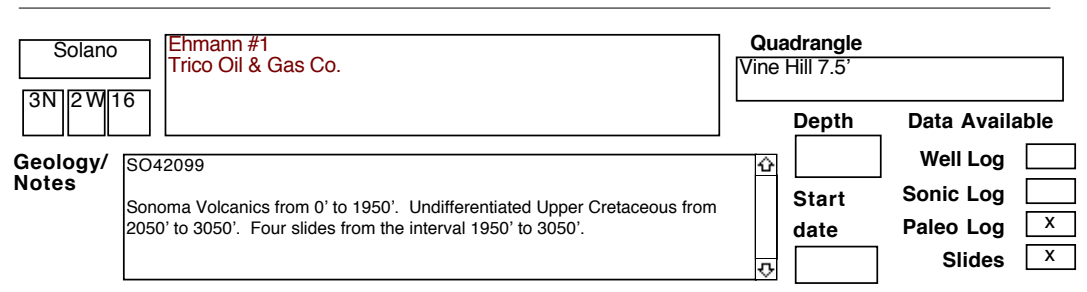




\section{SELECTED OIL TEST WELLS IN CENTRAL CALIFORNIA DATABASE}

County

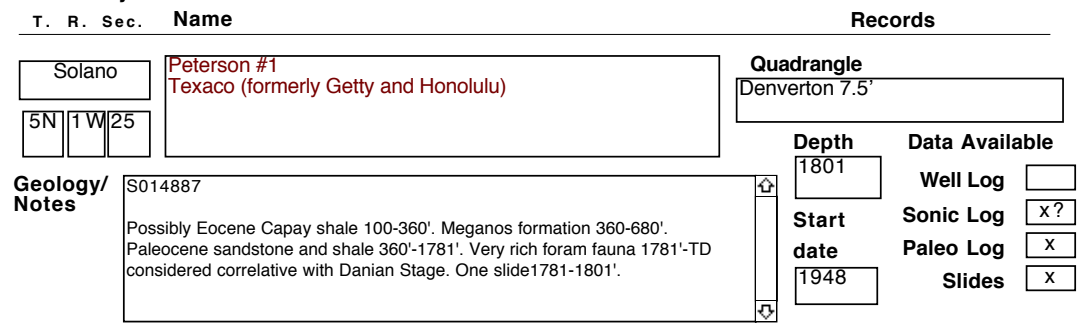

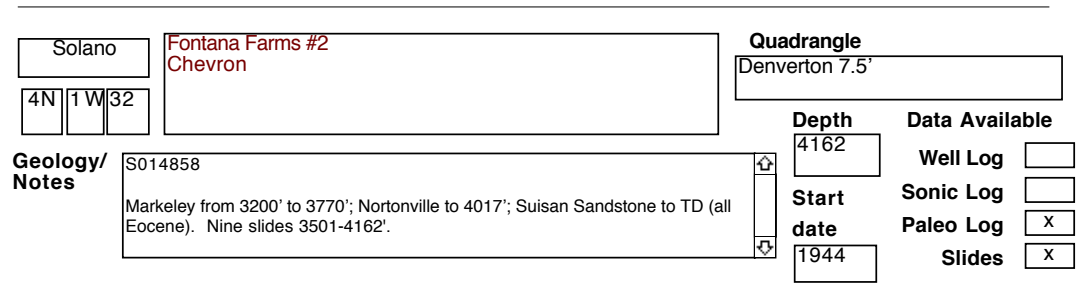

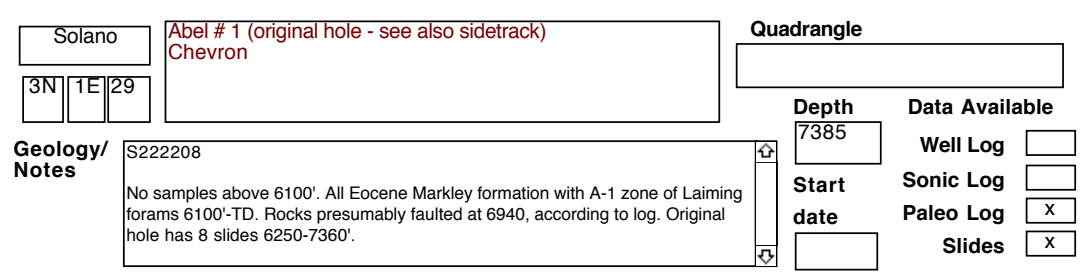

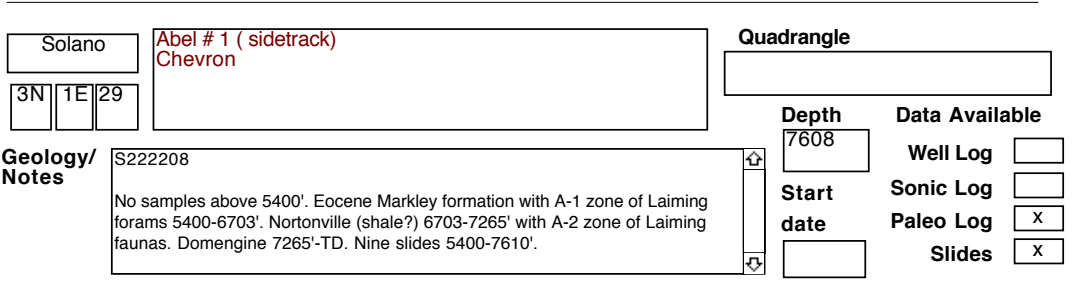

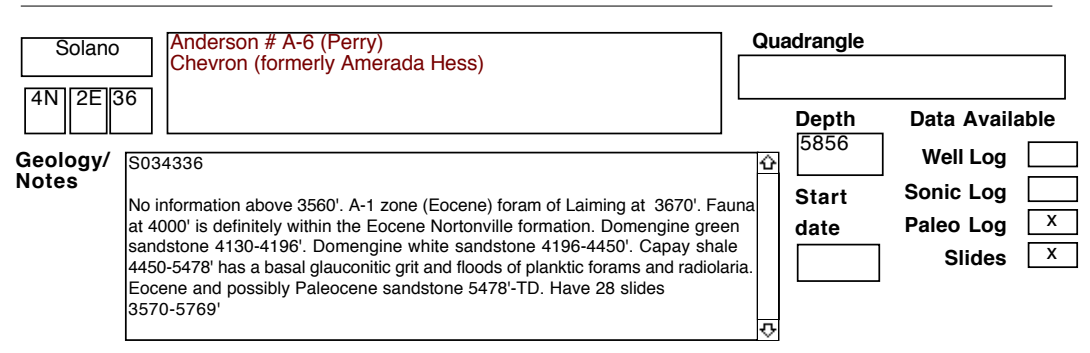


SELECTED OIL TEST WELLS IN CENTRAL CALIFORNIA DATABASE County

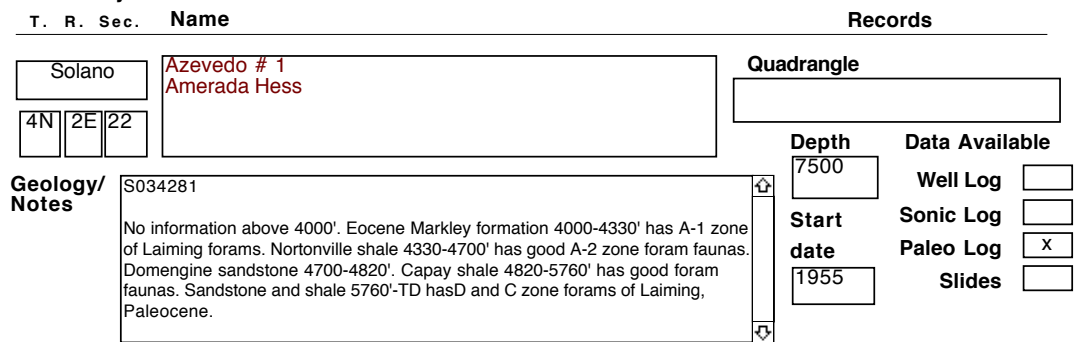

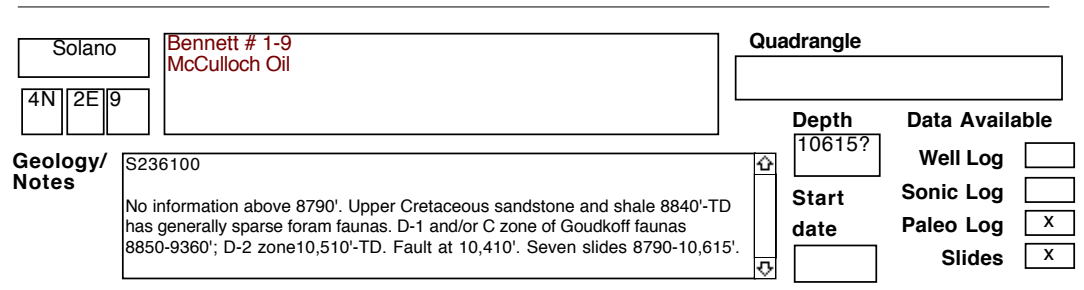

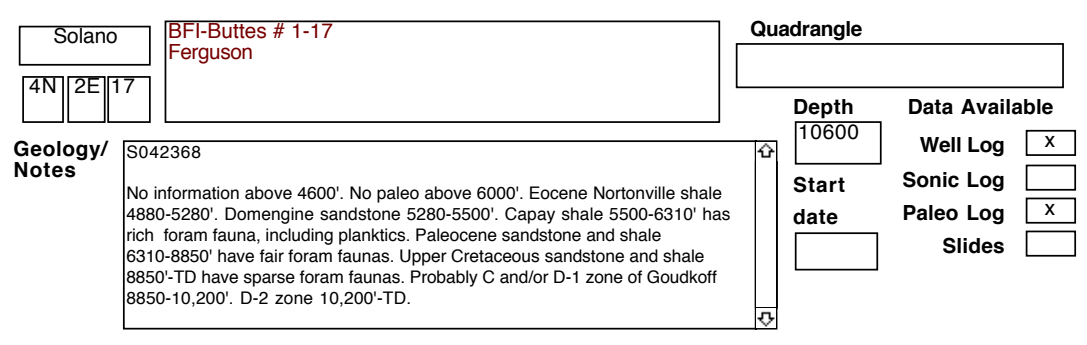

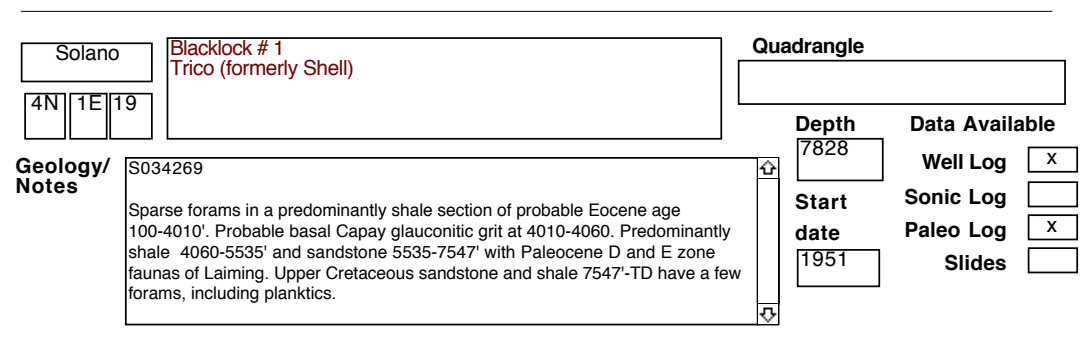

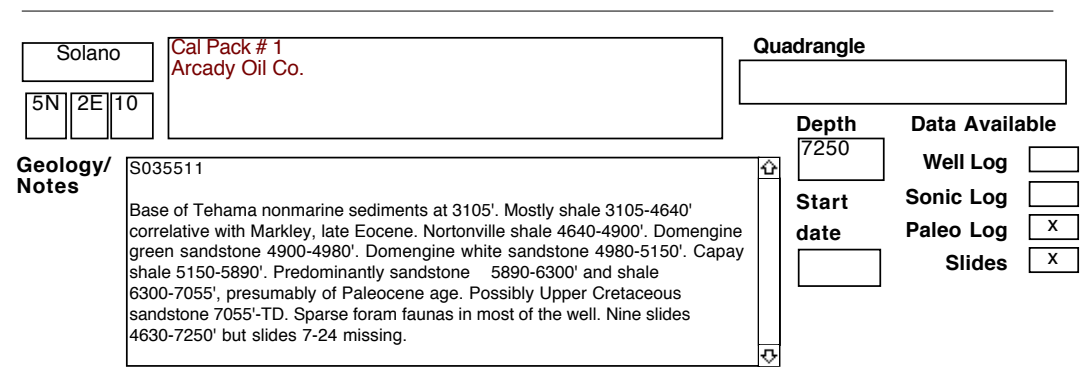


SELECTED OIL TEST WELLS IN CENTRAL CALIFORNIA DATABASE County

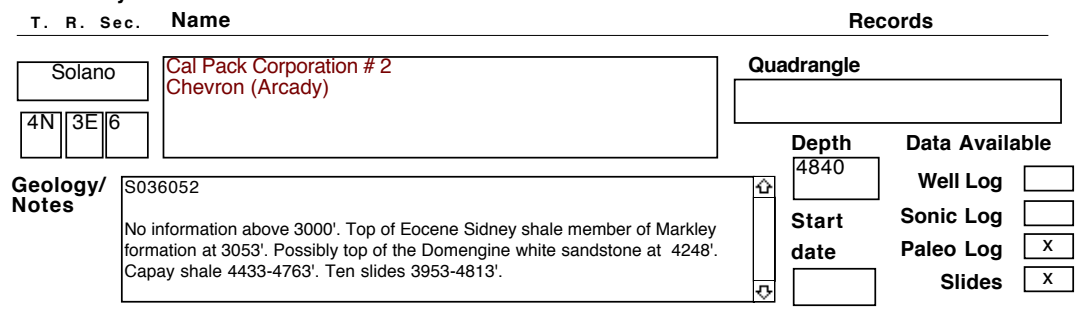

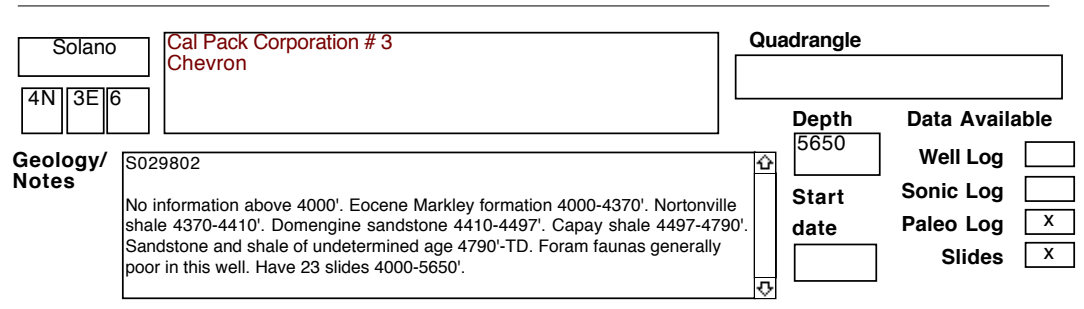

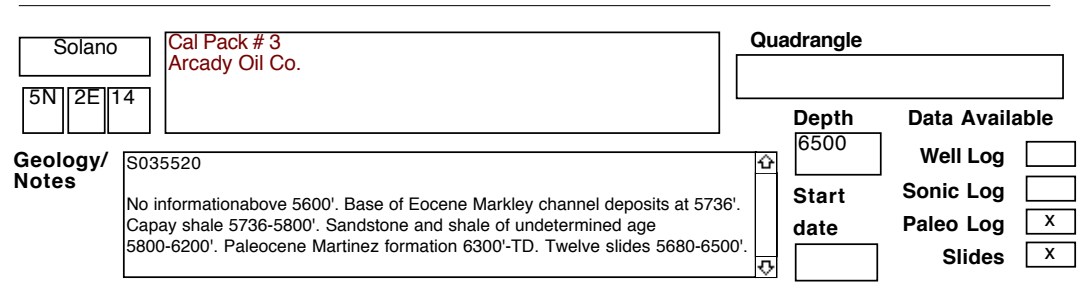

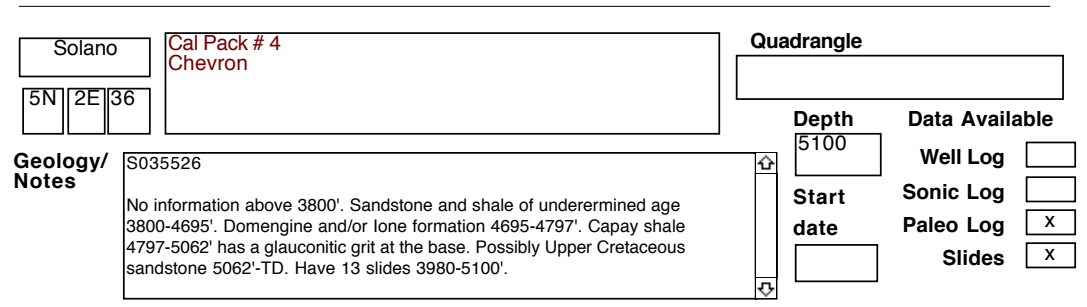

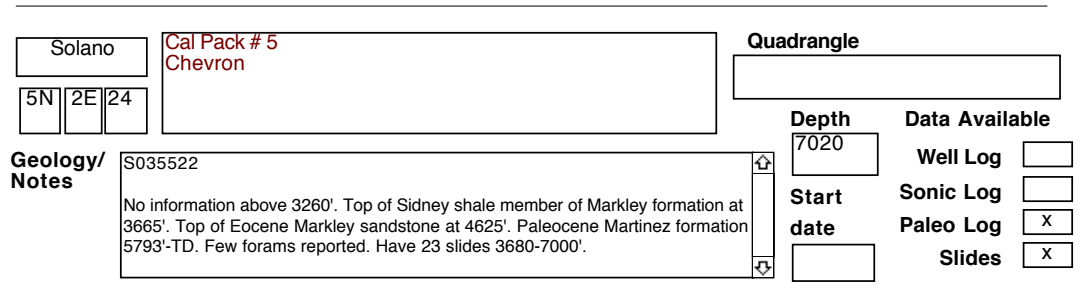




\section{SELECTED OIL TEST WELLS IN CENTRAL CALIFORNIA DATABASE}

County

\begin{tabular}{|c|c|}
\hline Solano & $\begin{array}{l}\text { Chevron-Hamilton 14-10 \#1 } \\
\text { Pan Canadian Petroleum Co. }\end{array}$ \\
\hline \begin{tabular}{|l|||l||l|}
$4 \mathrm{~N}$ & $2 \mathrm{E}$ & 10
\end{tabular} & \\
\hline
\end{tabular}

\section{Geology/ S252136}

Notes

No information above $4560^{\prime}$. Base of Eocene Markley channel deposits at $4625^{\prime}$. Nortonville shale $4525-4770^{\prime}$. Domengine sandstone $4770-4937^{\prime}$. Capay shale with good foram faunas, including planktics, $4937-5880$ '. Paleocene sandstone and shale 5880-7050'. No samples washed 7050-9300'. Upper Cretaceous sandstone and shale 9300'-TD. D-2 zone of Goudkoff 9300-11,650'. E zone 11,650'-TD. Have 41 slides 4560-12,610'.

\begin{tabular}{|c|c|c|c|}
\hline \multicolumn{4}{|c|}{ Records } \\
\hline \multicolumn{4}{|c|}{ Quadrangle } \\
\hline & Depth & Data Availal & \\
\hline & 12702 & Well Log & \\
\hline & Start & Sonic Log & \\
\hline & date & Paleo Log & $x$ \\
\hline & & Slides & $x$ \\
\hline
\end{tabular}

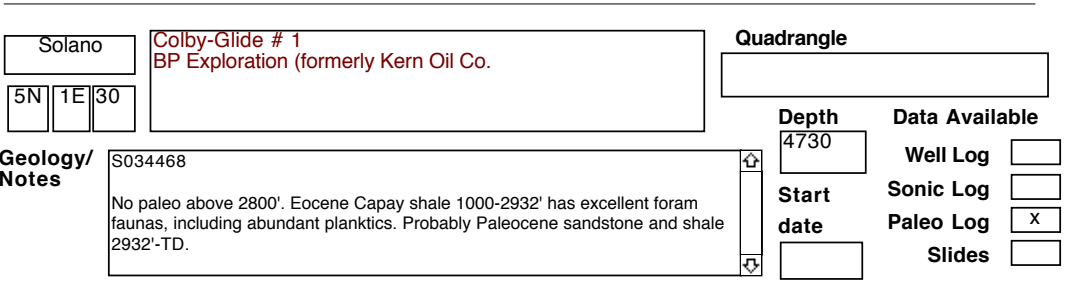

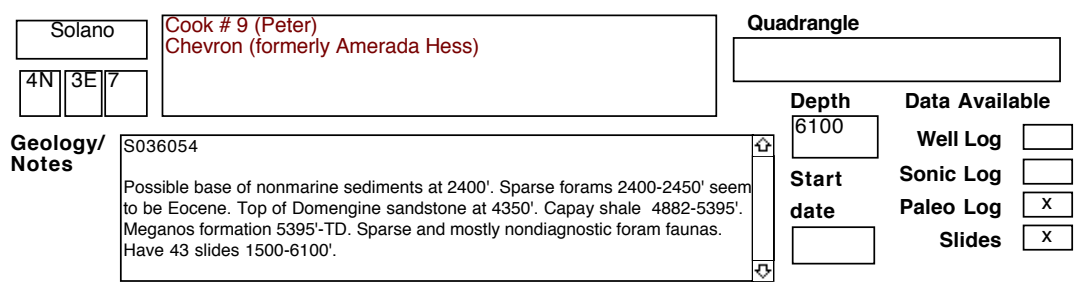

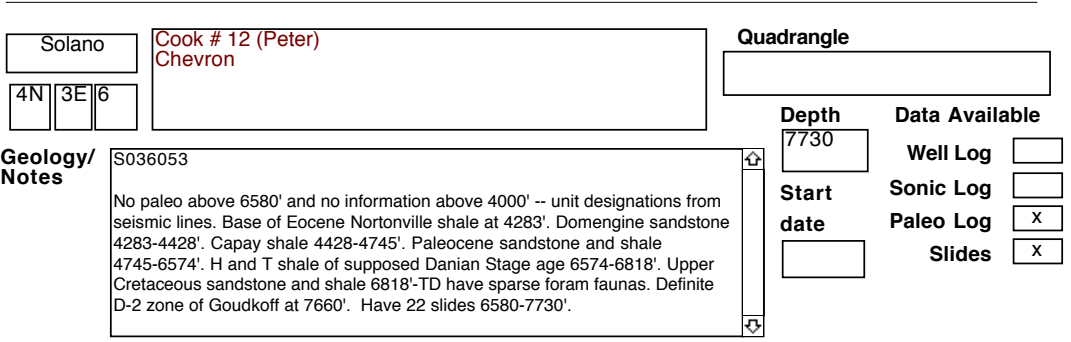

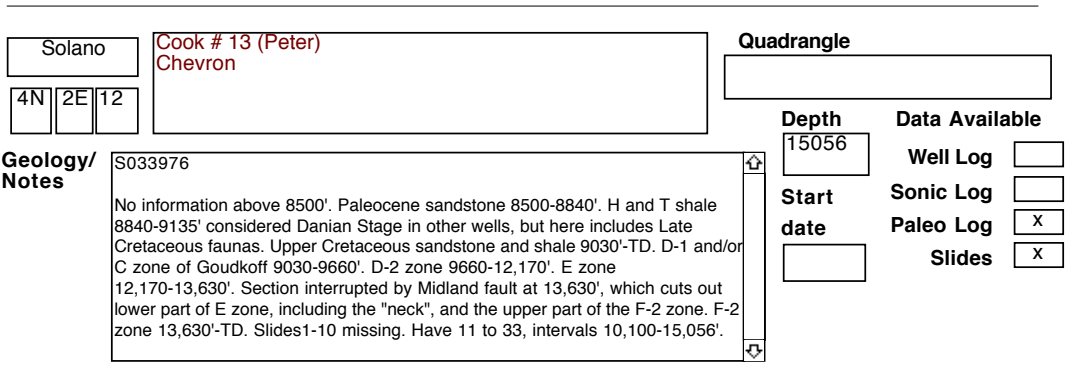




\section{SELECTED OIL TEST WELLS IN CENTRAL CALIFORNIA DATABASE}

County

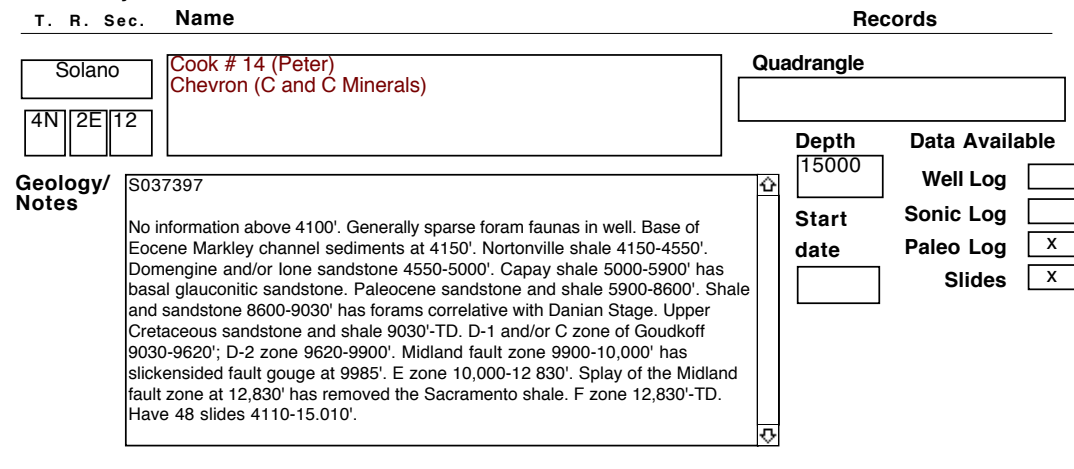

\begin{tabular}{l|l|l|l|l|l|}
\hline Solano & $\begin{array}{l}\text { Cook \# 15 (Peter) } \\
\text { Chevron }\end{array}$ \\
\hline
\end{tabular}

\begin{tabular}{l|l|l|l|l|l|}
\hline Solano & $\begin{array}{l}\text { Peter Cook \# 16 } \\
\text { Chevron }\end{array}$ \\
\hline
\end{tabular}

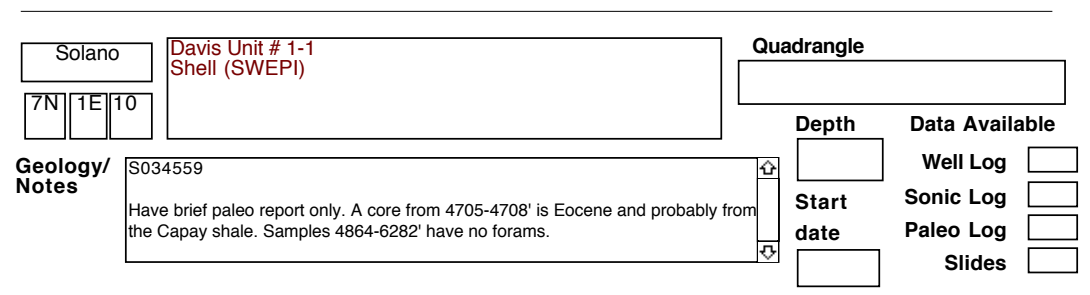

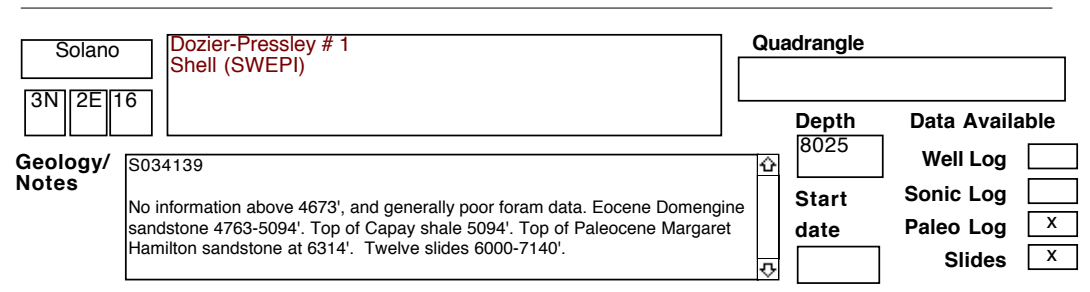


SELECTED OIL TEST WELLS IN CENTRAL CALIFORNIA DATABASE County

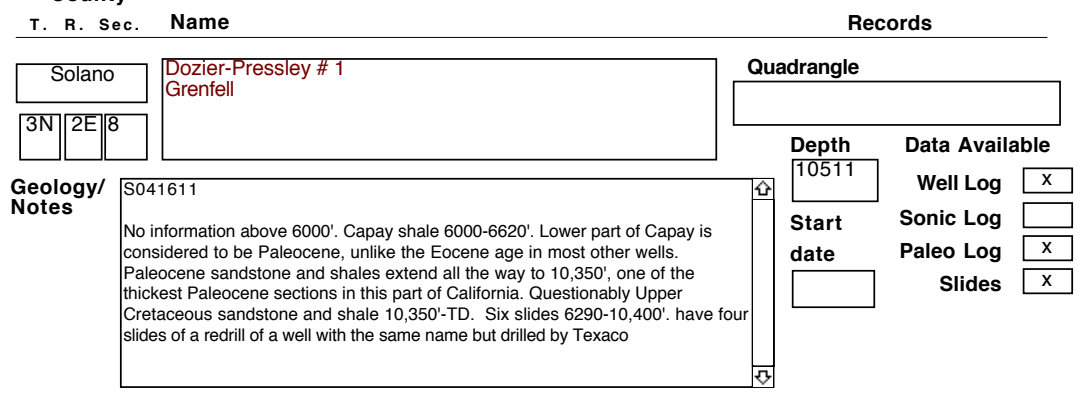

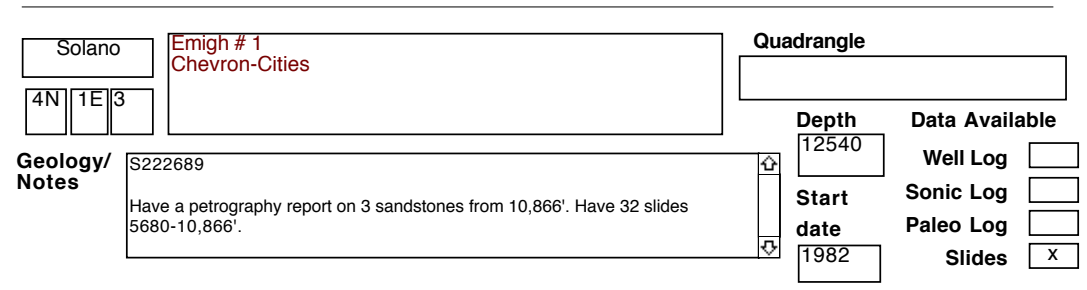

\begin{tabular}{l|l|l|l|l|l|l|}
\hline Solano & $\begin{array}{l}\text { Emigh \# 3 } \\
\text { Chevron }\end{array}$ \\
Geology/ & $\begin{array}{l}\text { C261257 } \\
\text { No information above 7000' and no paleo above 7970'. Base of margaret Hamilton } \\
\text { sandstone at 7958'. Paleocene sandstone and shale 7958-9460' have sparse } \\
\text { forams. Upper Cretaceous sandstone and shale 9460'-TD have sparse forams. } \\
\text { D-2 zone of Goudkoff 9890'-TD. Have 13 slides 8030-9950'. }\end{array}$ \\
\hline
\end{tabular}

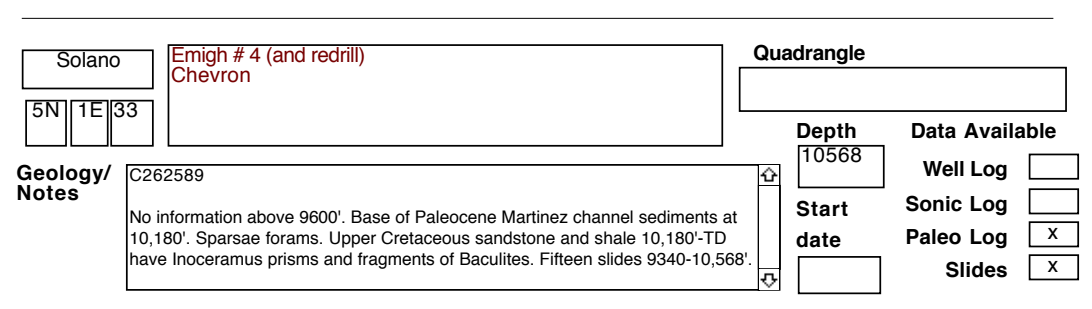

\begin{tabular}{l|l|l|l|l|l|}
\hline \multicolumn{1}{|c|}{ Solano } & $\begin{array}{l}\text { Emigh \# 7 } \\
\text { Chevron }\end{array}$ & \multicolumn{2}{l|}{ Quadrangle } \\
\hline
\end{tabular}




\section{SELECTED OIL TEST WELLS IN CENTRAL CALIFORNIA DATABASE}

County

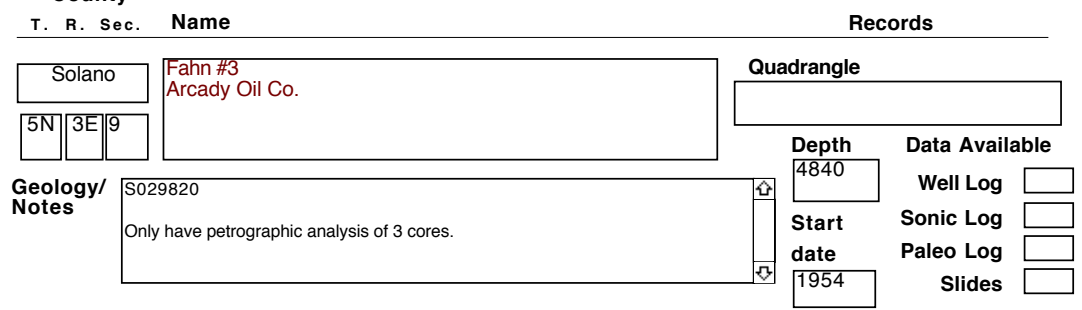

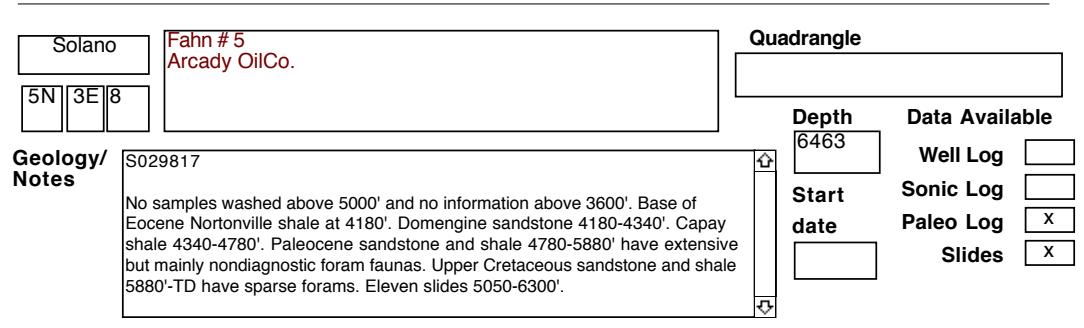

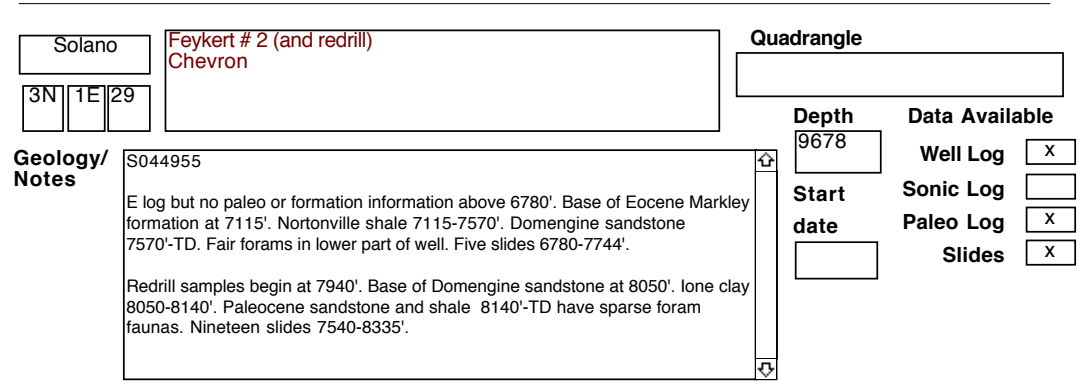

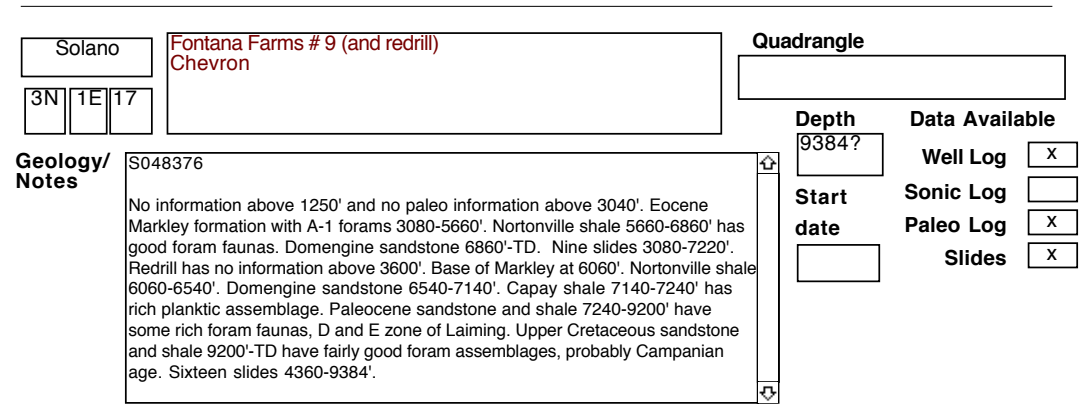

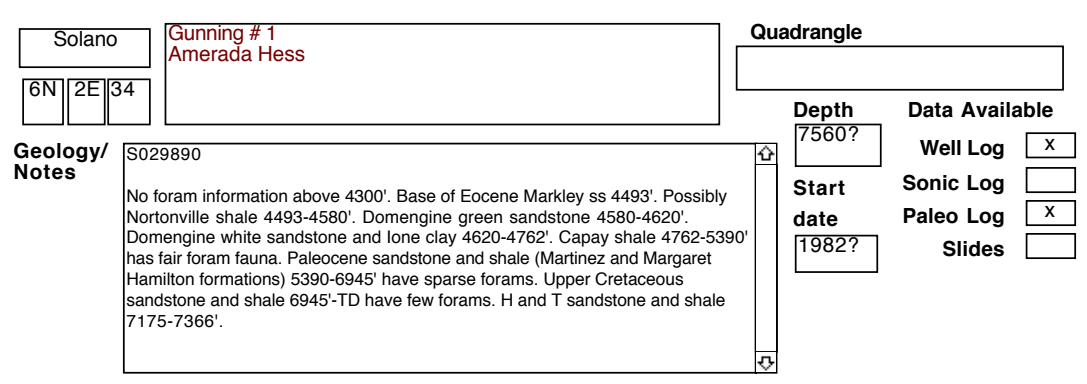


SELECTED OIL TEST WELLS IN CENTRAL CALIFORNIA DATABASE County

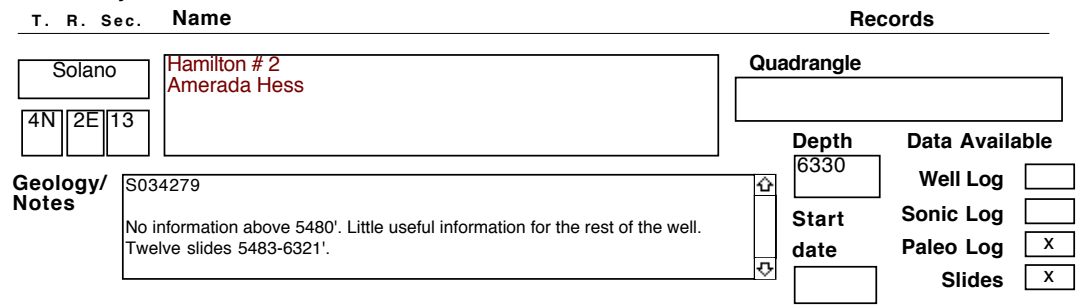

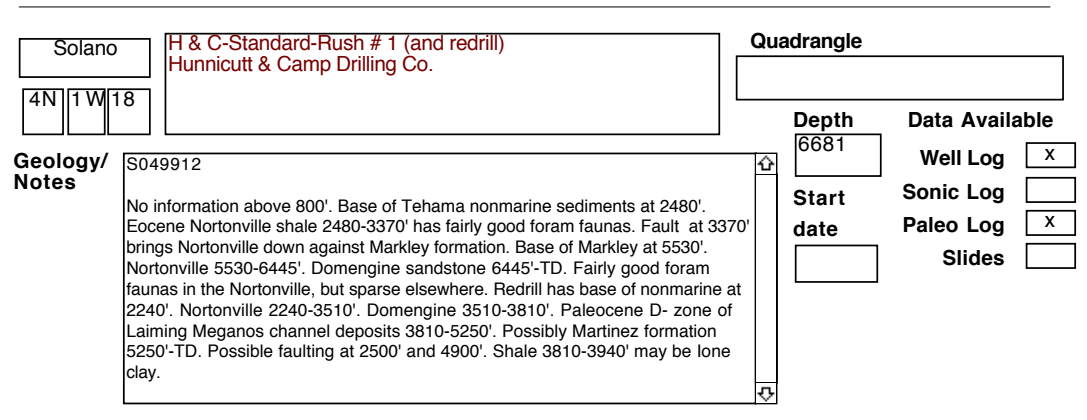

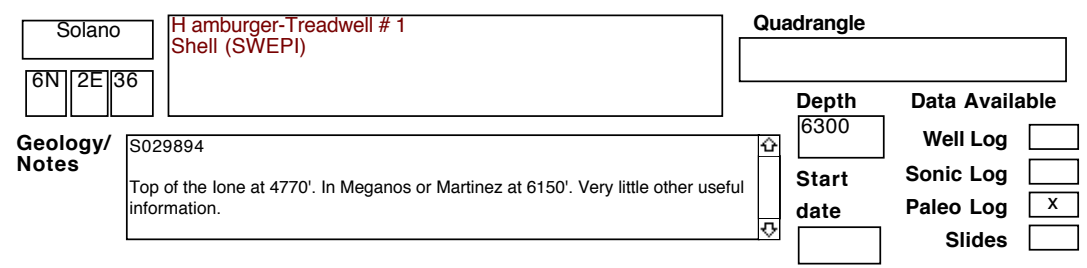

\begin{tabular}{l}
\hline Solano \\
\begin{tabular}{ll|l|l|}
\hline Geology/ \\
Notes
\end{tabular} \\
\end{tabular}

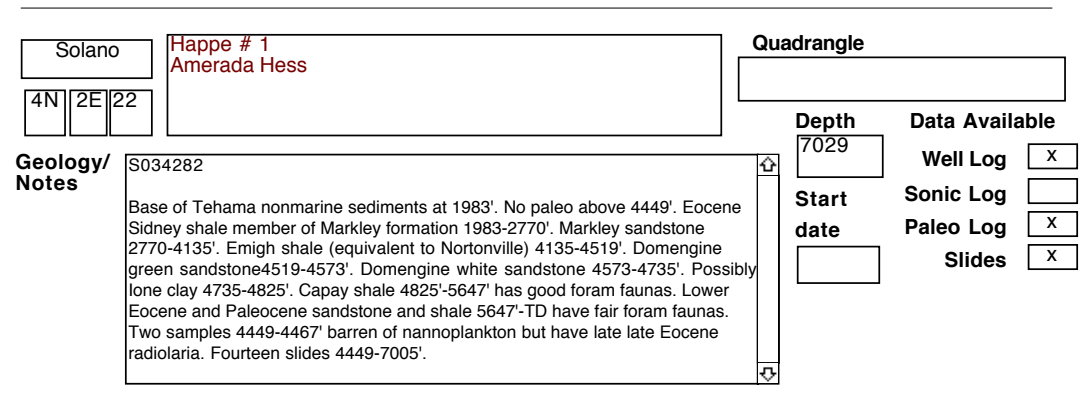




\section{SELECTED OIL TEST WELLS IN CENTRAL CALIFORNIA DATABASE}

County

\begin{tabular}{c|l|l|}
\hline T. & R. Sec. & Name \\
\hline Solano & $\begin{array}{l}\text { Harris \# 1 } \\
\text { Amerada Hess }\end{array}$ \\
\hline
\end{tabular}

\section{Geology/ S029915}

Notes

Base of Tehama nonmarine sediments at 3550'. Eocene sandstone and shale 3550-4240' probably includes both the Markley and Nortonville formations. Domengine green sandstone or a basal Nortonville glauconitic grit 4240-4275' Domengine white sandstone and lone clay 4275-4395' has Discocyclina. Capay shale 4395-4850'. Sandstone and shale 4850-5800' probably Paleocene but lacks distinctive forams. Upper Cretaceous sandstone and shale 5800'-TD also lacks distinctive forams. $\mathrm{H}$ and T shale 5990-6025'.

\begin{tabular}{|c|c|c|c|}
\hline \multicolumn{4}{|c|}{ Records } \\
\hline \multicolumn{4}{|c|}{ Quadrangle } \\
\hline & Depth & Data Availa & \\
\hline & 6250 & Well Log & $x$ \\
\hline & Start & Sonic Log & \\
\hline & date & Paleo Log & $x$ \\
\hline & 1955 & Slides & . \\
\hline
\end{tabular}

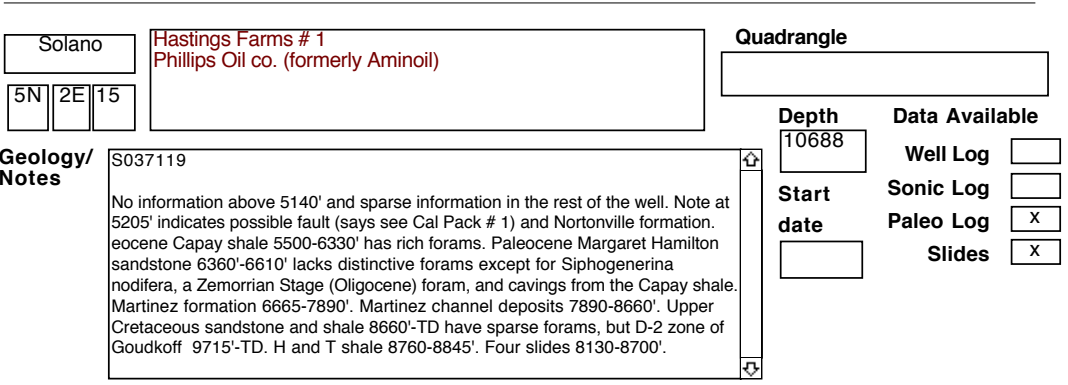

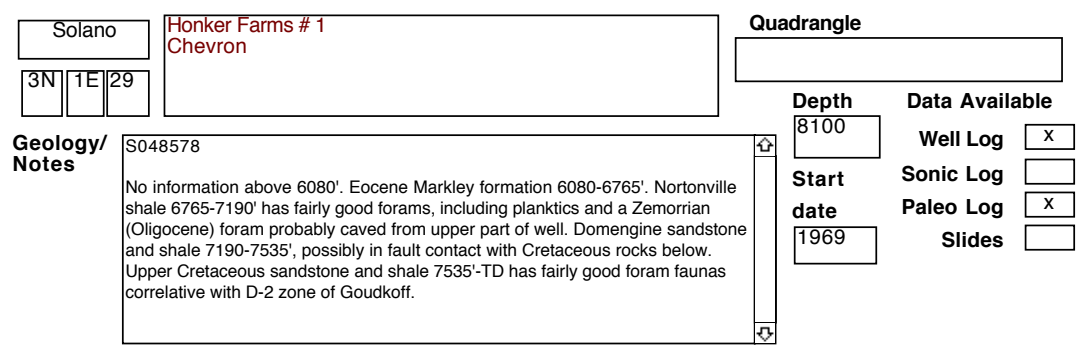

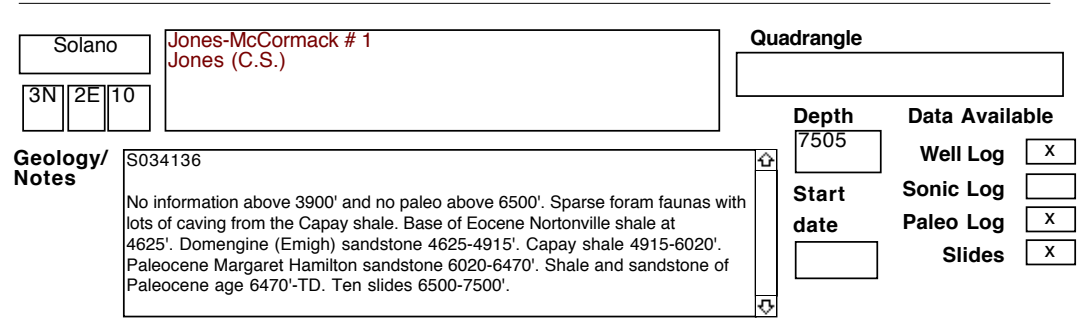

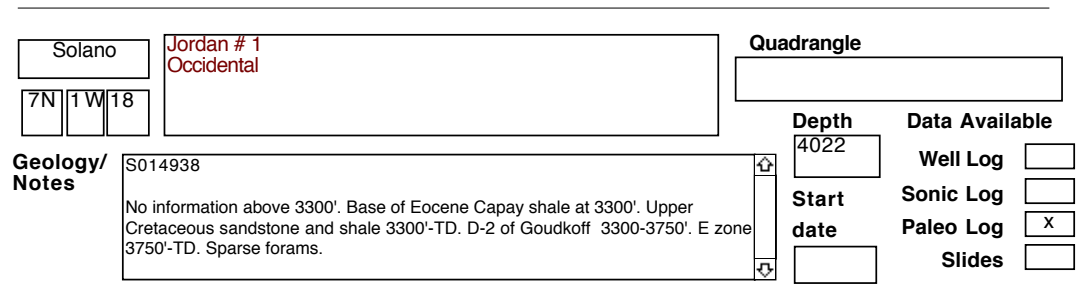


SELECTED OIL TEST WELLS IN CENTRAL CALIFORNIA DATABASE County

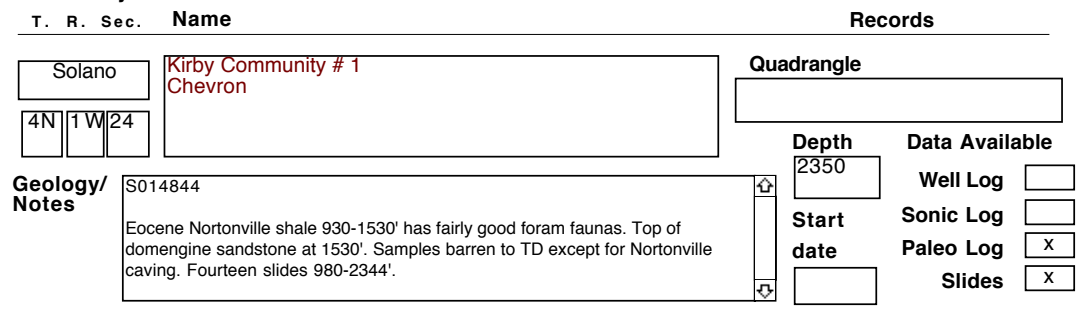

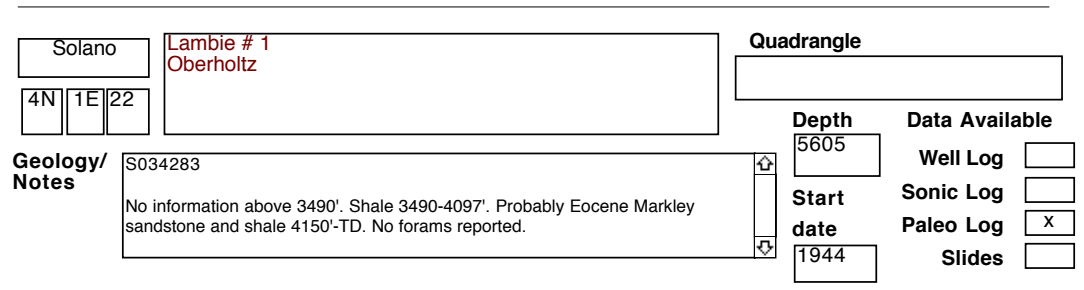

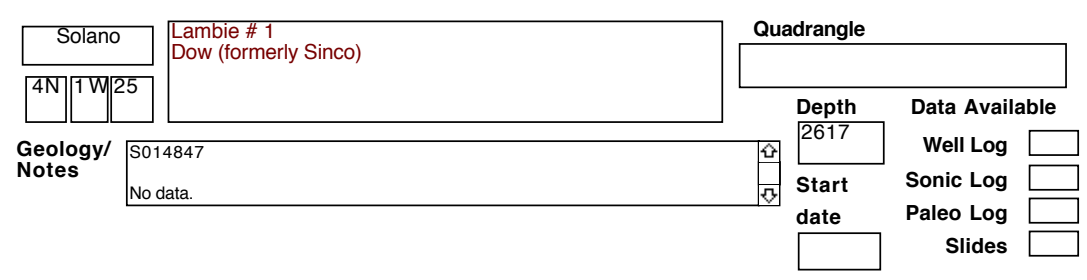

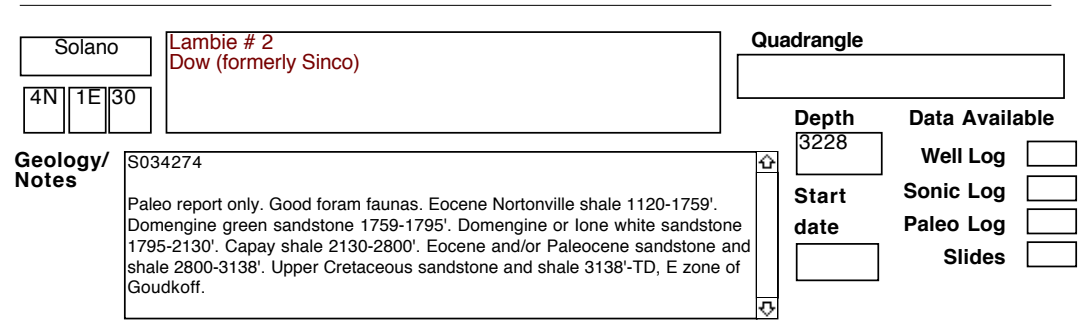

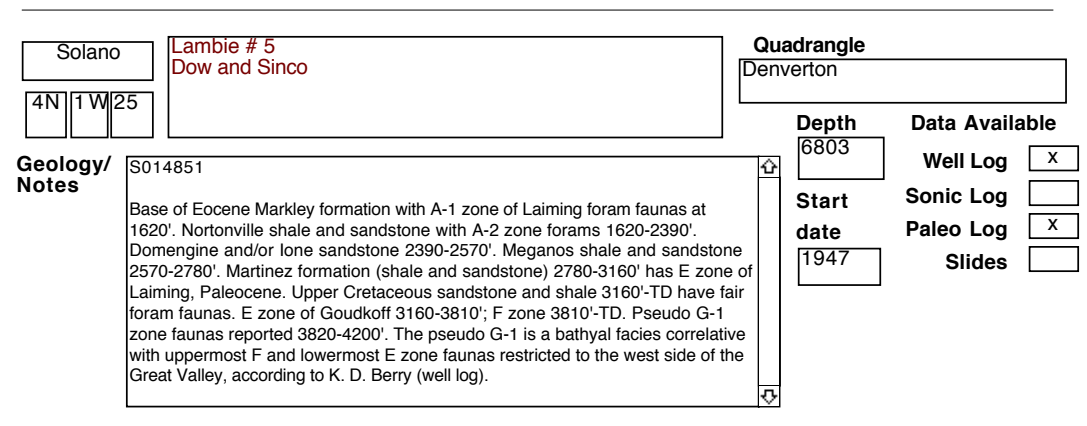




\section{SELECTED OIL TEST WELLS IN CENTRAL CALIFORNIA DATABASE}

County

\begin{tabular}{|c|l|l|}
\hline T. R. Sec. & Name \\
\hline Solano & Lambie \#6 (and redrills) \\
Shell (formerly Dow and Sinco?) \\
\hline $4 \mathrm{~N}$ & $1 \mathrm{E}$ & 30 \\
\hline
\end{tabular}

\section{Geology/ S034275}

Notes

No information above 2900' and no formation names above 5500'. Eocene Capay shale forams 3100-5240'. Paleocene Martinez foramation forams 5500-7400'.

Upper Cretaceous sandstone and shale 7400'-TD have sparse forams. Second

redrill. No information above 3400' Base of Eocene Capay shale at $4100^{\prime}$.

Meganos formation (log indicates mainly shale) $4100-4700$ ' has only one sparse

foram sample. Paleocene Martinez formation (log indicates mostly shale in upper

part and sandstone in lower part) $4700-5780^{\prime}$ has sparse forams. Upper

Cretaceous sandstone and shale $5780^{\prime}-\mathrm{TD}$ at $5900^{\prime}$ have sparse forams, F-2 zone

of Goudkoff.

Records

Quadrangle

\begin{tabular}{|c|c|c|}
\hline \multirow{2}{*}{$\begin{array}{l}\text { Depth } \\
7896\end{array}$} & \multicolumn{2}{|c|}{ Data Available } \\
\hline & Well Log & $\mathrm{x}$ \\
\hline Start & Sonic Log & \\
\hline date & Paleo Log & $x$ \\
\hline $1952 ?$ & Slides & \\
\hline
\end{tabular}

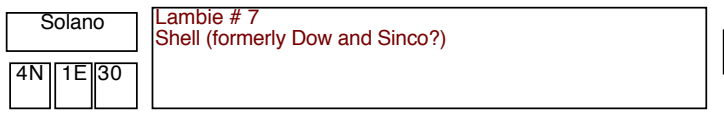

Geology/ S029732

Notes

No information above $1400^{\prime}$ and no samples above $3792^{\prime}$. Base of Eocene

Nortonville shale at 2180'. Domengine sandstone 2180-2530'. Capay shale

2530-3775'. Meganos formation (log indicates mostly shale with some sandsto

3775-4530 has fairly good forams, D zone of Laiming (Paleocene). Martinez

E zone of Laiming (Paleocene). Upper Cretaceous sandstone and shale

4770'-TD have fairly good foram faunas. E zone of Goudkoff 4770-4908'. F-1

zone 4908 '-TD.

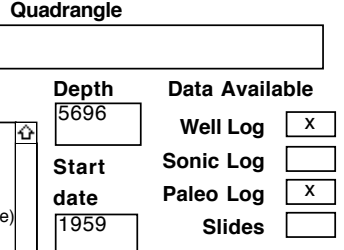

\begin{tabular}{|c|l|l|}
\hline Solano & $\begin{array}{l}\text { Lambie \# } 8 \\
\text { Shell (formerly Dow and Simco?) }\end{array}$ \\
\hline $4 \mathrm{~N}$ & $1 \mathrm{E}$ & 30 \\
\hline
\end{tabular}

\section{Geology/ S034276}

Notes

Base of Eocene Markley formation (sandstone and shale) at 1465'. Nortonville

shale 1465-2247'. Domengine sandstone 2247-2430'. Little paleo information in

hese Eocene units. Martinez formation (log indicates mainly shale with some

sandstone) 2430-3095' probably in fault contact with Domengine above and

Capay shale below. Capay shale 3095-3650' has good foram faunas, C and B-4

zones of Laiming. Margaret Hamilton sandstone 3650-3985' has sparse forams.

Meganos shale and sandstone 3985'-TD have abundant forams, D zone of

aiming (Paleocene). Interesting and well documented well.

1959

Slides

\begin{tabular}{|c|c|c|}
\hline \multicolumn{3}{|c|}{ Quadrangle } \\
\hline \multirow{2}{*}{ 象 } & Depth & Data Available \\
\hline & & Well Log \\
\hline & \multirow{3}{*}{$\begin{array}{l}\text { Start } \\
\text { date }\end{array}$} & Sonic Log \\
\hline & & Paleo Log \\
\hline & & Slides $\square$ \\
\hline
\end{tabular}

\begin{tabular}{|c|c|c|c|c|}
\hline Solano & Lambie \# 9-2 & \multicolumn{3}{|c|}{ luadrangle } \\
\hline $4 \mathrm{~N} / \mathrm{E} \mid 9$ & & \multirow{2}{*}{$\begin{array}{l}\text { Depth } \\
10643\end{array}$} & \multicolumn{2}{|c|}{ Data Available } \\
\hline \multirow{3}{*}{$\begin{array}{l}\text { Geology/ } \\
\text { Votes }\end{array}$} & S037664 & & Well Log & $\mathrm{x}$ \\
\hline & No information above $7300^{\prime}$. Base of Eocene Capay shale with forams correlative & Start & Sonic Log & \\
\hline & $\begin{array}{l}\text { with C zone of Laiming at } 7600^{\prime} \text {. Paleocene sandstones and shales with local } \\
\text { names } 7600-9880^{\prime} \text {. Probably Upper Cretaceous sandstone and shale } 9880^{\prime}-\text { TD. }\end{array}$ & $\begin{array}{l}\text { date } \\
1963\end{array}$ & $\begin{array}{r}\text { Paleo Log } \\
\text { Slides }\end{array}$ & $\mathrm{x}$ \\
\hline
\end{tabular}

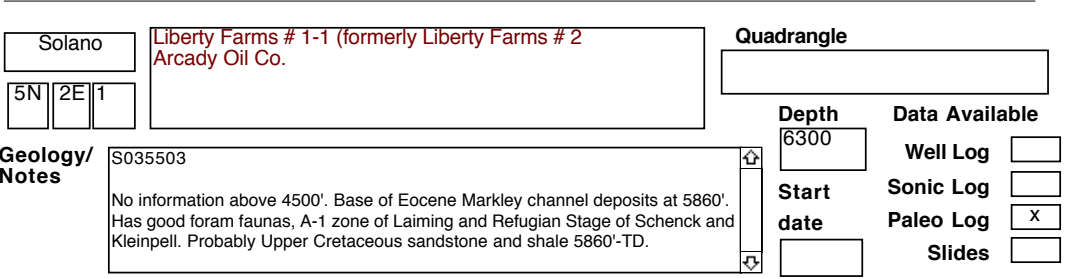




\section{SELECTED OIL TEST WELLS IN CENTRAL CALIFORNIA DATABASE}

County

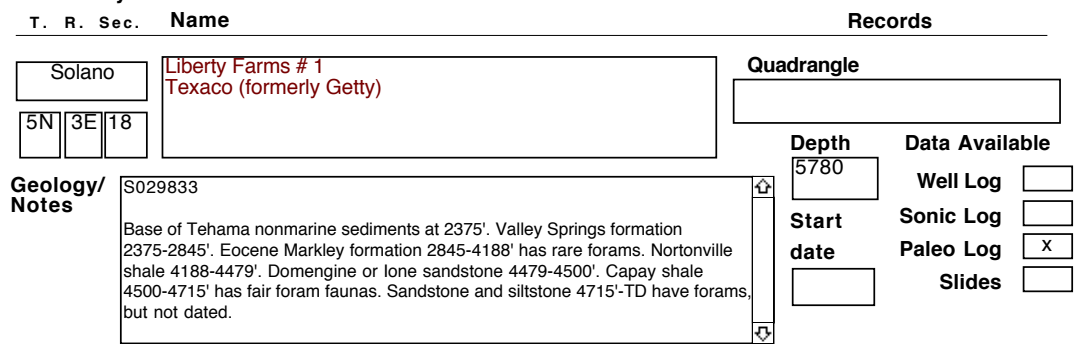

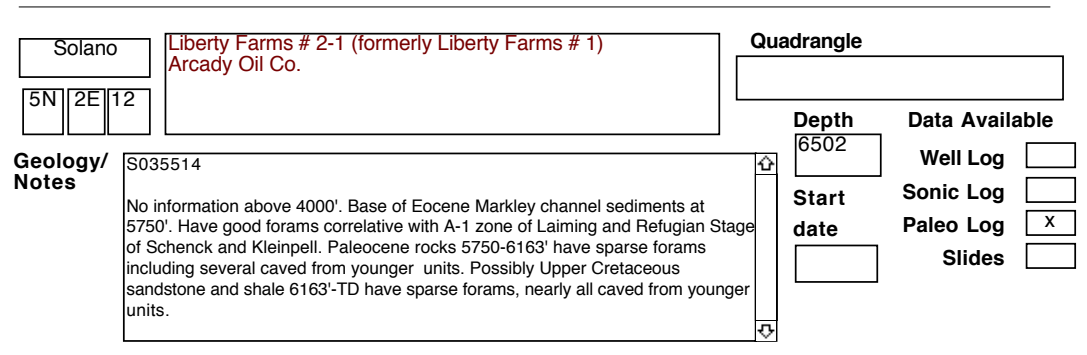

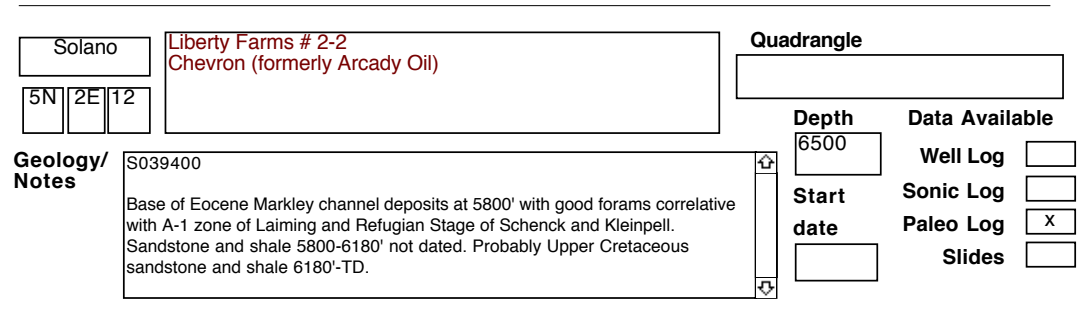

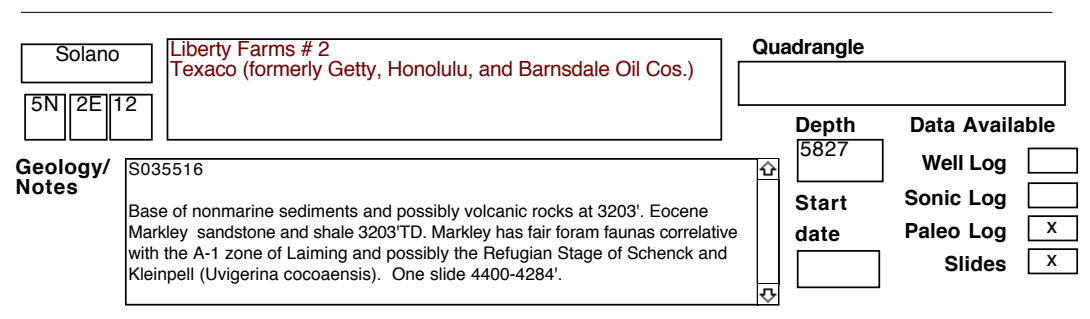

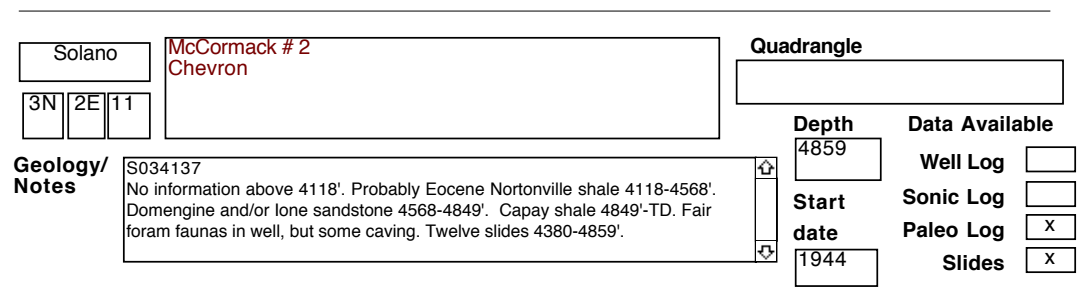


SELECTED OIL TEST WELLS IN CENTRAL CALIFORNIA DATABASE County

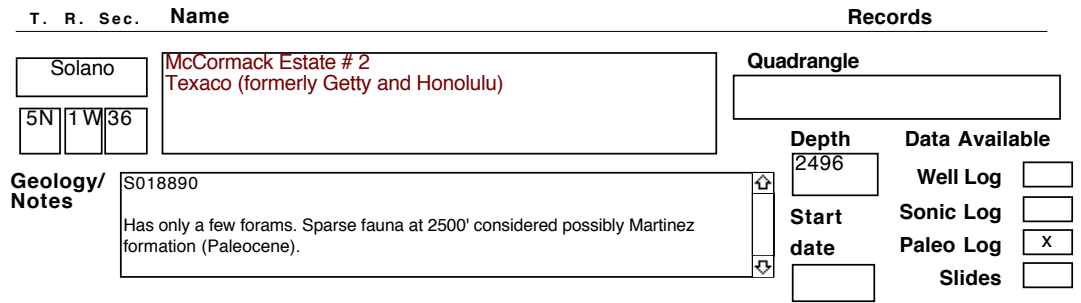

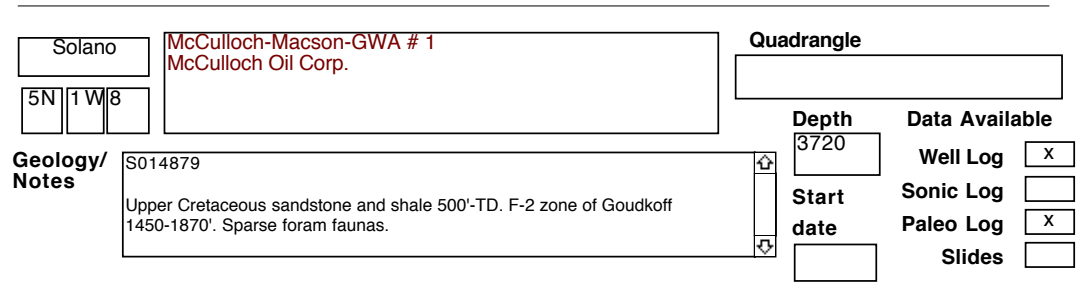

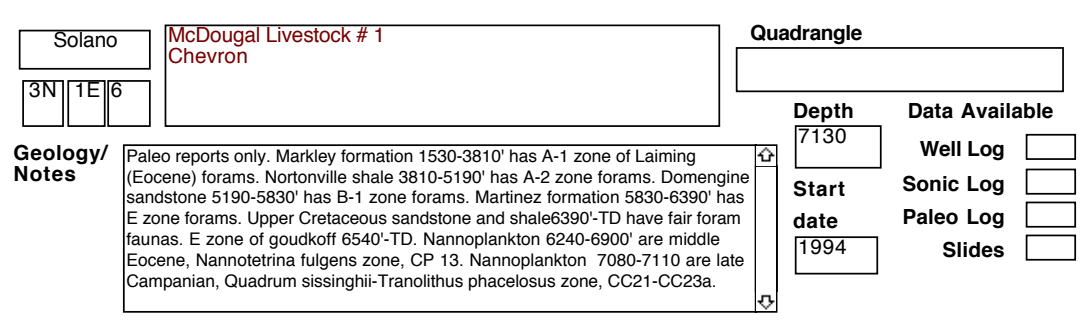

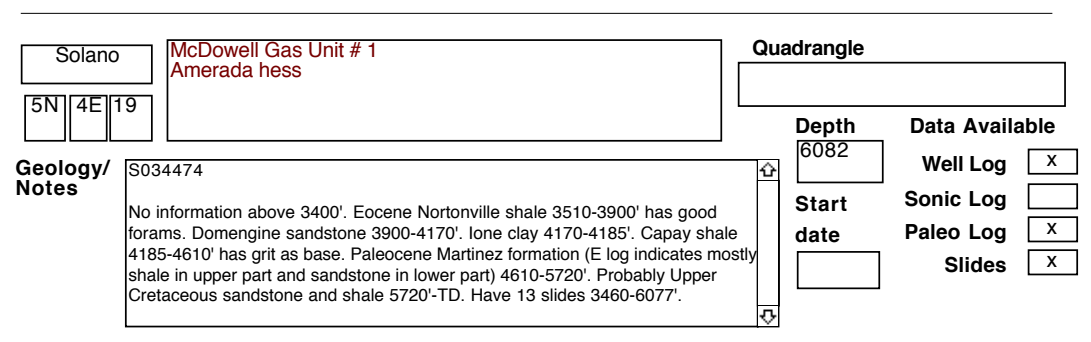

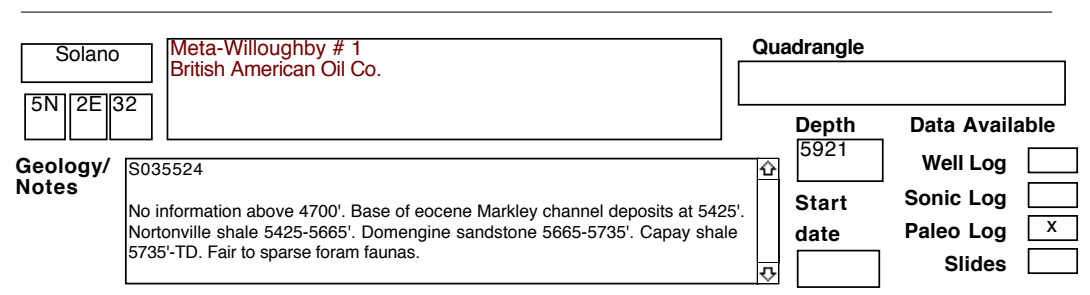




\section{SELECTED OIL TEST WELLS IN CENTRAL CALIFORNIA DATABASE}

County

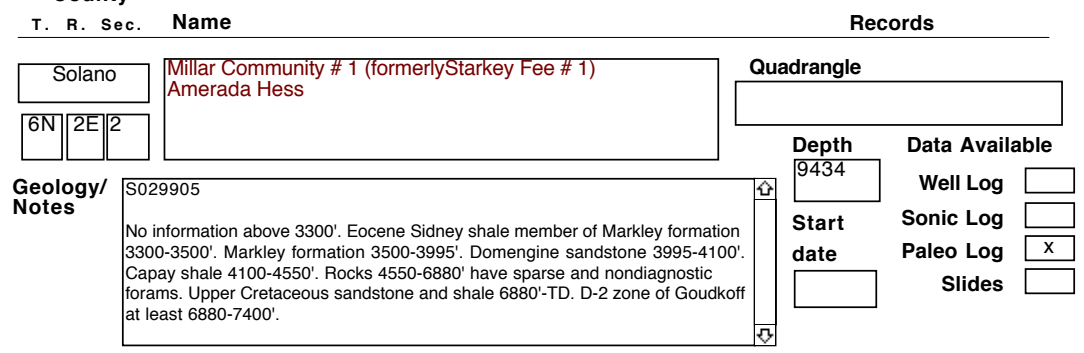

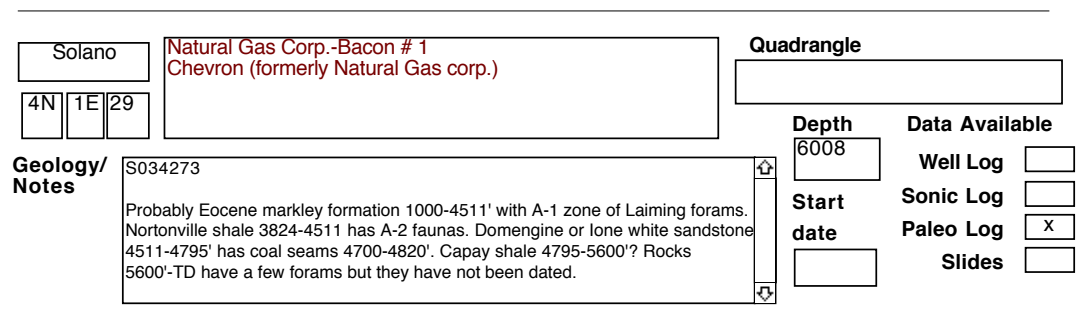

\begin{tabular}{|c|c|c|c|c|}
\hline Solano & \multirow{2}{*}{$\begin{array}{l}\text { Nixon Community \# } 1 \\
\text { Cities Service }\end{array}$} & \multicolumn{3}{|c|}{ Quadrangle } \\
\hline $5 \mathrm{~N} / 3 \mathrm{E}$ & & \multirow{2}{*}{\multicolumn{2}{|c|}{$\begin{array}{l}\text { Depth } \\
9000\end{array}$}} & \\
\hline \multirow{3}{*}{$\begin{array}{l}\text { Geology/ } \\
\text { Notes }\end{array}$} & 05130 & & & Well Log \\
\hline & $\begin{array}{l}\text { Iy have petrography report and SCM photos from sandstone core at } \\
55-8882^{\prime} \text {. }\end{array}$ & & Start & Sonic Log \\
\hline & & & 1974 & Slides \\
\hline
\end{tabular}

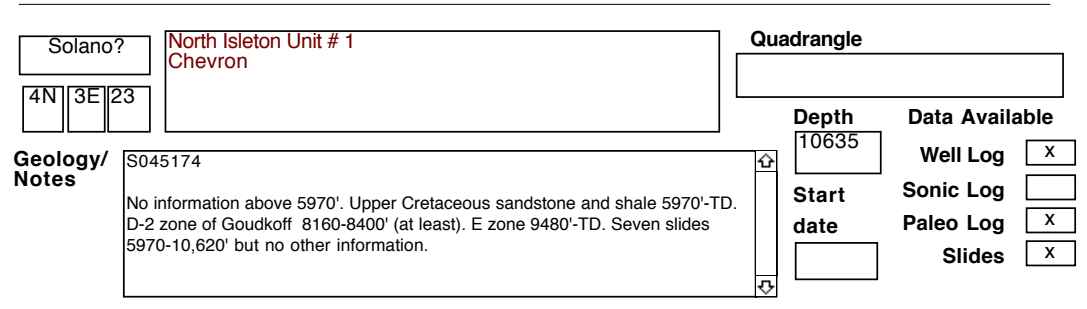

\begin{tabular}{l|l|l|l|l|l|}
\hline \multicolumn{1}{|c|}{$\begin{array}{l}\text { Solano } \\
\text { Cerry-Anderson \# 29 }\end{array}$} & \multicolumn{2}{l|}{ Quadrangle } \\
\hline
\end{tabular}


SELECTED OIL TEST WELLS IN CENTRAL CALIFORNIA DATABASE County

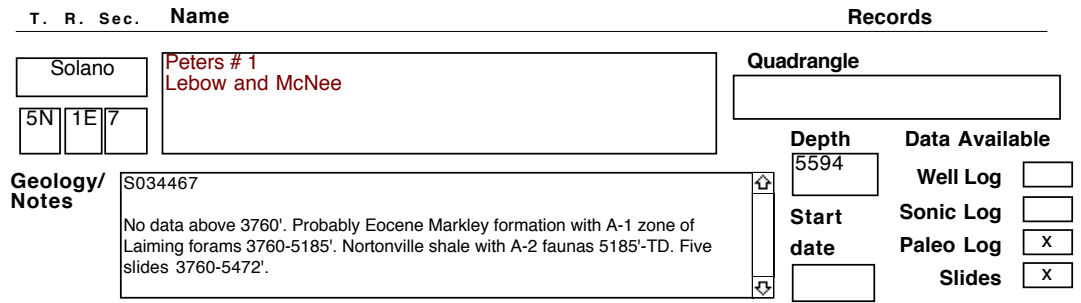

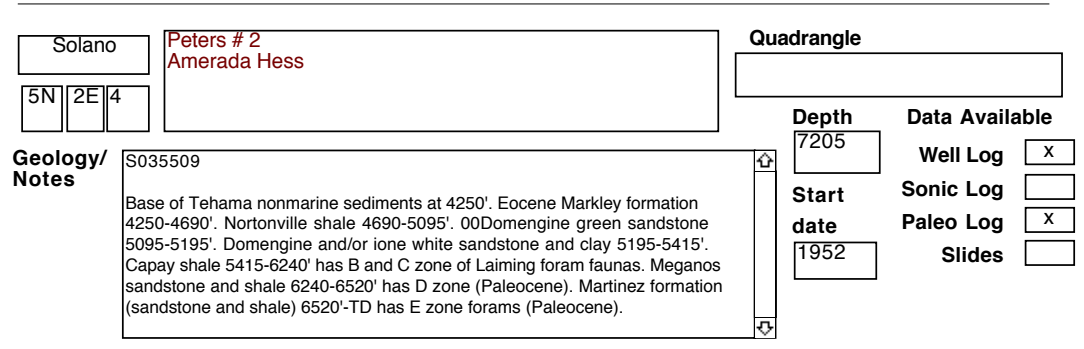

\begin{tabular}{|l|l|l|l|l|l|l|}
\hline Solano & $\begin{array}{l}\text { Peters \# 1 } \\
\text { Amerada Hess }\end{array}$ \\
\hline
\end{tabular}

\begin{tabular}{|l|l|l|l|l|l|}
\hline Seterson \#1 1 \\
Shell (SWEPI) \\
beware well with same name in section 35
\end{tabular}


SELECTED OIL TEST WELLS IN CENTRAL CALIFORNIA DATABASE County

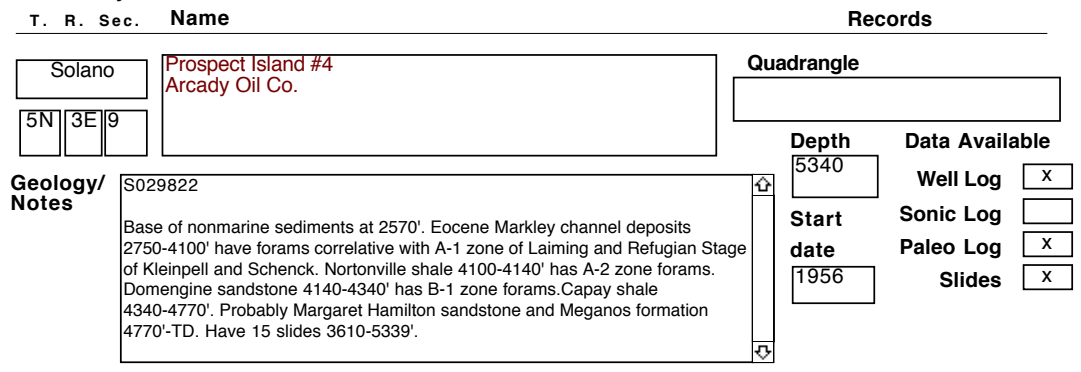

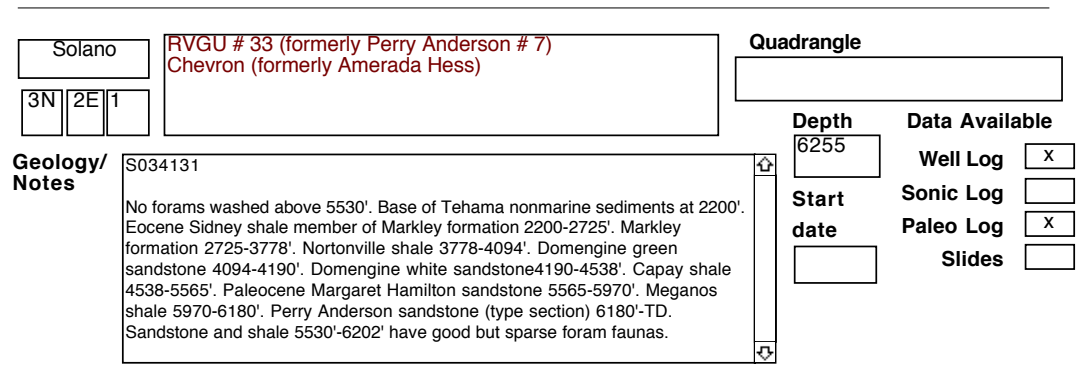

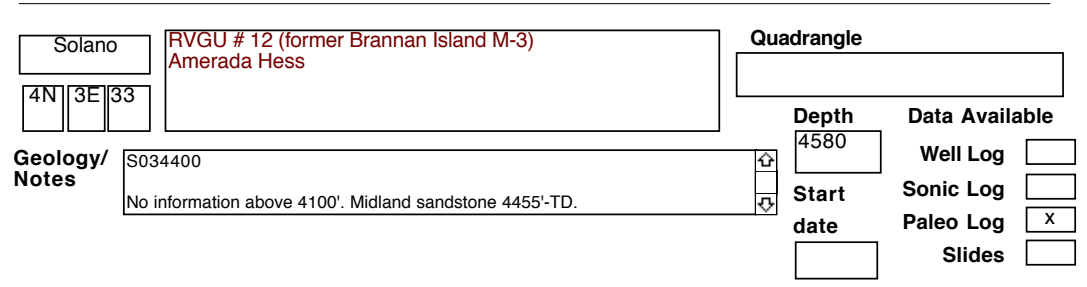

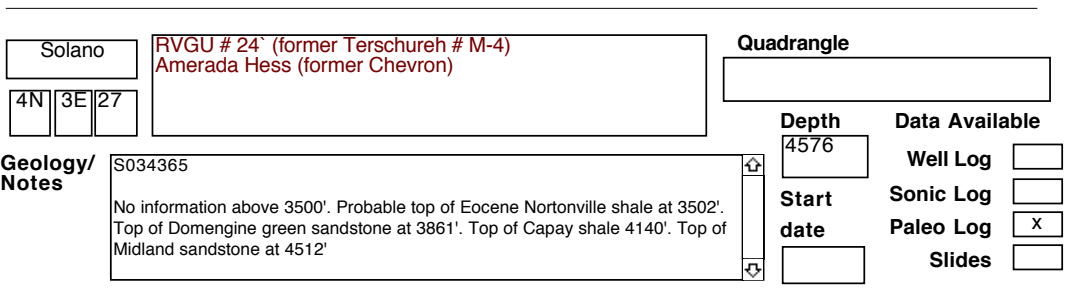

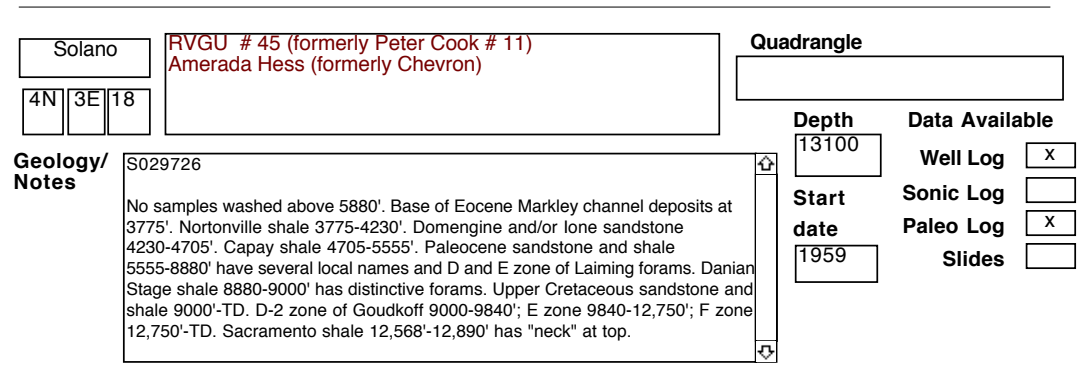


SELECTED OIL TEST WELLS IN CENTRAL CALIFORNIA DATABASE County

\begin{tabular}{|c|c|}
\hline Solano & $\begin{array}{l}\text { RVGU \# } 62 \text { (formerly Margaret Hamilton \# 1) } \\
\text { Amerada Hess }\end{array}$ \\
\hline $4 \mathrm{~N}|2 \mathrm{E}| \mid 26$ & \\
\hline
\end{tabular}

\section{Geology/ S034308}

Notes

No samples available above $4350^{\prime}$. Base of nonmarine sediments at $1920^{\prime}$. Eocene Sidney shale member of the Markley formation 1920-2700'. Markley ormation 2700-3955'. Nortonville shale 3955-4330'. Domengine green sandstone 4330-4485'. Domengine or lone white sandstone 4485-4720'. Capay shale $4720-5525^{\prime}$ has good forams. Meganos sandstone and shale $5525-6352$ has Margaret Hamilton sandstone (type?) 5525-5795 and Anderson sandstone $5875-6170$ '. Martinez formation (mostly shale on log) 6352'-TD has E zone of Laiming (Paleocene) forams. Five slides 5425-6664'.

\begin{tabular}{|c|c|c|}
\hline \multicolumn{3}{|c|}{ Records } \\
\hline \multicolumn{3}{|l|}{ uadrangle } \\
\hline Depth & Data Availal & \\
\hline 6791 & Well Log & $x$ \\
\hline Start & Sonic Log & \\
\hline date & Paleo Log & $\mathrm{x}$ \\
\hline & Slides & $\mathrm{x}$ \\
\hline
\end{tabular}

\begin{tabular}{|c|c|}
\hline Solano & RVGU \# 83 (formerly McCormick Estate \# 4) \\
\hline $4 \mathrm{~N} / 2 \mathrm{E} / 36$ & \\
\hline
\end{tabular}

Geology/ $\mathbf{5 0 3 4 3 3 5}$

Notes

No paleo information above $6420^{\prime}$, and sparse faunas $6420^{\prime}-T D$. Base of nonmarinesediments at $2110^{\prime}$. Eocene Markley formation 2110-3590'. Nortonville shale $3590-4040^{\prime}$. Domengine sandstone $4040-4380^{\prime}$. Capay shale 4380-5380.' Margaret Hamil. Dom sandstone 5380-5680'. Meganos shale formation 6365-8000'. Upper Cretaceous sandstone and shale 8000'-TD. D-1 zone of Goudkoff 9050-9800', D-2 zone 9800'-TD.

\begin{tabular}{|c|c|c|c|}
\hline \multicolumn{4}{|c|}{ Quadrangle } \\
\hline & \multirow{2}{*}{$\begin{array}{l}\text { Depth } \\
11051\end{array}$} & \multicolumn{2}{|c|}{ Data Available } \\
\hline 0 & & Well Log & $x$ \\
\hline & Start & Sonic Log & \\
\hline & date & Paleo Log & $x$ \\
\hline & 1948 & Slides & \\
\hline
\end{tabular}

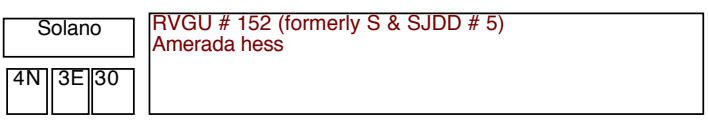

\section{Geology/ 5034376}

Notes

No useful information on log.

Quadrangle

\begin{tabular}{|c|c|c|c|}
\hline & Depth & Data Availabl & \\
\hline 0 & $5 / 80$ & Well Log & \\
\hline$\pi$ & Start & Sonic Log & \\
\hline & date & Paleo Log & $\mathrm{x}$ \\
\hline & & Slides & \\
\hline
\end{tabular}

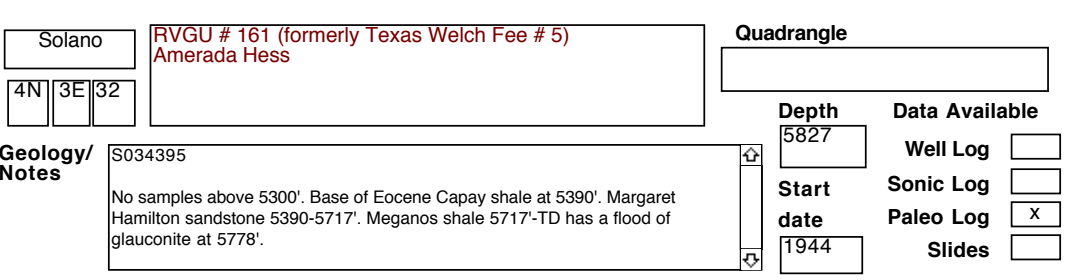

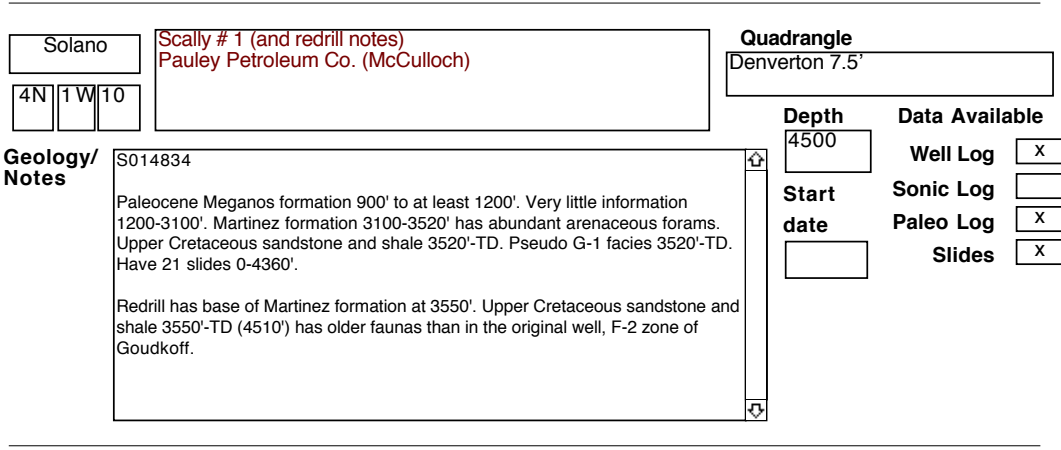




\section{SELECTED OIL TEST WELLS IN CENTRAL CALIFORNIA DATABASE}

County

\begin{tabular}{|c|c|}
\hline Solano & $\begin{array}{l}\text { Shell-Ryer \# } 2 \text { (and redrill) } \\
\text { Chevron }\end{array}$ \\
\hline $3 \mathrm{~N}|1 \mathrm{~W}| 30$ & \\
\hline
\end{tabular}

\section{Geology/ S043174}

Notes

No ditch samples above $5030^{\prime}$. Ditch samples not sufficient for ages or formation picks.

Redrill is much the same thing above $4100^{\prime}$. Nortonville shale $4100-5420^{\prime}$ has fair Eocene A-2 zone of Laiming foram faunas. Domengine sandstone 5420'-TD $\left(5830^{\prime}\right)$.

\begin{tabular}{|c|c|c|c|}
\hline \multicolumn{3}{|c|}{ Records } & \\
\hline \multicolumn{4}{|c|}{ Quadrangle } \\
\hline & Depth & Data Availa & \\
\hline & 7267 & Well Log & $\mathrm{x}$ \\
\hline & Start & Sonic Log & \\
\hline & date & Paleo Log & $x$ \\
\hline & & Slides & 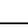 \\
\hline
\end{tabular}

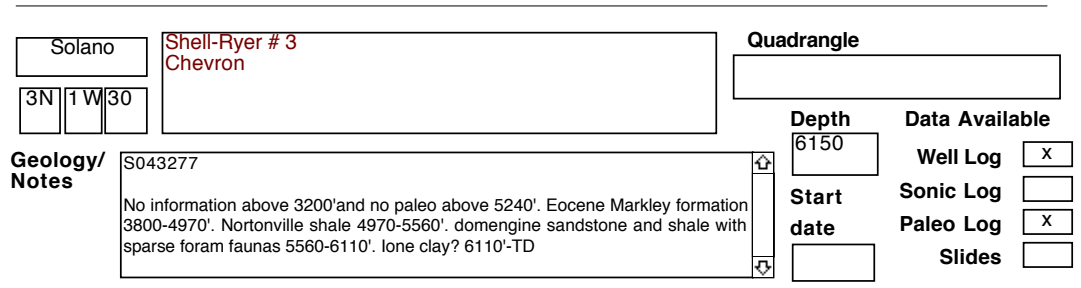

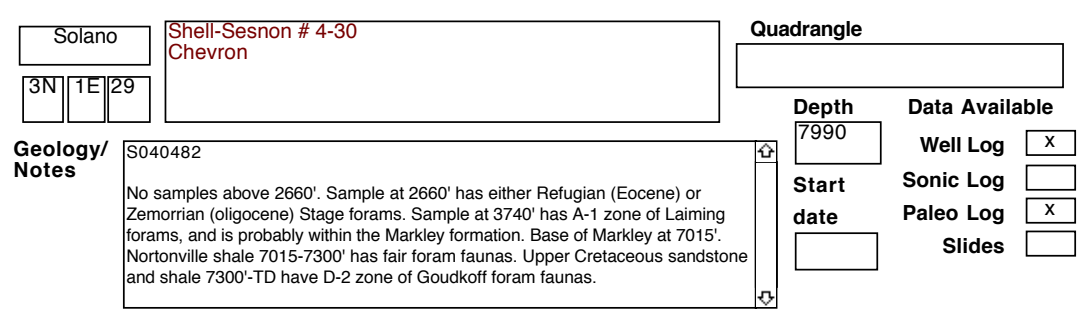

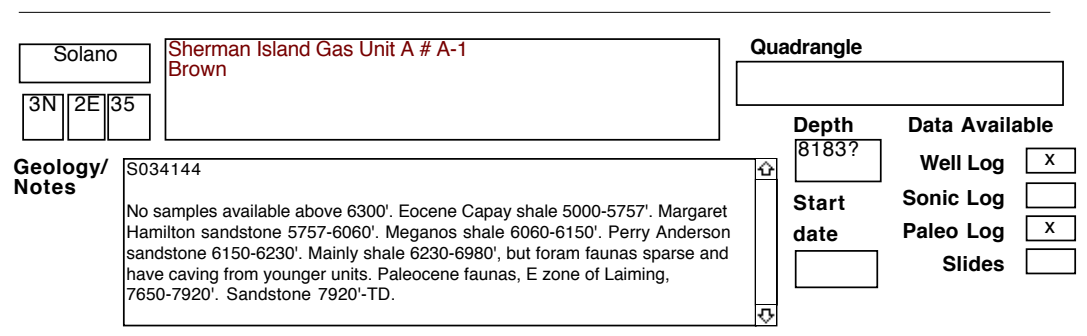

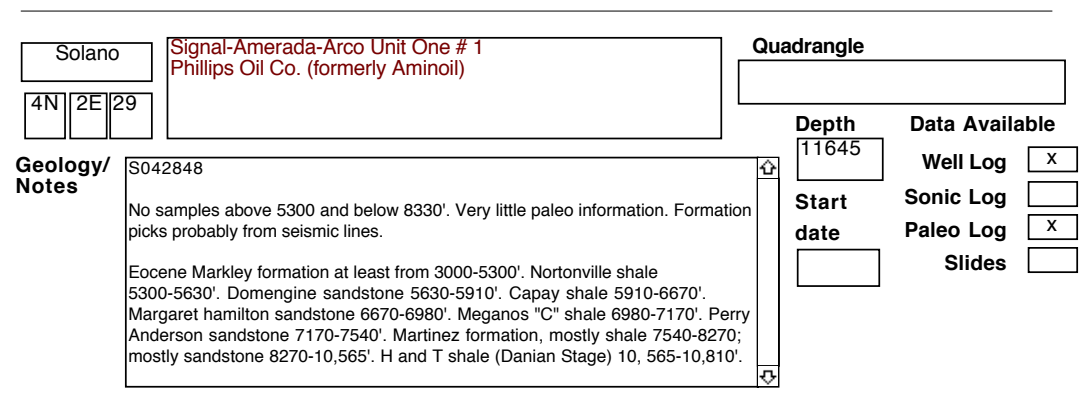


SELECTED OIL TEST WELLS IN CENTRAL CALIFORNIA DATABASE County

\begin{tabular}{ll|l|}
\hline T. & R. Sec. & Name \\
\hline Solano & $\begin{array}{l}\text { Standaard-Fontana Farms \# 5 (redrill) } \\
\text { Kadane and Sons }\end{array}$ \\
\hline $3 N$ & $1 \mathrm{E}$ & 5 \\
\hline
\end{tabular}

\section{Geology/ S034121}

Notes

No paleo information above3572'. Base of Eocene Markley formation at 3512'. Nortonville shale $3512-3675$ '. Wagenet fault at $3675^{\prime}$ brings Markley formation below the Nortonville, 3675-4710'. Repeated Nortonville 4710-5236'. Domengine sandstone 5236-5525' with a flood of lignite at 5415'. Capay shale 5525-6310' has fairly good forams, including Discocyclina near the base. Meganos formation 6310'-TD.

\begin{tabular}{|c|c|c|c|}
\hline \multicolumn{4}{|c|}{ Records } \\
\hline \multicolumn{4}{|c|}{ Quadrangle } \\
\hline & Depth & Data Availa & \\
\hline & 6510 & Well Log & $x$ \\
\hline & Start & Sonic Log & \\
\hline & date & Paleo Log & $x$ \\
\hline & 1958 & Slides & 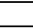 \\
\hline
\end{tabular}

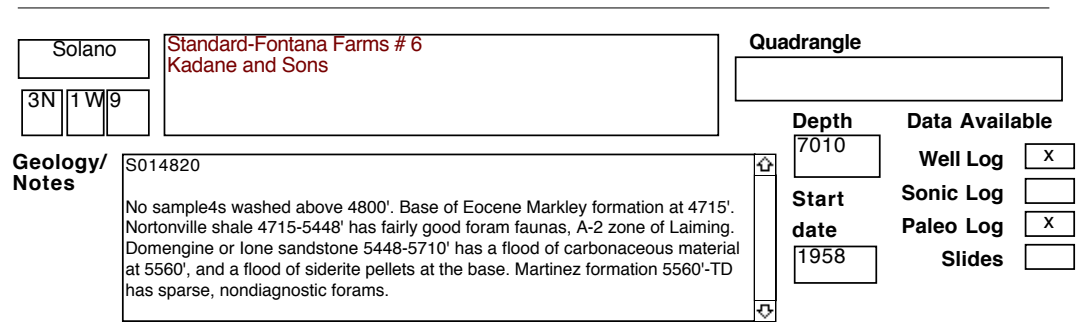

\begin{tabular}{|c|c|}
\hline Solano & $\begin{array}{l}\text { Standard-King \# } 1 \\
\text { Phillips (formerly Aminoil) }\end{array}$ \\
\hline $3 \mathrm{~N}][1 \mathrm{~W} \mid 2$ & \\
\hline
\end{tabular}

\section{Geology/ S202979}

Notes

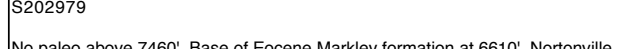
No paleo above $7460^{\prime}$. Base of Eocene Markley formation at $6610^{\prime}$. Nortonville and siderite pellets forams, E zone (Paleocene) of Laiming. Upper Cretaceous sandstone and shale 7995'-TD have fairly good foram faunas, including planktics. E zone of Goudkoff 7995 ' to perhaps 8530 '. F zone $8530-10,660$ '. Fault at 10,660 ' has removed several thousand feet of G-1 zone and possibly lower part of F-1 zone rocks. G-1 zone 10.660-10,780'. G-2 zone 10,780'-TD. Pseudo G-1 biofacies 8280-8870'. 
SELECTED OIL TEST WELLS IN CENTRAL CALIFORNIA DATABASE County

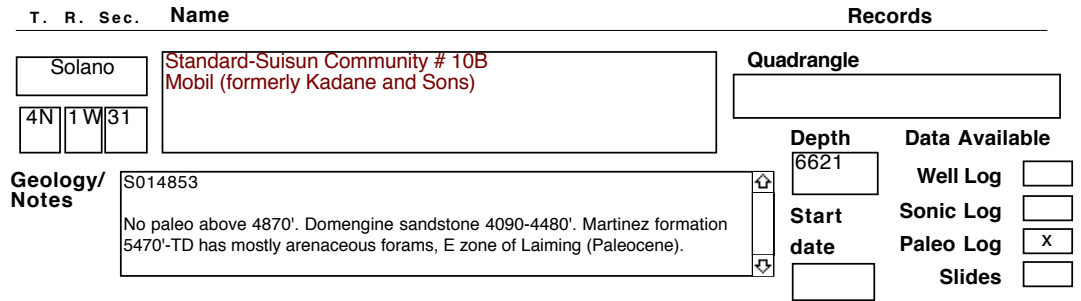

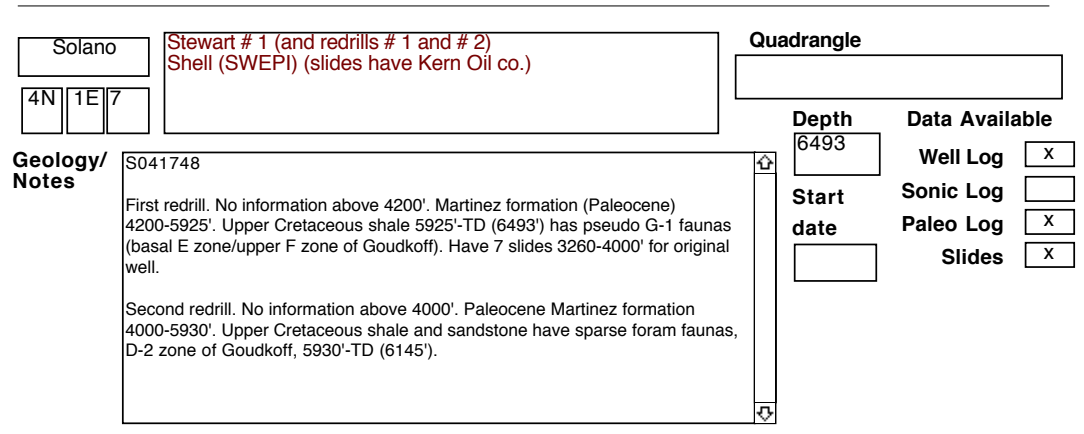

\begin{tabular}{|c|l|l|}
\hline Solano & $\begin{array}{l}\text { Stewart \# } 1 \\
\text { Shell (SWEPI) }\end{array}$ \\
\hline $4 \mathrm{~N}$ & $1 \mathrm{E}$ & 7 \\
\hline
\end{tabular}

Geology/ S041748
Notes

Base of Eocene Markley formation at 960'. Nortonville shale 960-1635'. Rare

forams indicate A-2 zone of Laiming. Domengine sandstone 1635-1810'. Capay

shale 1810-3020'. Mainly shale 3020-4390'; sandstone 4390-5430'; and shale

5430-6050'. Foram faunas correlative with E zone of Laiming (Paleocene)

4096-5994'. Upper Cretaceous, mainly shale, 6050'-TD has fairly good foram

faunas, including planktics. . D-2 zone of Goudkoff (Maastrichtian) 6050-7500'; E

zone (Campanian) 7500-8530'; F zone 8900-9320'; H zone (Turonian) 9320'-TD.

Pseudo G-1 biofacies 8320-9270'. Sacramento shale 8320-9195'. Normal fault at

$9320^{\circ}$

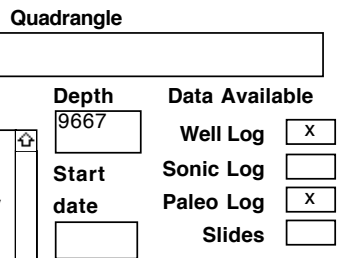

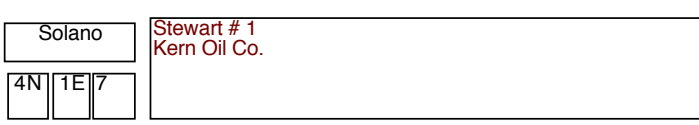

Geology/ 5034263

Notes

Eocene Nortonville shale $700-1300^{\prime}$ has some forams, A-2 zone of Laiming. No data 1300-3260'. Capay shale (part) 3260-3340'. Martinez formation 3340-3700.

No information $3700^{\prime}-\mathrm{TD}$.

\begin{tabular}{|c|c|c|c|}
\hline & Irangle & & \\
\hline & Depth & Data Availab & \\
\hline tis & 4627 & Well Log & \\
\hline & Start & Sonic Log & \\
\hline & date & Paleo Log & $x$ \\
\hline 0 & & Slides & \\
\hline
\end{tabular}

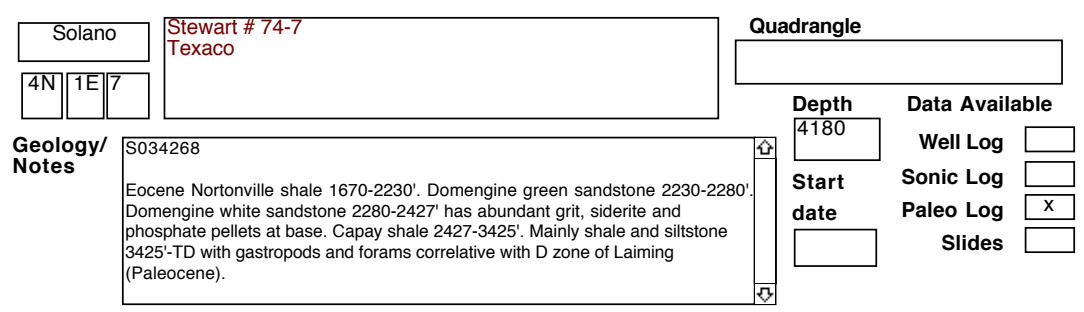




\section{SELECTED OIL TEST WELLS IN CENTRAL CALIFORNIA DATABASE}

County

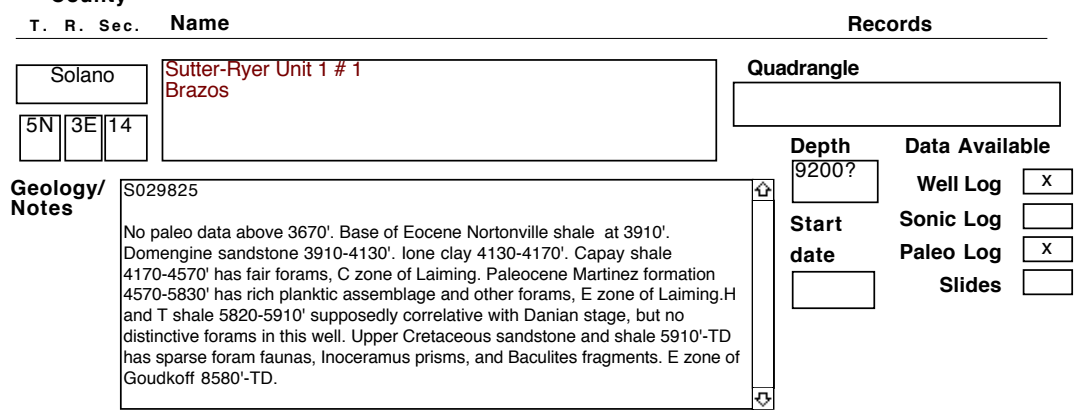

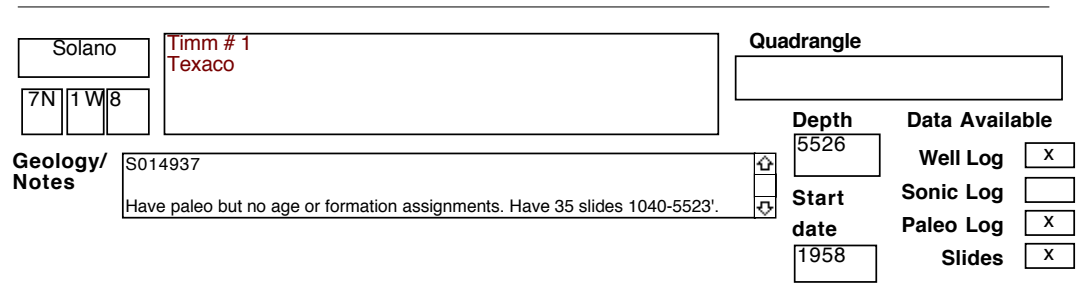

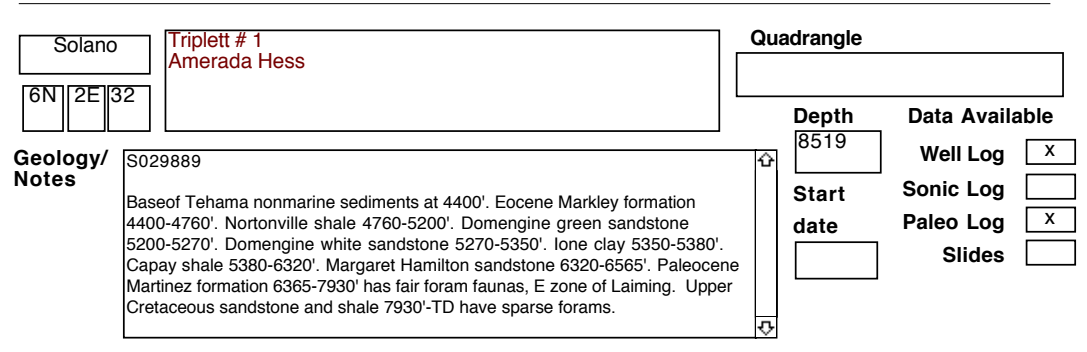

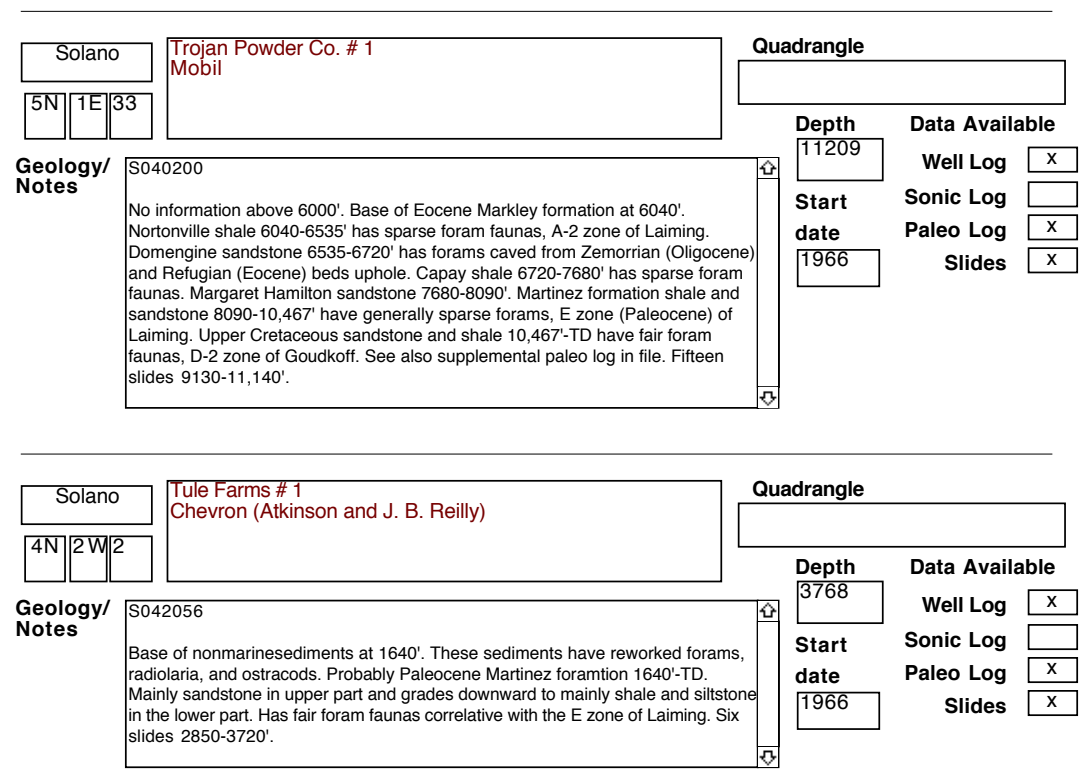


SELECTED OIL TEST WELLS IN CENTRAL CALIFORNIA DATABASE County

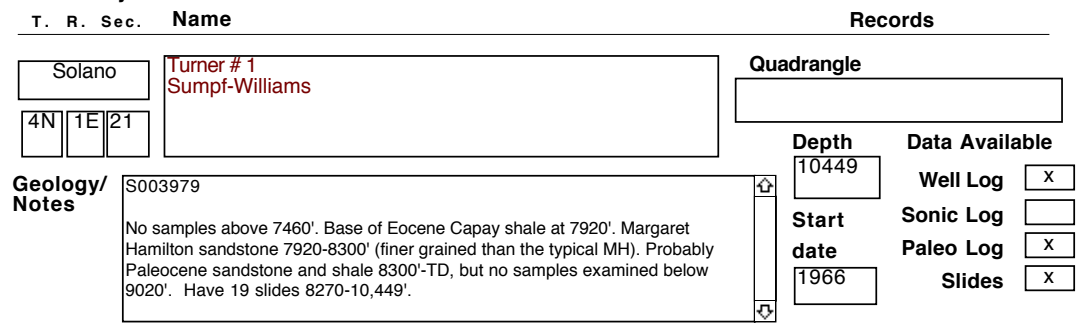

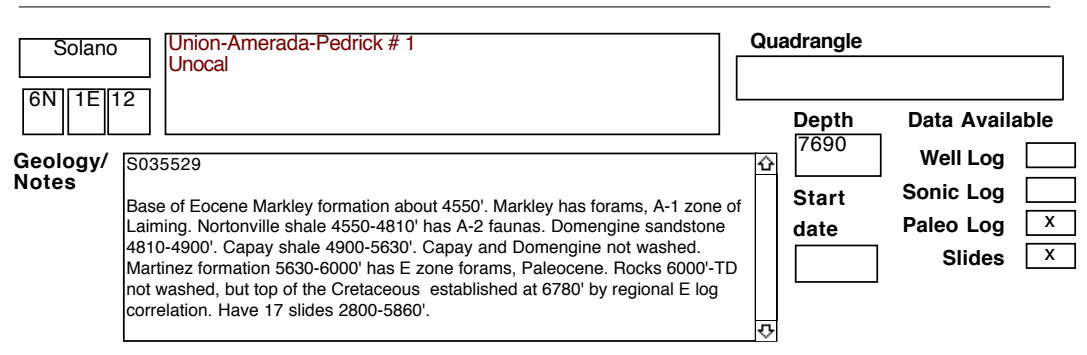

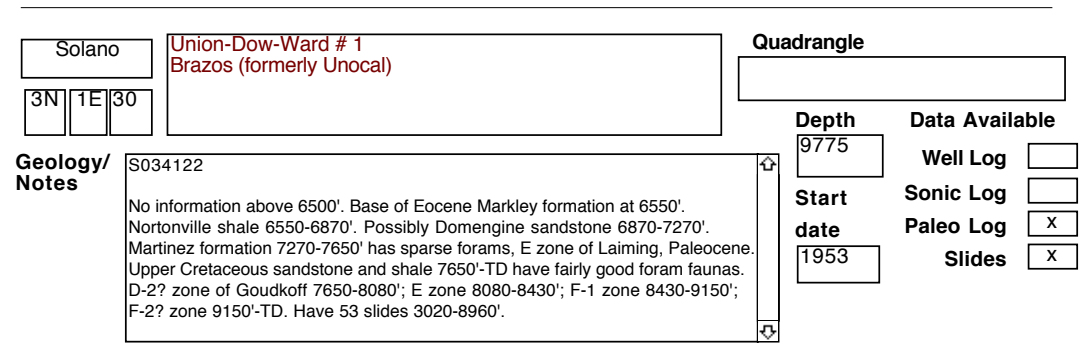

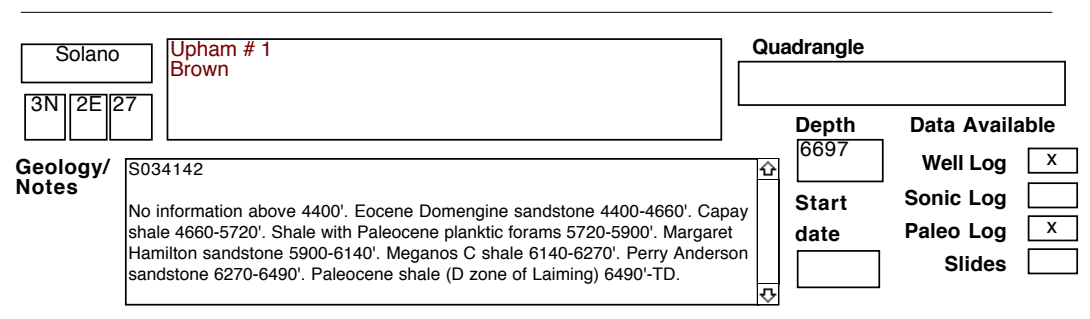

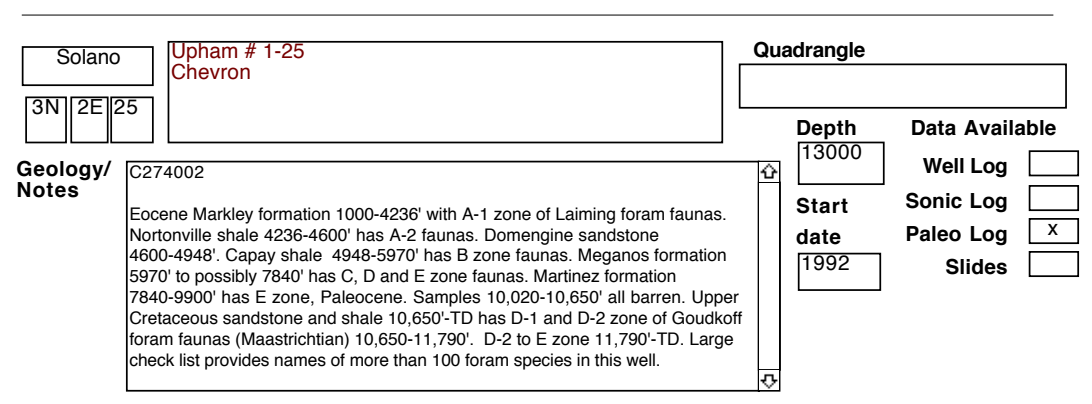


SELECTED OIL TEST WELLS IN CENTRAL CALIFORNIA DATABASE County

\begin{tabular}{|c|c|c|c|c|}
\hline T. R. Sec. & Name & \multicolumn{3}{|c|}{ Records } \\
\hline Solano & \multirow{2}{*}{$\begin{array}{l}\text { Upham \# } 4 \\
\text { Amerada Hess }\end{array}$} & \multicolumn{3}{|c|}{ Quadrangle } \\
\hline \begin{tabular}{|l|l|l|}
$3 N$ & $2 E$ & 13 \\
\end{tabular} & & \multirow{2}{*}{\multicolumn{2}{|c|}{$\begin{array}{l}\text { Depth } \\
9651\end{array}$}} & Data Available \\
\hline \multirow{3}{*}{$\begin{array}{l}\text { Geology/ } \\
\text { Notes }\end{array}$} & 07248 & & & Well Log \\
\hline & e petrography report and thin section photos for 7 cores $9098-9393$ '. & & Start & Sonic Log \\
\hline & & & $\begin{array}{l}\text { date } \\
1983\end{array}$ & $\begin{array}{l}\text { Paleo Log } \\
\text { Slides }\end{array}$ \\
\hline
\end{tabular}

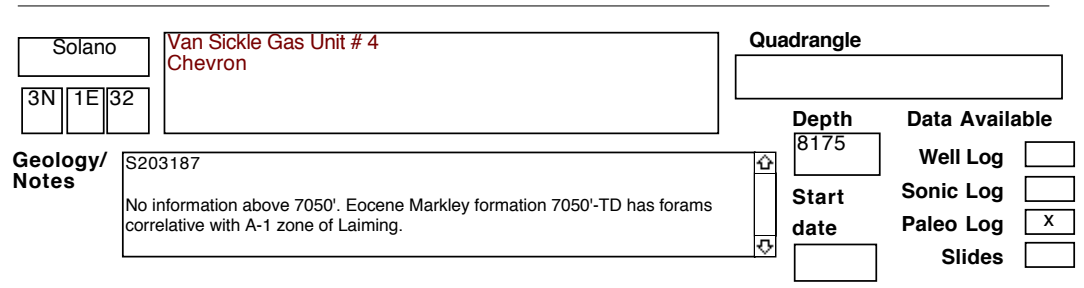

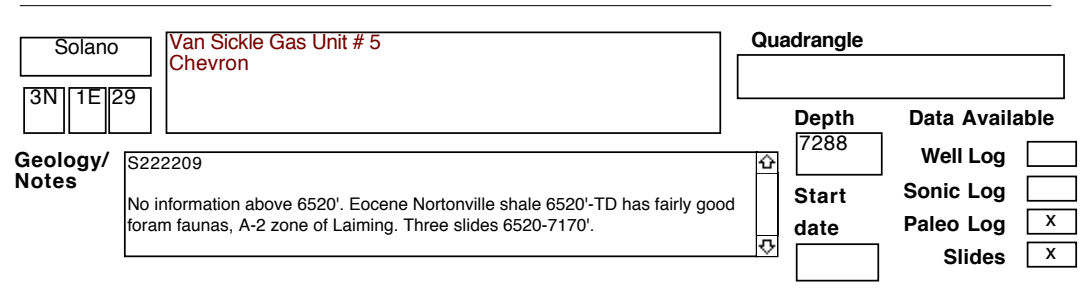

\begin{tabular}{l|l|l|l|l|l|}
\hline Solano & $\begin{array}{l}\text { Van Sickle Gas Unit \# } 6 \\
\text { Chevron }\end{array}$ \\
\hline
\end{tabular}

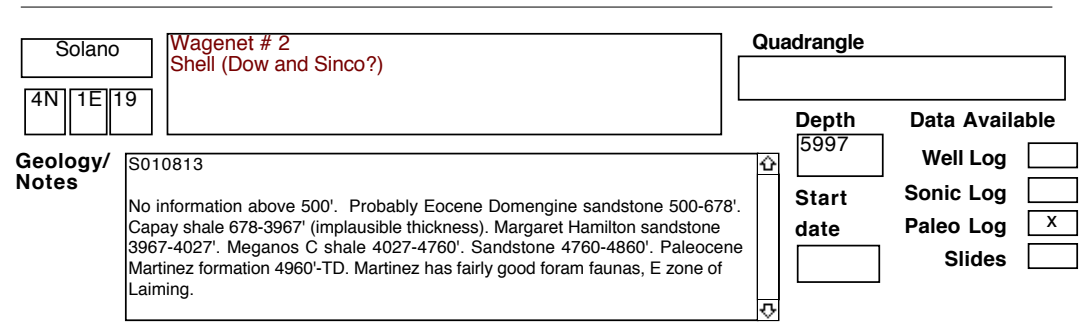


SELECTED OIL TEST WELLS IN CENTRAL CALIFORNIA DATABASE County

\begin{tabular}{|c|c|}
\hline Solano & $\begin{array}{l}\text { Wineman \# } 2 \\
\text { Amerada Hess }\end{array}$ \\
\hline \begin{tabular}{|l||l||l|}
$6 \mathrm{~N}$ & $2 \mathrm{E}$ & 27
\end{tabular} & \\
\hline
\end{tabular}

\section{Geology/ $\$ 029885$}

Notes

No paleo above 4500 '. Eocene Markley channel sediments 3500-4485'. Capay shale 4485-4760' has good foram faunas, B-4 zone of Laiming. Markley probably sits unconformably on the Capay, removing the Nortonville and Domengine.

Paleocene Martinez formation (sandstone and shale) $4760-5880^{\prime}$ has sparse and nondiagnostic foram faunas. Upper Cretaceous sandstone and shale 5880'-TD have sparse forams.

\begin{tabular}{|c|c|c|}
\hline \multicolumn{3}{|c|}{ Records } \\
\hline \multicolumn{3}{|l|}{ Quadrangle } \\
\hline Depth & Data Availab & \\
\hline $6350 ?$ & Well Log & \\
\hline Start & Sonic Log & \\
\hline date & Paleo Log & $\mathrm{x}$ \\
\hline & Slides & 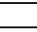 \\
\hline
\end{tabular}

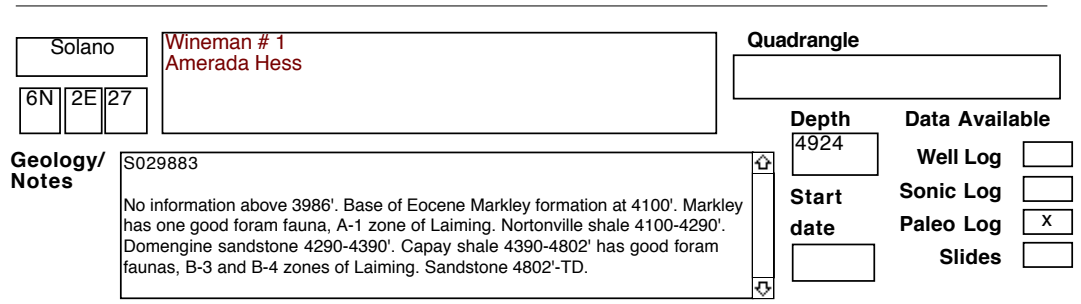

\begin{tabular}{l|l|l|l|l|l|}
\hline Solano & $\begin{array}{l}\text { WZU \# 4X } \\
\text { Amerada Hess }\end{array}$ \\
\hline Geology/ & $\begin{array}{l}\text { S029882 } \\
\text { No information above 3000'. Eocene Markley channel sediments 3000-4605'. } \\
\text { The Markley sits unconformably on the Capay shale. The Capay hasgood foram } \\
\text { faunas, B-4 and C zones of Laiming, and a basal grit at 4790'. Rocks 4800'-TD } \\
\text { called Meganos, but have no forams or lithology provided. }\end{array}$ & $\begin{array}{l}\text { Start } \\
\text { date }\end{array}$ \\
\hline
\end{tabular}

\begin{tabular}{|l|l|l|l|l|}
\hline Solano & $\begin{array}{l}\text { WZU \# 10 (also called H \& T 23-2) } \\
\text { Amerada Hess }\end{array}$ \\
Notes & $\begin{array}{l}\text { So information above 4200'. Base of Eocene Markley channel sediments at } \\
\text { 4470'. Markley sits unconformably on the Capay shale. The Capay, 4470-4690' } \\
\text { has good foram faunas, B zones of Laiming, and a basal grit. Paleocene Martinez } \\
\text { formation 469'-TD has some unusual forams, E zone of Laiming. Capay shale } \\
\text { probably sits unconformably on the Martinez, with all of the C and D zones of } \\
\text { Laiming and part of the E zone removed by erosion before the Capay was } \\
\text { deposited. }\end{array}$ \\
\hline
\end{tabular}

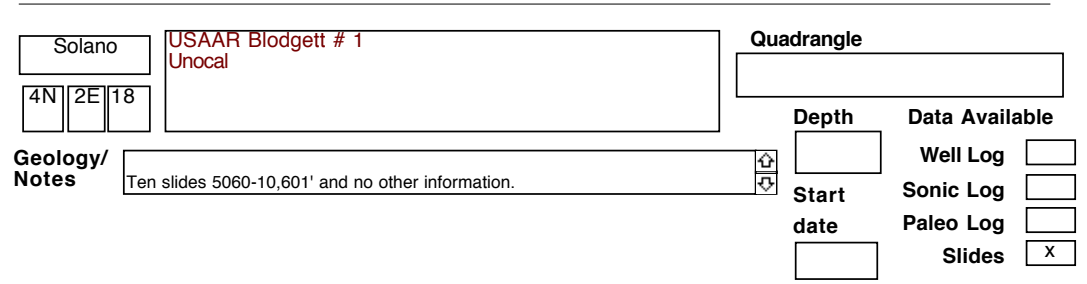


SELECTED OIL TEST WELLS IN CENTRAL CALIFORNIA DATABASE County

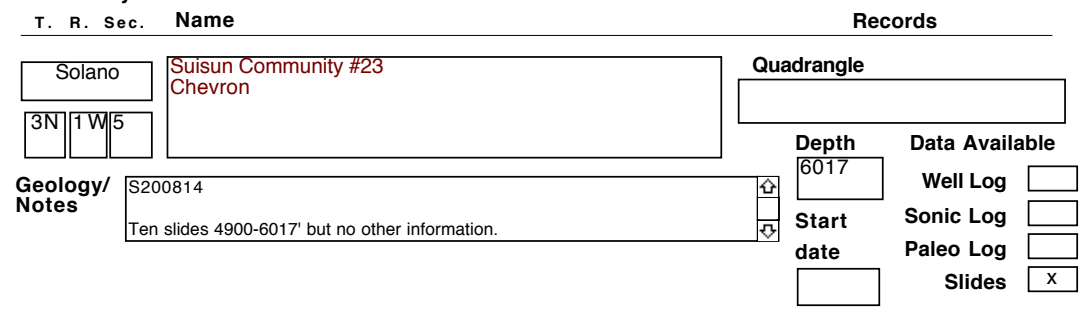

\begin{tabular}{|c|c|c|c|c|c|}
\hline Solano & Suisun community \#24 & Qu & idrangle & & \\
\hline $3 \mathrm{~N}$ & & \multirow{2}{*}{\multicolumn{2}{|c|}{$\begin{array}{l}\text { Depth } \\
7707\end{array}$}} & \multicolumn{2}{|c|}{ Data Available } \\
\hline \multirow{3}{*}{$\begin{array}{l}\text { Geology/ } \\
\text { Notes }\end{array}$} & 9248 & & & Well Log & \\
\hline & slides $5560-6400$ ' but no other information. & a & Start & Sonic Log & \\
\hline & & & & Slides & $\mathrm{x}$ \\
\hline
\end{tabular}

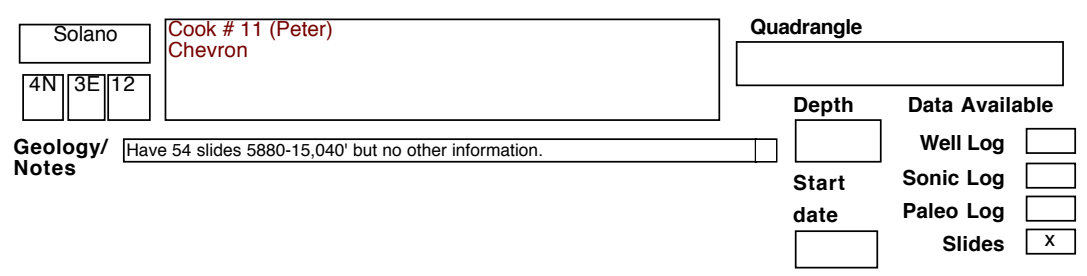

\begin{tabular}{|c|c|c|c|c|}
\hline Solano & \multirow{3}{*}{$\begin{array}{l}\text { Cook Lower Unit \# } 1 \\
\text { Cook }\end{array}$} & \multicolumn{3}{|l|}{ Quadrangle } \\
\hline \begin{tabular}{|l|l|l|}
44 & $3 \mathrm{E}$ \\
\end{tabular} & & \multirow{3}{*}{ Depth } & \multirow{2}{*}{\multicolumn{2}{|c|}{ Data Available }} \\
\hline & & & & \\
\hline \multirow{4}{*}{$\begin{array}{l}\text { Geology/ } \\
\text { Notes }\end{array}$} & slides $3640-5800^{\prime}$ but no other information. & & Well Log & \\
\hline & & Start & Sonic Log & \\
\hline & & date & Paleo Log & \\
\hline & & & Slides & $\mathrm{x}$ \\
\hline
\end{tabular}

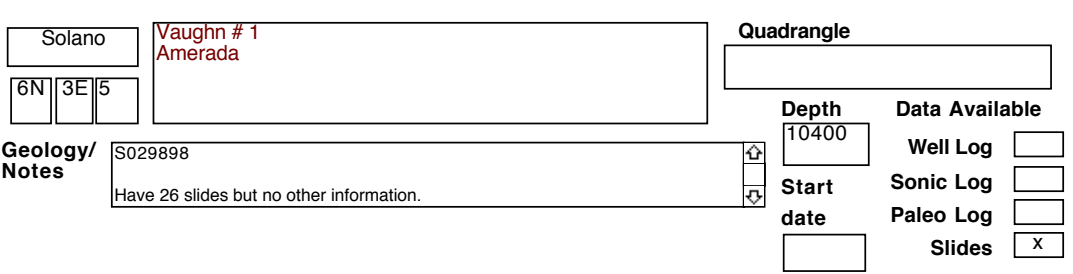


SELECTED OIL TEST WELLS IN CENTRAL CALIFORNIA DATABASE County

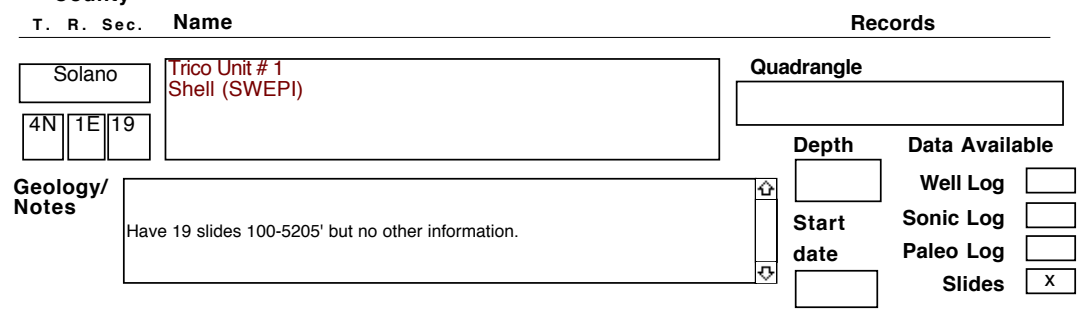

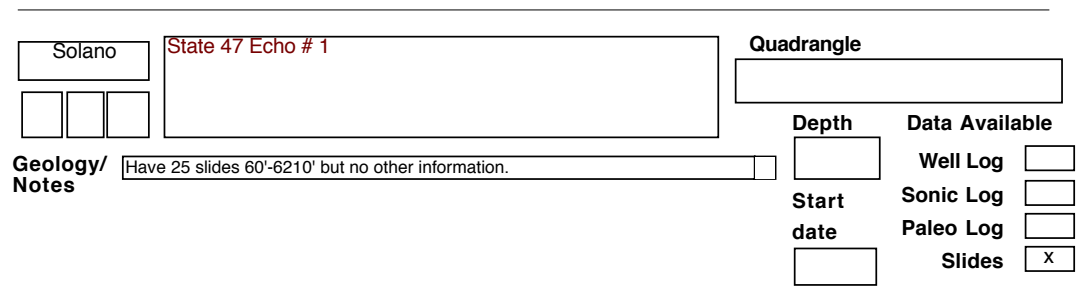

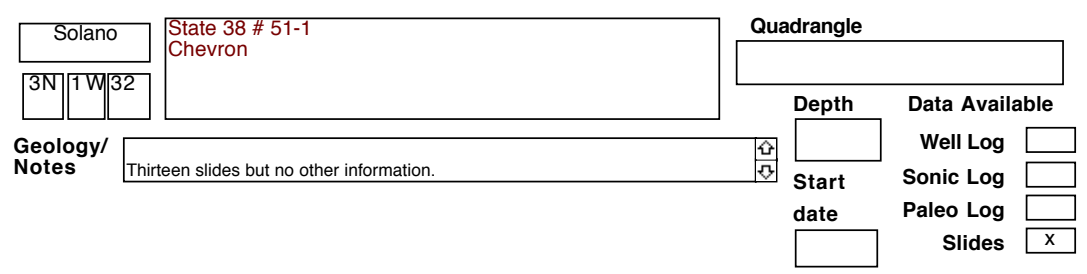

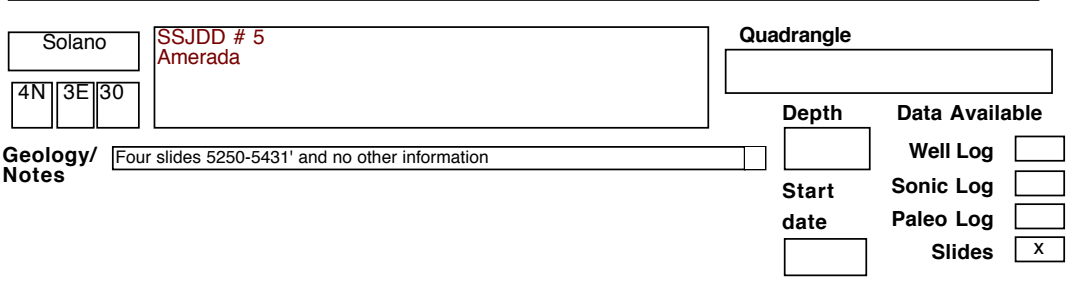

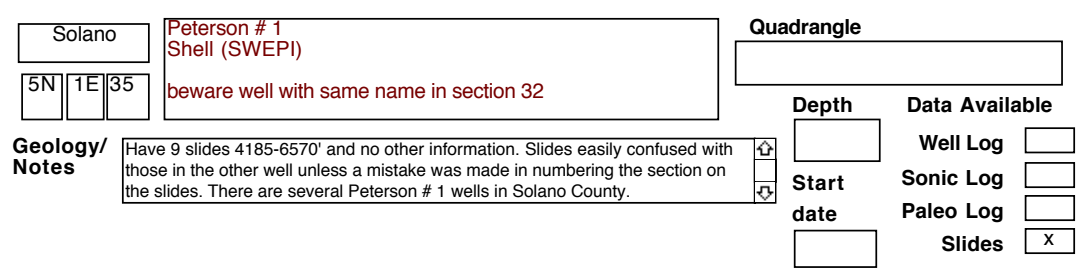


SELECTED OIL TEST WELLS IN CENTRAL CALIFORNIA DATABASE County

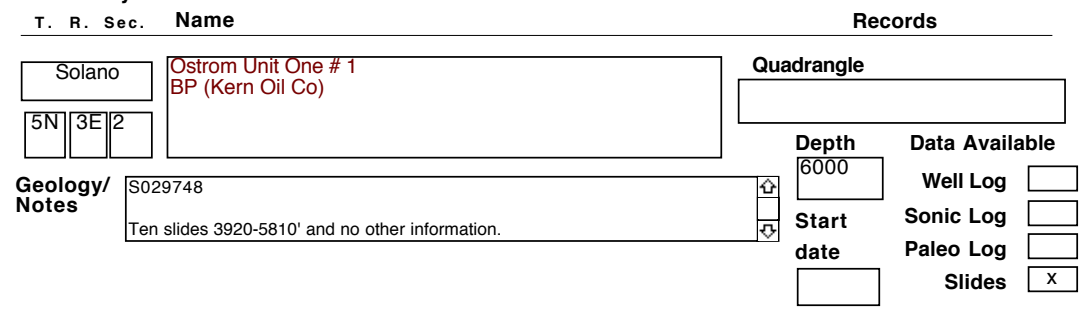

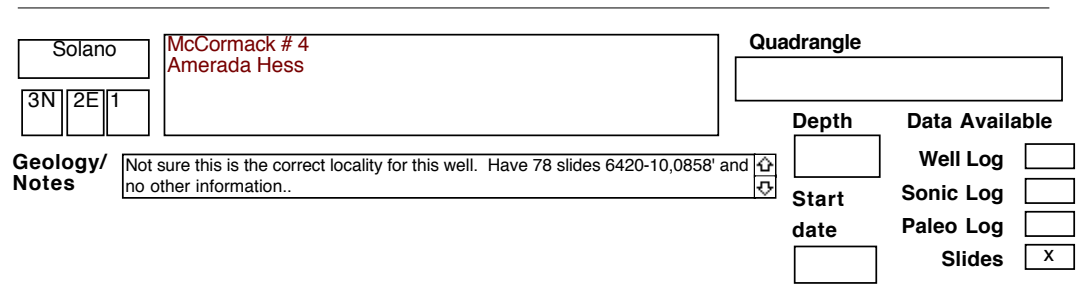

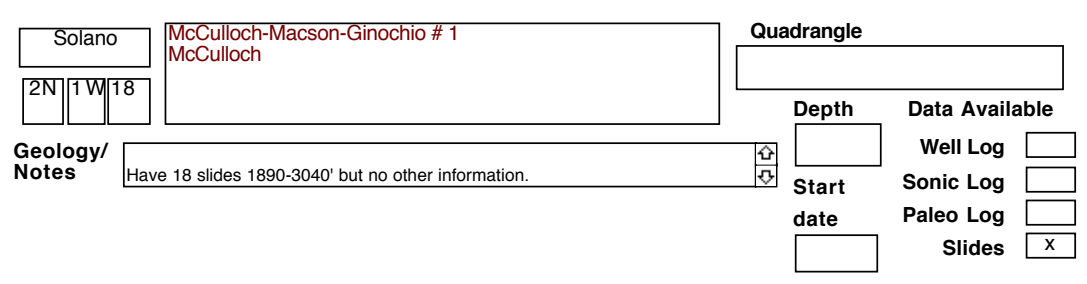

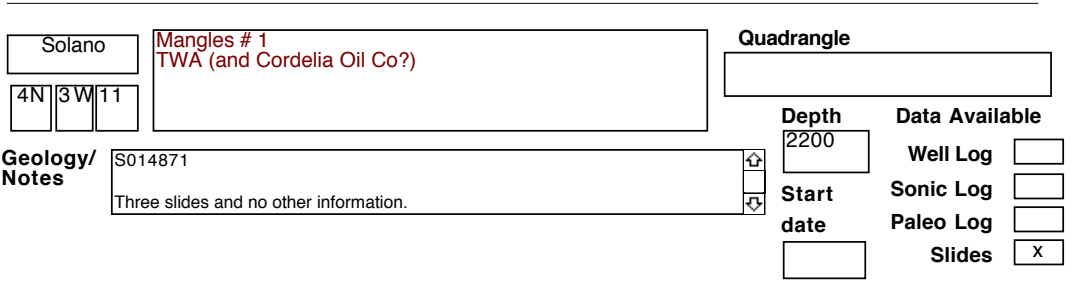

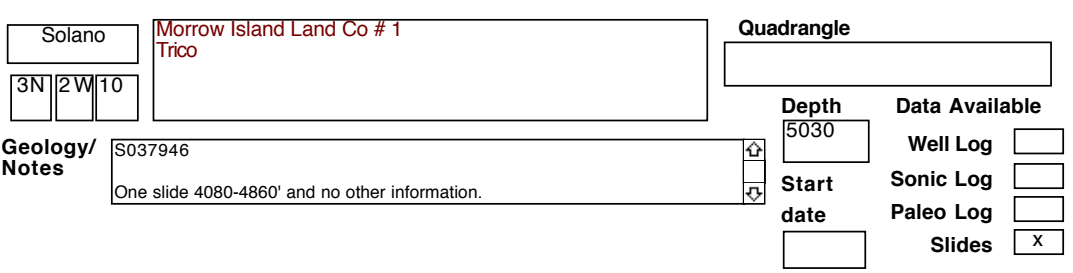


SELECTED OIL TEST WELLS IN CENTRAL CALIFORNIA DATABASE County

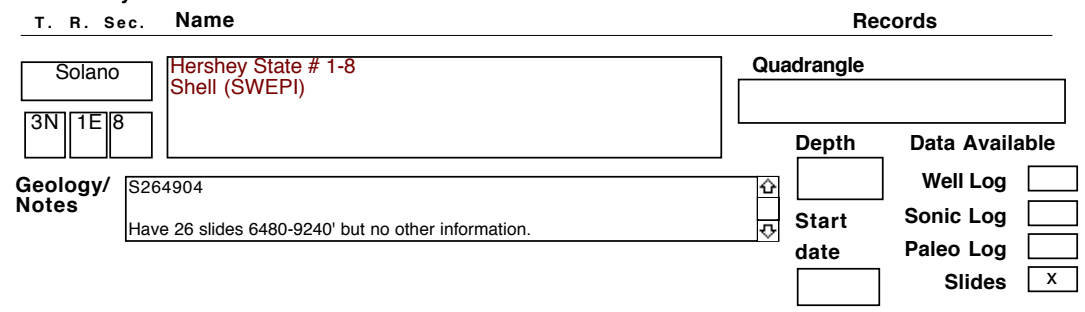

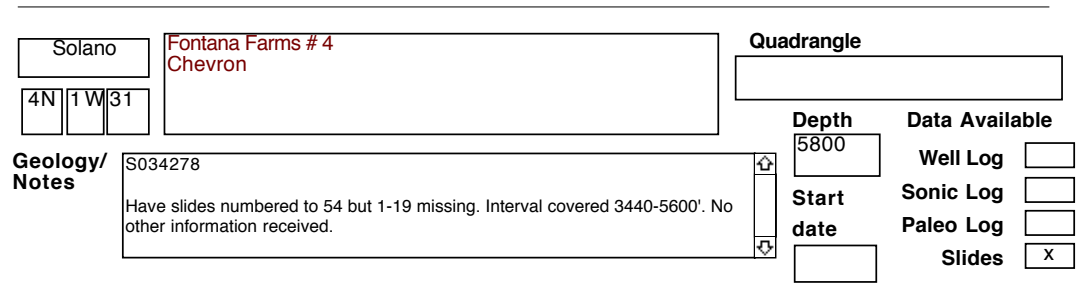

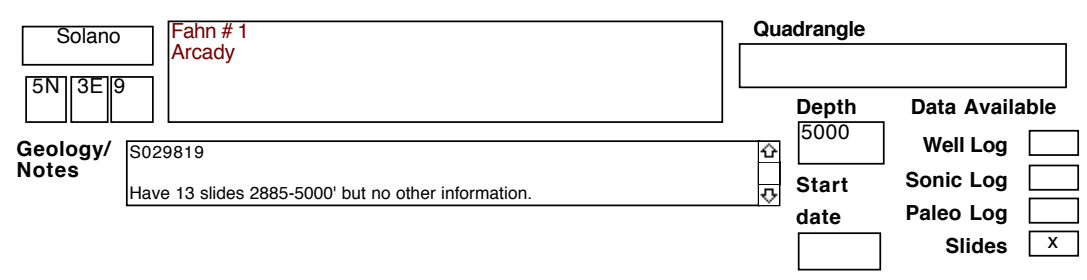

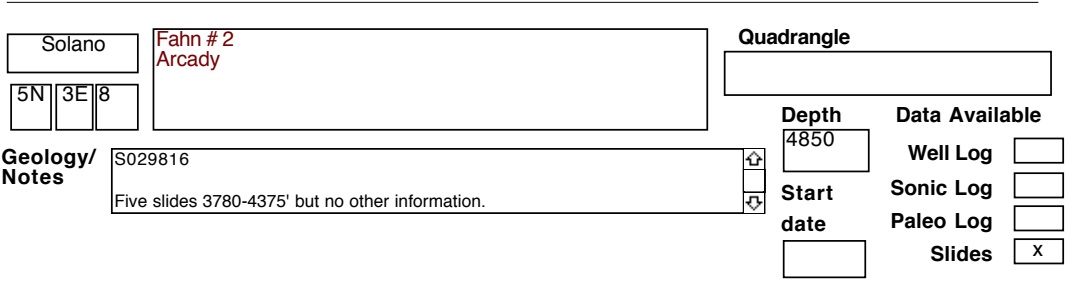

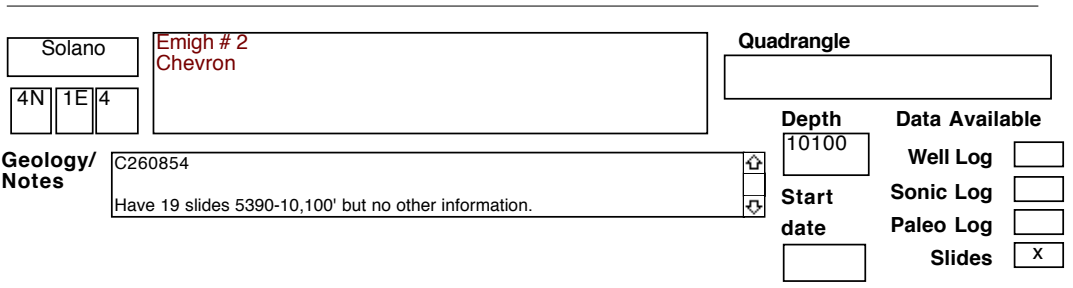


SELECTED OIL TEST WELLS IN CENTRAL CALIFORNIA DATABASE County

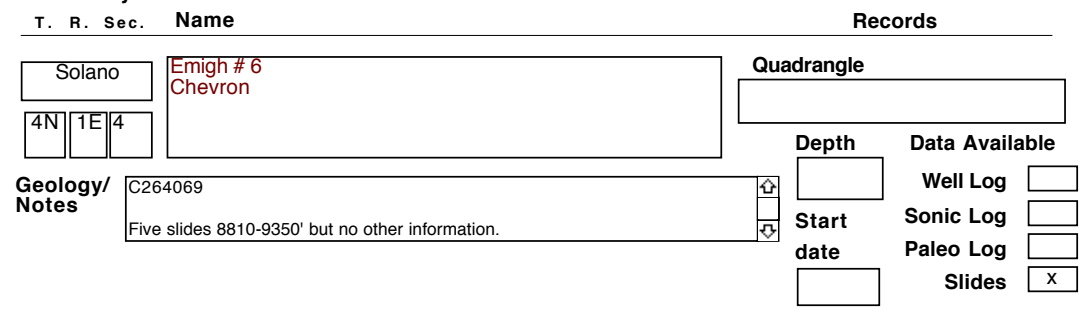

\begin{tabular}{|c|c|c|c|c|c|}
\hline Solano & \multirow{2}{*}{$\begin{array}{l}\text { Emigh \# 1 } \\
\text { Amerada }\end{array}$} & \multicolumn{4}{|c|}{ Quadrangle } \\
\hline$\left.4 N|| 2 ?\right|^{2}$ & & & & \multicolumn{2}{|c|}{ Data Available } \\
\hline \multirow{3}{*}{$\begin{array}{l}\text { Geology/ } \\
\text { Notes }\end{array}$} & & 6 & & Well Log & \\
\hline & e slides $2100-4348$ but no other information & . & \multirow{2}{*}{$\begin{array}{l}\text { Start } \\
\text { date }\end{array}$} & Sonic Log & \\
\hline & & & & Slides & $x$ \\
\hline
\end{tabular}

\begin{tabular}{|c|c|c|c|c|}
\hline Solano & \multirow{2}{*}{$\begin{array}{l}\text { Emigh \# 1-2 } \\
\text { Channel Expl;oration co. }\end{array}$} & \multicolumn{3}{|l|}{ Quadrangle } \\
\hline $4 \mathrm{~N}\left\|\left.\right|^{1 \mathrm{E}}\right\|^{2}$ & & \multirow[t]{2}{*}{ Depth } & \multicolumn{2}{|c|}{ Data Available } \\
\hline \multirow{4}{*}{$\begin{array}{l}\text { Geology/ } \\
\text { Notes }\end{array}$} & 33 slides $3740-12,990$ ' but no other information. & & Well Log & \\
\hline & & Start & Sonic Log & \\
\hline & & date & Paleo Log & \\
\hline & & & Slides & $x$ \\
\hline
\end{tabular}

\begin{tabular}{|c|c|c|c|c|c|}
\hline Solano & \multirow{2}{*}{$\begin{array}{l}\text { Elmira \# 1-21 } \\
\text { Kadane }\end{array}$} & \multicolumn{4}{|c|}{ Quadrangle } \\
\hline \begin{tabular}{|l||l|l|}
$6 N$ & $2 E$ & 21 \\
\end{tabular} & & \multirow{2}{*}{\multicolumn{2}{|c|}{$\begin{array}{l}\text { Depth } \\
5243\end{array}$}} & \multicolumn{2}{|c|}{ Data Available } \\
\hline \multirow{3}{*}{$\begin{array}{l}\text { Geology/ } \\
\text { Notes }\end{array}$} & 9873 & & & Well Log & \\
\hline & slides $5060-5240^{\prime}$ but no other information. & & Start & Sonic Log & \\
\hline & & & & Slides & $x$ \\
\hline
\end{tabular}

\begin{tabular}{|c|c|c|c|c|}
\hline Solano & DH \& P \# 1 & Quadrangle & & \\
\hline${ }^{6 N}\left\|^{3 E}\right\|^{28}$ & & Depth & \multicolumn{2}{|l|}{ Data Available } \\
\hline \multirow{3}{*}{\multicolumn{2}{|c|}{$\begin{array}{l}\text { Geology/ Seven slides } 4161-8580^{\prime} \text { but no other information. } \\
\text { Notes }\end{array}$}} & & Well Log & \\
\hline & & Start & Sonic Log & \\
\hline & & date & $\begin{array}{r}\text { Paleo Log } \\
\text { Slides }\end{array}$ & $\bar{x}$ \\
\hline
\end{tabular}


SELECTED OIL TEST WELLS IN CENTRAL CALIFORNIA DATABASE County

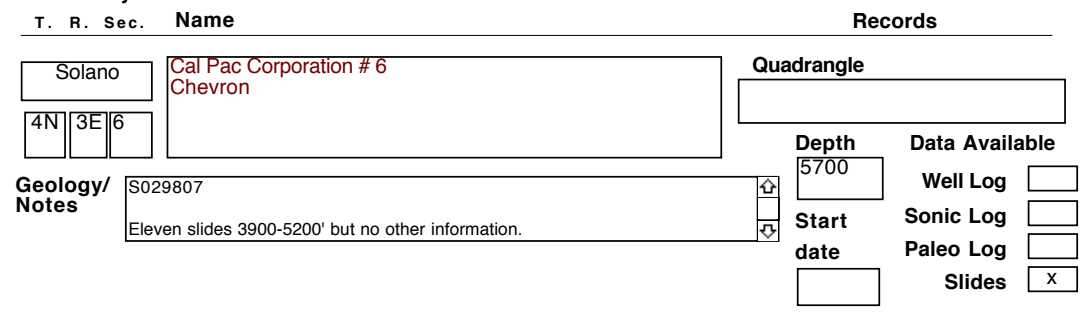

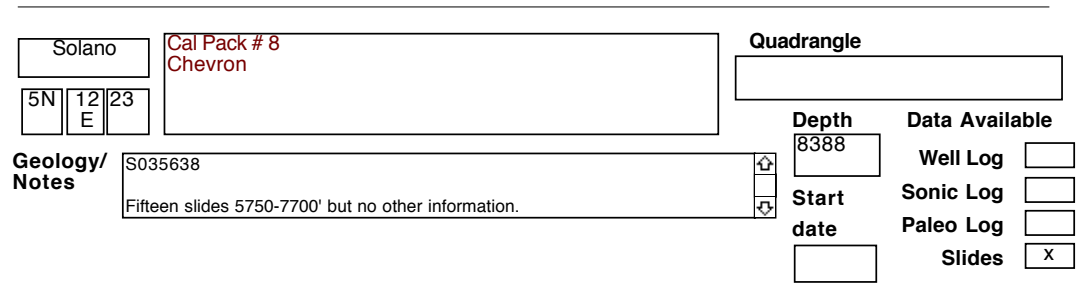

\begin{tabular}{l|l|l|l|l|l|}
\hline \multicolumn{1}{|c|}{ Solano } & $\begin{array}{l}\text { Arnold Ranch \#2 } \\
\text { Chevron }\end{array}$ & \multicolumn{2}{l|}{ Quadrangle } \\
\hline
\end{tabular}

\begin{tabular}{|l|l|l|l|l|l|}
\hline Solano & $\begin{array}{l}\text { Anderson \# 7 (Perry) } \\
\text { Chevron }\end{array}$ \\
Geology/ \\
Notes
\end{tabular}

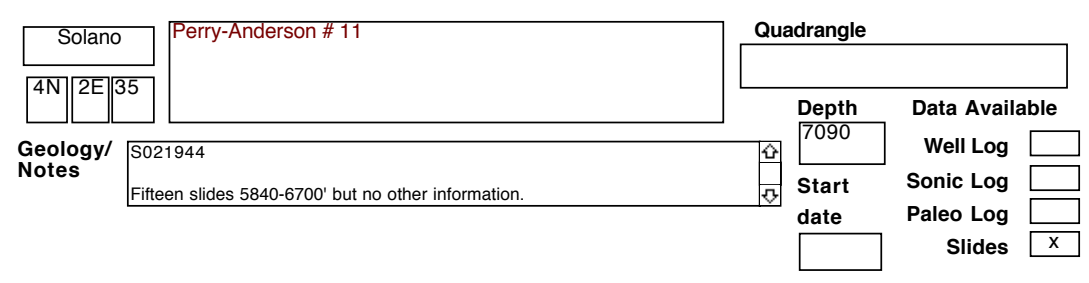


SELECTED OIL TEST WELLS IN CENTRAL CALIFORNIA DATABASE County

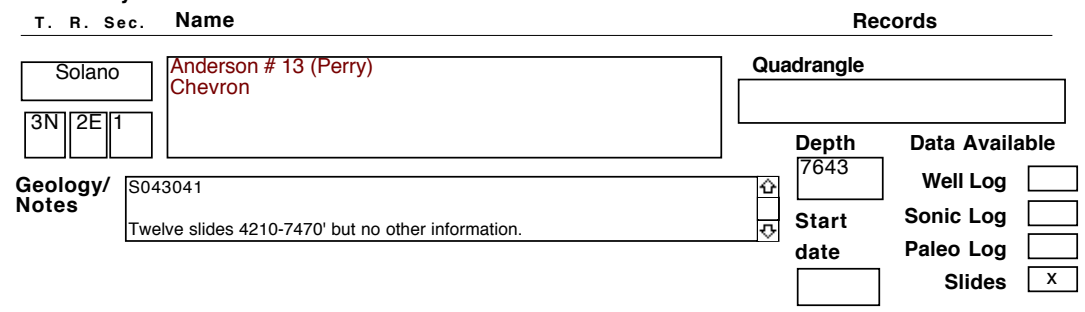

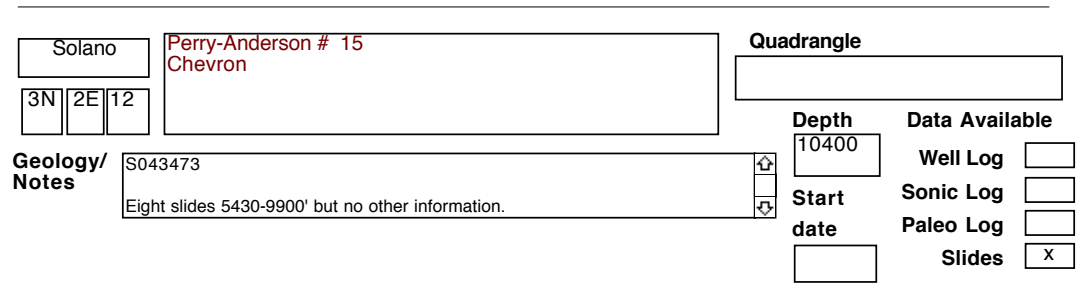

\begin{tabular}{|c|c|c|c|c|}
\hline \multirow[t]{2}{*}{ Solano } & \multirow{2}{*}{$\begin{array}{l}\text { Anderson \# H-10 (Perry) } \\
\text { Chevron }\end{array}$} & \multicolumn{3}{|l|}{ Quadrangle } \\
\hline & & Depth & Data Availab & \\
\hline \multirow{4}{*}{$\begin{array}{l}\text { Geology/ } \\
\text { Notes }\end{array}$} & ht slides $5660-6900^{\prime}$ but no other information. & T & Well Log & \\
\hline & & Start & Sonic Log & \\
\hline & & date & Paleo Log & \\
\hline & & & Slides & $\mathrm{x}$ \\
\hline
\end{tabular}

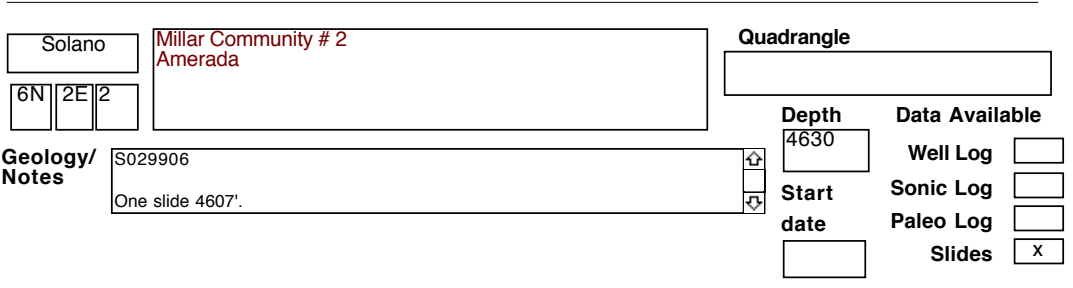

\begin{tabular}{|c|c|c|c|c|c|}
\hline Solano & \multirow{3}{*}{$\begin{array}{l}\text { Moresco Brothers \# } 1 \\
\text { ARCO (Atlantic) }\end{array}$} & \multicolumn{4}{|c|}{ Quadrangle } \\
\hline \multicolumn{5}{|l|}{\begin{tabular}{|l|l|l|}
$5 N$ & $2 E$ & 12 \\
\end{tabular}} & \\
\hline & & & 9490 & \multicolumn{2}{|c|}{ Data Available } \\
\hline \multirow{3}{*}{$\begin{array}{l}\text { Geology/ } \\
\text { Notes }\end{array}$} & 6165 & 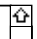 & & Well Log & \\
\hline & ee slides 9490-9670'. & $\theta$ & Start & Sonic Log & \\
\hline & & & date & $\begin{array}{r}\text { Paleo Log } \\
\text { Slides }\end{array}$ & $x$ \\
\hline
\end{tabular}


SELECTED OIL TEST WELLS IN CENTRAL CALIFORNIA DATABASE County

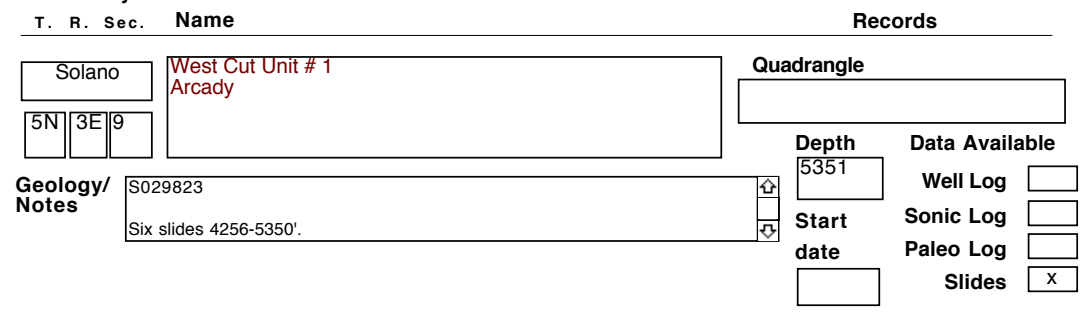

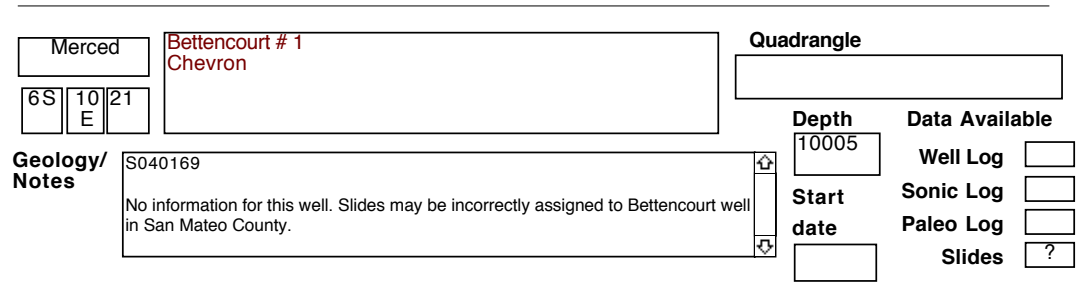

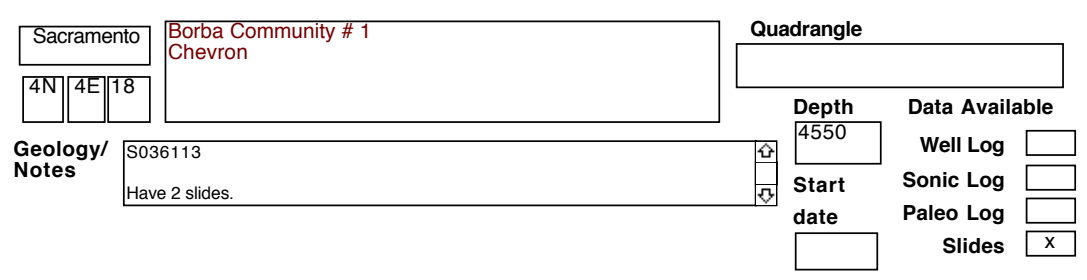

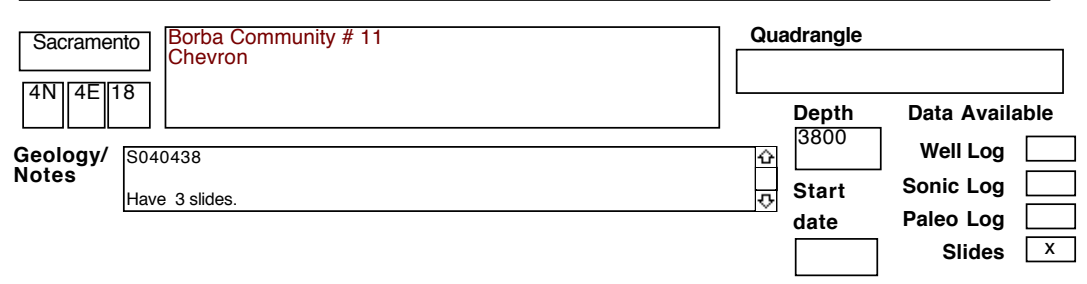

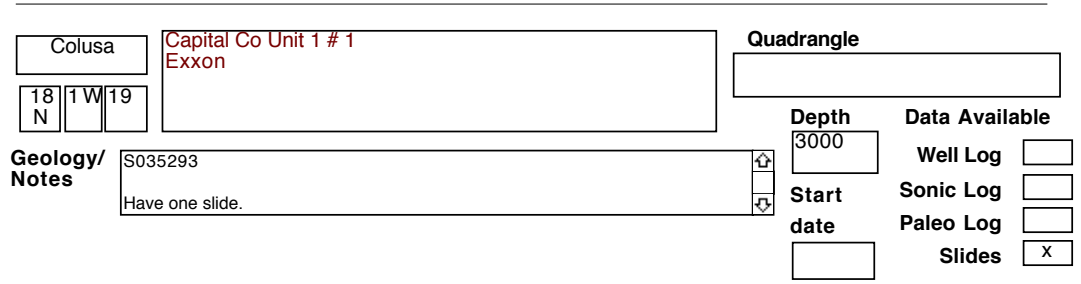


SELECTED OIL TEST WELLS IN CENTRAL CALIFORNIA DATABASE County

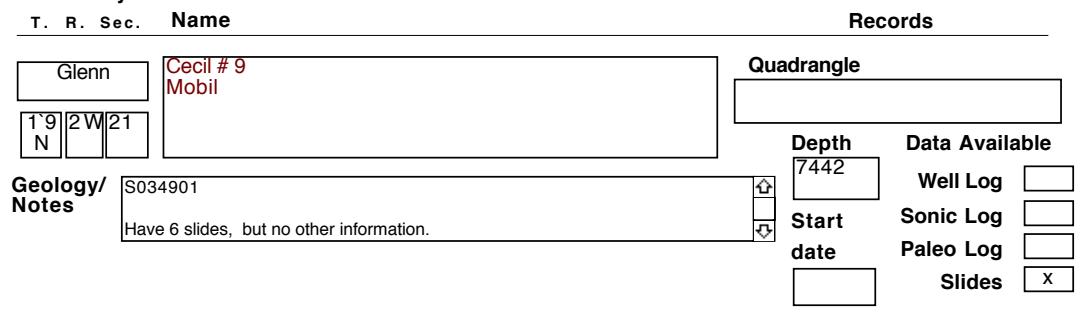

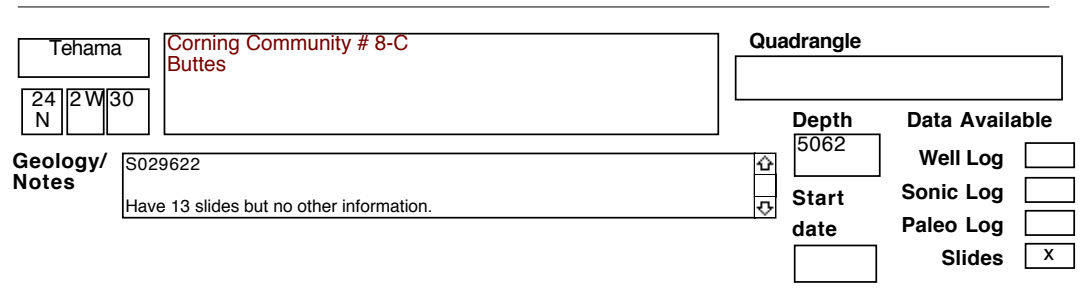

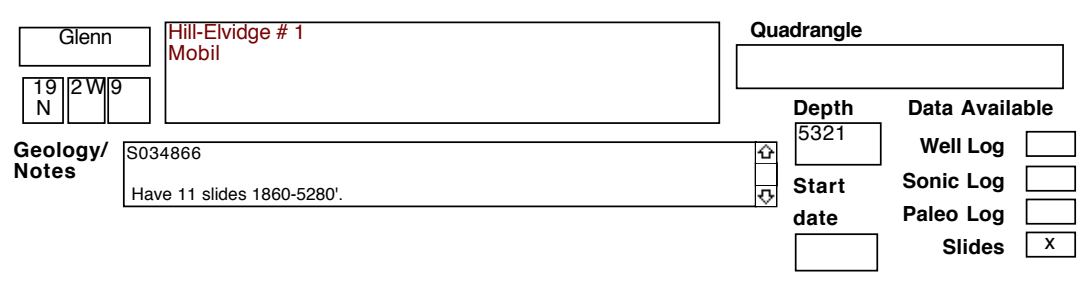

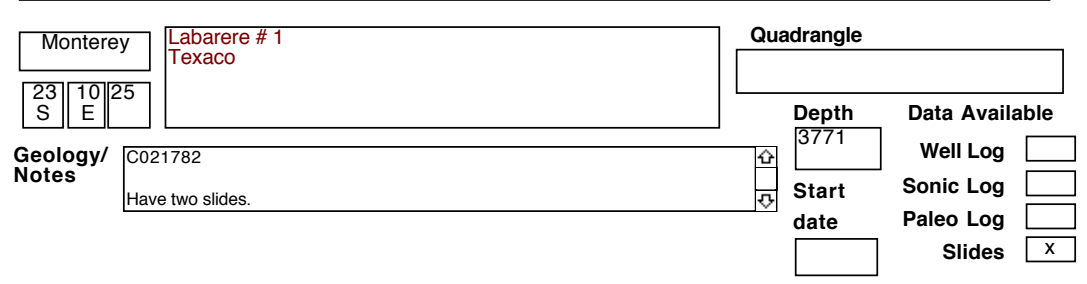

\begin{tabular}{l|l|l|l|l|l|}
\hline \multicolumn{1}{|c|}{ Glenn } & $\begin{array}{l}\text { Miner Jones \#2 } \\
\text { Mobil (formerly General Petroleum) }\end{array}$ & \multicolumn{2}{l|}{ Quadrangle } \\
& & & \\
\hline
\end{tabular}


SELECTED OIL TEST WELLS IN CENTRAL CALIFORNIA DATABASE County

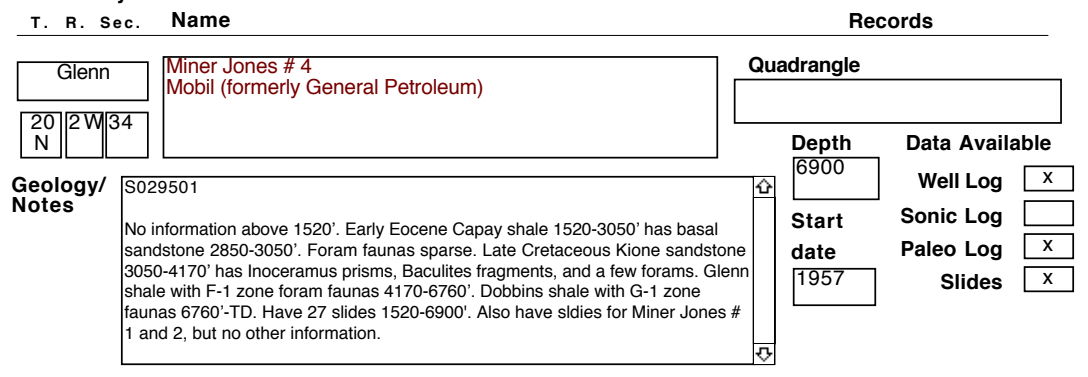

\begin{tabular}{l|l|l|l|l|l|}
\hline Tehama & $\begin{array}{l}\text { Saldubehere \# 2 } \\
\text { Dewitt (formerly Superior) }\end{array}$ & \multicolumn{2}{l|}{ Quadrangle } \\
\hline
\end{tabular}

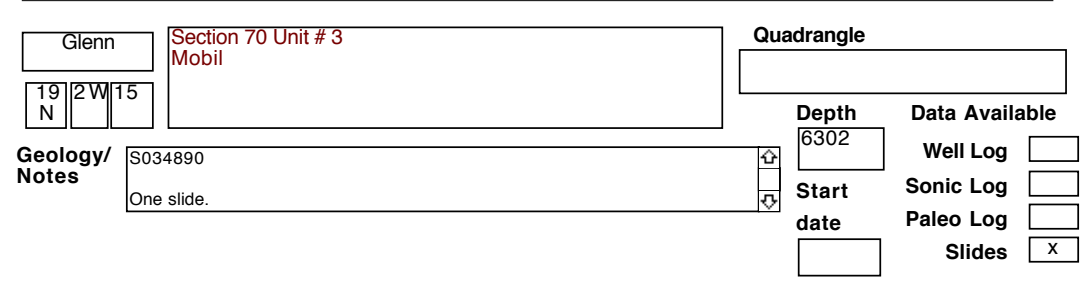

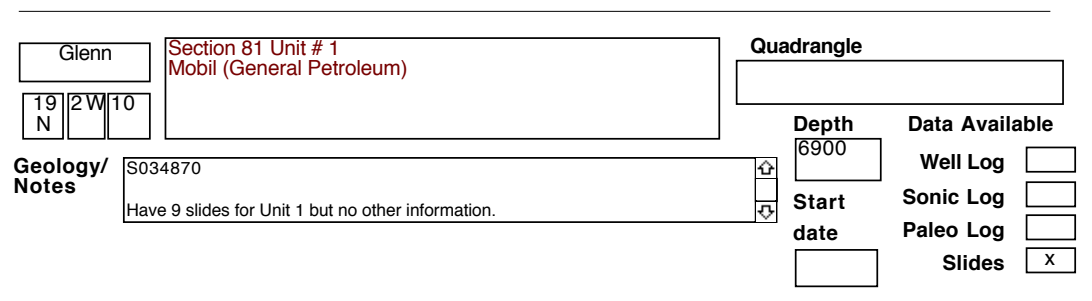

\begin{tabular}{|c|c|c|c|c|c|}
\hline Monterey & Shell B (NCT-1) \# 1 & \multicolumn{4}{|c|}{ Quadrangle } \\
\hline \begin{tabular}{|c|c||c|}
22 & $9 \mathrm{E}$ & 22 \\
$\mathrm{~S}$ & & \\
\end{tabular} & & \multirow{2}{*}{\multicolumn{2}{|c|}{$\begin{array}{l}\text { Depth } \\
6564\end{array}$}} & \multicolumn{2}{|l|}{ Data Available } \\
\hline \multirow{3}{*}{$\begin{array}{l}\text { Geology/ } \\
\text { Notes }\end{array}$} & 24277 & & & Well Log & \\
\hline & o slides but no other data. & $\theta$ & Start & Sonic Log & \\
\hline & & & & Slides & $\mathrm{x}$ \\
\hline
\end{tabular}


SELECTED OIL TEST WELLS IN CENTRAL CALIFORNIA DATABASE

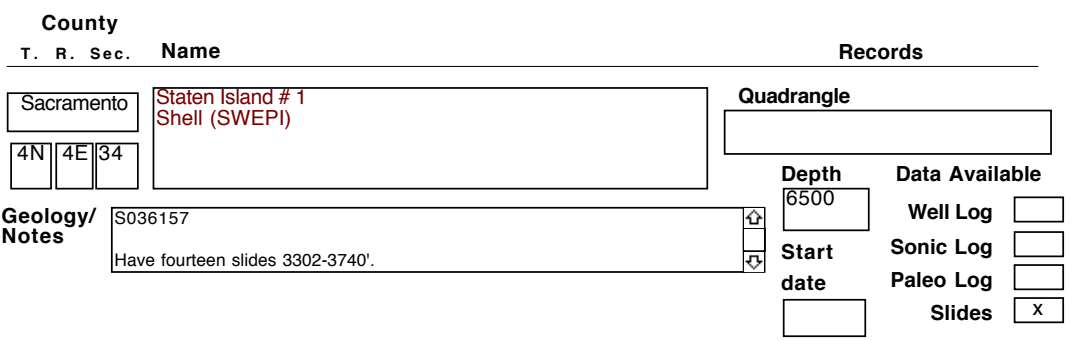

\begin{tabular}{|c|c|c|c|c|c|}
\hline Solano & \multirow[t]{2}{*}{$\begin{array}{l}\text { Suisun Community \#1 } \\
\text { Chevron }\end{array}$} & \multicolumn{4}{|c|}{$\begin{array}{l}\text { Quadrangle } \\
\text { Fairfield South 7.5' }\end{array}$} \\
\hline \multicolumn{2}{|l|}{\begin{tabular}{|l|l|}
$4 \mathrm{~N}$ & $2 \mathrm{~V}$ \\
\end{tabular}} & & Depth & Data Availab & \\
\hline \multirow{3}{*}{$\begin{array}{l}\text { Geology/ } \\
\text { Notes }\end{array}$} & 14868 & 今 & & Well Log & \\
\hline & ve 66 slides. & a & Start & Sonic Log & \\
\hline & & & & Slides & $\mathrm{x}$ \\
\hline
\end{tabular}

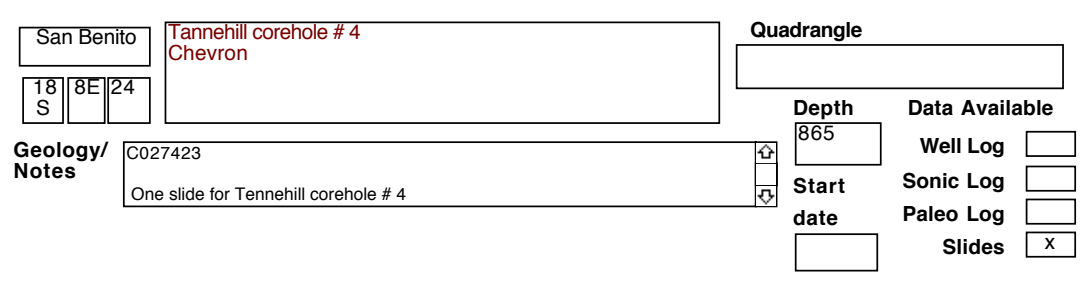

\begin{tabular}{l|l|l|l|l|l|}
\hline \multicolumn{1}{|c|}{ Colusa } & $\begin{array}{l}\text { Welch Unit One \# 1 } \\
\text { Loyal Resources (Honolulu) }\end{array}$ & \multicolumn{2}{|c|}{ Quadrangle } \\
\hline
\end{tabular}

\begin{tabular}{|c|c|c|c|c|c|}
\hline Solano & Chevron corehole \# 1 (Tolenas Hills) & \multicolumn{4}{|c|}{ Quadrangle } \\
\hline $5 \mathrm{~N} / \mathrm{W}$ & & \multirow{2}{*}{\multicolumn{2}{|c|}{$\begin{array}{l}\text { Depth } \\
190\end{array}$}} & \multicolumn{2}{|c|}{ Data Available } \\
\hline \multirow{3}{*}{$\begin{array}{l}\text { Geology/ } \\
\text { Notes }\end{array}$} & 14888 & & & Well Log & \\
\hline & ven slides $10-190^{\prime}$. & ⿹ & Start & Sonic Log & \\
\hline & & & & Slides & $\mathrm{x}$ \\
\hline
\end{tabular}


SELECTED OIL TEST WELLS IN CENTRAL CALIFORNIA DATABASE County

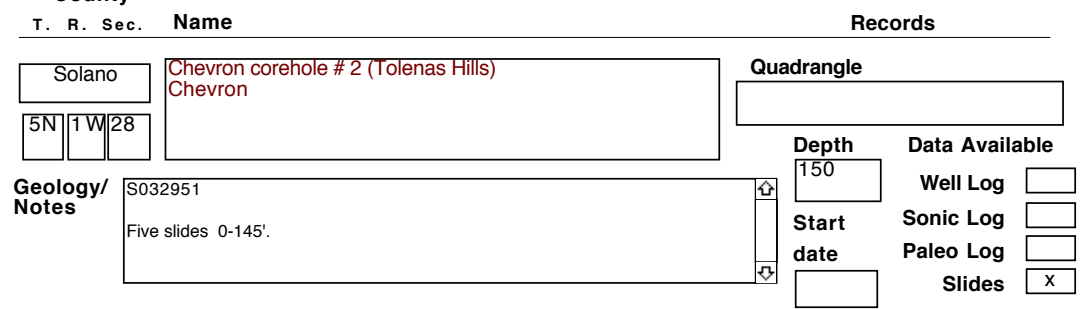

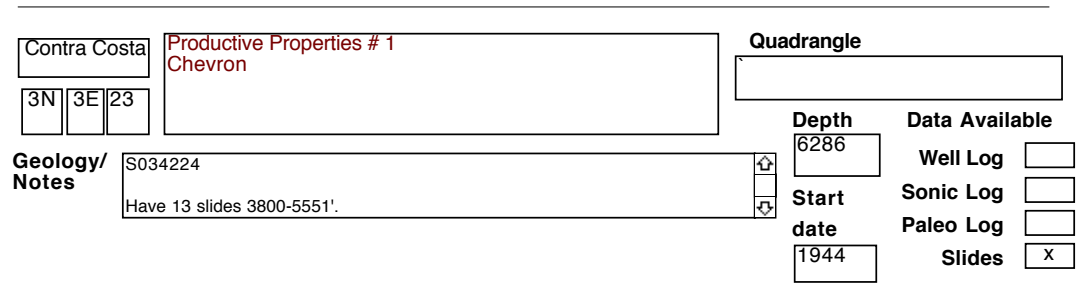

\begin{tabular}{l|l|l|l|l|l|}
\hline Contra Costs & $\begin{array}{l}\text { Stoeckle \# 1 } \\
\text { Western Petroleum }\end{array}$ & \multicolumn{2}{l|}{ Quadrangle } \\
\hline
\end{tabular}

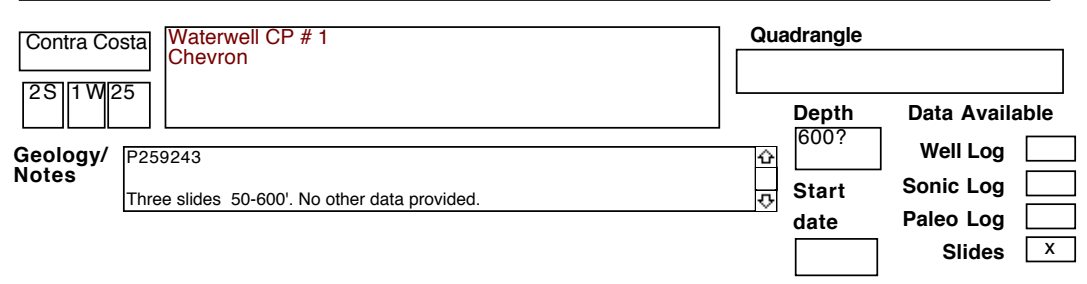

\begin{tabular}{|c|c|c|c|c|c|}
\hline Contra Costa & Chevron-CWS \# 1 & Qua & drangle & & \\
\hline $2 \mathrm{~N} / 2 \mathrm{~W} / 3$ & & & & & \\
\hline & & & Depth & Data Availab & \\
\hline Geology/ & & 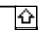 & & Well Log & \\
\hline Notes & r slides 3430-3700'. Some data from Munger Map 20. & 田 & Start & Sonic Log & \\
\hline & & & $\begin{array}{l}\text { date } \\
1962\end{array}$ & $\begin{array}{r}\text { Paleo Log } \\
\text { Slides }\end{array}$ & $\mathrm{X}$ \\
\hline
\end{tabular}


SELECTED OIL TEST WELLS IN CENTRAL CALIFORNIA DATABASE County

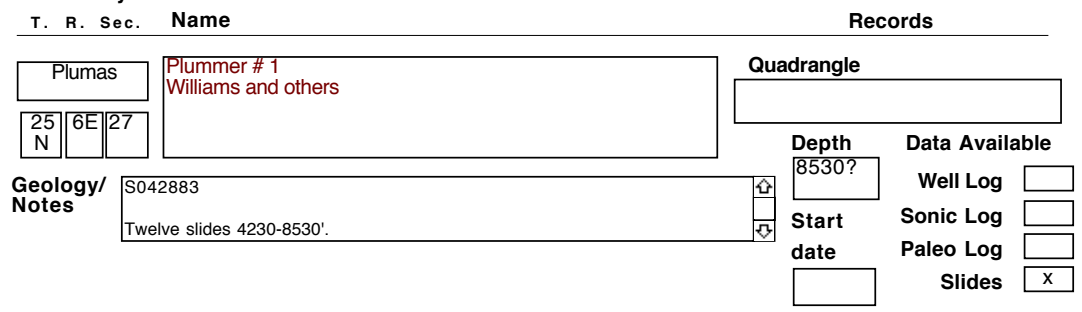

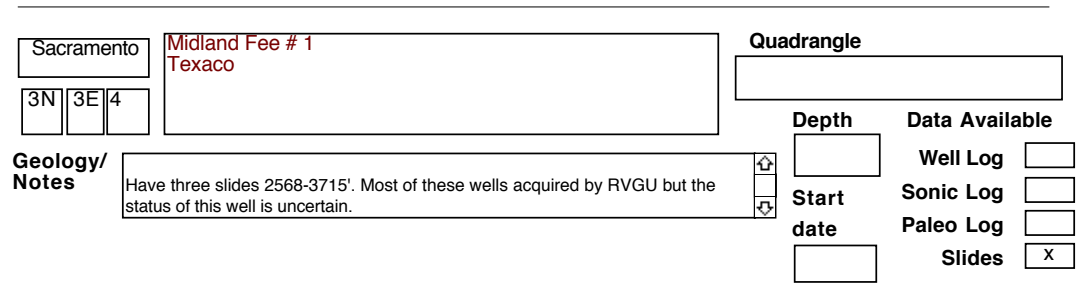

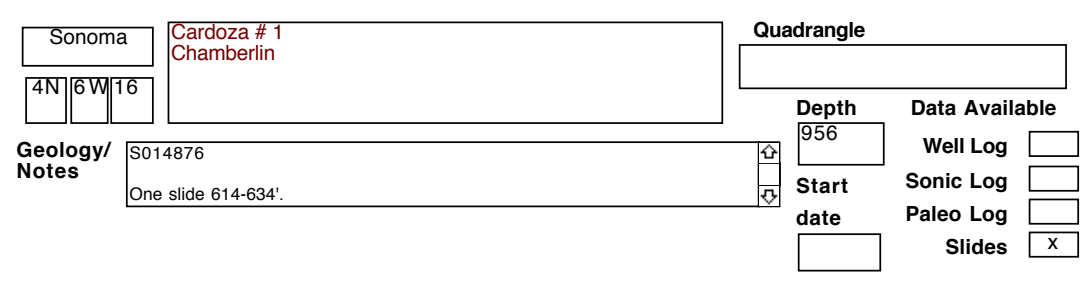

\begin{tabular}{l|l|l|l|l|l|}
\hline Alameda & $\begin{array}{l}\text { Nissen \# 1 } \\
\text { Brown }\end{array}$ & \multicolumn{2}{l|}{ Quadrangle } \\
\hline
\end{tabular}

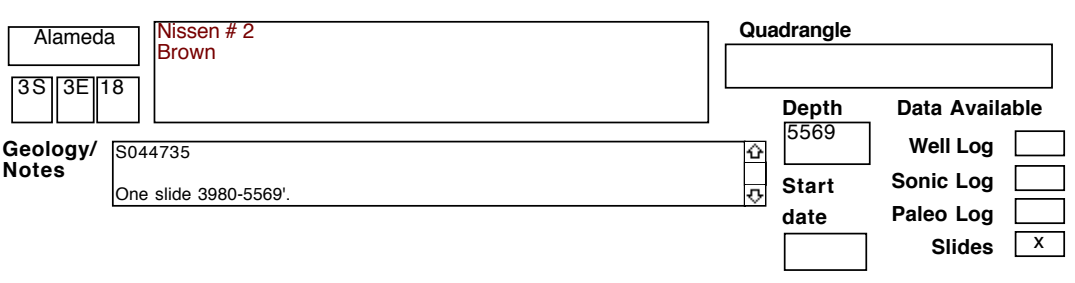


SELECTED OIL TEST WELLS IN CENTRAL CALIFORNIA DATABASE

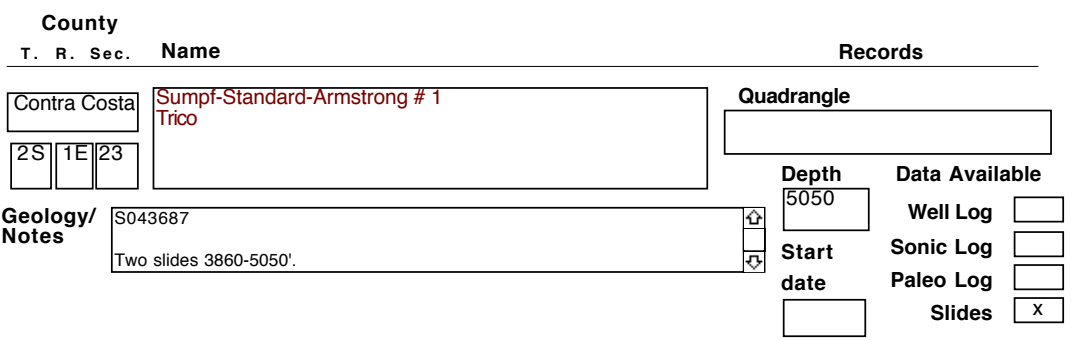

\begin{tabular}{l|l|l|l|l|l|}
\hline Santa Clara & $\begin{array}{l}\text { Strader \# 1 } \\
\text { Strader Oil Co }\end{array}$ & \multicolumn{2}{|l|}{ Quadrangle } \\
\hline
\end{tabular}

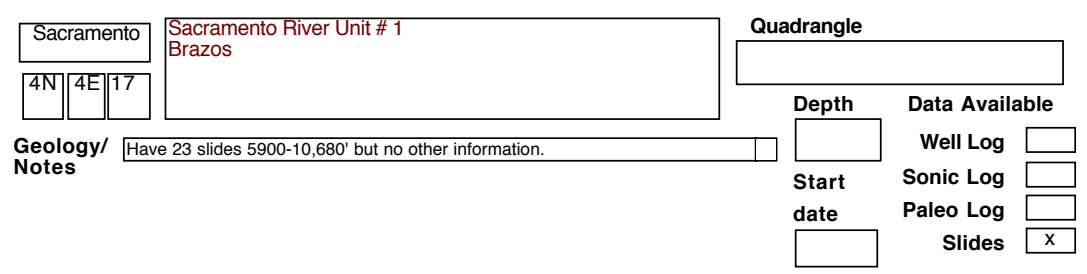

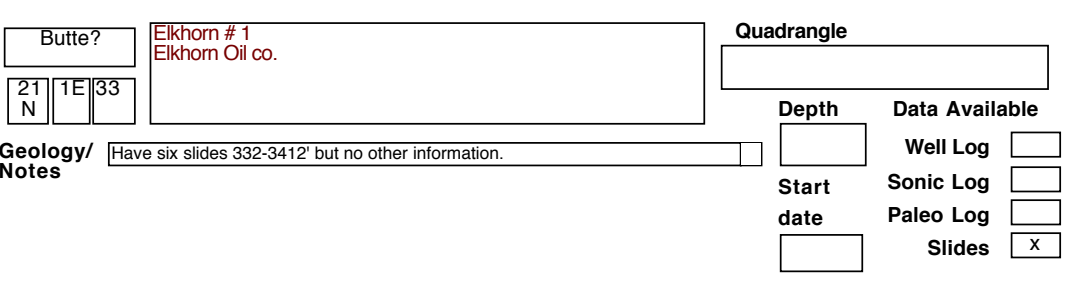

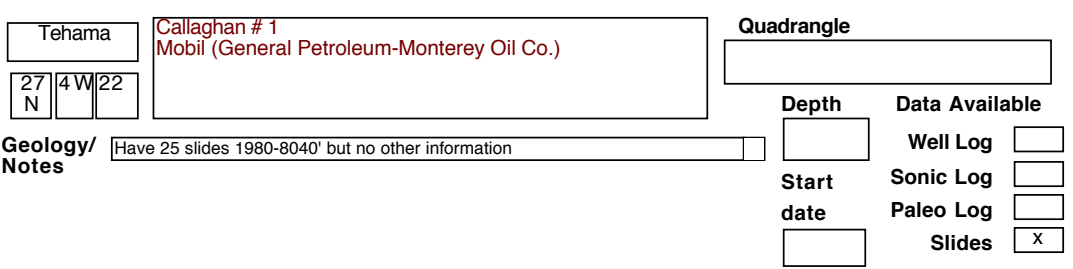


SELECTED OIL TEST WELLS IN CENTRAL CALIFORNIA DATABASE County

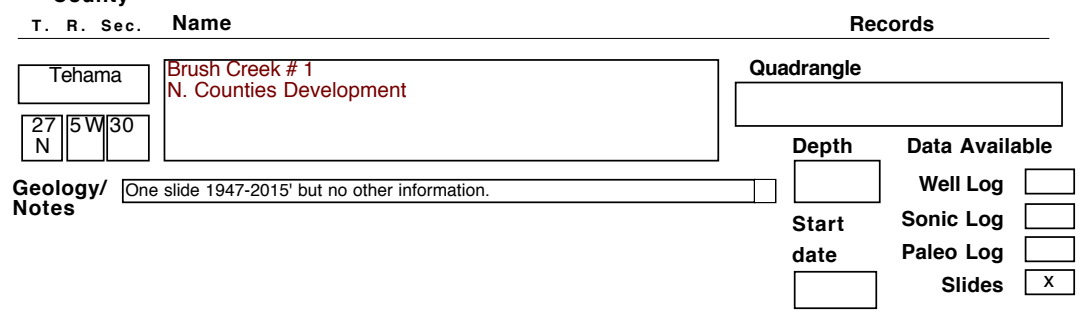

\begin{tabular}{|c|l|l|l|l|}
\hline Tehama & $\begin{array}{l}\text { Casady \# 1 } \\
\text { Hooker Dome Syn. }\end{array}$ & \multicolumn{2}{l|}{ Quadrangle } \\
\hline
\end{tabular}

\begin{tabular}{l|l|l|l|l|l|}
\hline Solano & $\begin{array}{l}\text { Chevron coreholes } 1450 \text { to } 1459 \\
\text { Chevron }\end{array}$ \\
Geology/ \\
Notes
\end{tabular}

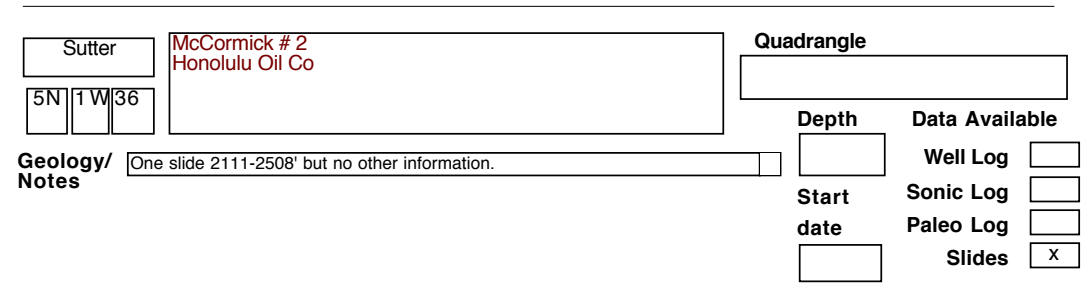

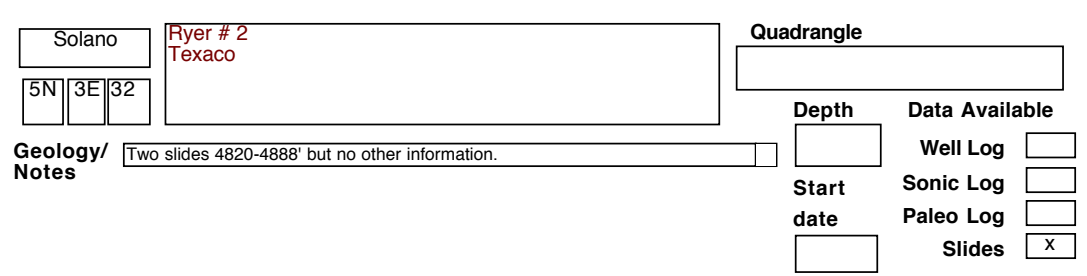


SELECTED OIL TEST WELLS IN CENTRAL CALIFORNIA DATABASE County

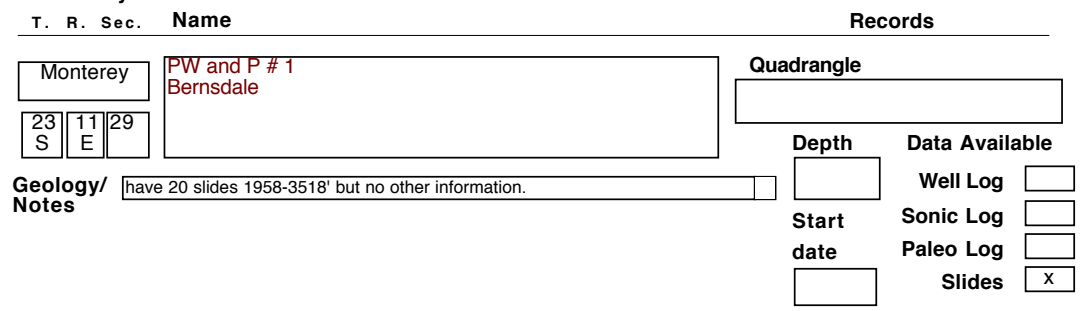

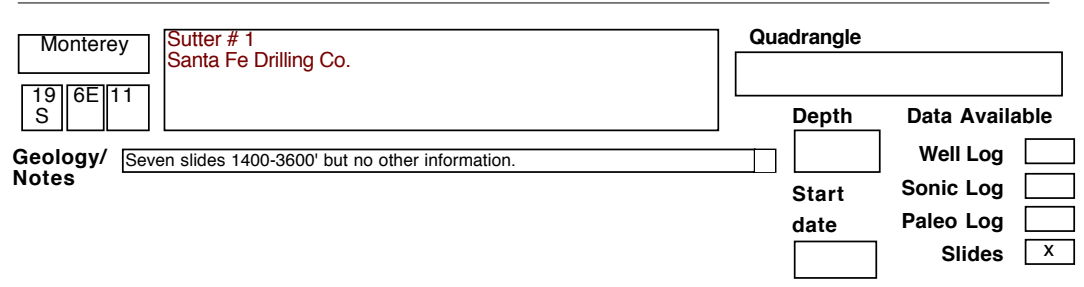

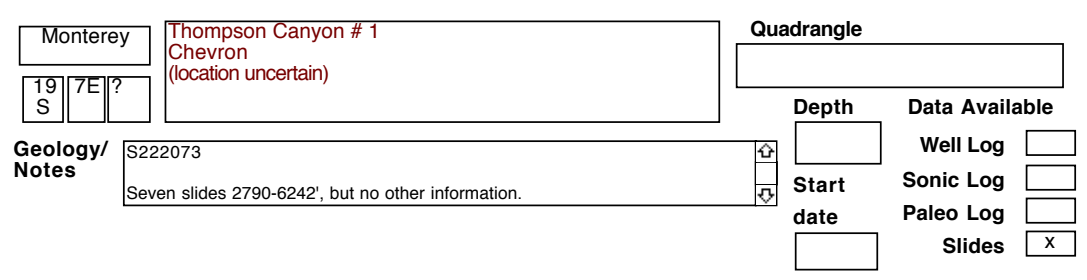

\begin{tabular}{l|l|l|l|l|}
\hline \multicolumn{2}{|c|}{$\begin{array}{l}\text { Corehole A } \\
\text { Amerada }\end{array}$} & \multicolumn{2}{l|}{ Quadrangle } \\
\hline
\end{tabular}

\begin{tabular}{l|l|l|l|l|}
\hline Colusa & $\begin{array}{l}\text { Corehole B } \\
\text { Amerada }\end{array}$ & \multicolumn{2}{l|}{ Quadrangle } \\
\hline
\end{tabular}


SELECTED OIL TEST WELLS IN CENTRAL CALIFORNIA DATABASE County

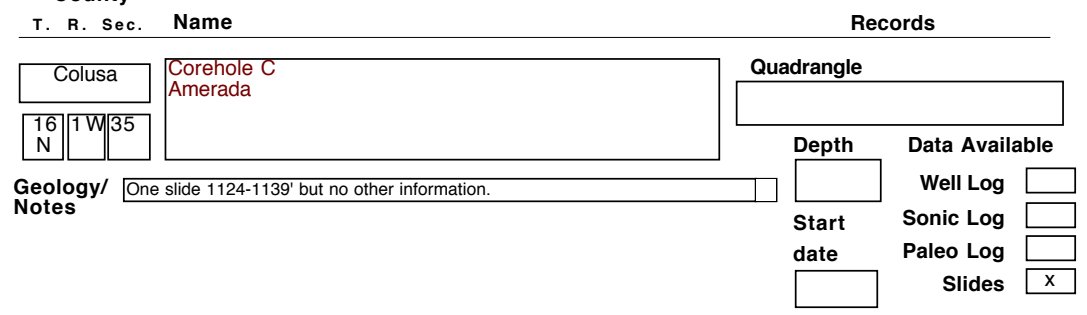

\begin{tabular}{|c|c|c|c|c|}
\hline Colusa & Corehole D & Quadrangle & & \\
\hline \begin{tabular}{|l|l|l}
16 & $1 \mathrm{~V}$ & 10 \\
$\mathrm{~N}$ & & \\
\end{tabular} & & \multirow{2}{*}{ Depth } & \multicolumn{2}{|c|}{ Data Available } \\
\hline \multirow{4}{*}{\multicolumn{2}{|c|}{$\begin{array}{l}\text { Geology/ Two slides } 1389-1532 \text { ' but no other information. } \\
\text { Notes }\end{array}$}} & & Well Log & \\
\hline & & \multirow{3}{*}{$\begin{array}{l}\text { Start } \\
\text { date }\end{array}$} & Sonic Log & \\
\hline & & & Paleo Log & \\
\hline & & & Slides & $\mathrm{x}$ \\
\hline
\end{tabular}

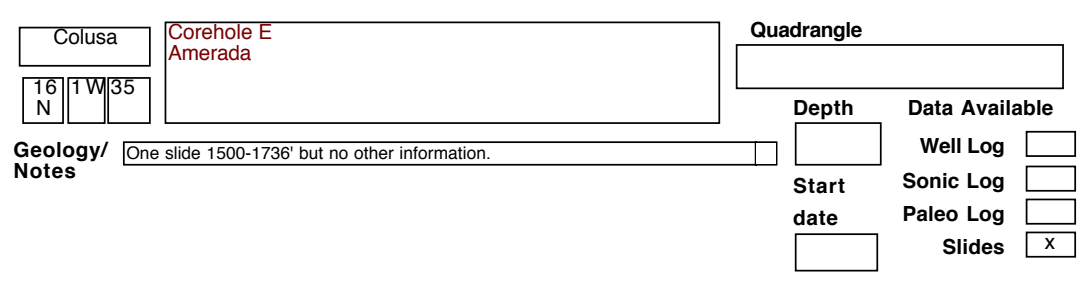

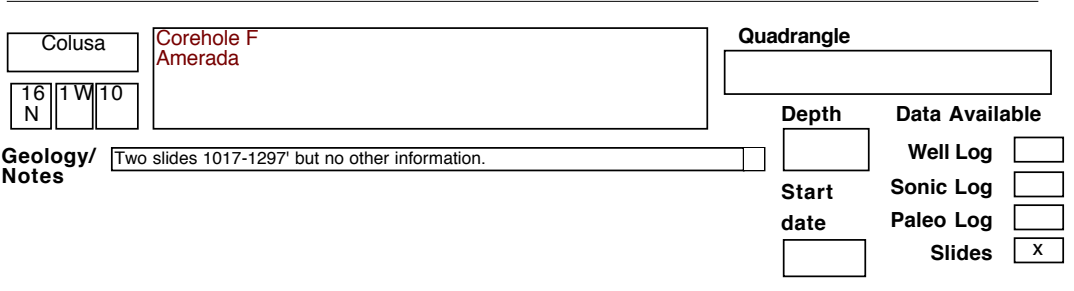

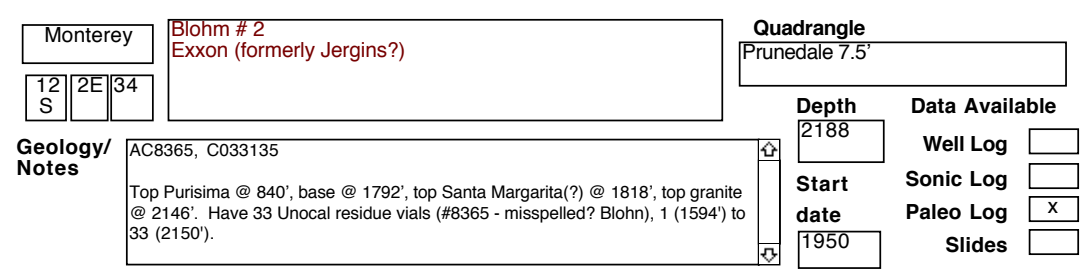


SELECTED OIL TEST WELLS IN CENTRAL CALIFORNIA DATABASE County

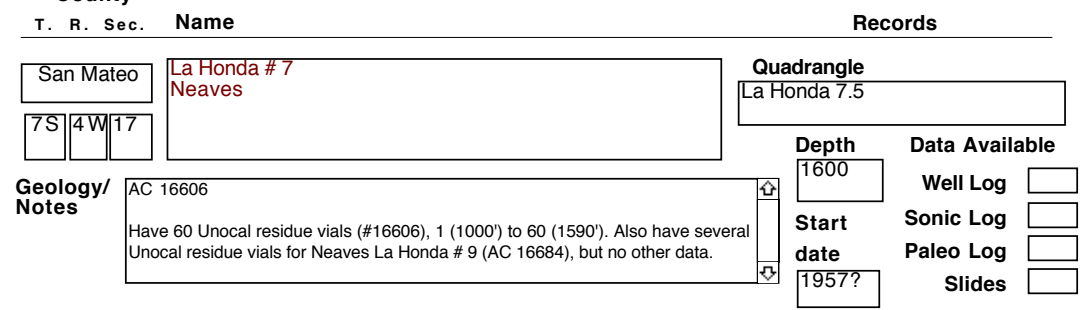

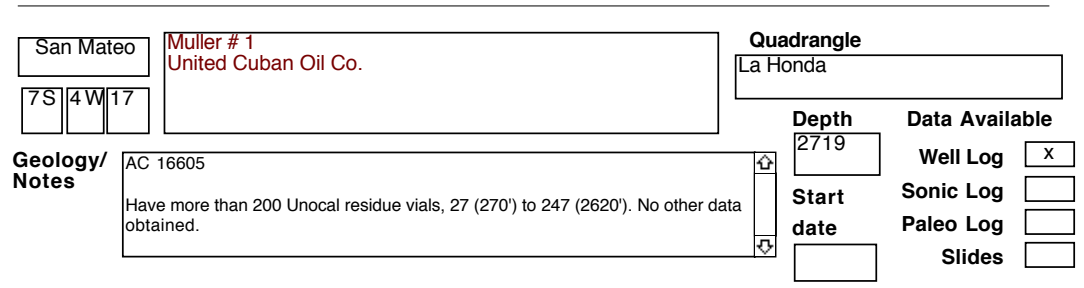

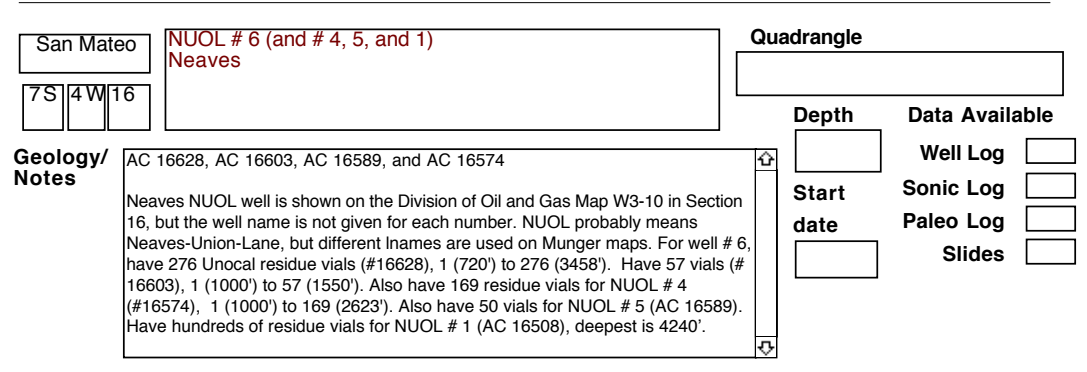

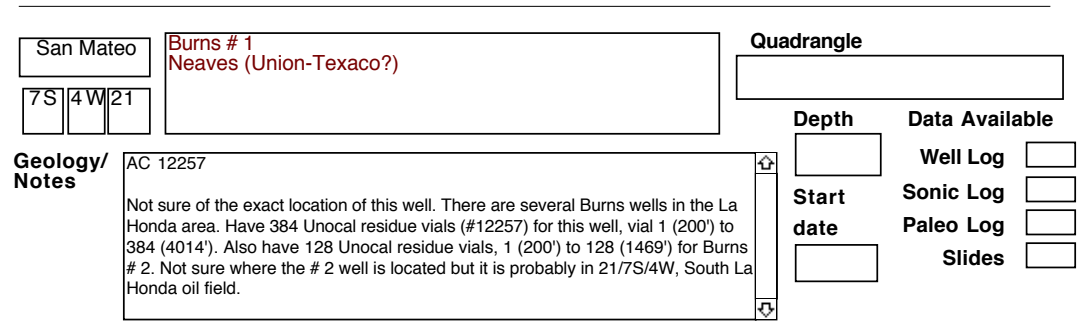

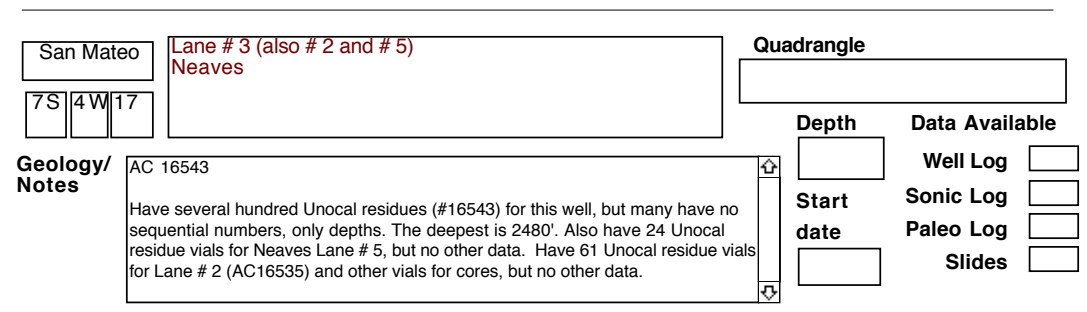


SELECTED OIL TEST WELLS IN CENTRAL CALIFORNIA DATABASE County

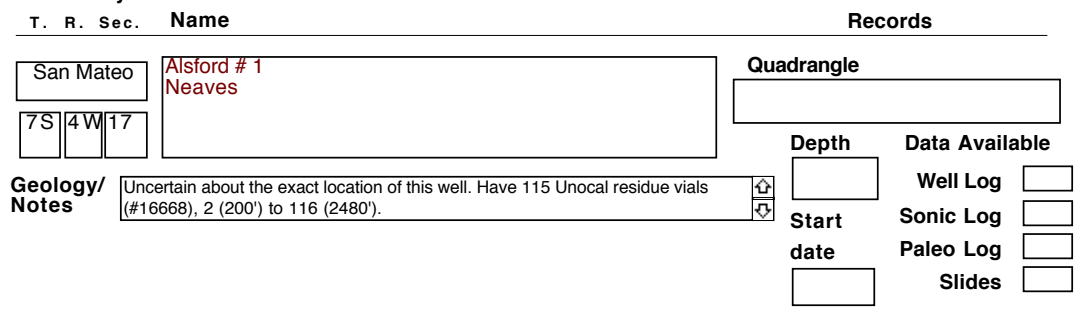

\begin{tabular}{l|l|l|l|l|l|}
\hline San Mateo & $\begin{array}{l}\text { Escloses \#1 } \\
\text { Neaves }\end{array}$ & \multicolumn{2}{l|}{ Quadrangle } \\
\hline
\end{tabular}

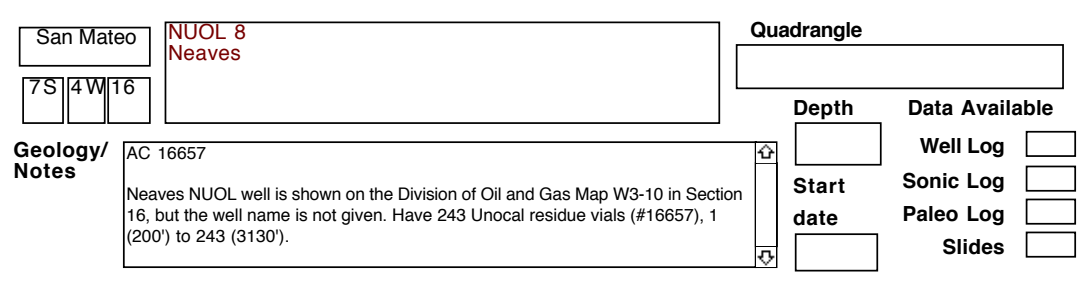

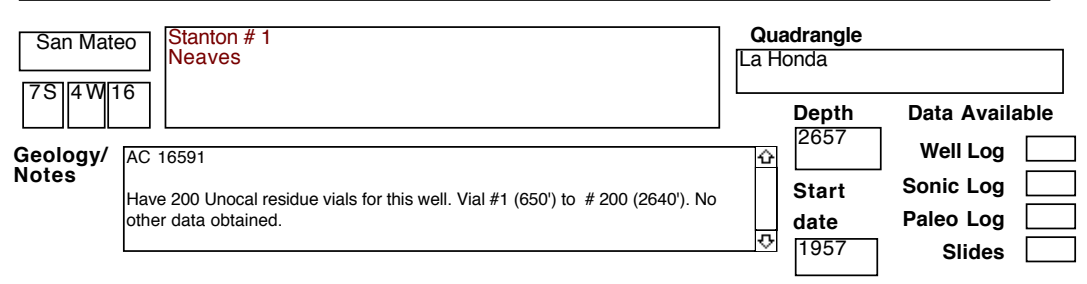

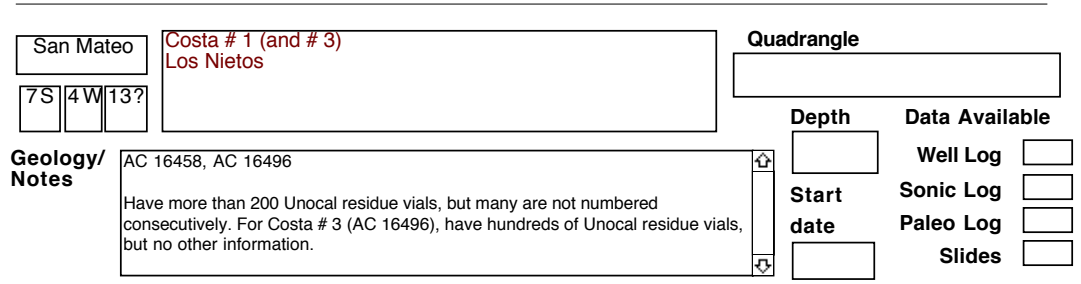


SELECTED OIL TEST WELLS IN CENTRAL CALIFORNIA DATABASE County

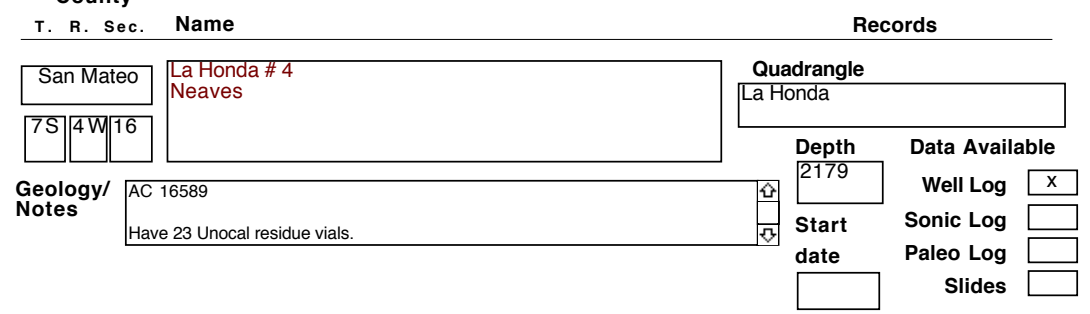

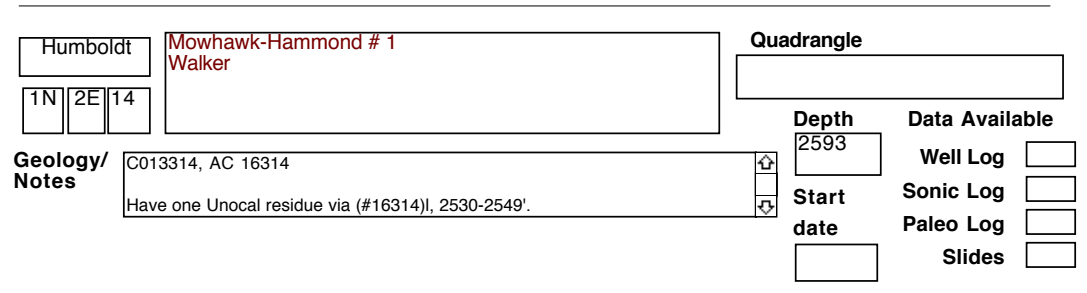

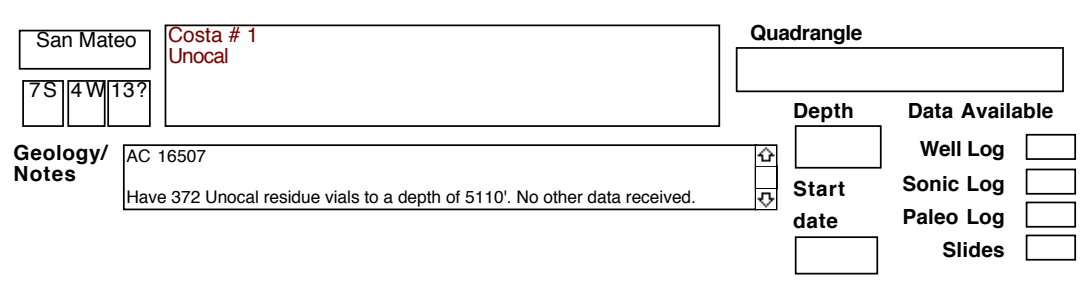

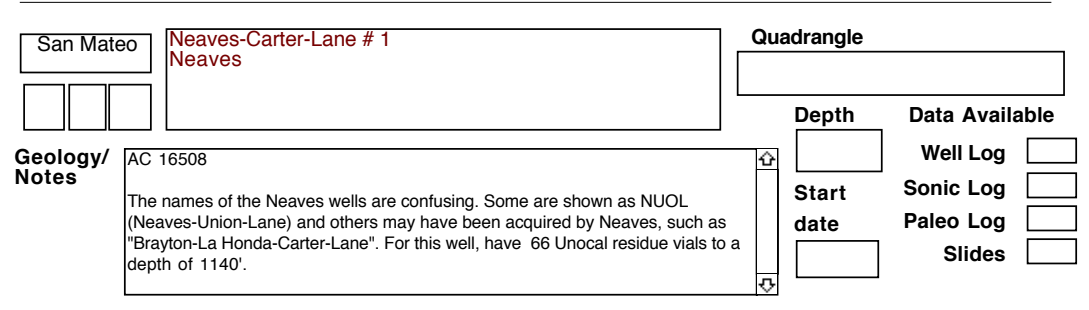

